\title{
Extensions of the Ramanujan-Mordell Formula
}

by

\author{
Mathieu Lemire \\ B. Math (Université de Montréal), M. Sc (Carleton University)
}

\author{
A Dissertation submitted to \\ the Faculty of Graduate and Postdoctoral Affairs \\ in partial fulfilment of \\ the requirements for the degree of \\ Doctor of Philosophy
}

\author{
School of Mathematics and Statistics \\ Ottawa-Carleton Institute for Mathematics and Statistics \\ Carleton University \\ Ottawa, Ontario \\ December, 2010 \\ (C) Copyright \\ 2010, Mathieu Lemire
}


Library and Archives

Canada

Published Heritage

Branch

395 Wellington Street

Ottawa ON K1A ON4

Canada
Bibliothèque et

Archives Canada

Direction du

Patrimoine de l'édition

395 , rue Wellington

Ottawa ON K1A 0N4

Canada
Your file Votre référence

ISBN: 978-0-494-79633-7

Our file Notre référence

ISBN: 978-0-494-79633-7
NOTICE:

The author has granted a nonexclusive license allowing Library and Archives Canada to reproduce, publish, archive, preserve, conserve, communicate to the public by telecommunication or on the Internet, loan, distribute and sell theses worldwide, for commercial or noncommercial purposes, in microform, paper, electronic and/or any other formats.

The author retains copyright ownership and moral rights in this thesis. Neither the thesis nor substantial extracts from it may be printed or otherwise reproduced without the author's permission.
AVIS:

L'auteur a accordé une licence non exclusive permettant à la Bibliothèque et Archives Canada de reproduire, publier, archiver, sauvegarder, conserver, transmettre au public par télécommunication ou par l'Internet, prêter, distribuer et vendre des thèses partout dans le monde, à des fins commerciales ou autres, sur support microforme, papier, électronique et/ou autres formats.

L'auteur conserve la propriété du droit d'auteur et des droits moraux qui protège cette thèse. Ni la thèse ni des extraits substantiels de celle-ci ne doivent être imprimés ou autrement reproduits sans son autorisation.
In compliance with the Canadian Privacy Act some supporting forms may have been removed from this thesis.

While these forms may be included in the document page count, their removal does not represent any loss of content from the thesis.
Conformément à la loi canadienne sur la protection de la vie privée, quelques formulaires secondaires ont été enlevés de cette thèse.

Bien que ces formulaires aient inclus dans la pagination, il n'y aura aucun contenu manquant. 


\section{Abstract}

Let $\mathbb{N}$ denote the set of all positive integers and $\mathbb{Z}$ the set of all integers. Explicit formulae are derived for the number of representations of $n \in \mathbb{N}$ by the quadratic form

$$
x_{1}^{2}+\cdots+x_{r}^{2}+2 x_{r+1}^{2}+\cdots+2 x_{r+s}^{2}+4 x_{r+s+1}^{2}+\cdots+4 x_{r+s+t}^{2}
$$

under certain conditions on $r, s$ and $t$, that is, we give formulae for the quantity

$\operatorname{card}\left\{\left(x_{1}, \ldots, x_{r+s+t}\right) \in \mathbb{Z}^{r+s+t} \mid n=x_{1}^{2}+\cdots+x_{r}^{2}+2 x_{r+1}^{2}+\cdots+2 x_{r+s}^{2}+4 x_{r+s+1}^{2}+\cdots+4 x_{r+s+t}^{2}\right\}$. 


\section{Acknowledgments}

The completion of my thesis has been a long, difficult but wonderful journey. There are many people that I would like to thank for making this adventure so memorable. I will forever be thankful to my supervisor Dr. Kenneth S. Williams for his incredible patience with me, his support and all the help that he provided for my thesis. Thank you for suggesting this beautiful project. I would like to thank the School of Mathematics and Statistics for giving me the chance to be a teaching assistant, a sessional lecturer and for the financial support that really helped me. Many thanks to everyone in my family for their continuous support over the years. Finally, I would like to thank my wife Katrina Rogers-Stewart for all her love, support and encouragement. I am so glad that I found a soulmate like you who makes me happy everyday. 


\section{Table of Contents}

$\begin{array}{ll}\text { Abstract } & \text { ii }\end{array}$

Acknowledgments

Table of Contents

1 Introduction 1

2 The theta function $\varphi(q)$ and the Eisenstein series $E_{k}(q) \quad 12$

2.1 The arithmetic function $\sigma_{k}(n) \ldots \ldots \ldots \ldots \ldots \ldots$

2.2 The Bernoulli numbers $B_{n} \ldots \ldots \ldots \ldots \ldots \ldots$

2.3 The theta function $\varphi(q) \ldots \ldots \ldots \ldots$

2.4 Analytic properties of $\varphi(q) \ldots \ldots \ldots \ldots \ldots$

2.5 The duplication principle . . . . . . . . . . . . . 21

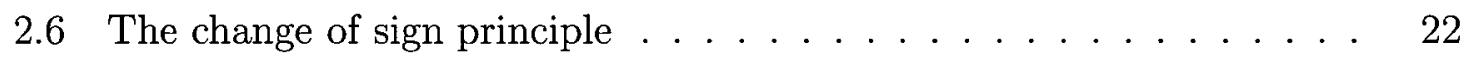

2.7 The rotation principle $\ldots \ldots \ldots \ldots \ldots \ldots$

2.8 The arithmetic function $N([a, b, c] ; n) \ldots \ldots \ldots \ldots$

2.9 The Eisenstein series $E_{k}(q) \ldots \ldots \ldots \ldots \ldots \ldots$

2.10 Ramanujan's discriminant function . . . . . . . . . . 32

2.11 The function $\tau_{k}(n)$ and six special values of $\delta(a, b, c ; n) \ldots \ldots 37$

3 The function $\left(\frac{1+(1-x)^{1 / 2}}{2}\right)^{r}\left(\frac{1+(1-x)^{1 / 4}}{2}\right)^{s} \quad 43$ 
3.1 The polynomials $A_{r, s}(x), B_{r, s}(x), C_{r, s}(x), D_{r, s}(x) \ldots \ldots \ldots 43$

3.2 The values of $(1+i)^{s} \pm(1-i)^{s} \ldots \ldots \ldots \ldots \ldots$

3.3 The values of $A_{r, s}(0), B_{r, s}(0), C_{r, s}(0), D_{r, s}(0) \ldots \ldots \ldots$

4 The functions $e_{k, r}(x) \quad 58$

4.1 The polynomials $e_{k, 1}(x), e_{k, 2}(x)$ and $e_{k, 4}(x) \ldots \ldots \ldots$

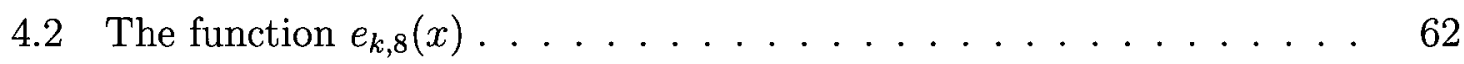

4.3 The function $e_{k, 16}(x) \ldots \ldots \ldots \ldots \ldots \ldots$

4.4 The identity $E_{k}\left(q^{r}\right)=e_{k, r}(x) z^{2 k} \ldots \ldots \ldots \ldots \ldots$

5 The polynomials $P_{k, r, s}(x), Q_{k, r, s}(x), R_{k, r, s}(x)$ and $S_{k, r, s}(x) \quad 80$

5.1 The polynomials $P_{k, r, 0}(x), Q_{k, r, 0}(x), R_{k, r, 0}(x)$ and $S_{k, r, 0}(x) \ldots \ldots 1$

5.2 The polynomials $P_{k, r, s}(x), Q_{k, r, s}(x), R_{k, r, s}(x)$ and $S_{k, r, s}(x) \ldots 85$

5.3 The polynomials $P_{k, 0, s}(x), Q_{k, 0, s}(x), R_{k, 0, s}(x)$ and $S_{k, 0, s}(x) \ldots 91$

6 Extensions of the Ramanujan-Mordell formula 100

6.1 Number of representations by the quadratic form $x_{1}^{2}+\cdots+x_{4 k-2 r}^{2}+$ $2 x_{4 k-2 r+1}^{2}+\ldots+2 x_{4 k}^{2} \ldots \ldots \ldots \ldots \ldots \ldots 1$

6.2 Number of representations by the quadratic form $x_{1}^{2}+\cdots+x_{4 k-2 r-s}^{2}+$ $2 x_{4 k-2 r-s+1}^{2}+\cdots+2 x_{4 k-s}^{2}+4 x_{4 k-s+1}^{2}+\cdots+4 x_{4 k}^{2} \ldots \ldots \ldots 107$

6.3 Number of representations by the quadratic form $x_{1}^{2}+\cdots+x_{4 k-s}^{2}+$ $4 x_{4 k-s+1}^{2}+\ldots+4 x_{4 k}^{2} \ldots \ldots \ldots \ldots \ldots \ldots \ldots$

7 Sums with 8,12 and 16 squares - I $\quad \mathbf{1 2 5}$

7.1 Sums with 8 squares $\ldots \ldots \ldots \ldots \ldots \ldots$

7.2 Sums with 12 squares . . . . . . . . . . . . . . . . 134

7.3 Sums with 16 squares . . . . . . . . . . . . . . . 147

$\begin{array}{lll}8 & \delta \text { identities } & \mathbf{1 7 6}\end{array}$ 
8.1 Change of sign, duplication and rotation principles for $\Delta(a, b, c ; q) \ldots 177$

8.2 Identity for the sums of 4 squares . . . . . . . . . . . . . . 184

8.3 Identities for the sums of 8 squares $\ldots \ldots \ldots \ldots$

8.4 Identities for the sums of 12 squares . . . . . . . . . . . 190

8.5 Identities for the sums of 16 squares . . . . . . . . . . 199

8.6 An important identity . . . . . . . . . . . . . . . . 207

9 Sums with 8,12 and 16 squares - II $\quad \mathbf{2 1 9}$

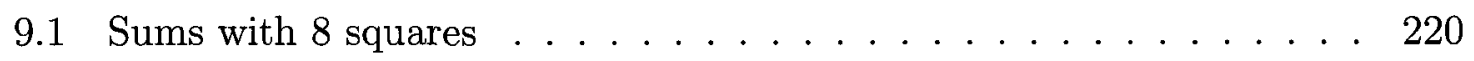

9.2 Sums with 12 squares . . . . . . . . . . . . . . . . . . . 231

9.3 Sums with 16 squares $\ldots \ldots \ldots \ldots \ldots \ldots$

$\begin{array}{ll}\text { Conclusion } & 274\end{array}$

List of References $\quad 275$ 


\section{Chapter 1}

\section{Introduction}

A central problem in the theory of numbers is the determination of an explicit formula for the quantity

$$
r_{h}(n):=\operatorname{card}\left\{\left(x_{1}, \ldots, x_{h}\right) \in \mathbb{Z}^{h} \mid n=x_{1}^{2}+\cdots+x_{h}^{2}\right\}
$$

which counts the number of representations of a positive integer $n$ as a sum of $h$ squares. This problem has a long and interesting history, which is described in the two books [46], [66]. Relevant references include [9], [12], [13], [15], [16], [22][26], [31], [32], [37]-[45], [47]-[52], [60], [61], [63]-[65], [67], [74]-[77], [79], [80], [82], [85].

When $h \equiv 0(\bmod 2)$, say $h=2 m(m \in \mathbb{N})$, Ramanujan [71], [72, pp. 136-162] gave a formula for $r_{2 m}(n)$, which was later proved by Mordell [63]. The Ramanujan-Mordell formula expresses $r_{2 m}(n)$ as a linear combination of elementary arithmetic functions and the coefficients in the power series expansions of certain

modular forms. A proof of this formula has recently been given by Cooper [31] using Ramanujan's ${ }_{1} \psi_{1}$ summation formula and elliptic functions.

When $m \equiv 0(\bmod 2)$, say $m=2 k(k \in \mathbb{N})$, so $h=4 k$, the elementary arithmetic functions in the Ramanujan-Mordell formula are $\sigma_{2 k-1}(n), \sigma_{2 k-1}(n / 2)$ 
and $\sigma_{2 k-1}(n / 4)$, where

$$
\sigma_{2 k-1}(l):= \begin{cases}\sum_{d \in \mathbb{N}} d^{2 k-1}, & \text { if } l \in \mathbb{N}, \\ d \mid l & \text { if } l \notin \mathbb{N},\end{cases}
$$

and $\sigma(l):=\sigma_{1}(l)$. Different functions are required when $m \equiv 1(\bmod 2)$. Recently Alaca, Alaca and Williams [9] have given a completely elementary proof of the Ramanujan-Mordell formula for $r_{4 k}(n)$. We state the Ramanujan-Mordell formula in the form given in their paper.

Theorem 1.0.1. (Ramanujan-Mordell formula) Let $k \in \mathbb{N}$. Then for $n \in \mathbb{N}$ there exist rational numbers $c(k, m) \quad(m=1,2, \ldots,[(k-1) / 2])$ such that

$$
\begin{aligned}
r_{4 k}(n)= & \frac{(-1)^{k+1} 4 k}{\left(2^{2 k}-1\right) B_{2 k}} \sigma_{2 k-1}(n)+\frac{\left(1+(-1)^{k}\right) 4 k}{\left(2^{2 k}-1\right) B_{2 k}} \sigma_{2 k-1}(n / 2) \\
& -\frac{2^{2 k+2} k}{\left(2^{2 k}-1\right) B_{2 k}} \sigma_{2 k-1}(n / 4)+\sum_{m=1}^{[(k-1) / 2]} c(k, m) a(k, m ; n),
\end{aligned}
$$

where the $B_{l} \quad\left(l \in \mathbb{N}_{0}\right)$ are the Bernoulli numbers given by

$$
\frac{t}{e^{t}-1}=\sum_{l=0}^{\infty} \frac{B_{l}}{l !} t^{l}, \quad|t|<2 \pi
$$

and

$$
q^{m} \prod_{n=1}^{\infty}\left(1-q^{n}\right)^{24 m-8 k}\left(1-q^{2 n}\right)^{20 k-48 m}\left(1-q^{4 n}\right)^{24 m-8 k}=\sum_{n=m}^{\infty} a(k, m ; n) q^{n} .
$$

Alaca, Alaca and Williams [9] have given the rational numbers $c(k, m)$ occurring in (1.0.3) as the coefficients in the expansion of a certain polynomial in $x[9$, eq. (4.11), p. 42] in powers of $x(1-x)$. Alternatively, as noted by Cooper [31], the $c(k, m)$ can be determined for a specific value of $k$ by taking $n=1,2,3, \ldots$ in (1.0.3) and solving the resulting equations. 
Taking $k=1,2,3,4,5,6$ in the formula (1.0.3), we obtain for $n \in \mathbb{N}$

$r_{4}(n)=8 \sigma(n)-32 \sigma(n / 4)$,

$r_{8}(n)=16 \sigma_{3}(n)-32 \sigma_{3}(n / 2)+256 \sigma_{3}(n / 4)$,

$r_{12}(n)=8 \sigma_{5}(n)-512 \sigma_{5}(n / 4)+16 a(3,1 ; n)$,

where

$$
q \prod_{n=1}^{\infty}\left(1-q^{2 n}\right)^{12}=\sum_{n=1}^{\infty} a(3,1 ; n) q^{n}
$$

$r_{16}(n)=\frac{32}{17} \sigma_{7}(n)-\frac{64}{17} \sigma_{7}(n / 2)+\frac{8192}{17} \sigma_{7}(n / 4)+\frac{512}{17} a(4,1 ; n)$,

where

$$
\begin{gathered}
q \prod_{n=1}^{\infty} \frac{\left(1-q^{2 n}\right)^{32}}{\left(1-q^{n}\right)^{8}\left(1-q^{4 n}\right)^{8}}=\sum_{n=1}^{\infty} a(4,1 ; n) q^{n}, \\
r_{20}(n)=\frac{8}{31} \sigma_{9}(n)-\frac{8192}{31} \sigma_{9}(n / 4)+\frac{1232}{31} a(5,1 ; n)-\frac{256}{31} a(5,2 ; n),
\end{gathered}
$$

where

$$
q \prod_{n=1}^{\infty} \frac{\left(1-q^{2 n}\right)^{52}}{\left(1-q^{n}\right)^{16}\left(1-q^{4 n}\right)^{16}}=\sum_{n=1}^{\infty} a(5,1 ; n) q^{n}
$$

and

$$
\begin{array}{r}
q \prod_{n=1}^{\infty}\left(1-q^{n}\right)^{8}\left(1-q^{2 n}\right)^{4}\left(1-q^{4 n}\right)^{8}=\sum_{n=1}^{\infty} a(5,2 ; n) q^{n}, \\
r_{24}(n)=\frac{16}{691} \sigma_{11}(n)-\frac{32}{691} \sigma_{11}(n / 2)+\frac{65536}{691} \sigma_{11}(n / 4)
\end{array}
$$




$$
+\frac{33152}{691} a(6,1 ; n)-\frac{65536}{691} a(6,2 ; n),
$$

where

$$
q \prod_{n=1}^{\infty} \frac{\left(1-q^{2 n}\right)^{72}}{\left(1-q^{n}\right)^{24}\left(1-q^{4 n}\right)^{24}}=\sum_{n=1}^{\infty} a(6,1 ; n) q^{n}
$$

and

$$
q^{2} \prod_{n=1}^{\infty}\left(1-q^{2 n}\right)^{24}=\sum_{n=1}^{\infty} a(6,2 ; n) q^{n} .
$$

The formulae for $r_{4}(n)$ and $r_{8}(n)$ first occured implicitly in the work of Jacobi [53]. Many proofs of these formulae are now known, see for example Carlitz [23], Ewell [40], Hirshhorn [50], Spearman and Williams [79], van der Pol [80] and Williams [82]. The formulae for $r_{12}(n)$ and $r_{16}(n)$ are due to Glaisher [43]. The formula for $r_{20}(n)$ has been treated by Rankin [76].

It is clear from the definition of $a(3,1 ; n)$ that

$$
a(3,1 ; n)=0, \text { if } n \equiv 0(\bmod 2) .
$$

Thus

$$
r_{12}(n)=8 \sigma_{5}(n)-512 \sigma_{5}(n / 4), \text { if } n \equiv 0(\bmod 2) .
$$

This elegant arithmetic formula was first given by Liouville [57]. A simple proof has recently been given by Williams [85].

It is known that

$$
a(6,1 ; n)=(-1)^{n-1} \tau(n)
$$

and

$$
a(6,2 ; n)=\tau(n / 2)
$$


where $\tau(n)$ is Ramanujan's tau function given by

$$
q \prod_{n=1}^{\infty}\left(1-q^{n}\right)^{24}=\sum_{n=1}^{\infty} \tau(n) q^{n} .
$$

Thus we have Ramanujan's beautiful formula [71], [72, p. 162]

$$
\begin{aligned}
r_{24}(n)= & \frac{16}{691} \sigma_{11}(n)-\frac{32}{691} \sigma_{11}(n / 2)+\frac{65536}{691} \sigma_{11}(n / 4) \\
& +\frac{33152}{691}(-1)^{n-1} \tau(n)-\frac{65536}{691} \tau(n / 2) .
\end{aligned}
$$

Rankin [75] has proved for $k \geq 3$ that the Ramanujan-Mordell formula for $r_{4 k}(n)$ must involve nonzero coefficients of modular forms. Liouville's formula (1.0.6) shows that this may not be the case if $n$ is restricted to an arithmetic progression.

Our purpose in this thesis is to extend the Ramanujan-Mordell theorem to the representation of a positive interger $n$ by the quadratic form

$$
x_{1}^{2}+\cdots+x_{r}^{2}+2 x_{r+1}^{2}+\cdots+2 x_{r+s}^{2}+4 x_{r+s+1}^{2}+\cdots+4 x_{r+s+t}^{2},
$$

where $r, s, t$ are integers satisfying

$$
r \geq 1, s \geq 0, t \geq 0, r+s+t=4 k, k \in \mathbb{N} .
$$

The Ramanujan-Mordell theorem is the special case $r=4 k, s=0, t=0$. We write $[r, s, t]$ for the form (1.0.9) so that for example we have

$$
[4,2,2]=x_{1}^{2}+x_{2}^{2}+x_{3}^{2}+x_{4}^{2}+2 x_{5}^{2}+2 x_{6}^{2}+4 x_{7}^{2}+4 x_{8}^{2} .
$$

In addition we write $N([r, s, t] ; n)$ for the number of representations of $n \in \mathbb{N}_{0}$ by the form $[r, s, t]$, that is

$$
N([r, s, t] ; n)=\operatorname{card}\left\{\left(x_{1}, \ldots, x_{4 k}\right) \in \mathbb{Z}^{4 k} \mid n=x_{1}^{2}+\cdots+x_{r}^{2}\right.
$$




$$
\left.+2 x_{r+1}^{2}+\cdots+2 x_{r+s}^{2}+4 x_{r+s+1}^{2}+\cdots+4 x_{r+s+t}^{2}\right\}
$$

so that $N([r, s, t] ; 0)=1$ and

$$
N([4 k, 0,0] ; n)=r_{4 k}(n) .
$$

When $k=1$ there are 10 forms of the the type (1.0.9). These are given in Table 1.1.

\begin{tabular}{|c|c|}
\hline quaternary quadratic forms & references \\
\hline$x_{1}^{2}+x_{2}^{2}+x_{3}^{2}+x_{4}^{2}$ & $N([4,0,0] ; n) \quad[2$, Theorem 1.6, p. 284$]$ \\
\hline$x_{1}^{2}+x_{2}^{2}+x_{3}^{2}+2 x_{4}^{2}$ & $N([3,1,0] ; n) \quad[84$, p. 225$]$ \\
\hline$x_{1}^{2}+x_{2}^{2}+x_{3}^{2}+4 x_{4}^{2}$ & $N([3,0,1] ; n) \quad[2$, Theorem 1.7, p. 284$]$ \\
\hline$x_{1}^{2}+x_{2}^{2}+2 x_{3}^{2}+2 x_{4}^{2}$ & $N([2,2,0] ; n) \quad[2$, Theorem 1.8, p. 284$]$ \\
\hline$x_{1}^{2}+x_{2}^{2}+2 x_{3}^{2}+4 x_{4}^{2}$ & $N([2,1,1] ; n) \quad[1$, Proposition 4.3, p. 160$]$ \\
\hline$x_{1}^{2}+x_{2}^{2}+4 x_{3}^{2}+4 x_{4}^{2}$ & $N([2,0,2] ; n) \quad[2$, Theorem 1.11, p. 285] \\
\hline$x_{1}^{2}+2 x_{2}^{2}+2 x_{3}^{2}+2 x_{4}^{2}$ & $N([1,3,0] ; n) \quad[84$, p. 225$]$ \\
\hline$x_{1}^{2}+2 x_{2}^{2}+2 x_{3}^{2}+4 x_{4}^{2}$ & $N([1,2,1] ; n) \quad[2$, Theorem 1.14, p. 285$]$ \\
\hline$x_{1}^{2}+2 x_{2}^{2}+4 x_{3}^{2}+4 x_{4}^{2}$ & $N([1,1,2] ; n) \quad[1$, Proposition 4.3, p. 161$]$ \\
\hline$x_{1}^{2}+4 x_{2}^{2}+4 x_{3}^{2}+4 x_{4}^{2}$ & {$[2$, Theorem 1.18, p. 286$]$} \\
\hline
\end{tabular}

Table 1.1

The second column in Table 1.1 gives a reference to where the determination of $N([r, s, t] ; n)(r+s+t=4)$ is given. As these determinations are all known we may suppose that $k \geq 2$ in this thesis.

Examination of the evaluations for the 10 forms in Table 1.1 shows that for the 6 forms $[4,0,0],[3,0,1],[2,2,0],[2,0,2],[1,2,1],[1,0,3]$ the evaluations only 
involve $\sigma(n), \sigma(n / 2), \sigma(n / 4), \sigma(n / 8)$ and $\sigma(n / 16)$, whereas for the 4 forms $[3,1,0]$, $[2,1,1],[1,3,0],[1,1,2]$ the evaluations require the function $\sum_{d \mid n} \frac{n}{d}\left(\frac{2}{d}\right)$. These two sets of forms are distinguished by the fact that $s$ is even for the first set and that $s$ is odd for the second set. Thus it is unlikely that for $k \geq 2$ the evaluation of $N([r, s, t] ; n)$ will only involve the divisor function unless we restrict $s$ to be even.

Under the restrictions

$$
r \geq 1, \quad s \geq 0, \quad t \geq 0, \quad r+s+t=4 k, \quad k \geq 2, \quad s \text { even, }
$$

we evaluate $N([r, s, t] ; n)$ for all $n \in \mathbb{N}$ in Theorems 6.1.3,6.2.3, 6.3.3. The evaluations are given in terms of $\sigma_{2 k-1}(n), \sigma_{2 k-1}(n / 2), \sigma_{2 k-1}(n / 4), \sigma_{2 k-1}(n / 8)$ and $\sigma_{2 k-1}(n / 16)$ and the coefficients in the power series expansions of certain infinite products. In Chapter 7 the formulae are given explicitly for forms of the type (1.0.9) in $8(k=2), 12$ $(k=3)$ and $16(k=4)$ variables. In Chapter 8 relationships between the coefficients $\delta$ in these formulae are determined and these are used in Chapter 9 to give simpler evaluations of the $N([r, s, t] ; n)$. Several instances were found where $N([r, s, t] ; n)$ can be evaluated just in terms of $\sigma_{2 k-1}(n), \sigma_{2 k-1}(n / 2), \sigma_{2 k-1}(n / 4), \sigma_{2 k-1}(n / 8)$ and $\sigma_{2 k-1}(n / 16)$ for certain residue classes of $n$, in analogy with Liouville's result (1.0.6). For example we find that

$$
\begin{aligned}
& \operatorname{card}\left\{\left(x_{1}, x_{2}, x_{3}, x_{4}, x_{5}, x_{6}, x_{7}, x_{8}, x_{9}, x_{10}, x_{11}, x_{12}\right) \in \mathbb{Z}^{12} \mid\right. \\
& \left.\quad n=x_{1}^{2}+x_{2}^{2}+x_{3}^{2}+x_{4}^{2}+x_{5}^{2}+x_{6}^{2}+2 x_{7}^{2}+2 x_{8}^{2}+2 x_{9}^{2}+2 x_{10}^{2}+2 x_{11}^{2}+2 x_{12}^{2}\right\} \\
& =N([6,6,0] ; n)=1032 \sigma_{5}(n / 4)-1536 \sigma_{5}(n / 8),
\end{aligned}
$$

for all $n \in \mathbb{N}$ with $n \equiv 0(\bmod 4)$, see Theorem 9.2.2.

In the case of 16 variables we did not find any evaluations of $N([r, s, t] ; n)$ 
$(r+s+t=16)$ that only depend upon $\sigma_{7}(n), \sigma_{7}(n / 2), \sigma_{7}(n / 4), \sigma_{7}(n / 8)$ and $\sigma_{7}(n / 16)$. However we found many that depend only upon the coefficients in the power series expansion of a single infinite product. For example

$$
\begin{aligned}
& \text { card }\left\{\left(x_{1}, \ldots, x_{16}\right) \in \mathbb{Z}^{16} \mid n=x_{1}^{2}+\cdots+x_{14}^{2}+2 x_{15}^{2}+2 x_{16}^{2}\right\}=N([14,2,0] ; n) \\
& =\frac{26112}{17} \sigma_{7}(n / 4)-\frac{253952}{17} \sigma_{7}(n / 8)-\frac{554}{17} a(n),
\end{aligned}
$$

where

$$
q \prod_{n=1}^{\infty} \frac{\left(1-q^{2 n}\right)^{32}}{\left(1-q^{n}\right)^{8}\left(1-q^{4 n}\right)^{8}}=\sum_{n=1}^{\infty} a(n) q^{n}
$$

for all $n \in \mathbb{N}$ with $n \equiv 0(\bmod 4)$, see Theorem 9.3.1. When $k=2$ a few of the evaluations of $N([r, s, t] ; n)$ had been carried out by other authors, see for example [5] and [11]. In every case the results of this thesis agreed with those evaluations.

The method used in this thesis follows the ideas of Alaca, Alaca and Williams in their evaluation of $r_{4 k}(n)$ [9]. However considerable modification of their argument must be made in order to extend their method to the evaluations of (1.0.11). We conclude this introduction by describing briefly the ideas involved.

We begin by introducing the theta function

$$
\varphi(q)=\sum_{n=-\infty}^{\infty} q^{n^{2}}
$$

in Chapter 2 and giving some of its properties. Then we show that

$$
\sum_{n=-\infty}^{\infty} N([r, s, t] ; n) q^{n}=\varphi(q)^{r} \varphi\left(q^{2}\right)^{s} \varphi\left(q^{4}\right)^{t}
$$


see Theorem 2.8.1, so that in order to determine $N([r, s, t] ; n)$ we require the coefficient of $q^{n}$ in $\varphi(q)^{r} \varphi\left(q^{2}\right)^{s} \varphi\left(q^{4}\right)^{t}$. To do this we introduce the parameters

$$
x=1-\frac{\varphi^{4}(-q)}{\varphi^{4}(q)}, \quad z=\varphi^{2}(q),
$$

see Definition 2.3.2, and show that

$$
\begin{aligned}
\varphi(q) & =z^{1 / 2} \\
\varphi\left(q^{2}\right) & =\left(\frac{1+(1-x)^{1 / 2}}{2}\right)^{1 / 2} z^{1 / 2}, \\
\varphi\left(q^{4}\right) & =\left(\frac{1+(1-x)^{1 / 4}}{2}\right) z^{1 / 2}
\end{aligned}
$$

see Theorem 2.5.2. Thus, for $r+s+t=4 k$, we have

$$
\varphi(q)^{r} \varphi\left(q^{2}\right)^{s} \varphi\left(q^{4}\right)^{t}=\left(\frac{1+(1-x)^{1 / 2}}{2}\right)^{s / 2}\left(\frac{1+(1-x)^{1 / 4}}{2}\right)^{t} z^{2 k}
$$

This leads us to study the function $\left(\frac{1+(1-x)^{1 / 2}}{2}\right)^{s / 2}\left(\frac{1+(1-x)^{1 / 4}}{2}\right)^{t}$, which is carried out in Chapter 3. In the sum of squares case considered by Alaca, Alaca and Williams [9], we have $s=t=0$, and the analysis is much simpler. In order to find the coefficient of $q^{n} \quad(n \in \mathbb{N})$ in the right hand side of (1.0.13) in terms of $\sigma_{2 k-1}(n), \sigma_{2 k-1}(n / 2), \sigma_{2 k-1}(n / 4), \sigma_{2 k-1}(n / 8)$ and $\sigma_{2 k-1}(n / 16)$, we require the Eisenstein series

$$
\left\{\begin{array}{l}
E_{k}(q)=1-\frac{4 k}{B_{2 k}} \sum_{n=1}^{\infty} \sigma_{2 k-1}(n) q^{n}, \\
E_{k}\left(q^{2}\right)=1-\frac{4 k}{B_{2 k}} \sum_{n=1}^{\infty} \sigma_{2 k-1}(n / 2) q^{n}, \\
E_{k}\left(q^{4}\right)=1-\frac{4 k}{B_{2 k}} \sum_{n=1}^{\infty} \sigma_{2 k-1}(n / 4) q^{n}, \\
E_{k}\left(q^{8}\right)=1-\frac{4 k}{B_{2 k}} \sum_{n=1}^{\infty} \sigma_{2 k-1}(n / 8) q^{n}, \\
E_{k}\left(q^{16}\right)=1-\frac{4 k}{B_{2 k}} \sum_{n=1}^{\infty} \sigma_{2 k-1}(n / 16) q^{n} .
\end{array}\right.
$$


In Theorem 4.4.1 we show that

$$
E_{k}\left(q^{r}\right)=e_{k, r}(x) z^{2 k}, \quad k \geq 2, \quad r \in\{1,2,4,8,16\},
$$

for some function $e_{k, r}(x)$, where $x$ and $z$ are given in (1.0.12).

In Section 2.10 Ramanujan's discriminant function

$$
\Delta(q):=q \prod_{n=1}^{\infty}\left(1-q^{n}\right)^{24}
$$

is recalled and a generalized discriminant function is defined by

$$
\begin{aligned}
\Delta(a, b, c ; q) & :=\Delta(q)^{a} \Delta\left(q^{2}\right)^{b} \Delta\left(q^{4}\right)^{c} \\
& =q^{a+2 b+4 c} \prod_{n=1}^{\infty}\left(1-q^{n}\right)^{24 a}\left(1-q^{2 n}\right)^{24 b}\left(1-q^{4 n}\right)^{24 c}
\end{aligned}
$$

for rational numbers $a, b, c$ satisfying certain conditions, see Theorem 2.10.2. For the rational numbers $a, b, c$ under consideration $\Delta(a, b, c ; q)$ has a power series expansion

$$
\Delta(a, b, c ; q)=\sum_{n=0}^{\infty} \delta(a, b, c ; n) q^{n} .
$$

Moreover we show that $\Delta(a, b, c ; q)$ is given in terms of the parameters $x$ and $z$ by

$$
\Delta(a, b, c ; q)=\frac{x^{a+2 b+4 c}(1-x)^{4 a+2 b+c} z^{12(a+b+c)}}{2^{4(a+2 b+4 c)}} .
$$

In (1.0.13) and (1.0.15) the exponent of $z$ is $2 k$, so we will be interested in those $\Delta(a, b, c ; q)$ for which $12(a+b+c)=2 k$, that is $a+b+c=k / 6$. The main body of this thesis consists of showing that for a complex variable $x$ there exist rational numbers $\alpha_{1}(r, s, t), \alpha_{2}(r, s, t), \alpha_{4}(r, s, t), \alpha_{8}(r, s, t)$ and $\alpha_{16}(r, s, t)$ such that

$$
\begin{aligned}
& \left(\frac{1+(1-x)^{1 / 2}}{2}\right)^{s / 2}\left(\frac{1+(1-x)^{1 / 4}}{2}\right)^{t}(r \geq 1, s \geq 0, t \geq 0, r+s+t=4 k, k \geq 2, s \text { even }) \\
& =\alpha_{1}(r, s, t) e_{k, 1}(x)+\alpha_{2}(r, s, t) e_{k, 2}(x)+\alpha_{4}(r, s, t) e_{k, 4}(x)
\end{aligned}
$$


$+\alpha_{8}(r, s, t) e_{k, 8}(x)+\alpha_{16}(r, s, t) e_{k, 16}(x)$

+ linear combination of terms of the form $x^{a+2 b+4 c}(1-x)^{4 a+2 b+c}$ with $a+b+c=k / 6$.

Then, taking $x$ and $z$ as in (1.0.12), and multiplying by $z^{2 k}$, we obtain by (1.0.13) and (1.0.15)

$$
\begin{aligned}
\varphi(q)^{r} \varphi & \left(q^{2}\right)^{s} \varphi\left(q^{4}\right)^{t} \\
& =\alpha_{1}(r, s, t) E_{k}(q)+\alpha_{2}(r, s, t) E_{k}\left(q^{2}\right)+\alpha_{4}(r, s, t) E_{k}\left(q^{4}\right) \\
& +\alpha_{8}(r, s, t) E_{k}\left(q^{8}\right)+\alpha_{16}(r, s, t) E_{k}\left(q^{16}\right) \\
& + \text { linear combination of terms } \Delta(a, b, c ; q) \text { with } a+b+c=k / 6 .
\end{aligned}
$$

Equating coefficients of $q^{n}(n \in \mathbb{N})$ in this identity, we obtain our extension of the Ramanujan-Mordell theorem, namely

$$
\begin{aligned}
N([r, s, t] ; n)= & \text { linear combination of } \sigma_{2 k-1}(n), \sigma_{2 k-1}(n / 2), \sigma_{2 k-1}(n / 4), \\
& \sigma_{2 k-1}(n / 8), \sigma_{2 k-1}(n / 16) \\
& + \text { linear combination of } \delta(a, b, c ; n) \text { with } a+b+c=k / 6 .
\end{aligned}
$$

The precise formulae are given in Theorems 6.1.3, 6.2.3 and 6.3.3. In Chapter 7 all such formulae are given when $k=2,3$ and 4 . In Chapter 8 relationships between the $\delta(a, b, c ; n)(a+b+c=k / 6)$ occurring in (1.0.19) are determined. These are used in Chapter 9 to simplify the formulae of Chapter 7 . 


\section{Chapter 2}

\section{The theta function $\varphi(q)$ and the Eisenstein series $E_{k}(q)$}

This chapter consists primarily of definitions and results from the literature. In particular we recall the definition of the theta function $\varphi(q)$, the Eisenstein series $E_{k}(q)$ and Ramanujan's discriminant function $\Delta(q)$, and we give those properties of these functions that we need in later chapters. Most of our work will be based on the results stated here.

\subsection{The arithmetic function $\sigma_{k}(n)$}

We begin by recalling the definition of the arithmetic function $\sigma_{k}(n)$.

Definition 2.1.1. For $k, n \in \mathbb{N}$ we set $\sigma_{k}(n)=\sum_{d \mid n} d^{k}$, where $d$ runs through the positive integers dividing $n$. If $n \notin \mathbb{N}$ we set $\sigma_{k}(n)=0$. We also set $\sigma_{1}(n)=\sigma(n)$.

It is well known that $\sigma_{k}(n)$ is a multiplicative function of $n$ for all $k$. We emphasize that if $n \in \mathbb{N}, a \in \mathbb{N}$ and $a$ does not divide $n$ then $n / a \notin \mathbb{N}$ and $\sigma_{k}(n / a)=0$. 


\subsection{The Bernoulli numbers $B_{n}$}

We recall the definition of the Bernouilli numbers. These numbers are used in the definition of the Eisenstein series $E_{k}(q)$.

Definition 2.2.1. The Bernoulli numbers $\left\{B_{n}\right\}_{n \in \mathbb{N}_{0}}$ are defined by

$$
\frac{x}{e^{x}-1}=\sum_{n=0}^{\infty} B_{n} \frac{x^{n}}{n !}, \quad x \in \mathbb{R},|x|<2 \pi .
$$

It is known that $B_{2 k+1}=0$ for $k \geq 1$. The first few values of the $B_{n}$ are given in Table 2.2.1.

Table 2.2.1

\begin{tabular}{|c|c|c|c|c|c|}
\hline$n$ & $B_{n}$ & $n$ & $B_{n}$ & $n$ & $B_{n}$ \\
\hline 0 & 1 & 8 & $-1 / 30$ & 18 & $43867 / 798$ \\
1 & $-1 / 2$ & 10 & $5 / 66$ & 20 & $-174611 / 330$ \\
2 & $1 / 6$ & 12 & $-691 / 2730$ & 22 & $854513 / 138$ \\
4 & $-1 / 30$ & 14 & $7 / 6$ & 24 & $-236364091 / 2730$ \\
6 & $1 / 42$ & 16 & $-3617 / 510$ & 26 & $8553103 / 3$ \\
\hline
\end{tabular}

\subsection{The theta function $\varphi(q)$}

In this section we define the theta function $\varphi(q)$ and prove some of its main properties. We then use $\varphi(q)$ to define the parameters $x(q)$ and $z(q)$, which will play a major role later in the thesis.

We begin by finding the radius of convergence of a certain power series.

Theorem 2.3.1. The radius of convergence of the complex power series $1+2 \sum_{n=1}^{\infty} q^{n^{2}}=\sum_{n=-\infty}^{\infty} q^{n^{2}}$ is equal to 1. 
Proof. Define the sequence $\left\{a_{n}\right\}_{n \in \mathbb{N}}$ by

$$
a_{n}= \begin{cases}1, & \text { if } n=m^{2} \text { for some } m \in \mathbb{N} \\ 0, & \text { otherwise }\end{cases}
$$

so that

$$
\sum_{n=1}^{\infty} q^{n^{2}}=\sum_{n=1}^{\infty} a_{n} q^{n}
$$

The radius of convergence of the power series $\sum_{n=1}^{\infty} a_{n} q^{n}$ is given by (see for example [29, Theorem 1.3, p. 31])

$$
\frac{1}{\limsup _{n \rightarrow+\infty}\left|a_{n}\right|^{-\frac{1}{n}}}=\frac{1}{1}=1 \text {. }
$$

Thus $\varphi(q)=1+2 \sum_{n=1}^{\infty} q^{n^{2}}=\sum_{n=-\infty}^{\infty} q^{n^{2}}$ has radius of convergence 1 .

We can now define the theta function $\varphi(q)$.

Definition 2.3.1. For $q \in \mathbb{C}$ such that $|q|<1$ we define the theta function $\varphi$ by

$$
\varphi(q):=\sum_{n=-\infty}^{\infty} q^{n^{2}}
$$

For the rest of this thesis, we let

$$
D:=\{q \in \mathbb{C}|| q \mid<1\}
$$

The next lemma gives three basic properties of the theta function $\varphi$.

Lemma 2.3.1. For $q \in D$, we have

(i) $\varphi(q)+\varphi(-q)=2 \varphi\left(q^{4}\right)$,

(ii) $\varphi^{2}(q)+\varphi^{2}(-q)=2 \varphi^{2}\left(q^{2}\right)$,

(iii) $\varphi(q) \varphi(-q)=\varphi^{2}\left(-q^{2}\right)$. 
Proof. All these identities are easy to prove.

(i) This is $[19$, eq. (3.6.1), p. 71].

(ii) This is $[19$, eq. (3.6.7), p. 72].

(iii) This is $[19$, eq. (1.3.32), p. 15].

Consider the function $f(q)=i q$. We note that $f(D)=D$. This implies that the composite function $\varphi(i q)$ is well-defined on $D$, and similarly for $\varphi(-i q)$. The next lemma relates the functions $\varphi(i q)$ and $\varphi(-i q)$ to the functions $\varphi(q)$ and $\varphi(-q)$.

Lemma 2.3.2. For $q \in D$, we have

(i) $\varphi(i q)=\left(\frac{\varphi(q)+\varphi(-q)}{2}\right)+i\left(\frac{\varphi(q)-\varphi(-q)}{2}\right)$,

(ii) $\varphi(-i q)=\left(\frac{\varphi(q)+\varphi(-q)}{2}\right)-i\left(\frac{\varphi(q)-\varphi(-q)}{2}\right)$.

Proof. We only prove (i) as the proof of (ii) is similar. Appealing to Definition 2.3.1 and Lemma 2.3.1(i), we obtain

$$
\begin{aligned}
\varphi(i q) & =\sum_{n=-\infty}^{\infty}(i q)^{n^{2}}=\sum_{n=-\infty}^{\infty} i^{n^{2}} q^{n^{2}}=\sum_{\substack{n=-\infty \\
n \equiv 0(\bmod 2)}}^{\infty} i^{n^{2}} q^{n^{2}}+\sum_{\substack{n=-\infty \\
n \equiv 1(\bmod 2)}}^{\infty} i^{n^{2}} q^{n^{2}} \\
& =\sum_{n=-\infty}^{\infty} q^{4 n^{2}}+i \sum_{\substack{n=-\infty \\
n \equiv 1(\bmod 2)}}^{\infty} q^{n^{2}}=\varphi\left(q^{4}\right)+i\left(\sum_{n=-\infty}^{\infty} q^{n^{2}}-\sum_{\substack{n=-\infty \\
n \equiv 0(\bmod 2)}}^{\infty} q^{n^{2}}\right) \\
& =\varphi\left(q^{4}\right)+i\left(\varphi(q)-\varphi\left(q^{4}\right)\right)=\left(\frac{\varphi(q)+\varphi(-q)}{2}\right)+i\left(\frac{\varphi(q)-\varphi(-q)}{2}\right)
\end{aligned}
$$

as asserted. 
We conclude this section with the definitions of the very important quantities $x(q)$ and $z(q)$.

Definition 2.3.2. For $q \in D$, we define

$$
x=x(q):=1-\frac{\varphi^{4}(-q)}{\varphi^{4}(q)}
$$

and

$$
z=z(q):=\varphi^{2}(q)
$$

We remark that in Theorem 2.4.2 we show that $\varphi(q) \neq 0$ for $q \in D$.

\subsection{Analytic properties of $\varphi(q)$}

A function $f(q)$, which is defined on $D$ and differentiable at every point of $D$ save possibly for a finite number of exceptional points, is said to be analytic on $D$. The exceptional points are called the singularities of $f$. If, however, no point of $D$ is a singularity of the analytic function $f$, we say that $f$ is regular on $D$.

Analytic properties of the functions $\varphi(q), \varphi(-q), \varphi(i q)$ and $\varphi(-i q)$ are stated and proved in this section. We first determine the domain where these functions are regular.

Theorem 2.4.1. (i) $\varphi(q)$ is a regular function on $D$.

(ii) $\varphi(-q)$ is a regular function on $D$.

(iii) $\varphi(i q)$ is a regular function on $D$.

(iv) $\varphi(-i q)$ is a regular function on $D$.

Proof. (i) This follows from the fact that a power series is regular within its circle of convergence, see for example [29, Corollary (2.9), p. 37] or [30, p. 38]. 
(ii) As the function $f(q)=-q$ is regular on $\mathbb{C}$ (so on $D$ ) and $f(D)=D$, we have that the composite function $\varphi(f(q))=\varphi(-q)$ is regular on $D$.

(iii) and (iv) are proved in the same way as (ii) with the functions $g(q)=i q$ and $h(q)=-i q$ respectively.

Next we show that all four functions are non-vanishing on $D$.

Theorem 2.4.2. For $q \in D$, we have

(i) $\varphi(q) \neq 0$,

(ii) $\varphi(-q) \neq 0$,

(iii) $\varphi(i q) \neq 0$,

(iv) $\varphi(-i q) \neq 0$.

Proof. (i) Suppose that there exists $q_{0} \in D$ with $\varphi\left(q_{0}\right)=0$. As $\varphi(0)=1 \neq 0$ we have $q_{0} \neq 0$. Set $q_{1}=-q_{0}^{2}$. Clearly $\left|q_{1}\right|=\left|-q_{0}^{2}\right|=\left|q_{0}\right|^{2}<1$ so $q_{1} \in D$. Also by Lemma 2.3.1 (iii) $\varphi^{2}\left(q_{1}\right)=\varphi^{2}\left(-q_{0}^{2}\right)=\varphi\left(q_{0}\right) \varphi\left(-q_{0}\right)=0$ so $\varphi\left(q_{1}\right)=0$. Clearly $q_{1} \neq q_{0}$.

Next we define $q_{2}=-q_{1}^{2}$. Clearly $\left|q_{2}\right|=\left|-q_{1}^{2}\right|=\left|q_{1}\right|^{2}<1$ so $q_{2} \in D$. Also $\varphi^{2}\left(q_{2}\right)=\varphi^{2}\left(-q_{1}^{2}\right)=\varphi\left(q_{1}\right) \varphi\left(-q_{1}\right)=0$ so $\varphi\left(q_{2}\right)=0$. Clearly $q_{2} \neq q_{0}, q_{1}$.

Continuing in this way we obtain an infinite sequence $q_{0}, q_{1}, q_{2}, \ldots$ of zeros of $\varphi(q)$ in $D$ having limit point $0(\in D)$. Thus, as $\varphi$ is a regular function on $D, \varphi(q)$ vanishes identically on $D$. This is a contradiction as $\varphi(0)=1 \neq 0$. Hence there is no point of $D$ at which $\varphi$ vanishes.

(ii)(iii)(iv) These follow from (i) and the fact that $q \in D \Leftrightarrow-q \in D \Leftrightarrow i q \in$ $D \Leftrightarrow-i q \in D$. 
We next deduce the following important results.

Theorem 2.4.3. Let $x=x(q)$ be the function defined in Definition 2.3 .2 for $q \in D$. Then the functions $1-x(q), 1-x(i q), \frac{\varphi(q)}{\varphi(-q)}, \frac{\varphi(i q)}{\varphi(-i q)}$ are regular on $D$.

Proof. By Theorem 2.4.1, the functions $\varphi(q)$ and $\varphi(-q)$ are regular on $D$. Hence $\varphi^{4}(q)$ and $\varphi^{4}(-q)$ are regular on $D$. Thus $\frac{\varphi^{4}(-q)}{\varphi^{4}(q)}$ is analytic on $D$. But, by Theorem 2.4.2, $\varphi(q) \neq 0$ on $D$. Hence $1-x(q)=\frac{\varphi^{4}(-q)}{\varphi^{4}(q)}$ is regular on $D$. The rest can be proved similarly.

The next result gives the region in $\mathbb{C}$ where the complex function $f(q)=q^{1 / 4}$ is regular.

Theorem 2.4.4. The function $f(q)=q^{1 / 4}$ is regular on $\mathbb{C} \backslash\{q \in \mathbb{C} \mid \operatorname{Re} q \leq$ 0 and $\operatorname{Im} q=0\}$.

Proof. The result can be found in [30, p. 49].

The following lemma is useful in the proof of Lemma 2.4.2.

Lemma 2.4.1. For $q \in D$, let $S=\operatorname{Re}\left(\frac{\varphi(-q)}{\varphi(q)}\right)$ and $T=\operatorname{Im}\left(\frac{\varphi(-q)}{\varphi(q)}\right)$. Then $\operatorname{Re}(1-x(q))>0$ if and only if $S^{4}-6 S^{2} T^{2}+T^{4}>0$.

Proof. As $\frac{\varphi(-q)}{\varphi(q)}=S+i T$, we have

$$
1-x(q)=\frac{\varphi^{4}(-q)}{\varphi^{4}(q)}=(S+i T)^{4}=\left(S^{4}-6 S^{2} T^{2}+T^{4}\right)+i\left(4 S^{3} T-4 S T^{3}\right) .
$$

Hence $\operatorname{Re}(1-x(q))=S^{4}-6 S^{2} T^{2}+T^{4}$ and the result follows.

Our next goal is to find a subset of $D$ on which the functions $(1-x(q))^{1 / 4}$ and $(1-x(i q))^{1 / 4}$ are regular. The following results are very important in order to reach that goal. 
Lemma 2.4.2. There exists $\delta \in \mathbb{R}$ with $0<\delta \leq 1$ such that

(i) $\operatorname{Re}(1-x(q))>0$, for all $q \in D_{\delta}$,

(ii) $\operatorname{Re}(1-x(i q))>0$, for all $q \in D_{\delta}$,

where $D_{\delta}:=\{q \in \mathbb{C}|| q \mid<\delta\}$.

Proof. (i) By Theorem 2.4.3 the function $\frac{\varphi(-q)}{\varphi(q)}$ is regular on D. Thus $\frac{\varphi(-q)}{\varphi(q)}$ is continuous at $q=0$. Moreover $\left.\frac{\varphi(-q)}{\varphi(q)}\right|_{q=0}=\frac{\varphi(0)}{\varphi(0)}=1$. Thus there exists $\delta \in \mathbb{R}$ with $0<\delta \leq 1$ such that

$$
\left|\frac{\varphi(-q)}{\varphi(q)}-1\right|<\frac{1}{8}
$$

for $|q|<\delta$. Then, with $S=\operatorname{Re}\left(\frac{\varphi(-q)}{\varphi(q)}\right)$ and $T=\operatorname{Im}\left(\frac{\varphi(-q)}{\varphi(q)}\right)$, we have

$$
|(S+i T)-1|<\frac{1}{8} \text { for }|q|<\delta .
$$

Therefore $|S-1|<\frac{1}{8},|T|<\frac{1}{8}$, for $|q|<\delta$. Thus $\frac{7}{8}<S<\frac{9}{8}, \quad-\frac{1}{8}<T<\frac{1}{8}$, for $|q|<\delta$. Then

$$
S^{4}-6 S^{2} T^{2}+T^{4}>\left(\frac{7}{8}\right)^{4}-6 \cdot \frac{9^{2}}{8^{2}} \cdot \frac{1}{8^{2}}=\frac{7^{4}-6 \cdot 81}{8^{4}}=\frac{2401-486}{8^{4}}>0 .
$$

Hence, by Lemma 2.4.1 this implies that $\operatorname{Re}(1-x(q))>0$.

(ii) If $f(q)=i q$, we have $f\left(D_{\delta}\right)=D_{\delta}$. Thus, from (i) we have that the composite function $1-x(i q)$ is such that $\operatorname{Re}(1-x(i q))>0$ for $|q|<\delta$.

For the remainder of this thesis we set

$$
D_{\delta}:=\{q \in \mathbb{C}|| q \mid<\delta\} \subseteq D,
$$

where $\delta$ is the constant determined by Lemma 2.4.2. 
We now answer our questions concerning the analyticity of the functions $(1-x(q))^{1 / 4}$ and $(1-x(i q))^{1 / 4}$.

Theorem 2.4.5. The functions $(1-x(q))^{1 / 4}$ and $(1-x(i q))^{1 / 4}$ are regular on $D_{\delta}$.

Proof. By Theorem 2.4.3(i) the function $1-x(q)$ is regular on $D_{\delta}$, and by Lemma $2.4 .2(\mathrm{i})$

$$
\left\{1-x(q) \mid q \in D_{\delta}\right\} \subseteq \mathbb{C} \backslash\{q \in \mathbb{C} \mid \operatorname{Re} q<0, \operatorname{Im} q=0\} .
$$

This implies that the composite function $(1-x(q))^{1 / 4}$ is a regular analytic function on $D_{\delta}$, see $\left[29, \S 2.4\right.$ p. 34]. Similarly for $(1-x(i q))^{1 / 4}$.

We are now ready to state and prove the main results of this section.

Theorem 2.4.6. For $q \in D_{\delta}$, we have

(i) $(1-x(q))^{1 / 4}=(1-x)^{1 / 4}=\frac{\varphi(-q)}{\varphi(q)}$,

(ii) $(1-x(i q))^{1 / 4}=\frac{\varphi(-i q)}{\varphi(i q)}=-i\left(\frac{1+i(1-x)^{1 / 4}}{1-i(1-x)^{1 / 4}}\right)$.

Proof. (i) By Definition 2.3.2 we have

$$
(1-x(q))^{1 / 4}=\left(\frac{\varphi^{4}(-q)}{\varphi^{4}(q)}\right)^{1 / 4}=\alpha(q) \frac{\varphi(-q)}{\varphi(q)}
$$

where $\alpha(q)=1, i,-1$ or $-i$. Now $\varphi(0)=1$ so $x(0)=0$. Taking $q=0$ in (2.4.1) we obtain $\alpha(0)=1^{1 / 4}=1$. In $D_{\delta}$ we have by Theorems 2.4 .3 and 2.4 .5 that

$$
\alpha(q)=\frac{\varphi(q)}{\varphi(-q)}(1-x(q))^{1 / 4}
$$

is regular in $D_{\delta}$. Thus $\alpha(q)$ is continuous on $D_{\delta}$. But $0 \in D_{\delta}, \alpha(0)=1$ and $\alpha(q)=1, i,-1$ or $-i$, so $\alpha(q)=1$ for all $q \in D_{\delta}$. Thus

$$
(1-x(q))^{1 / 4}=\frac{\varphi(-q)}{\varphi(q)} \quad \text { for all } q \in D_{\delta} .
$$

(ii) Using the same argument as (i) and appealing to Theorems 2.4.3 and 2.4.5, we find that $(1-x(i q))^{1 / 4}=\frac{\varphi(-i q)}{\varphi(i q)}$. 
By Lemmas 2.3.2(i)(ii) and 2.3.1(i), we have

$$
\begin{aligned}
& \frac{\varphi(-i q)}{\varphi(i q)}=\frac{\varphi\left(q^{4}\right)-i\left(\varphi(q)-\varphi\left(q^{4}\right)\right)}{\varphi\left(q^{4}\right)+i\left(\varphi(q)-\varphi\left(q^{4}\right)\right)}=\frac{\frac{\varphi(q)+\varphi(-q)}{2}-i\left(\frac{\varphi(q)-\varphi(-q)}{2}\right)}{\frac{\varphi(q)+\varphi(-q)}{2}+i\left(\frac{\varphi(q)-\varphi(-q)}{2}\right)} \\
= & \frac{\varphi(q)+\varphi(-q)-i(\varphi(q)-\varphi(-q))}{\varphi(q)+\varphi(-q)+i(\varphi(q)-\varphi(-q))}=\frac{1+\frac{\varphi(-q)}{\varphi(q)}-i\left(1-\frac{\varphi(-q)}{\varphi(q)}\right)}{1+\frac{\varphi(-q)}{\varphi(q)}+i\left(1-\frac{\varphi(-q)}{\varphi(q)}\right)} \\
= & \frac{(1-i)+(1+i) \frac{\varphi(-q)}{\varphi(q)}}{(1+i)+(1-i) \frac{\varphi(-q)}{\varphi(q)}}=\frac{1-i}{1+i}\left(\frac{1+i \frac{\varphi(-q)}{\varphi(q)}}{1-i \frac{\varphi(-q)}{\varphi(q)}}\right)=-i\left(\frac{1+i(1-x)^{1 / 4}}{1-i(1-x)^{1 / 4}}\right),
\end{aligned}
$$

where the last equality was obtained by using (i). This is the desired result.

\subsection{The duplication principle}

If $q \in D_{\delta}$ then $|q|<\delta$. Thus, as $\delta \leq 1$, we have $\left|q^{2}\right|=|q|^{2}<|q|<\delta$ so that $q^{2} \in D_{\delta}$. Now suppose we have $q \in D_{\delta}$ and we transform $q$ into $q^{2}$. How are $x\left(q^{2}\right)$ and $z\left(q^{2}\right)$ related to $x(q)$ and $z(q)$ ? The formulae giving this relationship comprise the "duplication principle", which was first discovered by Jacobi and is of crucial importance for the remainder of our work.

Theorem 2.5.1. (Duplication principle) For $q \in D_{\delta}$ we have

$$
x\left(q^{2}\right)=\left(\frac{1-(1-x(q))^{1 / 2}}{1+(1+x(q))^{1 / 2}}\right)^{2}
$$

and

$$
z\left(q^{2}\right)=\left(\frac{1+(1-x(q))^{1 / 2}}{2}\right) z(q)
$$

That is, if $q \rightarrow q^{2}$ then $x \rightarrow\left(\frac{1-(1-x)^{1 / 2}}{1+(1-x)^{1 / 2}}\right)^{2}$ and $z \rightarrow\left(\frac{1+(1-x)^{1 / 2}}{2}\right) z$.

Proof. By Definition 2.3.2, Lemma 2.3.1 (ii)(iii) and Theorem 2.4.6(i), we have

$$
x\left(q^{2}\right)=1-\frac{\varphi^{4}\left(-q^{2}\right)}{\varphi^{4}\left(q^{2}\right)}=1-\frac{\varphi^{2}(q) \varphi^{2}(-q)}{\left(\frac{\varphi^{2}(q)+\varphi^{2}(-q)}{2}\right)^{2}}
$$




$$
\begin{aligned}
& =\frac{\left(\varphi^{2}(q)+\varphi^{2}(-q)\right)^{2}-4 \varphi^{2}(q) \varphi^{2}(-q)}{\left(\varphi^{2}(q)+\varphi^{2}(-q)\right)^{2}}=\frac{\left(\varphi^{2}(q)-\varphi^{2}(-q)\right)^{2}}{\left(\varphi^{2}(q)+\varphi^{2}(-q)\right)^{2}} \\
& =\left(\frac{1-\frac{\varphi^{2}(-q)}{\varphi^{2}(q)}}{1+\frac{\varphi^{2}(-q)}{\varphi^{2}(q)}}\right)^{2}=\left(\frac{1-(1-x(q))^{1 / 2}}{1+(1-x(q))^{1 / 2}}\right)^{2} .
\end{aligned}
$$

By Definition 2.3.2, Lemma 2.3.1(ii) and Theorem 2.4.6(i) we have

$$
z\left(q^{2}\right)=\varphi^{2}\left(q^{2}\right)=\frac{\varphi^{2}(q)+\varphi^{2}(-q)}{2}=\frac{\left(1+\frac{\varphi^{2}(-q)}{\varphi^{2}(q)}\right) \varphi^{2}(q)}{2}=\left(\frac{1+(1-x(q))^{1 / 2}}{2}\right) z(q) .
$$

This completes the proof of the duplication principle.

Next, we use the duplication principle to find expressions for $\varphi\left(q^{2}\right)$ and $\varphi\left(q^{4}\right)$ in terms of $x$ and $z$ as defined in Definition 2.3.2.

Theorem 2.5.2. For $q \in D_{\delta}$ and $x$ and $z$ as defined in Definition 2.3.2, we have

(i) $\varphi(q)=z^{1 / 2}$,

(ii) $\varphi\left(q^{2}\right)=\left(\frac{1+(1-x)^{1 / 2}}{2}\right)^{1 / 2} z^{1 / 2}$

(iii) $\varphi\left(q^{4}\right)=\left(\frac{1+(1-x)^{1 / 4}}{2}\right) z^{1 / 2}$.

Proof. From Definition 2.3.2 we have $\varphi(q)=z^{1 / 2}$. Applying Theorem 2.5.1 to (i), we obtain (ii). Applying Theorem 2.5.1 to (ii), we obtain (iii).

\subsection{The change of sign principle}

Similarly, if $q \in D_{\delta}$ then $-q \in D_{\delta}$, and suppose we transform $q$ into $-q$. How are $x(-q)$ and $z(-q)$ related to $x(q)$ and $z(q)$ ? This result, also discovered by Jacobi, is called the "change of sign principle". 
Theorem 2.6.1. (Change of sign principle) For $q \in D_{\delta}$ we have

$$
x(-q)=\frac{x(q)}{x(q)-1}
$$

and

$$
z(-q)=(1-x(q))^{1 / 2} z(q)
$$

That is, if $q \rightarrow-q$ then $x \rightarrow \frac{x}{x-1}$ and $z \rightarrow(1-x)^{1 / 2} z$.

Proof. By Definition 2.3.2 we have

$$
\begin{aligned}
x(-q) & =1-\frac{\varphi^{4}(q)}{\varphi^{4}(-q)}=\frac{\varphi^{4}(-q)-\varphi^{4}(q)}{\varphi^{4}(-q)}=\frac{\frac{\varphi^{4}(-q)-\varphi^{4}(q)}{\varphi^{4}(q)}}{\frac{\varphi^{4}(-q)}{\varphi^{4}(q)}} \\
& =\frac{(1-x(q))-1}{1-x(q)}=\frac{-x(q)}{1-x(q)}=\frac{x(q)}{x(q)-1} .
\end{aligned}
$$

By Definition 2.3.2 and Theorem 2.4.6(i) we have

$$
z(-q)=\varphi^{2}(-q)=\frac{\varphi^{2}(-q)}{\varphi^{2}(q)} \varphi^{2}(q)=(1-x(q))^{1 / 2} z(q) .
$$

This completes the proof of the change of sign principle.

\subsection{The rotation principle}

If $q \in D_{\delta}$ then $i q \in D_{\delta}$. Our final result of this section determines what happens to $x(q)$ and $z(q)$ when $q$ is transformed into $i q$ for $q \in D_{\delta}$. This result is called the "rotation principle" and is due to Cheng [27].

Theorem 2.7.1. (Rotation principle) For $q \in D_{\delta}$ we have

$$
x(i q)=\frac{-8 i(1-x(q))^{1 / 4}\left(1-(1-x(q))^{1 / 2}\right)}{\left(1-i(1-x(q))^{1 / 4}\right)^{4}}
$$

and

$$
z(i q)=i \frac{z(q)}{2}\left(1-i(1-x(q))^{1 / 4}\right)^{2} .
$$


That is, if $q \rightarrow$ iq then $x \rightarrow \frac{-8 i(1-x)^{1 / 4}\left(1-(1-x)^{1 / 2}\right)}{\left(1-i(1-x)^{1 / 4}\right)^{4}}$ and $z \rightarrow$ $i \frac{z}{2}\left(1-i(1-x)^{1 / 4}\right)^{2}$.

Proof. Cheng's proof is given in [27]. Here we give a new proof based on some of the results that we proved earlier.

By Definition 2.3.2 and Theorem 2.4.6(ii), we have

$$
\begin{aligned}
x(i q) & =1-\frac{\varphi^{4}(-i q)}{\varphi^{4}(i q)}=1-\left(-i\left(\frac{1+i(1-x)^{1 / 4}}{1-i(1-x)^{1 / 4}}\right)\right)^{4} \\
& =1-\frac{\left(1+i(1-x)^{1 / 4}\right)^{4}}{\left(1-i(1-x)^{1 / 4}\right)^{4}}=\frac{\left(1-i(1-x)^{1 / 4}\right)^{4}-\left(1+i(1-x)^{1 / 4}\right)^{4}}{\left(1-i(1-x)^{1 / 4}\right)^{4}} \\
& =\frac{-8 i(1-x)^{1 / 4}+8 i(1-x)^{3 / 4}}{\left(1-i(1-x)^{1 / 4}\right)^{4}}=\frac{-8 i(1-x)^{1 / 4}\left(1-(1-x)^{1 / 2}\right)}{\left(1-i(1-x)^{1 / 4}\right)^{4}} .
\end{aligned}
$$

By Definition 2.3.2, Lemma 2.3.2(i) and Theorem 2.4.6(i), we have

$$
\begin{aligned}
z & (i q)=\varphi^{2}(i q)=\left(\left(\frac{\varphi(q)+\varphi(-q)}{2}\right)+i\left(\frac{\varphi(q)-\varphi(-q)}{2}\right)\right)^{2} \\
= & \frac{1}{4}((\varphi(q)+\varphi(-q))+i(\varphi(q)-\varphi(-q)))^{2} \\
= & \frac{1}{4}\left((\varphi(q)+\varphi(-q))^{2}-(\varphi(q)-\varphi(-q))^{2}+2 i(\varphi(q)+\varphi(-q))(\varphi(q)-\varphi(-q))\right) \\
= & \frac{1}{4}\left(4 \varphi(q) \varphi(-q)+2 i\left(\varphi^{2}(q)-\varphi^{2}(-q)\right)\right)=\varphi(q) \varphi(-q)+\frac{i}{2}\left(\varphi^{2}(q)-\varphi^{2}(-q)\right) \\
= & \left(\frac{\varphi(-q)}{\varphi(q)}+\frac{i}{2}\left(1-\frac{\varphi^{2}(-q)}{\varphi^{2}(q)}\right)\right) \varphi^{2}(q)=\frac{i}{2} z\left(-2 i(1-x)^{1 / 4}+\left(1-(1-x)^{1 / 2}\right)\right) \\
= & \frac{i}{2} z\left(1-i(1-x)^{1 / 4}\right)^{2} .
\end{aligned}
$$

This completes the proof of the rotation principle. 


\subsection{The arithmetic function $N([a, b, c] ; n)$}

The central problem that we address in this thesis is that of determining the number of representations of a positive integer $n$ by a quadratic form of the type

$$
x_{1}^{2}+\cdots+x_{a}^{2}+2 x_{a+1}^{2}+\cdots+2 x_{a+b}^{2}+4 x_{a+b+1}^{2}+\cdots+4 x_{t}^{2},
$$

where $a \in \mathbb{N}, b, c \in \mathbb{N}_{0}, b \equiv 0(\bmod 2), b$ or $c \in \mathbb{N}$ and $t=a+b+c \equiv 0(\bmod 4)$. To this end we make the following definition.

Definition 2.8.1. For $a, b, c, n \in \mathbb{N}_{0}$ and $t=a+b+c \geq 1$, we define

$N([a, b, c] ; n):=$

$\operatorname{card}\left\{\left(x_{1}, \ldots, x_{t}\right) \in \mathbb{Z}^{t} \mid n=x_{1}^{2}+\cdots+x_{a}^{2}+2 x_{a+1}^{2}+\cdots+2 x_{a+b}^{2}+4 x_{a+b+1}^{2}+\cdots+4 x_{t}^{2}\right\}$.

We note that as $(0, \ldots, 0) \in \mathbb{Z}^{t}$ is the only solution to

$$
0=x_{1}^{2}+\cdots+x_{a}^{2}+2 x_{a+1}^{2}+\cdots+2 x_{a+b}^{2}+4 x_{a+b+1}^{2}+\cdots+4 x_{t}^{2},
$$

we have $N([a, b, c] ; 0)=1$.

The next theorem expresses the series $1+\sum_{n=1}^{\infty} N([a, b, c] ; n) q^{n}$ in terms of the theta function $\varphi$.

Theorem 2.8.1. For $q \in \mathbb{C}$ such that $|q|<1$ and $a, b, c \in \mathbb{N}_{0}$ with $a+b+c \geq 1$, we have

$$
\varphi(q)^{a} \varphi\left(q^{2}\right)^{b} \varphi\left(q^{4}\right)^{c}=1+\sum_{n=1}^{\infty} N([a, b, c] ; n) q^{n} .
$$

Proof. We have by Definition 2.3.1

$$
\varphi(q)^{a} \varphi\left(q^{2}\right)^{b} \varphi\left(q^{4}\right)^{c}=\left(\sum_{k=-\infty}^{\infty} q^{k^{2}}\right)^{a}\left(\sum_{l=-\infty}^{\infty} q^{2 l^{2}}\right)^{b}\left(\sum_{m=-\infty}^{\infty} q^{4 m^{2}}\right)^{c}
$$




$$
\begin{aligned}
& =\left(\sum_{k_{1}, \ldots, k_{a}=-\infty}^{\infty} q^{k_{1}^{2}+\cdots+k_{a}^{2}}\right)\left(\sum_{l_{1}, \ldots, l_{b}=-\infty}^{\infty} q^{2 l_{1}^{2}+\cdots+2 l_{b}^{2}}\right)\left(\sum_{m_{1}, \ldots, m_{c}=-\infty}^{\infty} q^{4 m_{1}^{2}+\cdots+4 m_{c}^{2}}\right) \\
& =\sum_{k_{1}, \ldots, k_{a}, l_{1}, \ldots, l_{b}, m_{1}, \ldots, m_{c}=-\infty}^{\infty} q^{k_{1}^{2}+\cdots+k_{a}^{2}+2 l_{1}^{2}+\cdots+2 l_{b}^{2}+4 m_{1}^{2}+\cdots+4 m_{c}^{2}} \\
& =\sum_{n=0}^{\infty} \sum_{k_{1}^{2}+\cdots+k_{a}^{2}+2 l_{1}^{2}+\cdots+2 l_{b}^{2}+4 m_{1}^{2}+\cdots+4 m_{c}^{2}=n}^{\infty} q^{n} \\
& =\sum_{n=0}^{\infty} N([a, b, c] ; n) q^{n}=1+\sum_{n=1}^{\infty} N([a, b, c] ; n) q^{n},
\end{aligned}
$$

as asserted.

\subsection{The Eisenstein series $E_{k}(q)$}

We begin this section by defining the (normalized) Eisenstein series $E_{k}(q)(k \in \mathbb{N}$, $q \in \mathbb{C},|q|<1)$ of weight $2 k$.

Definition 2.9.1. For $k \in \mathbb{N}$ and $q \in \mathbb{C}$ with $|q|<1$, we define the Eisenstein series $E_{k}(q)$ by

$$
E_{k}(q):=1-\frac{4 k}{B_{2 k}} \sum_{n=1}^{\infty} \sigma_{2 k-1}(n) q^{n} .
$$

We note that $E_{k}(0)=1$. For $k \geq 2 \quad E_{k}$ is a modular form of weight $2 k$ on the full modular group. On the other hand $E_{1}$ is not a modular form.

A very important result for our work is the following result of Ramanujan, which was probably known to Weierstrass. 
Theorem 2.9.1. For $k \in \mathbb{N}$ with $k \geq 2$ and $q \in \mathbb{C}$ with $|q|<1$ there exist rational numbers $a_{k}(t, u)$ which depend upon $t$ and $u$ but not on $q$ such that

$$
E_{k}(q)=\sum_{\substack{(t, u) \in \mathbb{N}_{0}^{2} \\ 2 t+3 u=k}} a_{k}(t, u) E_{2}(q)^{t} E_{3}(q)^{u} .
$$

Proof. See Berndt [19, eq. (4.2.40), p. 96] or Kilford [54, Prop. 3.6, p. 48].

It is known that the rational numbers $a_{k}(t, u)$ are all positive, see for example Apostol [14, Theorem 1.13 and Ex. 1.11].

Taking $q=0$ in Theorem 2.9.1 we find that

$$
\sum_{\substack{(t, u) \in \mathbb{N}_{0}^{2} \\ 2 t+3 u=k}} a_{k}(t, u)=1, k \in \mathbb{N}, k \geq 2 .
$$

Using Definition 2.9.1 in Theorem 2.9.1, and equating small powers of $q$, we obtain the values of the $a_{k}(t, u) \quad\left(t, u \in \mathbb{N}_{0}, 2 t+3 u=k\right)$ for $2 \leq k \leq 10$.

Table 2.4.1

\begin{tabular}{|c|c|}
\hline$k$ & $a_{k}(t, u)$ \\
\hline 2 & $a_{2}(1,0)=1$ \\
\hline 3 & $a_{3}(0,1)=1$ \\
\hline 4 & $a_{4}(2,0)=1$ \\
\hline 5 & $a_{5}(1,1)=1$ \\
\hline 6 & $a_{6}(0,2)=\frac{250}{691}, a_{6}(3,0)=\frac{441}{691}$ \\
\hline 7 & $a_{7}(2,1)=1$ \\
\hline
\end{tabular}




\begin{tabular}{|c|c|}
\hline 8 & $a_{8}(1,2)=\frac{2000}{3617}, a_{8}(4,0)=\frac{1617}{3617}$ \\
\hline 9 & $a_{9}(0,3)=\frac{5500}{43867}, a_{9}(3,1)=\frac{38367}{43867}$ \\
\hline 10 & $a_{10}(2,2)=\frac{121250}{174611}, a_{10}(5,0)=\frac{53361}{174611}$ \\
\hline
\end{tabular}

We now state three theorems that express $E_{k}\left(q^{r}\right)$, where $k \in\{1,2,3\}$ and $r \in$ $\{1,2,4\}$, in terms of $x$ and $z$.

Theorem 2.9.2. For $q \in \mathbb{C}$ with $|q|<1$ define $x$ and $z$ as in Definition 2.3.2. Then

$$
E_{1}(q)=(1-5 x) z^{2}+12 x(1-x) z \frac{d z}{d x}
$$

$$
E_{1}\left(q^{2}\right)=(1-2 x) z^{2}+6 x(1-x) z \frac{d z}{d x}
$$

Proof. Formulae (2.9.2) and (2.9.3) can be found in [19, Theorem 5.4.9, p. 125].

Formula (2.9.4) can be found in [19, Exercise 5.4.13, p. 128].

Theorem 2.9.3. For $q \in \mathbb{C}$ with $|q|<1$ define $x$ and $z$ as in Definition 2.3.2. Then

$$
E_{1}\left(q^{4}\right)=\left(1-\frac{5}{4} x\right) z^{2}+3 x(1-x) z \frac{d z}{d x}
$$

$$
\begin{aligned}
& E_{2}(q)=\left(1+14 x+x^{2}\right) z^{4}, \\
& E_{2}\left(q^{2}\right)=\left(1-x+x^{2}\right) z^{4}, \\
& E_{2}\left(q^{4}\right)=\left(1-x+\frac{1}{16} x^{2}\right) z^{4} .
\end{aligned}
$$


Proof. Formulae (2.9.5) and (2.9.6) can be found in [19, Theorem 5.4.11, p. 126]. Formula (2.9.7) can be found in [19, Exercise 5.4.13, p. 128].

Theorem 2.9.4. For $q \in \mathbb{C}$ with $|q|<1$ define $x$ and $z$ as in Definition 2.3.2. Then

$$
\begin{aligned}
& E_{3}(q)=\left(1-33 x-33 x^{2}+x^{3}\right) z^{6}, \\
& E_{3}\left(q^{2}\right)=\left(1-\frac{3}{2} x-\frac{3}{2} x^{2}+x^{3}\right) z^{6}, \\
& E_{3}\left(q^{4}\right)=\left(1-\frac{3}{2} x+\frac{15}{32} x^{2}+\frac{1}{64} x^{3}\right) z^{6} .
\end{aligned}
$$

Proof. Formulae (2.9.8) and (2.9.9) can be found in [19, Theorem 5.4.12, p. 127]. Formula (2.9.10) can be found in [19, Exercise 5.4.13, p. 128].

Next we are interested in expressing the Eisenstein series $E_{1}\left(q^{8}\right)$ and $E_{1}\left(q^{16}\right)$ in terms of $x, z$ and $\frac{d z}{d x}$. In order to do this, we first prove the following simple lemma.

Lemma 2.9.1. For $q \in \mathbb{C}$ with $|q|<1$, define $x$ and $z$ as in Definition 2.3.2. Then

$$
2 E_{1}\left(q^{4}\right)-E_{1}\left(q^{2}\right)=\left(1-\frac{1}{2} x\right) z^{2}
$$

Proof. This result follows immediately from (2.9.3) and (2.9.4).

We now use Lemma 2.9.1 to prove the following theorem.

Theorem 2.9.5. For $q \in \mathbb{C}$ with $|q|<1$ define $x$ and $z$ as in Definition 2.3.2. Then for $q \in D_{\delta}$ we have

$E_{1}\left(q^{8}\right)=\left(\frac{5}{8}-\frac{11}{16} x+\frac{3}{8}(1-x)^{1 / 2}\right) z^{2}+\frac{3}{2} x(1-x) z \frac{d z}{d x}$, 
$E_{1}\left(q^{16}\right)=\left(\frac{11}{32}-\frac{23}{64} x+\frac{3}{16}(1-x)^{1 / 4}+\frac{9}{32}(1-x)^{1 / 2}+\frac{3}{16}(1-x)^{3 / 4}\right) z^{2}+\frac{3}{4} x(1-x) z \frac{d z}{d x}$.

Proof. Using the duplication principle (Theorem 2.5.1) in Lemma 2.9.1, we obtain for $q \in D_{\delta}$

$$
2 E_{1}\left(q^{8}\right)-E_{1}\left(q^{4}\right)=\left(\frac{1}{4}+\frac{3}{4}(1-x)^{1 / 2}-\frac{x}{8}\right) z^{2}
$$

Replacing $E_{1}\left(q^{4}\right)$ by its value given in (2.9.4), and simplifying, we obtain the desired result for $E_{1}\left(q^{8}\right)$.

Using the duplication principle in (2.9.11), we obtain for $q \in D_{\delta}$

$$
2 E_{1}\left(q^{16}\right)-E_{1}\left(q^{8}\right)=\left(\frac{1}{16}-\frac{1}{32} x+\frac{3}{8}(1-x)^{1 / 4}+\frac{3}{16}(1-x)^{1 / 2}+\frac{3}{8}(1-x)^{3 / 4}\right) z^{2} .
$$

Replacing $E_{1}\left(q^{8}\right)$ by its value given in the first part of Theorem 2.9.5, and simplifying, we obtain the desired result for $E_{1}\left(q^{16}\right)$.

In the next theorem we obtain new identities involving the Eisenstein series $E_{1}(q)$ in terms of $x, z$ and $\frac{d z}{d x}$.

Theorem 2.9.6. For $q \in D_{\delta}, x$ and $z$ as defined in Definttıon 2.3 .2 and the Eısenstern series $E_{1}(q)$, we have

(i) $2 E_{1}\left(q^{2}\right)-E_{1}(q)=(1+x) z^{2}$

(ii) $E_{1}(-q)=(1-2 x) z^{2}+12 x(1-x) z \frac{d z}{d x}$

(iii) $E_{1}(q)-E_{1}(-q)=-3 x z^{2}$

Proof. (i) Follows from Theorem 2.9.2.

(ii) Using the change of sign principle (Theorem 2.6.1) in (i), we obtain for $q \in D_{\delta}$

$$
2 E_{1}\left(q^{2}\right)-E_{1}(-q)=\left(1+\frac{x}{x-1}\right)(1-x) z^{2}=(1-2 x) z^{2}
$$


Replacing $E_{1}\left(q^{2}\right)$ by its value given in (2.9.3), and simplifying, we obtain the desired result for $E_{1}(-q)$.

(iii) This follows from (2.9.2) and (ii).

We conclude this section with two theorems that express the Eisenstein series $E_{2}\left(q^{8}\right), E_{2}\left(q^{16}\right), E_{3}\left(q^{8}\right)$ and $E_{3}\left(q^{16}\right)$ in terms of $x$ and $z$.

Theorem 2.9.7. For $q \in \mathbb{C}$ with $|q|<1$ define $x$ and $z$ as in Definition 2.3.2. Then for $q \in D_{\delta}$ we have

$$
\begin{aligned}
& E_{2}\left(q^{8}\right)=\frac{1}{256}\left(136-136 x+x^{2}+(120-60 x)(1-x)^{1 / 2}\right) z^{4} \\
& E_{2}\left(q^{16}\right)=\frac{1}{4096}\left(p_{2}(x)+q_{2}(x)(1-x)^{1 / 4}+r_{2}(x)(1-x)^{1 / 2}\right. \\
& \left.+s_{2}(x)(1-x)^{3 / 4}\right) z^{4},
\end{aligned}
$$

where

$$
\begin{aligned}
& p_{2}(x)=1096-1096+x^{2}, \\
& q_{2}(x)=960-840 x, \\
& r_{2}(x)=1080-540 x, \\
& s_{2}(x)=960-120 x .
\end{aligned}
$$

Proof. The formulae follow by applying Theorem 2.5 .1 to $E_{2}\left(q^{4}\right)$ and $E_{2}\left(q^{8}\right)$.

Theorem 2.9.8. For $q \in \mathbb{C}$ with $|q|<1$ define $x$ and $z$ as in Definition 2.3.2. Then for $q \in D_{\delta}$ we have

$$
\begin{aligned}
E_{3}\left(q^{8}\right)=\frac{1}{4096}(2080- & 3120 x+1038 x^{2}+x^{3} \\
+ & \left.\left(2016-2016 x+126 x^{2}\right)(1-x)^{1 / 2}\right) z^{6},
\end{aligned}
$$




$$
\begin{aligned}
E_{3}\left(q^{16}\right)=\frac{1}{262144} & \left(p_{3}(x)+q_{3}(x)(1-x)^{1 / 4}\right. \\
& \left.+r_{3}(x)(1-x)^{1 / 2}+s_{3}(x)(1-x)^{3 / 4}\right) z^{6}
\end{aligned}
$$

where

$$
\begin{aligned}
& p_{3}(x)=66592-99888 x+33294 x^{2}+x^{3}, \\
& q_{3}(x)=64512-79632 x+15372 x^{2}, \\
& r_{3}(x)=66528-66528 x+4158 x^{2}, \\
& s_{3}(x)=64512-49332 x+252 x^{2} .
\end{aligned}
$$

Proof. The formulae follow by applying Theorem 2.5.1 to $E_{3}\left(q^{4}\right)$ and $E_{3}\left(q^{8}\right)$.

\subsection{Ramanujan's discriminant function}

We begin this section with the definitions of Ramanujan's discriminant function and Ramanujan's tau function.

Definition 2.10.1. Ramanujan's discrimınant functıon $\Delta(q)$ is defined for $q \in \mathbb{C}$ with $|q|<1$ by

$$
\Delta(q):=q \prod_{n=1}^{\infty}\left(1-q^{n}\right)^{24} .
$$

Ramanujan's tau function $\tau(n)$ is defined for all $n \in \mathbb{N}$ by

$$
\sum_{n=1}^{\infty} \tau(n) q^{n}:=\Delta(q)
$$

With $q=e^{2 \pi \imath z}, z \in \mathbb{C}, \operatorname{Im} z>0$ and setting $\tilde{\Delta}(z):=\Delta\left(e^{2 \pi \imath z}\right)=$ $e^{2 \pi \imath z} \prod_{n=1}^{\infty}\left(1-e^{2 \pi \imath n z}\right)^{24}$, it is known that the action on $\tilde{\Delta}$ by the modular group

$$
\Gamma=S L(2, \mathbb{Z})=\left\{\left[\begin{array}{ll}
a & b \\
c & d
\end{array}\right] \mid a, b, c, d \in \mathbb{Z}, a d-b c=1\right\}
$$


is given by

$$
\tilde{\Delta}\left(\frac{a z+b}{c z+d}\right)=(c z+d)^{12} \tilde{\Delta}(z),
$$

see $[54$, p. 47$] . \tilde{\Delta}$ is a cusp form of weight 12 on $\Gamma$.

The first five values of $\tau(n)$ are

$$
\tau(1)=1, \tau(2)=-24, \tau(3)=252, \tau(4)=-1472, \tau(5)=4830 .
$$

Mordell [62] has shown that $\tau$ is multiplicative, that is

$$
\tau\left(n_{1} n_{2}\right)=\tau\left(n_{1}\right) \tau\left(n_{2}\right), \quad n_{1}, n_{2} \in \mathbb{N}, \quad\left(n_{1}, n_{2}\right)=1
$$

and that

$$
\tau\left(p^{n+1}\right)=\tau\left(p^{n}\right) \tau(p)-p^{11} \tau\left(p^{n-1}\right), n \in \mathbb{N}, p \text { prime. }
$$

Both (2.10.2) and (2.10.3) had been conjectured by Ramanujan [71]. Mordell's proofs were later generalized by Hecke and led to the definition and theory of what we now call Hecke operators. Ramanujan also conjectured that $|\tau(p)| \leq 2 p^{1 / 2}$ for all primes $p$. This estimate was proved by Deligne [33], [34] in the 1970's.

The relationship between $\Delta(q)$ and Eisenstein series is due to Ramanujan [71], [72], namely

$$
\Delta(q)=\frac{1}{1728}\left(E_{2}(q)^{3}-E_{3}(q)^{2}\right) .
$$

As $E_{2}(q)$ is a modular form of weight 4 on the full modular group $\Gamma, E_{2}(q)^{3}$ is a modular form of weight 12 on $\Gamma$. Similarly, as $E_{3}(q)$ is a modular form of weight 6 on $\Gamma, E_{3}(q)^{2}$ is also a modular form of weight 12 on $\Gamma$. It is easy to check that $E_{2}(q)^{3}$ and $E_{3}(q)^{2}$ are linearly independent over $\mathbb{Q}$. Thus, as the space of modular forms of 
weight 12 has dimension $2,\left\{E_{2}(q)^{3}, E_{3}(q)^{2}\right\}$ is a basis for this space. But $\Delta(q)$ is a modular form of weight 12 so

$$
\Delta(q)=a E_{2}(q)^{3}+b E_{3}(q)^{2}
$$

for some rational numbers $a$ and $b$. Equating the coefficients of 1 and $q$ we obtain

$$
\left\{\begin{array}{l}
0=a+b, \\
1=720 a-1008 b,
\end{array}\right.
$$

so $a=-b=\frac{1}{1728}$, which gives Ramanujan's formula.

The next theorem gives the parametrization of $\Delta(q), \Delta\left(q^{2}\right)$ and $\Delta\left(q^{4}\right)$ in terms of $x$ and $z$ as defined in Definition 2.3.2.

Theorem 2.10.1. For $q \in \mathbb{C}$ such that $|q|<1$, and $x$ and $z$ as defined in Definition 2.3.2, we have

$$
\begin{gathered}
\Delta(q)=\frac{x(1-x)^{4} z^{12}}{2^{4}} \\
\Delta\left(q^{2}\right)=\frac{x^{2}(1-x)^{2} z^{12}}{2^{8}} \\
\Delta\left(q^{4}\right)=\frac{x^{4}(1-x) z^{12}}{2^{16}} .
\end{gathered}
$$

Proof. These results are proved in [28].

We next define a generalized Ramanujan discriminant function.

Definition 2.10.2. For $a, b, c \in \mathbb{Q}$ with $24 a, 24 b, 24 c \in \mathbb{Z}, q \in \mathbb{C}$ and $|q|<1$, we define a generalized Ramanujan discriminant function $\Delta(a, b, c ; q)$ by

$$
\Delta(a, b, c ; q):=\Delta(q)^{a} \Delta\left(q^{2}\right)^{b} \Delta\left(q^{4}\right)^{c} .
$$


Clearly $\Delta(1,0,0 ; q)=\Delta(q), \Delta(0,1,0 ; q)=\Delta\left(q^{2}\right), \Delta(0,0,1 ; q)=\Delta\left(q^{4}\right)$.

In 1877 Dedekind introduced the eta function $\eta(z)$ defined by

$$
\eta(z)=e^{\pi i z / 12} \prod_{n=1}^{\infty}\left(1-e^{2 \pi i n z}\right), z \in \mathbb{C}, \operatorname{Im}(z)>0 .
$$

Setting $q=e^{2 \pi i z}$ we have $|q|<1$ so that

$$
\eta(z)=q^{1 / 24} \prod_{n=1}^{\infty}\left(1-q^{n}\right) .
$$

The infinite product $\prod_{n=1}^{\infty}\left(1-q^{n}\right)$ converges absolutely and is nonzero. The convergence is uniform on compact subsets of $\{z \mid \operatorname{Im}(z)>0\}$ so $\eta(z)$ is analytic on $\{z \mid \operatorname{Im}(z)>0\}$. Clearly

$$
\eta^{24}(z)=q \prod_{n=1}^{\infty}\left(1-q^{n}\right)^{24}=\Delta(q)
$$

Thus

$$
\Delta(a, b, c ; q)=\eta(z)^{24 a} \eta(2 z)^{24 b} \eta(4 z)^{24 c} .
$$

A product of the form

$$
\eta\left(t_{1} z\right)^{r_{1}} \eta\left(t_{2} z\right)^{r_{2}} \cdots \eta\left(t_{s} z\right)^{r_{s}}
$$

where $s, t_{1}, \ldots, t_{s} \in \mathbb{N}$ and $r_{1}, \ldots, r_{s} \in \mathbb{Z}$ is called a $\eta$-quotient, see for example [59, $\mathrm{p}$. 4825]. Thus, as $24 a, 24 b, 24 c \in \mathbb{Z}$, the generalized discriminant function $\Delta(a, b, c ; q)$ is an $\eta$-quotient.

The next theorem gives a generating function for $\Delta(a, b, c ; q)$ and a parametrization in terms of $x$ and $z$. 
Theorem 2.10.2. For $a, b, c \in \mathbb{Q}$ with $24 a, 24 b, 24 c \in \mathbb{Z}, q \in \mathbb{C}$ such that $|q|<1$, and $x$ and $z$ as defined in Definition 2.3.2, we have

(i) $\Delta(a, b, c ; q)=q^{a+2 b+4 c} \prod_{n=1}^{\infty}\left(1-q^{n}\right)^{24 a}\left(1-q^{2 n}\right)^{24 b}\left(1-q^{4 n}\right)^{24 c}$,

(ii) $\Delta(a, b, c ; q)=\frac{x^{a+2 b+4 c}(1-x)^{4 a+2 b+c} z^{12(a+b+c)}}{2^{4(a+2 b+4 c)}}$.

Proof. These results follow from Definitions 2.10.1 and 2.10.2, and Theorem 2.10 .1 .

Given $r, s, t \in \mathbb{Q}$, with $4 r, 4 s, 4 t \in \mathbb{Z}$ it is possible to find $a, b, c \in \mathbb{Q}$ with $24 a, 24 b, 24 c \in \mathbb{Z}$ such that $\Delta(a, b, c ; q)=\frac{x^{r}(1-x)^{s} z^{2 t}}{2^{4 r}}$. The following theorem shows us how.

Theorem 2.10.3. For $r, s, t \in \mathbb{Q}$ with $4 r, 4 s, 4 t \in \mathbb{Z}, q \in \mathbb{C}$ such that $|q|<1$, and $x, z$ as defined in Definition 2.3.2, we have

$$
\Delta\left(\frac{1}{3}(r+2 s-t), \frac{1}{6}(-6 r-6 s+5 t), \frac{1}{3}(2 r+s-t) ; q\right)=\frac{x^{r}(1-x)^{s} z^{2 t}}{2^{4 r}} .
$$

Proof. This follows from Theorem 2.10.2.

From Theorem $2.10 .2(\mathrm{i})$ we see that $\Delta(a, b, c ; q)$ has a power series expansion in nonnegative powers of $q$ if and only if $a+2 b+4 c \in \mathbb{N}_{0}$. When this condition holds we define the arithmetic function $\delta(a, b, c ; n)$ for all $n \in \mathbb{N}_{0}$ as follows.

Definition 2.10.3. For $a, b, c \in \mathbb{Q}$ with $24 a, 24 b, 24 c \in \mathbb{Z}$, and $a+2 b+4 c \in \mathbb{N}_{0}$, we define $\delta(a, b, c ; n)\left(n \in \mathbb{N}_{0}\right)$ by

$$
\Delta(a, b, c ; q)=\sum_{n=0}^{\infty} \delta(a, b, c ; n) q^{n}, q \in \mathbb{C},|q|<1 .
$$


Clearly, we always have

$$
\delta(a, b, c ; n)= \begin{cases}0, & \text { if } n<a+2 b+4 c \\ 1, & \text { if } n=a+2 b+4 c\end{cases}
$$

\subsection{The function $\tau_{k}(n)$ and six special values of}

$$
\delta(a, b, c ; n)
$$

We begin with the definition of the function $\tau_{k}(n)$.

Definition 2.11.1. For $k \in \mathbb{N}$ with $k \mid 24$, we define $\tau_{k}(n)$ for all $n \in \mathbb{N}$ by

$$
q \prod_{n=1}^{\infty}\left(1-q^{k n}\right)^{24 / k}=\sum_{n=1}^{\infty} \tau_{k}(n) q^{n}
$$

From Definitions 2.10.1 and 2.11.1 we see that $\tau_{1}=\tau$. Also it is clear that

$$
\tau_{k}(n)=0, \text { if } n \not \equiv 1 \quad(\bmod k)
$$

Thus in particular we have $\tau_{2}(n)=0$, if $n \equiv 0(\bmod 2)$, and $\tau_{4}(n)=0$, if $n \equiv 0,2,3$ $(\bmod 4)$.

It is the purpose of this section to determine the values of $\delta(1,0,0 ; n), \delta(-1,3,-1 ; n)$, $\delta\left(0, \frac{1}{2}, 0 ; n\right), \delta\left(0,0, \frac{1}{4} ; n\right), \delta\left(0,0, \frac{1}{2} ; n\right)$ and $\delta\left(-\frac{1}{3}, \frac{4}{3},-\frac{1}{3} ; n\right)$.

From (2.10.10) we see that

$$
\begin{aligned}
& \delta(1,0,0 ; 0)=\delta(-1,3,-1 ; 0)=\delta\left(0, \frac{1}{2}, 0 ; 0\right) \\
= & \delta\left(0,0, \frac{1}{4} ; 0\right)=\delta\left(0,0, \frac{1}{2} ; 0\right)=\delta\left(-\frac{1}{3}, \frac{4}{3},-\frac{1}{3} ; 0\right)=0
\end{aligned}
$$

so that we may exclude $n=0$ and restrict $n$ to be a positive integer. 
Theorem 2.11.1. For $n \in \mathbb{N}$ we have $\delta(1,0,0 ; n)=\tau(n)$.

Proof. Taking $a=1, b=0$ and $c=0$ in (2.10.9), we see that

$$
\sum_{n=0}^{\infty} \delta(1,0,0 ; n) q^{n}=\Delta(1,0,0 ; q)=q \prod_{n=1}^{\infty}\left(1-q^{n}\right)^{24}=\sum_{n=1}^{\infty} \tau(n) q^{n} .
$$

Equating coefficients of $q^{n}(n \in \mathbb{N})$, we obtain the asserted result.

Theorem 2.11.2. For $n \in \mathbb{N}$ we have $\delta(-1,3,-1 ; n)=(-1)^{n-1} \tau(n)$.

Proof. We have

$$
\prod_{n=1}^{\infty}\left(1-(-q)^{n}\right)=\prod_{n=1}^{\infty}\left(1+q^{2 n-1}\right)\left(1-q^{2 n}\right)
$$

Now

$$
\begin{aligned}
\prod_{n=1}^{\infty}\left(1+q^{2 n-1}\right) \prod_{n=1}^{\infty} \frac{1-q^{4 n}}{1-q^{2 n}} & =\prod_{n=1}^{\infty}\left(1+q^{2 n-1}\right) \prod_{n=1}^{\infty}\left(1+q^{2 n}\right)=\prod_{n=1}^{\infty}\left(1+q^{2 n-1}\right)\left(1+q^{2 n}\right) \\
& =\prod_{n=1}^{\infty}\left(1+q^{n}\right)=\prod_{n=1}^{\infty} \frac{1-q^{2 n}}{1-q^{n}},
\end{aligned}
$$

so

$$
\prod_{n=1}^{\infty}\left(1+q^{2 n-1}\right)=\prod_{n=1}^{\infty} \frac{\left(1-q^{2 n}\right)^{2}}{\left(1-q^{n}\right)\left(1-q^{4 n}\right)}
$$

and thus

$$
\prod_{n=1}^{\infty}\left(1-(-q)^{n}\right)=\prod_{n=1}^{\infty} \frac{\left(1-q^{2 n}\right)^{3}}{\left(1-q^{n}\right)\left(1-q^{4 n}\right)} .
$$

Replacing $q$ by $-q$ in Definition 2.10.1, we obtain

$$
-q \prod_{n=1}^{\infty}\left(1-(-q)^{n}\right)^{24}=\sum_{n=1}^{\infty} \tau(n)(-q)^{n}
$$

so

$$
\sum_{n=1}^{\infty}(-1)^{n-1} \tau(n) q^{n}=q \prod_{n=1}^{\infty}\left(1-(-q)^{n}\right)^{24}
$$




$$
\begin{aligned}
& =q \prod_{n=1}^{\infty}\left(1-q^{n}\right)^{-24}\left(1-q^{2 n}\right)^{72}\left(1-q^{4 n}\right)^{-24} \\
& =\sum_{n=0}^{\infty} \delta(-1,3,-1 ; n) q^{n} .
\end{aligned}
$$

Equating coefficients of $q^{n}(n \in \mathbb{N})$, we obtain the assertion of the theorem.

Theorem 2.11.3. For $n \in \mathbb{N}$ we have

$$
\delta\left(0, \frac{1}{2}, 0 ; n\right)=\tau_{2}(n)=\frac{1}{2} \sum_{\substack{\left(x_{1}, x_{2}, x_{3}, x_{4}\right) \in \mathbb{Z}^{4} \\ x_{1}^{2}+x_{2}^{2}+x_{3}^{2}+x_{4}^{2}=n}}\left(x_{1}^{4}-3 x_{1}^{2} x_{2}^{2}\right) .
$$

Moreover if $n$ is even we have

$$
\delta\left(0, \frac{1}{2}, 0 ; n\right)=\tau_{2}(n)=0 .
$$

Proof. Taking $a=0, b=\frac{1}{2}$ and $c=0$ in (2.10.9), we obtain

$$
\begin{aligned}
\sum_{n=0}^{\infty} \delta\left(0, \frac{1}{2}, 0 ; n\right) q^{n}=\Delta\left(0, \frac{1}{2}, 0 ; q\right) & =q \prod_{n=1}^{\infty}\left(1-q^{2 n}\right)^{2} \\
& =\sum_{n=1}^{\infty} \tau_{2}(n) q^{n}
\end{aligned}
$$

so that

$$
\delta\left(0, \frac{1}{2}, 0 ; n\right)=\tau_{2}(n), \quad n \in \mathbb{N} .
$$

Bulygin (Boulyguine) [21], see for example Carlitz [24] and Lomadze [58], has shown that for $n \in \mathbb{N}$

$$
r_{12}(n)=8 \sigma_{5}(n)-512 \sigma_{5}(n / 4)+8 \sum_{\substack{\left(x_{1}, x_{2}, x_{3}, x_{4}\right) \in \mathbb{Z}^{4} \\ x_{1}^{2}+x_{2}^{2}+x_{3}^{2}+x_{4}^{2}=n}}\left(x_{1}^{4}-3 x_{1}^{2} x_{2}^{2}\right) .
$$

From the case $k=3$ of the Ramanujan-Mordell formula, we have for $n \in \mathbb{N}$

$$
r_{12}(n)=8 \sigma_{5}(n)-512 \sigma_{5}(n / 4)+16 \delta\left(0, \frac{1}{2}, 0 ; n\right) .
$$


Thus

$$
\delta\left(0, \frac{1}{2}, 0 ; n\right)=\frac{1}{2} \sum_{\substack{\left(x_{1}, x_{2}, x_{3}, x_{4}\right) \in \mathbb{Z}^{4} \\ x_{1}^{2}+x_{2}^{2}+x_{3}^{2}+x_{4}^{2}=n}}\left(x_{1}^{4}-3 x_{1}^{2} x_{2}^{2}\right) .
$$

This completes the proof of the first assertion of the theorem.

Now suppose that $n$ is even. Then $\tau_{2}(n)=0$ by the remark following Definition 2.11.1. This proves the second assertion.

Theorem 2.11.4. For $n \in \mathbb{N}$

$$
\delta\left(0,0, \frac{1}{4} ; n\right)=\tau_{4}(n)= \begin{cases}\frac{1}{2} \sum_{\substack{\left.(x, y) \in \mathbb{Z}^{2} \\ x=1 \text { (mod } 2\right) \\ y=0 \\ n=x^{2}+y^{2}}}\left(x^{2}-y^{2}\right), & \text { if } n \equiv 1 \quad(\bmod 4), \\ 0, & \text { if } n \equiv 1 \quad(\bmod 4) .\end{cases}
$$

Proof. Taking $a=0, b=0$ and $c=1 / 4$ in (2.10.9), we obtain

$$
\sum_{n=0}^{\infty} \delta\left(0,0, \frac{1}{4} ; n\right) q^{n}=\Delta\left(0,0, \frac{1}{4} ; q\right)=q \prod_{n=1}^{\infty}\left(1-q^{4 n}\right)^{6}=\sum_{n=1}^{\infty} \tau_{4}(n) q^{n} .
$$

Equating coefficients of $q^{n}(n \in \mathbb{N})$, we deduce

$$
\delta\left(0,0, \frac{1}{4} ; n\right)=\tau_{4}(n), \quad n \in \mathbb{N} .
$$

Klein and Fricke [55, Vol. II, p. 377] have shown that

$$
\tau_{4}(n)=\left\{\begin{array}{lll}
\frac{1}{2} \sum_{\substack{\left.(x, y) \in \mathbb{Z}^{2} \\
x=1(\bmod 2) \\
y=0 \text { (mod } 2\right) \\
n=x^{2}+y^{2}}}\left(x^{2}-y^{2}\right), & \text { if } n \equiv 1 \quad(\bmod 4) \\
0, & \text { if } n \neq 1 \quad(\bmod 4) .
\end{array}\right.
$$

This completes the proof of the theorem. 
Theorem 2.11.5. For $n \in \mathbb{N}$ we have

$$
\delta\left(0,0, \frac{1}{2} ; n\right)=0, \quad \text { if } n \equiv 1(\bmod 2),
$$

and

$$
\delta\left(0,0, \frac{1}{2} ; n\right)=\tau_{2}\left(\frac{n}{2}\right)=\frac{1}{2} \sum_{\substack{\left(x_{1}, x_{2}, x_{3}, x_{4}\right) \in \mathbb{Z}^{4} \\ x_{1}^{2}+x_{2}^{2}+x_{3}^{2}+x_{4}^{2}=\frac{n}{2}}}\left(x_{1}^{4}-3 x_{1}^{2} x_{2}^{2}\right), \quad \text { if } n \equiv 0(\bmod 2) .
$$

Proof. By Definition 2.10.3 we have

$$
\sum_{n=2}^{\infty} \delta\left(0,0, \frac{1}{2} ; n\right) q^{n}=q^{2} \prod_{n=1}^{\infty}\left(1-q^{4 n}\right)^{12}
$$

and

$$
\sum_{n=1}^{\infty} \delta\left(0, \frac{1}{2}, 0 ; n\right) q^{n}=q \prod_{n=1}^{\infty}\left(1-q^{2 n}\right)^{12}
$$

Thus

$$
\sum_{n=1}^{\infty} \delta\left(0, \frac{1}{2}, 0 ; n\right) q^{2 n}=q^{2} \prod_{n=1}^{\infty}\left(1-q^{4 n}\right)^{12}=\sum_{n=2}^{\infty} \delta\left(0,0, \frac{1}{2} ; n\right) q^{n}
$$

Hence

$$
\delta\left(0,0, \frac{1}{2} ; n\right)=0, \text { if } n \equiv 1(\bmod 2)
$$

and

$$
\delta\left(0,0, \frac{1}{2} ; n\right)=\delta\left(0, \frac{1}{2}, 0 ; \frac{n}{2}\right), \text { if } n \equiv 0(\bmod 2) .
$$

Finally, by Theorem 2.11.3, we have

$$
\delta\left(0,0, \frac{1}{2} ; n\right)=\tau_{2}\left(\frac{n}{2}\right)=\frac{1}{2} \sum_{\substack{\left(x_{1}, x_{2}, x_{3}, x_{4}\right) \in \mathbb{Z}^{4} \\ x_{1}^{2}+x_{2}^{2}+x_{3}^{2}+x_{4}^{2}=\frac{n}{2}}}\left(x_{1}^{4}-3 x_{1}^{2} x_{2}^{2}\right), \quad \text { if } n \equiv 0(\bmod 2) .
$$


Theorem 2.11.6. For $n \in \mathbb{N}$ we have

$$
\delta\left(-\frac{1}{3}, \frac{4}{3},-\frac{1}{3} ; n\right)=\frac{1}{2} \sum_{\substack{\left.x_{1}, \ldots, x_{8}\right) \in \mathbb{Z}^{8} \\ x_{1}^{2}++x_{8}^{2}=n}}\left(x_{1}^{4}-3 x_{1}^{2} x_{2}^{2}\right) .
$$

Proof. Bulygin (Boulyguine) [21], see for example Carlitz [24] and Lomadze [58], has shown that for $n \in \mathbb{N}$

$$
r_{16}(n)=\frac{32}{17} \sigma_{7}(n)-\frac{64}{17} \sigma_{7}(n / 2)+\frac{8192}{17} \sigma_{7}(n / 4)+\frac{256}{17} \sum_{\substack{\left(x_{1}, \ldots, x_{8}\right) \in \mathbb{Z}^{8} \\ x_{1}^{2}++x_{8}^{2}=n}}\left(x_{1}^{4}-3 x_{1}^{2} x_{2}^{2}\right)
$$

and the Ramanujan-Mordell formula for $k=4$ gives

$$
r_{16}(n)=\frac{32}{17} \sigma_{7}(n)-\frac{64}{17} \sigma_{7}(n / 2)+\frac{8192}{17} \sigma_{7}(n / 4)+\frac{512}{17} \delta\left(-\frac{1}{3}, \frac{4}{3},-\frac{1}{3} ; n\right) .
$$

Equating these expressions, we obtain the asserted formula. 


\section{Chapter 3}

The function $\left(\frac{1+(1-x)^{1 / 2}}{2}\right)^{r}\left(\frac{1+(1-x)^{1 / 4}}{2}\right)^{s}$

Throughout this chapter $x$ denotes a variable. In this chapter we determine some of the properties of the function $\left(\frac{1+(1-x)^{1 / 2}}{2}\right)^{r}\left(\frac{1+(1-x)^{1 / 4}}{2}\right)^{s}$, where $r, s \in \mathbb{N}_{0}$, which arises naturally in Chapter 6 when we study the number of representations of a positive integer by the positive-definite, diagonal, integral quadratic form in $4 k$ variables

$$
x_{1}^{2}+\cdots+x_{4 k-2 r-s}^{2}+2 x_{4 k-2 r-s+1}^{2}+\cdots+2 x_{4 k-s}^{2}+4 x_{4 k-s+1}^{2}+\cdots+4 x_{4 k}^{2} .
$$

This form has 2 as coefficient of $2 r$ variables and 4 as the coefficient of $s$ variables. The remaining $4 k-2 r-s$ variables have coefficient 1 . We always assume that $2 r+s<4 k$.

\subsection{The polynomials $A_{r, s}(x), B_{r, s}(x), C_{r, s}(x), D_{r, s}(x)$}

We begin by defining the four polynomials studied throughout this chapter. The relationship of these polynomials to the function $\left(\frac{1+(1-x)^{1 / 2}}{2}\right)^{r}\left(\frac{1+(1-x)^{1 / 4}}{2}\right)^{s}$ is given in Lemma 3.1.3.

Definition 3.1.1. For $r, s \in \mathbb{N}_{0}$ we define

(i) $A_{r, s}(x):=\frac{1}{2^{r+s}} \sum_{\substack{0 \leq i \leq r \\ 0 \leq j \leq s \\ 2 \imath+\jmath}}\left(\begin{array}{l}r \\ i\end{array}\right)\left(\begin{array}{l}s \\ j\end{array}\right)(1-x)^{(2 \imath+\jmath) / 4}$, 
CHAPTER 3. THE FUNCTION $\left(\frac{1+(1-X)^{1 / 2}}{2}\right)^{R}\left(\frac{1+(1-X)^{1 / 4}}{2}\right)^{S}$

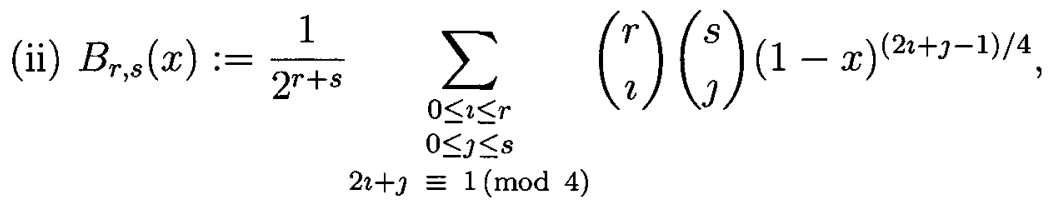

(iii) $C_{r, s}(x):=\frac{1}{2^{r+s}} \sum_{\substack{0 \leq \imath \leq r \\ 0 \leq \jmath \leq s \\ 0 \leq j}}\left(\begin{array}{l}r \\ \imath\end{array}\right)\left(\begin{array}{l}s \\ j\end{array}\right)(1-x)^{(2 \imath+\jmath-2) / 4}$

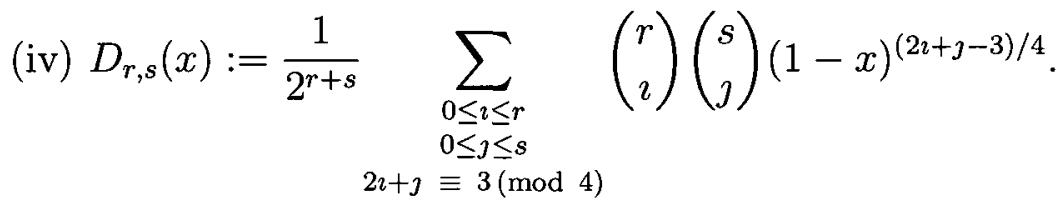

From Definition 3.1.1 we see that for $r \in \mathbb{N}_{0}$ we have

$$
\begin{gathered}
B_{r, 0}(x)=\frac{1}{2^{r}} \sum_{\substack{\imath=0 \\
2 \imath \equiv 1(\bmod 4)}}^{r}\left(\begin{array}{l}
r \\
\imath
\end{array}\right)(1-x)^{(2 \imath-1) / 4}=0, \\
D_{r, 0}(x)=\frac{1}{2^{r}} \sum_{\substack{\imath=0 \\
2 \imath}}^{r}\left(\begin{array}{l}
r \\
\imath
\end{array}\right)(1-x)^{(2 \imath-3) / 4}=0 .
\end{gathered}
$$

The next lemma gives an upper bound in terms of $r$ and $s$ for the degree of each of the polynomials $A_{r, s}(x), B_{r, s}(x), C_{r, s}(x)$ and $D_{r, s}(x)$.

Lemma 3.1.1. For $r, s \in \mathbb{N}_{0}$ we have

(i) $\operatorname{deg}\left(A_{r, s}(x)\right) \leq\left[\frac{2 r+s}{4}\right]$,

(ii) $\operatorname{deg}\left(B_{r, s}(x)\right) \leq\left[\frac{2 r+s-1}{4}\right]$, 
CHAPTER 3. THE FUNCTION $\left(\frac{1+(1-X)^{1 / 2}}{2}\right)^{R}\left(\frac{1+(1-X)^{1 / 4}}{2}\right)^{S}$

(iii) $\operatorname{deg}\left(C_{r, s}(x)\right) \leq\left[\frac{2 r+s-2}{4}\right]$,

(iv) $\operatorname{deg}\left(D_{r, s}(x)\right) \leq\left[\frac{2 r+s-3}{4}\right]$.

Proof. As

$$
\max _{\substack{0 \leq \imath \leq r \\ 0 \leq J \leq s \\ \jmath \leq 0(\bmod 4)}} \frac{2 \imath+\jmath}{4} \leq \frac{2 r+s}{4},
$$

we have, from Definition 3.1.1(i), that

$$
\operatorname{deg}\left(A_{r, s}(x)\right) \leq\left[\frac{2 r+s}{4}\right]
$$

The proofs of (ii), (iii), (iv) follow in a similar way.

The next lemma gives an upper bound for the degree of the polynomials $A_{r, s}(x)$, $B_{r, s}(x), C_{r, s}(x)$ and $D_{r, s}(x)$ in terms of an integer $k$ when $k \geq 2$ and $2 r+s<4 k$.

Lemma 3.1.2. For $k \in \mathbb{N}, r, s \in \mathbb{N}_{0}$ with $k \geq 2$ and $2 r+s<4 k$, we have

(i) $\operatorname{deg}\left(A_{r, s}(x)\right) \leq k-1$,

(ii) $\operatorname{deg}\left(B_{r, s}(x)\right) \leq k-1$,

(iii) $\operatorname{deg}\left(C_{r, s}(x)\right) \leq k-1$,

(iv) $\operatorname{deg}\left(D_{r, s}(x)\right) \leq k-1$.

Proof. (i) As $2 r+s<4 k$ implies $\frac{2 r+s}{4}<\frac{4 k}{4}=k$, we have, from Lemma 3.1.1(i), that

$$
\operatorname{deg}\left(A_{r, s}(x)\right) \leq\left[\frac{2 r+s}{4}\right] \leq k-1 .
$$

(ii) As $2 r+s<4 k$ implies $\frac{2 r+s-1}{4}<\frac{4 k-1}{4}=k-\frac{1}{4}$, we have, from Lemma 3.1.1 (ii), that

$$
\operatorname{deg}\left(B_{r, s}(x)\right) \leq\left[\frac{2 r+s-1}{4}\right] \leq k-1
$$


CHAPTER 3. THE FUNCTION $\left(\frac{1+(1-X)^{1 / 2}}{2}\right)^{R}\left(\frac{1+(1-X)^{1 / 4}}{2}\right)^{S}$

(iii) As $2 r+s<4 k$ implies $\frac{2 r+s-2}{4}<\frac{4 k-2}{4}=k-\frac{1}{2}$, we have, from Lemma 3.1.1 (iii), that

$$
\operatorname{deg}\left(C_{r, s}(x)\right) \leq\left[\frac{2 r+s-2}{4}\right] \leq k-1
$$

(iv) As $2 r+s<4 k$ implies $\frac{2 r+s-3}{4}<\frac{4 k-3}{4}=k-\frac{3}{4}$, we have, from Lemma 3.1 .1 (iv), that

$$
\operatorname{deg}\left(D_{r, s}(x)\right) \leq\left[\frac{2 r+s-3}{4}\right] \leq k-1 .
$$

This completes the proof of Lemma 3.1.2.

The next lemma relates the polynomials $A_{r, s}(x), B_{r, s}(x), C_{r, s}(x)$ and $D_{r, s}(x)$ to the function $\left(\frac{1+(1-x)^{1 / 2}}{2}\right)^{r}\left(\frac{1+(1-x)^{1 / 4}}{2}\right)^{s}$.

Lemma 3.1.3. For $r, s \in \mathbb{N}_{0}$, the polynomials $A_{r, s}(x), B_{r, s}(x), C_{r, s}(x)$ and $D_{r, s}(x)$ are such that

$$
\begin{aligned}
& \text { (i) }\left(\frac{1+(1-x)^{1 / 2}}{2}\right)^{r}\left(\frac{1+(1-x)^{1 / 4}}{2}\right)^{s} \\
& =A_{r, s}(x)+B_{r, s}(x)(1-x)^{1 / 4}+C_{r, s}(x)(1-x)^{1 / 2}+D_{r, s}(x)(1-x)^{3 / 4} \\
& \text { (ii) }\left(\frac{1+(1-x)^{1 / 2}}{2}\right)^{r}\left(\frac{1-(1-x)^{1 / 4}}{2}\right)^{s} \\
& =A_{r, s}(x)-B_{r, s}(x)(1-x)^{1 / 4}+C_{r, s}(x)(1-x)^{1 / 2}-D_{r, s}(x)(1-x)^{3 / 4} \\
& \text { (iii) }\left(\frac{1-(1-x)^{1 / 2}}{2}\right)^{r}\left(\frac{1+i(1-x)^{1 / 4}}{2}\right)^{s} \\
& =A_{r, s}(x)+i B_{r, s}(x)(1-x)^{1 / 4}-C_{r, s}(x)(1-x)^{1 / 2}-i D_{r, s}(x)(1-x)^{3 / 4}
\end{aligned}
$$


CHAPTER 3. THE FUNCTION $\left(\frac{1+(1-X)^{1 / 2}}{2}\right)^{R}\left(\frac{1+(1-X)^{1 / 4}}{2}\right)^{S}$

$$
\text { (iv) } \begin{aligned}
\left(\frac{1-(1-x)^{1 / 2}}{2}\right)^{r}\left(\frac{1-i(1-x)^{1 / 4}}{2}\right)^{s} \\
\quad=A_{r, s}(x)-i B_{r, s}(x)(1-x)^{1 / 4}-C_{r, s}(x)(1-x)^{1 / 2}+i D_{r, s}(x)(1-x)^{3 / 4}
\end{aligned}
$$

Proof. We begin by noting that for $h, k, l \in \mathbb{N}_{0}$ we have

$$
(-1)^{h k} i^{h l}=i^{2 h k+h l}=\left(i^{2 k+l}\right)^{h}= \begin{cases}1, & \text { if } 2 k+l \equiv 0(\bmod 4) \\ i^{h}, & \text { if } 2 k+l \equiv 1(\bmod 4) \\ (-1)^{h}, & \text { if } 2 k+l \equiv 2(\bmod 4) \\ (-i)^{h}, & \text { if } 2 k+l \equiv 3(\bmod 4)\end{cases}
$$

By the binomial theorem, we have for $h=0,1,2,3$

$$
\begin{aligned}
& \left(\frac{1+(-1)^{h}(1-x)^{1 / 2}}{2}\right)^{r}\left(\frac{1+i^{h}(1-x)^{1 / 4}}{2}\right)^{s} \\
& =\left(\frac{1}{2^{r}} \sum_{k=0}^{r}\left(\begin{array}{l}
r \\
k
\end{array}\right)(-1)^{h k}(1-x)^{k / 2}\right)\left(\frac{1}{2^{s}} \sum_{l=0}^{s}\left(\begin{array}{l}
s \\
l
\end{array}\right) i^{h l}(1-x)^{l / 4}\right) \\
& =\frac{1}{2^{r+s}} \sum_{\substack{0 \leq k \leq r \\
0 \leq l \leq s}}\left(\begin{array}{l}
r \\
k
\end{array}\right)\left(\begin{array}{l}
s \\
l
\end{array}\right)(-1)^{h k} i^{h l}(1-x)^{(2 k+l) / 4} \\
& =\frac{1}{2^{r+s}} \sum_{\substack{0 \leq k \leq r \\
0 \leq l \leq s \\
0 \leq 0(\bmod 4)}}\left(\begin{array}{l}
r \\
k
\end{array}\right)\left(\begin{array}{l}
s \\
l
\end{array}\right)(-1)^{h k} i^{h l}(1-x)^{(2 k+1) / 4} \\
& +\left(\frac{1}{2^{r+s}} \sum_{\substack{0 \leq k \leq r \\
0 \leq l \leq s \\
0 \leq l}}\left(\begin{array}{l}
r \\
k
\end{array}\right)\left(\begin{array}{l}
s \\
l
\end{array}\right)(-1)^{h k} i^{h l}(1-x)^{(2 k+l-1) / 4}\right)(1-x)^{1 / 4} \\
& +\left(\frac{1}{2^{r+s}} \sum_{\substack{0 \leq k \leq r \\
0 \leq l \leq s}}\left(\begin{array}{l}
r \\
k
\end{array}\right)\left(\begin{array}{l}
s \\
l
\end{array}\right)(-1)^{h k} i^{h l}(1-x)^{(2 k+l-2) / 4}\right)(1-x)^{1 / 2} \\
& 2 k+l \equiv 2(\bmod 4)
\end{aligned}
$$


CHAPTER 3. THE FUNCTION $\left(\frac{1+(1-X)^{1 / 2}}{2}\right)^{R}\left(\frac{1+(1-X)^{1 / 4}}{2}\right)^{S}$

$$
\begin{aligned}
& +\left(\frac{1}{2^{r+s}} \sum_{\substack{0 \leq k \leq r \\
0 \leq l \leq s}}\left(\begin{array}{l}
r \\
k
\end{array}\right)\left(\begin{array}{l}
s \\
l
\end{array}\right)(-1)^{h k} i^{h l}(1-x)^{(2 k+l-3) / 4}\right)(1-x)^{3 / 4} . \\
& 2 k+l \equiv 3(\bmod 4) \\
& =\frac{1}{2^{r+s}} \sum_{\substack{0 \leq k \leq r \\
0 \leq l \leq s}}\left(\begin{array}{l}
r \\
k
\end{array}\right)\left(\begin{array}{l}
s \\
l
\end{array}\right)(1-x)^{(2 k+l) / 4} \\
& 2 k+l \equiv 0(\bmod 4) \\
& +\left(\frac{1}{2^{r+s}} \sum_{\substack{0 \leq k \leq r \\
0 \leq l \leq s}}\left(\begin{array}{l}
r \\
k
\end{array}\right)\left(\begin{array}{l}
s \\
l
\end{array}\right) i^{h}(1-x)^{(2 k+l-1) / 4}\right)(1-x)^{1 / 4} \\
& 2 k+l \equiv \mathbf{1}(\bmod 4) \\
& +\left(\frac{1}{2^{r+s}} \sum_{\substack{0 \leq k \leq r \\
0 \leq l \leq s}}\left(\begin{array}{l}
r \\
k
\end{array}\right)\left(\begin{array}{l}
s \\
l
\end{array}\right)(-1)^{h}(1-x)^{(2 k+l-2) / 4}\right)(1-x)^{1 / 2} \\
& 2 k+l \equiv 2(\bmod 4) \\
& \begin{array}{c}
+\left(\frac{1}{2^{r+s}} \sum_{\substack{0 \leq k \leq r \\
0 \leq l \leq s \\
0 \leq+l}}\left(\begin{array}{l}
r \\
k
\end{array}\right)\left(\begin{array}{l}
s \\
l
\end{array}\right)(-i)^{h}(1-x)^{(2 k+l-3) / 4}\right)(1-x)^{3 / 4} . \\
2 k+\bmod 4)
\end{array} \\
& =A_{r, s}(x)+i^{h} B_{r, s}(x)+(-1)^{h} C_{r, s}(x)+(-i)^{h} D_{r, s}(x),
\end{aligned}
$$

which gives the asserted results.

Using Lemma 3.1 .3 we obtain explicit expressions for each of $A_{r, s}(x), B_{r, s}(x), C_{r, s}(x)$ and $D_{r, s}(x)$.

Lemma 3.1.4. For $r, s \in \mathbb{N}_{0}$ we have

$$
\begin{aligned}
& A_{r, s}(x)= \\
& \frac{1}{4}\left(\left(\frac{1+(1-x)^{1 / 2}}{2}\right)^{r}\left(\frac{1+(1-x)^{1 / 4}}{2}\right)^{s}+\left(\frac{1-(1-x)^{1 / 2}}{2}\right)^{r}\left(\frac{1+i(1-x)^{1 / 4}}{2}\right)^{s}\right. \\
& \left.\quad+\left(\frac{1+(1-x)^{1 / 2}}{2}\right)^{r}\left(\frac{1-(1-x)^{1 / 4}}{2}\right)^{s}+\left(\frac{1-(1-x)^{1 / 2}}{2}\right)^{r}\left(\frac{1-i(1-x)^{1 / 4}}{2}\right)^{s}\right),
\end{aligned}
$$


CHAPTER 3. THE FUNCTION $\left(\frac{1+(1-X)^{1 / 2}}{2}\right)^{R}\left(\frac{1+(1-X)^{1 / 4}}{2}\right)^{S}$

$$
\begin{aligned}
& B_{r, s}(x)= \\
& \frac{1}{4}\left(\left(\frac{1+(1-x)^{1 / 2}}{2}\right)^{r}\left(\frac{1+(1-x)^{1 / 4}}{2}\right)^{s}-i\left(\frac{1-(1-x)^{1 / 2}}{2}\right)^{r}\left(\frac{1+i(1-x)^{1 / 4}}{2}\right)^{s}\right. \\
& \left.-\left(\frac{1+(1-x)^{1 / 2}}{2}\right)^{r}\left(\frac{1-(1-x)^{1 / 4}}{2}\right)^{s}+i\left(\frac{1-(1-x)^{1 / 2}}{2}\right)^{r}\left(\frac{1-i(1-x)^{1 / 4}}{2}\right)^{s}\right) \\
& C_{r, s}(x)= \\
& \frac{1}{4}\left(\left(\frac{1+(1-x)^{1 / 2}}{2}\right)^{r}\left(\frac{1+(1-x)^{1 / 4}}{2}\right)^{s}-\left(\frac{1-(1-x)^{1 / 2}}{2}\right)^{r}\left(\frac{1+i(1-x)^{1 / 4}}{2}\right)^{s}\right. \\
& \left.+\left(\frac{1+(1-x)^{1 / 2}}{2}\right)^{r}\left(\frac{1-(1-x)^{1 / 4}}{2}\right)^{s}-\left(\frac{1-(1-x)^{1 / 2}}{2}\right)^{r}\left(\frac{1-i(1-x)^{1 / 4}}{2}\right)^{s}\right) \\
& D_{r, s}(x)= \\
& \frac{1}{4}\left(\left(\frac{1+(1-x)^{1 / 2}}{2}\right)^{r}\left(\frac{1+(1-x)^{1 / 4}}{2}\right)^{s}+i\left(\frac{1-(1-x)^{1 / 2}}{2}\right)^{r}\left(\frac{1+i(1-x)^{1 / 4}}{2}\right)^{s}\right. \\
& \left.-\left(\frac{1+(1-x)^{1 / 2}}{2}\right)^{r}\left(\frac{1-(1-x)^{1 / 4}}{2}\right)^{s}-i\left(\frac{1-(1-x)^{1 / 2}}{2}\right)^{r}\left(\frac{1-i(1-x)^{1 / 4}}{2}\right)^{s}\right)
\end{aligned}
$$

Proof. This follows by solving the four equations in Lemma 3.1 .3 for $A_{r, s}(x), B_{r, s}(x)$, $C_{r, s}(x)$ and $D_{r, s}(x)$.

The main objective of this chapter is to find the values of the polynomials 
CHAPTER 3. THE FUNCTION $\left(\frac{1+(1-X)^{1 / 2}}{2}\right)^{R}\left(\frac{1+(1-X)^{1 / 4}}{2}\right)^{S}$

$A_{r, s}(x), B_{r, s}(x), C_{r, s}(x)$ and $D_{r, s}(x)$ at $x=0$. From Lemma 3.1.4 we have

Lemma 3.1.5. For $r, s \in \mathbb{N}_{0}$

$$
\begin{aligned}
& A_{r, s}(0)=\frac{1}{4}\left(1+0^{r}\left(\frac{1+i}{2}\right)^{s}+0^{s}+0^{r}\left(\frac{1-i}{2}\right)^{s}\right) \\
& B_{r, s}(0)=\frac{1}{4}\left(1-i 0^{r}\left(\frac{1+i}{2}\right)^{s}-0^{s}+i 0^{r}\left(\frac{1-i}{2}\right)^{s}\right) \\
& C_{r, s}(0)=\frac{1}{4}\left(1-0^{r}\left(\frac{1+i}{2}\right)^{s}+0^{s}-0^{r}\left(\frac{1-i}{2}\right)^{s}\right) \\
& D_{r, s}(0)=\frac{1}{4}\left(1+i 0^{r}\left(\frac{1+i}{2}\right)^{s}-0^{s}-i 0^{r}\left(\frac{1-i}{2}\right)^{s}\right)
\end{aligned}
$$

where

$$
0^{r}= \begin{cases}0, & \text { if } r \geq 1, \\ 1, & \text { if } r=0 .\end{cases}
$$

Proof. This follows by replacing $x$ by 0 in Lemma 3.1.4.

It is clear from Lemma 3.1.5 that we require the values of $(1+i)^{s} \pm(1-i)^{s}$. This is the subject of the next section.

\subsection{The values of $(1+i)^{s} \pm(1-i)^{s}$}

The first lemma gives a formula for a sum involving binomial coefficients over an arithmetic progression.

Lemma 3.2.1. For $a, r, n \in \mathbb{N}_{0}$ such that $0 \leq a<r$ and $x \in \mathbb{C}$, we have

$$
\sum_{\substack{t=0 \\
\equiv(\bmod r)}}^{n}\left(\begin{array}{l}
n \\
t
\end{array}\right) x^{t}=\frac{1}{r} \sum_{j=1}^{r} e^{\frac{-2 \pi \imath a_{j}}{r}}\left(1+x e^{\frac{2 \pi \imath \jmath}{r}}\right)^{n} .
$$


CHAPTER 3. THE FUNCTION $\left(\frac{1+(1-X)^{1 / 2}}{2}\right)^{R}\left(\frac{1+(1-X)^{1 / 4}}{2}\right)^{S}$

Proof. For $r \in \mathbb{N}$ and $u \in \mathbb{Z}$ we have

$$
\sum_{\jmath=1}^{r} e^{\frac{2 \pi z u_{j}}{r}}= \begin{cases}r, & \text { if } u \equiv 0(\bmod r) \\ 0, & \text { if } u \neq \equiv(\bmod r)\end{cases}
$$

Thus

$$
\begin{aligned}
r \sum_{\substack{t=0 \\
t \equiv a(\bmod r)}}^{n}\left(\begin{array}{l}
n \\
t
\end{array}\right) x^{t} & =\sum_{t=0}^{n}\left(\begin{array}{l}
n \\
t
\end{array}\right) x^{t} \sum_{j=1}^{r} e^{\frac{2 \pi \imath \jmath(t-a)}{r}} \\
& =\sum_{j=1}^{r} e^{\frac{-2 \pi 2 a_{j}}{r}} \sum_{t=0}^{n}\left(\begin{array}{l}
n \\
t
\end{array}\right) x^{t} e^{\frac{2 \pi 2 \jmath t}{r}} \\
& =\sum_{j=1}^{r} e^{\frac{-2 \pi 2 a j}{r}}\left(1+x e^{\left.\frac{2 \pi r_{j}}{r}\right)^{n}}\right.
\end{aligned}
$$

and the asserted result follows.

The next lemma summarizes four simple but useful results involving the greatest integer function.

Lemma 3.2.2. For $k \in \mathbb{N}_{0}$, we have

(i) $k+2=\left[\frac{k+1}{2}\right]+\left[\frac{k+2}{2}\right]+1$,

(ii) $\left[\frac{k+2}{2}\right]= \begin{cases}\frac{k+2}{2}, & \text { if } k \equiv 0(\bmod 2), \\ \frac{k+1}{2}, & \text { if } k \equiv 1(\bmod 2),\end{cases}$ 
CHAPTER 3. THE FUNCTION $\left(\frac{1+(1-X)^{1 / 2}}{2}\right)^{R}\left(\frac{1+(1-X)^{1 / 4}}{2}\right)^{S}$

(iii) $\left[\frac{k}{4}\right]= \begin{cases}\frac{k}{4}, & \text { if } k \equiv 0(\bmod 4), \\ \frac{k-1}{4}, & \text { if } k \equiv 1(\bmod 4), \\ \frac{k-2}{4}, & \text { if } k \equiv 2(\bmod 4), \\ \frac{k-3}{4}, & \text { if } k \equiv 3(\bmod 4),\end{cases}$

(iv) $\left[\frac{k+1}{4}\right]= \begin{cases}\frac{k}{4}, & \text { if } k \equiv 0(\bmod 4), \\ \frac{k-1}{4}, & \text { if } k \equiv 1(\bmod 4), \\ \frac{k-2}{4}, & \text { if } k \equiv 2(\bmod 4), \\ \frac{k+1}{4}, & \text { if } k \equiv 3(\bmod 4) .\end{cases}$

Proof. (i) This identity is well-known and is easily verified by considering $k$ modulo 2 .

(ii) This is easily checked by considering $k$ modulo 2 .

(iii) This is easily checked by considering $k$ modulo 4 .

(iv) This follows from (iii) by replacing $k$ by $k+1$.

We next define an arithmetic function that will be useful throughout this thesis.

Definition 3.2.1. Let $n \in \mathbb{N}_{0}$. For $r \in \mathbb{N}_{0}$ and $s \in \mathbb{N}$ with $0 \leq r<s$, we define the arithmetic function $\varepsilon_{r, s}(n)$ by

$$
\varepsilon_{r, s}(n)= \begin{cases}0, & \text { if } n \equiv r(\bmod s) \\ 1, & \text { otherwise }\end{cases}
$$


CHAPTER 3. THE FUNCTION $\left(\frac{1+(1-X)^{1 / 2}}{2}\right)^{R}\left(\frac{1+(1-X)^{1 / 4}}{2}\right)^{S}$

To conclude this section, we state and prove a lemma that we will need in the proof of Lemma 3.3.3.

Lemma 3.2.3. For $s \in \mathbb{N}_{0}$, we have

(i) $(1+i)^{s}+(1-i)^{s}=(-1)^{\left[\frac{s+1}{4}\right]} 2^{\left[\frac{s+2}{2}\right]} \varepsilon_{2,4}(s)$,

(ii) $(1+i)^{s}-(1-i)^{s}=(-1)^{\left[\frac{s}{4}\right]} 2^{\left[\frac{s+2}{2}\right]} i \varepsilon_{0,4}(s)$.

Proof. As $(1+i)^{2}=2 i,(1+i)^{3}=-2(1-i)$ and $(1+i)^{4}=-4$, we have

$$
(1+i)^{s}= \begin{cases}(-1)^{\frac{s}{4}} 2^{\frac{s}{2}}, & \text { if } s \equiv 0(\bmod 4) \\ (-1)^{\frac{s-1}{4}} 2^{\frac{s-1}{2}}(1+i), & \text { if } s \equiv 1(\bmod 4) \\ (-1)^{\frac{s-2}{4}} 2^{\frac{s}{2}} i, & \text { if } s \equiv 2(\bmod 4) \\ -(-1)^{\frac{s-3}{4}} 2^{\frac{s-1}{2}}(1-i), & \text { if } s \equiv 3(\bmod 4)\end{cases}
$$

Similarly, as $(1-i)^{2}=-2 i,(1-i)^{3}=-2(1+i)$ and $(1-i)^{4}=-4$, we have

$$
(1-i)^{s}= \begin{cases}(-1)^{\frac{s}{4}} 2^{\frac{s}{2}}, & \text { if } s \equiv 0(\bmod 4), \\ (-1)^{\frac{s-1}{4}} 2^{\frac{s-1}{2}}(1-i), & \text { if } s \equiv 1(\bmod 4), \\ -(-1)^{\frac{s-2}{4}} 2^{\frac{s}{2}} i, & \text { if } s \equiv 2(\bmod 4), \\ -(-1)^{\frac{s-3}{4}} 2^{\frac{s-1}{2}}(1+i), & \text { if } s \equiv 3(\bmod 4) .\end{cases}
$$

This implies that

$$
(1+i)^{s}+(1-i)^{s}= \begin{cases}(-1)^{\frac{s}{4} 2^{\frac{s+2}{2}},} & \text { if } s \equiv 0(\bmod 4) \\ (-1)^{\frac{s-1}{4}} 2^{\frac{s+1}{2}}, & \text { if } s \equiv 1(\bmod 4) \\ 0, & \text { if } s \equiv 2(\bmod 4) \\ (-1)^{\frac{s+1}{4}} 2^{\frac{s+1}{2}}, & \text { if } s \equiv 3(\bmod 4)\end{cases}
$$


CHAPTER 3. THE FUNCTION $\left(\frac{1+(1-X)^{1 / 2}}{2}\right)^{R}\left(\frac{1+(1-X)^{1 / 4}}{2}\right)^{S}$

$$
=(-1)^{\left[\frac{s+1}{4}\right]} 2^{\left[\frac{s+2}{2}\right]} \varepsilon_{2,4}(s)
$$

by Definition 3.2.1 and Lemma 3.2.2(ii)(iv). Similarly, appealing to Definition 3.2.1 and Lemma 3.2.2(ii)(iii), we find that

$$
\begin{aligned}
& (1+i)^{s}-(1-i)^{s}= \begin{cases}0, & \text { if } s \equiv 0(\bmod 4), \\
(-1)^{\frac{s-1}{4}} 2^{\frac{s+1}{2}} i, & \text { if } s \equiv 1(\bmod 4), \\
(-1)^{\frac{s-2}{4} 2^{\frac{s+2}{2}} i,} & \text { if } s \equiv 2(\bmod 4), \\
(-1)^{\frac{s-3}{4} 2^{\frac{s+1}{2}} i,} & \text { if } s \equiv 3(\bmod 4),\end{cases} \\
& =(-1)^{\left[\frac{s}{4}\right]} 2^{\left[\frac{s+2}{2}\right]} \varepsilon_{0,4}(s) i,
\end{aligned}
$$

as required.

\subsection{The values of $A_{r, s}(0), B_{r, s}(0), C_{r, s}(0), D_{r, s}(0)$}

We are now ready to evaluate the four polynomials of Definition 3.1.1 when $x=0$. When $r=s=0$ we have

$$
A_{0,0}(x)=1, B_{0,0}(x)=C_{0,0}(x)=D_{0,0}(x)=0
$$

so

$$
A_{0,0}(0)=1, B_{0,0}(0)=C_{0,0}(0)=D_{0,0}(0)=0 .
$$

Thus we need only consider those $r$ and $s$ for which either $r \geq 1$ or $s \geq 1$. We consider three cases: 
CHAPTER 3. THE FUNCTION $\left(\frac{1+(1-X)^{1 / 2}}{2}\right)^{R}\left(\frac{1+(1-X)^{1 / 4}}{2}\right)^{S}$

(i) $r \geq 1, s=0 \quad$ (Lemma 3.3.1),

(ii) $r \geq 1, s \geq 1$ (Lemma 3.3.2).

(iii) $r=0, s \geq 1 \quad$ (Lemma 3.3.3),

We begin with case (i), namely $r \geq 1$ and $s=0$.

Lemma 3.3.1. For $r \in \mathbb{N}$, we have

(i) $A_{r, 0}(0)=\frac{1}{2}$

(ii) $B_{r, 0}(0)=0$,

(iii) $C_{r, 0}(0)=\frac{1}{2}$,

(iv) $D_{r, 0}(0)=0$.

Proof. Setting $r \geq 1$ and $s=0$ in Lemma 3.1 .5 we have

$$
\begin{aligned}
& A_{r, 0}(0)=\frac{1}{4}(1+1)=\frac{1}{2} \\
& B_{r, 0}(0)=\frac{1}{4}(1-1)=0 \\
& C_{r, 0}(0)=\frac{1}{4}(1+1)=\frac{1}{2} \\
& D_{r, 0}(0)=\frac{1}{4}(1-1)=0
\end{aligned}
$$

completing the proof of the lemma. 
CHAPTER 3. THE FUNCTION $\left(\frac{1+(1-X)^{1 / 2}}{2}\right)^{R}\left(\frac{1+(1-X)^{1 / 4}}{2}\right)^{S}$

Next we turn to case (ii), namely $r \geq 1, s \geq 1$.

Lemma 3.3.2. For $r, s \in \mathbb{N}$, we have

$$
A_{r, s}(0)=B_{r, s}(0)=C_{r, s}(0)=D_{r, s}(0)=\frac{1}{4}
$$

Proof. Setting $r \geq 1$ and $s \geq 1$ in Lemma 3.1 .5 we have

$$
\begin{aligned}
& A_{r, s}(0)=\frac{1}{4}(1+0+0+0)=\frac{1}{4} \\
& B_{r, s}(0)=\frac{1}{4}(1-i \cdot 0-0+i \cdot 0)=\frac{1}{4}, \\
& C_{r, s}(0)=\frac{1}{4}(1-0+0-0)=\frac{1}{4}, \\
& D_{r, s}(0)=\frac{1}{4}(1+i \cdot 0-0-i \cdot 0)=\frac{1}{4}
\end{aligned}
$$

as asserted.

Finally, we turn to case (iii), namely $r=0, s \geq 1$.

Lemma 3.3.3. For $s \in \mathbb{N}$, we have

(i) $A_{0, s}(0)=\frac{1}{4}+\frac{(-1)^{\left[\frac{s+1}{4}\right]} \varepsilon_{2,4}(s)}{2^{\left[\frac{s+1}{2}\right]+1}}$,

(ii) $B_{0, s}(0)=\frac{1}{4}+\frac{(-1)^{\left[\frac{s}{4}\right]} \varepsilon_{0,4}(s)}{2^{\left[\frac{s+1}{2}\right]+1}}$,

(iii) $C_{0, s}(0)=\frac{1}{4}-\frac{(-1)^{\left[\frac{s+1}{4}\right]} \varepsilon_{2,4}(s)}{2^{\left[\frac{s+1}{2}\right]+1}}$, 
CHAPTER 3. THE FUNCTION $\left(\frac{1+(1-X)^{1 / 2}}{2}\right)^{R}\left(\frac{1+(1-X)^{1 / 4}}{2}\right)^{S}$

(iv) $D_{0, s}(0)=\frac{1}{4}-\frac{(-1)^{\left[\frac{s}{4}\right]} \varepsilon_{0,4}(s)}{2^{\left[\frac{s+1}{2}\right]+1}}$.

Proof. By Lemmas 3.1.5, 3.2.2 and 3.2.3 we have

$$
\begin{aligned}
& A_{0, s}(0)=\frac{1}{4}\left(1+\left(\frac{1+i}{2}\right)^{s}+\left(\frac{1-i}{2}\right)^{s}\right)=\frac{1}{4}\left(1+\frac{1}{2^{s}}\left((1+i)^{s}+(1-i)^{s}\right)\right) \\
& =\frac{1}{4}+\frac{(-1)^{\left[\frac{s+1}{4}\right]} 2^{\left[\frac{s+2}{2}\right]} \varepsilon_{2,4}(s)}{2^{s+2}}=\frac{1}{4}+\frac{(-1)^{\left[\frac{s+1}{4}\right]} \varepsilon_{2,4}(s)}{2^{\left[\frac{s+1}{2}\right]+1}} \\
& B_{0, s}(0)=\frac{1}{4}\left(1-i\left(\frac{1+i}{2}\right)^{s}+i\left(\frac{1-i}{2}\right)^{s}\right)=\frac{1}{4}\left(1-\frac{i}{2^{s}}\left((1+i)^{s}-(1-i)^{s}\right)\right) \\
& =\frac{1}{4}+\frac{(-1)^{\left[\frac{s}{4}\right]} 2^{\left[\frac{s+2}{2}\right]} \varepsilon_{0,4}(s)}{2^{s+2}}=\frac{1}{4}+\frac{(-1)^{\left[\frac{s}{4}\right]} \varepsilon_{0,4}(s)}{2^{\left[\frac{s+1}{2}\right]+1}} \\
& C_{0, s}(0)=\frac{1}{4}\left(1-\left(\frac{1+i}{2}\right)^{s}-\left(\frac{1-i}{2}\right)^{s}\right) \\
& =\frac{1}{4}-\frac{(-1)^{\left[\frac{s+1}{4}\right]} 2^{\left[\frac{s+2}{2}\right]} \varepsilon_{2,4}(s)}{2^{s+2}}=\frac{1}{4}-\frac{(-1)^{\left[\frac{s+1}{4}\right]} \varepsilon_{2,4}(s)}{2^{\left[\frac{s+1}{2}\right]+1}}, \\
& D_{0, s}(0)=\frac{1}{4}\left(1+i\left(\frac{1+i}{2}\right)^{s}-i\left(\frac{1-i}{2}\right)^{s}\right)=\frac{1}{4}\left(1+\frac{i}{2^{s}}\left((1+i)^{s}-(1-i)^{s}\right)\right) \\
& =\frac{1}{4}-\frac{(-1)^{\left[\frac{s}{4}\right]} 2^{\left[\frac{s+2}{2}\right]} \varepsilon_{0,4}(s)}{2^{s+2}}=\frac{1}{4}-\frac{(-1)^{\left[\frac{s}{4}\right]} \varepsilon_{0,4}(s)}{2^{\left[\frac{s+1}{2}\right]+1}} .
\end{aligned}
$$

This completes the proof of the lemma. 


\section{Chapter 4}

\section{The functions $e_{k, r}(x)$}

Throughout this chapter $x$ denotes a complex variable, except in Section 4.4 where $x$ is taken to be $1-\frac{\varphi^{4}(-q)}{\varphi^{4}(q)}$.

In this chapter we define the polynomials $e_{k, 1}(x), e_{k, 2}(x), e_{k, 4}(x)$ (Section 4.1) and functions $e_{k, 8}(x), e_{k, 16}(x)$ (Sections 4.2 and 4.3) for $k \in \mathbb{N}$ with $k \geq 2$ and show in Section 4.4 that

$$
E_{k}\left(q^{r}\right)=e_{k, r}(x) z^{2 k}, k \geq 2, r \in\{1,2,4,8,16\}
$$

with

$$
x=1-\frac{\varphi^{4}(-q)}{\varphi^{4}(q)}, \quad z=\varphi^{2}(q) .
$$

We note that the exponent of $z$ in (4.0.1) is the weight of the Eisenstein series $E_{k}$.

\subsection{The polynomials $e_{k, 1}(x), e_{k, 2}(x)$ and $e_{k, 4}(x)$}

We begin by defining the polynomials $e_{k, 1}(x), e_{k, 2}(x)$ and $e_{k, 4}(x)$.

Definition 4.1.1. For $k \in \mathbb{N}$ with $k \geq 2$ we define

$$
e_{k, 1}(x):=\sum_{\substack{(t, u) \in \mathbb{N}_{0}^{2} \\ 2 t+3 u=k}} a_{k}(t, u)\left(1+14 x+x^{2}\right)^{t}\left(1-33 x-33 x^{2}+x^{3}\right)^{u},
$$




$$
\begin{aligned}
& e_{k, 2}(x):=\sum_{\substack{(t, u) \in \mathbb{N}_{0}^{2} \\
2 t+3 u=k}} a_{k}(t, u)\left(1-x+x^{2}\right)^{t}\left(1-\frac{3}{2} x-\frac{3}{2} x^{2}+x^{3}\right)^{u}, \\
& e_{k, 4}(x):=\sum_{\substack{(t, u) \in \mathbb{N}_{0}^{2} \\
2 t+3 u=k}} a_{k}(t, u)\left(1-x+\frac{1}{16} x^{2}\right)^{t}\left(1-\frac{3}{2} x+\frac{15}{32} x^{2}+\frac{1}{64} x^{3}\right)^{u},
\end{aligned}
$$

where the coefficients $a_{k}(t, u)$ are given in Theorem 2.9.1.

The objective of this section is to determine the values of $e_{k, r}(0)$ and $e_{k, r}(1)$ for $k \geq 2$ and $r \in\{1,2,4\}$. We begin with the polynomial $e_{k, 1}(x)$.

Lemma 4.1.1. For $k \in \mathbb{N}$ with $k \geq 2$, we have

(i) $e_{k, 1}(0)=1$,

(ii) $e_{k, 1}(1)=(-1)^{k} 2^{2 k}$,

(iii) $\operatorname{deg}\left(e_{k, 1}(x)\right)=k$,

(iv) the coefficient of $x^{k}$ in $e_{k, 1}(x)$ is 1 .

Proof. (i) By (4.1.1) and (2.9.1) we have

$$
e_{k, 1}(0)=\sum_{\substack{(t, u) \in \mathbb{N}_{0}^{2} \\ 2 t+3 u=k}} a_{k}(t, u)(1)^{t}(1)^{u}=\sum_{\substack{(t, u) \in \mathbb{N}_{0}^{2} \\ 2 t+3 u=k}} a_{k}(t, u)=1 .
$$

(ii) By (4.1.1) and (2.9.1) we have

$$
e_{k, 1}(1)=\sum_{\substack{(t, u) \in \mathbb{N}_{0}^{2} \\ 2 t+3 u=k}} a_{k}(t, u) 2^{4 t}(-1)^{u} 2^{6 u}=(-1)^{k} 2^{2 k} .
$$

as $2 t+3 u=k$ implies $u \equiv k(\bmod 2)$ so $(-1)^{u}=(-1)^{k}$.

(iii) (iv) For each $(t, u) \in \mathbb{N}_{0}^{2}$ with $2 t+3 u=k$, we have

$$
\operatorname{deg}\left(\left(1+14 x+x^{2}\right)^{t}\left(1-33 x-33 x^{2}+x^{3}\right)^{u}\right)=k,
$$


and the coefficient of $x^{k}$ in $\left(1+14 x+x^{2}\right)^{t}\left(1-33 x-33 x^{2}+x^{3}\right)^{u}$ is 1 . Thus by (4.1.1) the coefficient of $x^{k}$ in $e_{k, 1}(x)$ is

$$
\sum_{\substack{(t, u) \in \mathbb{N}_{0}^{2} \\ 2 t+3 u=k}} a_{k}(t, u)=1
$$

and

$$
\operatorname{deg}\left(e_{k, 1}(x)\right)=k
$$

This completes the proof of Lemma 4.1.1.

The next lemma gives the corresponding results for $e_{k, 2}(x)$.

Lemma 4.1.2. For $k \in \mathbb{N}$ with $k \geq 2$, we have

(i) $e_{k, 2}(0)=1$,

(ii) $e_{k, 2}(1)=(-1)^{k}$,

(iii) $\operatorname{deg}\left(e_{k, 2}(x)\right)=k$,

(iv) the coefficient of $x^{k}$ in $e_{k, 2}(x)$ is 1 .

Proof. By (4.1.2) and (2.9.1) we have

$$
e_{k, 2}(0)=\sum_{\substack{(t, u) \in \mathbb{N}_{0}^{2} \\ 2 t+3 u=k}} a_{k}(t, u)(1)^{t}(1)^{u}=\sum_{\substack{(t, u) \in \mathbb{N}_{0}^{2} \\ 2 t+3 u=k}} a_{k}(t, u)=1 .
$$

(ii) By (4.1.2) and (2.9.1) we have

$$
e_{k, 2}(1)=\sum_{\substack{(t, u) \in \mathbb{N}_{0}^{2} \\ 2 t+3 u=k}} a_{k}(t, u)(1)^{t}(-1)^{u}=(-1)^{k} \sum_{\substack{(t, u) \in \mathbb{N}_{0}^{2} \\ 2 t+3 u=k}} a_{k}(t, u)=(-1)^{k} .
$$

(iii) (iv) For each $(t, u) \in \mathbb{N}_{0}$ with $2 t+3 u=k$, we have

$$
\operatorname{deg}\left(\left(1-x+x^{2}\right)^{t}\left(1-\frac{3}{2} x-\frac{3}{2} x^{2}+x^{3}\right)^{u}\right)=k,
$$


and the coefficient of $x^{k}$ in $\left(1-x+x^{2}\right)^{t}\left(1-\frac{3}{2} x-\frac{3}{2} x^{2}+x^{3}\right)^{u}$ is 1 . Thus, by (4.1.2), the coefficient of $x^{k}$ in $e_{k, 2}(x)$ is

$$
\sum_{\substack{(t, u) \in \mathbb{N}_{0}^{2} \\ 2 t+3 u=k}} a_{k}(t, u)=1
$$

and

$$
\operatorname{deg}\left(e_{k, 2}(x)\right)=k
$$

This completes the proof of Lemma 4.1.2

Our final lemma of this section gives the corresponding results for $e_{k, 4}(x)$.

Lemma 4.1.3. For $k \in \mathbb{N}$ with $k \geq 2$, we have

(i) $e_{k, 4}(0)=1$,

(ii) $e_{k, 4}(1)=\frac{(-1)^{k}}{2^{2 k}}$

(iii) $\operatorname{deg}\left(e_{k, 4}(x)\right)=k$,

(iv) the coefficient of $x^{k}$ in $e_{k, 4}(x)$ is $\frac{1}{2^{2 k}}$.

Proof. (i) By (4.1.3) and (2.9.1) we have

$$
e_{k, 4}(0)=\sum_{\substack{(t, u) \in \mathbb{N}_{0}^{2} \\ 2 t+3 u=k}} a_{k}(t, u)(1)^{t}(1)^{u}=\sum_{\substack{(t, u) \in \mathbb{N}_{0}^{2} \\ 2 t+3 u=k}} a_{k}(t, u)=1 .
$$

(ii) By (4.1.3) and (2.9.1) we have

$$
e_{k, 4}(1)=\sum_{\substack{(t, u) \in \mathbb{N}_{0}^{2} \\ 2 t+3 u=k}} a_{k}(t, u)(2)^{-4 t}(-1)^{u}(2)^{-6 u}=\frac{(-1)^{k}}{2^{2 k}} \sum_{\substack{(t, u) \in \mathbb{N}_{0}^{2} \\ 2 t+3 u=k}} a_{k}(t, u)=\frac{(-1)^{k}}{2^{2 k}} .
$$

(iii) (iv) For each $(t, u) \in \mathbb{N}_{0}^{2}$ with $2 t+3 u=k$, we have

$$
\operatorname{deg}\left(\left(1-x+\frac{1}{16} x^{2}\right)^{t}\left(1-\frac{3}{2} x+\frac{15}{32} x^{2}+\frac{1}{64} x^{3}\right)^{u}\right)=k
$$


and the coefficient of $x^{k}$ in $\left(1-x+\frac{1}{16} x^{2}\right)^{t}\left(1-\frac{3}{2} x+\frac{15}{32} x^{2}+\frac{1}{64} x^{3}\right)^{u}$ is $\frac{1}{16^{t} 64^{u}}=$ $\frac{1}{2^{4 t+6 u}}=\frac{1}{2^{k}}$. Thus by (4.1.3) the coefficient of $x^{k}$ in $e_{k, 4}(x)$ is

$$
\sum_{\substack{(t, u) \in \mathbb{N}_{0}^{2} \\ 2 t+3 u=k}} \frac{a_{k}(t, u)}{2^{2 k}}=\frac{1}{2^{2 k}}
$$

and

$$
\operatorname{deg}\left(e_{k, 2}(x)\right)=k
$$

This completes the proof of Lemma 4.1.3.

\subsection{The function $e_{k, 8}(x)$}

In this section we define the function $e_{k, 8}(x)$ for $k \in \mathbb{N}$ with $k \geq 2$.

Definition 4.2.1. For $k \in \mathbb{N}$ with $k \geq 2$, we define

$$
\begin{aligned}
& e_{k, 8}(x):=\frac{1}{2^{4 k}} \sum_{\substack{(t, u) \in \mathbb{N}_{0}^{2} \\
2 t+3 u=k}} a_{k}(t, u)\left(136-136 x+x^{2}+(120-60 x)(1-x)^{1 / 2}\right)^{t} \\
& \times\left(2080-3120 x+1038 x^{2}+x^{3}+\left(2016-2016 x+126 x^{2}\right)(1-x)^{1 / 2}\right)^{u} \\
& \quad=e_{k, 8}^{(0)}(x)+e_{k, 8}^{(1)}(x)(1-x)^{1 / 2}
\end{aligned}
$$

where $e_{k, 8}^{(0)}(x), e_{k, 8}^{(1)}(x) \in \mathbb{Q}[x]$ and the coefficients $a_{k}(t, u)$ are given in Theorem 2.9.1.

Taking $k=2$ in (4.2.1) we see that (as $t=1, u=0, a_{2}(1,0)=1$ )

$$
e_{2,8}^{(0)}(x)=\frac{136-136 x+x^{2}}{256}
$$


and

$$
e_{2,8}^{(1)}(x)=\frac{120-60 x}{256}=\frac{30-15 x}{64}
$$

Taking $k=3$ in (4.2.1) we see that (as $t=0, u=1, a_{3}(0,1)=1$ )

$$
e_{3,8}^{(0)}(x)=\frac{2080-3120 x+1038 x^{2}+x^{3}}{4096}
$$

and

$$
e_{3,8}^{(1)}(x)=\frac{2016-2016 x+126 x^{2}}{4096}=\frac{1008-1008 x+63 x^{2}}{2048}
$$

The next lemma gives the values of the polynomials $e_{k, 8}^{(0)}(x)$ and $e_{k, 8}^{(1)}(x)$ at $x=0$.

Lemma 4.2.1. For $k \in \mathbb{N}$ with $k \geq 2$, we have

(i) $e_{k, 8}(0)=1$,

(ii) $e_{k, 8}^{(0)}(0)=\frac{2^{2 k}+1}{2^{2 k+1}}$,

(iii) $e_{k, 8}^{(1)}(0)=\frac{2^{2 k}-1}{2^{2 k+1}}$.

Proof. We have by (4.2.1), (4.2.2) and (2.9.1)

$$
\begin{aligned}
e_{k, 8}(0) & =e_{k, 8}^{(0)}(0)+e_{k, 8}^{(1)}(0)=\frac{1}{2^{4 k}} \sum_{\substack{(t, u) \in \mathbb{N}_{0}^{2} \\
2 t+3 u=k}} a_{k}(t, u)(256)^{t}(4096)^{u} \\
& =\frac{1}{2^{4 k}} \sum_{\substack{(t, u) \in \mathbb{N}_{0}^{2} \\
2 t+3 u=k}} a_{k}(t, u) 2^{8 t} 2^{12 u}=\frac{1}{2^{4 k}} \sum_{\substack{(t, u) \in \mathbb{N}_{0}^{2} \\
2 t+3 u=k}} a_{k}(t, u) 2^{4 k}=\sum_{\substack{(t, u) \in \mathbb{N}_{0}^{2} \\
2 t+3 u=k}} a_{k}(t, u)=1,
\end{aligned}
$$

which proves (i).

By Definition 4.2.1, we have

$$
2 e_{k, 8}^{(0)}(x)=\frac{1}{2^{4 k}} \sum_{\substack{(t, u) \in \mathbb{N}_{0}^{2} \\ 2 t+3 u=k}} a_{k}(t, u)\left(\left(136-136 x+x^{2}+(120-60 x)(1-x)^{1 / 2}\right)^{t}\right.
$$




$$
\begin{aligned}
& \times\left(2080-3120 x+1038 x^{2}+x^{3}+\left(2016-2016 x+126 x^{2}\right)(1-x)^{1 / 2}\right)^{u} \\
& +\left(136-136 x+x^{2}-(120-60 x)(1-x)^{1 / 2}\right)^{t} \\
& \left.\times\left(2080-3120 x+1038 x^{2}+x^{3}-\left(2016-2016 x+126 x^{2}\right)(1-x)^{1 / 2}\right)^{u}\right) .
\end{aligned}
$$

Thus $2 e_{k, 8}^{(0)}(0)=\frac{1}{2^{4 k}} \sum_{\substack{(t, u) \in \mathbb{N}_{0}^{2} \\ 2 t+3 u=k}} a_{k}(t, u)\left(2^{8 t+12 u}+2^{4 t+6 u}\right)=\frac{1}{2^{4 k}}\left(2^{4 k}+2^{2 k}\right)=\frac{2^{2 k}+1}{2^{2 k}}$, so that $e_{k, 8}^{(0)}(0)=\frac{2^{2 k}+1}{2^{2 k+1}}$, which is (ii).

Finally we deduce

$$
e_{k, 8}^{(1)}(0)=e_{k, 8}(0)-e_{k, 8}^{(0)}(0)=1-\left(\frac{1}{2}+\frac{1}{2^{2 k+1}}\right)=\frac{2^{2 k}-1}{2^{2 k+1}},
$$

which is (iii).

The next lemma gives the value of the polynomial $e_{k, 0}^{(0)}(x)$ at $x=1$.

Lemma 4.2.2. For $k \in \mathbb{N}$ with $k \geq 2$, we have

$$
e_{k, 8}(1)=e_{k, 8}^{(0)}(1)=\frac{(-1)^{k}}{2^{4 k}}
$$

Proof. We first note that by (4.2.2), we have $e_{k, 8}(1)=e_{k, 8}^{(0)}(1)+e_{k, 8}^{(1)}(1) \sqrt{1-1}=$ $e_{k, 8}^{(0)}(1)$.

Moreover, by (2.9.1) and (4.2.1), we have

$$
e_{k, 8}(1)=\frac{1}{2^{4 k}} \sum_{\substack{(t, u) \in \mathbb{N}_{0}^{2} \\ 2 t+3 u=k}} a_{k}(t, u)(1)^{t}(-1)^{u}=\frac{(-1)^{k}}{2^{4 k}},
$$

completing the proof of Lemma 4.2.2. 
We conclude this section with a lemma that gives information about the degrees of the polynomials $e_{k, 8}^{(0)}(x)$ and $e_{k, 8}^{(1)}(x)$.

Lemma 4.2.3. For $k \in \mathbb{N}$ with $k \geq 2$, we have

(i) $\operatorname{deg}\left(e_{k, 8}^{(0)}(x)\right)=k$,

(ii) $\operatorname{deg}\left(e_{k, 8}^{(1)}(x)\right) \leq k-1$,

(iii) the coefficient of $x^{k}$ in $e_{k, 8}^{(0)}(x)$ is $\frac{1}{2^{4 k}}$.

Proof. Using the binomial theorem, the right hand side of (4.2.1) becomes

$$
\begin{aligned}
& \frac{1}{2^{4 k}} \sum_{\substack{(t, u) \in \mathbb{N}_{0}^{2} \\
2 t+3 u=k}} a_{k}(t, u) \sum_{\substack{(d, e) \in \mathbb{N}_{0}^{2} \\
d+e=t}}\left(\begin{array}{l}
t \\
d
\end{array}\right)\left(136-136 x+x^{2}\right)^{d}(120-60 x)^{e}(1-x)^{e / 2} \\
& \quad \times \sum_{\substack{(f, g) \in \mathbb{N}_{0}^{2} \\
f+g=u}}\left(\begin{array}{c}
u \\
f
\end{array}\right)\left(2080-3120 x+1038 x^{2}+x^{3}\right)^{f}\left(2016-2016 x+126 x^{2}\right)^{g}(1-x)^{g / 2} \\
& =\frac{1}{2^{4 k}} \sum_{\substack{(d, e, f, g) \in \mathbb{N}_{0}^{4} \\
2 d+2 e+3 f+3 g=k}} a_{k}(d+e, f+g)\left(\begin{array}{c}
d+e \\
d
\end{array}\right)\left(\begin{array}{c}
f+g \\
f
\end{array}\right)\left(136-136 x+x^{2}\right)^{d}(120-60 x)^{e} \\
& \quad \times\left(2080-3120 x+1038 x^{2}+x^{3}\right)^{f}\left(2016-2016 x+126 x^{2}\right)^{g}(1-x)^{(e+g) / 2} .
\end{aligned}
$$

Thus

$$
e_{k, 8}^{(1)}(x)=\frac{1}{2^{4 k}} \sum_{\substack{(d, e, f, g) \in \mathbb{N}_{0}^{4} \\
2 d+2 e+3 f+3=k \\
e+g \equiv 1(\bmod 2)}} a_{k}(d+e, f+g)\left(\begin{array}{c}
d+e \\
d
\end{array}\right)\left(\begin{array}{c}
f+g \\
f
\end{array}\right)\left(136-136 x+x^{2}\right)^{d}(120-60 x)^{e}
$$




$$
\times\left(2080-3120 x+1038 x^{2}+x^{3}\right)^{f}\left(2016-2016 x+126 x^{2}\right)^{g}(1-x)^{(e+g-1) / 2} .
$$

The highest power of $x$ in $e_{k, 8}^{(1)}(x)$ is

$$
\begin{aligned}
& \max _{\substack{(d, e, f, g) \in \mathbb{N}_{0}^{4} \\
2 d+2 e+3 f+3 g=k \\
e+g \equiv 1(\bmod 2)}}\left(2 d+e+3 f+2 g+\frac{e+g-1}{2}\right)=\frac{1}{2} \max _{\substack{d, e, f, g \geq 0 \\
2 d+2 e+3 f+3 g=k \\
e+g \equiv 1(\bmod 2)}}(4 d+2 e+6 f+4 g+e+g-1) \\
& =\frac{1}{2} \max _{\substack{d, e, f, g \geq 0 \\
2 d+2 e+3 f+3 g=k \\
e+g \equiv 1(\bmod 2)}}(4 d+3 e+6 f+5 g-1)=\frac{1}{2} \max _{\substack{d, e, f, g \geq 0 \\
2 d+2 e+3 f+3 g=k \\
e+g \equiv 1(\bmod 2)}}(2 k-e-g-1) \\
& =\frac{1}{2}(2 k-2)=k-1,
\end{aligned}
$$

proving (ii).

Also

$$
\begin{aligned}
e_{k, 8}^{(0)}(x)= & \frac{1}{2^{4 k}} \sum_{\substack{(d, e, f, g) \in \mathbb{N}_{0}^{4} \\
2 d+2 e+3 f+3 g=k \\
e+g \equiv 0(\bmod 2)}} a_{k}(d+e, f+g)\left(\begin{array}{c}
d+e \\
d
\end{array}\right)\left(\begin{array}{c}
f+g \\
f
\end{array}\right) \\
& \times\left(136-136 x+x^{2}\right)^{d}(120-60 x)^{e} \\
& \times\left(2080-3120 x+1038 x^{2}+x^{3}\right)^{f} \\
& \times\left(2016-2016 x+126 x^{2}\right)^{g}(1-x)^{(e+g) / 2} .
\end{aligned}
$$


The highest power of $x$ in $e_{k, 8}^{(0)}(x)$ is

$$
\begin{aligned}
& \max _{\substack{(d, e, f, g) \in \mathbb{N}_{0}^{4} \\
2 d+2 e+3 f+3 g=k \\
e+g \equiv 0(\bmod 2)}}\left(2 d+e+3 f+2 g+\frac{e+g}{2}\right)=\frac{1}{2} \max _{\substack{d, e, f, g \geq 0 \\
2 d+2 e+3 f+3 g=k \\
e+g \equiv 0(\bmod 2)}}(4 d+3 e+6 f+5 g) \\
& =\frac{1}{2} \max _{\substack{d, e, f, g \geq 0 \\
2 d+2 e+3 f+3 g=k \\
e+g \equiv 0(\bmod 2)}}(2 k-e-g)=\frac{1}{2} 2 k=k .
\end{aligned}
$$

From the last equation we note that the highest power of $e_{k, 8}^{(0)}(x)$ occurs when $e+g=0$, that is when $e=g=0$. Thus by (4.2.4) and (2.9.1) the coefficient of $x^{k}$ in $e_{k, 8}^{(0)}(x)$ is

$$
\frac{1}{2^{4 k}} \sum_{\substack{(d, f) \in \mathbb{N}_{0}^{2} \\ 2 d+3 f=k}} a_{k}(d, f)=\frac{1}{2^{4 k}},
$$

proving (iii).

\subsection{The function $e_{k, 16}(x)$}

Definition 4.3.1. For $k \in \mathbb{N}$ with $k \geq 2$, we define

$$
\begin{gathered}
e_{k, 16}(x):=\frac{1}{2^{6 k}} \sum_{\substack{(t, u) \in \mathbb{N}_{0} \\
2 t+3 u=k}} a_{k}(t, u)\left(p_{2}(x)+q_{2}(x)(1-x)^{1 / 4}\right. \\
\left.\quad+r_{2}(x)(1-x)^{1 / 2}+s_{2}(x)(1-x)^{3 / 4}\right)^{t} \\
\times\left(p_{3}(x)+q_{3}(x)(1-x)^{1 / 4}+r_{3}(x)(1-x)^{1 / 2}+s_{3}(x)(1-x)^{3 / 4}\right)^{u} \\
=e_{k, 16}^{(0)}(x)+e_{k, 16}^{(1)}(x)(1-x)^{1 / 4}+e_{k, 16}^{(2)}(x)(1-x)^{1 / 2}+e_{k, 16}^{(3)}(x)(1-x)^{3 / 4},
\end{gathered}
$$

where

$$
p_{2}(x)=1096-1096 x+x^{2}
$$




$$
\begin{aligned}
& q_{2}(x)=960-840 x, \\
& r_{2}(x)=1080-540 x, \\
& s_{2}(x)=960-120 x, \\
& p_{3}(x)=66592-99888 x+33294 x^{2}+x^{3}, \\
& q_{3}(x)=64512-79632 x+15372 x^{2}, \\
& r_{3}(x)=66528-66528 x+4158 x^{2}, \\
& s_{3}(x)=64512-49332 x+252 x^{2},
\end{aligned}
$$

where $e_{k, 16}^{(j)}(x) \in \mathbb{Q}[x]$ for $j \in\{0,1,2,3\}$ and the coefficients $a_{k}(t, u)$ are given in Theorem 2.9.1.

We note that for $k=2$, we have

$$
\begin{aligned}
& e_{2,16}^{(0)}(x)=p_{2}(x)=1096-1096 x+x^{2}, \quad e_{2,16}^{(0)}(0)=p_{2}(0)=1096, \\
& e_{2,16}^{(1)}(x)=q_{2}(x)=960-840 x, \quad e_{2,16}^{(1)}(0)=q_{2}(0)=960, \\
& e_{2,16}^{(2)}(x)=r_{2}(x)=1080-540 x, \quad e_{2,16}^{(2)}(0)=r_{2}(0)=1080, \\
& e_{2,16}^{(3)}(x)=s_{2}(x)=960-120 x, \quad e_{2,16}^{(3)}(0)=s_{2}(0)=960, \\
& e_{3,16}^{(0)}(x)=p_{3}(x)=66592-99888 x+33294 x^{2}+x^{3}, \quad e_{3,16}^{(0)}(0)=66592, \\
& e_{3,16}^{(1)}(x)=q_{3}(x)=64512-79632 x+15372 x^{2}, \quad e_{3,16}^{(1)}(0)=q_{3}(0)=64512, \\
& e_{3,16}^{(2)}(x)=r_{3}(x)=66528-66528 x+4158 x^{2}, \quad e_{3,16}^{(2)}(0)=r_{3}(0)=66528,
\end{aligned}
$$




$$
e_{3,16}^{(3)}(x)=s_{3}(x)=64512-49332 x+252 x^{2}, \quad e_{3,16}^{(3)}(0)=s_{3}(0)=64512 \text {. }
$$

Lemma 4.3.1. For $k \in \mathbb{N}$ with $k \geq 2$, we have

(i)

$$
\begin{array}{r}
\frac{1}{2^{6 k}} \sum_{\substack{(t, u) \in \mathbb{N}_{0} \\
2 t+3 u=k}} a_{k}(t, u)\left(p_{2}(0)+q_{2}(0)+r_{2}(0)+s_{2}(0)\right)^{t} \\
\quad \times\left(p_{3}(0)+q_{3}(0)+r_{3}(0)+s_{3}(0)\right)^{u}=1
\end{array}
$$

(ii) $\frac{1}{2^{6 k}} \sum_{\substack{(t, u) \in \mathbb{N}_{0} \\ 2 t+3 u=k}} a_{k}(t, u)\left(p_{2}(0)+q_{2}(0)-r_{2}(0)-s_{2}(0)\right)^{t}$

$$
\times\left(p_{3}(0)+q_{3}(0)-r_{3}(0)-s_{3}(0)\right)^{u}=\frac{1}{2^{4 k}}
$$

(iii)

$$
\begin{aligned}
& \frac{1}{2^{6 k}} \sum_{\substack{(t, u) \in \mathbb{N}_{0} \\
2 t+3 u=k}} a_{k}(t, u)\left(p_{2}(0)-q_{2}(0)-r_{2}(0)+s_{2}(0)\right)^{t} \\
& \quad \times\left(p_{3}(0)-q_{3}(0)-r_{3}(0)+s_{3}(0)\right)^{u}=\frac{1}{2^{4 k}}
\end{aligned}
$$

$$
\text { (iv) } \begin{aligned}
\frac{1}{2^{6 k}} \sum_{\substack{(t, u) \in \mathbb{N}_{0} \\
2 t+3 u=k}} a_{k}(t, u)\left(p_{2}(0)-q_{2}(0)+r_{2}(0)-s_{2}(0)\right)^{t} \\
\\
\quad \times\left(p_{3}(0)-q_{3}(0)+r_{3}(0)-s_{3}(0)\right)^{u}=\frac{1}{2^{2 k}}
\end{aligned}
$$

Proof. We have

$$
\begin{aligned}
& \frac{1}{2^{6 k}} \sum_{\substack{(t, u) \in \mathbb{N}_{0} \\
2 t+3 u=k}} a_{k}(t, u)\left(p_{2}(0)+q_{2}(0)+r_{2}(0)+s_{2}(0)\right)^{t}\left(p_{3}(0)+q_{3}(0)+r_{3}(0)+s_{3}(0)\right)^{u} \\
& =\frac{1}{2^{6 k}} \sum_{\substack{(t, u) \in \mathbb{N}_{0} \\
2 t+3 u=k}} a_{k}(t, u)(1096+960+1080+960)^{t}(66592+64512+66528+64512)^{u}
\end{aligned}
$$




$$
\begin{aligned}
& =\frac{1}{2^{6 k}} \sum_{\substack{(t, u) \in \mathbb{N}_{0} \\
2 t+3 u=k}} a_{k}(t, u)(4096)^{t}(262144)^{u} \\
& =\frac{1}{2^{6 k}} \sum_{\substack{(t, u) \in \mathbb{N}_{0} \\
2 t+3 u=k}} a_{k}(t, u)(2)^{12 t}(2)^{18 u}=\frac{1}{2^{6 k}} \sum_{\substack{(t, u) \in \mathbb{N}_{0} \\
2 t+3 u=k}} a_{k}(t, u) 2^{6 k}=\sum_{\substack{(t, u) \in \mathbb{N}_{0} \\
2 t+3 u=k}} a_{k}(t, u)=1,
\end{aligned}
$$

which proves (i). Next

$$
\begin{aligned}
& \frac{1}{2^{6 k}} \sum_{\substack{(t, u) \in \mathbb{N}_{0} \\
2 t+3 u=k}} a_{k}(t, u)\left(p_{2}(0)+q_{2}(0)-r_{2}(0)-s_{2}(0)\right)^{t}\left(p_{3}(0)+q_{3}(0)-r_{3}(0)-s_{3}(0)\right)^{u} \\
& =\frac{1}{2^{6 k}} \sum_{\substack{(t, u) \in \mathbb{N}_{0} \\
2 t+3 u=k}} a_{k}(t, u)(1096+960-1080-960)^{t}(66592+64512-66528-64512)^{u} \\
& =\frac{1}{2^{6 k}} \sum_{(t, u) \in \mathbb{N}_{0}} a_{k}(t, u)(16)^{t}(64)^{u} \\
& =\frac{1}{2^{6 k}} \sum_{\substack{2 t+3 u=k \\
2 t, u) \in \mathbb{N}_{0}}} a_{k}(t, u)(2)^{4 t}(2)^{6 u}=\frac{1}{2^{6 k}} \sum_{\substack{(t, u) \in \mathbb{N}_{0} \\
2 t+3 u=k}} a_{k}(t, u) 2^{2 k}=\frac{1}{2^{4 k}} \sum_{\substack{(t, u) \in \mathbb{N}_{0} \\
2 t+3 u=k}} a_{k}(t, u)=\frac{1}{2^{4 k}}
\end{aligned}
$$

which proves (ii). Further

$$
\begin{aligned}
& \frac{1}{2^{6 k}} \sum_{\substack{(t, u) \in \mathbb{N}_{0} \\
2 t+3 u=k}} a_{k}(t, u)\left(p_{2}(0)-q_{2}(0)-r_{2}(0)+s_{2}(0)\right)^{t}\left(p_{3}(0)-q_{3}(0)-r_{3}(0)+s_{3}(0)\right)^{u} \\
& =\frac{1}{2^{6 k}} \sum_{\substack{(t, u) \in \mathbb{N}_{0} \\
2 t+3 u=k}} a_{k}(t, u)(1096-960-1080+960)^{t}(66592-64512-66528+64512)^{u} \\
& =\frac{1}{2^{6 k}} \sum_{(t, u) \in \mathbb{N}_{0}} a_{k}(t, u)(16)^{t}(64)^{u} \\
& =\frac{1}{2^{6 k}} \sum_{\substack{(t, u) \in \mathbb{N}_{0} \\
2 t+3 u=k}} a_{k}(t, u)(2)^{4 t}(2)^{6 u}=\frac{1}{2^{6 k}} \sum_{\substack{(t, u) \in \mathbb{N}_{0} \\
2 t+3 u=k}} a_{k}(t, u) 2^{2 k}=\frac{1}{2^{4 k}} \sum_{\substack{(t, u) \in \mathbb{N}_{0} \\
2 t+3 u=k}} a_{k}(t, u)=\frac{1}{2^{4 k}}
\end{aligned}
$$

which proves (iii). Finally 


$$
\begin{aligned}
& \frac{1}{2^{6 k}} \sum_{(t, u) \in \mathbb{N}_{0}} a_{k}(t, u)\left(p_{2}(0)-q_{2}(0)+r_{2}(0)-s_{2}(0)\right)^{t}\left(p_{3}(0)-q_{3}(0)+r_{3}(0)-s_{3}(0)\right)^{u} \\
& =\frac{1}{2^{6 k}} \sum_{(t, u) \in \mathbb{N}_{0}} a_{k}(t, u)(1096-960+1080-960)^{t}(66592-64512+66528-64512)^{u} \\
& =\frac{1}{2^{6 k}} \sum_{(t, u) \in \mathbb{N}_{0}}^{2 t+3 u=k} a_{k}(t, u)(256)^{t}(4096)^{u} \\
& =\frac{1}{2^{6 k}} \sum_{\substack{2 t+3 u=k \\
(t, u) \in \mathbb{N}_{0}}} a_{k}(t, u)(2)^{8 t}(2)^{12 u}=\frac{1}{2^{6 k}} \sum_{\substack{(t, u) \in \mathbb{N}_{0} \\
2 t+3 u=k}} a_{k}(t, u) 2^{4 k}=\frac{1}{2^{2 k}} \sum_{\substack{(t, u) \in \mathbb{N}_{0} \\
2 t+3 u=k}} a_{k}(t, u)=\frac{1}{2^{2 k}}
\end{aligned}
$$

which proves (iv).

The next lemma gives the values of the polynomials $e_{k, 16}^{(0)}(x), e_{k, 16}^{(1)}(x), e_{k, 16}^{(2)}(x)$ and $e_{k, 16}^{(3)}(x)$ at $x=0$.

Lemma 4.3.2. For $k \in \mathbb{N}$ with $k \geq 2$, we have

(i) $e_{k, 16}(0)=1$,

(ii) $e_{k, 16}^{(0)}(0)=\frac{2^{2 k-1}\left(2^{2 k}+1\right)+1}{2^{4 k+1}}$

(iii) $e_{k, 16}^{(1)}(0)=\frac{2^{2 k}-1}{2^{2 k+2}}$

(iv) $e_{k, 16}^{(2)}(0)=\frac{2^{2 k-1}\left(2^{2 k}+1\right)-1}{2^{4 k+1}}$

(v) $e_{k, 16}^{(3)}(0)=\frac{2^{2 k}-1}{2^{2 k+2}}$.

Proof. (i) By Definition 4.3.1 and Lemma 4.3.1(i), we have

$$
\begin{aligned}
& e_{k, 16}(0)=e_{k, 16}^{(0)}(0)+e_{k, 16}^{(1)}(0)+e_{k, 16}^{(2)}(0)+e_{k, 16}^{(3)}(0) \\
& =\frac{1}{2^{6 k}} \sum_{\substack{(t, u) \in \mathbb{N}_{0} \\
2 t+3 u=k}} a_{k}(t, u)\left(p_{2}(0)+q_{2}(0)+r_{2}(0)+s_{2}(0)\right)^{t}\left(p_{3}(0)+q_{3}(0)+r_{3}(0)+s_{3}(0)\right)^{u}
\end{aligned}
$$


$=1$.

(ii) By Definition 4.3.1 we have

$$
\begin{aligned}
& 4 e_{k, 16}^{(0)}(x)=e_{k, 16}^{(0)}(x)+e_{k, 16}^{(1)}(x)(1-x)^{1 / 4}+e_{k, 16}^{(2)}(x)(1-x)^{1 / 2}+e_{k, 16}^{(3)}(x)(1-x)^{3 / 4} \\
& +e_{k, 16}^{(0)}(x)-e_{k, 16}^{(1)}(x)(1-x)^{1 / 4}-e_{k, 16}^{(2)}(x)(1-x)^{1 / 2}+e_{k, 16}^{(3)}(x)(1-x)^{3 / 4} \\
& +e_{k, 16}^{(0)}(x)-e_{k, 16}^{(1)}(x)(1-x)^{1 / 4}+e_{k, 16}^{(2)}(x)(1-x)^{1 / 2}-e_{k, 16}^{(3)}(x)(1-x)^{3 / 4} \\
& +e_{k, 16}^{(0)}(x)+e_{k, 16}^{(1)}(x)(1-x)^{1 / 4}-e_{k, 16}^{(2)}(x)(1-x)^{1 / 2}-e_{k, 16}^{(3)}(x)(1-x)^{3 / 4} \\
& =\frac{1}{2^{6 k}} \sum_{\substack{(t, u) \in \mathbb{N}_{0}^{2} \\
2 t+3 u=k}} a_{k}(t, u)\left(p_{2}(x)+q_{2}(x)(1-x)^{1 / 4}+r_{2}(x)(1-x)^{1 / 2}+s_{2}(x)(1-x)^{3 / 4}\right)^{t} \\
& \times\left(p_{3}(x)+q_{3}(x)(1-x)^{1 / 4}+r_{3}(x)(1-x)^{1 / 2}+s_{3}(x)(1-x)^{3 / 4}\right)^{u} \\
& +\frac{1}{2^{6 k}} \sum_{\substack{(t, u) \in \mathbb{N}_{0}^{2} \\
2 t+3 u=k}} a_{k}(t, u)\left(p_{2}(x)-q_{2}(x)(1-x)^{1 / 4}-r_{2}(x)(1-x)^{1 / 2}+s_{2}(x)(1-x)^{3 / 4}\right)^{t} \\
& \times\left(p_{3}(x)-q_{3}(x)(1-x)^{1 / 4}-r_{3}(x)(1-x)^{1 / 2}+s_{3}(x)(1-x)^{3 / 4}\right)^{u} \\
& +\frac{1}{2^{6 k}} \sum_{\substack{(t, u) \in \mathbb{N}_{0}^{2} \\
2 t+3 u=k}} a_{k}(t, u)\left(p_{2}(x)-q_{2}(x)(1-x)^{1 / 4}+r_{2}(x)(1-x)^{1 / 2}-s_{2}(x)(1-x)^{3 / 4}\right)^{t} \\
& \times\left(p_{3}(x)-q_{3}(x)(1-x)^{1 / 4}+r_{3}(x)(1-x)^{1 / 2}-s_{3}(x)(1-x)^{3 / 4}\right)^{u} \\
& +\frac{1}{2^{6 k}} \sum_{\substack{(t, u) \in \mathbb{N}_{0}^{2} \\
2 t+3 u=k}} a_{k}(t, u)\left(p_{2}(x)+q_{2}(x)(1-x)^{1 / 4}-r_{2}(x)(1-x)^{1 / 2}-s_{2}(x)(1-x)^{3 / 4}\right)^{t} \\
& \times\left(p_{3}(x)+q_{3}(x)(1-x)^{1 / 4}-r_{3}(x)(1-x)^{1 / 2}-s_{3}(x)(1-x)^{3 / 4}\right)^{u} .
\end{aligned}
$$

Thus, by Lemma 4.3.1, we obtain

$$
\begin{aligned}
& 4 e_{k, 16}^{(0)}(0) \\
& =\frac{1}{2^{6 k}} \sum_{\substack{(t, u) \in \mathbb{N}_{0}^{2} \\
2 t+3 u=k}} a_{k}(t, u)\left(p_{2}(0)+q_{2}(0)+r_{2}(0)+s_{2}(0)\right)^{t}\left(p_{3}(0)+q_{3}(0)+r_{3}(0)+s_{3}(0)\right)^{u}
\end{aligned}
$$




$$
\begin{aligned}
& +\frac{1}{2^{6 k}} \sum_{\substack{(t, u) \in \mathbb{N}_{0}^{2} \\
2 t+3 u=k}} a_{k}(t, u)\left(p_{2}(0)-q_{2}(0)-r_{2}(0)+s_{2}(0)\right)^{t}\left(p_{3}(0)-q_{3}(0)-r_{3}(0)+s_{3}(0)\right)^{u} \\
& +\frac{1}{2^{6 k}} \sum_{\substack{(t, u) \in \mathbb{N}_{0}^{2} \\
2 t+3 u=k}} a_{k}(t, u)\left(p_{2}(0)-q_{2}(0)+r_{2}(0)-s_{2}(0)\right)^{t}\left(p_{3}(0)-q_{3}(0)+r_{3}(0)-s_{3}(0)\right)^{u} \\
& +\frac{1}{2^{6 k}} \sum_{\substack{(t, u) \in \mathbb{N}_{0}^{2} \\
2 t+3 u=k}} a_{k}(t, u)\left(p_{2}(0)+q_{2}(0)-r_{2}(0)-s_{2}(0)\right)^{t}\left(p_{3}(0)+q_{3}(0)-r_{3}(0)-s_{3}(0)\right)^{u} \\
& =1+\frac{1}{2^{4 k}}+\frac{1}{2^{2 k}}+\frac{1}{2^{4 k}}=\frac{2^{2 k-1}\left(2^{2 k}+1\right)+1}{2^{4 k-1}}
\end{aligned}
$$

Hence we have

$$
e_{k, 16}^{(0)}(0)=\frac{2^{2 k-1}\left(2^{2 k}+1\right)+1}{2^{4 k+1}} .
$$

(iii) By Definition 4.3.1 we have

$$
\begin{aligned}
& 2 e_{k, 16}^{(0)}(x)+2 e_{k, 16}^{(1)}(x) \\
& =\frac{1}{2^{6 k}} \sum_{\begin{array}{r}
(t, u) \in \mathbb{N}_{0}^{2} \\
2 t+3 u=k
\end{array}} a_{k}(t, u)\left(p_{2}(x)+q_{2}(x)(1-x)^{1 / 4}+r_{2}(x)(1-x)^{1 / 2}+s_{2}(x)(1-x)^{3 / 4}\right)^{t} \\
& \quad \times\left(p_{3}(x)+q_{3}(x)(1-x)^{1 / 4}+r_{3}(x)(1-x)^{1 / 2}+s_{3}(x)(1-x)^{3 / 4}\right)^{u} \\
& +\frac{1}{2^{6 k}} \sum_{\substack{(t, u) \in \mathbb{N}_{0}^{2} \\
2 t+3 u=k}} a_{k}(t, u)\left(p_{2}(x)+q_{2}(x)(1-x)^{1 / 4}-r_{2}(x)(1-x)^{1 / 2}-s_{2}(x)(1-x)^{3 / 4}\right)^{t} \\
& \quad \times\left(p_{3}(x)+q_{3}(x)(1-x)^{1 / 4}-r_{3}(x)(1-x)^{1 / 2}-s_{3}(x)(1-x)^{3 / 4}\right)^{u} .
\end{aligned}
$$

Thus, by Lemma 4.3.1(i)(ii), we deduce

$$
\begin{aligned}
& 2 e_{k, 16}^{(0)}(0)+2 e_{k, 16}^{(1)}(0) \\
= & \frac{1}{2^{6 k}} \sum_{\substack{(t, u) \in \mathbb{N}_{0}^{2} \\
2 t+3 u=k}} a_{k}(t, u)\left(p_{2}(0)+q_{2}(0)+r_{2}(0)+s_{2}(0)\right)^{t}\left(p_{3}(0)+q_{3}(0)+r_{3}(0)+s_{3}(0)\right)^{u} \\
+ & \frac{1}{2^{6 k}} \sum_{\substack{(t, u) \in \mathbb{N}_{0}^{2} \\
2 t+3 u=k}} a_{k}(t, u)\left(p_{2}(0)+q_{2}(0)-r_{2}(0)-s_{2}(0)\right)^{t}\left(p_{3}(0)+q_{3}(0)-r_{3}(0)-s_{3}(0)\right)^{u}
\end{aligned}
$$




$$
=1+\frac{1}{2^{4 k}} \text {. }
$$

This implies that

$$
2 e_{k, 16}^{(1)}(0)=\left(1+\frac{1}{2^{4 k}}\right)-2 e_{k, 16}^{(0)}(0)=\frac{2^{2 k}-1}{2^{2 k+1}} .
$$

Hence we have

$$
e_{k, 16}^{(1)}(0)=\frac{2^{2 k}-1}{2^{2 k+2}} .
$$

(iv) By Definition 4.3.1 we have

$$
\begin{aligned}
& 2 e_{k, 16}^{(0)}(x)+2 e_{k, 16}^{(2)}(x) \\
&=\frac{1}{2^{6 k}} \sum_{\substack{(t, u) \in \mathbb{N}_{0}^{2} \\
2 t+3 u=k}} a_{k}(t, u)\left(p_{2}(x)+q_{2}(x)(1-x)^{1 / 4}+r_{2}(x)(1-x)^{1 / 2}+s_{2}(x)(1-x)^{3 / 4}\right)^{t} \\
& \quad \times\left(p_{3}(x)+q_{3}(x)(1-x)^{1 / 4}+r_{3}(x)(1-x)^{1 / 2}+s_{3}(x)(1-x)^{3 / 4}\right)^{u} \\
&+\frac{1}{2^{6 k}} \sum_{\substack{(t, u) \in \mathbb{N}_{0}^{2} \\
2 t+3 u=k}} a_{k}(t, u)\left(p_{2}(x)-q_{2}(x)(1-x)^{1 / 4}+r_{2}(x)(1-x)^{1 / 2}-s_{2}(x)(1-x)^{3 / 4}\right)^{t} \\
& \quad \times\left(p_{3}(x)-q_{3}(x)(1-x)^{1 / 4}+r_{3}(x)(1-x)^{1 / 2}-s_{3}(x)(1-x)^{3 / 4}\right)^{u} .
\end{aligned}
$$

Thus, by Lemma 4.3.1(i)(iv), we have

$$
\begin{aligned}
& 2 e_{k, 16}^{(0)}(0)+2 e_{k, 16}^{(2)}(0) \\
& =\frac{1}{2^{6 k}} \sum_{\substack{(t, u) \in \mathbb{N}_{0} \\
2 t+3 u=k}} a_{k}(t, u)\left(p_{2}(0)+q_{2}(0)+r_{2}(0)+s_{2}(0)\right)^{t}\left(p_{3}(0)+q_{3}(0)+r_{3}(0)+s_{3}(0)\right)^{u} \\
& +\frac{1}{2^{6 k}} \sum_{\substack{(t, u) \in \mathbb{N}_{0} \\
2 t+3 u=k}} a_{k}(t, u)\left(p_{2}(0)-q_{2}(0)+r_{2}(0)-s_{2}(0)\right)^{t}\left(p_{3}(0)-q_{3}(0)+r_{3}(0)-s_{3}(0)\right)^{u} \\
& =1+\frac{1}{2^{2 k}} .
\end{aligned}
$$

This implies that

$$
2 e_{k, 16}^{(2)}(0)=\left(1+\frac{1}{2^{2 k}}\right)-2 e_{k, 16}^{(0)}=\frac{2^{2 k-1}\left(2^{2 k}+1\right)-1}{2^{4 k}} .
$$


Hence we have

$$
e_{k, 16}^{(2)}(0)=\frac{2^{2 k-1}\left(2^{2 k}+1\right)-1}{2^{4 k+1}}
$$

(v) Finally as $1=e_{k, 16}(0)=e_{k, 16}^{(0)}(0)+e_{k, 16}^{(1)}(0)+e_{k, 16}^{(2)}(0)+e_{k, 16}^{(3)}(0)$, we have

$$
e_{k, 16}^{(3)}(0)=1-e_{k, 16}^{(0)}(0)-e_{k, 16}^{(1)}(0)-e_{k, 16}^{(2)}(0)=\frac{2^{2 k}-1}{2^{2 k+2}} .
$$

This completes the proof of Lemma 4.3.2.

The next lemma gives the value of the polynomial $e_{k, 16}^{(0)}(x)$ at $x=1$.

Lemma 4.3.3. For $k \in \mathbb{N}$ with $k \geq 2$, we have

$$
e_{k, 16}(1)=e_{k, 16}^{(0)}(1)=\frac{(-1)^{k}}{2^{6 k}}
$$

Proof. We first note from Definition 4.3.1 that

$$
e_{k, 16}(1)=e_{k, 16}^{(0)}(1)+e_{k, 16}^{(1)}(1)(1-1)^{1 / 4}+e_{k, 16}^{(2)}(1)(1-1)^{1 / 2}+e_{k, 16}^{(0)}(1)(1-1)^{3 / 4}=e_{k, 16}^{(1)}(1)
$$

Moreover, from Definition 4.3.1 and (2.9.1), we have

$$
\begin{aligned}
e_{k, 16}(1)=\frac{1}{2^{6 k}} \sum_{\substack{(t, u) \in \mathbb{N}_{0}^{2} \\
2 t+3 u=k}} a_{k}(t, u)\left(p_{2}(1)+q_{2}(1)(1-1)^{1 / 4}+r_{2}(1)(1-1)^{1 / 2}+s_{2}(1)(1-1)^{3 / 4}\right)^{t} \\
\times\left(p_{3}(1)+q_{3}(1)(1-1)^{1 / 4}+r_{3}(1)(1-1)^{1 / 2}+s_{3}(1)(1-1)^{3 / 4}\right)^{u} \\
=\frac{1}{2^{6 k}} \sum_{\substack{(t, u) \in \mathbb{N}_{0}^{2} \\
2 t+3 u=k}} a_{k}(t, u)\left(p_{2}(1)\right)^{t}\left(p_{3}(1)\right)^{u}=\frac{1}{2^{6 k}} \sum_{\substack{(t, u) \in \mathbb{N}_{0} \\
2 t+3 u=k}} a_{k}(t, u)(1)^{t}(-1)^{u} \\
=\frac{(-1)^{k}}{2^{6 k}},
\end{aligned}
$$

as asserted. 
The final lemma of this section gives information on the degree of the polynomial $e_{k, 16}^{(i)}(x)$ for $i \in\{0,1,2,3\}$.

Lemma 4.3.4. For $k \in \mathbb{N}$ with $k \geq 2$, we have

i) $\operatorname{deg}\left(e_{k, 16}^{(i)}(x)\right) \leq k-1$ for $i \in\{1,2,3\}$,

ii) $\operatorname{deg}\left(e_{k, 16}^{(0)}(x)\right)=k$,

iii) the coefficient of $x^{k}$ in $e_{k, 16}^{(0)}(x)$ is $\frac{1}{2^{6 k}}$.

Proof. By the multinomial theorem we have

$$
\begin{aligned}
& \sum_{\substack{(t, u) \in \mathbb{N}_{0}^{2} \\
2 t+3 u=k}} a_{k}(t, u)\left(p_{2}(x)+q_{2}(x)(1-x)^{1 / 4}+r_{2}(x)(1-x)^{1 / 2}+s_{2}(x)(1-x)^{3 / 4}\right)^{t} \\
& \times\left(p_{3}(x)+q_{3}(x)(1-x)^{1 / 4}+r_{3}(x)(1-x)^{1 / 2}+s_{3}(x)(1-x)^{3 / 4}\right)^{u} \\
& =\sum_{\substack{(t, u) \in \mathbb{N}_{0}^{2} \\
2 t+3 u=k}} a_{k}(t, u)\left(\sum_{\substack{\left(t_{0}, t_{1}, t_{2}, t_{3}\right) \in \mathbb{N}_{0}^{4} \\
t_{0}+t_{1}+t_{2}+t_{3}=t}}\left(\begin{array}{c}
t \\
t_{0}, t_{1}, t_{2}, t_{3}
\end{array}\right) p_{2}(x)^{t_{0}} q_{2}(x)^{t_{1}} r_{2}(x)^{t_{2}} s_{2}(x)^{t_{3}}(1-x)^{\left(t_{1}+2 t_{2}+3 t_{3}\right) / 4}\right) \\
& \times\left(\sum_{\left(u_{0}, u_{1}, u_{2}, u_{3}\right) \in \mathbb{N}_{0}^{4}}\left(\begin{array}{c}
u \\
u_{0}, u_{1}, u_{2}, u_{3}
\end{array}\right) p_{3}(x)^{u_{0}} q_{3}(x)^{u_{1}} r_{3}(x)^{u_{2}} s_{3}(x)^{u_{3}}(1-x)^{\left(u_{1}+2 u_{2}+3 u_{3}\right) / 4}\right) \\
& =\sum_{\substack{\left(t_{0}, t_{1}, t_{2}, t_{3}, u_{0}, u_{1}, u_{2}, u_{3}\right) \in \mathbb{N}_{0}^{8} \\
2\left(t_{0}+t_{1}+t_{2}+t_{3}\right)+3\left(u_{0}+u_{1}+u_{2}+u_{3}\right)=k}} a_{k}\left(t_{0}+\cdots+t_{3}, u_{0}+\cdots+u_{3}\right)\left(\begin{array}{c}
t_{0}+\cdots+t_{3} \\
t_{0}, t_{1}, t_{2}, t_{3}
\end{array}\right)\left(\begin{array}{c}
u_{0}+\cdots+u_{3} \\
u_{0}, u_{1}, u_{2}, u_{3}
\end{array}\right) \\
& \times p_{2}(x)^{t_{0}} q_{2}(x)^{t_{1}} r_{2}(x)^{t_{2}} s_{2}(x)^{t_{3}} p_{3}(x)^{u_{0}} q_{3}(x)^{u_{1}} r_{3}(x)^{u_{2}} s_{3}(x)^{u_{3}}(1-x)^{\left(t_{1}+2 t_{2}+3 t_{3}+u_{1}+2 u_{2}+3 u_{3}\right) / 4} .
\end{aligned}
$$

Thus, by Definition 4.3.1, for $i \in\{0,1,2,3\}$ we have $e_{k, 16}^{(i)}(x)$ 


$$
\begin{aligned}
& =\frac{1}{2^{6 k}} \sum_{\substack{\left(t_{0}, t_{1}, t_{2}, t_{3}, u_{0}, u_{1}, u_{2}, u_{3}\right) \in \mathbb{N}_{0}^{8} \\
2\left(t_{0}+t_{1}+t_{2}+t_{3}\right)+3\left(u_{0}+u_{1}+u_{2}+u_{3}\right)=k \\
t_{1}+2 t_{2}+3 t_{3}+u_{1}+2 u_{2}+3 u_{3} \equiv \imath(\bmod 4)}} a\left(t_{3}+\cdots+t_{3}, u_{0}+\cdots+u_{3}\right)\left(\begin{array}{c}
t_{0}+\cdots+t_{3} \\
t_{0}, t_{1}, t_{2}, t_{3}
\end{array}\right)\left(\begin{array}{c}
u_{0}+\cdots+u_{3} \\
u_{0}, u_{1}, u_{2}, u_{3}
\end{array}\right) \\
& \times p_{2}(x)^{t_{0}} q_{2}(x)^{t_{1}} r_{2}(x)^{t_{2}} s_{2}(x)^{t_{3}} p_{3}(x)^{u_{0}} q_{3}(x)^{u_{1}} r_{3}(x)^{u_{2}} s_{3}(x)^{u_{3}}(1-x)^{\left(t_{1}+2 t_{2}+3 t_{3}+u_{1}+2 u_{2}+3 u_{3}-\imath\right) / 4} .
\end{aligned}
$$

The highest power of $x$ in $e_{k, 16}^{(2)}(x)$ is at most

$$
\max _{\substack{\left(t_{0}, t_{1}, t_{2}, t_{3}, u_{0}, u_{1}, u_{2}, u_{3}\right) \in \mathbb{N}_{0}^{8} \\ 2\left(t_{0}+t_{1}+t_{2}+t_{3}\right)+3\left(u_{0}+u_{1}+u_{2}+u_{3}\right)=k \\ t_{1}+2 t_{2}+3 t_{3}+u_{1}+2 u_{2}+3 u_{3} \equiv \imath(\bmod 4)}}\left(2 t_{0}+t_{1}+t_{2}+t_{3}+3 u_{0}+2 u_{1}+2 u_{2}+2 u_{3}\right.
$$

$$
\begin{aligned}
& \left.+\frac{t_{1}+2 t_{2}+3 t_{3}+u_{1}+2 u_{2}+3 u_{3}-\imath}{4}\right) \\
= & \frac{1}{4} \max _{\substack{\left(t_{0}, t_{1}, t_{2}, t_{3}, u_{0}, u_{1}, u_{2}, u_{3}\right) \in \mathbb{N}_{0}^{8} \\
2\left(t_{0}+t_{1}+t_{2}+t_{3}\right)+3\left(u_{0}+u_{1}+u_{2}+u_{3}\right)=k \\
t_{1}+2 t_{2}+3 t_{3}+u_{1}+2 u_{2}+3 u_{3} \equiv 2(\bmod 4)}}\left(8 t_{0}+5 t_{1}+6 t_{2}+7 t_{3}+12 u_{0}+9 u_{1}+10 u_{2}+11 u_{3}-i\right) \\
= & \frac{1}{4} \quad \max _{\substack{\left(t_{0}, t_{1}, t_{2}, t_{3}, u_{0}, u_{1}, u_{2}, u_{3}\right) \in \mathbb{N}_{0}^{8} \\
2\left(t_{0}+t_{1}+t_{2}+t_{3}\right)+3\left(u_{0}+u_{1}+u_{2}+u_{3}\right)=k \\
t_{1}+2 t_{2}+3 t_{3}+u_{1}+2 u_{2}+3 u_{3} \equiv 2(\bmod 4)}}\left(4 k-3 t_{1}-2 t_{2}-t_{3}-3 u_{1}-2 u_{2}-u_{3}-i\right) \\
= & \begin{cases}\frac{1}{4}(4 k-4)=k-1, & \text { if } i \in\{1,2,3\}, \\
\frac{1}{4}(4 k)=k, & \text { if } i=0 .\end{cases}
\end{aligned}
$$

From the last equation we note that the highest power of $x$ in $e_{k, 16}^{(0)}(x)$ occurs when $t_{1}+2 t_{2}+3 t_{3}+u_{1}+2 u_{2}+3 u_{3}=0$, that is when $t_{1}=t_{2}=t_{3}=u_{1}=u_{2}=u_{3}=0$. This shows that the coefficient of $x^{k}$ is $\frac{1}{2^{6 k}}$. 


\subsection{The identity $E_{k}\left(q^{r}\right)=e_{k, r}(x) z^{2 k}$}

In this section we show that for $k \in \mathbb{N}$ with $k \geq 2$ and $r \in\{1,2,4,8,16\}$, the Eisenstein series $E_{k}\left(q^{r}\right)=e_{k, r}(x) z^{2 k}$ with $x=1-\frac{\varphi^{4}(-q)}{\varphi^{4}(q)}$ and $z=\varphi^{2}(q)$.

Theorem 4.4.1. For $q \in \mathbb{C}$ with $|q|<1$ define $x=1-\frac{\varphi^{4}(-q)}{\varphi^{4}(q)}$ and $z=\varphi^{2}(q)$. Then, for $k \in \mathbb{N}$ with $k \geq 2$ and $q \in D_{\delta}$, we have

(i) $E_{k}(q)=e_{k, 1}(x) z^{2 k}$,

(ii) $E_{k}\left(q^{2}\right)=e_{k, 2}(x) z^{2 k}$,

(iii) $E_{k}\left(q^{4}\right)=e_{k, 4}(x) z^{2 k}$

(iv) $E_{k}\left(q^{8}\right)=e_{k, 8}(x) z^{2 k}$

(v) $E_{k}\left(q^{16}\right)=e_{k, 16}(x) z^{2 k}$.

Proof. (i) This follows from Theorem 2.9.1, (2.9.5), (2.9.8), (4.1.1) and the fact that $z^{4 t+6 u}=z^{2 k}$ when $2 t+3 u=k$.

(ii) This follows from Theorem 2.9.1, (2.9.6), (2.9.9), (4.1.2) and the fact that $z^{4 t+6 u}=z^{2 k}$ when $2 t+3 u=k$.

(iii) This follows from Theorem 2.9.1, (2.9.7), (2.9.10), (4.1.3) and the fact that $z^{4 t+6 u}=z^{2 k}$ when $2 t+3 u=k$.

(iv) This follows from Theorem 2.9.1, (2.9.12), (2.9.14), (4.2.1) and the fact that $z^{4 t+6 u}=z^{2 k}$ when $2 t+3 u=k$. Note that $q \in D_{\delta}$ is needed for (2.9.12) and $(2.9 .14)$.

(v) This follows from Theorem 2.9.1, (2.9.13), (2.9.15), (4.3.1) and the fact 
that $z^{4 t+6 u}=z^{2 k}$ when $2 t+3 u=k$. Note that $q \in D_{\delta}$ is needed for (2.9.13) and (2.9.15). 


\section{Chapter 5}

\section{The polynomials $P_{k, r, s}(x), Q_{k, r, s}(x), R_{k, r, s}(x)$ \\ and $S_{k, r, s}(x)$}

Let $r, s \in \mathbb{N}_{0}$ with $(r, s) \neq(0,0)$. Let $k \in \mathbb{N}$ be such that $k \geq 2$ and $2 r+s<4 k$. In this chapter we define four polynomials $P_{k, r, s}(x), Q_{k, r, s}(x), R_{k, r, s}(x)$ and $S_{k, r, s}(x)$. These polynomials play an important role in the proofs of our main theorems in Chapter 6. As in Section 3.3 we consider three cases.

(i) $r \geq 1, s=0$ and $r<2 k$ (Section 5.1),

(ii) $r \geq 1, s \geq 1$ and $2 r+s<4 k$ (Section 5.2),

(iii) $r=0, s \geq 1$ and $s<4 k$ (Section 5.3).

Each section of this chapter considers one of these three particular cases. For each case some properties of the four polynomials are given. In particular, we are interested in the values of $P_{k, r, s}(0), P_{k, r, s}(1), Q_{k, r, s}(0), R_{k, r, s}(0), S_{k, r, s}(0)$ as well as an upper bound for the degree of each of these polynomials.

Let $h \in \mathbb{N}_{0}$. Throughout this chapter, if $f(x)$ is a polynomial in $x$ over $\mathbb{Q}$ 
CHAPTER 5. $P_{K, R, S}(X), Q_{K, R, S}(X), R_{K, R, S}(X)$ AND $S_{K, R, S}(X)$

of degree at most $h$, then

$$
f(x)=a_{0}+a_{1} x+\cdots+a_{h} x^{h}, \quad a_{0}, \ldots, a_{h} \in \mathbb{Q} .
$$

We abbreviate this by writing

$$
f(x)=a_{h} x^{h}+\cdots
$$

We emphasize with this notation that $a_{h}$ may be 0 .

\subsection{The polynomials $P_{k, r, 0}(x), Q_{k, r, 0}(x), R_{k, r, 0}(x)$ and $S_{k, r, 0}(x)$}

We begin this section with the definition of the polynomials $P_{k, r, 0}(x), Q_{k, r, 0}(x)$, $R_{k, r, 0}(x)$ and $S_{k, r, 0}(x)$. Recall that the polynomials $A_{r, s}(x), B_{r, s}(x), C_{r, s}(x)$ and $D_{r, s}(x)$ are defined in Definition 3.1.1.

Definition 5.1.1. For $k, r \in \mathbb{N}$ with $k \geq 2$ and $r<2 k$, we define

(i) $P_{k, r, 0}(x)=A_{r, 0}(x)-\frac{(-1)^{k}}{2^{r}\left(2^{2 k}-1\right)} e_{k, 1}(x)+\frac{(-1)^{k}}{2^{r}\left(2^{2 k}-1\right)} e_{k, 2}(x)$

$$
+\frac{1}{2^{2 k}-1} e_{k, 4}(x)-\frac{2^{2 k}}{2^{2 k}-1} e_{k, 8}^{(0)}(x)
$$

(ii) $Q_{k, r, 0}(x)=0$,

(iii) $R_{k, r, 0}(x)=C_{r, 0}(x)-\frac{2^{2 k}}{2^{2 k}-1} e_{k, 8}^{(1)}(x)$,

(iv) $S_{k, r, 0}(x)=0$. 
CHAPTER 5. $P_{K, R, S}(X), Q_{K, R, S}(X), R_{K, R, S}(X)$ AND $S_{K, R, S}(X)$

The following lemma justifies our definition of these four polynomials and plays a key part in the proof of our main theorems.

Lemma 5.1.1. For $k, r \in \mathbb{N}$ with $k \geq 2$ and $r<2 k$, we have

$$
\begin{aligned}
& P_{k, r, 0}(x)+Q_{k, r, 0}(x)(1-x)^{1 / 4}+R_{k, r, 0}(x)(1-x)^{1 / 2}+S_{k, r, 0}(x)(1-x)^{3 / 4} \\
& =\left(\frac{1+(1-x)^{1 / 2}}{2}\right)^{r}-\frac{(-1)^{k}}{2^{r}\left(2^{2 k}-1\right)} e_{k, 1}(x)+\frac{(-1)^{k}}{2^{r}\left(2^{2 k}-1\right)} e_{k, 2}(x)+\frac{1}{2^{2 k}-1} e_{k, 4}(x) \\
& \quad-\frac{2^{2 k}}{2^{2 k}-1}\left(e_{k, 8}^{(0)}(x)+e_{k, 8}^{(1)}(x)(1-x)^{1 / 2}\right) .
\end{aligned}
$$

Proof. From Definition 5.1.1 and Lemma 3.1.3, we have

$$
\begin{aligned}
& \left(\frac{1+(1-x)^{1 / 2}}{2}\right)^{r}-\frac{(-1)^{k}}{2^{r}\left(2^{2 k}-1\right)} e_{k, 1}(x)+\frac{(-1)^{k}}{2^{r}\left(2^{2 k}-1\right)} e_{k, 2}(x) \\
& \quad+\frac{1}{2^{2 k}-1} e_{k, 4}(x)-\frac{2^{2 k}}{2^{2 k}-1}\left(e_{k, 8}^{(0)}(x)+e_{k, 8}^{(1)}(x)(1-x)^{1 / 2}\right) \\
& =A_{r, 0}(x)+C_{r, 0}(x)(1-x)^{1 / 2}-\frac{(-1)^{k}}{2^{r}\left(2^{2 k}-1\right)} e_{k, 1}(x)+\frac{(-1)^{k}}{2^{r}\left(2^{2 k}-1\right)} e_{k, 2}(x) \\
& \quad+\frac{1}{2^{2 k}-1} e_{k, 4}(x)-\frac{2^{2 k}}{2^{2 k}-1}\left(e_{k, 8}^{(0)}(x)+e_{k, 8}^{(1)}(x)(1-x)^{1 / 2}\right) \\
& =P_{k, r, 0}(x)+Q_{k, r, 0}(x)(1-x)^{1 / 4}+R_{k, r, 0}(x)(1-x)^{1 / 2}+S_{k, r, 0}(x)(1-x)^{3 / 4},
\end{aligned}
$$

which completes the proof.

In the next lemma we evaluate the polynomial $P_{k, r, 0}(x)$ at $x=0$ and $x=1$, and give an upper bound for the degree of $P_{k, r, 0}(x)$ when $r<2 k$. 
Lemma 5.1.2. For $k, r \in \mathbb{N}, k \geq 2$ and $r<2 k$, we have

(i) $P_{k, r, 0}(0)=0$,

(ii) $P_{k, r, 0}(1)=0$,

(iii) $\operatorname{deg}\left(P_{k, r, 0}(x)\right) \leq k-1$.

Proof. (i) By Definition 5.1.1(i), Lemmas 3.3.1, 4.1.1(i), 4.1.2(i), 4.1.3(i) and 4.2.1 (ii), we have

$$
\begin{aligned}
P_{k, r, 0}(0)= & A_{r, 0}(0)-\frac{(-1)^{k}}{2^{r}\left(2^{2 k}-1\right)} e_{k, 1}(0)+\frac{(-1)^{k}}{2^{r}\left(2^{2 k}-1\right)} e_{k, 2}(0) \\
& +\frac{1}{2^{2 k}-1} e_{k, 4}(0)-\frac{2^{2 k}}{2^{2 k}-1} e_{k, 8}^{(0)}(0) \\
= & \frac{1}{2}-\frac{(-1)^{k}}{2^{r}\left(2^{2 k}-1\right)}+\frac{(-1)^{k}}{2^{r}\left(2^{2 k}-1\right)}+\frac{1}{2^{2 k}-1}-\frac{2^{2 k}}{2^{2 k}-1} \frac{2^{2 k}+1}{2^{2 k+1}} \\
= & \frac{1}{2}+\frac{1}{2^{2 k}-1}-\frac{2^{2 k}+1}{2\left(2^{2 k}-1\right)}=0,
\end{aligned}
$$

which proves (i).

(ii) By Lemmas 5.1.1, 4.1.1(ii), 4.1.2(ii), 4.1.3(ii) and 4.2.2, we have

$$
\begin{aligned}
P_{k, r, 0}(1)= & \frac{1}{2^{r}}-\frac{(-1)^{k}}{2^{r}\left(2^{2 k}-1\right)} e_{k, 1}(1)+\frac{(-1)^{k}}{2^{r}\left(2^{2 k}-1\right)} e_{k, 2}(1) \\
& +\frac{1}{2^{2 k}-1} e_{k, 4}(1)-\frac{2^{2 k}}{2^{2 k}-1} e_{k, 8}^{(0)}(1) \\
= & \frac{1}{2^{r}}-\frac{2^{2 k}}{2^{r}\left(2^{2 k}-1\right)}+\frac{1}{2^{r}\left(2^{2 k}-1\right)}+\frac{(-1)^{k}}{2^{2 k}\left(2^{2 k}-1\right)}-\frac{2^{2 k}}{2^{2 k}-1} \frac{(-1)^{k}}{2^{4 k}}
\end{aligned}
$$




$$
=0,
$$

which proves (ii).

(iii) By Lemmas 3.1 .2 (i), 4.1 .1 (iii)(iv), 4.1 .2 (iii)(iv), 4.1 .3 (iii)(iv) and 4.2.3 (i)(iii), we have

$$
\begin{aligned}
A_{r, 0}(x) & =a_{k-1} x^{k-1}+\cdots, \\
e_{k, 1}(x) & =x^{k}+\cdots, \\
e_{k, 2}(x) & =x^{k}+\cdots, \\
e_{k, 4}(x) & =\frac{1}{2^{2 k}} x^{k}+\cdots, \\
e_{k, 8}^{(0)}(x) & =\frac{1}{2^{4 k}} x^{k}+\cdots
\end{aligned}
$$

Then, by Definition 5.1.1(i), we obtain

$$
\begin{aligned}
P_{k, r, 0}(x) & =\left(\frac{-(-1)^{k}}{2^{r}\left(2^{2 k}-1\right)}+\frac{(-1)^{k}}{2^{r}\left(2^{2 k}-1\right)}+\frac{1}{2^{2 k}-1} \frac{1}{2^{2 k}}-\frac{2^{2 k}}{2^{2 k}-1} \frac{1}{2^{4 k}}\right) x^{k}+\cdots \\
& =0 x^{k}+\cdots,
\end{aligned}
$$

which shows that $\operatorname{deg}\left(P_{k, r, 0}(x)\right) \leq k-1$.

We conclude this section by evaluating the polynomial $R_{k, r, 0}(x)$ at $x=0$ and giving an upper bound for the degree of $R_{k, r, 0}(x)$.

Lemma 5.1.3. For $k, r \in \mathbb{N}, k \geq 2$ and $r<2 k$, we have

(i) $R_{k, r, 0}(0)=0$,

(ii) $\operatorname{deg}\left(R_{k, r, 0}(x)\right) \leq k-1$. 
CHAPTER 5. $P_{K, R, S}(X), Q_{K, R, S}(X), R_{K, R, S}(X) A N D S_{K, R, S}(X)$

Proof. (i) By Definition 5.1.1(iii), Lemmas 3.3.1 and 4.2.1(iii), we have

$$
R_{k, r, 0}(0)=C_{r, 0}(0)-\frac{2^{2 k}}{2^{2 k}-1} e_{k, 8}^{(1)}(0)=\frac{1}{2}-\frac{2^{2 k}}{2^{2 k}-1} \frac{2^{2 k}-1}{2^{2 k+1}}=\frac{1}{2}-\frac{1}{2}=0,
$$

which proves (i).

(ii) This follows from Definition 5.1.1(iii), Lemmas 3.1.2(iii) and 4.2.3(ii).

\subsection{The polynomials $P_{k, r, s}(x), Q_{k, r, s}(x), R_{k, r, s}(x)$ and $S_{k, r, s}(x)$}

We begin this section with the definition of the polynomials $P_{k, r, s}(x), Q_{k, r, s}(x)$, $R_{k, r, s}(x)$ and $S_{k, r, s}(x)$.

Definition 5.2.1. For $k, r, s \in \mathbb{N}$ with $k \geq 2$ and $2 r+s<4 k$, we define

(i) $P_{k, r, s}(x)=A_{r, s}(x)-\frac{(-1)^{k}}{2^{r+s}\left(2^{2 k}-1\right)} e_{k, 1}(x)+\frac{(-1)^{k}}{2^{r+s}\left(2^{2 k}-1\right)} e_{k, 2}(x)$

$$
+\frac{1}{2^{2 k}-1} e_{k, 8}^{(0)}(x)-\frac{2^{2 k}}{2^{2 k}-1} e_{k, 16}^{(0)}(x)
$$

(ii) $Q_{k, r, s}(x)=B_{r, s}(x)-\frac{2^{2 k}}{2^{2 k}-1} e_{k, 16}^{(1)}(x)$,

(iii) $R_{k, r, s}(x)=C_{r, s}(x)+\frac{1}{2^{2 k}-1} e_{k, 8}^{(1)}(x)-\frac{2^{2 k}}{2^{2 k}-1} e_{k, 16}^{(2)}(x)$,

(iv) $S_{k, r, s}(x)=D_{r, s}(x)-\frac{2^{2 k}}{2^{2 k}-1} e_{k, 16}^{(3)}(x)$.

The following lemma justifies our definition of the four polynomials and plays a key part in the proof of our main theorems. 
Lemma 5.2.1. For $k, r, s \in \mathbb{N}$ with $k \geq 2$ and $2 r+s<4 k$, we have

$$
\begin{aligned}
P_{k, r, s}(x) & +Q_{k, r, s}(x)(1-x)^{1 / 4}+R_{k, r, s}(x)(1-x)^{1 / 2}+S_{k, r, s}(x)(1-x)^{3 / 4} \\
= & \left(\frac{1+(1-x)^{1 / 2}}{2}\right)^{r}\left(\frac{1+(1-x)^{1 / 4}}{2}\right)^{s}-\frac{(-1)^{k}}{2^{r+s}\left(2^{2 k}-1\right)} e_{k, 1}(x) \\
& +\frac{(-1)^{k}}{2^{r+s}\left(2^{2 k}-1\right)} e_{k, 2}(x)+\frac{1}{2^{2 k}-1}\left(e_{k, 8}^{(0)}(x)+e_{k, 8}^{(1)}(x)(1-x)^{1 / 2}\right) \\
& -\frac{2^{2 k}}{2^{2 k}-1}\left(e_{k, 16}^{(0)}(x)+e_{k, 16}^{(1)}(x)(1-x)^{1 / 4}+e_{k, 16}^{(2)}(1-x)^{1 / 2}+e_{k, 16}^{(3)}(1-x)^{3 / 4}\right) .
\end{aligned}
$$

Proof. From Lemma 3.1.3(i) and Definition 5.2.1, we have

$$
\begin{aligned}
& \left(\frac{1+(1-x)^{1 / 2}}{2}\right)^{r}\left(\frac{1+(1-x)^{1 / 4}}{2}\right)^{s}-\frac{(-1)^{k}}{2^{r+s}\left(2^{2 k}-1\right)} e_{k, 1}(x) \\
& +\frac{(-1)^{k}}{2^{r+s}\left(2^{2 k}-1\right)} e_{k, 2}(x)+\frac{1}{2^{2 k}-1}\left(e_{k, 8}^{(0)}(x)+e_{k, 8}^{(1)}(x)(1-x)^{1 / 2}\right) \\
& \quad-\frac{2^{2 k}}{2^{2 k}-1}\left(e_{k, 16}^{(0)}(x)+e_{k, 16}^{(1)}(x)(1-x)^{1 / 4}+e_{k, 16}^{(2)}(1-x)^{1 / 2}+e_{k, 16}^{(3)}(1-x)^{3 / 4}\right) \\
& =A_{r, s}(x)+B_{r, s}(x)(1-x)^{1 / 4}+C_{r, s}(x)(1-x)^{1 / 2}+D_{r, s}(x)(1-x)^{3 / 4} \\
& \quad-\frac{(-1)^{k}}{2^{r+s}\left(2^{2 k}-1\right)} e_{k, 1}(x)+\frac{(-1)^{k}}{2^{r+s}\left(2^{2 k}-1\right)} e_{k, 2}(x) \\
& \quad+\frac{1}{2^{2 k}-1}\left(e_{k, 8}^{(0)}(x)+e_{k, 8}^{(1)}(x)(1-x)^{1 / 2}\right)
\end{aligned}
$$


CHAPTER 5. $P_{K, R, S}(X), Q_{K, R, S}(X), R_{K, R, S}(X)$ AND $S_{K, R, S}(X)$

$$
\begin{aligned}
& -\frac{2^{2 k}}{2^{2 k}-1}\left(e_{k, 16}^{(0)}(x)+e_{k, 16}^{(1)}(x)(1-x)^{1 / 4}+e_{k, 16}^{(2)}(1-x)^{1 / 2}+e_{k, 16}^{(3)}(1-x)^{3 / 4}\right) \\
= & P_{k, r, s}(x)+Q_{k, r, s}(x)(1-x)^{1 / 4}+R_{k, r, s}(x)(1-x)^{1 / 2}+S_{k, r, s}(x)(1-x)^{3 / 4},
\end{aligned}
$$

as asserted.

In the next lemma we evaluate the polynomial $P_{k, r, s}(x)$ at $x=0$ and $x=1$, and give an upper bound for the degree of $P_{k, r, s}(x)$ when $2 r+s<4 k$.

Lemma 5.2.2. For $k, r, s \in \mathbb{N}$ with $k \geq 2$ and $2 r+s<4 k$, we have

(i) $P_{k, r, s}(0)=0$,

(ii) $P_{k, r, s}(1)=0$,

(iii) $\operatorname{deg}\left(P_{k, r, s}(x)\right) \leq k-1$.

Proof. (i) By Definition 5.2.1(i), Lemmas 3.3.2, 4.1.1(i), 4.1.2(i), 4.2.1(ii) and 4.3.2 (ii), we have

$$
\begin{aligned}
P_{k, r, s}(0)= & A_{r, s}(0)-\frac{(-1)^{k}}{2^{r+s}\left(2^{2 k}-1\right)} e_{k, 1}(0)+\frac{(-1)^{k}}{2^{r+s}\left(2^{2 k}-1\right)} e_{k, 2}(0) \\
& +\frac{1}{2^{2 k}-1} e_{k, 8}^{(0)}(0)-\frac{2^{2 k}}{2^{2 k}-1} e_{k, 16}^{(0)}(0) \\
= & \frac{1}{4}-\frac{(-1)^{k}}{2^{r+s}\left(2^{2 k}-1\right)}+\frac{(-1)^{k}}{2^{r+s}\left(2^{2 k}-1\right)} \\
& +\frac{1}{2^{2 k}-1}\left(\frac{2^{2 k}+1}{2^{2 k+1}}\right)-\frac{2^{2 k}}{2^{2 k}-1}\left(\frac{2^{2 k-1}\left(2^{2 k}+1\right)+1}{2^{4 k+1}}\right) \\
= & \frac{1}{4}+\frac{\left(2^{2 k}+1\right)-\left(2^{2 k-1}\left(2^{2 k}+1\right)+1\right)}{\left(2^{2 k}-1\right) 2^{2 k+1}}
\end{aligned}
$$




$$
=\frac{1}{4}+\frac{2-\left(2^{2 k}+1\right)}{4\left(2^{2 k}-1\right)}=\frac{1}{4}+\frac{1-2^{2 k}}{4\left(2^{2 k}-1\right)}=\frac{1}{4}-\frac{1}{4}=0,
$$

which proves (i).

(ii) By Lemmas 5.2.1, 4.1.1(ii), 4.1.2(ii), 4.2.2 and 4.3.3, we have

$$
\begin{aligned}
P_{k, r, s}(1)= & \frac{1}{2^{r+s}}-\frac{(-1)^{k}}{2^{r+s}\left(2^{2 k}-1\right)} e_{k, 1}(1)+\frac{(-1)^{k}}{2^{r+s}\left(2^{2 k}-1\right)} e_{k, 2}(1) \\
& +\frac{1}{2^{2 k}-1} e_{k, 8}^{(0)}(1)-\frac{2^{2 k}}{2^{2 k}-1} e_{k, 16}^{(0)}(1) \\
= & \frac{1}{2^{r+s}}-\frac{2^{2 k}}{2^{r+s}\left(2^{2 k}-1\right)}+\frac{1}{2^{r+s}\left(2^{2 k}-1\right)} \\
& +\frac{1}{2^{2 k}-1} \frac{(-1)^{k}}{2^{4 k}}-\frac{2^{2 k}}{2^{2 k}-1} \frac{(-1)^{k}}{2^{6 k}} \\
= & 0,
\end{aligned}
$$

which proves (ii).

(iii) By Lemmas 3.1.2 (i), 4.1.1 (iii)(iv), 4.1 .2 (iii)(iv), 4.2 .3 (i)(iii) and 4.3.4 (ii)(iii), we have

$$
\begin{aligned}
A_{r, s}(x) & =a_{k-1} x^{k-1}+\cdots, \\
e_{k, 1}(x) & =x^{k}+\cdots, \\
e_{k, 2}(x) & =x^{k}+\cdots, \\
e_{k, 8}^{(0)}(x) & =\frac{1}{2^{4 k}} x^{k}+\cdots,
\end{aligned}
$$


CHAPTER 5. $P_{K, R, S}(X), Q_{K, R, S}(X), R_{K, R, S}(X)$ AND $S_{K, R, S}(X)$

$$
e_{k, 16}^{(0)}(x)=\frac{1}{2^{6 k}} x^{k}+\cdots
$$

Then, by Definition 5.2.1(i), we obtain

$$
\begin{aligned}
P_{k, r, s}(x) & =\left(-\frac{(-1)^{k}}{2^{r+s}\left(2^{2 k}-1\right)}+\frac{(-1)^{k}}{2^{r+s}\left(2^{2 k}-1\right)}+\frac{1}{2^{2 k}-1} \frac{1}{2^{4 k}}-\frac{2^{2 k}}{2^{2 k}-1} \frac{1}{2^{6 k}}\right) x^{k}+\cdots \\
& =0 x^{k}+\cdots,
\end{aligned}
$$

which shows that $\operatorname{deg}\left(P_{k, r, s}(x)\right) \leq k-1$.

The next three lemmas evaluate the polynomials $Q_{k, r, s}(x), R_{k, r, s}(x)$ and $S_{k, r, s}(x)$ at $x=0$ and give upper bounds for their degrees.

Lemma 5.2.3. For $k, r, s \in \mathbb{N}$ with $k \geq 2$ and $2 r+s<4 k$, we have

(i) $Q_{k, r, s}(0)=0$,

(ii) $\operatorname{deg}\left(Q_{k, r, s}(x)\right) \leq k-1$.

Proof. (i) By Definition 5.2.1(ii), Lemmas 3.3.2 and 4.3.2(iii), we have

$$
Q_{k, r, s}(0)=B_{r, s}(0)-\frac{2^{2 k}}{2^{2 k}-1} e_{k, 16}^{(1)}(0)=\frac{1}{4}-\frac{2^{2 k}}{2^{2 k}-1} \frac{2^{2 k}-1}{2^{2 k+2}}=0 .
$$

(ii) This follows from Definition 5.2.1(ii), Lemmas 3.1.2(ii) and 4.3.4(i).

Lemma 5.2.4. For $k, r, s \in \mathbb{N}$ with $k \geq 2$ and $2 r+s<4 k$, we have

(i) $R_{k, r, s}(0)=0$,

(ii) $\operatorname{deg}\left(R_{k, r, s}(x)\right) \leq k-1$.

Proof. (i) By Definition 5.2.1(iii), Lemmas 3.3.2, 4.2.1(iii) and 4.3.2(iv), we have

$$
R_{k, r, s}(0)=C_{r, s}(0)+\frac{1}{2^{2 k}-1} e_{k, 8}^{(1)}(0)-\frac{2^{2 k}}{2^{2 k}-1} e_{k, 16}^{(2)}(0)
$$




$$
\begin{aligned}
& =\frac{1}{4}+\frac{1}{2^{2 k}-1} \frac{2^{2 k}-1}{2^{2 k+1}}-\frac{2^{2 k}}{2^{2 k}-1} \frac{\left(2^{2 k-1}\left(2^{2 k}+1\right)-1\right)}{2^{4 k+1}} \\
& =\frac{1}{4}+\frac{1}{2^{2 k+1}}-\frac{\left(2^{2 k}-1\right)\left(2^{2 k-1}+1\right)}{\left(2^{2 k}-1\right) 2^{2 k+1}} \\
& =\frac{1}{4}+\frac{1}{2^{2 k+1}}-\frac{\left(2^{2 k-1}+1\right)}{2^{2 k+1}}=\frac{1}{4}-\frac{1}{4}=0,
\end{aligned}
$$

which proves (i).

(ii) This follows from Definition 5.2.1 (iii), Lemmas 3.1.2 (iii), 4.2 .3 (ii) and 4.3.4(i).

Lemma 5.2.5. For $k, r, s \in \mathbb{N}$ with $k \geq 2$ and $2 r+s<4 k$, we have

(i) $S_{k, r, s}(0)=0$

(ii) $\operatorname{deg}\left(S_{k, r, s}(x)\right) \leq k-1$.

Proof. (i) By Definition 5.2.1(iv), Lemmas 3.3.2 and 4.3.2(v), we have

$$
S_{k, r, s}(0)=D_{r, s}(0)-\frac{2^{2 k}}{2^{2 k}-1} e_{k, 16}^{(3)}(0)=\frac{1}{4}-\frac{2^{2 k}}{2^{2 k}-1} \frac{2^{2 k}-1}{2^{2 k+2}}=0 .
$$

(ii) This follows from Definition 5.2.1(iv), Lemmas 3.1.2(iv) and 4.3.4(i). 
CHAPTER 5. $P_{K, R, S}(X), Q_{K, R, S}(X), R_{K, R, S}(X)$ AND $S_{K, R, S}(X)$

5.3 The polynomials $P_{k, 0, s}(x), Q_{k, 0, s}(x), R_{k, 0, s}(x)$ and $S_{k, 0, s}(x)$

We begin this section with the definition of the polynomials $P_{k, 0, s}(x), Q_{k, 0, s}(x)$, $R_{k, 0, s}(x)$ and $S_{k, 0, s}(x)$.

Definition 5.3.1. For $k, s \in \mathbb{N}$ with $k \geq 2$ and $s<4 k$, we define

(i) $P_{k, 0, s}(x)=A_{0, s}(x)-\frac{(-1)^{k}}{2^{s}\left(2^{2 k}-1\right)} e_{k, 1}(x)$

$$
\begin{aligned}
& +\frac{\varepsilon_{2,4}(s)(-1)^{\left[\frac{s+1}{4}\right]} 2^{\left[\frac{s}{2}\right]}+(-1)^{k}}{2^{s}\left(2^{2 k}-1\right)} e_{k, 2}(x) \\
& -\frac{\varepsilon_{2,4}(s)(-1)^{\left[\frac{s+1}{4}\right]}\left(2^{2 k}+1\right)}{2^{\left[\frac{s+1}{2}\right]}\left(2^{2 k}-1\right)} e_{k, 4}(x) \\
& +\frac{\varepsilon_{2,4}(s)(-1)^{\left[\frac{s+1}{4}\right]} 2^{2 k-\left[\frac{s+1}{2}\right]}+1}{2^{2 k}-1} e_{k, 8}^{(0)}(x)-\frac{2^{2 k}}{2^{2 k}-1} e_{k, 16}^{(0)}(x),
\end{aligned}
$$

(ii) $Q_{k, 0, s}(x)=B_{0, s}(x)-\frac{2^{2 k}}{2^{2 k}-1} e_{k, 16}^{(1)}(x)$,

(iii) $R_{k, 0, s}(x)=C_{0, s}(x)+\frac{\varepsilon_{2,4}(s)(-1)^{\left[\frac{s+1}{4}\right]} 2^{2 k-\left[\frac{s+1}{2}\right]}+1}{2^{2 k}-1} e_{k, 8}^{(1)}(x)-\frac{2^{2 k}}{2^{2 k}-1} e_{k, 16}^{(2)}(x)$,

(iv) $S_{k, 0, s}(x)=D_{0, s}(x)-\frac{2^{2 k}}{2^{2 k}-1} e_{k, 16}^{(3)}(x)$.

The following lemma justifies our definition of the four polynomials and plays a key part in the proof of our main theorems. 
CHAPTER 5. $P_{K, R, S}(X), Q_{K, R, S}(X), R_{K, R, S}(X)$ AND $S_{K, R, S}(X)$

Lemma 5.3.1. For $k, s \in \mathbb{N}$ with $k \geq 2$ and $s<4 k$, we have

$$
\begin{aligned}
& P_{k, 0, s}(x)+Q_{k, 0, s}(x)(1-x)^{1 / 4}+R_{k, 0, s}(x)(1-x)^{1 / 2}+S_{k, 0, s}(x)(1-x)^{3 / 4} \\
& =\left(\frac{1+(1-x)^{1 / 4}}{2}\right)^{s}-\frac{(-1)^{k}}{2^{s}\left(2^{2 k}-1\right)} e_{k, 1}(x) \\
& +\frac{\varepsilon_{2,4}(s)(-1)^{\left[\frac{s+1}{4}\right]} 2^{\left[\frac{s}{2}\right]}+(-1)^{k}}{2^{s}\left(2^{2 k}-1\right)} e_{k, 2}(x) \\
& -\frac{\varepsilon_{2,4}(s)(-1)^{\left[\frac{s+1}{4}\right]}\left(2^{2 k}+1\right)}{2^{\left[\frac{s+1}{2}\right]}\left(2^{2 k}-1\right)} e_{k, 4}(x) \\
& +\frac{\varepsilon_{2,4}(s)(-1)^{\left[\frac{s+1}{4}\right]} 2^{2 k-\left[\frac{s+1}{2}\right]}+1}{2^{2 k}-1}\left(e_{k, 8}^{(0)}(x)+e_{k, 8}^{(1)}(x)(1-x)^{1 / 2}\right) \\
& -\frac{2^{2 k}}{2^{2 k}-1}\left(e_{k, 16}^{(0)}(x)+e_{k, 16}^{(1)}(x)(1-x)^{1 / 4}+e_{k, 16}^{(2)}(1-x)^{1 / 2}+e_{k, 16}^{(3)}(1-x)^{3 / 4}\right)
\end{aligned}
$$

Proof. From Definition 5.3.1 and Lemma 3.1.3, we have

$$
\begin{aligned}
& \left(\frac{1+(1-x)^{1 / 4}}{2}\right)^{s}-\frac{(-1)^{k}}{2^{s}\left(2^{2 k}-1\right)} e_{k, 1}(x) \\
& +\frac{\varepsilon_{2,4}(s)(-1)^{\left[\frac{s+1}{4}\right]} 2^{\left[\frac{s}{2}\right]}+(-1)^{k}}{2^{s}\left(2^{2 k}-1\right)} e_{k, 2}(x) \\
& -\frac{\varepsilon_{2,4}(s)(-1)^{\left[\frac{s+1}{4}\right]}\left(2^{2 k}+1\right)}{2^{\left[\frac{s+1}{2}\right]}\left(2^{2 k}-1\right)} e_{k, 4}(x) \\
& +\frac{\varepsilon_{2,4}(s)(-1)^{\left[\frac{s+1}{4}\right]} 2^{2 k-\left[\frac{s+1}{2}\right]}+1}{2^{2 k}-1}\left(e_{k, 8}^{(0)}(x)+e_{k, 8}^{(1)}(x)(1-x)^{1 / 2}\right)
\end{aligned}
$$




$$
\begin{aligned}
& -\frac{2^{2 k}}{2^{2 k}-1}\left(e_{k, 16}^{(0)}(x)+e_{k, 16}^{(1)}(x)(1-x)^{1 / 4}+e_{k, 16}^{(2)}(1-x)^{1 / 2}+e_{k, 16}^{(3)}(1-x)^{3 / 4}\right) \\
= & A_{0, s}(x)+B_{0, s}(x)(1-x)^{1 / 4}+C_{0, s}(x)(1-x)^{1 / 2}+D_{0, s}(x)(1-x)^{3 / 4} \\
& -\frac{(-1)^{k}}{2^{s}\left(2^{2 k}-1\right)} e_{k, 1}(x)+\frac{\varepsilon_{2,4}(s)(-1)^{\left[\frac{s+1}{4}\right]} 2^{\left[\frac{s}{2}\right]}+(-1)^{k}}{2^{s}\left(2^{2 k}-1\right)} e_{k, 2}(x) \\
& -\frac{\varepsilon_{2,4}(s)(-1)^{\left[\frac{s+1}{4}\right]}\left(2^{2 k}+1\right)}{2^{\left[\frac{s+1}{2}\right]}\left(2^{2 k}-1\right)} e_{k, 4}(x) \\
& +\frac{\varepsilon_{2,4}(s)(-1)^{\left[\frac{s+1}{4}\right]} 2^{2 k-\left[\frac{s+1}{2}\right]}+1}{2^{2 k}-1}\left(e_{k, 8}^{(0)}(x)+e_{k, 8}^{(1)}(x)(1-x)^{1 / 2}\right) \\
& -\frac{2^{2 k}}{2^{2 k}-1}\left(e_{k, 16}^{(0)}(x)+e_{k, 16}^{(1)}(x)(1-x)^{1 / 4}+e_{k, 16}^{(2)}(1-x)^{1 / 2}+e_{k, 16}^{(3)}(1-x)^{3 / 4}\right) \\
= & P_{k, 0, s}(x)+Q_{k, 0, s}(x)(1-x)^{1 / 4}+R_{k, 0, s}(x)(1-x)^{1 / 2}+S_{k, 0, s}(x)(1-x)^{3 / 4},
\end{aligned}
$$

as required.

In the next lemma we evaluate the polynomial $P_{k, 0, s}(x)$ at $x=0$ and $x=1$. We also give an upper bound for the degree of the polynomial $P_{k, 0, s}(x)$ when $s<4 k$.

Lemma 5.3.2. For $k, s \in \mathbb{N}$ with $k \geq 2$ and $s<4 k$, we have

(i) $P_{k, 0, s}(0)=0$

(ii) $P_{k, 0, s}(1)=0$,

(iii) $\operatorname{deg}\left(P_{k, 0, s}(x)\right) \leq k-1$. 
CHAPTER 5. $P_{K, R, S}(X), Q_{K, R, S}(X), R_{K, R, S}(X)$ AND $S_{K, R, S}(X)$

Proof. (i) From Definition 5.3.1(i), Lemmas 3.3.3(i), 4.1.1(i), 4.1.2(i), 4.1.3(i), 4.2.1

(ii) and 4.3.2(ii), we have

$$
\begin{aligned}
& P_{k, 0, s}(0)=A_{0, s}(0)-\frac{(-1)^{k}}{2^{s}\left(2^{2 k}-1\right)} e_{k, 1}(0)+\frac{\varepsilon_{2,4}(s)(-1)^{\left[\frac{s+1}{4}\right]} 2^{\left[\frac{s}{2}\right]}+(-1)^{k}}{2^{s}\left(2^{2 k}-1\right)} e_{k, 2}(0) \\
& -\frac{\varepsilon_{2,4}(n)(-1)^{\left[\frac{s+1}{4}\right]}\left(2^{2 k}+1\right)}{2^{\left[\frac{s+1}{2}\right]}\left(2^{2 k}-1\right)} e_{k, 4}(0) \\
& +\frac{\varepsilon_{2,4}(s)(-1)^{\left[\frac{s+1}{4}\right]} 2^{2 k-\left[\frac{s+1}{2}\right]}+1}{2^{2 k}-1} e_{k, 8}^{(0)}(0)-\frac{2^{2 k}}{2^{2 k}-1} e_{k, 16}^{(0)}(0) \\
& =\frac{1}{4}+\frac{\varepsilon_{2,4}(x)(-1)^{\left[\frac{s+1}{4}\right]}}{2^{1+\left[\frac{s+1}{2}\right]}}-\frac{(-1)^{k}}{2^{s}\left(2^{2 k}-1\right)}+\frac{\varepsilon_{2,4}(s)(-1)^{\left[\frac{s+1}{4}\right]} 2^{\left[\frac{s}{2}\right]}+(-1)^{k}}{2^{s}\left(2^{2 k}-1\right)} \\
& -\frac{\varepsilon_{2,4}(s)(-1)^{\left[\frac{s+1}{4}\right]}\left(2^{2 k}+1\right)}{2^{\left[\frac{s+1}{2}\right]}\left(2^{2 k}-1\right)}+\frac{\varepsilon_{2,4}(s)(-1)^{\left[\frac{s+1}{4}\right]} 2^{2 k-\left[\frac{s+1}{2}\right]}+1}{2^{2 k}-1} \frac{2^{2 k}+1}{2^{2 k+1}} \\
& -\frac{2^{2 k}}{2^{2 k}-1} \frac{2^{2 k-1}\left(2^{2 k}+1\right)+1}{2^{4 k+1}} \\
& =\frac{1}{4}-\frac{(-1)^{k}}{2^{s}\left(2^{2 k}-1\right)}+\frac{(-1)^{k}}{2^{s}\left(2^{2 k}-1\right)}+\frac{2^{2 k}+1}{\left(2^{2 k}-1\right) 2^{2 k+1}}-\frac{2^{2 k-1}\left(2^{2 k}+1\right)+1}{\left(2^{2 k}-1\right) 2^{2 k+1}} \\
& +\varepsilon_{2,4}(s)(-1)^{\left[\frac{s+1}{4}\right]}\left(\frac{1}{2^{1+\left[\frac{s+1}{2}\right]}}+\frac{2^{\left[\frac{s}{2}\right]}}{2^{s}\left(2^{2 k}-1\right)}-\frac{2^{2 k}+1}{2^{\left[\frac{s+1}{2}\right]}\left(2^{2 k}-1\right)}\right. \\
& \left.+\frac{2^{2 k-\left[\frac{s+1}{2}\right]}\left(2^{2 k}+1\right)}{\left(2^{2 k}-1\right) 2^{2 k+1}}\right)
\end{aligned}
$$




$$
\begin{aligned}
= & \frac{1}{4}+\frac{2^{2 k}-2^{4 k-1}-2^{2 k-1}}{\left(2^{2 k}-1\right)\left(2^{2 k+1}\right)} \\
& +\varepsilon_{2,4}(s)(-1)^{\left[\frac{s+1}{4}\right]}\left(\frac{1}{2^{1+\left[\frac{s+1}{2}\right]}}+\frac{1}{2^{\left[\frac{s+1}{2}\right]}\left(2^{2 k}-1\right)}-\frac{2^{2 k}+1}{2^{\left[\frac{s+1}{2}\right]}\left(2^{2 k}-1\right)}\right. \\
= & \left.\frac{1}{4}+\frac{2^{2 k-1}-2^{4 k-1}}{\left(2^{2 k}-1\right) 2^{2 k+1}}+\frac{2^{2 k}+1}{\left(2^{2 k}-1\right) 2^{\left[\frac{s+1}{2}\right]+1}}\right) \\
& +\varepsilon_{2,4}(s)(-1)^{\left[\frac{s+1}{4}\right]}\left(\frac{\left(2^{2 k}-1\right)+2-2^{2 k+1}-2+2^{2 k}+1}{2\left(2^{2 k}-1\right) 2^{\left[\frac{s+1}{2}\right]}}\right) \\
= & \frac{1}{4}+\frac{2^{2 k-1}\left(1-2^{2 k}\right)}{\left(2^{2 k}-1\right) 2^{2 k+1}+\varepsilon_{2,4}(s)(-1)^{\left[\frac{s+1}{4}\right]}(0)=\frac{1}{4}-\frac{1}{4}=0,} \frac{1}{2}-1
\end{aligned}
$$

which proves (i).

(ii) By Lemmas 5.3.1, 4.1.1(ii), 4.1.2(ii), 4.1.3(ii), 4.2.2 and 4.3.3, we have

$$
\begin{aligned}
P_{k, 0, s}(1)= & \frac{1}{2^{s}}-\frac{(-1)^{k}}{2^{s}\left(2^{2 k}-1\right)} e_{k, 1}(1)+\frac{\varepsilon_{2,4}(s)(-1)^{\left[\frac{s+1}{4}\right]} 2^{\left[\frac{s}{2}\right]}+(-1)^{k}}{2^{s}\left(2^{2 k}-1\right)} e_{k, 2}(1) \\
& -\frac{\varepsilon_{2,4}(s)(-1)^{\left[\frac{s+1}{4}\right]}\left(2^{2 k}+1\right)}{2^{\left[\frac{s+1}{2}\right]}\left(2^{2 k}-1\right)} e_{k, 4}(1) \\
& +\frac{\varepsilon_{2,4}(s)(-1)^{\left[\frac{s+1}{4}\right]} 2^{2 k-\left[\frac{s+1}{2}\right]}+1}{2^{2 k}-1} e_{k, 8}^{(0)}(1)-\frac{2^{2 k}}{2^{2 k}-1} e_{k, 16}^{(0)}(1),
\end{aligned}
$$




$$
\begin{aligned}
= & \frac{1}{2^{s}}-\frac{(-1)^{k}}{2^{s}\left(2^{2 k}-1\right)} e_{k, 1}(1)+\frac{(-1)^{k}}{2^{s}\left(2^{2 k}-1\right)} e_{k, 2}(1)+\frac{e_{k, 8}^{(0)}(x)}{2^{2 k}-1} \\
& -\frac{2^{2 k}}{2^{2 k}-1} e_{k, 16}^{(0)}(1)+(-1)^{\left[\frac{s+1}{4}\right]} \varepsilon_{2,4}(s)\left(\frac{1}{2^{\left[\frac{s+1}{2}\right]}\left(2^{2 k}-1\right)} e_{k, 2}(1)\right. \\
& \left.-\frac{\left(2^{2 k}+1\right)}{2^{\left.\frac{s+1}{2}\right]}\left(2^{2 k}-1\right)} e_{k, 4}(1)+\frac{2^{2 k-\left[\frac{s+1}{2}\right]}}{2^{2 k}-1} e_{k, 8}^{(0)}(1)\right) \\
= & \frac{1}{2^{s}}-\frac{2^{2 k}}{2^{s}\left(2^{2 k}-1\right)}+\frac{1}{2^{s}\left(2^{2 k}-1\right)}+\frac{(-1)^{k}}{2^{4 k}\left(2^{2 k}-1\right)}-\frac{2^{2 k}}{2^{2 k}-1} \frac{(-1)^{k}}{2^{6 k}} \\
& +(-1)^{\left[\frac{s+1}{4}\right]} \varepsilon_{2,4}(s)\left(\frac{(-1)^{k}}{2^{\left[\frac{s+1}{2}\right]}\left(2^{2 k}-1\right)}-\frac{\left(2^{2 k}+1\right)(-1)^{k}}{2^{\left[\frac{s+1}{2}\right]}\left(2^{2 k}-1\right) 2^{2 k}}+\frac{2^{2 k-\left[\frac{s+1}{2}\right]}}{2^{2 k}-1} \frac{(-1)^{k}}{2^{4 k}}\right) \\
= & \frac{\left(2^{2 k}-1\right) 2^{4 k}-2^{6 k}+2^{4 k}+(-1)^{k} 2^{s}-(-1)^{k} 2^{s}}{2^{4 k+s}\left(2^{2 k}-1\right)} \\
& +(-1)^{\left[\frac{s+1}{4}\right]} \varepsilon_{2,4}(s)\left(\frac{(-1)^{k} 2^{2 k}-\left(2^{2 k}+1\right)(-1)^{k}+(-1)^{k}}{2^{2 k+\left[\frac{s+1}{2}\right]}\left(2^{2 k}-1\right)}\right) \\
& +(-1)^{\left[\frac{s+1}{4}\right]} \varepsilon_{2,4}(s)(0)=0,
\end{aligned}
$$

which proves (ii).

(iii) By Lemmas 3.1.2(i), 4.1.1(iii)(iv), 4.1.2 (iii)(iv), 4.1.3 (iii)(iv), 4.2.3(i)(iii) and 4.3.4(ii)(iii), we have

$$
\begin{aligned}
A_{0, s}(x) & =a_{k-1} x^{k-1}+\cdots, \\
e_{k, 1}(x) & =x^{k}+\cdots,
\end{aligned}
$$


CHAPTER 5. $P_{K, R, S}(X), Q_{K, R, S}(X), R_{K, R, S}(X)$ AND $S_{K, R, S}(X)$

$$
\begin{aligned}
e_{k, 2}(x) & =x^{k}+\cdots \\
e_{k, 4}(x) & =\frac{1}{2^{2 k}} x^{k}+\cdots \\
e_{k, 8}^{(0)}(x) & =\frac{1}{2^{4 k}} x^{k}+\cdots \\
e_{k, 16}^{(0)}(x) & =\frac{1}{2^{6 k}} x^{k}+\cdots
\end{aligned}
$$

Then, by Definition 5.3.1(i) we have

$$
\begin{aligned}
P_{k, 0, s}(x)= & \left(-\frac{(-1)^{k}}{2^{s}\left(2^{2 k}-1\right)}+\frac{\varepsilon_{2,4}(s)(-1)^{\left[\frac{s+1}{4}\right]} 2^{\left[\frac{s}{2}\right]}+(-1)^{k}}{2^{s}\left(2^{2 k}-1\right)}\right. \\
& -\frac{\varepsilon_{2,4}(s)(-1)^{\left.\frac{s+1}{4}\right]}\left(2^{2 k}+1\right)}{2^{\left[\frac{s+1}{2}\right]}\left(2^{2 k}-1\right)} \frac{1}{2^{2 k}}+\frac{\varepsilon_{2,4}(s)(-1)^{\left[\frac{s+1}{4}\right]} 2^{2 k-\left[\frac{s+1}{2}\right]}+1}{2^{2 k}-1} \frac{1}{2^{4 k}} \\
& \left.-\frac{2^{2 k}}{2^{2 k}-1} \frac{1}{2^{6 k}}\right) x^{k}+\cdots \\
= & \left(-\frac{(-1)^{k}}{2^{s}\left(2^{2 k}-1\right)}+\frac{(-1)^{k}}{2^{s}\left(2^{2 k}-1\right)}+\frac{1}{2^{2 k}-1} \frac{1}{2^{4 k}}-\frac{2^{2 k}}{2^{2 k}-1} \frac{1}{2^{6 k}}\right. \\
& +(-1)^{\left[\frac{s+1}{4}\right]} \varepsilon_{2,4}(s)\left(\frac{2^{\left[\frac{s}{2}\right]}}{2^{s}\left(2^{2 k}-1\right)}-\frac{2^{2 k}+1}{2^{\left.\frac{s+1}{2}\right]}\left(2^{2 k}-1\right)} \frac{1}{2^{2 k}}\right. \\
& \left.\left.+\frac{2^{2 k-\left[\frac{s+1}{2}\right]}}{2^{2 k}-1} \frac{1}{2^{4 k}}\right)\right) x^{k}+\cdots, \\
& \left.\left.+\frac{1}{2^{\left[\frac{s+1}{2}\right]+2 k}\left(2^{2 k}-1\right)}\right)\right) x^{k}+\cdots \\
= & \left(0+(-1)^{\left[\frac{s+1}{4}\right]} \varepsilon_{2,4}(s)\left(\frac{1}{2^{\left[\frac{s+1}{2}\right]}\left(2^{2 k}-1\right)}-\frac{2^{2 k}+1}{2^{\left[\frac{s+1}{2}\right]+2 k}\left(2^{2 k}-1\right)}\right.\right. \\
& \\
& \\
& \\
& \\
&
\end{aligned}
$$




$$
=0 x^{k}+\cdots,
$$

which shows that $\operatorname{deg}\left(P_{k, 0, s}(x)\right) \leq k-1$.

The next lemma evaluates the polynomial $R_{k, 0, s}(x)$ at $x=0$ and gives an upper bound for the degrees of $Q_{k, 0, s}(x), R_{k, 0, s}(x)$ and $S_{k, 0, s}(x)$.

Lemma 5.3.3. For $k, s \in \mathbb{N}$ with $k \geq 2$ and $s<4 k$, we have

(i) $R_{k, 0, s}(0)=0$

(ii) $\operatorname{deg}\left(R_{k, 0, s}(x)\right) \leq k-1$,

(iii) $\operatorname{deg}\left(Q_{k, 0, s}(x)\right) \leq k-1$,

(iv) $\operatorname{deg}\left(S_{k, 0, s}(x)\right) \leq k-1$.

Proof. (i) From Definition 5.3.1(iii), Lemmas 3.3.3(iii), 4.2.1(iii) and 4.3.2(iv), we have

$$
\begin{aligned}
R_{k, 0, s}(0)= & C_{0, s}(0)+\frac{\varepsilon_{2,4}(s)(-1)^{\left[\frac{s+1}{4}\right]} 2^{2 k-\left[\frac{s+1}{2}\right]}+1}{2^{2 k}-1} e_{k, 8}^{(1)}(0)-\frac{2^{2 k}}{2^{2 k}-1} e_{k, 16}^{(2)}(0) \\
= & \frac{1}{4}-\frac{\varepsilon_{2,4}(s)(-1)^{\left[\frac{s+1}{4}\right]}}{2^{\left[\frac{s+1}{2}\right]+1}}+\left(\frac{\varepsilon_{2,4}(s)(-1)^{\left[\frac{s+1}{4}\right]} 2^{2 k-\left[\frac{s+1}{2}\right]}+1}{2^{2 k}-1}\right)\left(\frac{2^{2 k}-1}{2^{2 k+1}}\right) \\
& -\frac{2^{2 k}}{2^{2 k}-1}\left(\frac{2^{2 k-1}\left(2^{2 k}+1\right)-1}{2^{4 k+1}}\right) \\
= & \frac{1}{4}+\frac{1}{2^{2 k+1}}-\frac{\left(2^{2 k-1}\left(2^{2 k}+1\right)-1\right)}{\left(2^{2 k}-1\right) 2^{2 k+1}} \\
& +\varepsilon_{2,4}(s)(-1)^{\left[\frac{s+1}{4}\right]}\left(\frac{-1}{2^{\left[\frac{s+1}{2}\right]+1}}+\frac{1}{2^{\left[\frac{s+1}{2}\right]+1}}\right)
\end{aligned}
$$


CHAPTER 5. $P_{K, R, S}(X), Q_{K, R, S}(X), R_{K, R, S}(X) A N D S_{K, R, S}(X)$

$$
=\frac{2^{2 k-1}\left(2^{2 k}-1\right)+2^{2 k}-1-2^{2 k-1}\left(2^{2 k}+1\right)+1}{\left(2^{2 k}-1\right) 2^{2 k+1}}=0 .
$$

(ii) This follows from Definition 5.3.1(ii), Lemmas 3.1.2(ii) and 4.3.4(i).

(iii) This follows from Definition 5.3.1(iii), Lemmas 3.1.2(iii), 4.2 .3 (ii) and 4.3.4(i).

(iv) This follows from Definition 5.3.1(iv), Lemmas 3.1.2(iv) and 4.3.4(i). 


\section{Chapter 6}

\section{Extensions of the Ramanujan-Mordell formula}

We recall that the main problem that we address in this thesis is that of determining the number of representations of a positive integer $n$ by a quadratic form of the type

$$
x_{1}^{2}+\cdots+x_{a}^{2}+2 x_{a+1}^{2}+\cdots+2 x_{a+b}^{2}+4 x_{a+b+1}^{2}+\cdots+4 x_{t}^{2},
$$

where $a \in \mathbb{N}, b, c \in \mathbb{N}_{0}$ and $t=a+b+c$. In this chapter, we give formulae that determine the number of representations for any positive integer $n$ and any $a, b, c \in \mathbb{N}_{0}$, where $b$ is an even number and $t=4 k$. These formulae give our extensions of the Ramanujan-Mordell formula (Theorem 1.0.1). As in previous chapters, we have three cases. Each section covers one such case.

In Section 6.1 we obtain explicit formulae for $N([4 k-2 r, 2 r, 0] ; n)$ when $k, r \in \mathbb{N}$ and $r<2 k$.

In Section 6.2 we obtain explicit formulae for $N([4 k-2 r-s, 2 r, s] ; n)$ when $k, r, s \in \mathbb{N}$ and $2 r+s<4 k$.

In Section 6.3 we obtain explicit formulae for $N([4 k-s, 0, s] ; n)$ when $k, s \in \mathbb{N}$ and $s<4 k$. 
In order to include the case $k=1$ in the theorems of this chapter, we define the polynomials

$$
\begin{aligned}
e_{1,1}(x) & :=1-5 x \\
e_{1,2}(x) & :=1-2 x \\
e_{1,4}(x) & :=1-\frac{5}{4} x \\
e_{1,8}(x) & :=\frac{5}{8}-\frac{11}{16} x+\frac{3}{8}(1-x)^{1 / 2} \\
e_{1,16}(x) & :=\frac{11}{32}-\frac{23}{64} x+\frac{3}{16}(1-x)^{1 / 4}+\frac{9}{32}(1-x)^{1 / 2}+\frac{3}{16}(1-x)^{3 / 4}
\end{aligned}
$$

We emphasize that for $x$ and $z$ as defined in Definition 2.3.2 we have

$$
E_{1}\left(q^{r}\right) \neq e_{1, r}(x) z^{2} \quad \text { for every } r \in\{1,2,4,8,16\} .
$$

\subsection{Number of representations by the quadratic form $x_{1}^{2}+\cdots+x_{4 k-2 r}^{2}+2 x_{4 k-2 r+1}^{2}+\cdots+2 x_{4 k}^{2}$.}

To begin this section we use all the results that we found in Section 5.1 to obtain the following theorem that is valid for any variables $x$ and $z$.

Theorem 6.1.1. For $k, r \in \mathbb{N}$ with $r<2 k$ and variables $x$ and $z$, we have

$$
\begin{aligned}
& \left(\frac{1+(1-x)^{1 / 2}}{2}\right)^{r} z^{2 k} \\
& \quad=\frac{(-1)^{k}}{2^{r}\left(2^{2 k}-1\right)} e_{k, 1}(x) z^{2 k}-\frac{(-1)^{k}}{2^{r}\left(2^{2 k}-1\right)} e_{k, 2}(x) z^{2 k}-\frac{1}{2^{2 k}-1} e_{k, 4}(x) z^{2 k} \\
& +\frac{2^{2 k}}{2^{2 k}-1} e_{k, 8}(x) z^{2 k}
\end{aligned}
$$




$$
+(1-x) \sum_{i=1}^{k-2} a_{k, r, 0}^{\prime}(i) x^{\imath} z^{2 k}+(1-x)^{1 / 2} \sum_{i=1}^{k-1} c_{k, r, 0}^{\prime}(i) x^{2} z^{2 k},
$$

where $a_{k, r, 0}^{\prime}(i) \in \mathbb{Q}$ for $i \in\{1, \ldots, k-2\}$ and $c_{k, r, 0}^{\prime}(i) \in \mathbb{Q}$ for $i \in\{1, \ldots, k-1\}$.

Proof. When $k=1$ we have $r=1$. From (6.0.1)-(6.0.4) we have

$$
\frac{1+(1-x)^{1 / 2}}{2} z^{2}=-\frac{1}{6} e_{1,1}(x) z^{2}+\frac{1}{6} e_{1,2}(x) z^{2}-\frac{1}{3} e_{1,4}(x) z^{2}+\frac{4}{3} e_{1,8}(x) z^{2}
$$

For $k \geq 2$, by Definition 5.1.1, Lemmas 5.1.2 and 5.1.3 we have

$$
\begin{aligned}
& P_{k, r, 0}(x)=(1-x)\left(a_{k, r, 0}^{\prime}(1) x+\cdots+a_{k, r, 0}^{\prime}(k-2) x^{k-2}\right) \\
& R_{k, r, 0}(x)=c_{k, r, 0}^{\prime}(1) x+\cdots+c_{k, r, 0}^{\prime}(k-1) x^{k-1}
\end{aligned}
$$

where $a_{k, r, 0}^{\prime}(i) \in \mathbb{Q}$ for $i \in\{1, \ldots, k-2\}$ and $c_{k, r, 0}^{\prime}(i) \in \mathbb{Q}$ for $i \in\{1, \ldots, k-1\}$.

This implies, by Lemma 5.1.1 and (4.2.2), that

$$
\begin{aligned}
& \left(\frac{1+(1-x)^{1 / 2}}{2}\right)^{r} \\
& =\frac{(-1)^{k}}{2^{r}\left(2^{2 k}-1\right)} e_{k, 1}(x)-\frac{(-1)^{k}}{2^{r}\left(2^{2 k}-1\right)} e_{k, 2}(x)-\frac{1}{2^{2 k}-1} e_{k, 4}(x) \\
& \quad+\frac{2^{2 k}}{2^{2 k}-1} e_{k, 8}(x) \\
& \quad+(1-x) \sum_{i=1}^{k-2} a_{k, r, 0}^{\prime}(i) x^{2}+(1-x)^{1 / 2} \sum_{\imath=1}^{k-1} c_{k, r, 0}^{\prime}(i) x^{\imath} .
\end{aligned}
$$

Finally, we obtain the asserted result by multiplying both sides of (6.1.1) by $z^{2 k}$. 
Replacing $x$ and $z$ in Theorem 6.1.1 by their values given in Definition 2.3.2, we obtain the following identities involving the theta function $\varphi$, Eisenstein series $E_{k}\left(q^{r}\right)$ with $r \in\{1,2,4,8\}$ and the generalized Ramanujan discriminant function $\Delta(a, b, c ; q)$.

Theorem 6.1.2. For $k, r \in \mathbb{N}$ with $r<2 k$ and $q \in D_{\delta}$, we have

$$
\begin{aligned}
& \varphi(q)^{4 k-2 r} \varphi\left(q^{2}\right)^{2 r} \\
& \quad=\frac{(-1)^{k}}{2^{r}\left(2^{2 k}-1\right)} E_{k}(q)-\frac{(-1)^{k}}{2^{r}\left(2^{2 k}-1\right)} E_{k}\left(q^{2}\right)-\frac{1}{2^{2 k}-1} E_{k}\left(q^{4}\right)+\frac{2^{2 k}}{2^{2 k}-1} E_{k}\left(q^{8}\right) \\
& \quad+\sum_{\imath=1}^{k-2} a_{k, r, 0}(\imath) \Delta\left(\frac{\imath-k+2}{3}, \frac{-6 \imath+5 k-6}{6}, \frac{2 \imath-k+1}{3} ; q\right) \\
& \quad+\sum_{\imath=1}^{k-1} c_{k, r, 0}(\imath) \Delta\left(\frac{\imath-k+1}{3}, \frac{-6 \imath+5 k-3}{6}, \frac{4 \imath-2 k+1}{6} ; q\right),
\end{aligned}
$$

where $a_{k, r, 0}(\imath)=2^{4 \imath} a_{k, r, 0}^{\prime}(\imath)$ for $\imath \in\{1, \ldots, k-2\}, c_{k, r, 0}(\imath)=2^{4 \imath} c_{k, r, 0}^{\prime}(\imath)$ for $\imath \in$ $\{1, \ldots, k-1\}$ and the coefficients $a_{k, r, 0}^{\prime}(\imath), c_{k, r, 0}^{\prime}(\imath)$ are given in Theorem 6.1.1.

Proof. Let $q \in D_{\delta}$. Set $x=1-\frac{\varphi^{4}(-q)}{\varphi^{4}(q)}, z=\varphi^{2}(q)$. For $k=1$, we have by Theorems 2.5.2 (i) (ii), 2.9.2 and 2.9.5

$$
\varphi(q)^{2} \varphi\left(q^{2}\right)^{2}+\frac{1}{6} E_{1}(q)-\frac{1}{6} E_{1}\left(q^{2}\right)+\frac{1}{3} E_{1}\left(q^{4}\right)-\frac{4}{3} E_{1}\left(q^{8}\right)=0,
$$

which gives the asserted result on isolating the term $\varphi(q)^{2} \varphi\left(q^{2}\right)^{2}$.

For $k \geq 2$, we note that by Theorem 2.10 .3 we have for $\imath \in\{1, \ldots, k-2\}$

$$
a_{k, r, 0}^{\prime}(\imath) x^{2}(1-x) z^{2 k}=2^{4 \imath} a_{k, r, 0}^{\prime}(\imath) \frac{x^{2}(1-x)}{2^{4 \imath}} z^{2 k}
$$




$$
\begin{aligned}
& =2^{4 \imath} a_{k, r, 0}^{\prime}(\imath) \Delta\left(\frac{i-k+2}{3}, \frac{-6 i+5 k-6}{6}, \frac{2 i-k+1}{3} ; q\right) \\
& =a_{k, r, 0}(i) \Delta\left(\frac{i-k+2}{3}, \frac{-6 i+5 k-6}{6}, \frac{2 i-k+1}{3} ; q\right),
\end{aligned}
$$

where $a_{k, r, 0}(i)=2^{4 \imath} a_{k, r, 0}^{\prime}(i)$. Similarly, we obtain for $i \in\{1, \ldots, k-1\}$

$$
c_{k, r, 0}^{\prime}(i) x^{2}(1-x)^{1 / 2} z^{2 k}=c_{k, r, 0}(i) \Delta\left(\frac{i-k+1}{3}, \frac{-6 i+5 k-3}{6}, \frac{4 i-2 k+1}{6} ; q\right),
$$

where $c_{k, r, 0}(i)=2^{4 \imath} c_{k, r, 0}^{\prime}(i)$. Finally, from Theorems 2.5.2, 4.4.1 and 6.1.1, we obtain the desired results.

Our next result gives the number of representations of $n \in \mathbb{N}$ by the quadratic form

$$
x_{1}^{2}+\cdots+x_{4 k-2 r}^{2}+2 x_{4 k-2 r+1}^{2}+\cdots+2 x_{4 k}^{2},
$$

where $r<2 k$.

Theorem 6.1.3. For $k, r, n \in \mathbb{N}$ with $r<2 k$, we have

$$
\begin{aligned}
& N([4 k-2 r, 2 r, 0] ; n) \\
& =-\frac{(-1)^{k} 4 k}{2^{r}\left(2^{2 k}-1\right) B_{2 k}} \sigma_{2 k-1}(n)+\frac{(-1)^{k} 4 k}{2^{r}\left(2^{2 k}-1\right) B_{2 k}} \sigma_{2 k-1}\left(\frac{n}{2}\right)+\frac{4 k}{\left(2^{2 k}-1\right) B_{2 k}} \sigma_{2 k-1}\left(\frac{n}{4}\right) \\
& \quad-\frac{2^{2 k+2} k}{\left(2^{2 k}-1\right) B_{2 k}} \sigma_{2 k-1}\left(\frac{n}{8}\right) \\
& +\sum_{i=1}^{k-2} a_{k, r, 0}(i) \delta\left(\frac{i-k+2}{3}, \frac{-6 i+5 k-6}{6}, \frac{2 i-k+1}{3} ; n\right) \\
& +\sum_{i=1}^{k-1} c_{k, r, 0}(i) \delta\left(\frac{i-k+1}{3}, \frac{-6 i+5 k-3}{6}, \frac{4 i-2 k+1}{6} ; n\right),
\end{aligned}
$$


where $a_{k, r, 0}(i)=2^{4 i} a_{k, r, 0}^{\prime}(i)$ for $i \in\{1, \ldots, k-2\}, c_{k, r, 0}(i)=2^{4 i} c_{k, r, 0}^{\prime}(i)$ for $i \in$ $\{1, \ldots, k-1\}$ and the coefficients $a_{k, r, 0}^{\prime}(i), c_{k, r, 0}^{\prime}(i)$ are given in Theorem 6.1.1.

Proof. From Theorem 2.8.1, Definition 2.9.1, Definition 2.10.3 and Theorem 6.1.2 we have for $q \in D_{\delta}$

$$
\begin{aligned}
1+ & \sum_{n=1}^{\infty} N([4 k-2 r, 2 r, 0] ; n) q^{n}=\varphi(q)^{4 k-2 r} \varphi\left(q^{2}\right)^{2 r} \\
& =\frac{(-1)^{k}}{2^{r}\left(2^{2 k}-1\right)}\left(1-\frac{4 k}{B_{2 k}} \sum_{n=1}^{\infty} \sigma_{2 k-1}(n) q^{n}\right)-\frac{(-1)^{k}}{2^{r}\left(2^{2 k}-1\right)}\left(1-\frac{4 k}{B_{2 k}} \sum_{n=1}^{\infty} \sigma_{2 k-1}(n) q^{2 n}\right) \\
& -\frac{1}{2^{2 k}-1}\left(1-\frac{4 k}{B_{2 k}} \sum_{n=1}^{\infty} \sigma_{2 k-1}(n) q^{4 n}\right)+\frac{2^{2 k}}{2^{2 k}-1}\left(1-\frac{4 k}{B_{2 k}} \sum_{n=1}^{\infty} \sigma_{2 k-1}(n) q^{8 n}\right) \\
& +\sum_{i=1}^{k-2} a_{k, r, 0}(i) \sum_{n=1}^{\infty} \delta\left(\frac{i-k+2}{3}, \frac{-6 i+5 k-6}{6}, \frac{2 i-k+1}{3} ; n\right) q^{n} \\
& +\sum_{i=1}^{k-1} c_{k, r, 0}(i) \sum_{n=1}^{\infty} \delta\left(\frac{i-k+1}{3}, \frac{-6 i+5 k-3}{6}, \frac{4 i-2 k+1}{6} ; q\right) q^{n} \\
& +\sum_{n=1}^{\infty}\left(\sum_{i=1}^{k-2} a_{k, r, 0}(i) \delta\left(\frac{i-k+2}{3}, \frac{-6 i+5 k-6}{6}, \frac{2 i-k+1}{3} ; n\right) q^{n}\right. \\
& +\frac{1}{2^{2 k}-1}\left(\frac{4 k}{B_{2 k}} \sum_{n=1}^{\infty} \sigma_{2 k-1}(n) q^{4 n}\right)-\frac{2^{2 k}}{2^{2 k}-1}\left(\frac{4 k}{B_{2 k}} \sum_{n=1}^{\infty} \sigma_{2 k-1}(n) q^{8 n}\right) \\
& =1-\frac{(-1)^{k}}{2^{r}\left(2^{2 k}-1\right)}\left(\frac{4 k}{B_{2 k}} \sum_{n=1}^{\infty} \sigma_{2 k-1}(n) q^{n}\right)+\frac{(-1)^{k}}{2^{r}\left(2^{2 k}-1\right)}\left(\frac{4 k}{B_{2 k}} \sum_{n=1}^{\infty} \sigma_{2 k-1}(n) q^{2 n}\right)
\end{aligned}
$$




$$
\begin{aligned}
& +\sum_{n=1}^{\infty}\left(\sum_{i=1}^{k-1} c_{k, r, 0}(i) \delta\left(\frac{i-k+1}{3}, \frac{-6 i+5 k-3}{6}, \frac{4 i-2 k+1}{6} ; n\right)\right) q^{n} \\
& =1+\sum_{n=1}^{\infty}\left(-\frac{(-1)^{k} 4 k}{2^{r}\left(2^{2 k}-1\right) B_{2 k}} \sigma_{2 k-1}(n)+\frac{(-1)^{k} 4 k}{2^{r}\left(2^{2 k}-1\right) B_{2 k}} \sigma_{2 k-1}\left(\frac{n}{2}\right)\right. \\
& +\frac{4 k}{\left(2^{2 k}-1\right) B_{2 k}} \sigma_{2 k-1}\left(\frac{n}{4}\right)-\frac{2^{2 k+2} k}{\left(2^{2 k}-1\right) B_{2 k}} \sigma_{2 k-1}\left(\frac{n}{8}\right) \\
& +\sum_{i=1}^{k-2} a_{k, r, 0}(i) \delta\left(\frac{i-k+2}{3}, \frac{-6 i+5 k-6}{6}, \frac{2 i-k+1}{3} ; n\right) \\
& \left.+\sum_{i=1}^{k-1} c_{k, r, 0}(i) \delta\left(\frac{i-k+1}{3}, \frac{-6 i+5 k-3}{6}, \frac{4 i-2 k+1}{6} ; n\right)\right) q^{n} .
\end{aligned}
$$

Equating coefficients of $q^{n} \quad(n \in \mathbb{N})$, we obtain the desired result.

When $k=1$ we have $r=1$ (as $1 \leq r<2 k$ ). In this case Theorem 6.1.3 gives

$$
N([2,2,0] ; n)=4 \sigma(n)-4 \sigma(n / 2)+8 \sigma(n / 4)-32 \sigma(n / 8), \quad n \in \mathbb{N}
$$

in agreement with $[2$, Theorem 1.8, p. 297]. 


\subsection{Number of representations by the quadratic} form $x_{1}^{2}+\cdots+x_{4 k-2 r-s}^{2}+2 x_{4 k-2 r-s+1}^{2}+\cdots+2 x_{4 k-s}^{2}+$

$$
4 x_{4 k-s+1}^{2}+\cdots+4 x_{4 k}^{2}
$$

To begin this section we use all the results that we found in Section 5.2 to obtain the following theorem that is valid for any variables $x$ and $z$.

Theorem 6.2.1. For $k, r, s \in \mathbb{N}$ with $2 r+s<4 k$, we have

$$
\begin{aligned}
& \left(\frac{1+(1-x)^{1 / 2}}{2}\right)^{r}\left(\frac{1+(1-x)^{1 / 4}}{2}\right)^{s} z^{2 k} \\
& =\frac{(-1)^{k}}{2^{r+s}\left(2^{2 k}-1\right)} e_{k, 1}(x) z^{2 k}-\frac{(-1)^{k}}{2^{r+s}\left(2^{2 k}-1\right)} e_{k, 2}(x) z^{2 k}-\frac{1}{2^{2 k}-1} e_{k, 8}(x) z^{2 k} \\
& +\frac{2^{2 k}}{2^{2 k}-1} e_{k, 16}(x) z^{2 k}+(1-x) \sum_{\imath=1}^{k-2} a_{k, r, s}^{\prime}(i) x^{2} z^{2 k}+(1-x)^{1 / 4} \sum_{\imath=1}^{k-1} b_{k, r, s}^{\prime}(i) x^{\imath} z^{2 k} \\
& \quad+(1-x)^{1 / 2} \sum_{i=1}^{k-1} c_{k, r, s}^{\prime}(i) x^{\imath} z^{2 k}+(1-x)^{3 / 4} \sum_{i=1}^{k-1} d_{k, r, s}^{\prime}(i) x^{\imath} z^{2 k},
\end{aligned}
$$

where $a_{k, r, s}^{\prime}(i) \in \mathbb{Q}$ for $i \in\{1, \ldots, k-2\}$ and $b_{k, r, s}^{\prime}(i), c_{k, r, s}^{\prime}(i), d_{k, r, s}^{\prime}(\imath) \in \mathbb{Q}$ for $i \in$ $\{1, \ldots, k-1\}$.

Proof. When $k=1$ we have $r=1$ and $s=1$. From (6.0.1)-(6.0.5) we have

$$
\begin{aligned}
\left(\frac{1+(1-x)^{1 / 2}}{2}\right)\left(\frac{1+(1-x)^{1 / 4}}{2}\right) z^{2}= & -\frac{1}{12} e_{1,1}(x) z^{2}+\frac{1}{12} e_{1,2}(x) z^{2}-\frac{1}{3} e_{1,8}(x) z^{2} \\
& +\frac{4}{3} e_{1,16}(x) z^{2}
\end{aligned}
$$

For $k \geq 2$, by Definition 5.2.1 and Lemmas 5.2.2 - 5.2.5, we have

$$
P_{k, r, s}(x)=(1-x)\left(a_{k, r, s}(1) x+\cdots+a_{k, r, s}(k-2) x^{k-2}\right)
$$




$$
\begin{aligned}
& Q_{k, r, s}(x)=b_{k, r, s}^{\prime}(1) x+\cdots+b_{k, r, s}^{\prime}(k-1) x^{k-1}, \\
& R_{k, r, s}(x)=c_{k, r, s}^{\prime}(1) x+\cdots+c_{k, r, s}^{\prime}(k-1) x^{k-1} \\
& S_{k, r, s}(x)=d_{k, r, s}^{\prime}(1) x+\cdots+d_{k, r, s}^{\prime}(k-1) x^{k-1},
\end{aligned}
$$

where $a_{k, r, s}^{\prime}(i) \in \mathbb{Q}$ for $i \in\{1, \ldots, k-2\}$ and $b_{k, r, s}^{\prime}(i), c_{k, r, s}^{\prime}(i), d_{k, r, s}^{\prime}(i) \in \mathbb{Q}$ for $i \in$ $\{1, \ldots, k-1\}$. This implies, by Lemma 5.2.1, (4.2.2) and (4.3.2) that

$$
\begin{aligned}
& \left(\frac{1+(1-x)^{1 / 2}}{2}\right)^{r}\left(\frac{1+(1-x)^{1 / 4}}{2}\right)^{s} \\
& =\frac{(-1)^{k}}{2^{r+s}\left(2^{2 k}-1\right)} e_{k, 1}(x)-\frac{(-1)^{k}}{2^{r+s}\left(2^{2 k}-1\right)} e_{k, 2}(x)-\frac{1}{2^{2 k}-1} e_{k, 8}(x) \\
& +\frac{2^{2 k}}{2^{2 k}-1} e_{k, 16}(x) \\
& +(1-x) \sum_{\imath=1}^{k-2} a_{k, r, s}^{\prime}(i) x^{2}+(1-x)^{1 / 4} \sum_{i=1}^{k-1} b_{k, r, s}^{\prime}(i) x^{2} \\
& +(1-x)^{1 / 2} \sum_{i=1}^{k-1} c_{k, r, s}^{\prime}(i) x^{\imath}+(1-x)^{3 / 4} \sum_{i=1}^{k-1} d_{k, r, s}^{\prime}(i) x^{\imath}
\end{aligned}
$$

Finally, we obtain the asserted result by multiplying both sides of (6.2.1) by $z^{2 k}$.

Replacing $x$ and $z$ in Theorem 6.2 .1 by their values given in Definition 2.3 .2 we obtain the following identities involving the theta function $\varphi$, Eisenstein series $E_{k}\left(q^{r}\right)$ with $r \in\{1,2,4,8,16\}$ and the generalized Ramanujan discriminant function 
$\Delta(a, b, c ; q)$

Theorem 6.2.2. For $k, r, s \in \mathbb{N}$ with $2 r+s<4 k$ and $q \in D_{\delta}$, we have

$$
\begin{aligned}
\varphi^{4 k-2 r-s}(q) \varphi^{2 r}\left(q^{2}\right) \varphi^{s}\left(q^{4}\right) \\
=\frac{(-1)^{k}}{2^{r+s}\left(2^{2 k}-1\right)} E_{k}(q)-\frac{(-1)^{k}}{2^{r+s}\left(2^{2 k}-1\right)} E_{k}\left(q^{2}\right)-\frac{1}{2^{2 k}-1} E_{k}\left(q^{8}\right)+\frac{2^{2 k}}{2^{2 k}-1} E_{k}\left(q^{16}\right) \\
+\sum_{\imath=1}^{k-2} a_{k, r, s}(\imath) \Delta\left(\frac{\imath-k+2}{3}, \frac{-6 \imath+5 k-6}{6}, \frac{2 \imath-k+1}{3} ; q\right) \\
+\sum_{\imath=1}^{k-1} b_{k, r, s}(\imath) \Delta\left(\frac{2 \imath-2 k+1}{6}, \frac{-12 \imath+10 k-3}{12}, \frac{8 \imath-4 k+1}{12} ; q\right) \\
+\sum_{\imath=1}^{k-1} c_{k, r, s}(\imath) \Delta\left(\frac{\imath-k+1}{3}, \frac{-6 \imath+5 k-3}{6}, \frac{4 \imath-2 k+1}{6} ; q\right) \\
+\sum_{\imath=1}^{k-1} d_{k, r, s}(\imath) \Delta\left(\frac{2 \imath-2 k+3}{6}, \frac{-12 \imath+10 k-9}{12}, \frac{8 \imath-4 k+3}{12} ; q\right)
\end{aligned}
$$

where $a_{k, r, s}(\imath)=2^{4 \imath} a_{k, r, s}^{\prime}(\imath)$ for $\imath \in\{1, \ldots, k-2\}, b_{k, r, s}(\imath)=2^{4 \imath} b_{k, r, s}^{\prime}(\imath)$ for $\imath \in$ $\{1, \ldots, k-1\}, c_{k, r, s}(\imath)=2^{4 \imath} c_{k, r, s}^{\prime}(\imath)$ for $\imath \in\{1, \ldots, k-1\}, d_{k, r, s}(\imath)=2^{4 \imath} d_{k, r, s}^{\prime}(\imath)$ for $\imath \in\{1, \ldots, k-1\}$ and the coefficıents $a_{k, r, s}^{\prime}(\imath), b_{k, r, s}^{\prime}(\imath), c_{k, r, s}^{\prime}(\imath), d_{k, r, s}^{\prime}(\imath)$ are given $\imath n$ Theorem 6.2.1.

Proof. Let $q \in D_{\delta}$. Set $x=1-\frac{\varphi^{4}(-q)}{\varphi^{4}(q)}, z=\varphi^{2}(q)$. For $k=1$, we have by Theorems 2.5.2 (i)(ii)(iii), 2.9.2 and 2.9.5

$$
\varphi(q) \varphi\left(q^{2}\right)^{2} \varphi\left(q^{4}\right)+\frac{1}{12} E_{1}(q)-\frac{1}{12} E_{1}\left(q^{2}\right)+\frac{1}{3} E_{1}\left(q^{8}\right)-\frac{4}{3} E_{1}\left(q^{16}\right)=0,
$$


which is the asserted result if we isolate the term $\varphi(q) \varphi\left(q^{2}\right)^{2} \varphi\left(q^{4}\right)$.

For $k \geq 2$, we note that by Theorem 2.10 .3 we have for $i \in\{1, \ldots, k-2\}$

$$
\begin{aligned}
a_{k, r, s}^{\prime}(i) x^{2}(1-x) z^{2 k} & =2^{4 \imath} a_{k, r, s}^{\prime}(i) \frac{x^{2}(1-x)}{2^{4 \imath}} z^{2 k} \\
& =2^{4 \imath} a_{k, r, s}^{\prime}(i) \Delta\left(\frac{i-k+2}{3}, \frac{-6 i+5 k-6}{6}, \frac{2 i-k+1}{3} ; q\right) \\
& =a_{k, r, s}(i) \Delta\left(\frac{i-k+2}{3}, \frac{-6 i+5 k-6}{6}, \frac{2 i-k+1}{3} ; q\right)
\end{aligned}
$$

where $a_{k, r, s}(i)=2^{4 \imath} a_{k, r, s}^{\prime}(i)$. Similarly, we obtain for $i \in\{1, \ldots, k-1\}$

$$
\begin{aligned}
& b_{k, r, s}^{\prime}(i) x^{2}(1-x)^{1 / 4} z^{2 k}=b_{k, r, s}(i) \Delta\left(\frac{2 i-2 k+1}{6}, \frac{-12 i+10 k-3}{12}, \frac{8 i-4 k+1}{12} ; q\right), \\
& c_{k, r, s}^{\prime}(i) x^{\imath}(1-x)^{1 / 2} z^{2 k}=c_{k, r, s}(i) \Delta\left(\frac{i-k+1}{3}, \frac{-6 i+5 k-3}{6}, \frac{4 i-2 k+1}{6} ; q\right), \\
& d_{k, r, s}^{\prime}(i) x^{2}(1-x)^{3 / 4} z^{2 k}=d_{k, r, s}(i) \Delta\left(\frac{2 i-2 k+3}{6}, \frac{-12 i+10 k-9}{12}, \frac{8 i-4 k+3}{12} ; q\right), \\
& \text { where } b_{k, r, s}(i)=2^{4 \imath} b_{k, r, s}^{\prime}(i), c_{k, r, s}(i)=2^{42} c_{k, r, s}^{\prime}(i) \text { and } d_{k, r, s}(i)=2^{42} d_{k, r, s}^{\prime}(i) .
\end{aligned}
$$

Finally, from Theorems 2.5.2, 4.4.1 and 6.2.1 we obtain the desired results.

The final result of this section gives the number of representations of $n \in \mathbb{N}$ by the quadratic form

$$
x_{1}^{2}+\cdots+x_{4 k-2 r-s}^{2}+2 x_{4 k-2 r-s+1}^{2}+2 x_{4 k-s}^{2}+4 x_{4 k-s+1}^{2}+\cdots+4 x_{4 k}^{2},
$$

where $2 r+s<4 k$. 
Theorem 6.2.3. For $k, r, s, n \in \mathbb{N}$ with $2 r+s<4 k$, we have

$$
\begin{aligned}
N & ([4 k-2 r-s, 2 r, s] ; n) \\
& =-\frac{(-1)^{k} 4 k}{2^{r+s}\left(2^{2 k}-1\right) B_{2 k}} \sigma_{2 k-1}(n)+\frac{(-1)^{k} 4 k}{2^{r+s}\left(2^{2 k}-1\right) B_{2 k}} \sigma_{2 k-1}\left(\frac{n}{2}\right) \\
+ & \frac{4 k}{\left(2^{2 k}-1\right) B_{2 k}} \sigma_{2 k-1}\left(\frac{n}{8}\right)-\frac{2^{2 k+2} k}{\left(2^{2 k}-1\right) B_{2 k}} \sigma_{2 k-1}\left(\frac{n}{16}\right) \\
+ & \sum_{\imath=1}^{k-2} a_{k, r, s}(\imath) \delta\left(\frac{\imath-k+2}{3}, \frac{-6 \imath+5 k-6}{6}, \frac{2 \imath-k+1}{3} ; n\right) \\
+ & \sum_{\imath=1}^{k-1} b_{k, r, s}(\imath) \delta\left(\frac{2 \imath-2 k+1}{6}, \frac{-12 \imath+10 k-3}{12}, \frac{8 \imath-4 k+1}{12} ; n\right) \\
+ & \sum_{\imath=1}^{k-1} c_{k, r, s}(\imath) \delta\left(\frac{\imath-k+1}{3}, \frac{-6 \imath+5 k-3}{6}, \frac{4 \imath-2 k+1}{6} ; n\right) \\
& +\sum_{\imath=1}^{k-1} d_{k, r, s}(\imath) \delta\left(\frac{2 \imath-2 k+3}{6}, \frac{-12 \imath+10 k-9}{12}, \frac{8 \imath-4 k+3}{12} ; n\right)
\end{aligned}
$$

where $a_{k, r, s}(\imath)=2^{4 \imath} a_{k, r, s}^{\prime}(\imath)$ for $\imath \in\{1, \ldots, k-2\}, b_{k, r, s}(\imath)=2^{4 \imath} b_{k, r, s}^{\prime}(\imath)$ for $\imath \in$ $\{1, \ldots, k-1\}, c_{k, r, s}(\imath)=2^{4 \imath} c_{k, r, s}^{\prime}(\imath)$ for $\imath \in\{1, \ldots, k-1\}, d_{k, r, s}(\imath)=2^{4 \imath} d_{k, r, s}^{\prime}(\imath)$ for $\imath \in\{1, \ldots, k-1\}$ and the coefficients $a_{k, r, s}^{\prime}(\imath), b_{k, r, s}^{\prime}(\imath), c_{k, r, s}^{\prime}(\imath), d_{k, r, s}^{\prime}(\imath)$ are given $\imath n$ Theorem 6.2.1.

Proof. From Theorem 2.8.1, Definition 2.9.1, Definition 2.10.3 and Theorem 6.2.2 we have for $q \in D_{\delta}$

$$
1+\sum_{n=1}^{\infty} N([4 k-2 r-s, 2 r, s] ; n) q^{n}=\varphi(q)^{4 k-2 r-s} \varphi\left(q^{2}\right)^{2 r} \varphi\left(q^{4}\right)^{s}
$$




$$
\begin{aligned}
= & \frac{(-1)^{k}}{2^{r+s}\left(2^{2 k}-1\right)}\left(1-\frac{4 k}{B_{2 k}} \sum_{n=1}^{\infty} \sigma_{2 k-1}(n) q^{n}\right) \\
& -\frac{(-1)^{k}}{2^{r+s}\left(2^{2 k}-1\right)}\left(1-\frac{4 k}{B_{2 k}} \sum_{n=1}^{\infty} \sigma_{2 k-1}(n) q^{2 n}\right) \\
& -\frac{1}{2^{2 k}-1}\left(1-\frac{4 k}{B_{2 k}} \sum_{n=1}^{\infty} \sigma_{2 k-1}(n) q^{8 n}\right) \\
& +\frac{2^{2 k}}{2^{2 k}-1}\left(1-\frac{4 k}{B_{2 k}} \sum_{n=1}^{\infty} \sigma_{2 k-1}(n) q^{16 n}\right) \\
& +\sum_{i=1}^{k-2} a_{k, r, s}(i) \sum_{n=1}^{\infty} \delta\left(\frac{i-k+2}{3}, \frac{-6 i+5 k-6}{6}, \frac{2 i-k+1}{3} ; n\right) q^{n} \\
& +\sum_{i=1}^{k-1} d_{k, r, s}(i) \sum_{n=1}^{\infty} \delta\left(\frac{2 i-2 k+3}{6}, \frac{-12 i+10 k-9}{12}, \frac{8 i-4 k+3}{12} ; n\right) q^{n} \\
& +\sum_{i=1}^{k-1} b_{k, r, s}(i) \sum_{n=1}^{\infty} \delta\left(\frac{2 i-2 k+1}{6}, \frac{-12 i+10 k-3}{12}, \frac{8 i-4 k+1}{12} ; n\right) q^{n} \\
& +\sum_{i=1}^{k-1} c_{k, r, s}(i) \sum_{n=1}^{\infty} \delta\left(\frac{i-k+1}{3}, \frac{-6 i+5 k-3}{6}, \frac{4 i-2 k+1}{6} ; n\right) q^{n} \\
& \\
& \\
&
\end{aligned}
$$




$$
\begin{aligned}
& +\frac{(-1)^{k}}{2^{r+s}\left(2^{2 k}-1\right)}\left(\frac{4 k}{B_{2 k}} \sum_{n=1}^{\infty} \sigma_{2 k-1}(n) q^{2 n}\right) \\
& +\frac{1}{2^{2 k}-1}\left(\frac{4 k}{B_{2 k}} \sum_{n=1}^{\infty} \sigma_{2 k-1}(n) q^{8 n}\right) \\
& +\frac{2^{2 k}}{2^{2 k}-1}\left(\frac{4 k}{B_{2 k}} \sum_{n=1}^{\infty} \sigma_{2 k-1}(n) q^{16 n}\right) \\
& +\sum_{n=1}^{\infty}\left(\sum_{i=1}^{k-2} a_{k, r, s}(i) \delta\left(\frac{i-k+2}{3}, \frac{-6 i+5 k-6}{6}, \frac{2 i-k+1}{3} ; n\right)\right) q^{n} \\
& +\sum_{n=1}^{\infty}\left(\sum_{i=1}^{k-1} b_{k, r, s}(i) \delta\left(\frac{2 i-2 k+1}{6}, \frac{-12 i+10 k-3}{12}, \frac{8 i-4 k+1}{12} ; n\right)\right) q^{n} \\
& +\sum_{n=1}^{\infty}\left(-\frac{(-1)^{k} 4 k}{2^{r+s}\left(2^{2 k}-1\right) B_{2 k}} \sigma_{2 k-1}(n)+\frac{(-1)^{k} 4 k}{2^{r+s}\left(2^{2 k}-1\right) B_{2 k}} \sigma_{2 k-1}\left(\frac{n}{2}\right)\right. \\
& +\sum_{n=1}^{\infty}\left(\sum_{i=1}^{k-1} c_{k, r, s}(i) \delta\left(\frac{i-k+1}{3}, \frac{-6 i+5 k-3}{6}, \frac{4 i-2 k+1}{6} ; n\right)\right) q^{n} \\
& \left.+2_{k=1}^{2 k}-1\right) B_{2 k} \sigma_{2 k-1}\left(\frac{n}{8}\right)-\frac{2^{2 k+2} k}{\left(2^{2 k}-1\right) B_{2 k}} \sigma_{2 k-1}\left(\frac{n}{16}\right) \\
& \left.+\frac{2 i-2 k+3}{6}, \frac{-12 i+10 k-9}{12}, \frac{8 i-4 k+3}{12}\right) q^{n}
\end{aligned}
$$




$$
\begin{aligned}
& +\sum_{i=1}^{k-2} a_{k, r, s}(i) \delta\left(\frac{i-k+2}{3}, \frac{-6 i+5 k-6}{6}, \frac{2 i-k+1}{3} ; n\right) \\
& +\sum_{i=1}^{k-1} b_{k, r, s}(i) \delta\left(\frac{2 i-2 k+1}{6}, \frac{-12 i+10 k-3}{12}, \frac{8 i-4 k+1}{12} ; n\right) \\
& +\sum_{i=1}^{k-1} c_{k, r, s}(i) \delta\left(\frac{i-k+1}{3}, \frac{-6 i+5 k-3}{6}, \frac{4 i-2 k+1}{6} ; n\right) \\
& \left.+\sum_{i=1}^{k-1} d_{k, r, s}(i) \delta\left(\frac{2 i-2 k+3}{6}, \frac{-12 i+10 k-9}{12}, \frac{8 i-4 k+3}{12} ; n\right)\right) q^{n} .
\end{aligned}
$$

Equating coefficients of $q^{n}(n \in \mathbb{N})$, we obtain the desired results.

When $k=1$ we have $r=s=1$ (as $r, s \in \mathbb{N}$ and $r+2 s<4 k$ ). Then Theorem gives 6.2 .3 gives

$$
N([1,2,1] ; n)=2 \sigma(n)-2 \sigma(n / 2)+8 \sigma(n / 8)-32 \sigma(n / 16), \quad n \in \mathbb{N},
$$

in agreement with [2, Theorem 1.14, p. 300].

\subsection{Number of representations by the quadratic}

$$
\text { form } x_{1}^{2}+\cdots+x_{4 k-s}^{2}+4 x_{4 k-s+1}^{2}+\cdots+4 x_{4 k}^{2}
$$

We begin this section by using all the results that we found in Section 5.3 to obtain the following theorem that is valid for any variables $x$ and $z$.

Theorem 6.3.1. For $k, s \in \mathbb{N}$ with $s<4 k$, we have

$$
\left(\frac{1+(1-x)^{1 / 4}}{2}\right)^{s} z^{2 k}
$$




$$
\begin{aligned}
& =\frac{(-1)^{k}}{2^{s}\left(2^{2 k}-1\right)} e_{k, 1}(x) z^{2 k}-\frac{\varepsilon_{2,4}(s)(-1)^{\left[\frac{s+1}{4}\right]} 2^{\left[\frac{s}{2}\right]}+(-1)^{k}}{2^{s}\left(2^{2 k}-1\right)} e_{k, 2}(x) z^{2 k} \\
& +\frac{\varepsilon_{2,4}(s)(-1)^{\left[\frac{s+1}{4}\right]}\left(2^{2 k}+1\right)}{2^{\left[\frac{s+1}{2}\right]}\left(2^{2 k}-1\right)} e_{k, 4}(x) z^{2 k}-\frac{\varepsilon_{2,4}(s)(-1)^{\left[\frac{s+1}{4}\right]} 2^{2 k-\left[\frac{s+1}{2}\right]}+1}{2^{2 k}-1} e_{k, 8}(x) z^{2 k} \\
& +\frac{2^{2 k}}{2^{2 k}-1} e_{k, 16}(x) z^{2 k} \\
& +(1-x) \sum_{i=1}^{k-2} a_{k, 0, s}^{\prime}(i) x^{2} z^{2 k}+(1-x)^{1 / 4} \sum_{i=0}^{k-1} b_{k, 0, s}^{\prime}(i) x^{2} z^{2 k} \\
& +(1-x)^{1 / 2} \sum_{i=1}^{k-1} c_{k, 0, s}^{\prime}(i) x^{2} z^{2 k}+(1-x)^{3 / 4} \sum_{i=0}^{k-1} d_{k, 0, s}^{\prime}(i) x^{i} z^{2 k}
\end{aligned}
$$

where $a_{k, 0, s}^{\prime}(i) \in \mathbb{Q}$ for $i \in\{1, \ldots, k-2\}, b_{k, 0, s}^{\prime}(i), d_{k, 0, s}^{\prime}(i) \in \mathbb{Q}$ for $i \in\{0, \ldots, k-1\}$ and $c_{k, 0, s}^{\prime}(i) \in \mathbb{Q}$ for $i \in\{1, \ldots, k-1\}$.

Proof. When $k=1$ we have $s=1,2$ or 3 as $s<4 k$. From (6.0.1)-(6.0.5) we have

$$
\begin{aligned}
\left(\frac{1+(1-x)^{1 / 4}}{2}\right)= & -\frac{1}{6} e_{1,1}(x) z^{2}+\frac{5}{6} e_{1,4}(x) z^{2}-e_{1,8}(x) z^{2}+\frac{4}{3} e_{1,16}(x) z^{2} \\
& +\frac{1}{4}(1-x)^{1 / 4} z^{2}-\frac{1}{4}(1-x)^{3 / 4} z^{2}, \\
\left(\frac{1+(1-x)^{1 / 4}}{2}\right)^{2}= & -\frac{1}{12} e_{1,1}(x) z^{2}-\frac{1}{12} e_{1,2}(x) z^{2}-\frac{1}{3} e_{1,8}(x) z^{2}+\frac{4}{3} e_{1,16}(x) z^{2} \\
& +\frac{1}{4}(1-x)^{1 / 4} z^{2}-\frac{1}{4}(1-x)^{3 / 4} z^{2}, \\
\left(\frac{1+(1-x)^{1 / 4}}{2}\right)^{3}= & -\frac{1}{24} e_{1,1}(x) z^{2}+\frac{1}{8} e_{1,2}(x) z^{2}-\frac{5}{12} e_{1,8}(x) z^{2}+\frac{4}{3} e_{1,16}(x) z^{2}
\end{aligned}
$$




$$
+\frac{1}{8}(1-x)^{1 / 4} z^{2}-\frac{1}{8}(1-x)^{3 / 4} z^{2}
$$

For $k \geq 2$, by Definition 5.3.1, Lemmas 5.3.2 and 5.3.3 we have

$$
\begin{aligned}
& P_{k, 0, s}(x)=(1-x)\left(a_{k, 0, s}(1) x+\cdots+a_{k, 0, s}(k-2) x^{k-2}\right) \\
& Q_{k, 0, s}(x)=b_{k, 0, s}^{\prime}(0)+b_{k, 0, s}^{\prime}(1) x+\cdots+b_{k, 0, s}^{\prime}(k-1) x^{k-1}, \\
& R_{k, 0, s}(x)=c_{k, 0, s}^{\prime}(1) x+\cdots+c_{k, 0, s}^{\prime}(k-1) x^{k-1} \\
& S_{k, 0, s}(x)=d_{k, 0, s}^{\prime}(0)+d_{k, 0, s}^{\prime}(1) x+\cdots+d_{k, 0, s}^{\prime}(k-1) x^{k-1},
\end{aligned}
$$

where $a_{k, 0, s}^{\prime}(i) \in \mathbb{Q}$ for $i \in\{1, \ldots, k-2\}, b_{k, 0, s}^{\prime}(i), d_{k, 0, s}^{\prime}(i) \in \mathbb{Q}$ for $i \in\{0, \ldots, k-1\}$ and $c_{k, 0, s}^{\prime}(i) \in \mathbb{Q}$ for $i \in\{1, \ldots, k-1\}$. This implies, by Lemma 5.3.1, that

$$
\begin{aligned}
& \left(\frac{1+(1-x)^{1 / 4}}{2}\right)^{s} \\
& =\frac{(-1)^{k}}{2^{s}\left(2^{2 k}-1\right)} e_{k, 1}(x)-\frac{\varepsilon_{2,4}(s)(-1)^{\left[\frac{s+1}{4}\right]} 2^{\left[\frac{s}{2}\right]}+(-1)^{k}}{2^{s}\left(2^{2 k}-1\right)} e_{k, 2}(x) \\
& +\frac{\varepsilon_{2,4}(s)(-1)^{\left[\frac{s+1}{4}\right]}\left(2^{2 k}+1\right)}{2^{\left[\frac{s+1}{2}\right]}\left(2^{2 k}-1\right)} e_{k, 4}(x) \\
& \quad-\frac{\varepsilon_{2,4}(s)(-1)^{\left[\frac{s+1}{4}\right]} 2^{2 k-\left[\frac{s+1}{2}\right]}+1}{2^{2 k}-1} e_{k, 8}(x)+\frac{2^{2 k}}{2^{2 k}-1} e_{k, 16}(x) \\
& \quad+(1-x) \sum_{i=1}^{k-2} a_{k, 0, s}^{\prime}(i) x^{\imath}+(1-x)^{1 / 4} \sum_{i=0}^{k-1} b_{k, 0, s}^{\prime}(i) x^{\imath}
\end{aligned}
$$




$$
+(1-x)^{1 / 2} \sum_{i=1}^{k-1} c_{k, 0, s}^{\prime}(i) x^{\imath}+(1-x)^{3 / 4} \sum_{i=0}^{k-1} d_{k, 0, s}^{\prime}(i) x^{\imath} .
$$

Finally, we obtain the asserted result by multiplying both sides of (6.3.1) by $z^{2 k}$.

Replacing $x$ and $z$ in Theorem 6.3 .1 by their values given in Definition 2.3.2 we obtain the following identities involving the theta function $\varphi$, Eisenstein series $E_{k}\left(q^{r}\right)$ with $r \in\{1,2,4,8,16\}$ and the generalized Ramanujan discriminant function $\Delta(a, b, c ; q)$.

Theorem 6.3.2. For $k, s \in \mathbb{N}$ with $s<4 k$ and $q \in D_{\delta}$, we have

$$
\begin{aligned}
& \varphi(q)^{4 k-s} \varphi\left(q^{4}\right)^{s} \\
& =\frac{(-1)^{k}}{2^{s}\left(2^{2 k}-1\right)} E_{k}(q)-\frac{\varepsilon_{2,4}(s)(-1)^{\left[\frac{s+1}{4}\right]} 2^{\left[\frac{s}{2}\right]}+(-1)^{k}}{2^{s}\left(2^{2 k}-1\right)} E_{k}\left(q^{2}\right) \\
& +\frac{\varepsilon_{2,4}(s)(-1)^{\left[\frac{s+1}{4}\right]}\left(2^{2 k}+1\right)}{2^{\left[\frac{s+1}{2}\right]}\left(2^{2 k}-1\right)} E_{k}\left(q^{4}\right)-\frac{\varepsilon_{2,4}(s)(-1)^{\left[\frac{s+1}{4}\right]} 2^{2 k-\left[\frac{s+1}{2}\right]}+1}{2^{2 k}-1} E_{k}\left(q^{8}\right) \\
& +\frac{2^{2 k}}{2^{2 k}-1} E_{k}\left(q^{16}\right) \\
& +\sum_{i=1}^{k-2} a_{k, 0, s}(i) \Delta\left(\frac{i-k+2}{3}, \frac{-6 i+5 k-6}{6}, \frac{2 i-k+1}{3} ; q\right) \\
& +\sum_{i=0}^{k-1} b_{k, 0, s}(i) \Delta\left(\frac{2 i-2 k+1}{6}, \frac{-12 i+10 k-3}{12}, \frac{8 i-4 k+1}{12} ; q\right) \\
& +\sum_{i=1}^{k-1} c_{k, 0, s}(i) \Delta\left(\frac{i-k+1}{3}, \frac{-6 i+5 k-3}{6}, \frac{4 i-2 k+1}{6} ; q\right) \\
&
\end{aligned}
$$




$$
+\sum_{\imath=0}^{k-1} d_{k, 0, s}(i) \Delta\left(\frac{2 i-2 k+3}{6}, \frac{-12 i+10 k-9}{12}, \frac{8 i-4 k+3}{12} ; q\right)
$$

where $a_{k, 0, s}(i)=2^{4 \imath} a_{k, 0, s}^{\prime}(i)$ for $i \in\{1, \ldots, k-2\}, b_{k, 0, s}(i)=2^{4 \imath} b_{k, 0, s}^{\prime}(i)$ for $i \in$ $\{0, \ldots, k-1\}, c_{k, 0, s}(i)=2^{4 \imath} c_{k, 0, s}^{\prime}(i)$ for $i \in\{1, \ldots, k-1\}, d_{k, 0, s}(i)=2^{4 \imath} d_{k, 0, s}^{\prime}(i)$ for $i \in\{0, \ldots, k-1\}$ and the coefficients $a_{k, 0, s}^{\prime}(i), b_{k, 0, s}^{\prime}(i), c_{k, 0, s}^{\prime}(i), d_{k, 0, s}^{\prime}(i)$ are given in Theorem 6.3.1.

Proof. Let $q \in D_{\delta}$. Set $x=1-\frac{\varphi^{4}(-q)}{\varphi^{4}(q)}, z=\varphi^{2}(q)$. For $k=1$, we have by Theorems 2.5.2(ii), 2.9.2 and 2.9.5

$$
\begin{gathered}
\varphi(q)^{3} \varphi\left(q^{4}\right)+\frac{1}{6} E_{1}(q)-\frac{5}{6} E_{1}\left(q^{4}\right)+E_{1}\left(q^{8}\right)-\frac{4}{3} E_{1}\left(q^{16}\right)= \\
\frac{1}{4}(1-x)^{1 / 4} z^{2}-\frac{1}{4}(1-x)^{3 / 4} z^{2} \\
\varphi(q)^{2} \varphi\left(q^{4}\right)^{2}+\frac{1}{12} E_{1}(q)-\frac{1}{12} E_{1}\left(q^{2}\right)+\frac{1}{3} E_{1}\left(q^{8}\right)-\frac{4}{3} E_{1}\left(q^{16}\right)= \\
\frac{1}{4}(1-x)^{1 / 4} z^{2}-\frac{1}{4}(1-x)^{3 / 4} z^{2} \\
\varphi(q) \varphi\left(q^{4}\right)^{3}+\frac{1}{24} E_{1}(q)-\frac{1}{8} E_{1}\left(q^{2}\right)+\frac{5}{12} E_{1}\left(q^{4}\right)-\frac{4}{3} E_{1}\left(q^{16}\right)= \\
\frac{1}{8}(1-x)^{1 / 4} z^{2}-\frac{1}{8}(1-x)^{3 / 4} z^{2}
\end{gathered}
$$

which give the asserted results on isolating $\varphi(q)^{3} \varphi\left(q^{4}\right), \varphi(q)^{2} \varphi\left(q^{4}\right)^{2}$ and $\varphi(q) \varphi\left(q^{4}\right)^{3}$.

For $k \geq 2$, we note that from Theorem 2.10 .3 we have for $i \in\{1, \ldots, k-2\}$

$$
\begin{aligned}
a_{k, 0, s}^{\prime}(i) x^{2}(1-x) z^{2 k} & =2^{4 \imath} a_{k, 0, s}^{\prime}(i) \frac{x^{2}(1-x)}{2^{4 \imath}} z^{2 k} \\
& =2^{4 \imath} a_{k, 0, s}^{\prime}(i) \Delta\left(\frac{i-k+2}{3}, \frac{-6 i+5 k-6}{6}, \frac{2 i-k+1}{3} ; q\right)
\end{aligned}
$$




$$
=a_{k, 0, s}(i) \Delta\left(\frac{i-k+2}{3}, \frac{-6 i+5 k-6}{6}, \frac{2 i-k+1}{3} ; q\right),
$$

where $a_{k, 0, s}(i)=2^{4 \imath} a_{k, 0, s}^{\prime}(i)$. Similarly, we obtain for $i \in\{0, \ldots, k-1\}$

$$
\begin{aligned}
& b_{k, 0, s}^{\prime}(i) x^{2}(1-x)^{1 / 4} z^{2 k}=b_{k, 0, s}(i) \Delta\left(\frac{2 i-2 k+1}{6}, \frac{-12 i+10 k-3}{12}, \frac{8 i-4 k+1}{12} ; q\right), \\
& d_{k, 0, s}^{\prime}(i) x^{2}(1-x)^{3 / 4} z^{2 k}=d_{k, 0, s}(i) \Delta\left(\frac{2 i-2 k+3}{6}, \frac{-12 i+10 k-9}{12}, \frac{8 i-4 k+3}{12} ; q\right),
\end{aligned}
$$

where $b_{k, 0, s}(i)=2^{4 \imath} b_{k, 0, s}^{\prime}(i)$ and $d_{k, 0, s}(i)=2^{4 \imath} d_{k, 0, s}^{\prime}(i)$. For $i \in\{1, \ldots, k-1\}$ we have

$$
c_{k, 0, s}^{\prime}(i) x^{2}(1-x)^{1 / 2} z^{2 k}=c_{k, 0, s}(i) \Delta\left(\frac{i-k+1}{3}, \frac{-6 i+5 k-3}{6}, \frac{4 i-2 k+1}{6} ; q\right) .
$$

Finally, from Theorems 4.4 .1 and 6.3.1 we obtain the desired results.

Our final result gives the number of representations of $n \in \mathbb{N}$ by the quadratic form

$$
x_{1}^{2}+\cdots+x_{4 k-s}^{2}+4 x_{4 k-s+1}^{2}+\cdots+4 x_{4 k}^{2},
$$

where $s<4 k$.

Theorem 6.3.3. For $k, s, n \in \mathbb{N}$ with $s<4 k$, we have

$$
\begin{aligned}
& N([4 k-s, 0, s] ; n) \\
& =-\frac{(-1)^{k} 4 k}{2^{s}\left(2^{2 k}-1\right) B_{2 k}} \sigma_{2 k-1}(n)+\frac{\left(\varepsilon_{2,4}(s)(-1)^{\left[\frac{s+1}{4}\right]} 2^{\left[\frac{s}{2}\right]}+(-1)^{k}\right) 4 k}{2^{s}\left(2^{2 k}-1\right) B_{2 k}} \sigma_{2 k-1}\left(\frac{n}{2}\right) \\
& \quad-\frac{\varepsilon_{2,4}(s)(-1)^{\left[\frac{s+1}{4}\right]} 4 k\left(2^{2 k}+1\right)}{2^{\left[\frac{s+1}{2}\right]}\left(2^{2 k}-1\right) B_{2 k}} \sigma_{2 k-1}\left(\frac{n}{4}\right)
\end{aligned}
$$




$$
\begin{aligned}
& +\frac{\left(\varepsilon_{2,4}(s)(-1)^{\left[\frac{s+1}{4}\right]} 2^{2 k-\left[\frac{s+1}{2}\right]}+1\right) 4 k}{\left(2^{2 k}-1\right) B_{2 k}} \sigma_{2 k-1}\left(\frac{n}{8}\right)-\frac{2^{2 k+2} k}{\left(2^{2 k}-1\right) B_{2 k}} \sigma_{2 k-1}\left(\frac{n}{16}\right) \\
& +\sum_{i=1}^{k-2} a_{k, 0, s}(i) \delta\left(\frac{i-k+2}{3}, \frac{-6 i+5 k-6}{6}, \frac{2 i-k+1}{3} ; n\right) \\
& +\sum_{i=0}^{k-1} b_{k, 0, s}(i) \delta\left(\frac{2 i-2 k+1}{6}, \frac{-12 i+10 k-3}{12}, \frac{8 i-4 k+1}{12} ; n\right) \\
& +\sum_{i=1}^{k-1} c_{k, 0, s}(i) \delta\left(\frac{i-k+1}{3}, \frac{-6 i+5 k-3}{6}, \frac{4 i-2 k+1}{6} ; n\right) \\
& +\sum_{i=0}^{k-1} d_{k, 0, s}(i) \delta\left(\frac{2 i-2 k+3}{6}, \frac{-12 i+10 k-9}{12}, \frac{8 i-4 k+3}{12} ; n\right)
\end{aligned}
$$

where $a_{k, 0, s}(i)=2^{4 \imath} a_{k, 0, s}^{\prime}(i)$ for $i \in\{1, \ldots, k-2\}, b_{k, 0, s}(i)=2^{4 \imath} b_{k, 0, s}^{\prime}(i)$ for $i \in$ $\{0, \ldots, k-1\}, c_{k, 0, s}(i)=2^{4 i} c_{k, 0, s}^{\prime}(i)$ for $i \in\{1, \ldots, k-1\}, d_{k, 0, s}(i)=2^{4 \imath} d_{k, 0, s}^{\prime}(i)$ for $i \in\{0, \ldots, k-1\}$ and the coefficients $a_{k, 0, s}^{\prime}(i), b_{k, 0, s}^{\prime}(i), c_{k, 0, s}^{\prime}(i), d_{k, 0, s}^{\prime}(i)$ are given in Theorem 6.3.1.

Proof. From Theorem 2.8.1, Theorem 6.3.2 and Definition 2.10.3, we have for $q \in D_{\delta}$

$$
\begin{aligned}
& 1+\sum_{n=1}^{\infty} N([4 k-s, 0, s] ; n) q^{n}=\varphi(q)^{4 k-s} \varphi\left(q^{4}\right)^{s} \\
& =\frac{(-1)^{k}}{2^{s}\left(2^{2 k}-1\right)}\left(1-\frac{4 k}{B_{2 k}} \sum_{n=1}^{\infty} \sigma_{2 k-1}(n) q^{n}\right) \\
& \quad-\frac{\varepsilon_{2,4}(s)(-1)^{\left[\frac{s+1}{4}\right]} 2^{\left[\frac{s}{2}\right]}+(-1)^{k}}{2^{s}\left(2^{2 k}-1\right)}\left(1-\frac{4 k}{B_{2 k}} \sum_{n=1}^{\infty} \sigma_{2 k-1}(n) q^{2 n}\right)
\end{aligned}
$$




$$
\begin{aligned}
& +\frac{\varepsilon_{2,4}(s)(-1)^{\left[\frac{s+1}{4}\right]}\left(2^{2 k}+1\right)}{2^{\left[\frac{s+1}{2}\right]}\left(2^{2 k}-1\right)}\left(1-\frac{4 k}{B_{2 k}} \sum_{n=1}^{\infty} \sigma_{2 k-1}(n) q^{4 n}\right) \\
& -\frac{\varepsilon_{2,4}(n)(-1)^{\left[\frac{s+1}{4}\right]} 2^{2 k-\left[\frac{s+1}{2}\right]}+1}{2^{2 k}-1}\left(1-\frac{4 k}{B_{2 k}} \sum_{n=1}^{\infty} \sigma_{2 k-1}(n) q^{8 n}\right) \\
& +\frac{2^{2 k}}{2^{2 k}-1}\left(1-\frac{4 k}{B_{2 k}} \sum_{n=1}^{\infty} \sigma_{2 k-1}(n) q^{16 n}\right) \\
& +\sum_{i=1}^{k-2} a_{k, 0, s}(i) \sum_{n=1}^{\infty} \delta\left(\frac{i-k+2}{3}, \frac{-6 i+5 k-6}{6}, \frac{2 i-k+1}{3} ; n\right) q^{n} \\
& +\sum_{i=0}^{k-1} b_{k, 0, s}(i) \sum_{n=1}^{\infty} \delta\left(\frac{2 i-2 k+1}{6}, \frac{-12 i+10 k-3}{12}, \frac{8 i-4 k+1}{12} ; n\right) q^{n} \\
& +\sum_{\imath=1}^{k-1} c_{k, 0, s}(i) \sum_{n=1}^{\infty} \delta\left(\frac{i-k+1}{3}, \frac{-6 i+5 k-3}{6}, \frac{4 i-2 k+1}{6} ; n\right) q^{n} \\
& +\sum_{i=0}^{k-1} d_{k, 0, s}(i) \sum_{n=1}^{\infty} \delta\left(\frac{2 i-2 k+3}{6}, \frac{-12 i+10 k-9}{12}, \frac{8 i-4 k+3}{12} ; n\right) q^{n} \\
& =1-\frac{(-1)^{k}}{2^{s}\left(2^{2 k}-1\right)}\left(\frac{4 k}{B_{2 k}} \sum_{n=1}^{\infty} \sigma_{2 k-1}(n) q^{n}\right) \\
& +\frac{\varepsilon_{2,4}(s)(-1)^{\left[\frac{s+1}{4}\right]} 2^{\left[\frac{s}{2}\right]}+(-1)^{k}}{2^{s}\left(2^{2 k}-1\right)}\left(\frac{4 k}{B_{2 k}} \sum_{n=1}^{\infty} \sigma_{2 k-1}(n) q^{2 n}\right)
\end{aligned}
$$




$$
\begin{aligned}
& -\frac{\varepsilon_{2,4}(n)(-1)^{\left[\frac{s+1}{4}\right]}\left(2^{2 k}+1\right)}{2^{\left[\frac{s+1}{2}\right]}\left(2^{2 k}-1\right)}\left(\frac{4 k}{B_{2 k}} \sum_{n=1}^{\infty} \sigma_{2 k-1}(n) q^{4 n}\right) \\
& +\frac{\varepsilon_{2,4}(s)(-1)^{\left[\frac{s+1}{4}\right]} 2^{2 k-\left[\frac{s+1}{2}\right]}+1}{2^{2 k}-1}\left(\frac{4 k}{B_{2 k}} \sum_{n=1}^{\infty} \sigma_{2 k-1}(n) q^{8 n}\right) \\
& -\frac{2^{2 k}}{2^{2 k}-1}\left(\frac{4 k}{B_{2 k}} \sum_{n=1}^{\infty} \sigma_{2 k-1}(n) q^{16 n}\right) \\
& +\sum_{n=1}^{\infty}\left(\sum_{\imath=1}^{k-2} a_{k, 0, s}(i) \delta\left(\frac{i-k+2}{3}, \frac{-6 i+5 k-6}{6}, \frac{2 i-k+1}{3} ; n\right)\right) q^{n} \\
& +\sum_{n=1}^{\infty}\left(\sum_{\imath=0}^{k-1} b_{k, 0, s}(i) \delta\left(\frac{2 i-2 k+1}{6}, \frac{-12 i+10 k-3}{12}, \frac{8 i-4 k+1}{12} ; n\right)\right) q^{n} \\
& +\sum_{n=1}^{\infty}\left(-\frac{(-1)^{k} 4 k}{2^{s}\left(2^{2 k}-1\right) B_{2 k}} \sigma_{2 k-1}(n)\right. \\
& +\sum_{n=1}^{\infty}\left(\sum_{\imath=0}^{\infty}\left(\sum_{\imath=1}^{k-1} c_{k, 0, s}(i) \delta\left(\frac{i-k+1}{3}, \frac{-6 i+5 k-3}{6}, \frac{4 i-2 k+1}{6} ; n\right)\right) q^{n}\right. \\
& +d_{k, 0, s}(i) \delta\left(\frac{2 i-2 k+3}{6}, \frac{-12 i+10 k-9}{12}, \frac{8 i-4 k+3}{12} ; n\right) q^{n} \\
& \left.\left.+\frac{s+1}{4}\right] 2^{\left[\frac{s}{2}\right]}+(-1)^{k}\right) 4 k \\
& +2_{2 k-1}\left(\frac{n}{2}\right) \\
& +1) B_{2 k}
\end{aligned}
$$




$$
\begin{aligned}
& -\frac{\varepsilon_{2,4}(s)(-1)^{\left[\frac{s+1}{4}\right]} 4 k\left(2^{2 k}+1\right)}{2^{\left[\frac{s+1}{2}\right]}\left(2^{2 k}-1\right) B_{2 k}} \sigma_{2 k-1}\left(\frac{n}{4}\right) \\
& +\frac{\left(\varepsilon_{2,4}(s)(-1)^{\left[\frac{s+1}{4}\right]} 2^{2 k-\left[\frac{s+1}{2}\right]}+1\right) 4 k}{\left(2^{2 k}-1\right) B_{2 k}} \sigma_{2 k-1}\left(\frac{n}{8}\right)-\frac{2^{2 k+2} k}{\left(2^{2 k}-1\right) B_{2 k}} \sigma_{2 k-1}\left(\frac{n}{16}\right) \\
& +\sum_{i=1}^{k-2} a_{k, 0, s}(2) \delta\left(\frac{i-k+2}{3}, \frac{-6 i+5 k-6}{6}, \frac{2 i-k+1}{3} ; n\right) \\
& +\sum_{i=0}^{k-1} b_{k, 0, s}(i) \delta\left(\frac{2 i-2 k+1}{6}, \frac{-12 i+10 k-3}{12}, \frac{8 i-4 k+1}{12} ; n\right) \\
& +\sum_{i=1}^{k-1} c_{k, 0, s}(i) \delta\left(\frac{i-k+1}{3}, \frac{-6 i+5 k-3}{6}, \frac{4 i-2 k+1}{6} ; n\right) \\
& \left.+\sum_{i=0}^{k-1} d_{k, 0, s}(i) \delta\left(\frac{2 i-2 k+3}{6}, \frac{-12 i+10 k-9}{12}, \frac{8 i-4 k+3}{12} ; n\right)\right) q^{n} .
\end{aligned}
$$

Equating coefficients of $q^{n} \quad\left(n \in \mathbb{N}_{0}\right)$, we obtain the desired results.

When $k=1$ we have $s=1,2$ or 3 as $s<4 k$. With $k=s=1$ Theorem 6.3 .3 gives

$$
\begin{aligned}
N([3,0,1] ; n)= & 4 \sigma(n)-20 \sigma(n / 4)+24 \sigma(n / 8)-32 \sigma(n / 16) \\
& +\frac{1}{4} \delta\left(-\frac{1}{6}, \frac{7}{12},-\frac{1}{4} ; n\right)-\frac{1}{4} \delta\left(\frac{1}{6}, \frac{1}{12},-\frac{1}{12} ; n\right) .
\end{aligned}
$$

With $k=1, s=2$ Theorem 6.3.3 gives

$$
\begin{aligned}
N([2,0,2] ; n)= & 2 \sigma(n)-2 \sigma(n / 2)+8 \sigma(n / 8)-32 \sigma(n / 16) \\
& +\frac{1}{4} \delta\left(-\frac{1}{6}, \frac{7}{12},-\frac{1}{4} ; n\right)-\frac{1}{4} \delta\left(\frac{1}{6}, \frac{1}{12},-\frac{1}{12} ; n\right) .
\end{aligned}
$$


With $k=1, s=3$ Theorem 6.3 .3 gives

$$
\begin{aligned}
N([1,0,3] ; n)= & \sigma(n)-3 \sigma(n / 2)+10 \sigma(n / 4)-32 \sigma(n / 16) \\
& +\frac{1}{8} \delta\left(-\frac{1}{6}, \frac{7}{12},-\frac{1}{4} ; n\right)-\frac{1}{8} \delta\left(\frac{1}{6}, \frac{1}{12},-\frac{1}{12} ; n\right) .
\end{aligned}
$$

We show in Chapter 8 that

$$
\delta\left(-\frac{1}{6}, \frac{7}{12},-\frac{1}{4} ; n\right)-\delta\left(-\frac{1}{6}, \frac{1}{12},-\frac{1}{12} ; n\right)=8\left(\frac{-4}{n}\right) \sigma(n), \quad n \in \mathbb{N}
$$

so that

$$
\begin{aligned}
& N([3,0,1] ; n)=\left(4+2\left(\frac{-4}{n}\right)\right) \sigma(n)-20 \sigma(n / 4)+24 \sigma(n / 8)-32 \sigma(n / 16), \\
& N([2,0,2] ; n)=\left(2+2\left(\frac{-4}{n}\right)\right) \sigma(n)-2 \sigma(n / 2)+8 \sigma(n / 8)-32 \sigma(n / 16), \\
& N([1,0,3] ; n)=\left(1+\left(\frac{-4}{n}\right)\right) \sigma(n)-3 \sigma(n / 2)+10 \sigma(n / 4)-32 \sigma(n / 16),
\end{aligned}
$$

in agreement with [2, Theorems 1.7, 1.11 and 1.18]. 


\section{Chapter 7}

\section{Sums with 8,12 and 16 squares - I}

In this chapter we apply Theorems $6.1 .3,6.2 .3$ and 6.3 .3 with $k=2,3,4$ to obtain the number of representations of an integer $n \in \mathbb{N}$ by the quadratic forms

$x_{1}^{2}+\cdots+x_{4 k-2 r}^{2}+2 x_{4 k-2 r+1}^{2}+\cdots+2 x_{4 k}^{2}$, where $r \geq 1$ and $r<2 k$,

$x_{1}^{2}+\cdots+x_{4 k-2 r-s}^{2}+2 x_{4 k-2 r-s+1}^{2}+2 x_{4 k-s}^{2}+4 x_{4 k-s+1}^{2}+\cdots+4 x_{4 k}^{2}$, where $r \geq 1, s \geq 1$ and $2 r+s<4 k$,

$x_{1}^{2}+\cdots+x_{4 k-s}^{2}+4 x_{4 k-s+1}^{2}+\cdots+4 x_{4 k}^{2}$, where $s \geq 1$ and $s<4 k$.

In Section 2, we apply the three theorems for sums with 8 squares. In Section 3, we apply the three theorems for sums with 12 squares and in Section 4, we apply the three theorems for sums with 16 squares.

Explicit formulae in terms of the sigma function $\sigma_{k}(n)$ and functions of the form $\delta(a, b, c ; n)$ are thus obtained for all three cases when $k=2, k=3$ and $k=4$. 


\subsection{Sums with 8 squares}

We begin by applying Theorems $6.1 .3,6.2 .3$ and 6.3 .3 with $k=2$. We first note that from Definition 4.1.1 we have

$$
\begin{aligned}
& e_{2,1}(x)=1+14 x+x^{2} \\
& e_{2,2}(x)=1-x+x^{2} \\
& e_{2,4}(x)=1-x+\frac{1}{16} x^{2}
\end{aligned}
$$

From Definition 4.2.1 we have

$$
e_{2,8}(x)=e_{2,8}^{(0)}(x)+e_{2,8}^{(1)}(x)(1-x)^{1 / 2}
$$

where

$$
\begin{aligned}
& e_{2,8}^{(0)}(x)=\frac{17}{32}-\frac{17}{32} x+\frac{1}{256} x^{2} \\
& e_{2,8}^{(1)}(x)=\frac{15}{32}-\frac{15}{64} x
\end{aligned}
$$

From Definition 4.3.1 we deduce

$$
e_{2,16}(x)=\left(e_{2,16}^{(0)}(x)+e_{2,16}^{(1)}(x)(1-x)^{1 / 4}+e_{2,16}^{(2)}(x)(1-x)^{1 / 2}+e_{2,16}^{(3)}(x)(1-x)^{3 / 4}\right)
$$

where

$$
\begin{aligned}
& e_{2,16}^{(0)}(x)=\frac{137}{512}-\frac{137}{512} x+\frac{1}{4096} x^{2} \\
& e_{2,16}^{(1)}(x)=\frac{15}{64}-\frac{105}{512} x \\
& e_{2,16}^{(2)}(x)=\frac{135}{512}-\frac{135}{1024} x \\
& e_{2,16}^{(3)}(x)=\frac{15}{64}-\frac{15}{512} x
\end{aligned}
$$

For our first result, we apply Theorem 6.1 .3 to the case when $k=2$ to obtain the number of representations of a positive integer $n$ by the sum of $8-2 r$ squares and $2 r$ squares each multiplied by 2 , where $r \in \mathbb{N}$ and $0<r<4$. These results were previously obtained in [2]. 
Theorem 7.1.1. Let $n \in \mathbb{N}$. Then

$$
\begin{aligned}
N([6,2,0] ; n)= & 8 \sigma_{3}(n)-8 \sigma_{3}(n / 2)-16 \sigma_{3}(n / 4)+256 \sigma_{3}(n / 8) \\
& +4 \delta\left(0, \frac{1}{6}, \frac{1}{6} ; n\right) \\
N([4,4,0] ; n)= & 4 \sigma_{3}(n)-4 \sigma_{3}(n / 2)-16 \sigma_{3}(n / 4)+256 \sigma_{3}(n / 8) \\
& +4 \delta\left(0, \frac{1}{6}, \frac{1}{6} ; n\right), \\
N([2,6,0] ; n)= & 2 \sigma_{3}(n)-2 \sigma_{3}(n / 2)-16 \sigma_{3}(n / 4)+256 \sigma_{3}(n / 8) \\
& +2 \delta\left(0, \frac{1}{6}, \frac{1}{6} ; n\right) .
\end{aligned}
$$

Proof. We only prove the formula for $N([2,6,0] ; n)$. This is the case $k=2, r=3$.

The proofs for the other cases follow in a similar way.

From (7.1.1)-(7.1.5)

$$
\begin{aligned}
\left(\frac{1+(1-x)^{1 / 2}}{2}\right)^{r}= & \left(\frac{1+(1-x)^{1 / 2}}{2}\right)^{3} \\
= & \left(\frac{1}{2}-\frac{3}{8} x\right)+\left(\frac{1}{2}-\frac{1}{8} x\right)(1-x)^{1 / 2} \\
= & \frac{1}{120}\left(1+14 x+x^{2}\right)-\frac{1}{120}\left(1-x+x^{2}\right)-\frac{1}{15}\left(1-x+\frac{1}{16} x^{2}\right) \\
& +\frac{16}{15}\left(\frac{17}{32}-\frac{17}{32} x+\frac{1}{256} x^{2}+\left(\frac{15}{32}-\frac{15}{64} x\right)(1-x)^{1 / 2}\right) \\
& +\frac{1}{8} x(1-x)^{1 / 2} \\
= & \frac{1}{120} e_{2,1}(x)-\frac{1}{120} e_{2,2}(x)-\frac{1}{15} e_{2,4}(x) \\
& +\frac{16}{15}\left(e_{2,8}^{(0)}(x)+e_{2,8}^{(1)}(x)(1-x)^{1 / 2}\right)+\frac{1}{8} x(1-x)^{1 / 2} .
\end{aligned}
$$

Then, by Theorem 6.1.1, we deduce

$$
c_{2,3,0}^{\prime}(1)=\frac{1}{8} .
$$


Hence

$$
c_{2,3,0}(1)=16 c_{2,3,0}^{\prime}(1)=2
$$

Then, by Theorem 6.1.3, we have

$$
\begin{aligned}
N([2,6,0] ; n)= & 2 \sigma_{3}(n)-2 \sigma_{3}(n / 2)-16 \sigma_{3}(n / 4)+256 \sigma_{3}(n / 8) \\
& +2 \delta\left(0, \frac{1}{6}, \frac{1}{6} ; n\right)
\end{aligned}
$$

as asserted.

The second formula of Theorem 7.1.1 was first proved by Williams [83, Theorem 2, p. 388] in 2006 as a consequence of his determination of the convolution sum $\sum_{m<n / 8} \sigma(m) \sigma(n-8 m)$ for all $n \in \mathbb{N}$. All three formulae of Theorem 7.1.1 were proved in 2010 by Alaca, Alaca and Williams [11, Theorem 1.1 (ii)(iv)(ix), pp. 38-39].

For our second result, we apply Theorem 6.2 .3 to the case when $k=2$ to obtain the number of representations of each positive integer $n$ by the sum of $8-2 r-s$ squares, $2 r$ squares each multiplied by 2 and $s$ squares each multiplied by 4 , where $r, s \in \mathbb{N}$ and $0<2 r+s<8$.

Theorem 7.1.2. Let $n \in \mathbb{N}$. Then

$$
\begin{aligned}
N([1,2,5] ; n)= & \frac{1}{4} \sigma_{3}(n)-\frac{1}{4} \sigma_{3}(n / 2)-16 \sigma_{3}(n / 8)+256 \sigma_{3}(n / 16) \\
& +\frac{3}{4} \delta\left(0, \frac{1}{6}, \frac{1}{6} ; n\right)+\frac{3}{4} \delta\left(-\frac{1}{6}, \frac{5}{12}, \frac{1}{12} ; n\right) \\
& +\frac{1}{4} \delta\left(\frac{1}{6},-\frac{1}{12}, \frac{1}{4} ; n\right) \\
N([2,2,4] ; n)= & \frac{1}{2} \sigma_{3}(n)-\frac{1}{2} \sigma_{3}(n / 2)-16 \sigma_{3}(n / 8)+256 \sigma_{3}(n / 16) \\
& +\frac{3}{2} \delta\left(0, \frac{1}{6}, \frac{1}{6} ; n\right)+\frac{3}{2} \delta\left(-\frac{1}{6}, \frac{5}{12}, \frac{1}{12} ; n\right)
\end{aligned}
$$




$$
\begin{aligned}
& +\frac{1}{2} \delta\left(\frac{1}{6},-\frac{1}{12}, \frac{1}{4} ; n\right) \\
& N([3,2,3] ; n)=\sigma_{3}(n)-\sigma_{3}(n / 2)-16 \sigma_{3}(n / 8)+256 \sigma_{3}(n / 16) \\
& +2 \delta\left(0, \frac{1}{6}, \frac{1}{6} ; n\right)+\frac{5}{2} \delta\left(-\frac{1}{6}, \frac{5}{12}, \frac{1}{12} ; n\right) \\
& +\frac{1}{2} \delta\left(\frac{1}{6},-\frac{1}{12}, \frac{1}{4} ; n\right) \text {, } \\
& N([4,2,2] ; n)=2 \sigma_{3}(n)-2 \sigma_{3}(n / 2)-16 \sigma_{3}(n / 8)+256 \sigma_{3}(n / 16) \\
& +2 \delta\left(0, \frac{1}{6}, \frac{1}{6} ; n\right)+\frac{7}{2} \delta\left(-\frac{1}{6}, \frac{5}{12}, \frac{1}{12} ; n\right) \\
& +\frac{1}{2} \delta\left(\frac{1}{6},-\frac{1}{12}, \frac{1}{4} ; n\right) \text {, } \\
& N([5,2,1] ; n)=4 \sigma_{3}(n)-4 \sigma_{3}(n / 2)-16 \sigma_{3}(n / 8)+256 \sigma_{3}(n / 16) \\
& +2 \delta\left(0, \frac{1}{6}, \frac{1}{6} ; n\right)+\frac{7}{2} \delta\left(-\frac{1}{6}, \frac{5}{12}, \frac{1}{12} ; n\right) \\
& +\frac{1}{2} \delta\left(\frac{1}{6},-\frac{1}{12}, \frac{1}{4} ; n\right) \text {, } \\
& N([1,4,3] ; n)=\frac{1}{2} \sigma_{3}(n)-\frac{1}{2} \sigma_{3}(n / 2)-16 \sigma_{3}(n / 8)+256 \sigma_{3}(n / 16) \\
& +\frac{1}{2} \delta\left(0, \frac{1}{6}, \frac{1}{6} ; n\right)+\delta\left(-\frac{1}{6}, \frac{5}{12}, \frac{1}{12} ; n\right), \\
& N([2,4,2] ; n)=\sigma_{3}(n)-\sigma_{3}(n / 2)-16 \sigma_{3}(n / 8)+256 \sigma_{3}(n / 16) \\
& +\delta\left(0, \frac{1}{6}, \frac{1}{6} ; n\right)+\frac{3}{2} \delta\left(-\frac{1}{6}, \frac{5}{12}, \frac{1}{12} ; n\right) \\
& +\frac{1}{2} \delta\left(\frac{1}{6},-\frac{1}{12}, \frac{1}{4} ; n\right) \text {, }
\end{aligned}
$$




$$
\begin{aligned}
N([3,4,1] ; n)= & 2 \sigma_{3}(n)-2 \sigma_{3}(n / 2)-16 \sigma_{3}(n / 8)+256 \sigma_{3}(n / 16) \\
& +2 \delta\left(0, \frac{1}{6}, \frac{1}{6} ; n\right)+\frac{3}{2} \delta\left(-\frac{1}{6}, \frac{5}{12}, \frac{1}{12} ; n\right) \\
& +\frac{1}{2} \delta\left(\frac{1}{6},-\frac{1}{12}, \frac{1}{4} ; n\right), \\
N([1,6,1] ; n)= & \sigma_{3}(n)-\sigma_{3}(n / 2)-16 \sigma_{3}(n / 8)+256 \sigma_{3}(n / 16) \\
& +\delta\left(0, \frac{1}{6}, \frac{1}{6} ; n\right)+\frac{1}{2} \delta\left(-\frac{1}{6}, \frac{5}{12}, \frac{1}{12} ; n\right) \\
& -\frac{1}{2} \delta\left(\frac{1}{6},-\frac{1}{12}, \frac{1}{4} ; n\right) .
\end{aligned}
$$

Proof. We only prove the formula for $N([2,4,2] ; n)$. Here $r=s=k=2$. The proofs for the other cases follow in a similar way.

By (7.1.1), (7.1.2) and (7.1.4) - (7.1.9) we have

$$
\begin{aligned}
& \left(\frac{1+(1-x)^{1 / 2}}{2}\right)^{2}\left(\frac{1+(1-x)^{1 / 4}}{2}\right)^{2}-\frac{1}{240} e_{2,1}(x)+\frac{1}{240} e_{2,2}(x) \\
& \quad+\frac{1}{15}\left(e_{2,8}^{(0)}(x)+e_{2,8}^{(1)}(x)(1-x)^{1 / 2}\right) \\
& \quad-\frac{16}{15}\left(e_{2,16}^{(0)}(x)+e_{2,16}^{(1)}(x)(1-x)^{1 / 4}+e_{2,16}^{(2)}(1-x)^{1 / 2}+e_{2,16}^{(3)}(1-x)^{3 / 4}\right) \\
& =(1-x)^{1 / 4}\left(\frac{3}{32} x\right)+(1-x)^{1 / 2}\left(\frac{1}{16} x\right) \\
& +(1-x)^{3 / 4}\left(\frac{1}{32} x\right) .
\end{aligned}
$$

Thus, by Theorem 6.2.1, we have

$$
b_{2,2,2}^{\prime}(1)=\frac{3}{32}, \quad c_{2,2,2}^{\prime}(1)=\frac{1}{16}, \quad d_{2,2,2}^{\prime}(1)=\frac{1}{32}
$$

Hence

$$
b_{2,2,2}(1)=\frac{3}{2}, \quad c_{2,2,2}(1)=1, \quad d_{2,2,2}(1)=\frac{1}{2} .
$$


Using Theorem 6.2.3 with $k=2, r=2$ and $s=2$, we obtain the desired result.

These formulae are simplified in Theorem 9.1.3. The simplified formulae agree with those obtained by Alaca, Alaca and Williams [11, Theorem 1.1 (iii)(v)(vii)(viii)(x)(xi)(xii)(xiii)(xiv)] in 2010 .

For the final result of this section, we apply Theorem 6.3.3 to the case when $k=2$ to obtain the number of representations of a positive integer $n$ by the sum of $8-s$ squares and $s$ squares multiplied by 4 , where $s \in \mathbb{N}$ and $0<s<8$.

Theorem 7.1.3. Let $n \in \mathbb{N}$. Then

$$
\begin{aligned}
N([1,0,7] ; n)= & \frac{1}{8} \sigma_{3}(n)-\frac{9}{8} \sigma_{3}(n / 2)+17 \sigma_{3}(n / 4)-32 \sigma_{3}(n / 8)+256 \sigma_{3}(n / 16) \\
& +\frac{7}{8} \delta\left(0, \frac{1}{6}, \frac{1}{6} ; n\right)-\frac{1}{32} \delta\left(-\frac{1}{2}, \frac{17}{12},-\frac{7}{12} ; n\right) \\
& +\frac{1}{32} \delta\left(-\frac{1}{6}, \frac{11}{12},-\frac{5}{12} ; n\right)+\frac{7}{8} \delta\left(-\frac{1}{6}, \frac{5}{12}, \frac{1}{12} ; n\right) \\
& +\frac{3}{8} \delta\left(\frac{1}{6},-\frac{1}{12}, \frac{1}{4} ; n\right) \\
N([2,0,6] ; n)= & \frac{1}{4} \sigma_{3}(n)-\frac{1}{4} \sigma_{3}(n / 2)-16 \sigma_{3}(n / 8)+256 \sigma_{3}(n / 16) \\
& +\frac{7}{4} \delta\left(0, \frac{1}{6}, \frac{1}{6} ; n\right)-\frac{1}{16} \delta\left(-\frac{1}{2}, \frac{17}{12},-\frac{7}{12} ; n\right) \\
& +\frac{1}{16} \delta\left(-\frac{1}{6}, \frac{11}{12},-\frac{5}{12} ; n\right)+2 \delta\left(-\frac{1}{6}, \frac{5}{12}, \frac{1}{12} ; n\right) \\
& +\frac{1}{2} \delta\left(\frac{1}{6},-\frac{1}{12}, \frac{1}{4} ; n\right) \\
& +\frac{5}{2} \delta\left(0, \frac{1}{6}, \frac{1}{6} ; n\right)-\frac{1}{16} \delta\left(-\frac{1}{2}, \frac{17}{12},-\frac{7}{12} ; n\right) \\
N([3,0,5] ; n)= & \frac{1}{2} \sigma_{3}(n)+\frac{3}{2} \sigma_{3}(n / 2)-34 \sigma_{3}(n / 4)+16 \sigma_{3}(n / 8)+256 \sigma_{3}(n / 16) \\
& 5.50
\end{aligned}
$$




$$
\begin{aligned}
& +\frac{1}{16} \delta\left(-\frac{1}{6}, \frac{11}{12},-\frac{5}{12} ; n\right)+3 \delta\left(-\frac{1}{6}, \frac{5}{12}, \frac{1}{12} ; n\right) \\
& +\frac{1}{2} \delta\left(\frac{1}{6},-\frac{1}{12}, \frac{1}{4} ; n\right)
\end{aligned}
$$

$N([4,0,4] ; n)=\sigma_{3}(n)+3 \sigma_{3}(n / 2)-68 \sigma_{3}(n / 4)+48 \sigma_{3}(n / 8)+256 \sigma_{3}(n / 16)$

$$
\begin{aligned}
& +3 \delta\left(0, \frac{1}{6}, \frac{1}{6} ; n\right)+\frac{7}{2} \delta\left(-\frac{1}{6}, \frac{5}{12}, \frac{1}{12} ; n\right) \\
& +\frac{1}{2} \delta\left(\frac{1}{6},-\frac{1}{12}, \frac{1}{4} ; n\right),
\end{aligned}
$$

$$
\begin{aligned}
N([5,0,3] ; n)= & 2 \sigma_{3}(n)+2 \sigma_{3}(n / 2)-68 \sigma_{3}(n / 4)+48 \sigma_{3}(n / 8)+256 \sigma_{3}(n / 16) \\
& +3 \delta\left(0, \frac{1}{6}, \frac{1}{6} ; n\right)+\frac{1}{8} \delta\left(-\frac{1}{2}, \frac{17}{12},-\frac{7}{12} ; n\right) \\
& -\frac{1}{8} \delta\left(-\frac{1}{6}, \frac{11}{12},-\frac{5}{12} ; n\right)+\frac{7}{2} \delta\left(-\frac{1}{6}, \frac{5}{12}, \frac{1}{12} ; n\right) \\
& +\frac{1}{2} \delta\left(\frac{1}{6},-\frac{1}{12}, \frac{1}{4} ; n\right),
\end{aligned}
$$

$$
\begin{aligned}
N([6,0,2] ; n)= & 4 \sigma_{3}(n)-4 \sigma_{3}(n / 2)-16 \sigma_{3}(n / 8)+256 \sigma_{3}(n / 16) \\
& +2 \delta\left(0, \frac{1}{6}, \frac{1}{6} ; n\right)+\frac{1}{4} \delta\left(-\frac{1}{2}, \frac{17}{12},-\frac{7}{12} ; n\right) \\
& -\frac{1}{4} \delta\left(-\frac{1}{6}, \frac{11}{12},-\frac{5}{12} ; n\right)+\frac{7}{2} \delta\left(-\frac{1}{6}, \frac{5}{12}, \frac{1}{12} ; n\right) \\
& +\frac{1}{2} \delta\left(\frac{1}{6},-\frac{1}{12}, \frac{1}{4} ; n\right), \\
N([7,0,1] ; n)= & 8 \sigma_{3}(n)-16 \sigma_{3}(n / 2)+136 \sigma_{3}(n / 4)-144 \sigma_{3}(n / 8)+256 \sigma_{3}(n / 16) \\
& +\frac{1}{4} \delta\left(-\frac{1}{2}, \frac{17}{12},-\frac{7}{12} ; n\right)-\frac{1}{4} \delta\left(-\frac{1}{6}, \frac{11}{12},-\frac{5}{12} ; n\right) \\
& +\frac{7}{2} \delta\left(-\frac{1}{6}, \frac{5}{12}, \frac{1}{12} ; n\right)+\frac{1}{2} \delta\left(\frac{1}{6},-\frac{1}{12}, \frac{1}{4} ; n\right) .
\end{aligned}
$$

Proof. We only prove the formula for $N([3,0,5] ; n)$. In this case $k=2$ and $s=5$. 
The proofs for the other cases follow in a similar way.

$$
\begin{aligned}
& \text { By (7.1.1)-(7.1.9) we have } \\
& \left(\frac{1+(1-x)^{1 / 4}}{2}\right)^{5}-\frac{1}{480} e_{2,1}(x)-\frac{1}{160} e_{2,2}(x)+\frac{17}{120} e_{2,4}(x) \\
& \quad-\frac{1}{15}\left(e_{2,8}^{(0)}(x)+e_{2,8}^{(1)}(x)(1-x)^{1 / 2}\right) \\
& \quad-\frac{16}{15}\left(e_{2,16}^{(0)}(x)+e_{2,16}^{(1)}(x)(1-x)^{1 / 4}+e_{2,16}^{(2)}(1-x)^{1 / 2}+e_{2,16}^{(3)}(1-x)^{3 / 4}\right) \\
& =(1-x)^{1 / 4}\left(-\frac{1}{16}+\frac{3}{16} x\right)+(1-x)^{1 / 2}\left(\frac{5}{32} x\right) \\
& +(1-x)^{3 / 4}\left(\frac{1}{16}+\frac{1}{32} x\right)
\end{aligned}
$$

Thus we have

$b_{2,0,5}^{\prime}(0)=-\frac{1}{16}, b_{2,0,5}^{\prime}(1)=\frac{3}{16}, c_{2,0,5}^{\prime}(1)=\frac{5}{32}, d_{2,0,5}^{\prime}(0)=\frac{1}{16}, d_{2,0,5}^{\prime}(1)=\frac{1}{32}$

Using Theorem 6.3 .3 with $k=2$ and $s=5$, we obtain the desired result.

The seven formulae of Theorem 7.1.3 are simplified in Theorem 9.1.6. The simplified formulae agree with those of Alaca, Alaca and Williams [5, Theorem 1.1, pp. 343-344] obtained in 2008. 


\subsection{Sums with 12 squares}

In this section, we apply Theorem 6.1.3, Theorem 6.2.3 and Theorem 6.3.3 when $k=3$. We first note that from Definition 4.1 .1 we have

$$
\begin{aligned}
& e_{3,1}(x)=1-33 x-33 x^{2}+x^{3} \\
& e_{3,2}(x)=1-\frac{3}{2} x-\frac{3}{2} x^{2}+x^{3} \\
& e_{3,4}(x)=1-\frac{3}{2} x+\frac{15}{32} x^{2}+\frac{1}{64} x^{3} .
\end{aligned}
$$

From Definition 4.2.1 we have

$$
e_{3,8}(x)=e_{3,8}^{(0)}(x)+e_{3,8}^{(1)}(x)(1-x)^{1 / 2}
$$

where

$$
\begin{aligned}
& e_{3,8}^{(0)}(x)=\frac{65}{128}-\frac{195}{256} x+\frac{519}{2048} x^{2}+\frac{1}{4096} x^{3} \\
& e_{3,8}^{(1)}(x)=\frac{65}{128}-\frac{63}{128} x+\frac{63}{2048} x^{2}
\end{aligned}
$$

From Definition 4.3.1 we have

$$
e_{3,16}(x)=\left(e_{3,16}^{(0)}(x)+e_{3,16}^{(1)}(x)(1-x)^{1 / 4}+e_{3,16}^{(2)}(x)(1-x)^{1 / 2}+e_{3,16}^{(3)}(x)(1-x)^{3 / 4}\right)
$$

where

$$
\begin{aligned}
& e_{3,16}^{(0)}(x)=\frac{2081}{8192}-\frac{6243}{16384} x+\frac{16647}{131072} x^{2}+\frac{1}{262144} x^{3} \\
& e_{3,16}^{(1)}(x)=\frac{63}{256}-\frac{4977}{16384} x+\frac{3843}{65536} x^{2} \\
& e_{3,16}^{(2)}(x)=\frac{2079}{8192}-\frac{2079}{8192} x+\frac{2079}{131072} x^{2} \\
& e_{3,16}^{(3)}(x)=\frac{63}{256}-\frac{3087}{16384} x+\frac{63}{65536} x^{2}
\end{aligned}
$$

For our first result, we apply Theorem 6.1 .3 to the case when $k=3$ to obtain the number of representations of an integer $n$ by the sum of $12-2 r$ squares and $2 r$ squares multiplied by 2 , where $r \in \mathbb{N}$ and $0<r<6$. 
Theorem 7.2.1. Let $n \in \mathbb{N}$. Then

$$
\begin{aligned}
N([10,2,0] ; n)= & 4 \sigma_{5}(n)-4 \sigma_{5}(n / 2)+8 \sigma_{5}(n / 4)-512 \sigma_{5}(n / 8) \\
& +8 \delta\left(0, \frac{1}{2}, 0 ; n\right)+8 \delta\left(-\frac{1}{3}, 1,-\frac{1}{6} ; n\right)-8 \delta\left(0,0, \frac{1}{2} ; n\right), \\
N([8,4,0] ; n)= & 2 \sigma_{5}(n)-2 \sigma_{5}(n / 2)+8 \sigma_{5}(n / 4)-512 \sigma_{5}(n / 8) \\
& +6 \delta\left(0, \frac{1}{2}, 0 ; n\right)+8 \delta\left(-\frac{1}{3}, 1,-\frac{1}{6} ; n\right)-8 \delta\left(0,0, \frac{1}{2} ; n\right), \\
N([6,6,0] ; n)= & \sigma_{5}(n)-\sigma_{5}(n / 2)+8 \sigma_{5}(n / 4)-512 \sigma_{5}(n / 8) \\
& +5 \delta\left(0, \frac{1}{2}, 0 ; n\right)+6 \delta\left(-\frac{1}{3}, 1,-\frac{1}{6} ; n\right)-8 \delta\left(0,0, \frac{1}{2} ; n\right), \\
N([4,8,0] ; n)= & \frac{1}{2} \sigma_{5}(n)-\frac{1}{2} \sigma_{5}(n / 2)+8 \sigma_{5}(n / 4)-512 \sigma_{5}(n / 8) \\
& +\frac{7}{2} \delta\left(0, \frac{1}{2}, 0 ; n\right)+4 \delta\left(-\frac{1}{3}, 1,-\frac{1}{6} ; n\right)-8 \delta\left(0,0, \frac{1}{2} ; n\right), \\
& +\frac{7}{4} \delta\left(0, \frac{1}{2}, 0 ; n\right)+2 \delta\left(-\frac{1}{3}, 1,-\frac{1}{6} ; n\right) . \\
N([2,10,0] ; n)= & \frac{1}{4} \sigma_{5}(n)-\frac{1}{4} \sigma_{5}(n / 2)+8 \sigma_{5}(n / 4)-512 \sigma_{5}(n / 8) \\
&
\end{aligned}
$$

Proof. We only prove the formula for $N([10,2,0] ; n)$. The proofs for the other cases follow in a similar way.

From (7.2.1)-(7.2.9) and Lemma 5.1.1 we have

$$
\begin{aligned}
& \left(\frac{1+(1-x)^{1 / 2}}{2}\right)+\frac{1}{126} e_{3,1}(x)-\frac{1}{126} e_{3,2}(x)+\frac{1}{63} e_{3,4}(x) \\
& -\frac{64}{63}\left(e_{3,8}^{(0)}(x)+e_{3,8}^{(1)}(x)(1-x)^{1 / 2}\right)
\end{aligned}
$$


$=(1-x)\left(\frac{1}{2} x\right)+(1-x)^{1 / 2}\left(\frac{1}{2} x-\frac{1}{32} x^{2}\right)$.

Thus we have

$a_{3,2,0}^{\prime}(1)=\frac{1}{2}, c_{3,2,0}^{\prime}(1)=\frac{1}{2}, c_{3,2,0}^{\prime}(2)=-\frac{1}{32}$

Using Theorem 6.1.3 with $k=3$ and $r=2$, we obtain the desired result.

We recall from Theorems 2.11 .2 and 2.11 .4 that $\delta\left(0, \frac{1}{2}, 0 ; n\right)=\tau_{2}(n)$ and $\delta\left(0,0, \frac{1}{2} ; n\right)=\tau_{2}(n / 2)$ for $n \in \mathbb{N}$.

For our second result, we apply Theorem 6.2 .3 to the case when $k=3$ to obtain the number of representations of an integer $n$ by the sum of $12-2 r-s$ squares, $2 r$ squares multiplied by 2 and $s$ squares multiplied by 4 , where $r, s \in \mathbb{N}$ and $0<2 r+s<12$.

Theorem 7.2.2. Let $n \in \mathbb{N}$. Then

$$
\begin{aligned}
N([1,2,9] ; n)= & \frac{1}{128} \sigma_{5}(n)-\frac{1}{128} \sigma_{5}(n / 2)+8 \sigma_{5}(n / 8)-512 \sigma_{5}(n / 16) \\
& +\frac{71}{128} \delta\left(0, \frac{1}{2}, 0 ; n\right)+\frac{7}{16} \delta\left(-\frac{1}{3}, 1,-\frac{1}{6} ; n\right)-\frac{7}{4} \delta\left(0,0, \frac{1}{2} ; n\right) \\
& +\frac{1}{2} \delta\left(-\frac{1}{2}, \frac{5}{4},-\frac{1}{4} ; n\right)-6 \delta\left(-\frac{1}{6}, \frac{1}{4}, \frac{5}{12} ; n\right) \\
& +\frac{1}{2} \delta\left(-\frac{1}{6}, \frac{3}{4},-\frac{1}{12} ; n\right) \\
N([2,2,8] ; n)= & \frac{1}{64} \sigma_{5}(n)-\frac{1}{64} \sigma_{5}(n / 2)+8 \sigma_{5}(n / 8)-512 \sigma_{5}(n / 16) \\
& +\frac{71}{64} \delta\left(0, \frac{1}{2}, 0 ; n\right)+\frac{7}{8} \delta\left(-\frac{1}{3}, 1,-\frac{1}{6} ; n\right)-\frac{7}{2} \delta\left(0,0, \frac{1}{2} ; n\right)
\end{aligned}
$$




$$
\begin{aligned}
& +\frac{15}{16} \delta\left(-\frac{1}{2}, \frac{5}{4},-\frac{1}{4} ; n\right)-\frac{45}{4} \delta\left(-\frac{1}{6}, \frac{1}{4}, \frac{5}{12} ; n\right) \\
& +\frac{17}{16} \delta\left(-\frac{1}{6}, \frac{3}{4},-\frac{1}{12} ; n\right)-\frac{1}{4} \delta\left(\frac{1}{6},-\frac{1}{4}, \frac{7}{12} ; n\right), \\
& N([3,2,7] ; n)=\frac{1}{32} \sigma_{5}(n)-\frac{1}{32} \sigma_{5}(n / 2)+8 \sigma_{5}(n / 8)-512 \sigma_{5}(n / 16) \\
& +\frac{51}{32} \delta\left(0, \frac{1}{2}, 0 ; n\right)+\frac{11}{8} \delta\left(-\frac{1}{3}, 1,-\frac{1}{6} ; n\right)-4 \delta\left(0,0, \frac{1}{2} ; n\right) \\
& +\frac{21}{16} \delta\left(-\frac{1}{2}, \frac{5}{4},-\frac{1}{4} ; n\right)-\frac{57}{4} \delta\left(-\frac{1}{6}, \frac{1}{4}, \frac{5}{12} ; n\right) \\
& +\frac{27}{16} \delta\left(-\frac{1}{6}, \frac{3}{4},-\frac{1}{12} ; n\right)-\frac{1}{4} \delta\left(\frac{1}{6},-\frac{1}{4}, \frac{7}{12} ; n\right) \text {, } \\
& N([4,2,6] ; n)=\frac{1}{16} \sigma_{5}(n)-\frac{1}{16} \sigma_{5}(n / 2)+8 \sigma_{5}(n / 8)-512 \sigma_{5}(n / 16) \\
& +\frac{31}{16} \delta\left(0, \frac{1}{2}, 0 ; n\right)+2 \delta\left(-\frac{1}{3}, 1,-\frac{1}{6} ; n\right)-4 \delta\left(0,0, \frac{1}{2} ; n\right) \\
& +\frac{27}{16} \delta\left(-\frac{1}{2}, \frac{5}{4},-\frac{1}{4} ; n\right)-\frac{61}{4} \delta\left(-\frac{1}{6}, \frac{1}{4}, \frac{5}{12} ; n\right) \\
& +\frac{37}{16} \delta\left(-\frac{1}{6}, \frac{3}{4},-\frac{1}{12} ; n\right)-\frac{1}{4} \delta\left(\frac{1}{6},-\frac{1}{4}, \frac{7}{12} ; n\right), \\
& N([5,2,5] ; n)=\frac{1}{8} \sigma_{5}(n)-\frac{1}{8} \sigma_{5}(n / 2)+8 \sigma_{5}(n / 8)-512 \sigma_{5}(n / 16) \\
& +\frac{17}{8} \delta\left(0, \frac{1}{2}, 0 ; n\right)+\frac{11}{4} \delta\left(-\frac{1}{3}, 1,-\frac{1}{6} ; n\right)-4 \delta\left(0,0, \frac{1}{2} ; n\right) \\
& +\frac{35}{16} \delta\left(-\frac{1}{2}, \frac{5}{4},-\frac{1}{4} ; n\right)-\frac{61}{4} \delta\left(-\frac{1}{6}, \frac{1}{4}, \frac{5}{12} ; n\right) \\
& +\frac{45}{16} \delta\left(-\frac{1}{6}, \frac{3}{4},-\frac{1}{12} ; n\right)-\frac{1}{4} \delta\left(\frac{1}{6},-\frac{1}{4}, \frac{7}{12} ; n\right), \\
& N([6,2,4] ; n)=\frac{1}{4} \sigma_{5}(n)-\frac{1}{4} \sigma_{5}(n / 2)+8 \sigma_{5}(n / 8)-512 \sigma_{5}(n / 16) \\
& +\frac{9}{4} \delta\left(0, \frac{1}{2}, 0 ; n\right)+\frac{7}{2} \delta\left(-\frac{1}{3}, 1,-\frac{1}{6} ; n\right)-4 \delta\left(0,0, \frac{1}{2} ; n\right)
\end{aligned}
$$




$$
\begin{aligned}
& +\frac{47}{16} \delta\left(-\frac{1}{2}, \frac{5}{4},-\frac{1}{4} ; n\right)-\frac{61}{4} \delta\left(-\frac{1}{6}, \frac{1}{4}, \frac{5}{12} ; n\right) \\
& +\frac{49}{16} \delta\left(-\frac{1}{6}, \frac{3}{4},-\frac{1}{12} ; n\right)-\frac{1}{4} \delta\left(\frac{1}{6},-\frac{1}{4}, \frac{7}{12} ; n\right) \text {, } \\
& N([7,2,3] ; n)=\frac{1}{2} \sigma_{5}(n)-\frac{1}{2} \sigma_{5}(n / 2)+8 \sigma_{5}(n / 8)-512 \sigma_{5}(n / 16) \\
& +\frac{5}{2} \delta\left(0, \frac{1}{2}, 0 ; n\right)+4 \delta\left(-\frac{1}{3}, 1,-\frac{1}{6} ; n\right)-4 \delta\left(0,0, \frac{1}{2} ; n\right) \\
& +\frac{63}{16} \delta\left(-\frac{1}{2}, \frac{5}{4},-\frac{1}{4} ; n\right)-\frac{61}{4} \delta\left(-\frac{1}{6}, \frac{1}{4}, \frac{5}{12} ; n\right) \\
& +\frac{49}{16} \delta\left(-\frac{1}{6}, \frac{3}{4},-\frac{1}{12} ; n\right)-\frac{1}{4} \delta\left(\frac{1}{6},-\frac{1}{4}, \frac{7}{12} ; n\right), \\
& N([8,2,2] ; n)=\sigma_{5}(n)-\sigma_{5}(n / 2)+8 \sigma_{5}(n / 8)-512 \sigma_{5}(n / 16) \\
& +3 \delta\left(0, \frac{1}{2}, 0 ; n\right)+4 \delta\left(-\frac{1}{3}, 1,-\frac{1}{6} ; n\right)-4 \delta\left(0,0, \frac{1}{2} ; n\right) \\
& +\frac{79}{16} \delta\left(-\frac{1}{2}, \frac{5}{4},-\frac{1}{4} ; n\right)-\frac{61}{4} \delta\left(-\frac{1}{6}, \frac{1}{4}, \frac{5}{12} ; n\right) \\
& +\frac{49}{16} \delta\left(-\frac{1}{6}, \frac{3}{4},-\frac{1}{12} ; n\right)-\frac{1}{4} \delta\left(\frac{1}{6},-\frac{1}{4}, \frac{7}{12} ; n\right), \\
& N([9,2,1] ; n)=2 \sigma_{5}(n)-2 \sigma_{5}(n / 2)+8 \sigma_{5}(n / 8)-512 \sigma_{5}(n / 16) \\
& +4 \delta\left(0, \frac{1}{2}, 0 ; n\right)+4 \delta\left(-\frac{1}{3}, 1,-\frac{1}{6} ; n\right)-4 \delta\left(0,0, \frac{1}{2} ; n\right) \\
& +\frac{79}{16} \delta\left(-\frac{1}{2}, \frac{5}{4},-\frac{1}{4} ; n\right)-\frac{61}{4} \delta\left(-\frac{1}{6}, \frac{1}{4}, \frac{5}{12} ; n\right) \\
& +\frac{49}{16} \delta\left(-\frac{1}{6}, \frac{3}{4},-\frac{1}{12} ; n\right)-\frac{1}{4} \delta\left(\frac{1}{6},-\frac{1}{4}, \frac{7}{12} ; n\right) \text {, } \\
& N([1,4,7] ; n)=\frac{1}{64} \sigma_{5}(n)-\frac{1}{64} \sigma_{5}(n / 2)+8 \sigma_{5}(n / 8)-512 \sigma_{5}(n / 16) \\
& +\frac{31}{64} \delta\left(0, \frac{1}{2}, 0 ; n\right)+\frac{1}{2} \delta\left(-\frac{1}{3}, 1,-\frac{1}{6} ; n\right)-\frac{1}{2} \delta\left(0,0, \frac{1}{2} ; n\right)
\end{aligned}
$$




$$
\begin{aligned}
& +\frac{7}{16} \delta\left(-\frac{1}{2}, \frac{5}{4},-\frac{1}{4} ; n\right)-\frac{15}{4} \delta\left(-\frac{1}{6}, \frac{1}{4}, \frac{5}{12} ; n\right) \\
& +\frac{9}{16} \delta\left(-\frac{1}{6}, \frac{3}{4},-\frac{1}{12} ; n\right)+\frac{1}{4} \delta\left(\frac{1}{6},-\frac{1}{4}, \frac{7}{12} ; n\right) \text {, } \\
& N([2,4,6] ; n)=\frac{1}{32} \sigma_{5}(n)-\frac{1}{32} \sigma_{5}(n / 2)+8 \sigma_{5}(n / 8)-512 \sigma_{5}(n / 16) \\
& +\frac{31}{32} \delta\left(0, \frac{1}{2}, 0 ; n\right)+\delta\left(-\frac{1}{3}, 1,-\frac{1}{6} ; n\right)-3 \delta\left(0,0, \frac{1}{2} ; n\right) \\
& +\frac{15}{16} \delta\left(-\frac{1}{2}, \frac{5}{4},-\frac{1}{4} ; n\right)-\frac{37}{4} \delta\left(-\frac{1}{6}, \frac{1}{4}, \frac{5}{12} ; n\right) \\
& +\frac{17}{16} \delta\left(-\frac{1}{6}, \frac{3}{4},-\frac{1}{12} ; n\right)-\frac{1}{4} \delta\left(\frac{1}{6},-\frac{1}{4}, \frac{7}{12} ; n\right) \text {, } \\
& N([3,4,5] ; n)=\frac{1}{16} \sigma_{5}(n)-\frac{1}{16} \sigma_{5}(n / 2)+8 \sigma_{5}(n / 8)-512 \sigma_{5}(n / 16) \\
& +\frac{23}{16} \delta\left(0, \frac{1}{2}, 0 ; n\right)+\frac{3}{2} \delta\left(-\frac{1}{3}, 1,-\frac{1}{6} ; n\right)-4 \delta\left(0,0, \frac{1}{2} ; n\right) \\
& +\frac{23}{16} \delta\left(-\frac{1}{2}, \frac{5}{4},-\frac{1}{4} ; n\right)-\frac{53}{4} \delta\left(-\frac{1}{6}, \frac{1}{4}, \frac{5}{12} ; n\right) \\
& +\frac{25}{16} \delta\left(-\frac{1}{6}, \frac{3}{4},-\frac{1}{12} ; n\right)-\frac{1}{4} \delta\left(\frac{1}{6},-\frac{1}{4}, \frac{7}{12} ; n\right) \text {, } \\
& N([4,4,4] ; n)=\frac{1}{8} \sigma_{5}(n)-\frac{1}{8} \sigma_{5}(n / 2)+8 \sigma_{5}(n / 8)-512 \sigma_{5}(n / 16) \\
& +\frac{15}{8} \delta\left(0, \frac{1}{2}, 0 ; n\right)+2 \delta\left(-\frac{1}{3}, 1,-\frac{1}{6} ; n\right)-4 \delta\left(0,0, \frac{1}{2} ; n\right) \\
& +\frac{31}{16} \delta\left(-\frac{1}{2}, \frac{5}{4},-\frac{1}{4} ; n\right)-\frac{61}{4} \delta\left(-\frac{1}{6}, \frac{1}{4}, \frac{5}{12} ; n\right) \\
& +\frac{33}{16} \delta\left(-\frac{1}{6}, \frac{3}{4},-\frac{1}{12} ; n\right)-\frac{1}{4} \delta\left(\frac{1}{6},-\frac{1}{4}, \frac{7}{12} ; n\right) \text {, } \\
& N([5,4,3] ; n)=\frac{1}{4} \sigma_{5}(n)-\frac{1}{4} \sigma_{5}(n / 2)+8 \sigma_{5}(n / 8)-512 \sigma_{5}(n / 16) \\
& +\frac{9}{4} \delta\left(0, \frac{1}{2}, 0 ; n\right)+\frac{5}{2} \delta\left(-\frac{1}{3}, 1,-\frac{1}{6} ; n\right)-4 \delta\left(0,0, \frac{1}{2} ; n\right)
\end{aligned}
$$




$$
\begin{aligned}
& +\frac{39}{16} \delta\left(-\frac{1}{2}, \frac{5}{4},-\frac{1}{4} ; n\right)-\frac{61}{4} \delta\left(-\frac{1}{6}, \frac{1}{4}, \frac{5}{12} ; n\right) \\
& +\frac{41}{16} \delta\left(-\frac{1}{6}, \frac{3}{4},-\frac{1}{12} ; n\right)-\frac{1}{4} \delta\left(\frac{1}{6},-\frac{1}{4}, \frac{7}{12} ; n\right), \\
N([6,4,2] ; n)= & \frac{1}{2} \sigma_{5}(n)-\frac{1}{2} \sigma_{5}(n / 2)+8 \sigma_{5}(n / 8)-512 \sigma_{5}(n / 16) \\
& +\frac{5}{2} \delta\left(0, \frac{1}{2}, 0 ; n\right)+3 \delta\left(-\frac{1}{3}, 1,-\frac{1}{6} ; n\right)-4 \delta\left(0,0, \frac{1}{2} ; n\right) \\
& +\frac{47}{16} \delta\left(-\frac{1}{2}, \frac{5}{4},-\frac{1}{4} ; n\right)-\frac{61}{4} \delta\left(-\frac{1}{6}, \frac{1}{4}, \frac{5}{12} ; n\right) \\
& +\frac{49}{16} \delta\left(-\frac{1}{6}, \frac{3}{4},-\frac{1}{12} ; n\right)-\frac{1}{4} \delta\left(\frac{1}{6},-\frac{1}{4}, \frac{7}{12} ; n\right),
\end{aligned}
$$

$$
\begin{aligned}
N([7,4,1] ; n)= & \sigma_{5}(n)-\sigma_{5}(n / 2)+8 \sigma_{5}(n / 8)-512 \sigma_{5}(n / 16) \\
& +3 \delta\left(0, \frac{1}{2}, 0 ; n\right)+4 \delta\left(-\frac{1}{3}, 1,-\frac{1}{6} ; n\right)-4 \delta\left(0,0, \frac{1}{2} ; n\right) \\
& +\frac{47}{16} \delta\left(-\frac{1}{2}, \frac{5}{4},-\frac{1}{4} ; n\right)-\frac{61}{4} \delta\left(-\frac{1}{6}, \frac{1}{4}, \frac{5}{12} ; n\right) \\
& +\frac{49}{16} \delta\left(-\frac{1}{6}, \frac{3}{4},-\frac{1}{12} ; n\right)-\frac{1}{4} \delta\left(\frac{1}{6},-\frac{1}{4}, \frac{7}{12} ; n\right),
\end{aligned}
$$

$$
\begin{aligned}
N([1,6,5] ; n)= & \frac{1}{32} \sigma_{5}(n)-\frac{1}{32} \sigma_{5}(n / 2)+8 \sigma_{5}(n / 8)-512 \sigma_{5}(n / 16) \\
& +\frac{15}{32} \delta\left(0, \frac{1}{2}, 0 ; n\right)+\frac{1}{2} \delta\left(-\frac{1}{3}, 1,-\frac{1}{6} ; n\right)+\delta\left(0,0, \frac{1}{2} ; n\right) \\
& +\frac{7}{16} \delta\left(-\frac{1}{2}, \frac{5}{4},-\frac{1}{4} ; n\right)-\frac{9}{4} \delta\left(-\frac{1}{6}, \frac{1}{4}, \frac{5}{12} ; n\right) \\
& +\frac{9}{16} \delta\left(-\frac{1}{6}, \frac{3}{4},-\frac{1}{12} ; n\right)+\frac{3}{4} \delta\left(\frac{1}{6},-\frac{1}{4}, \frac{7}{12} ; n\right),
\end{aligned}
$$

$$
\begin{aligned}
N([2,6,4] ; n)= & \frac{1}{16} \sigma_{5}(n)-\frac{1}{16} \sigma_{5}(n / 2)+8 \sigma_{5}(n / 8)-512 \sigma_{5}(n / 16) \\
& +\frac{15}{16} \delta\left(0, \frac{1}{2}, 0 ; n\right)+\delta\left(-\frac{1}{3}, 1,-\frac{1}{6} ; n\right)-2 \delta\left(0,0, \frac{1}{2} ; n\right)
\end{aligned}
$$




$$
\begin{aligned}
& +\frac{15}{16} \delta\left(-\frac{1}{2}, \frac{5}{4},-\frac{1}{4} ; n\right)-\frac{29}{4} \delta\left(-\frac{1}{6}, \frac{1}{4}, \frac{5}{12} ; n\right) \\
& +\frac{17}{16} \delta\left(-\frac{1}{6}, \frac{3}{4},-\frac{1}{12} ; n\right)-\frac{1}{4} \delta\left(\frac{1}{6},-\frac{1}{4}, \frac{7}{12} ; n\right),
\end{aligned}
$$

$$
\begin{aligned}
N([3,6,3] ; n)= & \frac{1}{8} \sigma_{5}(n)-\frac{1}{8} \sigma_{5}(n / 2)+8 \sigma_{5}(n / 8)-512 \sigma_{5}(n / 16) \\
& +\frac{11}{8} \delta\left(0, \frac{1}{2}, 0 ; n\right)+\frac{3}{2} \delta\left(-\frac{1}{3}, 1,-\frac{1}{6} ; n\right)-4 \delta\left(0,0, \frac{1}{2} ; n\right) \\
& +\frac{23}{16} \delta\left(-\frac{1}{2}, \frac{5}{4},-\frac{1}{4} ; n\right)-\frac{45}{4} \delta\left(-\frac{1}{6}, \frac{1}{4}, \frac{5}{12} ; n\right) \\
& +\frac{25}{16} \delta\left(-\frac{1}{6}, \frac{3}{4},-\frac{1}{12} ; n\right)-\frac{1}{4} \delta\left(\frac{1}{6},-\frac{1}{4}, \frac{7}{12} ; n\right)
\end{aligned}
$$

$$
\begin{aligned}
N([4,6,2] ; n)= & \frac{1}{4} \sigma_{5}(n)-\frac{1}{4} \sigma_{5}(n / 2)+8 \sigma_{5}(n / 8)-512 \sigma_{5}(n / 16) \\
& +\frac{7}{4} \delta\left(0, \frac{1}{2}, 0 ; n\right)+2 \delta\left(-\frac{1}{3}, 1,-\frac{1}{6} ; n\right)-4 \delta\left(0,0, \frac{1}{2} ; n\right) \\
& +\frac{31}{16} \delta\left(-\frac{1}{2}, \frac{5}{4},-\frac{1}{4} ; n\right)-\frac{61}{4} \delta\left(-\frac{1}{6}, \frac{1}{4}, \frac{5}{12} ; n\right) \\
& +\frac{33}{16} \delta\left(-\frac{1}{6}, \frac{3}{4},-\frac{1}{12} ; n\right)-\frac{1}{4} \delta\left(\frac{1}{6},-\frac{1}{4}, \frac{7}{12} ; n\right),
\end{aligned}
$$

$$
\begin{aligned}
N([5,6,1] ; n)= & \frac{1}{2} \sigma_{5}(n)-\frac{1}{2} \sigma_{5}(n / 2)+8 \sigma_{5}(n / 8)-512 \sigma_{5}(n / 16) \\
& +\frac{5}{2} \delta\left(0, \frac{1}{2}, 0 ; n\right)+3 \delta\left(-\frac{1}{3}, 1,-\frac{1}{6} ; n\right)-4 \delta\left(0,0, \frac{1}{2} ; n\right) \\
& +\frac{31}{16} \delta\left(-\frac{1}{2}, \frac{5}{4},-\frac{1}{4} ; n\right)-\frac{61}{4} \delta\left(-\frac{1}{6}, \frac{1}{4}, \frac{5}{12} ; n\right) \\
& +\frac{33}{16} \delta\left(-\frac{1}{6}, \frac{3}{4},-\frac{1}{12} ; n\right)-\frac{1}{4} \delta\left(\frac{1}{6},-\frac{1}{4}, \frac{7}{12} ; n\right),
\end{aligned}
$$

$$
\begin{aligned}
N([1,8,3] ; n)= & \frac{1}{16} \sigma_{5}(n)-\frac{1}{16} \sigma_{5}(n / 2)+8 \sigma_{5}(n / 8)-512 \sigma_{5}(n / 16) \\
& +\frac{7}{16} \delta\left(0, \frac{1}{2}, 0 ; n\right)+\frac{1}{2} \delta\left(-\frac{1}{3}, 1,-\frac{1}{6} ; n\right)+2 \delta\left(0,0, \frac{1}{2} ; n\right)
\end{aligned}
$$




$$
\begin{aligned}
& +\frac{7}{16} \delta\left(-\frac{1}{2}, \frac{5}{4},-\frac{1}{4} ; n\right)-\frac{5}{4} \delta\left(-\frac{1}{6}, \frac{1}{4}, \frac{5}{12} ; n\right) \\
& +\frac{9}{16} \delta\left(-\frac{1}{6}, \frac{3}{4},-\frac{1}{12} ; n\right)+\frac{7}{4} \delta\left(\frac{1}{6},-\frac{1}{4}, \frac{7}{12} ; n\right) \\
N([2,8,2] ; n)= & \frac{1}{8} \sigma_{5}(n)-\frac{1}{8} \sigma_{5}(n / 2)+8 \sigma_{5}(n / 8)-512 \sigma_{5}(n / 16) \\
& +\frac{7}{8} \delta\left(0, \frac{1}{2}, 0 ; n\right)+\delta\left(-\frac{1}{3}, 1,-\frac{1}{6} ; n\right)+\frac{15}{16} \delta\left(-\frac{1}{2}, \frac{5}{4},-\frac{1}{4} ; n\right) \\
& -\frac{29}{4} \delta\left(-\frac{1}{6}, \frac{1}{4}, \frac{5}{12} ; n\right)+\frac{17}{16} \delta\left(-\frac{1}{6}, \frac{3}{4},-\frac{1}{12} ; n\right) \\
& -\frac{1}{4} \delta\left(\frac{1}{6},-\frac{1}{4}, \frac{7}{12} ; n\right), \\
N([3,8,1] ; n)= & \frac{1}{4} \sigma_{5}(n)-\frac{1}{4} \sigma_{5}(n / 2)+8 \sigma_{5}(n / 8)-512 \sigma_{5}(n / 16) \\
& +\frac{7}{4} \delta\left(0, \frac{1}{2}, 0 ; n\right)+2 \delta\left(-\frac{1}{3}, 1,-\frac{1}{6} ; n\right)-4 \delta\left(0,0, \frac{1}{2} ; n\right) \\
& +\frac{15}{16} \delta\left(-\frac{1}{2}, \frac{5}{4},-\frac{1}{4} ; n\right)-\frac{29}{4} \delta\left(-\frac{1}{6}, \frac{1}{4}, \frac{5}{12} ; n\right) \\
& +\frac{17}{16} \delta\left(-\frac{1}{6}, \frac{3}{4},-\frac{1}{12} ; n\right)-\frac{1}{4} \delta\left(\frac{1}{6},-\frac{1}{4}, \frac{7}{12} ; n\right), \\
& +\frac{1}{16} \delta\left(-\frac{1}{6}, \frac{3}{4},-\frac{1}{12} ; n\right)+\frac{15}{4} \delta\left(\frac{1}{6},-\frac{1}{4}, \frac{7}{12} ; n\right) . \\
N([1,10,1] ; n)= & \frac{1}{8} \sigma_{5}(n)-\frac{1}{8} \sigma_{5}(n / 2)+8 \sigma_{5}(n / 8)-512 \sigma_{5}(n / 16) \\
& +\frac{7}{8} \delta\left(0, \frac{1}{2}, 0 ; n\right)+\delta\left(-\frac{1}{3}, 1,-\frac{1}{6} ; n\right) \\
& \left.-\frac{1}{2}, \frac{5}{4},-\frac{1}{4} ; n\right)+\frac{19}{4} \delta\left(-\frac{1}{6}, \frac{1}{4}, \frac{5}{12} ; n\right) \\
& \\
&
\end{aligned}
$$

Proof. We only prove the formula for $N([1,6,5] ; n)$. The proofs for the other cases follow in a similar way.

From (7.2.1)-(7.2.9) and Lemma 5.2.1 we have 


$$
\begin{aligned}
& \left(\frac{1+(1-x)^{1 / 2}}{2}\right)^{3}\left(\frac{1+(1-x)^{1 / 4}}{2}\right)^{5}+\frac{1}{16128} e_{3,1}(x)-\frac{1}{16128} e_{3,2}(x) \\
& \quad+\frac{1}{63}\left(e_{3,8}^{(0)}(x)+e_{3,8}^{(1)}(x)(1-x)^{1 / 2}\right) \\
& \quad-\frac{64}{63}\left(e_{3,16}^{(0)}(x)+e_{3,16}^{(1)}(x)(1-x)^{1 / 4}+e_{3,16}^{(2)}(1-x)^{1 / 2}+e_{3,16}^{(3)}(1-x)^{3 / 4}\right) \\
& =(1-x)\left(\frac{15}{512} x\right)+(1-x)^{1 / 4}\left(\frac{7}{256} x-\frac{9}{1024} x^{2}\right)+(1-x)^{1 / 2}\left(\frac{1}{32} x+\frac{1}{256} x^{2}\right) \\
& \quad+(1-x)^{3 / 4}\left(\frac{9}{256} x+\frac{3}{1024} x^{2}\right) .
\end{aligned}
$$

Thus we have

$$
\begin{aligned}
& a_{3,6,5}^{\prime}(1)=\frac{15}{512}, b_{3,6,5}^{\prime}(1)=\frac{7}{256}, b_{3,6,5}^{\prime}(2)=-\frac{9}{1024}, c_{3,6,5}^{\prime}(1)=\frac{1}{32}, c_{3,6,5}^{\prime}(2)=\frac{1}{256} \\
& d_{3,6,5}^{\prime}(1)=\frac{9}{256}, d_{3,6,5}^{\prime}(2)=\frac{3}{1024}
\end{aligned}
$$

Using Theorem 6.2.3 with $k=3, r=6$ and $s=5$, we obtain the desired result.

For the final result of this section, we apply Theorem 6.3.3 to the case when $k=3$ to obtain the number of representations of an integer $n$ by the sum of $12-s$ squares and $s$ squares multiplied by 4 , where $s \in \mathbb{N}$ and $0<s<12$.

Theorem 7.2.3. Let $n \in \mathbb{N}$. Then

$$
\begin{aligned}
N([1,0,11] ; n)= & \frac{1}{256} \sigma_{5}(n)-\frac{33}{256} \sigma_{5}(n / 2)+\frac{65}{8} \sigma_{5}(n / 4)-512 \sigma_{5}(n / 16) \\
& +\frac{167}{256} \delta\left(0, \frac{1}{2}, 0 ; n\right)+\frac{11}{32} \delta\left(-\frac{1}{3}, 1,-\frac{1}{6} ; n\right)-\frac{11}{4} \delta\left(0,0, \frac{1}{2} ; n\right) \\
& +\frac{1}{128} \delta\left(-\frac{5}{6}, \frac{9}{4},-\frac{11}{12} ; n\right)+\frac{15}{32} \delta\left(-\frac{1}{2}, \frac{5}{4},-\frac{1}{4} ; n\right)
\end{aligned}
$$




$$
\begin{aligned}
& -\frac{67}{8} \delta\left(-\frac{1}{6}, \frac{1}{4}, \frac{5}{12} ; n\right)-\frac{1}{128} \delta\left(-\frac{1}{2}, \frac{7}{4},-\frac{3}{4} ; n\right) \\
& +\frac{15}{32} \delta\left(-\frac{1}{6}, \frac{3}{4},-\frac{1}{12} ; n\right)-\frac{1}{8} \delta\left(\frac{1}{6},-\frac{1}{4}, \frac{7}{12} ; n\right) \\
N([2,0,10] ; n)= & \frac{1}{128} \sigma_{5}(n)-\frac{1}{128} \sigma_{5}(n / 2)+8 \sigma_{5}(n / 8)-512 \sigma_{5}(n / 16) \\
& +\frac{167}{256} \delta\left(0, \frac{1}{2}, 0 ; n\right)+\frac{11}{16} \delta\left(-\frac{1}{3}, 1,-\frac{1}{6} ; n\right)-\frac{15}{4} \delta\left(0,0, \frac{1}{2} ; n\right) \\
& +\frac{1}{64} \delta\left(-\frac{5}{6}, \frac{9}{4},-\frac{11}{12} ; n\right)+\frac{11}{16} \delta\left(-\frac{1}{2}, \frac{5}{4},-\frac{1}{4} ; n\right) \\
& -\frac{51}{4} \delta\left(-\frac{1}{6}, \frac{1}{4}, \frac{5}{12} ; n\right)-\frac{1}{64} \delta\left(-\frac{1}{2}, \frac{7}{4},-\frac{3}{4} ; n\right) \\
& +\frac{19}{16} \delta\left(-\frac{1}{6}, \frac{3}{4},-\frac{1}{12} ; n\right)-\frac{1}{4} \delta\left(\frac{1}{6},-\frac{1}{4}, \frac{7}{12} ; n\right) \\
N([3,0,9] ; n)= & \frac{1}{64} \sigma_{5}(n)+\frac{15}{64} \sigma_{5}(n / 2)-\frac{65}{4} \sigma_{5}(n / 4)+24 \sigma_{5}(n / 8)-512 \sigma_{5}(n / 16) \\
& +\frac{119}{64} \delta\left(0, \frac{1}{2}, 0 ; n\right)+\frac{9}{8} \delta\left(-\frac{1}{3}, 1,-\frac{1}{6} ; n\right)-\frac{15}{4} \delta\left(0,0, \frac{1}{2} ; n\right) \\
& +\frac{1}{64} \delta\left(-\frac{5}{6}, \frac{9}{4},-\frac{11}{12} ; n\right)+\frac{15}{16} \delta\left(-\frac{1}{2}, \frac{5}{4},-\frac{1}{4} ; n\right) \\
& +\frac{59}{4} \delta\left(-\frac{1}{6}, \frac{1}{4}, \frac{5}{12} ; n\right)-\frac{1}{64} \delta\left(-\frac{1}{2}, \frac{7}{4},-\frac{3}{4} ; n\right) \\
& +\frac{31}{16} \delta\left(-\frac{1}{6}, \frac{3}{4},-\frac{1}{12} ; n\right)-\frac{1}{4} \delta\left(\frac{1}{6},-\frac{1}{4}, \frac{7}{12} ; n\right), \\
& +\frac{71}{32} \delta\left(0, \frac{1}{2}, 0 ; n\right)+\frac{7}{4} \delta\left(-\frac{1}{3}, 1,-\frac{1}{6} ; n\right)-\frac{7}{2} \delta\left(0,0, \frac{1}{2} ; n\right) \\
& \frac{1}{32} \sigma_{5}(n)+\frac{15}{32} \sigma_{5}(n / 2)-\frac{65}{2} \sigma_{5}(n / 4)+40 \sigma_{5}(n / 8)-512 \sigma_{5}(n / 16) \\
& \\
&
\end{aligned}
$$




$$
\begin{aligned}
N([5,0,7] ; n)= & \frac{1}{16} \sigma_{5}(n)+\frac{7}{64} \sigma_{5}(n / 2)-\frac{65}{2} \sigma_{5}(n / 4)+40 \sigma_{5}(n / 8)-512 \sigma_{5}(n / 16) \\
& +\frac{37}{16} \delta\left(0, \frac{1}{2}, 0 ; n\right)+\frac{21}{8} \delta\left(-\frac{1}{3}, 1,-\frac{1}{6} ; n\right)-\frac{7}{2} \delta\left(0,0, \frac{1}{2} ; n\right) \\
& -\frac{1}{32} \delta\left(-\frac{5}{6}, \frac{9}{4},-\frac{11}{12} ; n\right)+\frac{37}{16} \delta\left(-\frac{1}{2}, \frac{5}{4},-\frac{1}{4} ; n\right) \\
& -\frac{61}{4} \delta\left(-\frac{1}{6}, \frac{1}{4}, \frac{5}{12} ; n\right)+\frac{1}{32} \delta\left(-\frac{1}{2}, \frac{7}{4},-\frac{3}{4} ; n\right) \\
& +\frac{47}{16} \delta\left(-\frac{1}{6}, \frac{3}{4},-\frac{1}{12} ; n\right)-\frac{1}{4} \delta\left(\frac{1}{6},-\frac{1}{4}, \frac{7}{12} ; n\right)
\end{aligned}
$$

$$
\begin{aligned}
N([6,0,6] ; n)= & \frac{1}{8} \sigma_{5}(n)-\frac{1}{8} \sigma_{5}(n / 2)+8 \sigma_{5}(n / 8)-512 \sigma_{5}(n / 16) \\
& +\frac{17}{8} \delta\left(0, \frac{1}{2}, 0 ; n\right)+\frac{15}{4} \delta\left(-\frac{1}{3}, 1,-\frac{1}{6} ; n\right)-4 \delta\left(0,0, \frac{1}{2} ; n\right) \\
& -\frac{1}{16} \delta\left(-\frac{5}{6}, \frac{9}{4},-\frac{11}{12} ; n\right)+\frac{55}{16} \delta\left(-\frac{1}{2}, \frac{5}{4},-\frac{1}{4} ; n\right) \\
& -\frac{61}{4} \delta\left(-\frac{1}{6}, \frac{1}{4}, \frac{5}{12} ; n\right)+\frac{1}{16} \delta\left(-\frac{1}{2}, \frac{7}{4},-\frac{3}{4} ; n\right) \\
& +\frac{49}{16} \delta\left(-\frac{1}{6}, \frac{3}{4},-\frac{1}{12} ; n\right)-\frac{1}{4} \delta\left(\frac{1}{6},-\frac{1}{4}, \frac{7}{12} ; n\right),
\end{aligned}
$$

$$
\begin{aligned}
N([7,0,5] ; n)= & \frac{1}{4} \sigma_{5}(n)-\frac{5}{4} \sigma_{5}(n / 2)+65 \sigma_{5}(n / 4)-56 \sigma_{5}(n / 8)-512 \sigma_{5}(n / 16) \\
& +\frac{7}{4} \delta\left(0, \frac{1}{2}, 0 ; n\right)+5 \delta\left(-\frac{1}{3}, 1,-\frac{1}{6} ; n\right)-5 \delta\left(0,0, \frac{1}{2} ; n\right) \\
& -\frac{1}{16} \delta\left(-\frac{5}{6}, \frac{9}{4},-\frac{11}{12} ; n\right)+\frac{71}{16} \delta\left(-\frac{1}{2}, \frac{5}{4},-\frac{1}{4} ; n\right) \\
& -\frac{61}{4} \delta\left(-\frac{1}{6}, \frac{1}{4}, \frac{5}{12} ; n\right)+\frac{1}{16} \delta\left(-\frac{1}{2}, \frac{7}{4},-\frac{3}{4} ; n\right) \\
& +\frac{49}{16} \delta\left(-\frac{1}{6}, \frac{3}{4},-\frac{1}{12} ; n\right)-\frac{1}{4} \delta\left(\frac{1}{6},-\frac{1}{4}, \frac{7}{12} ; n\right) \\
N([8,0,4] ; n)= & \frac{1}{2} \sigma_{5}(n)-\frac{5}{2} \sigma_{5}(n / 2)+130 \sigma_{5}(n / 4)-120 \sigma_{5}(n / 8)-512 \sigma_{5}(n / 16) \\
& +\frac{3}{2} \delta\left(0, \frac{1}{2}, 0 ; n\right)+6 \delta\left(-\frac{1}{3}, 1,-\frac{1}{6} ; n\right)-6 \delta\left(0,0, \frac{1}{2} ; n\right)
\end{aligned}
$$




$$
\begin{aligned}
& +\frac{79}{16} \delta\left(-\frac{1}{2}, \frac{5}{4},-\frac{1}{4} ; n\right)-\frac{61}{4} \delta\left(-\frac{1}{6}, \frac{1}{4}, \frac{5}{12} ; n\right) \\
& +\frac{49}{16} \delta\left(-\frac{1}{6}, \frac{3}{4},-\frac{1}{12} ; n\right)-\frac{1}{4} \delta\left(\frac{1}{6},-\frac{1}{4}, \frac{7}{12} ; n\right), \\
& N([9,0,3] ; n)=\sigma_{5}(n)-3 \sigma_{5}(n / 2)+130 \sigma_{5}(n / 4)-120 \sigma_{5}(n / 8)-512 \sigma_{5}(n / 16) \\
& +2 \delta\left(0, \frac{1}{2}, 0 ; n\right)+6 \delta\left(-\frac{1}{3}, 1,-\frac{1}{6} ; n\right)-6 \delta\left(0,0, \frac{1}{2} ; n\right) \\
& +\frac{1}{8} \delta\left(-\frac{5}{6}, \frac{9}{4},-\frac{11}{12} ; n\right)+\frac{79}{16} \delta\left(-\frac{1}{2}, \frac{5}{4},-\frac{1}{4} ; n\right) \\
& -\frac{61}{4} \delta\left(-\frac{1}{6}, \frac{1}{4}, \frac{5}{12} ; n\right)-\frac{1}{8} \delta\left(-\frac{1}{2}, \frac{7}{4},-\frac{3}{4} ; n\right) \\
& +\frac{49}{16} \delta\left(-\frac{1}{6}, \frac{3}{4},-\frac{1}{12} ; n\right)-\frac{1}{4} \delta\left(\frac{1}{6},-\frac{1}{4}, \frac{7}{12} ; n\right) \text {, } \\
& N([10,0,2] ; n)=2 \sigma_{5}(n)-2 \sigma_{5}(n / 2)+8 \sigma_{5}(n / 8)-512 \sigma_{5}(n / 16) \\
& +4 \delta\left(0, \frac{1}{2}, 0 ; n\right)+4 \delta\left(-\frac{1}{3}, 1,-\frac{1}{6} ; n\right)-4 \delta\left(0,0, \frac{1}{2} ; n\right) \\
& +\frac{1}{4} \delta\left(-\frac{5}{6}, \frac{9}{4},-\frac{11}{12} ; n\right)+\frac{79}{16} \delta\left(-\frac{1}{2}, \frac{5}{4},-\frac{1}{4} ; n\right) \\
& -\frac{61}{4} \delta\left(-\frac{1}{6}, \frac{1}{4}, \frac{5}{12} ; n\right)-\frac{1}{4} \delta\left(-\frac{1}{2}, \frac{7}{4},-\frac{3}{4} ; n\right) \\
& +\frac{49}{16} \delta\left(-\frac{1}{6}, \frac{3}{4},-\frac{1}{12} ; n\right)-\frac{1}{4} \delta\left(\frac{1}{6},-\frac{1}{4}, \frac{7}{12} ; n\right) \text {, } \\
& N([11,0,1] ; n)=4 \sigma_{5}(n)-260 \sigma_{5}(n / 4)+264 \sigma_{5}(n / 8)-512 \sigma_{5}(n / 16) \\
& +8 \delta\left(0, \frac{1}{2}, 0 ; n\right)+\frac{1}{4} \delta\left(-\frac{5}{6}, \frac{9}{4},-\frac{11}{12} ; n\right)+\frac{79}{16} \delta\left(-\frac{1}{2}, \frac{5}{4},-\frac{1}{4} ; n\right) \\
& -\frac{61}{4} \delta\left(-\frac{1}{6}, \frac{1}{4}, \frac{5}{12} ; n\right)-\frac{1}{4} \delta\left(-\frac{1}{2}, \frac{7}{4},-\frac{3}{4} ; n\right) \\
& +\frac{49}{16} \delta\left(-\frac{1}{6}, \frac{3}{4},-\frac{1}{12} ; n\right)-\frac{1}{4} \delta\left(\frac{1}{6},-\frac{1}{4}, \frac{7}{12} ; n\right) \text {. }
\end{aligned}
$$


Proof. We only prove the formula for $N([4,0,8] ; n)$. The proofs for the other cases follow in a similar way.

From (7.2.1)-(7.2.9) and Lemma 5.3.1 we have

$$
\begin{aligned}
( & \left.\frac{1+(1-x)^{1 / 4}}{2}\right)^{8}+\frac{1}{16128} e_{3,1}(x)+\frac{5}{5376} e_{3,2}(x)-\frac{65}{1008} e_{3,4}(x) \\
& +\frac{5}{63}\left(e_{3,8}^{(0)}(x)+e_{3,8}^{(1)}(x)(1-x)^{1 / 2}\right) \\
& -\frac{64}{63}\left(e_{3,16}^{(0)}(x)+e_{3,16}^{(1)}(x)(1-x)^{1 / 4}+e_{3,16}^{(2)}(1-x)^{1 / 2}+e_{3,16}^{(3)}(1-x)^{3 / 4}\right) \\
= & (1-x)\left(\frac{71}{512} x\right)+(1-x)^{1 / 4}\left(\frac{23}{256} x-\frac{61}{1024} x^{2}\right)+(1-x)^{1 / 2}\left(\frac{7}{64} x-\frac{7}{512} x^{2}\right) \\
& +(1-x)^{3 / 4}\left(\frac{41}{256} x-\frac{1}{1024} x^{2}\right) .
\end{aligned}
$$

Thus we have

$$
\begin{aligned}
& a_{3,0,8}^{\prime}(1)=\frac{71}{512}, b_{3,0,8}^{\prime}(0)=0, b_{3,0,8}^{\prime}(1)=\frac{23}{256}, b_{3,0,8}^{\prime}(2)=-\frac{61}{1024}, c_{3,0,8}^{\prime}(1)=\frac{7}{64}, \\
& c_{3,0,8}^{\prime}(2)=-\frac{7}{512}, d_{3,0,8}^{\prime}(0)=0, d_{3,0,8}^{\prime}(1)=\frac{41}{256}, d_{3,0,8}^{\prime}(2)=-\frac{1}{1024} .
\end{aligned}
$$

Using Theorem 6.3.3 with $k=3$ and $s=8$, we obtain the desired result.

\subsection{Sums with 16 squares}

In this section, we apply Theorem 6.1.3, Theorem 6.2.3 and Theorem 6.3.3 when $k=4$. We first note that from Definition 4.1.1 we have

$$
\begin{aligned}
& e_{4,1}(x)=\left(e_{2,1}(x)\right)^{2}=1+28 x+198 x^{2}+28 x^{3}+x^{4}, \\
& e_{4,2}(x)=\left(e_{2,2}(x)\right)^{2}=1-2 x+3 x^{2}-2 x^{3}+x^{4}, \\
& e_{4,4}(x)=\left(e_{2,4}(x)\right)^{2}=1-2 x+\frac{9}{8} x^{2}-\frac{1}{8} x^{3}+\frac{1}{256} x^{4} .
\end{aligned}
$$


From Definition 4.2 .1 we have

$$
e_{4,8}(x)=\left(e_{2,8}(x)\right)^{2}=\left(e_{2,8}^{(0)}(x)+e_{2,8}^{(1)}(x)(1-x)^{1 / 2}\right)^{2}=\left(e_{4,8}^{(0)}(x)+e_{4,8}^{(1)}(x)(1-x)^{1 / 2}\right)
$$

where

$$
\begin{aligned}
& e_{4,8}^{(0)}(x)=\frac{257}{512}-\frac{257}{256} x+\frac{1149}{2048} x^{2}-\frac{121}{2048} x^{3}+\frac{1}{65536} x^{4} \\
& e_{4,8}^{(1)}(x)=\frac{255}{512}-\frac{765}{1024} x+\frac{1035}{4096} x^{2}-\frac{15}{8192} x^{3}
\end{aligned}
$$

From Definition 4.3.1 we have

$$
\begin{aligned}
e_{4,16}(x) & =\left(e_{2,16}(x)\right)^{2} \\
& =\left(e_{2,16}^{(0)}(x)+e_{2,16}^{(1)}(x)(1-x)^{1 / 4}+e_{2,16}^{(2)}(x)(1-x)^{1 / 2}+e_{2,16}^{(3)}(x)(1-x)^{3 / 4}\right)^{2},
\end{aligned}
$$

where

$$
\begin{aligned}
& e_{2,16}^{(0)}(x)=\frac{32897}{131072}-\frac{32897}{65536} x+\frac{147069}{524288} x^{2}-\frac{15481}{524288} x^{3}+\frac{1}{16777216} x^{4}, \\
& e_{2,16}^{(1)}(x)=\frac{255}{1024}-\frac{28575}{65536} x+\frac{51075}{262144} x^{2}-\frac{8205}{1048576} x^{3} \\
& e_{2,16}^{(2)}(x)=\frac{32895}{131072}-\frac{98685}{262144} x+\frac{133515}{1048576} x^{2}-\frac{1935}{2097152} x^{3}, \\
& e_{2,16}^{(3)}(x)=\frac{255}{1024}-\frac{20385}{65536} x+\frac{18315}{262144} x^{2}-\frac{8205}{1048576} x^{3} .
\end{aligned}
$$

For the first result of this section, we apply Theorem 6.1 .3 to the case when $k=4$ to obtain the number of representations of an integer $n$ by the sum of $16-2 r$ squares and $2 r$ squares multiplied by 2 , where $r \in \mathbb{N}$ and $0<r<8$.

Theorem 7.3.1. Let $n \in \mathbb{N}$. Then

$$
\begin{aligned}
& N([14,2,0] ; n) \\
& =\frac{16}{17} \sigma_{7}(n)-\frac{16}{17} \sigma_{7}(n / 2)-\frac{32}{17} \sigma_{7}(n / 4)+\frac{8192}{17} \sigma_{7}(n / 8) \\
& \quad+\frac{256}{17} \delta\left(-\frac{1}{3}, \frac{4}{3},-\frac{1}{3} ; n\right)+12 \delta\left(-\frac{2}{3}, \frac{11}{6},-\frac{1}{2} ; n\right)-\frac{1104}{17} \delta\left(-\frac{1}{3}, \frac{5}{6}, \frac{1}{6} ; n\right)
\end{aligned}
$$




$$
+\frac{128}{17} \delta\left(0,-\frac{1}{6}, \frac{5}{6} ; n\right)
$$

$$
\begin{aligned}
& N([12,4,0] ; n) \\
& =\frac{8}{17} \sigma_{7}(n)-\frac{8}{17} \sigma_{7}(n / 2)-\frac{32}{17} \sigma_{7}(n / 4)+\frac{8192}{17} \sigma_{7}(n / 8) \\
& \quad+\frac{196}{17} \delta\left(-\frac{1}{3}, \frac{4}{3},-\frac{1}{3} ; n\right)-\frac{128}{17} \delta\left(0, \frac{1}{3}, \frac{1}{3} ; n\right)+12 \delta\left(-\frac{2}{3}, \frac{11}{6},-\frac{1}{2} ; n\right) \\
& \quad-\frac{1104}{17} \delta\left(-\frac{1}{3}, \frac{5}{6}, \frac{1}{6} ; n\right)+\frac{128}{17} \delta\left(0,-\frac{1}{6}, \frac{5}{6} ; n\right), \\
& N([10,6,0] ; n) \\
& =\frac{4}{17} \sigma_{7}(n)-\frac{4}{17} \sigma_{7}(n / 2)-\frac{32}{17} \sigma_{7}(n / 4)+\frac{8192}{17} \sigma_{7}(n / 8) \\
& \quad+\frac{166}{17} \delta\left(-\frac{1}{3}, \frac{4}{3},-\frac{1}{3} ; n\right)-\frac{192}{17} \delta\left(0, \frac{1}{3}, \frac{1}{3} ; n\right)+10 \delta\left(-\frac{2}{3}, \frac{11}{6},-\frac{1}{2} ; n\right) \\
& \quad-\frac{1104}{17} \delta\left(-\frac{1}{3}, \frac{5}{6}, \frac{1}{6} ; n\right)+\frac{128}{17} \delta\left(0,-\frac{1}{6}, \frac{5}{6} ; n\right),
\end{aligned}
$$

$N([8,8,0] ; n)$

$$
\begin{aligned}
= & \frac{2}{17} \sigma_{7}(n)-\frac{2}{17} \sigma_{7}(n / 2)-\frac{32}{17} \sigma_{7}(n / 4)+\frac{8192}{17} \sigma_{7}(n / 8) \\
& +\frac{134}{17} \delta\left(-\frac{1}{3}, \frac{4}{3},-\frac{1}{3} ; n\right)-\frac{224}{17} \delta\left(0, \frac{1}{3}, \frac{1}{3} ; n\right)+8 \delta\left(-\frac{2}{3}, \frac{11}{6},-\frac{1}{2} ; n\right) \\
& -\frac{1104}{17} \delta\left(-\frac{1}{3}, \frac{5}{6}, \frac{1}{6} ; n\right)+\frac{128}{17} \delta\left(0,-\frac{1}{6}, \frac{5}{6} ; n\right),
\end{aligned}
$$

$$
\begin{aligned}
& N([6,10,0] ; n) \\
& =\frac{1}{17} \sigma_{7}(n)-\frac{1}{17} \sigma_{7}(n / 2)-\frac{32}{17} \sigma_{7}(n / 4)+\frac{8192}{17} \sigma_{7}(n / 8) \\
& \quad+\frac{101}{17} \delta\left(-\frac{1}{3}, \frac{4}{3},-\frac{1}{3} ; n\right)-\frac{240}{17} \delta\left(0, \frac{1}{3}, \frac{1}{3} ; n\right)+6 \delta\left(-\frac{2}{3}, \frac{11}{6},-\frac{1}{2} ; n\right) \\
& \quad-\frac{968}{17} \delta\left(-\frac{1}{3}, \frac{5}{6}, \frac{1}{6} ; n\right)+\frac{128}{17} \delta\left(0,-\frac{1}{6}, \frac{5}{6} ; n\right),
\end{aligned}
$$




$$
\begin{aligned}
& N([4,12,0] ; n) \\
&= \frac{1}{34} \sigma_{7}(n)-\frac{1}{34} \sigma_{7}(n / 2)-\frac{32}{17} \sigma_{7}(n / 4)+\frac{8192}{17} \sigma_{7}(n / 8) \\
&+\frac{135}{34} \delta\left(-\frac{1}{3}, \frac{4}{3},-\frac{1}{3} ; n\right)-\frac{180}{17} \delta\left(0, \frac{1}{3}, \frac{1}{3} ; n\right)+4 \delta\left(-\frac{2}{3}, \frac{11}{6},-\frac{1}{2} ; n\right) \\
&-\frac{696}{17} \delta\left(-\frac{1}{3}, \frac{5}{6}, \frac{1}{6} ; n\right)+\frac{128}{17} \delta\left(0,-\frac{1}{6}, \frac{5}{6} ; n\right), \\
& N([2,14,0] ; n) \\
&= \frac{1}{68} \sigma_{7}(n)-\frac{1}{68} \sigma_{7}(n / 2)-\frac{32}{17} \sigma_{7}(n / 4)+\frac{8192}{17} \sigma_{7}(n / 8) \\
&+\frac{135}{68} \delta\left(-\frac{1}{3}, \frac{4}{3},-\frac{1}{3} ; n\right)-\frac{14}{17} \delta\left(0, \frac{1}{3}, \frac{1}{3} ; n\right)+2 \delta\left(-\frac{2}{3}, \frac{11}{6},-\frac{1}{2} ; n\right) \\
&-\frac{288}{17} \delta\left(-\frac{1}{3}, \frac{5}{6}, \frac{1}{6} ; n\right)+\frac{-416}{17} \delta\left(0,-\frac{1}{6}, \frac{5}{6} ; n\right) .
\end{aligned}
$$

Proof. We only prove the formula for $N([6,10,0] ; n)$. The proofs for the other cases follow in a similar way.

From (7.3.1)-(7.3.9) and Lemma 5.1.1 we have

$$
\begin{aligned}
& \left(\frac{1+(1-x)^{1 / 2}}{2}\right)^{5}-\frac{1}{8160} e_{4,1}(x)+\frac{1}{8160} e_{4,2}(x)+\frac{1}{255} e_{4,4}(x) \\
& \quad-\frac{256}{255}\left(e_{4,8}^{(0)}(x)+e_{4,8}^{(1)}(x)(1-x)^{1 / 2}\right) \\
& =(1-x)\left(\frac{101}{272} x-\frac{15}{272} x^{2}\right)+(1-x)^{1 / 2}\left(\frac{3}{8} x-\frac{121}{544} x^{2}+\frac{1}{544} x^{3}\right) .
\end{aligned}
$$

Thus we have 
$a_{6,10,0}^{\prime}(1)=\frac{101}{272}, a_{6,10,0}^{\prime}(2)=-\frac{15}{272}, c_{6,10,0}^{\prime}(1)=\frac{3}{8}, c_{6,10,0}^{\prime}(2)=-\frac{121}{544}, c_{6,10,0}^{\prime}(3)=\frac{1}{544}$.

Using Theorem 6.1.3 with $k=4, r=10$ and $s=0$, we obtain the desired result.

For the second result of this section, we apply Theorem 6.2 .3 to the case when $k=4$ to obtain the number of representations of an integer $n$ by the sum of $16-2 r-s$ squares, $2 r$ squares multiplied by 2 and $s$ squares multiplied by 4 , where $r, s \in \mathbb{N}$ and $0<2 r+s<16$.

Theorem 7.3.2. Let $n \in \mathbb{N}$. Then

$$
\begin{aligned}
N & ([1,2,13] ; n) \\
= & \frac{1}{8704} \sigma_{7}(n)-\frac{1}{8704} \sigma_{7}(n / 2)-\frac{32}{17} \sigma_{7}(n / 8)+\frac{8192}{17} \sigma_{7}(n / 16) \\
& +\frac{4215}{8704} \delta\left(-\frac{1}{3}, \frac{4}{3},-\frac{1}{3} ; n\right)-\frac{3107}{1088} \delta\left(0, \frac{1}{3}, \frac{1}{3} ; n\right)+\frac{33}{64} \delta\left(-\frac{2}{3}, \frac{11}{6},-\frac{1}{2} ; n\right) \\
& -\frac{1981}{272} \delta\left(-\frac{1}{3}, \frac{5}{6}, \frac{1}{6} ; n\right)+\frac{35}{68} \delta\left(0,-\frac{1}{6}, \frac{5}{6} ; n\right)+\frac{531}{1088} \delta\left(-\frac{5}{6}, \frac{25}{12},-\frac{7}{12} ; n\right) \\
& -\frac{2281}{272} \delta\left(-\frac{1}{2}, \frac{13}{12}, \frac{1}{12} ; n\right)+\frac{845}{68} \delta\left(-\frac{1}{6}, \frac{1}{12}, \frac{3}{4} ; n\right)+\frac{557}{1088} \delta\left(-\frac{1}{2}, \frac{19}{12},-\frac{5}{12} ; n\right) \\
& -\frac{1501}{272} \delta\left(-\frac{1}{6}, \frac{7}{12}, \frac{1}{4} ; n\right)-\frac{13}{68} \delta\left(\frac{1}{6},-\frac{5}{12}, \frac{11}{12} ; n\right),
\end{aligned}
$$

$$
\begin{aligned}
N & ([2,2,12] ; n) \\
= & \frac{1}{4352} \sigma_{7}(n)-\frac{1}{4352} \sigma_{7}(n / 2)-\frac{32}{17} \sigma_{7}(n / 8)+\frac{8192}{17} \sigma_{7}(n / 16) \\
& +\frac{4215}{4352} \delta\left(-\frac{1}{3}, \frac{4}{3},-\frac{1}{3} ; n\right)-\frac{2955}{544} \delta\left(0, \frac{1}{3}, \frac{1}{3} ; n\right)+\frac{33}{32} \delta\left(-\frac{2}{3}, \frac{11}{6},-\frac{1}{2} ; n\right) \\
& -\frac{2019}{136} \delta\left(-\frac{1}{3}, \frac{5}{6}, \frac{1}{6} ; n\right)+\frac{111}{34} \delta\left(0,-\frac{1}{6}, \frac{5}{6} ; n\right)+\frac{273}{272} \delta\left(-\frac{5}{6}, \frac{25}{12},-\frac{7}{12} ; n\right) \\
& -\frac{589}{34} \delta\left(-\frac{1}{2}, \frac{13}{12}, \frac{1}{12} ; n\right)+\frac{445}{17} \delta\left(-\frac{1}{6}, \frac{1}{12}, \frac{3}{4} ; n\right)+\frac{271}{272} \delta\left(-\frac{1}{2}, \frac{19}{12},-\frac{5}{12} ; n\right)
\end{aligned}
$$




$$
-\frac{182}{17} \delta\left(-\frac{1}{6}, \frac{7}{12}, \frac{1}{4} ; n\right)+\frac{1}{17} \delta\left(\frac{1}{6},-\frac{5}{12}, \frac{11}{12} ; n\right),
$$

$N([3,2,11] ; n)$

$$
\begin{aligned}
= & \frac{1}{2176} \sigma_{7}(n)-\frac{1}{2176} \sigma_{7}(n / 2)-\frac{32}{17} \sigma_{7}(n / 8)+\frac{8192}{17} \sigma_{7}(n / 16) \\
& +\frac{3195}{2176} \delta\left(-\frac{1}{3}, \frac{4}{3},-\frac{1}{3} ; n\right)-\frac{1859}{272} \delta\left(0, \frac{1}{3}, \frac{1}{3} ; n\right)+\frac{49}{32} \delta\left(-\frac{2}{3}, \frac{11}{6},-\frac{1}{2} ; n\right) \\
& -\frac{365}{17} \delta\left(-\frac{1}{3}, \frac{5}{6}, \frac{1}{6} ; n\right)+\frac{64}{17} \delta\left(0,-\frac{1}{6}, \frac{5}{6} ; n\right)+\frac{835}{544} \delta\left(-\frac{5}{6}, \frac{25}{12},-\frac{7}{12} ; n\right) \\
& -\frac{439}{17} \delta\left(-\frac{1}{2}, \frac{13}{12}, \frac{1}{12} ; n\right)+\frac{530}{17} \delta\left(-\frac{1}{6}, \frac{1}{12}, \frac{3}{4} ; n\right)+\frac{797}{544} \delta\left(-\frac{1}{2}, \frac{19}{12},-\frac{5}{12} ; n\right) \\
& -\frac{983}{68} \delta\left(-\frac{1}{6}, \frac{7}{12}, \frac{1}{4} ; n\right)+\frac{1}{17} \delta\left(\frac{1}{6},-\frac{5}{12}, \frac{11}{12} ; n\right),
\end{aligned}
$$

$$
\begin{aligned}
& N([4,2,10] ; n) \\
&= \frac{1}{1088} \sigma_{7}(n)-\frac{1}{1088} \sigma_{7}(n / 2)-\frac{32}{17} \sigma_{7}(n / 8)+\frac{8192}{17} \sigma_{7}(n / 16) \\
&+\frac{2175}{1088} \delta\left(-\frac{1}{3}, \frac{4}{3},-\frac{1}{3} ; n\right)-\frac{1005}{136} \delta\left(0, \frac{1}{3}, \frac{1}{3} ; n\right)+2 \delta\left(-\frac{2}{3}, \frac{11}{6},-\frac{1}{2} ; n\right) \\
&-\frac{1817}{68} \delta\left(-\frac{1}{3}, \frac{5}{6}, \frac{1}{6} ; n\right)+\frac{64}{17} \delta\left(0,-\frac{1}{6}, \frac{5}{6} ; n\right)+\frac{281}{136} \delta\left(-\frac{5}{6}, \frac{25}{12},-\frac{7}{12} ; n\right) \\
&-\frac{575}{17} \delta\left(-\frac{1}{2}, \frac{13}{12}, \frac{1}{12} ; n\right)+\frac{547}{17} \delta\left(-\frac{1}{6}, \frac{1}{12}, \frac{3}{4} ; n\right)+\frac{263}{136} \delta\left(-\frac{1}{2}, \frac{19}{12},-\frac{5}{12} ; n\right) \\
&-\frac{284}{17} \delta\left(-\frac{1}{6}, \frac{7}{12}, \frac{1}{4} ; n\right)+\frac{1}{17} \delta\left(\frac{1}{6},-\frac{5}{12}, \frac{11}{12} ; n\right),
\end{aligned}
$$

$N([5,2,9] ; n)$

$$
\begin{aligned}
= & \frac{1}{544} \sigma_{7}(n)-\frac{1}{544} \sigma_{7}(n / 2)-\frac{32}{17} \sigma_{7}(n / 8)+\frac{8192}{17} \sigma_{7}(n / 16) \\
& +\frac{1393}{544} \delta\left(-\frac{1}{3}, \frac{4}{3},-\frac{1}{3} ; n\right)-\frac{15}{2} \delta\left(0, \frac{1}{3}, \frac{1}{3} ; n\right)+\frac{39}{16} \delta\left(-\frac{2}{3}, \frac{11}{6},-\frac{1}{2} ; n\right) \\
& -\frac{2055}{68} \delta\left(-\frac{1}{3}, \frac{5}{6}, \frac{1}{6} ; n\right)+\frac{64}{17} \delta\left(0,-\frac{1}{6}, \frac{5}{6} ; n\right)+\frac{349}{136} \delta\left(-\frac{5}{6}, \frac{25}{12},-\frac{7}{12} ; n\right)
\end{aligned}
$$




$$
\begin{aligned}
& -\frac{694}{17} \delta\left(-\frac{1}{2}, \frac{13}{12}, \frac{1}{12} ; n\right)+\frac{547}{17} \delta\left(-\frac{1}{6}, \frac{1}{12}, \frac{3}{4} ; n\right)+\frac{331}{136} \delta\left(-\frac{1}{2}, \frac{19}{12},-\frac{5}{12} ; n\right) \\
& -\frac{301}{17} \delta\left(-\frac{1}{6}, \frac{7}{12}, \frac{1}{4} ; n\right)+\frac{1}{17} \delta\left(\frac{1}{6},-\frac{5}{12}, \frac{11}{12} ; n\right),
\end{aligned}
$$

$N([6,2,8] ; n)$

$$
\begin{aligned}
= & \frac{1}{272} \sigma_{7}(n)-\frac{1}{272} \sigma_{7}(n / 2)-\frac{32}{17} \sigma_{7}(n / 8)+\frac{8192}{17} \sigma_{7}(n / 16) \\
& +\frac{849}{272} \delta\left(-\frac{1}{3}, \frac{4}{3},-\frac{1}{3} ; n\right)-\frac{127}{17} \delta\left(0, \frac{1}{3}, \frac{1}{3} ; n\right)+\frac{23}{8} \delta\left(-\frac{2}{3}, \frac{11}{6},-\frac{1}{2} ; n\right) \\
& -\frac{1087}{34} \delta\left(-\frac{1}{3}, \frac{5}{6}, \frac{1}{6} ; n\right)+\frac{64}{17} \delta\left(0,-\frac{1}{6}, \frac{5}{6} ; n\right)+\frac{817}{272} \delta\left(-\frac{5}{6}, \frac{25}{12},-\frac{7}{12} ; n\right) \\
& -\frac{3133}{68} \delta\left(-\frac{1}{2}, \frac{13}{12}, \frac{1}{12} ; n\right)+\frac{547}{17} \delta\left(-\frac{1}{6}, \frac{1}{12}, \frac{3}{4} ; n\right)+\frac{815}{272} \delta\left(-\frac{1}{2}, \frac{19}{12},-\frac{5}{12} ; n\right) \\
& -\frac{1221}{68} \delta\left(-\frac{1}{6}, \frac{7}{12}, \frac{1}{4} ; n\right)+\frac{1}{17} \delta\left(\frac{1}{6},-\frac{5}{12}, \frac{11}{12} ; n\right),
\end{aligned}
$$

$$
\begin{aligned}
& N([7,2,7] ; n) \\
&=\frac{1}{136} \sigma_{7}(n)-\frac{1}{136} \sigma_{7}(n / 2)-\frac{32}{17} \sigma_{7}(n / 8)+\frac{8192}{17} \sigma_{7}(n / 16) \\
&+\frac{123}{34} \delta\left(-\frac{1}{3}, \frac{4}{3},-\frac{1}{3} ; n\right)-\frac{126}{17} \delta\left(0, \frac{1}{3}, \frac{1}{3} ; n\right)+\frac{27}{8} \delta\left(-\frac{2}{3}, \frac{11}{6},-\frac{1}{2} ; n\right) \\
&-\frac{552}{17} \delta\left(-\frac{1}{3}, \frac{5}{6}, \frac{1}{6} ; n\right)+\frac{64}{17} \delta\left(0,-\frac{1}{6}, \frac{5}{6} ; n\right)+\frac{919}{272} \delta\left(-\frac{5}{6}, \frac{25}{12},-\frac{7}{12} ; n\right) \\
&-\frac{3337}{68} \delta\left(-\frac{1}{2}, \frac{13}{12}, \frac{1}{12} ; n\right)+\frac{547}{17} \delta\left(-\frac{1}{6}, \frac{1}{12}, \frac{3}{4} ; n\right)+\frac{985}{272} \delta\left(-\frac{1}{2}, \frac{19}{12},-\frac{5}{12} ; n\right) \\
&-\frac{1221}{68} \delta\left(-\frac{1}{6}, \frac{7}{12}, \frac{1}{4} ; n\right)+\frac{1}{17} \delta\left(\frac{1}{6},-\frac{5}{12}, \frac{11}{12} ; n\right),
\end{aligned}
$$

$N([8,2,6] ; n)$

$$
\begin{aligned}
= & \frac{1}{68} \sigma_{7}(n)-\frac{1}{68} \sigma_{7}(n / 2)-\frac{32}{17} \sigma_{7}(n / 8)+\frac{8192}{17} \sigma_{7}(n / 16) \\
& +\frac{271}{68} \delta\left(-\frac{1}{3}, \frac{4}{3},-\frac{1}{3} ; n\right)-\frac{124}{17} \delta\left(0, \frac{1}{3}, \frac{1}{3} ; n\right)+4 \delta\left(-\frac{2}{3}, \frac{11}{6},-\frac{1}{2} ; n\right)
\end{aligned}
$$




$$
\begin{aligned}
& -\frac{552}{17} \delta\left(-\frac{1}{3}, \frac{5}{6}, \frac{1}{6} ; n\right)+\frac{64}{17} \delta\left(0,-\frac{1}{6}, \frac{5}{6} ; n\right)+\frac{1021}{272} \delta\left(-\frac{5}{6}, \frac{25}{12},-\frac{7}{12} ; n\right) \\
& -\frac{3405}{68} \delta\left(-\frac{1}{2}, \frac{13}{12}, \frac{1}{12} ; n\right)+\frac{547}{17} \delta\left(-\frac{1}{6}, \frac{1}{12}, \frac{3}{4} ; n\right)+\frac{1155}{272} \delta\left(-\frac{1}{2}, \frac{19}{12},-\frac{5}{12} ; n\right) \\
& -\frac{1221}{68} \delta\left(-\frac{1}{6}, \frac{7}{12}, \frac{1}{4} ; n\right)+\frac{1}{17} \delta\left(\frac{1}{6},-\frac{5}{12}, \frac{11}{12} ; n\right)
\end{aligned}
$$

$N([9,2,5] ; n)$

$$
\begin{aligned}
= & \frac{1}{34} \sigma_{7}(n)-\frac{1}{34} \sigma_{7}(n / 2)-\frac{32}{17} \sigma_{7}(n / 8)+\frac{8192}{17} \sigma_{7}(n / 16) \\
& +\frac{287}{68} \delta\left(-\frac{1}{3}, \frac{4}{3},-\frac{1}{3} ; n\right)-\frac{120}{17} \delta\left(0, \frac{1}{3}, \frac{1}{3} ; n\right)+\frac{19}{4} \delta\left(-\frac{2}{3}, \frac{11}{6},-\frac{1}{2} ; n\right) \\
& -\frac{552}{17} \delta\left(-\frac{1}{3}, \frac{5}{6}, \frac{1}{6} ; n\right)+\frac{64}{17} \delta\left(0,-\frac{1}{6}, \frac{5}{6} ; n\right)+\frac{1157}{272} \delta\left(-\frac{5}{6}, \frac{25}{12},-\frac{7}{12} ; n\right) \\
& -\frac{3405}{68} \delta\left(-\frac{1}{2}, \frac{13}{12}, \frac{1}{12} ; n\right)+\frac{547}{17} \delta\left(-\frac{1}{6}, \frac{1}{12}, \frac{3}{4} ; n\right)+\frac{1291}{272} \delta\left(-\frac{1}{2}, \frac{19}{12},-\frac{5}{12} ; n\right) \\
& -\frac{1221}{68} \delta\left(-\frac{1}{6}, \frac{7}{12}, \frac{1}{4} ; n\right)+\frac{1}{17} \delta\left(\frac{1}{6},-\frac{5}{12}, \frac{11}{12} ; n\right),
\end{aligned}
$$

$N([10,2,4] ; n)$

$$
\begin{aligned}
= & \frac{1}{17} \sigma_{7}(n)-\frac{1}{17} \sigma_{7}(n / 2)-\frac{32}{17} \sigma_{7}(n / 8)+\frac{8192}{17} \sigma_{7}(n / 16) \\
& +\frac{151}{34} \delta\left(-\frac{1}{3}, \frac{4}{3},-\frac{1}{3} ; n\right)-\frac{112}{17} \delta\left(0, \frac{1}{3}, \frac{1}{3} ; n\right)+\frac{11}{2} \delta\left(-\frac{2}{3}, \frac{11}{6},-\frac{1}{2} ; n\right) \\
& -\frac{552}{17} \delta\left(-\frac{1}{3}, \frac{5}{6}, \frac{1}{6} ; n\right)+\frac{64}{17} \delta\left(0,-\frac{1}{6}, \frac{5}{6} ; n\right)+\frac{1361}{272} \delta\left(-\frac{5}{6}, \frac{25}{12},-\frac{7}{12} ; n\right) \\
& -\frac{3405}{68} \delta\left(-\frac{1}{2}, \frac{13}{12}, \frac{1}{12} ; n\right)+\frac{547}{17} \delta\left(-\frac{1}{6}, \frac{1}{12}, \frac{3}{4} ; n\right)+\frac{1359}{272} \delta\left(-\frac{1}{2}, \frac{19}{12},-\frac{5}{12} ; n\right) \\
& -\frac{1221}{68} \delta\left(-\frac{1}{6}, \frac{7}{12}, \frac{1}{4} ; n\right)+\frac{1}{17} \delta\left(\frac{1}{6},-\frac{5}{12}, \frac{11}{12} ; n\right),
\end{aligned}
$$

$N([11,2,3] ; n)$

$$
=\frac{2}{17} \sigma_{7}(n)-\frac{2}{17} \sigma_{7}(n / 2)-\frac{32}{17} \sigma_{7}(n / 8)+\frac{8192}{17} \sigma_{7}(n / 16)
$$




$$
\begin{aligned}
& +\frac{83}{17} \delta\left(-\frac{1}{3}, \frac{4}{3},-\frac{1}{3} ; n\right)-\frac{96}{17} \delta\left(0, \frac{1}{3}, \frac{1}{3} ; n\right)+6 \delta\left(-\frac{2}{3}, \frac{11}{6},-\frac{1}{2} ; n\right) \\
& -\frac{552}{17} \delta\left(-\frac{1}{3}, \frac{5}{6}, \frac{1}{6} ; n\right)+\frac{64}{17} \delta\left(0,-\frac{1}{6}, \frac{5}{6} ; n\right)+\frac{1633}{272} \delta\left(-\frac{5}{6}, \frac{25}{12},-\frac{7}{12} ; n\right) \\
& -\frac{3405}{68} \delta\left(-\frac{1}{2}, \frac{13}{12}, \frac{1}{12} ; n\right)+\frac{547}{17} \delta\left(-\frac{1}{6}, \frac{1}{12}, \frac{3}{4} ; n\right)+\frac{1359}{272} \delta\left(-\frac{1}{2}, \frac{19}{12},-\frac{5}{12} ; n\right) \\
& -\frac{1221}{68} \delta\left(-\frac{1}{6}, \frac{7}{12}, \frac{1}{4} ; n\right)+\frac{1}{17} \delta\left(\frac{1}{6},-\frac{5}{12}, \frac{11}{12} ; n\right),
\end{aligned}
$$

$N([12,2,2] ; n)$

$$
\begin{aligned}
= & \frac{4}{17} \sigma_{7}(n)-\frac{4}{17} \sigma_{7}(n / 2)-\frac{32}{17} \sigma_{7}(n / 8)+\frac{8192}{17} \sigma_{7}(n / 16) \\
& +\frac{98}{17} \delta\left(-\frac{1}{3}, \frac{4}{3},-\frac{1}{3} ; n\right)-\frac{64}{17} \delta\left(0, \frac{1}{3}, \frac{1}{3} ; n\right)+6 \delta\left(-\frac{2}{3}, \frac{11}{6},-\frac{1}{2} ; n\right) \\
& -\frac{552}{17} \delta\left(-\frac{1}{3}, \frac{5}{6}, \frac{1}{6} ; n\right)+\frac{64}{17} \delta\left(0,-\frac{1}{6}, \frac{5}{6} ; n\right)+\frac{1905}{272} \delta\left(-\frac{5}{6}, \frac{25}{12},-\frac{7}{12} ; n\right) \\
& -\frac{3405}{68} \delta\left(-\frac{1}{2}, \frac{13}{12}, \frac{1}{12} ; n\right)+\frac{547}{17} \delta\left(-\frac{1}{6}, \frac{1}{12}, \frac{3}{4} ; n\right)+\frac{1359}{272} \delta\left(-\frac{1}{2}, \frac{19}{12},-\frac{5}{12} ; n\right) \\
& -\frac{1221}{68} \delta\left(-\frac{1}{6}, \frac{7}{12}, \frac{1}{4} ; n\right)+\frac{1}{17} \delta\left(\frac{1}{6},-\frac{5}{12}, \frac{11}{12} ; n\right),
\end{aligned}
$$

$N([13,2,1] ; n)$

$$
\begin{aligned}
= & \frac{8}{17} \sigma_{7}(n)-\frac{8}{17} \sigma_{7}(n / 2)-\frac{32}{17} \sigma_{7}(n / 8)+\frac{8192}{17} \sigma_{7}(n / 16) \\
& +\frac{128}{17} \delta\left(-\frac{1}{3}, \frac{4}{3},-\frac{1}{3} ; n\right)+6 \delta\left(-\frac{2}{3}, \frac{11}{6},-\frac{1}{2} ; n\right) \\
& -\frac{552}{17} \delta\left(-\frac{1}{3}, \frac{5}{6}, \frac{1}{6} ; n\right)+\frac{64}{17} \delta\left(0,-\frac{1}{6}, \frac{5}{6} ; n\right)+\frac{1905}{272} \delta\left(-\frac{5}{6}, \frac{25}{12},-\frac{7}{12} ; n\right) \\
& -\frac{3405}{68} \delta\left(-\frac{1}{2}, \frac{13}{12}, \frac{1}{12} ; n\right)+\frac{547}{17} \delta\left(-\frac{1}{6}, \frac{1}{12}, \frac{3}{4} ; n\right)+\frac{1359}{272} \delta\left(-\frac{1}{2}, \frac{19}{12},-\frac{5}{12} ; n\right) \\
& -\frac{1221}{68} \delta\left(-\frac{1}{6}, \frac{7}{12}, \frac{1}{4} ; n\right)+\frac{1}{17} \delta\left(\frac{1}{6},-\frac{5}{12}, \frac{11}{12} ; n\right),
\end{aligned}
$$

$N([1,4,11] ; n)$

$$
=\frac{1}{4352} \sigma_{7}(n)-\frac{1}{4352} \sigma_{7}(n / 2)-\frac{32}{17} \sigma_{7}(n / 8)+\frac{8192}{17} \sigma_{7}(n / 16)
$$




$$
\begin{aligned}
& +\frac{2175}{4352} \delta\left(-\frac{1}{3}, \frac{4}{3},-\frac{1}{3} ; n\right)-\frac{915}{544} \delta\left(0, \frac{1}{3}, \frac{1}{3} ; n\right)+\frac{1}{2} \delta\left(-\frac{2}{3}, \frac{11}{6},-\frac{1}{2} ; n\right) \\
& -\frac{863}{136} \delta\left(-\frac{1}{3}, \frac{5}{6}, \frac{1}{6} ; n\right)-\frac{59}{34} \delta\left(0,-\frac{1}{6}, \frac{5}{6} ; n\right)+\frac{137}{272} \delta\left(-\frac{5}{6}, \frac{25}{12},-\frac{7}{12} ; n\right) \\
& -\frac{1081}{136} \delta\left(-\frac{1}{2}, \frac{13}{12}, \frac{1}{12} ; n\right)+\frac{125}{34} \delta\left(-\frac{1}{6}, \frac{1}{12}, \frac{3}{4} ; n\right)+\frac{135}{272} \delta\left(-\frac{1}{2}, \frac{19}{12},-\frac{5}{12} ; n\right) \\
& -\frac{555}{136} \delta\left(-\frac{1}{6}, \frac{7}{12}, \frac{1}{4} ; n\right)-\frac{15}{34} \delta\left(\frac{1}{6},-\frac{5}{12}, \frac{11}{12} ; n\right),
\end{aligned}
$$

$N([2,4,10] ; n)$

$$
\begin{aligned}
= & \frac{1}{2176} \sigma_{7}(n)-\frac{1}{2176} \sigma_{7}(n / 2)-\frac{32}{17} \sigma_{7}(n / 8)+\frac{8192}{17} \sigma_{7}(n / 16) \\
& +\frac{2175}{2176} \delta\left(-\frac{1}{3}, \frac{4}{3},-\frac{1}{3} ; n\right)-\frac{1247}{272} \delta\left(0, \frac{1}{3}, \frac{1}{3} ; n\right)+\delta\left(-\frac{2}{3}, \frac{11}{6},-\frac{1}{2} ; n\right) \\
& -\frac{229}{17} \delta\left(-\frac{1}{3}, \frac{5}{6}, \frac{1}{6} ; n\right)+\frac{47}{17} \delta\left(0,-\frac{1}{6}, \frac{5}{6} ; n\right)+\frac{273}{272} \delta\left(-\frac{5}{6}, \frac{25}{12},-\frac{7}{12} ; n\right) \\
& -\frac{286}{17} \delta\left(-\frac{1}{2}, \frac{13}{12}, \frac{1}{12} ; n\right)+\frac{377}{17} \delta\left(-\frac{1}{6}, \frac{1}{12}, \frac{3}{4} ; n\right)+\frac{271}{272} \delta\left(-\frac{1}{2}, \frac{19}{12},-\frac{5}{12} ; n\right) \\
& -\frac{313}{34} \delta\left(-\frac{1}{6}, \frac{7}{12}, \frac{1}{4} ; n\right)+\frac{1}{17} \delta\left(\frac{1}{6},-\frac{5}{12}, \frac{11}{12} ; n\right),
\end{aligned}
$$

$$
\begin{aligned}
N & ([3,4,9] ; n) \\
= & \frac{1}{1088} \sigma_{7}(n)-\frac{1}{1088} \sigma_{7}(n / 2)-\frac{32}{17} \sigma_{7}(n / 8)+\frac{8192}{17} \sigma_{7}(n / 16) \\
& +\frac{1631}{1088} \delta\left(-\frac{1}{3}, \frac{4}{3},-\frac{1}{3} ; n\right)-\frac{869}{136} \delta\left(0, \frac{1}{3}, \frac{1}{3} ; n\right)+\frac{3}{2} \delta\left(-\frac{2}{3}, \frac{11}{6},-\frac{1}{2} ; n\right) \\
& -\frac{1341}{68} \delta\left(-\frac{1}{3}, \frac{5}{6}, \frac{1}{6} ; n\right)+\frac{64}{17} \delta\left(0,-\frac{1}{6}, \frac{5}{6} ; n\right)+\frac{409}{272} \delta\left(-\frac{5}{6}, \frac{25}{12},-\frac{7}{12} ; n\right) \\
& -\frac{422}{17} \delta\left(-\frac{1}{2}, \frac{13}{12}, \frac{1}{12} ; n\right)+\frac{513}{17} \delta\left(-\frac{1}{6}, \frac{1}{12}, \frac{3}{4} ; n\right)+\frac{407}{272} \delta\left(-\frac{1}{2}, \frac{19}{12},-\frac{5}{12} ; n\right) \\
& -\frac{449}{34} \delta\left(-\frac{1}{6}, \frac{7}{12}, \frac{1}{4} ; n\right)+\frac{1}{17} \delta\left(\frac{1}{6},-\frac{5}{12}, \frac{11}{12} ; n\right),
\end{aligned}
$$

$N([4,4,8] ; n)$ 


$$
\begin{aligned}
= & \frac{1}{544} \sigma_{7}(n)-\frac{1}{544} \sigma_{7}(n / 2)-\frac{32}{17} \sigma_{7}(n / 8)+\frac{8192}{17} \sigma_{7}(n / 16) \\
& +\frac{1087}{544} \delta\left(-\frac{1}{3}, \frac{4}{3},-\frac{1}{3} ; n\right)-\frac{29}{4} \delta\left(0, \frac{1}{3}, \frac{1}{3} ; n\right)+2 \delta\left(-\frac{2}{3}, \frac{11}{6},-\frac{1}{2} ; n\right) \\
& -\frac{849}{34} \delta\left(-\frac{1}{3}, \frac{5}{6}, \frac{1}{6} ; n\right)+\frac{64}{17} \delta\left(0,-\frac{1}{6}, \frac{5}{6} ; n\right)+\frac{545}{272} \delta\left(-\frac{5}{6}, \frac{25}{12},-\frac{7}{12} ; n\right) \\
& -\frac{2181}{68} \delta\left(-\frac{1}{2}, \frac{13}{12}, \frac{1}{12} ; n\right)+\frac{547}{17} \delta\left(-\frac{1}{6}, \frac{1}{12}, \frac{3}{4} ; n\right)+\frac{543}{272} \delta\left(-\frac{1}{2}, \frac{19}{12},-\frac{5}{12} ; n\right) \\
& -\frac{1085}{68} \delta\left(-\frac{1}{6}, \frac{7}{12}, \frac{1}{4} ; n\right)+\frac{1}{17} \delta\left(\frac{1}{6},-\frac{5}{12}, \frac{11}{12} ; n\right),
\end{aligned}
$$

$$
\begin{aligned}
& N([5,4,7] ; n) \\
&=\frac{1}{272} \sigma_{7}(n)-\sigma_{7}(n / 2)-\frac{1}{272} \sigma_{7}(n / 8)+\frac{8192}{17} \sigma_{7}(n / 16) \\
&+\frac{679}{272} \delta\left(-\frac{1}{3}, \frac{4}{3},-\frac{1}{3} ; n\right)-\frac{127}{17} \delta\left(0, \frac{1}{3}, \frac{1}{3} ; n\right)+\frac{5}{2} \delta\left(-\frac{2}{3}, \frac{11}{6},-\frac{1}{2} ; n\right) \\
&-\frac{985}{34} \delta\left(-\frac{1}{3}, \frac{5}{6}, \frac{1}{6} ; n\right)+\frac{64}{17} \delta\left(0,-\frac{1}{6}, \frac{5}{6} ; n\right)+\frac{681}{272} \delta\left(-\frac{5}{6}, \frac{25}{12},-\frac{7}{12} ; n\right) \\
&-\frac{2633}{68} \delta\left(-\frac{1}{2}, \frac{13}{12}, \frac{1}{12} ; n\right)+\frac{547}{17} \delta\left(-\frac{1}{6}, \frac{1}{12}, \frac{3}{4} ; n\right)+\frac{679}{272} \delta\left(-\frac{1}{2}, \frac{19}{12},-\frac{5}{12} ; n\right) \\
&-\frac{1187}{68} \delta\left(-\frac{1}{6}, \frac{7}{12}, \frac{1}{4} ; n\right)+\frac{1}{17} \delta\left(\frac{1}{6},-\frac{5}{12}, \frac{11}{12} ; n\right),
\end{aligned}
$$

$N([6,4,6] ; n)$

$$
\begin{aligned}
= & \frac{1}{136} \sigma_{7}(n)-\frac{1}{136} \sigma_{7}(n / 2)-\frac{32}{17} \sigma_{7}(n / 8)+\frac{8192}{17} \sigma_{7}(n / 16) \\
& +\frac{407}{136} \delta\left(-\frac{1}{3}, \frac{4}{3},-\frac{1}{3} ; n\right)-\frac{126}{17} \delta\left(0, \frac{1}{3}, \frac{1}{3} ; n\right)+3 \delta\left(-\frac{2}{3}, \frac{11}{6},-\frac{1}{2} ; n\right) \\
& -\frac{535}{17} \delta\left(-\frac{1}{3}, \frac{5}{6}, \frac{1}{6} ; n\right)+\frac{64}{17} \delta\left(0,-\frac{1}{6}, \frac{5}{6} ; n\right)+\frac{817}{272} \delta\left(-\frac{5}{6}, \frac{25}{12},-\frac{7}{12} ; n\right) \\
& -\frac{2997}{68} \delta\left(-\frac{1}{2}, \frac{13}{12}, \frac{1}{12} ; n\right)+\frac{547}{17} \delta\left(-\frac{1}{6}, \frac{1}{12}, \frac{3}{4} ; n\right)+\frac{815}{272} \delta\left(-\frac{1}{2}, \frac{19}{12},-\frac{5}{12} ; n\right) \\
& -\frac{1221}{68} \delta\left(-\frac{1}{6}, \frac{7}{12}, \frac{1}{4} ; n\right)+\frac{1}{17} \delta\left(\frac{1}{6},-\frac{5}{12}, \frac{11}{12} ; n\right),
\end{aligned}
$$




$$
\begin{aligned}
& N([7,4,5] ; n) \\
&=\frac{1}{68} \sigma_{7}(n)-\frac{1}{68} \sigma_{7}(n / 2)-\frac{32}{17} \sigma_{7}(n / 8)+\frac{8192}{17} \sigma_{7}(n / 16) \\
&+\frac{237}{68} \delta\left(-\frac{1}{3}, \frac{4}{3},-\frac{1}{3} ; n\right)-\frac{124}{17} \delta\left(0, \frac{1}{3}, \frac{1}{3} ; n\right)+\frac{7}{2} \delta\left(-\frac{2}{3}, \frac{11}{6},-\frac{1}{2} ; n\right) \\
&-\frac{552}{17} \delta\left(-\frac{1}{3}, \frac{5}{6}, \frac{1}{6} ; n\right)+\frac{64}{17} \delta\left(0,-\frac{1}{6}, \frac{5}{6} ; n\right)+\frac{953}{272} \delta\left(-\frac{5}{6}, \frac{25}{12},-\frac{7}{12} ; n\right) \\
&-\frac{3269}{68} \delta\left(-\frac{1}{2}, \frac{13}{12}, \frac{1}{12} ; n\right)+\frac{547}{17} \delta\left(-\frac{1}{6}, \frac{1}{12}, \frac{3}{4} ; n\right)+\frac{951}{272} \delta\left(-\frac{1}{2}, \frac{19}{12},-\frac{5}{12} ; n\right) \\
&-\frac{1221}{68} \delta\left(-\frac{1}{6}, \frac{7}{12}, \frac{1}{4} ; n\right)+\frac{1}{17} \delta\left(\frac{1}{6},-\frac{5}{12}, \frac{11}{12} ; n\right),
\end{aligned}
$$

$$
\begin{aligned}
N & ([8,4,4] ; n) \\
= & \frac{1}{34} \sigma_{7}(n)-\frac{1}{34} \sigma_{7}(n / 2)-\frac{32}{17} \sigma_{7}(n / 8)+\frac{8192}{17} \sigma_{7}(n / 16) \\
& +\frac{135}{34} \delta\left(-\frac{1}{3}, \frac{4}{3},-\frac{1}{3} ; n\right)-\frac{120}{17} \delta\left(0, \frac{1}{3}, \frac{1}{3} ; n\right)+4 \delta\left(-\frac{2}{3}, \frac{11}{6},-\frac{1}{2} ; n\right) \\
& -\frac{552}{17} \delta\left(-\frac{1}{3}, \frac{5}{6}, \frac{1}{6} ; n\right)+\frac{64}{17} \delta\left(0,-\frac{1}{6}, \frac{5}{6} ; n\right)+\frac{1089}{272} \delta\left(-\frac{5}{6}, \frac{25}{12},-\frac{7}{12} ; n\right) \\
& -\frac{3405}{68} \delta\left(-\frac{1}{2}, \frac{13}{12}, \frac{1}{12} ; n\right)+\frac{547}{17} \delta\left(-\frac{1}{6}, \frac{1}{12}, \frac{3}{4} ; n\right)+\frac{951}{272} \delta\left(-\frac{1}{2}, \frac{19}{12},-\frac{5}{12} ; n\right) \\
& -\frac{1221}{68} \delta\left(-\frac{1}{6}, \frac{7}{12}, \frac{1}{4} ; n\right)+\frac{1}{17} \delta\left(\frac{1}{6},-\frac{5}{12}, \frac{11}{12} ; n\right),
\end{aligned}
$$

$$
\begin{aligned}
N & ([9,4,3] ; n) \\
= & \frac{1}{17} \sigma_{7}(n)-\frac{1}{17} \sigma_{7}(n / 2)-\frac{32}{17} \sigma_{7}(n / 8)+\frac{8192}{17} \sigma_{7}(n / 16) \\
& +\frac{151}{34} \delta\left(-\frac{1}{3}, \frac{4}{3},-\frac{1}{3} ; n\right)-\frac{112}{17} \delta\left(0, \frac{1}{3}, \frac{1}{3} ; n\right)+\frac{9}{2} \delta\left(-\frac{2}{3}, \frac{11}{6},-\frac{1}{2} ; n\right) \\
& -\frac{552}{17} \delta\left(-\frac{1}{3}, \frac{5}{6}, \frac{1}{6} ; n\right)+\frac{64}{17} \delta\left(0,-\frac{1}{6}, \frac{5}{6} ; n\right)+\frac{1089}{272} \delta\left(-\frac{5}{6}, \frac{25}{12},-\frac{7}{12} ; n\right) \\
& -\frac{3405}{68} \delta\left(-\frac{1}{2}, \frac{13}{12}, \frac{1}{12} ; n\right)+\frac{547}{17} \delta\left(-\frac{1}{6}, \frac{1}{12}, \frac{3}{4} ; n\right)+\frac{1223}{272} \delta\left(-\frac{1}{2}, \frac{19}{12},-\frac{5}{12} ; n\right) \\
& -\frac{1221}{68} \delta\left(-\frac{1}{6}, \frac{7}{12}, \frac{1}{4} ; n\right)+\frac{1}{17} \delta\left(\frac{1}{6},-\frac{5}{12}, \frac{11}{12} ; n\right),
\end{aligned}
$$




$$
\begin{aligned}
N( & {[10,4,2] ; n) } \\
= & \frac{2}{17} \sigma_{7}(n)-\frac{2}{17} \sigma_{7}(n / 2)-\frac{32}{17} \sigma_{7}(n / 8)+\frac{8192}{17} \sigma_{7}(n / 16) \\
& +\frac{83}{17} \delta\left(-\frac{1}{3}, \frac{4}{3},-\frac{1}{3} ; n\right)-\frac{96}{17} \delta\left(0, \frac{1}{3}, \frac{1}{3} ; n\right)+5 \delta\left(-\frac{2}{3}, \frac{11}{6},-\frac{1}{2} ; n\right) \\
& -\frac{552}{17} \delta\left(-\frac{1}{3}, \frac{5}{6}, \frac{1}{6} ; n\right)+\frac{64}{17} \delta\left(0,-\frac{1}{6}, \frac{5}{6} ; n\right)+\frac{1361}{272} \delta\left(-\frac{5}{6}, \frac{25}{12},-\frac{7}{12} ; n\right) \\
& -\frac{3405}{68} \delta\left(-\frac{1}{2}, \frac{13}{12}, \frac{1}{12} ; n\right)+\frac{547}{17} \delta\left(-\frac{1}{6}, \frac{1}{12}, \frac{3}{4} ; n\right)+\frac{1359}{272} \delta\left(-\frac{1}{2}, \frac{19}{12},-\frac{5}{12} ; n\right) \\
& -\frac{1221}{68} \delta\left(-\frac{1}{6}, \frac{7}{12}, \frac{1}{4} ; n\right)+\frac{1}{17} \delta\left(\frac{1}{6},-\frac{5}{12}, \frac{11}{12} ; n\right),
\end{aligned}
$$

$$
\begin{aligned}
& N([11,4,1] ; n) \\
&=\frac{4}{17} \sigma_{7}(n)-\frac{4}{17} \sigma_{7}(n / 2)-\frac{32}{17} \sigma_{7}(n / 8)+\frac{8192}{17} \sigma_{7}(n / 16) \\
&+\frac{98}{17} \delta\left(-\frac{1}{3}, \frac{4}{3},-\frac{1}{3} ; n\right)-\frac{64}{17} \delta\left(0, \frac{1}{3}, \frac{1}{3} ; n\right)+6 \delta\left(-\frac{2}{3}, \frac{11}{6},-\frac{1}{2} ; n\right) \\
&-\frac{552}{17} \delta\left(-\frac{1}{3}, \frac{5}{6}, \frac{1}{6} ; n\right)+\frac{64}{17} \delta\left(0,-\frac{1}{6}, \frac{5}{6} ; n\right)+\frac{1361}{272} \delta\left(-\frac{5}{6}, \frac{25}{12},-\frac{7}{12} ; n\right) \\
&-\frac{3405}{68} \delta\left(-\frac{1}{2}, \frac{13}{12}, \frac{1}{12} ; n\right)+\frac{547}{17} \delta\left(-\frac{1}{6}, \frac{1}{12}, \frac{3}{4} ; n\right)+\frac{1359}{272} \delta\left(-\frac{1}{2}, \frac{19}{12},-\frac{5}{12} ; n\right) \\
&-\frac{1221}{68} \delta\left(-\frac{1}{6}, \frac{7}{12}, \frac{1}{4} ; n\right)+\frac{1}{17} \delta\left(\frac{1}{6},-\frac{5}{12}, \frac{11}{12} ; n\right),
\end{aligned}
$$

$$
\begin{aligned}
& N([1,6,9] ; n) \\
&= \frac{1}{2176} \sigma_{7}(n)-\frac{1}{2176} \sigma_{7}(n / 2)-\frac{32}{17} \sigma_{7}(n / 8)+\frac{8192}{17} \sigma_{7}(n / 16) \\
&+\frac{1087}{2176} \delta\left(-\frac{1}{3}, \frac{4}{3},-\frac{1}{3} ; n\right)-\frac{159}{272} \delta\left(0, \frac{1}{3}, \frac{1}{3} ; n\right)+\frac{1}{2} \delta\left(-\frac{2}{3}, \frac{11}{6},-\frac{1}{2} ; n\right) \\
&-\frac{93}{17} \delta\left(-\frac{1}{3}, \frac{5}{6}, \frac{1}{6} ; n\right)-\frac{89}{17} \delta\left(0,-\frac{1}{6}, \frac{5}{6} ; n\right)+\frac{137}{272} \delta\left(-\frac{5}{6}, \frac{25}{12},-\frac{7}{12} ; n\right) \\
&-\frac{481}{68} \delta\left(-\frac{1}{2}, \frac{13}{12}, \frac{1}{12} ; n\right)-\frac{116}{17} \delta\left(-\frac{1}{6}, \frac{1}{12}, \frac{3}{4} ; n\right)+\frac{135}{272} \delta\left(-\frac{1}{2}, \frac{19}{12},-\frac{5}{12} ; n\right)
\end{aligned}
$$




$$
-\frac{201}{68} \delta\left(-\frac{1}{6}, \frac{7}{12}, \frac{1}{4} ; n\right)-\frac{16}{17} \delta\left(\frac{1}{6},-\frac{5}{12}, \frac{11}{12} ; n\right),
$$

$$
\begin{aligned}
N & ([2,6,8] ; n) \\
= & \frac{1}{1088} \sigma_{7}(n)-\frac{1}{1088} \sigma_{7}(n / 2)-\frac{32}{17} \sigma_{7}(n / 8)+\frac{8192}{17} \sigma_{7}(n / 16) \\
& +\frac{1087}{1088} \delta\left(-\frac{1}{3}, \frac{4}{3},-\frac{1}{3} ; n\right)-\frac{495}{136} \delta\left(0, \frac{1}{3}, \frac{1}{3} ; n\right)+\delta\left(-\frac{2}{3}, \frac{11}{6},-\frac{1}{2} ; n\right) \\
& -\frac{212}{17} \delta\left(-\frac{1}{3}, \frac{5}{6}, \frac{1}{6} ; n\right)+\frac{30}{17} \delta\left(0,-\frac{1}{6}, \frac{5}{6} ; n\right)+\frac{273}{272} \delta\left(-\frac{5}{6}, \frac{25}{12},-\frac{7}{12} ; n\right) \\
& -\frac{1093}{68} \delta\left(-\frac{1}{2}, \frac{13}{12}, \frac{1}{12} ; n\right)+\frac{275}{17} \delta\left(-\frac{1}{6}, \frac{1}{12}, \frac{3}{4} ; n\right)+\frac{271}{272} \delta\left(-\frac{1}{2}, \frac{19}{12},-\frac{5}{12} ; n\right) \\
& -\frac{541}{68} \delta\left(-\frac{1}{6}, \frac{7}{12}, \frac{1}{4} ; n\right)+\frac{1}{17} \delta\left(\frac{1}{6},-\frac{5}{12}, \frac{11}{12} ; n\right),
\end{aligned}
$$

$N([3,6,7] ; n)$

$$
\begin{aligned}
= & \frac{1}{544} \sigma_{7}(n)-\frac{1}{544} \sigma_{7}(n / 2)-\frac{32}{17} \sigma_{7}(n / 8)+\frac{8192}{17} \sigma_{7}(n / 16) \\
& +\frac{815}{544} \delta\left(-\frac{1}{3}, \frac{4}{3},-\frac{1}{3} ; n\right)-\frac{23}{4} \delta\left(0, \frac{1}{3}, \frac{1}{3} ; n\right)+\frac{3}{2} \delta\left(-\frac{2}{3}, \frac{11}{6},-\frac{1}{2} ; n\right) \\
& -\frac{314}{17} \delta\left(-\frac{1}{3}, \frac{5}{6}, \frac{1}{6} ; n\right)+\frac{64}{17} \delta\left(0,-\frac{1}{6}, \frac{5}{6} ; n\right)+\frac{409}{272} \delta\left(-\frac{5}{6}, \frac{25}{12},-\frac{7}{12} ; n\right) \\
& -\frac{1637}{68} \delta\left(-\frac{1}{2}, \frac{13}{12}, \frac{1}{12} ; n\right)+\frac{479}{17} \delta\left(-\frac{1}{6}, \frac{1}{12}, \frac{3}{4} ; n\right)+\frac{407}{272} \delta\left(-\frac{1}{2}, \frac{19}{12},-\frac{5}{12} ; n\right) \\
& -\frac{813}{68} \delta\left(-\frac{1}{6}, \frac{7}{12}, \frac{1}{4} ; n\right)+\frac{1}{17} \delta\left(\frac{1}{6},-\frac{5}{12}, \frac{11}{12} ; n\right),
\end{aligned}
$$

$N([4,6,6] ; n)$

$$
\begin{aligned}
= & \frac{1}{272} \sigma_{7}(n)-\frac{1}{272} \sigma_{7}(n / 2)-\frac{32}{17} \sigma_{7}(n / 8)+\frac{8192}{17} \sigma_{7}(n / 16) \\
& +\frac{543}{272} \delta\left(-\frac{1}{3}, \frac{4}{3},-\frac{1}{3} ; n\right)-\frac{237}{34} \delta\left(0, \frac{1}{3}, \frac{1}{3} ; n\right)+2 \delta\left(-\frac{2}{3}, \frac{11}{6},-\frac{1}{2} ; n\right) \\
& -\frac{399}{17} \delta\left(-\frac{1}{3}, \frac{5}{6}, \frac{1}{6} ; n\right)+\frac{64}{17} \delta\left(0,-\frac{1}{6}, \frac{5}{6} ; n\right)+\frac{545}{272} \delta\left(-\frac{5}{6}, \frac{25}{12},-\frac{7}{12} ; n\right)
\end{aligned}
$$




$$
\begin{aligned}
& -\frac{2113}{68} \delta\left(-\frac{1}{2}, \frac{13}{12}, \frac{1}{12} ; n\right)+\frac{547}{17} \delta\left(-\frac{1}{6}, \frac{1}{12}, \frac{3}{4} ; n\right)+\frac{543}{272} \delta\left(-\frac{1}{2}, \frac{19}{12},-\frac{5}{12} ; n\right) \\
& -\frac{1017}{68} \delta\left(-\frac{1}{6}, \frac{7}{12}, \frac{1}{4} ; n\right)+\frac{1}{17} \delta\left(\frac{1}{6},-\frac{5}{12}, \frac{11}{12} ; n\right)
\end{aligned}
$$

$N([5,6,5] ; n)$

$$
\begin{aligned}
= & \frac{1}{136} \sigma_{7}(n)-\frac{1}{136} \sigma_{7}(n / 2)-\frac{32}{17} \sigma_{7}(n / 8)+\frac{8192}{17} \sigma_{7}(n / 16) \\
& +\frac{339}{136} \delta\left(-\frac{1}{3}, \frac{4}{3},-\frac{1}{3} ; n\right)-\frac{126}{17} \delta\left(0, \frac{1}{3}, \frac{1}{3} ; n\right)+\frac{5}{2} \delta\left(-\frac{2}{3}, \frac{11}{6},-\frac{1}{2} ; n\right) \\
& -\frac{467}{17} \delta\left(-\frac{1}{3}, \frac{5}{6}, \frac{1}{6} ; n\right)+\frac{64}{17} \delta\left(0,-\frac{1}{6}, \frac{5}{6} ; n\right)+\frac{681}{272} \delta\left(-\frac{5}{6}, \frac{25}{12},-\frac{7}{12} ; n\right) \\
& -\frac{2521}{68} \delta\left(-\frac{1}{2}, \frac{13}{12}, \frac{1}{12} ; n\right)+\frac{547}{17} \delta\left(-\frac{1}{6}, \frac{1}{12}, \frac{3}{4} ; n\right)+\frac{679}{272} \delta\left(-\frac{1}{2}, \frac{19}{12},-\frac{5}{12} ; n\right) \\
& -\frac{1153}{68} \delta\left(-\frac{1}{6}, \frac{7}{12}, \frac{1}{4} ; n\right)+\frac{1}{17} \delta\left(\frac{1}{6},-\frac{5}{12}, \frac{11}{12} ; n\right),
\end{aligned}
$$

$N([6,6,4] ; n)$

$$
\begin{aligned}
= & \frac{1}{68} \sigma_{7}(n)-\frac{1}{68} \sigma_{7}(n / 2)-\frac{32}{17} \sigma_{7}(n / 8)+\frac{8192}{17} \sigma_{7}(n / 16) \\
& +\frac{203}{68} \delta\left(-\frac{1}{3}, \frac{4}{3},-\frac{1}{3} ; n\right)-\frac{124}{17} \delta\left(0, \frac{1}{3}, \frac{1}{3} ; n\right)+3 \delta\left(-\frac{2}{3}, \frac{11}{6},-\frac{1}{2} ; n\right) \\
& -\frac{518}{17} \delta\left(-\frac{1}{3}, \frac{5}{6}, \frac{1}{6} ; n\right)+\frac{64}{17} \delta\left(0,-\frac{1}{6}, \frac{5}{6} ; n\right)+\frac{817}{272} \delta\left(-\frac{5}{6}, \frac{25}{12},-\frac{7}{12} ; n\right) \\
& -\frac{2861}{68} \delta\left(-\frac{1}{2}, \frac{13}{12}, \frac{1}{12} ; n\right)+\frac{547}{17} \delta\left(-\frac{1}{6}, \frac{1}{12}, \frac{3}{4} ; n\right)+\frac{815}{272} \delta\left(-\frac{1}{2}, \frac{19}{12},-\frac{5}{12} ; n\right) \\
& -\frac{1221}{68} \delta\left(-\frac{1}{6}, \frac{7}{12}, \frac{1}{4} ; n\right)+\frac{1}{17} \delta\left(\frac{1}{6},-\frac{5}{12}, \frac{11}{12} ; n\right),
\end{aligned}
$$

$$
\begin{aligned}
& N([7,6,3] ; n) \\
& =\frac{1}{34} \sigma_{7}(n)-\frac{1}{34} \sigma_{7}(n / 2)-\frac{32}{17} \sigma_{7}(n / 8)+\frac{8192}{17} \sigma_{7}(n / 16) \\
& \quad+\frac{59}{17} \delta\left(-\frac{1}{3}, \frac{4}{3},-\frac{1}{3} ; n\right)-\frac{120}{17} \delta\left(0, \frac{1}{3}, \frac{1}{3} ; n\right)+\frac{7}{2} \delta\left(-\frac{2}{3}, \frac{11}{6},-\frac{1}{2} ; n\right)
\end{aligned}
$$




$$
\begin{aligned}
& -\frac{552}{17} \delta\left(-\frac{1}{3}, \frac{5}{6}, \frac{1}{6} ; n\right)+\frac{64}{17} \delta\left(0,-\frac{1}{6}, \frac{5}{6} ; n\right)+\frac{953}{272} \delta\left(-\frac{5}{6}, \frac{25}{12},-\frac{7}{12} ; n\right) \\
& -\frac{3133}{68} \delta\left(-\frac{1}{2}, \frac{13}{12}, \frac{1}{12} ; n\right)+\frac{547}{17} \delta\left(-\frac{1}{6}, \frac{1}{12}, \frac{3}{4} ; n\right)+\frac{951}{272} \delta\left(-\frac{1}{2}, \frac{19}{12},-\frac{5}{12} ; n\right) \\
& -\frac{1221}{68} \delta\left(-\frac{1}{6}, \frac{7}{12}, \frac{1}{4} ; n\right)+\frac{1}{17} \delta\left(\frac{1}{6},-\frac{5}{12}, \frac{11}{12} ; n\right)
\end{aligned}
$$

$N([8,6,2] ; n)$

$$
\begin{aligned}
= & \frac{1}{17} \sigma_{7}(n)-\frac{1}{17} \sigma_{7}(n / 2)-\frac{32}{17} \sigma_{7}(n / 8)+\frac{8192}{17} \sigma_{7}(n / 16) \\
& +\frac{67}{17} \delta\left(-\frac{1}{3}, \frac{4}{3},-\frac{1}{3} ; n\right)-\frac{112}{17} \delta\left(0, \frac{1}{3}, \frac{1}{3} ; n\right)+4 \delta\left(-\frac{2}{3}, \frac{11}{6},-\frac{1}{2} ; n\right) \\
& -\frac{552}{17} \delta\left(-\frac{1}{3}, \frac{5}{6}, \frac{1}{6} ; n\right)+\frac{64}{17} \delta\left(0,-\frac{1}{6}, \frac{5}{6} ; n\right)+\frac{1089}{272} \delta\left(-\frac{5}{6}, \frac{25}{12},-\frac{7}{12} ; n\right) \\
& -\frac{3405}{68} \delta\left(-\frac{1}{2}, \frac{13}{12}, \frac{1}{12} ; n\right)+\frac{547}{17} \delta\left(-\frac{1}{6}, \frac{1}{12}, \frac{3}{4} ; n\right)+\frac{1087}{272} \delta\left(-\frac{1}{2}, \frac{19}{12},-\frac{5}{12} ; n\right) \\
& -\frac{1221}{68} \delta\left(-\frac{1}{6}, \frac{7}{12}, \frac{1}{4} ; n\right)+\frac{1}{17} \delta\left(\frac{1}{6},-\frac{5}{12}, \frac{11}{12} ; n\right),
\end{aligned}
$$

$N([9,6,1] ; n)$

$$
\begin{aligned}
= & \frac{2}{17} \sigma_{7}(n)-\frac{2}{17} \sigma_{7}(n / 2)-\frac{32}{17} \sigma_{7}(n / 8)+\frac{8192}{17} \sigma_{7}(n / 16) \\
& +\frac{83}{17} \delta\left(-\frac{1}{3}, \frac{4}{3},-\frac{1}{3} ; n\right)-\frac{96}{17} \delta\left(0, \frac{1}{3}, \frac{1}{3} ; n\right)+5 \delta\left(-\frac{2}{3}, \frac{11}{6},-\frac{1}{2} ; n\right) \\
& -\frac{552}{17} \delta\left(-\frac{1}{3}, \frac{5}{6}, \frac{1}{6} ; n\right)+\frac{64}{17} \delta\left(0,-\frac{1}{6}, \frac{5}{6} ; n\right)+\frac{1089}{272} \delta\left(-\frac{5}{6}, \frac{25}{12},-\frac{7}{12} ; n\right) \\
& -\frac{3405}{68} \delta\left(-\frac{1}{2}, \frac{13}{12}, \frac{1}{12} ; n\right)+\frac{547}{17} \delta\left(-\frac{1}{6}, \frac{1}{12}, \frac{3}{4} ; n\right)+\frac{1087}{272} \delta\left(-\frac{1}{2}, \frac{19}{12},-\frac{5}{12} ; n\right) \\
& -\frac{1221}{68} \delta\left(-\frac{1}{6}, \frac{7}{12}, \frac{1}{4} ; n\right)+\frac{1}{17} \delta\left(\frac{1}{6},-\frac{5}{12}, \frac{11}{12} ; n\right),
\end{aligned}
$$

$N([1,8,7] ; n)$

$$
=\frac{1}{1088} \sigma_{7}(n)-\frac{1}{1088} \sigma_{7}(n / 2)-\frac{32}{17} \sigma_{7}(n / 8)+\frac{8192}{17} \sigma_{7}(n / 16)
$$




$$
\begin{aligned}
& +\frac{543}{1088} \delta\left(-\frac{1}{3}, \frac{4}{3},-\frac{1}{3} ; n\right)-\frac{49}{136} \delta\left(0, \frac{1}{3}, \frac{1}{3} ; n\right)+\frac{1}{2} \delta\left(-\frac{2}{3}, \frac{11}{6},-\frac{1}{2} ; n\right) \\
& -\frac{76}{17} \delta\left(-\frac{1}{3}, \frac{5}{6}, \frac{1}{6} ; n\right)-\frac{174}{17} \delta\left(0,-\frac{1}{6}, \frac{5}{6} ; n\right)+\frac{137}{272} \delta\left(-\frac{5}{6}, \frac{25}{12},-\frac{7}{12} ; n\right) \\
& -\frac{413}{68} \delta\left(-\frac{1}{2}, \frac{13}{12}, \frac{1}{12} ; n\right)-\frac{303}{17} \delta\left(-\frac{1}{6}, \frac{1}{12}, \frac{3}{4} ; n\right)+\frac{135}{272} \delta\left(-\frac{1}{2}, \frac{19}{12},-\frac{5}{12} ; n\right) \\
& -\frac{133}{68} \delta\left(-\frac{1}{6}, \frac{7}{12}, \frac{1}{4} ; n\right)-\frac{33}{17} \delta\left(\frac{1}{6},-\frac{5}{12}, \frac{11}{12} ; n\right),
\end{aligned}
$$

$N([2,8,6] ; n)$

$$
\begin{aligned}
= & \frac{1}{544} \sigma_{7}(n)-\frac{1}{544} \sigma_{7}(n / 2)-\frac{32}{17} \sigma_{7}(n / 8)+\frac{8192}{17} \sigma_{7}(n / 16) \\
& +\frac{543}{544} \delta\left(-\frac{1}{3}, \frac{4}{3},-\frac{1}{3} ; n\right)-\frac{11}{4} \delta\left(0, \frac{1}{3}, \frac{1}{3} ; n\right)+\delta\left(-\frac{2}{3}, \frac{11}{6},-\frac{1}{2} ; n\right) \\
& -\frac{195}{17} \delta\left(-\frac{1}{3}, \frac{5}{6}, \frac{1}{6} ; n\right)-\frac{4}{17} \delta\left(0,-\frac{1}{6}, \frac{5}{6} ; n\right)+\frac{273}{272} \delta\left(-\frac{5}{6}, \frac{25}{12},-\frac{7}{12} ; n\right) \\
& -\frac{1025}{68} \delta\left(-\frac{1}{2}, \frac{13}{12}, \frac{1}{12} ; n\right)+\frac{139}{17} \delta\left(-\frac{1}{6}, \frac{1}{12}, \frac{3}{4} ; n\right)+\frac{271}{272} \delta\left(-\frac{1}{2}, \frac{19}{12},-\frac{5}{12} ; n\right) \\
& -\frac{473}{68} \delta\left(-\frac{1}{6}, \frac{7}{12}, \frac{1}{4} ; n\right)+\frac{1}{17} \delta\left(\frac{1}{6},-\frac{5}{12}, \frac{11}{12} ; n\right),
\end{aligned}
$$

$N([3,8,5] ; n)$

$$
\begin{aligned}
= & \frac{1}{272} \sigma_{7}(n)-\frac{1}{272} \sigma_{7}(n / 2)-\frac{32}{17} \sigma_{7}(n / 8)+\frac{8192}{17} \sigma_{7}(n / 16) \\
& +\frac{407}{272} \delta\left(-\frac{1}{3}, \frac{4}{3},-\frac{1}{3} ; n\right)-\frac{169}{34} \delta\left(0, \frac{1}{3}, \frac{1}{3} ; n\right)+\frac{3}{2} \delta\left(-\frac{2}{3}, \frac{11}{6},-\frac{1}{2} ; n\right) \\
& -\frac{297}{17} \delta\left(-\frac{1}{3}, \frac{5}{6}, \frac{1}{6} ; n\right)+\frac{64}{17} \delta\left(0,-\frac{1}{6}, \frac{5}{6} ; n\right)+\frac{409}{272} \delta\left(-\frac{5}{6}, \frac{25}{12},-\frac{7}{12} ; n\right) \\
& -\frac{1569}{68} \delta\left(-\frac{1}{2}, \frac{13}{12}, \frac{1}{12} ; n\right)+\frac{411}{17} \delta\left(-\frac{1}{6}, \frac{1}{12}, \frac{3}{4} ; n\right)+\frac{407}{272} \delta\left(-\frac{1}{2}, \frac{19}{12},-\frac{5}{12} ; n\right) \\
& -\frac{745}{68} \delta\left(-\frac{1}{6}, \frac{7}{12}, \frac{1}{4} ; n\right)+\frac{1}{17} \delta\left(\frac{1}{6},-\frac{5}{12}, \frac{11}{12} ; n\right),
\end{aligned}
$$

$N([4,8,4] ; n)$ 


$$
\begin{aligned}
= & \frac{1}{136} \sigma_{7}(n)-\frac{1}{136} \sigma_{7}(n / 2)-\frac{32}{17} \sigma_{7}(n / 8)+\frac{8192}{17} \sigma_{7}(n / 16) \\
& +\frac{271}{136} \delta\left(-\frac{1}{3}, \frac{4}{3},-\frac{1}{3} ; n\right)-\frac{109}{17} \delta\left(0, \frac{1}{3}, \frac{1}{3} ; n\right)+2 \delta\left(-\frac{2}{3}, \frac{11}{6},-\frac{1}{2} ; n\right) \\
& -\frac{382}{17} \delta\left(-\frac{1}{3}, \frac{5}{6}, \frac{1}{6} ; n\right)+\frac{64}{17} \delta\left(0,-\frac{1}{6}, \frac{5}{6} ; n\right)+\frac{545}{272} \delta\left(-\frac{5}{6}, \frac{25}{12},-\frac{7}{12} ; n\right) \\
& -\frac{2045}{68} \delta\left(-\frac{1}{2}, \frac{13}{12}, \frac{1}{12} ; n\right)+\frac{547}{17} \delta\left(-\frac{1}{6}, \frac{1}{12}, \frac{3}{4} ; n\right)+\frac{543}{272} \delta\left(-\frac{1}{2}, \frac{19}{12},-\frac{5}{12} ; n\right) \\
& -\frac{949}{68} \delta\left(-\frac{1}{6}, \frac{7}{12}, \frac{1}{4} ; n\right)+\frac{1}{17} \delta\left(\frac{1}{6},-\frac{5}{12}, \frac{11}{12} ; n\right),
\end{aligned}
$$

$$
\begin{aligned}
& N([5,8,3] ; n) \\
&=\frac{1}{68} \sigma_{7}(n)-\frac{1}{68} \sigma_{7}(n / 2)-\frac{32}{17} \sigma_{7}(n / 8)+\frac{8192}{17} \sigma_{7}(n / 16) \\
&+\frac{169}{68} \delta\left(-\frac{1}{3}, \frac{4}{3},-\frac{1}{3} ; n\right)-\frac{124}{17} \delta\left(0, \frac{1}{3}, \frac{1}{3} ; n\right)+\frac{5}{2} \delta\left(-\frac{2}{3}, \frac{11}{6},-\frac{1}{2} ; n\right) \\
&-\frac{450}{17} \delta\left(-\frac{1}{3}, \frac{5}{6}, \frac{1}{6} ; n\right)+\frac{64}{17} \delta\left(0,-\frac{1}{6}, \frac{5}{6} ; n\right)+\frac{681}{272} \delta\left(-\frac{5}{6}, \frac{25}{12},-\frac{7}{12} ; n\right) \\
&-\frac{2453}{68} \delta\left(-\frac{1}{2}, \frac{13}{12}, \frac{1}{12} ; n\right)+\frac{547}{17} \delta\left(-\frac{1}{6}, \frac{1}{12}, \frac{3}{4} ; n\right)+\frac{679}{272} \delta\left(-\frac{1}{2}, \frac{19}{12},-\frac{5}{12} ; n\right) \\
&-\frac{1085}{68} \delta\left(-\frac{1}{6}, \frac{7}{12}, \frac{1}{4} ; n\right)+\frac{1}{17} \delta\left(\frac{1}{6},-\frac{5}{12}, \frac{11}{12} ; n\right),
\end{aligned}
$$

$$
\begin{aligned}
& N([6,8,2] ; n) \\
&= \frac{1}{34} \sigma_{7}(n)-\frac{1}{34} \sigma_{7}(n / 2)-\frac{32}{17} \sigma_{7}(n / 8)+\frac{8192}{17} \sigma_{7}(n / 16) \\
&+\frac{101}{34} \delta\left(-\frac{1}{3}, \frac{4}{3},-\frac{1}{3} ; n\right)-\frac{120}{17} \delta\left(0, \frac{1}{3}, \frac{1}{3} ; n\right)+3 \delta\left(-\frac{2}{3}, \frac{11}{6},-\frac{1}{2} ; n\right) \\
&-\frac{484}{17} \delta\left(-\frac{1}{3}, \frac{5}{6}, \frac{1}{6} ; n\right)+\frac{64}{17} \delta\left(0,-\frac{1}{6}, \frac{5}{6} ; n\right)+\frac{817}{272} \delta\left(-\frac{5}{6}, \frac{25}{12},-\frac{7}{12} ; n\right) \\
&-\frac{2861}{68} \delta\left(-\frac{1}{2}, \frac{13}{12}, \frac{1}{12} ; n\right)+\frac{547}{17} \delta\left(-\frac{1}{6}, \frac{1}{12}, \frac{3}{4} ; n\right)+\frac{815}{272} \delta\left(-\frac{1}{2}, \frac{19}{12},-\frac{5}{12} ; n\right) \\
&-\frac{1221}{68} \delta\left(-\frac{1}{6}, \frac{7}{12}, \frac{1}{4} ; n\right)+\frac{1}{17} \delta\left(\frac{1}{6},-\frac{5}{12}, \frac{11}{12} ; n\right),
\end{aligned}
$$




$$
\begin{aligned}
& N([7,8,1] ; n) \\
&=\frac{1}{17} \sigma_{7}(n)-\frac{1}{17} \sigma_{7}(n / 2)-\frac{32}{17} \sigma_{7}(n / 8)+\frac{8192}{17} \sigma_{7}(n / 16) \\
&+\frac{67}{17} \delta\left(-\frac{1}{3}, \frac{4}{3},-\frac{1}{3} ; n\right)-\frac{112}{17} \delta\left(0, \frac{1}{3}, \frac{1}{3} ; n\right)+4 \delta\left(-\frac{2}{3}, \frac{11}{6},-\frac{1}{2} ; n\right) \\
&-\frac{552}{17} \delta\left(-\frac{1}{3}, \frac{5}{6}, \frac{1}{6} ; n\right)+\frac{64}{17} \delta\left(0,-\frac{1}{6}, \frac{5}{6} ; n\right)+\frac{817}{272} \delta\left(-\frac{5}{6}, \frac{25}{12},-\frac{7}{12} ; n\right) \\
&-\frac{2861}{68} \delta\left(-\frac{1}{2}, \frac{13}{12}, \frac{1}{12} ; n\right)+\frac{547}{17} \delta\left(-\frac{1}{6}, \frac{1}{12}, \frac{3}{4} ; n\right)+\frac{815}{272} \delta\left(-\frac{1}{2}, \frac{19}{12},-\frac{5}{12} ; n\right) \\
&-\frac{1221}{68} \delta\left(-\frac{1}{6}, \frac{7}{12}, \frac{1}{4} ; n\right)+\frac{1}{17} \delta\left(\frac{1}{6},-\frac{5}{12}, \frac{11}{12} ; n\right),
\end{aligned}
$$

$$
\begin{aligned}
& N([1,10,5] ; n) \\
&=\frac{1}{544} \sigma_{7}(n)-\frac{1}{544} \sigma_{7}(n / 2)-\frac{32}{17} \sigma_{7}(n / 8)+\frac{8192}{17} \sigma_{7}(n / 16) \\
&+\frac{271}{544} \delta\left(-\frac{1}{3}, \frac{4}{3},-\frac{1}{3} ; n\right)+\frac{5}{4} \delta\left(0, \frac{1}{3}, \frac{1}{3} ; n\right)+\frac{1}{2} \delta\left(-\frac{2}{3}, \frac{11}{6},-\frac{1}{2} ; n\right) \\
&-\frac{59}{17} \delta\left(-\frac{1}{3}, \frac{5}{6}, \frac{1}{6} ; n\right)-\frac{276}{17} \delta\left(0,-\frac{1}{6}, \frac{5}{6} ; n\right)+\frac{137}{272} \delta\left(-\frac{5}{6}, \frac{25}{12},-\frac{7}{12} ; n\right) \\
&-\frac{345}{68} \delta\left(-\frac{1}{2}, \frac{13}{12}, \frac{1}{12} ; n\right)-\frac{473}{17} \delta\left(-\frac{1}{6}, \frac{1}{12}, \frac{3}{4} ; n\right)+\frac{135}{272} \delta\left(-\frac{1}{2}, \frac{19}{12},-\frac{5}{12} ; n\right) \\
&-\frac{65}{68} \delta\left(-\frac{1}{6}, \frac{7}{12}, \frac{1}{4} ; n\right)-\frac{67}{17} \delta\left(\frac{1}{6},-\frac{5}{12}, \frac{11}{12} ; n\right),
\end{aligned}
$$

$$
\begin{aligned}
N( & {[2,10,4] ; n) } \\
= & \frac{1}{272} \sigma_{7}(n)-\frac{1}{272} \sigma_{7}(n / 2)-\frac{32}{17} \sigma_{7}(n / 8)+\frac{8192}{17} \sigma_{7}(n / 16) \\
& +\frac{271}{272} \delta\left(-\frac{1}{3}, \frac{4}{3},-\frac{1}{3} ; n\right)-\frac{67}{34} \delta\left(0, \frac{1}{3}, \frac{1}{3} ; n\right)+\delta\left(-\frac{2}{3}, \frac{11}{6},-\frac{1}{2} ; n\right) \\
& -\frac{178}{17} \delta\left(-\frac{1}{3}, \frac{5}{6}, \frac{1}{6} ; n\right)-\frac{72}{71} \delta\left(0,-\frac{1}{6}, \frac{5}{6} ; n\right)+\frac{273}{272} \delta\left(-\frac{5}{6}, \frac{25}{12},-\frac{7}{12} ; n\right) \\
& -\frac{957}{68} \delta\left(-\frac{1}{2}, \frac{13}{12}, \frac{1}{12} ; n\right)+\frac{3}{17} \delta\left(-\frac{1}{6}, \frac{1}{12}, \frac{3}{4} ; n\right)+\frac{271}{272} \delta\left(-\frac{1}{2}, \frac{19}{12},-\frac{5}{12} ; n\right) \\
& -\frac{405}{68} \delta\left(-\frac{1}{6}, \frac{7}{12}, \frac{1}{4} ; n\right)+\frac{1}{17} \delta\left(\frac{1}{6},-\frac{5}{12}, \frac{11}{12} ; n\right),
\end{aligned}
$$


$N([3,10,3] ; n)$

$$
\begin{aligned}
= & \frac{1}{136} \sigma_{7}(n)-\frac{1}{136} \sigma_{7}(n / 2)-\frac{32}{17} \sigma_{7}(n / 8)+\frac{8192}{17} \sigma_{7}(n / 16) \\
& +\frac{203}{136} \delta\left(-\frac{1}{3}, \frac{4}{3},-\frac{1}{3} ; n\right)-\frac{75}{17} \delta\left(0, \frac{1}{3}, \frac{1}{3} ; n\right)+\frac{3}{2} \delta\left(-\frac{2}{3}, \frac{11}{6},-\frac{1}{2} ; n\right) \\
& -\frac{280}{17} \delta\left(-\frac{1}{3}, \frac{5}{6}, \frac{1}{6} ; n\right)+\frac{64}{17} \delta\left(0,-\frac{1}{6}, \frac{5}{6} ; n\right)+\frac{409}{272} \delta\left(-\frac{5}{6}, \frac{25}{12},-\frac{7}{12} ; n\right) \\
& -\frac{1501}{68} \delta\left(-\frac{1}{2}, \frac{13}{12}, \frac{1}{12} ; n\right)+\frac{275}{17} \delta\left(-\frac{1}{6}, \frac{1}{12}, \frac{3}{4} ; n\right)+\frac{407}{272} \delta\left(-\frac{1}{2}, \frac{19}{12},-\frac{5}{12} ; n\right) \\
& -\frac{677}{68} \delta\left(-\frac{1}{6}, \frac{7}{12}, \frac{1}{4} ; n\right)+\frac{1}{17} \delta\left(\frac{1}{6},-\frac{5}{12}, \frac{11}{12} ; n\right),
\end{aligned}
$$

$N([4,10,2] ; n)$

$$
\begin{aligned}
= & \frac{1}{68} \sigma_{7}(n)-\frac{1}{68} \sigma_{7}(n / 2)-\frac{32}{17} \sigma_{7}(n / 8)+\frac{8192}{17} \sigma_{7}(n / 16) \\
& +\frac{135}{68} \delta\left(-\frac{1}{3}, \frac{4}{3},-\frac{1}{3} ; n\right)-\frac{90}{17} \delta\left(0, \frac{1}{3}, \frac{1}{3} ; n\right)+2 \delta\left(-\frac{2}{3}, \frac{11}{6},-\frac{1}{2} ; n\right) \\
& -\frac{348}{17} \delta\left(-\frac{1}{3}, \frac{5}{6}, \frac{1}{6} ; n\right)+\frac{64}{17} \delta\left(0,-\frac{1}{6}, \frac{5}{6} ; n\right)+\frac{545}{272} \delta\left(-\frac{5}{6}, \frac{25}{12},-\frac{7}{12} ; n\right) \\
& -\frac{2045}{68} \delta\left(-\frac{1}{2}, \frac{13}{12}, \frac{1}{12} ; n\right)+\frac{547}{17} \delta\left(-\frac{1}{6}, \frac{1}{12}, \frac{3}{4} ; n\right)+\frac{543}{272} \delta\left(-\frac{1}{2}, \frac{19}{12},-\frac{5}{12} ; n\right) \\
& -\frac{949}{68} \delta\left(-\frac{1}{6}, \frac{7}{12}, \frac{1}{4} ; n\right)+\frac{1}{17} \delta\left(\frac{1}{6},-\frac{5}{12}, \frac{11}{12} ; n\right),
\end{aligned}
$$

$$
\begin{aligned}
& N([5,10,1] ; n) \\
&=\frac{1}{34} \sigma_{7}(n)-\frac{1}{34} \sigma_{7}(n / 2)-\frac{32}{17} \sigma_{7}(n / 8)+\frac{8192}{17} \sigma_{7}(n / 16) \\
&+\frac{101}{34} \delta\left(-\frac{1}{3}, \frac{4}{3},-\frac{1}{3} ; n\right)-\frac{120}{17} \delta\left(0, \frac{1}{3}, \frac{1}{3} ; n\right)+3 \delta\left(-\frac{2}{3}, \frac{11}{6},-\frac{1}{2} ; n\right) \\
&-\frac{484}{17} \delta\left(-\frac{1}{3}, \frac{5}{6}, \frac{1}{6} ; n\right)+\frac{64}{17} \delta\left(0,-\frac{1}{6}, \frac{5}{6} ; n\right)+\frac{545}{272} \delta\left(-\frac{5}{6}, \frac{25}{12},-\frac{7}{12} ; n\right) \\
&-\frac{2045}{68} \delta\left(-\frac{1}{2}, \frac{13}{12}, \frac{1}{12} ; n\right)+\frac{547}{272} \delta\left(-\frac{1}{6}, \frac{1}{12}, \frac{3}{4} ; n\right)+\frac{543}{272} \delta\left(-\frac{1}{2}, \frac{19}{12},-\frac{5}{12} ; n\right)
\end{aligned}
$$




$$
-\frac{949}{68} \delta\left(-\frac{1}{6}, \frac{7}{12}, \frac{1}{4} ; n\right)+\frac{1}{17} \delta\left(\frac{1}{6},-\frac{5}{12}, \frac{11}{12} ; n\right),
$$

$$
\begin{aligned}
& N([1,12,3] ; n) \\
&= \frac{1}{272} \sigma_{7}(n)-\frac{1}{272} \sigma_{7}(n / 2)-\frac{32}{17} \sigma_{7}(n / 8)+\frac{8192}{17} \sigma_{7}(n / 16) \\
&+\frac{135}{272} \delta\left(-\frac{1}{3}, \frac{4}{3},-\frac{1}{3} ; n\right)+\frac{69}{34} \delta\left(0, \frac{1}{3}, \frac{1}{3} ; n\right)+\frac{1}{2} \delta\left(-\frac{2}{3}, \frac{11}{6},-\frac{1}{2} ; n\right) \\
&-\frac{42}{17} \delta\left(-\frac{1}{3}, \frac{5}{6}, \frac{1}{6} ; n\right)-\frac{344}{17} \delta\left(0,-\frac{1}{6}, \frac{5}{6} ; n\right)+\frac{137}{272} \delta\left(-\frac{5}{6}, \frac{25}{12},-\frac{7}{12} ; n\right) \\
&-\frac{277}{68} \delta\left(-\frac{1}{2}, \frac{13}{12}, \frac{1}{12} ; n\right)-\frac{677}{17} \delta\left(-\frac{1}{6}, \frac{1}{12}, \frac{3}{4} ; n\right)+\frac{135}{272} \delta\left(-\frac{1}{2}, \frac{19}{12},-\frac{5}{12} ; n\right) \\
&+\frac{3}{68} \delta\left(-\frac{1}{6}, \frac{7}{12}, \frac{1}{4} ; n\right)-\frac{135}{17} \delta\left(\frac{1}{6},-\frac{5}{12}, \frac{11}{12} ; n\right),
\end{aligned}
$$

$$
\begin{aligned}
& N([2,12,2] ; n) \\
&=\frac{1}{136} \sigma_{7}(n)-\frac{1}{136} \sigma_{7}(n / 2)-\frac{32}{17} \sigma_{7}(n / 8)+\frac{8192}{17} \sigma_{7}(n / 16) \\
&+\frac{135}{136} \delta\left(-\frac{1}{3}, \frac{4}{3},-\frac{1}{3} ; n\right)-\frac{7}{17} \delta\left(0, \frac{1}{3}, \frac{1}{3} ; n\right)+\delta\left(-\frac{2}{3}, \frac{11}{6},-\frac{1}{2} ; n\right) \\
&-\frac{144}{17} \delta\left(-\frac{1}{3}, \frac{5}{6}, \frac{1}{6} ; n\right)-\frac{208}{17} \delta\left(0,-\frac{1}{6}, \frac{5}{6} ; n\right)+\frac{273}{272} \delta\left(-\frac{5}{6}, \frac{25}{12},-\frac{7}{12} ; n\right) \\
&-\frac{957}{68} \delta\left(-\frac{1}{2}, \frac{13}{12}, \frac{1}{12} ; n\right)+\frac{3}{17} \delta\left(-\frac{1}{6}, \frac{1}{12}, \frac{3}{4} ; n\right)+\frac{271}{272} \delta\left(-\frac{1}{2}, \frac{19}{12},-\frac{5}{12} ; n\right) \\
&-\frac{405}{68} \delta\left(-\frac{1}{6}, \frac{7}{12}, \frac{1}{4} ; n\right)+\frac{1}{17} \delta\left(\frac{1}{6},-\frac{5}{12}, \frac{11}{12} ; n\right),
\end{aligned}
$$

$N([3,12,1] ; n)$

$$
\begin{aligned}
= & \frac{1}{68} \sigma_{7}(n)-\frac{1}{68} \sigma_{7}(n / 2)-\frac{32}{17} \sigma_{7}(n / 8)+\frac{8192}{17} \sigma_{7}(n / 16) \\
& +\frac{135}{68} \delta\left(-\frac{1}{3}, \frac{4}{3},-\frac{1}{3} ; n\right)-\frac{90}{17} \delta\left(0, \frac{1}{3}, \frac{1}{3} ; n\right)+2 \delta\left(-\frac{2}{3}, \frac{11}{6},-\frac{1}{2} ; n\right) \\
& -\frac{348}{17} \delta\left(-\frac{1}{3}, \frac{5}{6}, \frac{1}{6} ; n\right)+\frac{64}{17} \delta\left(0,-\frac{1}{6}, \frac{5}{6} ; n\right)+\frac{273}{272} \delta\left(-\frac{5}{6}, \frac{25}{12},-\frac{7}{12} ; n\right)
\end{aligned}
$$




$$
\begin{aligned}
& -\frac{957}{68} \delta\left(-\frac{1}{2}, \frac{13}{12}, \frac{1}{12} ; n\right)+\frac{3}{17} \delta\left(-\frac{1}{6}, \frac{1}{12}, \frac{3}{4} ; n\right)+\frac{271}{272} \delta\left(-\frac{1}{2}, \frac{19}{12},-\frac{5}{12} ; n\right) \\
& -\frac{405}{68} \delta\left(-\frac{1}{6}, \frac{7}{12}, \frac{1}{4} ; n\right)+\frac{1}{7} \delta\left(\frac{1}{6},-\frac{5}{12}, \frac{11}{12} ; n\right),
\end{aligned}
$$

$$
\begin{aligned}
& N([1,14,1] ; n) \\
&=\frac{1}{136} \sigma_{7}(n)-\frac{1}{136} \sigma_{7}(n / 2)-\frac{32}{17} \sigma_{7}(n / 8)+\frac{8192}{17} \sigma_{7}(n / 16) \\
&+\frac{135}{136} \delta\left(-\frac{1}{3}, \frac{4}{3},-\frac{1}{3} ; n\right)-\frac{7}{17} \delta\left(0, \frac{1}{3}, \frac{1}{3} ; n\right)+\delta\left(-\frac{2}{3}, \frac{11}{6},-\frac{1}{2} ; n\right) \\
&-\frac{144}{17} \delta\left(-\frac{1}{3}, \frac{5}{6}, \frac{1}{6} ; n\right)-\frac{208}{17} \delta\left(0,-\frac{1}{6}, \frac{5}{6} ; n\right)+\frac{1}{272} \delta\left(-\frac{5}{6}, \frac{25}{12},-\frac{7}{12} ; n\right) \\
&+\frac{403}{68} \delta\left(-\frac{1}{2}, \frac{13}{12}, \frac{1}{12} ; n\right)-\frac{1357}{17} \delta\left(-\frac{1}{6}, \frac{1}{12}, \frac{3}{4} ; n\right)-\frac{1}{272} \delta\left(-\frac{1}{2}, \frac{19}{12},-\frac{5}{12} ; n\right) \\
&+\frac{411}{68} \delta\left(-\frac{1}{6}, \frac{7}{12}, \frac{1}{4} ; n\right)-\frac{271}{17} \delta\left(\frac{1}{6},-\frac{5}{12}, \frac{11}{12} ; n\right) .
\end{aligned}
$$

Proof. We only prove the formula for $N([7,2,7] ; n)$. The proofs for the other cases follow in a similar way.

From (7.3.1)-(7.3.9) and Lemma 5.2.1 we have

$$
\begin{aligned}
& \left(\frac{1+(1-x)^{1 / 2}}{2}\right)\left(\frac{1+(1-x)^{1 / 4}}{2}\right)^{7}-\frac{1}{65280} e_{4,1}(x)+\frac{1}{65280} e_{4,2}(x) \\
& \quad+\frac{1}{255}\left(e_{4,8}^{(0)}(x)+e_{4,8}^{(1)}(x)(1-x)^{1 / 2}\right) \\
& \quad-\frac{256}{255}\left(e_{4,16}^{(0)}(x)+e_{4,16}^{(1)}(x)(1-x)^{1 / 4}+e_{4,16}^{(2)}(1-x)^{1 / 2}+e_{4,16}^{(3)}(1-x)^{3 / 4}\right) \\
& =(1-x)\left(\frac{123}{544} x-\frac{63}{2176} x^{2}\right)+(1-x)^{1 / 4}\left(\frac{919}{4352} x-\frac{3337}{17408} x^{2}+\frac{547}{69632} x^{3}\right) \\
& \quad+(1-x)^{1 / 2}\left(\frac{27}{128} x-\frac{69}{544} x^{2}+\frac{1}{1088} x^{3}\right)
\end{aligned}
$$




$$
+(1-x)^{3 / 4}\left(\frac{985}{4352} x-\frac{1221}{17408} x^{2}+\frac{1}{69632} x^{3}\right) .
$$

Thus we have

$$
\begin{aligned}
& a_{4,2,7}^{\prime}(1)=\frac{123}{544}, a_{4,2,7}^{\prime}(2)=-\frac{63}{2176}, b_{4,2,7}^{\prime}(1)=\frac{919}{4352}, b_{4,2,7}^{\prime}(2)=-\frac{3337}{17408}, \\
& b_{4,2,7}^{\prime}(3)=\frac{547}{69632}, c_{4,2,7}^{\prime}(1)=\frac{27}{128}, c_{4,2,7}^{\prime}(2)=-\frac{69}{544}, c_{4,2,7}^{\prime}(3)=\frac{1}{1088}, d_{4,2,7}^{\prime}(1)=\frac{985}{4352}, \\
& d_{4,2,7}^{\prime}(2)=-\frac{1221}{17408}, d_{4,2,7}^{\prime}(3)=\frac{1}{69632} .
\end{aligned}
$$

Using Theorem 6.2.3 with $k=4, r=2$ and $s=7$, we obtain the desired result.

For the final result of this chapter, we apply Theorem 6.3 .3 to the case when $k=4$ to obtain the number of representations of an integer $n$ by the sum of $16-s$ squares and $s$ squares multiplied by 4 , where $s \in \mathbb{N}$ and $0<s<16$.

Theorem 7.3.3. Let $n \in \mathbb{N}$. Then

$$
\begin{aligned}
N & ([1,0,15] ; n) \\
= & \frac{1}{17408} \sigma_{7}(n)-\frac{129}{17408} \sigma_{7}(n / 2)+\frac{257}{136} \sigma_{7}(n / 4)-\frac{64}{17} \sigma_{7}(n / 8)+\frac{8192}{17} \sigma_{7}(n / 16) \\
& +\frac{7751}{17408} \delta\left(-\frac{1}{3}, \frac{4}{3},-\frac{1}{3} ; n\right)-\frac{8775}{2176} \delta\left(0, \frac{1}{3}, \frac{1}{3} ; n\right)+\frac{71}{128} \delta\left(-\frac{2}{3}, \frac{11}{6},-\frac{1}{2} ; n\right) \\
& -\frac{1143}{136} \delta\left(-\frac{1}{3}, \frac{5}{6}, \frac{1}{6} ; n\right)+\frac{253}{136} \delta\left(0,-\frac{1}{6}, \frac{5}{6} ; n\right)-\frac{1}{512} \delta\left(-\frac{7}{6}, \frac{37}{12},-\frac{5}{4} ; n\right) \\
& +\frac{1079}{2176} \delta\left(-\frac{5}{6}, \frac{25}{12},-\frac{7}{12} ; n\right)-\frac{2315}{272} \delta\left(-\frac{1}{2}, \frac{13}{12}, \frac{1}{12} ; n\right)+\frac{2591}{136} \delta\left(-\frac{1}{6}, \frac{1}{12}, \frac{3}{4} ; n\right) \\
& +\frac{1}{512} \delta\left(-\frac{5}{6}, \frac{31}{12},-\frac{13}{12} ; n\right)+\frac{1131}{2176} \delta\left(-\frac{1}{2}, \frac{19}{12},-\frac{5}{12} ; n\right)-\frac{1977}{272} \delta\left(-\frac{1}{6}, \frac{7}{12}, \frac{1}{4} ; n\right) \\
& -\frac{9}{136} \delta\left(\frac{1}{6},-\frac{5}{12}, \frac{11}{12} ; n\right),
\end{aligned}
$$


$N([2,0,14] ; n)$

$$
\begin{aligned}
= & \frac{1}{8704} \sigma_{7}(n)-\frac{1}{8704} \sigma_{7}(n / 2)-\frac{32}{17} \sigma_{7}(n / 8)+\frac{8192}{17} \sigma_{7}(n / 16) \\
& +\frac{7751}{8704} \delta\left(-\frac{1}{3}, \frac{4}{3},-\frac{1}{3} ; n\right)-\frac{6643}{1088} \delta\left(0, \frac{1}{3}, \frac{1}{3} ; n\right)+\frac{71}{64} \delta\left(-\frac{2}{3}, \frac{11}{6},-\frac{1}{2} ; n\right) \\
& -\frac{4565}{272} \delta\left(-\frac{1}{3}, \frac{5}{6}, \frac{1}{6} ; n\right)+\frac{239}{68} \delta\left(0,-\frac{1}{6}, \frac{5}{6} ; n\right)-\frac{1}{256} \delta\left(-\frac{7}{6}, \frac{37}{12},-\frac{5}{4} ; n\right) \\
& +\frac{597}{544} \delta\left(-\frac{5}{6}, \frac{25}{12},-\frac{7}{12} ; n\right)-\frac{4933}{272} \delta\left(-\frac{1}{2}, \frac{13}{12}, \frac{1}{12} ; n\right)+\frac{975}{34} \delta\left(-\frac{1}{6}, \frac{1}{12}, \frac{3}{4} ; n\right) \\
& +\frac{1}{256} \delta\left(-\frac{5}{6}, \frac{31}{12},-\frac{13}{12} ; n\right)+\frac{127}{136} \delta\left(-\frac{1}{2}, \frac{19}{12},-\frac{5}{12} ; n\right)-\frac{3337}{272} \delta\left(-\frac{1}{6}, \frac{7}{12}, \frac{1}{4} ; n\right) \\
& +\frac{1}{17} \delta\left(\frac{1}{6},-\frac{5}{12}, \frac{11}{12} ; n\right),
\end{aligned}
$$

$N([3,0,13] ; n)$

$$
\begin{aligned}
= & \frac{1}{4352} \sigma_{7}(n)+\frac{63}{4352} \sigma_{7}(n / 2)-\frac{257}{68} \sigma_{7}(n / 4)+\frac{32}{17} \sigma_{7}(n / 8)+\frac{8192}{17} \sigma_{7}(n / 16) \\
& +\frac{5983}{4352} \delta\left(-\frac{1}{3}, \frac{4}{3},-\frac{1}{3} ; n\right)-\frac{3809}{544} \delta\left(0, \frac{1}{3}, \frac{1}{3} ; n\right)+\frac{13}{8} \delta\left(-\frac{2}{3}, \frac{11}{6},-\frac{1}{2} ; n\right) \\
& -\frac{6539}{272} \delta\left(-\frac{1}{3}, \frac{5}{6}, \frac{1}{6} ; n\right)+\frac{65}{17} \delta\left(0,-\frac{1}{6}, \frac{5}{6} ; n\right)-\frac{1}{256} \delta\left(-\frac{7}{6}, \frac{37}{12},-\frac{5}{4} ; n\right) \\
& +\frac{115}{68} \delta\left(-\frac{5}{6}, \frac{25}{12},-\frac{7}{12} ; n\right)-\frac{7517}{272} \delta\left(-\frac{1}{2}, \frac{13}{12}, \frac{1}{12} ; n\right)+\frac{1077}{34} \delta\left(-\frac{1}{6}, \frac{1}{12}, \frac{3}{4} ; n\right) \\
& +\frac{1}{256} \delta\left(-\frac{5}{6}, \frac{31}{12},-\frac{13}{12} ; n\right)+\frac{729}{544} \delta\left(-\frac{1}{2}, \frac{19}{12},-\frac{5}{12} ; n\right)-\frac{4221}{272} \delta\left(-\frac{1}{6}, \frac{7}{12}, \frac{1}{4} ; n\right) \\
& +\frac{1}{17} \delta\left(\frac{1}{6},-\frac{5}{12}, \frac{11}{12} ; n\right), \\
N & ([4,0,12] ; n) \\
= & \frac{1}{2176} \sigma_{7}(n)+\frac{63}{2176} \sigma_{7}(n / 2)-\frac{257}{34} \sigma_{7}(n / 4)+\frac{96}{17} \sigma_{7}(n / 8)+\frac{8192}{17} \sigma_{7}(n / 16) \\
& +\frac{4215}{2176} \delta\left(-\frac{1}{3}, \frac{4}{3},-\frac{1}{3} ; n\right)-\frac{1965}{272} \delta\left(0, \frac{1}{3}, \frac{1}{3} ; n\right)+\frac{33}{16} \delta\left(-\frac{2}{3}, \frac{11}{6},-\frac{1}{2} ; n\right) \\
& -\frac{3993}{136} \delta\left(-\frac{1}{3}, \frac{5}{6}, \frac{1}{6} ; n\right)+\frac{66}{17} \delta\left(0,-\frac{1}{6}, \frac{5}{6} ; n\right)+\frac{149}{68} \delta\left(-\frac{5}{6}, \frac{25}{12},-\frac{7}{12} ; n\right) \\
& -\frac{1235}{34} \delta\left(-\frac{1}{2}, \frac{13}{12}, \frac{1}{12} ; n\right)+\frac{547}{17} \delta\left(-\frac{1}{6}, \frac{1}{12}, \frac{3}{4} ; n\right)+\frac{123}{68} \delta\left(-\frac{1}{2}, \frac{19}{12},-\frac{5}{12} ; n\right) \\
& -\frac{585}{34} \delta\left(-\frac{1}{6}, \frac{7}{12}, \frac{1}{4} ; n\right)+\frac{1}{17} \delta\left(\frac{1}{6},-\frac{5}{12}, \frac{11}{12} ; n\right),
\end{aligned}
$$




$$
\begin{aligned}
& N([5,0,11] ; n) \\
&= \frac{1}{1088} \sigma_{7}(n)+\frac{31}{1088} \sigma_{7}(n / 2)-\frac{257}{34} \sigma_{7}(n / 4)+\frac{96}{17} \sigma_{7}(n / 8)+\frac{8192}{17} \sigma_{7}(n / 16) \\
&+\frac{2821}{1088} \delta\left(-\frac{1}{3}, \frac{4}{3},-\frac{1}{3} ; n\right)-\frac{495}{68} \delta\left(0, \frac{1}{3}, \frac{1}{3} ; n\right)+\frac{77}{32} \delta\left(-\frac{2}{3}, \frac{11}{6},-\frac{1}{2} ; n\right) \\
&-\frac{4367}{136} \delta\left(-\frac{1}{3}, \frac{5}{6}, \frac{1}{6} ; n\right)+\frac{66}{17} \delta\left(0,-\frac{1}{6}, \frac{5}{6} ; n\right)+\frac{1}{128} \delta\left(-\frac{7}{6}, \frac{37}{12},-\frac{5}{4} ; n\right) \\
&+\frac{1379}{544} \delta\left(-\frac{5}{6}, \frac{25}{12},-\frac{7}{12} ; n\right)-\frac{5875}{136} \delta\left(-\frac{1}{2}, \frac{13}{12}, \frac{1}{12} ; n\right)+\frac{547}{17} \delta\left(-\frac{1}{6}, \frac{1}{12}, \frac{3}{4} ; n\right) \\
&-\frac{1}{128} \delta\left(-\frac{5}{6}, \frac{31}{12},-\frac{13}{12} ; n\right)+\frac{1307}{544} \delta\left(-\frac{1}{2}, \frac{19}{12},-\frac{5}{12} ; n\right)-\frac{2425}{136} \delta\left(-\frac{1}{6}, \frac{7}{12}, \frac{1}{4} ; n\right) \\
&+\frac{1}{17} \delta\left(\frac{1}{6},-\frac{5}{12}, \frac{11}{12} ; n\right),
\end{aligned}
$$

$N([6,0,10] ; n)$

$$
\begin{aligned}
= & \frac{1}{544} \sigma_{7}(n)-\frac{1}{544} \sigma_{7}(n / 2)-\frac{32}{17} \sigma_{7}(n / 8)+\frac{8192}{17} \sigma_{7}(n / 16) \\
& +\frac{1801}{544} \delta\left(-\frac{1}{3}, \frac{4}{3},-\frac{1}{3} ; n\right)-\frac{15}{2} \delta\left(0, \frac{1}{3}, \frac{1}{3} ; n\right)+\frac{43}{16} \delta\left(-\frac{2}{3}, \frac{11}{6},-\frac{1}{2} ; n\right) \\
& -\frac{2191}{68} \delta\left(-\frac{1}{3}, \frac{5}{6}, \frac{1}{6} ; n\right)+\frac{64}{17} \delta\left(0,-\frac{1}{6}, \frac{5}{6} ; n\right)+\frac{1}{64} \delta\left(-\frac{7}{6}, \frac{37}{12},-\frac{5}{4} ; n\right) \\
& +\frac{749}{272} \delta\left(-\frac{5}{6}, \frac{25}{12},-\frac{7}{12} ; n\right)-\frac{3235}{68} \delta\left(-\frac{1}{2}, \frac{13}{12}, \frac{1}{12} ; n\right)+\frac{547}{17} \delta\left(-\frac{1}{6}, \frac{1}{12}, \frac{3}{4} ; n\right) \\
& -\frac{1}{64} \delta\left(-\frac{5}{6}, \frac{31}{12},-\frac{13}{12} ; n\right)+\frac{849}{272} \delta\left(-\frac{1}{2}, \frac{19}{12},-\frac{5}{12} ; n\right)-\frac{1221}{68} \delta\left(-\frac{1}{6}, \frac{7}{12}, \frac{1}{4} ; n\right) \\
& +\frac{1}{17} \delta\left(\frac{1}{6},-\frac{5}{12}, \frac{11}{12} ; n\right), \\
N & ([7,0,9] ; n) \\
= & \frac{1}{272} \sigma_{7}(n)-\frac{1}{16} \sigma_{7}(n / 2)+\frac{257}{17} \sigma_{7}(n / 4)-\frac{288}{17} \sigma_{7}(n / 8)+\frac{8192}{17} \sigma_{7}(n / 16) \\
& +\frac{1087}{272} \delta\left(-\frac{1}{3}, \frac{4}{3},-\frac{1}{3} ; n\right)-\frac{135}{17} \delta\left(0, \frac{1}{3}, \frac{1}{3} ; n\right)+3 \delta\left(-\frac{2}{3}, \frac{11}{6},-\frac{1}{2} ; n\right) \\
& -\frac{1035}{34} \delta\left(-\frac{1}{3}, \frac{5}{6}, \frac{1}{6} ; n\right)+\frac{60}{17} \delta\left(0,-\frac{1}{6}, \frac{5}{6} ; n\right)+\frac{1}{64} \delta\left(-\frac{7}{6}, \frac{37}{12},-\frac{5}{4} ; n\right) \\
& +\frac{817}{272} \delta\left(-\frac{5}{6}, \frac{25}{12},-\frac{7}{12} ; n\right)-\frac{3371}{68} \delta\left(-\frac{1}{2}, \frac{13}{12}, \frac{1}{12} ; n\right)+\frac{547}{17} \delta\left(-\frac{1}{6}, \frac{1}{12}, \frac{3}{4} ; n\right) \\
& -\frac{1}{64} \delta\left(-\frac{5}{6}, \frac{31}{12},-\frac{13}{12} ; n\right)+\frac{1053}{272} \delta\left(-\frac{1}{2}, \frac{19}{12},-\frac{5}{12} ; n\right)-\frac{1221}{68} \delta\left(-\frac{1}{6}, \frac{7}{12}, \frac{1}{4} ; n\right)
\end{aligned}
$$




$$
+\frac{1}{17} \delta\left(\frac{1}{6},-\frac{5}{12}, \frac{11}{12} ; n\right),
$$

$N([8,0,8] ; n)$

$$
\begin{aligned}
= & \frac{1}{136} \sigma_{7}(n)-\frac{1}{8} \sigma_{7}(n / 2)+\frac{514}{17} \sigma_{7}(n / 4)-32 \sigma_{7}(n / 8)+\frac{8192}{17} \sigma_{7}(n / 16) \\
& +\frac{611}{136} \delta\left(-\frac{1}{3}, \frac{4}{3},-\frac{1}{3} ; n\right)-\frac{142}{17} \delta\left(0, \frac{1}{3}, \frac{1}{3} ; n\right)+\frac{7}{2} \delta\left(-\frac{2}{3}, \frac{11}{6},-\frac{1}{2} ; n\right) \\
& -\frac{483}{17} \delta\left(-\frac{1}{3}, \frac{5}{6}, \frac{1}{6} ; n\right)+\frac{56}{17} \delta\left(0,-\frac{1}{6}, \frac{5}{6} ; n\right)+\frac{953}{272} \delta\left(-\frac{5}{6}, \frac{25}{12},-\frac{7}{12} ; n\right) \\
& -\frac{3405}{68} \delta\left(-\frac{1}{2}, \frac{13}{12}, \frac{1}{12} ; n\right)+\frac{547}{17} \delta\left(-\frac{1}{6}, \frac{1}{12}, \frac{3}{4} ; n\right)+\frac{1223}{272} \delta\left(-\frac{1}{2}, \frac{19}{12},-\frac{5}{12} ; n\right) \\
& -\frac{1221}{68} \delta\left(-\frac{1}{6}, \frac{7}{12}, \frac{1}{4} ; n\right)+\frac{1}{17} \delta\left(\frac{1}{6},-\frac{5}{12}, \frac{11}{12} ; n\right),
\end{aligned}
$$

$N([9,0,7] ; n)$

$$
\begin{aligned}
= & \frac{1}{68} \sigma_{7}(n)-\frac{9}{68} \sigma_{7}(n / 2)+\frac{514}{17} \sigma_{7}(n / 4)-32 \sigma_{7}(n / 8)+\frac{8192}{17} \sigma_{7}(n / 16) \\
& +\frac{627}{136} \delta\left(-\frac{1}{3}, \frac{4}{3},-\frac{1}{3} ; n\right)-\frac{140}{17} \delta\left(0, \frac{1}{3}, \frac{1}{3} ; n\right)+\frac{35}{8} \delta\left(-\frac{2}{3}, \frac{11}{6},-\frac{1}{2} ; n\right) \\
& -\frac{483}{17} \delta\left(-\frac{1}{3}, \frac{5}{6}, \frac{1}{6} ; n\right)+\frac{56}{17} \delta\left(0,-\frac{1}{6}, \frac{5}{6} ; n\right)-\frac{1}{32} \delta\left(-\frac{7}{6}, \frac{37}{12},-\frac{5}{4} ; n\right) \\
& +\frac{1191}{272} \delta\left(-\frac{5}{6}, \frac{25}{12},-\frac{7}{12} ; n\right)-\frac{3405}{68} \delta\left(-\frac{1}{2}, \frac{13}{12}, \frac{1}{12} ; n\right)+\frac{547}{17} \delta\left(-\frac{1}{6}, \frac{1}{12}, \frac{3}{4} ; n\right) \\
& +\frac{1}{32} \delta\left(-\frac{5}{6}, \frac{31}{12},-\frac{13}{12} ; n\right)+\frac{1325}{272} \delta\left(-\frac{1}{2}, \frac{19}{12},-\frac{5}{12} ; n\right)-\frac{1221}{68} \delta\left(-\frac{1}{6}, \frac{7}{12}, \frac{1}{4} ; n\right) \\
& +\frac{1}{17} \delta\left(\frac{1}{6},-\frac{5}{12}, \frac{11}{12} ; n\right),
\end{aligned}
$$

$$
N([10,0,6] ; n)
$$

$$
\begin{aligned}
= & \frac{1}{34} \sigma_{7}(n)-\frac{1}{34} \sigma_{7}(n / 2)-\frac{32}{17} \sigma_{7}(n / 8)+\frac{8192}{17} \sigma_{7}(n / 16) \\
& +\frac{287}{68} \delta\left(-\frac{1}{3}, \frac{4}{3},-\frac{1}{3} ; n\right)-\frac{120}{17} \delta\left(0, \frac{1}{3}, \frac{1}{3} ; n\right)+\frac{23}{4} \delta\left(-\frac{2}{3}, \frac{11}{6},-\frac{1}{2} ; n\right) \\
& -\frac{552}{17} \delta\left(-\frac{1}{3}, \frac{5}{6}, \frac{1}{6} ; n\right)+\frac{64}{17} \delta\left(0,-\frac{1}{6}, \frac{5}{6} ; n\right)-\frac{1}{16} \delta\left(-\frac{7}{6}, \frac{37}{12},-\frac{5}{4} ; n\right) \\
& +\frac{1497}{272} \delta\left(-\frac{5}{6}, \frac{25}{12},-\frac{7}{12} ; n\right)-\frac{3405}{68} \delta\left(-\frac{1}{2}, \frac{13}{12}, \frac{1}{12} ; n\right)+\frac{547}{17} \delta\left(-\frac{1}{6}, \frac{1}{12}, \frac{3}{4} ; n\right) \\
& +\frac{1}{16} \delta\left(-\frac{5}{6}, \frac{31}{12},-\frac{13}{12} ; n\right)+\frac{1359}{272} \delta\left(-\frac{1}{2}, \frac{19}{12},-\frac{5}{12} ; n\right)-\frac{1221}{68} \delta\left(-\frac{1}{6}, \frac{7}{12}, \frac{1}{4} ; n\right)
\end{aligned}
$$




$$
+\frac{1}{17} \delta\left(\frac{1}{6},-\frac{5}{12}, \frac{11}{12} ; n\right),
$$

$N([11,0,5] ; n)$

$$
\begin{aligned}
= & \frac{1}{17} \sigma_{7}(n)+\frac{3}{17} \sigma_{7}(n / 2)-\frac{1028}{17} \sigma_{7}(n / 4)+\frac{992}{17} \sigma_{7}(n / 8)+\frac{8192}{17} \sigma_{7}(n / 16) \\
& +\frac{117}{34} \delta\left(-\frac{1}{3}, \frac{4}{3},-\frac{1}{3} ; n\right)-\frac{80}{17} \delta\left(0, \frac{1}{3}, \frac{1}{3} ; n\right)+\frac{15}{2} \delta\left(-\frac{2}{3}, \frac{11}{6},-\frac{1}{2} ; n\right) \\
& -\frac{690}{17} \delta\left(-\frac{1}{3}, \frac{5}{6}, \frac{1}{6} ; n\right)+\frac{80}{17} \delta\left(0,-\frac{1}{6}, \frac{5}{6} ; n\right)-\frac{1}{16} \delta\left(-\frac{7}{6}, \frac{37}{12},-\frac{5}{4} ; n\right) \\
& +\frac{1769}{272} \delta\left(-\frac{5}{6}, \frac{25}{12},-\frac{7}{12} ; n\right)-\frac{3405}{68} \delta\left(-\frac{1}{2}, \frac{13}{12}, \frac{1}{12} ; n\right)+\frac{547}{17} \delta\left(-\frac{1}{6}, \frac{1}{12}, \frac{3}{4} ; n\right) \\
& +\frac{1}{16} \delta\left(-\frac{5}{6}, \frac{31}{12},-\frac{13}{12} ; n\right)+\frac{1359}{272} \delta\left(-\frac{1}{2}, \frac{19}{12},-\frac{5}{12} ; n\right)-\frac{1221}{68} \delta\left(-\frac{1}{6}, \frac{7}{12}, \frac{1}{4} ; n\right) \\
& +\frac{1}{17} \delta\left(\frac{1}{6},-\frac{5}{12}, \frac{11}{12} ; n\right),
\end{aligned}
$$

$N([12,0,4] ; n)$

$$
\begin{aligned}
= & \frac{2}{17} \sigma_{7}(n)+\frac{6}{17} \sigma_{7}(n / 2)-\frac{2056}{17} \sigma_{7}(n / 4)+\frac{2016}{17} \sigma_{7}(n / 8)+\frac{8192}{17} \sigma_{7}(n / 16) \\
& +\frac{49}{17} \delta\left(-\frac{1}{3}, \frac{4}{3},-\frac{1}{3} ; n\right)-\frac{32}{17} \delta\left(0, \frac{1}{3}, \frac{1}{3} ; n\right)+9 \delta\left(-\frac{2}{3}, \frac{11}{6},-\frac{1}{2} ; n\right) \\
& -\frac{828}{17} \delta\left(-\frac{1}{3}, \frac{5}{6}, \frac{1}{6} ; n\right)+\frac{96}{17} \delta\left(0,-\frac{1}{6}, \frac{5}{6} ; n\right)+\frac{1905}{272} \delta\left(-\frac{5}{6}, \frac{25}{12},-\frac{7}{12} ; n\right) \\
& -\frac{3405}{68} \delta\left(-\frac{1}{2}, \frac{13}{12}, \frac{1}{12} ; n\right)+\frac{547}{17} \delta\left(-\frac{1}{6}, \frac{1}{12}, \frac{3}{4} ; n\right)+\frac{1359}{272} \delta\left(-\frac{1}{2}, \frac{19}{12},-\frac{5}{12} ; n\right) \\
& -\frac{1221}{68} \delta\left(-\frac{1}{6}, \frac{7}{12}, \frac{1}{4} ; n\right)+\frac{1}{17} \delta\left(\frac{1}{6},-\frac{5}{12}, \frac{11}{12} ; n\right),
\end{aligned}
$$

$N([13,0,3] ; n)$

$$
\begin{aligned}
= & \frac{4}{17} \sigma_{7}(n)+\frac{4}{17} \sigma_{7}(n / 2)-\frac{2056}{17} \sigma_{7}(n / 4)+\frac{2016}{17} \sigma_{7}(n / 8)+\frac{8192}{17} \sigma_{7}(n / 16) \\
& +\frac{64}{17} \delta\left(-\frac{1}{3}, \frac{4}{3},-\frac{1}{3} ; n\right)+9 \delta\left(-\frac{2}{3}, \frac{11}{6},-\frac{1}{2} ; n\right)-\frac{828}{17} \delta\left(-\frac{1}{3}, \frac{5}{6}, \frac{1}{6} ; n\right) \\
& +\frac{96}{17} \delta\left(0,-\frac{1}{6}, \frac{5}{6} ; n\right)+\frac{1}{8} \delta\left(-\frac{7}{6}, \frac{37}{12},-\frac{5}{4} ; n\right)+\frac{1905}{272} \delta\left(-\frac{5}{6}, \frac{25}{12},-\frac{7}{12} ; n\right) \\
& -\frac{3405}{68} \delta\left(-\frac{1}{2}, \frac{13}{12}, \frac{1}{12} ; n\right)+\frac{547}{17} \delta\left(-\frac{1}{6}, \frac{1}{12}, \frac{3}{4} ; n\right)-\frac{1}{8} \delta\left(-\frac{5}{6}, \frac{31}{12},-\frac{13}{12} ; n\right) \\
& +\frac{1359}{272} \delta\left(-\frac{1}{2}, \frac{19}{12},-\frac{5}{12} ; n\right)-\frac{1221}{68} \delta\left(-\frac{1}{6}, \frac{7}{12}, \frac{1}{4} ; n\right)+\frac{1}{17} \delta\left(\frac{1}{6},-\frac{5}{12}, \frac{11}{12} ; n\right),
\end{aligned}
$$




$$
\begin{aligned}
N & ([14,0,2] ; n) \\
= & \frac{8}{17} \sigma_{7}(n)-\frac{8}{17} \sigma_{7}(n / 2)-\frac{32}{17} \sigma_{7}(n / 8)+\frac{8192}{17} \sigma_{7}(n / 16) \\
& +\frac{128}{17} \delta\left(-\frac{1}{3}, \frac{4}{3},-\frac{1}{3} ; n\right)+6 \delta\left(-\frac{2}{3}, \frac{11}{6},-\frac{1}{2} ; n\right)-\frac{552}{17} \delta\left(-\frac{1}{3}, \frac{5}{6}, \frac{1}{6} ; n\right) \\
& +\frac{64}{17} \delta\left(0,-\frac{1}{6}, \frac{5}{6} ; n\right)+\frac{1}{4} \delta\left(-\frac{7}{6}, \frac{37}{12},-\frac{5}{4} ; n\right)+\frac{1905}{272} \delta\left(-\frac{5}{6}, \frac{25}{12},-\frac{7}{12} ; n\right) \\
& -\frac{3405}{68} \delta\left(-\frac{1}{2}, \frac{13}{12}, \frac{1}{12} ; n\right)+\frac{547}{17} \delta\left(-\frac{1}{6}, \frac{1}{12}, \frac{3}{4} ; n\right)-\frac{1}{4} \delta\left(-\frac{5}{6}, \frac{31}{12},-\frac{13}{12} ; n\right) \\
& +\frac{1359}{272} \delta\left(-\frac{1}{2}, \frac{19}{12},-\frac{5}{12} ; n\right)-\frac{1221}{68} \delta\left(-\frac{1}{6}, \frac{7}{12}, \frac{1}{4} ; n\right)+\frac{1}{17} \delta\left(\frac{1}{6},-\frac{5}{12}, \frac{11}{12} ; n\right) \\
N & ([15,0,1] ; n) \\
= & \frac{16}{17} \sigma_{7}(n)-\frac{32}{17} \sigma_{7}(n / 2)+\frac{4112}{17} \sigma_{7}(n / 4)-\frac{4128}{17} \sigma_{7}(n / 8)+\frac{8192}{17} \sigma_{7}(n / 16) \\
& +\frac{256}{17} \delta\left(-\frac{1}{3}, \frac{4}{3},-\frac{1}{3} ; n\right)+\frac{1}{4} \delta\left(-\frac{7}{6}, \frac{37}{12},-\frac{5}{4} ; n\right)+\frac{1905}{272} \delta\left(-\frac{5}{6}, \frac{25}{12},-\frac{7}{12} ; n\right) \\
& -\frac{3405}{68} \delta\left(-\frac{1}{2}, \frac{13}{12}, \frac{1}{12} ; n\right)+\frac{547}{17} \delta\left(-\frac{1}{6}, \frac{1}{12}, \frac{3}{4} ; n\right)-\frac{1}{4} \delta\left(-\frac{5}{6}, \frac{31}{12},-\frac{13}{12} ; n\right) \\
& +\frac{1359}{272} \delta\left(-\frac{1}{2}, \frac{19}{12},-\frac{5}{12} ; n\right)-\frac{1221}{68} \delta\left(-\frac{1}{6}, \frac{7}{12}, \frac{1}{4} ; n\right)+\frac{1}{17} \delta\left(\frac{1}{6},-\frac{5}{12}, \frac{11}{12} ; n\right)
\end{aligned}
$$

Proof. We only prove the formula for $N([13,0,3] ; n)$. The proofs for the other cases follow in a similar way.

From (7.3.1)-(7.3.9) and Lemma 5.3.1 we have

$$
\begin{aligned}
& \left(\frac{1+(1-x)^{1 / 4}}{2}\right)^{3}-\frac{1}{2040} e_{4,1}(x)-\frac{1}{2040} e_{4,2}(x)+\frac{257}{1020} e_{4,4}(x) \\
& -\frac{21}{85}\left(e_{4,8}^{(0)}(x)+e_{4,8}^{(1)}(x)(1-x)^{1 / 2}\right) \\
& \quad-\frac{256}{255}\left(e_{4,16}^{(0)}(x)+e_{4,16}^{(1)}(x)(1-x)^{1 / 4}+e_{4,16}^{(2)}(1-x)^{1 / 2}+e_{4,16}^{(3)}(1-x)^{3 / 4}\right)
\end{aligned}
$$




$$
\begin{aligned}
= & (1-x)\left(\frac{4}{17} x\right)+(1-x)^{1 / 4}\left(\frac{1}{8}+\frac{1905}{4352} x-\frac{3405}{17408} x^{2}+\frac{547}{69632} x^{3}\right) \\
& +(1-x)^{1 / 2}\left(\frac{9}{16} x-\frac{207}{1088} x^{2}+\frac{3}{2176} x^{3}\right) \\
& +(1-x)^{3 / 4}\left(-\frac{1}{8}+\frac{1359}{4352} x-\frac{1221}{17408} x^{2}+\frac{1}{69632} x^{3}\right) .
\end{aligned}
$$

Thus we have

$$
\begin{aligned}
& a_{4,0,3}^{\prime}(1)=\frac{4}{17}, a_{4,0,3}^{\prime}(2)=0, b_{4,0,3}^{\prime}(0)=\frac{1}{8}, b_{4,0,3}^{\prime}(1)=\frac{1905}{4352}, b_{4,0,3}^{\prime}(2)=-\frac{3405}{17408}, \\
& b_{4,0,3}^{\prime}(3)=\frac{547}{69632}, c_{4,0,3}^{\prime}(1)=\frac{9}{16}, c_{4,0,3}^{\prime}(2)=-\frac{207}{1088}, c_{4,0,3}^{\prime}(3)=\frac{3}{2176}, d_{4,0,3}^{\prime}(0)=-\frac{1}{8}, \\
& d_{4,0,3}^{\prime}(1)=\frac{1359}{4352}, d_{4,0,3}^{\prime}(2)=-\frac{1221}{17408}, d_{4,0,3}^{\prime}(3)=\frac{1}{69632} .
\end{aligned}
$$

Using Theorem 6.3.3 with $k=4$ and $s=3$, we obtain the desired result. 


\section{Chapter 8}

\section{$\delta$ identities}

In Chapter 7, we applied our main results (Theorems 6.1.3, 6.2.3 and 6.3.3) to sums with $8,12,16$ squares. Each result depends on a certain number of the arithmetic functions $\delta(a, b, c ; n)$. However these functions are not always linearly independent over $\mathbb{Q}$.

The purpose of this chapter is to prove several identities between the functions $\delta(a, b, c ; n)$. In Chapter 9 , we use the identities proved in this chapter to simplify the formulae obtain in Chapter 7.

In Section 1, we state and prove all the results that we need in order to prove these identities. In Section 2, we prove an identity needed for the sums of 4 squares. In Section 3, we prove all the needed identities for sums with 8 squares. In Section 4 , we prove all the needed identities for sums with 12 squares. In Section 5, we prove all the needed identities for the sums with 16 squares. In Section 6, we state and prove several lemmas in order to prove one special identity.

We recall that the set $D$ is defined by

$$
D:=\{q \in \mathbb{C}|| q \mid<1\},
$$


and that the set $D_{\delta}$ is defined by

$$
D_{\delta}:=\{q \in \mathbb{C}|| q \mid<\delta\}
$$

where the number $\delta$ was defined in Lemma 2.4.2.

\subsection{Change of sign, duplication and rotation prin- ciples for $\Delta(a, b, c ; q)$}

For $q \in D$ define $x$ and $z$ as in Definition 2.3.2. In this section, we apply the change of sign, the duplication and the rotation principles to obtain formulae for $\Delta(a, b, c ;-q), \Delta\left(a, b, c ; q^{2}\right), \Delta\left(a, b, c ;-q^{2}\right), \Delta(a, b, c ; i q)$ and $\Delta(a, b, c ;-i q)$, in terms of $x$ and $z$.

We also give formulae for the Eisenstein series $E_{k}(i q)$ and $E_{k}(-i q)$ when $k=2,3$ and 4.

Theorem 8.1.1. For $q \in D_{\delta}$ and $x$ and $z$ as defined in Definition 2.3.2, we have:

(i) if $q \rightarrow-q$ then $\frac{x^{r}(1-x)^{s}}{2^{4 r}} z^{2 t} \longrightarrow \frac{(-1)^{r} x^{r}(1-x)^{t-r-s}}{2^{4 r}} z^{2 t}$,

(ii) if $q \rightarrow q^{2}$ then

$$
\frac{x^{r}(1-x)^{s}}{2^{4 r}} z^{2 t} \longrightarrow \frac{(1-x)^{s / 2}(1-\sqrt{1-x})^{2 r}(1+\sqrt{1-x})^{2 t-2 r-2 s}}{2^{4 r-2 s+2 t}} z^{2 t},
$$

(iii) if $q \rightarrow-q^{2}$ then

$$
\frac{x^{r}(1-x)^{s}}{2^{4 r}} z^{2 t} \longrightarrow \frac{(-1)^{r}(1-x)^{\frac{t-r-s}{2}}(1-\sqrt{1-x})^{2 r}(1+\sqrt{1-x})^{2 s}}{2^{6 r+2 s}} z^{2 t} .
$$


Proof. (i) By Theorem 2.6.1, we have that if $q \rightarrow-q$ then $x \rightarrow \frac{x}{x-1}$ and $z \rightarrow$ $\sqrt{1-x} z$. Hence, if $q \rightarrow-q$ we have

$$
\begin{gathered}
\frac{x^{r}(1-x)^{s}}{2^{4 r}} z^{2 t} \longrightarrow \frac{\left(\frac{x}{x-1}\right)^{r}\left(1-\frac{x}{x-1}\right)^{s}(\sqrt{1-x} z)^{2 t}}{2^{4 r}}=\frac{\frac{(-1)^{r} x^{r}}{(1-x)^{r}}\left(\frac{-1}{x-1}\right)^{s}(1-x)^{t} z^{2 t}}{2^{4 r}} \\
=\frac{\frac{(-1)^{r} x^{r}}{(1-x)^{r}}\left(\frac{1}{1-x}\right)^{s}(1-x)^{t} z^{2 t}}{2^{4 r}}=\frac{(-1)^{r} x^{r}(1-x)^{t-r-s}}{2^{4 r}} z^{2 t} .
\end{gathered}
$$

(ii) By Theorem 2.5.1, we have that if $q \rightarrow q^{2}$, then $x \rightarrow\left(\frac{1-\sqrt{1-x}}{1+\sqrt{1-x}}\right)^{2}$ and $z \rightarrow\left(\frac{1+\sqrt{1-x}}{2}\right) z$. Hence, if $q \rightarrow q^{2}$, we have

$$
\begin{aligned}
\frac{x^{r}(1-x)^{s}}{2^{4 r}} z^{2 t} & \longrightarrow \frac{\left(\frac{1-\sqrt{1-x}}{1+\sqrt{1-x}}\right)^{2 r}\left(1-\left(\frac{1-\sqrt{1-x}}{1+\sqrt{1-x}}\right)^{2}\right)^{s}\left(\frac{1+\sqrt{1-x}}{2}\right)^{2 t} z^{2 t}}{2^{4 r}} \\
& =\frac{\frac{(1-\sqrt{1-x})^{2 r}}{(1+\sqrt{1-x})^{2 r}}\left(\frac{(1+\sqrt{1-x})^{2}-(1-\sqrt{1-x})^{2}}{(1+\sqrt{1-x})^{2}}\right)^{s} \frac{(1+\sqrt{1-x})^{2 t}}{2^{2 t}} z^{2 t}}{2^{4 r}} \\
& =\frac{\frac{(1-\sqrt{1-x})^{2 r}}{(1+\sqrt{1-x})^{2 r}} \frac{\left(4(1-x)^{1 / 2}\right)^{s}}{(1+\sqrt{1-x})^{2 s}}(1+\sqrt{1-x})^{2 t} z^{2 t}}{2^{4 r+2 t}} \\
& =\frac{(1-\sqrt{1-x})^{2 r} 2^{2 s}(1-x)^{x / 2}(1+\sqrt{1-x})^{2 t-2 r-2 s}}{2^{4 r+2 t-2 s}} z^{2 t} \\
= & \frac{(1-x)^{s / 2}(1-\sqrt{1-x})^{2 r}(1+\sqrt{1-x})^{2 t-2 r-2 s}}{2^{4 r+2 t-2 s}} z^{2 t}
\end{aligned}
$$

(iii) By (i) we have that if $q \rightarrow-q$, then $\frac{x^{r}(1-x)^{s}}{2^{4 r}} z^{2 t} \longrightarrow \frac{(-1)^{r} x^{r}(1-x)^{t-r-s}}{2^{4 r}} z^{2 t}$. 
If $q \rightarrow q^{2}$, then by Theorem 2.5.1 we have

$$
\begin{aligned}
\frac{(-1)^{r} x^{r}(1-x)^{t-r-s}}{2^{4 r}} z^{2 t} \rightarrow & \frac{(-1)^{r}\left(\frac{1-\sqrt{1-x}}{1+\sqrt{1-x}}\right)^{2 r}\left(1-\left(\frac{1-\sqrt{1-x}}{1+\sqrt{1-x}}\right)^{2}\right)^{t-r-s}\left(\frac{1+\sqrt{1-x}}{2}\right)^{2 t} z^{2 t}}{2^{4 r}} \\
& =\frac{(-1)^{r} \frac{(1-\sqrt{1-x})^{2 r}}{(1+\sqrt{1-x})^{2 r}} \frac{(4 \sqrt{1-x})^{t-r-s}}{(1+\sqrt{1-x})^{2 t-2 r-2 s}}(1+\sqrt{1-x})^{2 t} z^{2 t}}{2^{4 r+2 t}} \\
& =\frac{(-1)^{r}(1-x)^{\frac{t-r-s}{2}}(1-\sqrt{1-x})^{2 r}(1+\sqrt{1-x})^{2 s} z^{2 t}}{2^{6 r+2 s}}
\end{aligned}
$$

which proves (iii).

Next we prove the following lemma. We require this lemma in order to apply the rotation principle to $\Delta(a, b, c ; q)$.

Lemma 8.1.1. For $q \in D_{\delta}, x$ and $z$ as defined in Definition 2.3 .2 and $s \in\left\{\frac{1}{4}, \frac{1}{2}, \frac{3}{4}, 1\right\}$, we have

$$
\left(\frac{\left(1+i(1-x)^{1 / 4}\right)^{4}}{\left(1-i(1-x)^{1 / 4}\right)^{4}}\right)^{s}=a \frac{\left(1+i(1-x)^{1 / 4}\right)^{4 s}}{\left(1-i(1-x)^{1 / 4}\right)^{4 s}}
$$

where

$$
a=\left\{\begin{aligned}
-i, & \text { if } s=\frac{1}{4}, \\
-1, & \text { if } s=\frac{1}{2}, \\
i, & \text { if } s=\frac{3}{4}, \\
1, & \text { if } s=1 .
\end{aligned}\right.
$$

Proof. From Theorem 2.4.6(ii) we have

$$
(1-x(i q))^{1 / 4}=-i \frac{1+i(1-x)^{1 / 4}}{1-i(1-x)^{1 / 4}}
$$


Raising each side of (8.1.1) to the power 4 we obtain

$$
1-x(i q)=\frac{\left(1+i(1-x)^{1 / 4}\right)^{4}}{\left(1-i(1-x)^{1 / 4}\right)^{4}}
$$

Raising each side of equation (8.1.2) to the power $\frac{1}{4}$ we obtain

$$
(1-x(i q))^{1 / 4}=\left(\frac{\left(1+i(1-x)^{1 / 4}\right)^{4}}{\left(1-i(1-x)^{1 / 4}\right)^{4}}\right)^{1 / 4}
$$

Equating (8.1.1) and (8.1.3) we obtain

$$
\left(\frac{\left(1+i(1-x)^{1 / 4}\right)^{4}}{\left(1-i(1-x)^{1 / 4}\right)^{4}}\right)^{1 / 4}=-i \frac{1+i(1-x)^{1 / 4}}{1-i(1-x)^{1 / 4}},
$$

which is the desired result for $s=1 / 4$. Raising (8.1.4) to the powers 2,3 and 4 , we obtain the other results.

Theorem 8.1.2. For $q \in D_{\delta}, x$ and $z$ as defined in Definition 2.3 .2 and $s \in\left\{\frac{1}{4}, \frac{1}{2}, \frac{3}{4}, 1\right\}$, we have

(i) If $q \rightarrow i q$, then

$$
\begin{aligned}
& \frac{x^{r}(1-x)^{s}}{2^{4 t}} z^{2 t} \longrightarrow \\
& \frac{(-1)^{r+t} i^{r} a(1-x)^{r / 4}(1-\sqrt{1-x})^{r}\left(1+i(1-x)^{1 / 4}\right)^{4 s}\left(1-i(1-x)^{1 / 4}\right)^{4 t-4 r-4 s}}{2^{r+2 t}} z^{2 t} .
\end{aligned}
$$

(ii) If $q \rightarrow-i q$, then

$$
\frac{x^{r}(1-x)^{s}}{2^{4 t}} z^{2 t} \longrightarrow
$$




$$
\begin{aligned}
& \frac{1}{2^{r+2 t}}\left((-1)^{5 t-2 r-4 s} i^{4 t-3 r} a(1-x)^{r / 4}(1-\sqrt{1-x})^{r}\right. \\
& \left.\quad \times\left(1+i(1-x)^{1 / 4}\right)^{4 t-4 r-4 s}\left(1-i(1-x)^{1 / 4}\right)^{4 s}\right) z^{2 t}
\end{aligned}
$$

where

$$
a=\left\{\begin{aligned}
-i, & \text { if } s=\frac{1}{4} \\
-1, & \text { if } s=\frac{1}{2} \\
i, & \text { if } s=\frac{3}{4} \\
1, & \text { if } s=1 .
\end{aligned}\right.
$$

Proof. (i) By Theorem 2.7.1, we have that if $q \rightarrow i q$ then

$$
x \rightarrow \frac{-8 i(1-x)^{1 / 4}(1-\sqrt{1-x})}{\left(1-i(1-x)^{1 / 4}\right)^{4}}
$$

and

$$
z \rightarrow i \frac{z}{2}\left(1-i(1-x)^{1 / 4}\right)^{2} .
$$

Hence, if $q \rightarrow i q$, we have by Theorem 2.7.1 and Lemma 8.1.1

$$
\begin{aligned}
& \frac{x^{r}(1-x)^{s}}{2^{4 r}} z^{2 t} \longrightarrow \\
& =\frac{\left(\frac{-8 \imath(1-x)^{1 / 4}(1-\sqrt{1-x})}{\left(1-\imath(1-x)^{1 / 4}\right)^{4}}\right)^{r}\left(1+\frac{8 \imath(1-x)^{1 / 4}(1-\sqrt{1-x})}{\left(1-\imath(1-x)^{1 / 4}\right)^{4}}\right)^{s}\left(i \frac{z}{2}\left(1-i(1-x)^{1 / 4}\right)^{2}\right)^{2 t}}{2^{4 r}}
\end{aligned}
$$$$
=\frac{\left(\frac{(-1)^{r} \imath^{r} 2^{3 r}(1-x)^{r / 4}(1-\sqrt{1-x})^{r}}{\left(1-\imath(1-x)^{1 / 4}\right)^{4 r}}\right)\left(\frac{\left(1+\imath(1-x)^{1 / 4}\right)^{4}}{\left(1-\imath(1-x)^{1 / 4}\right)^{4}}\right)^{s} \frac{(-1)^{t} z^{2 t}\left(1-\imath(1-x)^{1 / 4}\right)^{4 t}}{2^{2 t}}}{2^{4 r}}
$$$$
=\frac{\frac{(-1)^{r+t} \imath^{r}(1-x)^{r / 4}(1-\sqrt{1-x})^{r}}{\left(1-\imath(1-x)^{1 / 4}\right)^{4 \tau}} a \frac{\left(1+\imath(1-x)^{1 / 4}\right)^{4 s}}{\left(1-\imath(1-x)^{1 / 4}\right)^{4 s}}\left(1-i(1-x)^{1 / 4}\right)^{4 t}}{2^{r+2 t}} z^{2 t}
$$ 


$$
=\frac{(-1)^{r+t} i^{r} a(1-x)^{r / 4}(1-\sqrt{1-x})^{r}\left(1+i(1-x)^{1 / 4}\right)^{4 s}\left(1-i(1-x)^{1 / 4}\right)^{4 t-4 r-4 s}}{2^{r+2 t}} z^{2 t}
$$

(ii) By Theorem 2.6.1, we have that if $q \rightarrow-q$ then $x \rightarrow \frac{x}{x-1}$ and $z \rightarrow \sqrt{1-x} z$.

Hence, if $q \rightarrow-q$ we have by (i) and Theorem 2.6 .1 that

$$
\frac{(-1)^{r+t} i^{r} a(1-x)^{r / 4}(1-\sqrt{1-x})^{r}\left(1+i(1-x)^{1 / 4}\right)^{4 s}\left(1-i(1-x)^{1 / 4}\right)^{4 t-4 r-4 s}}{2^{r+2 t}} z^{2 t}
$$

becomes

$$
\frac{(-1)^{r+t} i^{r} a \frac{1}{(1-x)^{r / 4}} \frac{(-1)^{r}}{(1-x)^{r / 2}}(1-\sqrt{1-x})^{r}\left(\frac{(1-x)^{1 / 4}+i}{(1-x)^{1 / 4}}\right)^{4 s}\left(\frac{(1-x)^{1 / 4}-i}{(1-x)^{1 / 4}}\right)^{4 t-4 r-4 s}}{2^{r+2 t}} z^{2 t}
$$$$
=\frac{1}{2^{r+2 t}}\left((-1)^{2 r+t} i^{r} a(1-x)^{r / 4}(1-\sqrt{1-x})^{r}(i)^{4 s}\left(1-i(1-x)^{1 / 4}\right)^{4 s}\right.
$$$$
\left.\times(-i)^{4 t-4 r-4 s}\left(1+i(1-x)^{1 / 4}\right)^{4 t-4 r-4 s}\right) z^{2 t}
$$

$$
\begin{aligned}
=\frac{1}{2^{r+2 t}}( & (-1)^{5 t-2 r-4 s}(i)^{4 t-3 r} a(1-x)^{r / 4}(1-\sqrt{1-x})^{r} \\
& \left.\times\left(1+i(1-x)^{1 / 4}\right)^{4 t-4 r-4 s}\left(1-i(1-x)^{1 / 4}\right)^{4 s}\right) z^{2 t}
\end{aligned}
$$

which is the asserted result

In the next theorem we express the quantity $E_{1}(i q)-E_{1}(-i q)$ in terms of $x$ and $z$. 
Theorem 8.1.3. Let $q \in D_{\delta}$. Define $x$ and $z$ as in Definition 2.3 .2 and the Eisenstein series $E_{1}(q)$ as in Definition 2.9.1. Then

$$
E_{1}(i q)-E_{1}(-i q)=-6 i\left((1-x)^{1 / 4}-(1-x)^{3 / 4}\right) z^{2}
$$

Proof. Using the rotation principle (Theorem 2.7.1) in Theorem 2.9.6 (iii), we obtain for $q \in D_{\delta}$

$$
\begin{aligned}
E_{1}(i q)-E_{1}(-i q) & =-3\left(\frac{-8 i(1-x)^{1 / 4}\left(1-(1-x)^{1 / 2}\right)}{\left(1-i(1-x)^{1 / 4}\right)^{4}}\right)\left(i \frac{z}{2}\left(1-i(1-x)^{1 / 4}\right)^{2}\right)^{2} \\
& =-6 i(1-x)^{1 / 4}\left(1-(1-x)^{1 / 2}\right) z^{2}
\end{aligned}
$$

which is the desired result.

We conclude this section with formulae for the Eisenstein series $E_{k}(i q)$ and $E_{k}(-i q)$ for $k \geq 2$.

Theorem 8.1.4. For $q \in D_{\delta}, x$ and $z$ as defined in Definition 2.3 .2 and the Eisenstein series $E_{2}(q)$ and $E_{3}(q)$ as in Definition 2.9.1, we have

(i) $E_{2}(i q)=\frac{1}{16}\left(1-120 i g-540 g^{2}+840 i g^{3}+1094 g^{4}\right.$

$$
\left.-840 i g^{5}-540 g^{6}+120 i g^{7}+g^{8}\right) z^{4},
$$

(ii) $E_{2}(-i q)=\frac{1}{16}\left(1+120 i g-540 g^{2}-840 i g^{3}+1094 g^{4}\right.$

$$
\left.+840 i g^{5}-540 g^{6}-120 i g^{7}+g^{8}\right) z^{4},
$$

(iii) $E_{3}(i q)=\frac{1}{64}\left(1-12 i g-6 g^{2}+12 i g^{3}+g^{4}\right)$ 


$$
\times\left(-1-264 i g-996 g^{2}+1848 i g^{3}+1978 g^{4}-1848 i g^{5}-996 g^{6}+264 i g^{7}-g^{8}\right) z^{6},
$$

(iv) $E_{3}(-i q)=\frac{1}{64}\left(1+12 i g-6 g^{2}-12 i g^{3}+g^{4}\right)$

$$
\times\left(-1+264 i g-996 g^{2}-1848 i g^{3}+1978 g^{4}+1848 i g^{5}-996 g^{6}-264 i g^{7}-g^{8}\right) z^{6},
$$

(v) $E_{4}(i q)=\left(\frac{1}{16}\left(1-120 i g-540 g^{2}+840 i g^{3}+1094 g^{4}\right.\right.$

$$
\left.\left.-840 i g^{5}-540 g^{6}+120 i g^{7}+g^{8}\right)\right)^{2} z^{8}
$$

(vi) $E_{4}(-i q)=\left(\frac{1}{16}\left(1+120 i g-540 g^{2}-840 i g^{3}+1094 g^{4}\right.\right.$

$$
\left.\left.+840 i g^{5}-540 g^{6}-120 i g^{7}+g^{8}\right)\right)^{2} z^{8}
$$

where $g=(1-x)^{1 / 4}$.

Proof. Results (i)-(iv) were found in [28]. (v) and (vi) follow by Theorem 2.9 .1 and (i) to (iv).

\subsection{Identity for the sums of 4 squares}

In this section we state and prove an identity relating the functions $\delta\left(-\frac{1}{6}, \frac{7}{12},-\frac{1}{4} ; n\right)$ and $\delta\left(\frac{1}{6}, \frac{1}{12},-\frac{1}{12} ; n\right)$, which occur in the formulae (6.3.2), (6.3.3) and (6.3.4), to the function $\sigma(n)$.

Theorem 8.2.1. For $n \in \mathbb{N}$, we have

$$
\left(\frac{-4}{n}\right) \sigma(n)=\frac{1}{8} \delta\left(-\frac{1}{6}, \frac{7}{12},-\frac{1}{4} ; n\right)-\frac{1}{8} \delta\left(-\frac{1}{6}, \frac{1}{12},-\frac{1}{12} ; n\right) .
$$


Proof. Let $q \in D_{\delta}$. As

$$
\begin{aligned}
\frac{i^{n}-(-i)^{n}}{2 i}= \begin{cases}1, & \text { if } n \equiv 1(\bmod 4) \\
-1, & \text { if } n \equiv 3(\bmod 4) \\
0, & \text { if } n \equiv 0(\bmod 4)\end{cases} \\
=\left(\frac{-4}{n}\right)
\end{aligned}
$$

we have

$$
\begin{aligned}
\frac{E_{1}(i q)-E_{1}(-i q)}{2 i} & =\frac{1}{2 i}\left(\left(1-24 \sum_{n=1}^{+\infty} \sigma(n)(i q)^{n}\right)-\left(1-24 \sum_{n=1}^{+\infty} \sigma(n)(-i q)^{n}\right)\right) \\
& =-24 \sum_{n=1}^{+\infty} \sigma(n)\left(\frac{i^{n}-(-i)^{n}}{2 i}\right) q^{n} \\
& =-24 \sum_{n=1}^{+\infty}\left(\frac{-4}{n}\right) \sigma(n) q^{n} .
\end{aligned}
$$

Moreover, by Theorem 8.1.3, we have

$$
\begin{aligned}
& \frac{E_{1}(i q)-E_{1}(-i q)}{2 i}=\frac{-6 i(1-x)^{1 / 4}\left(1-(1-x)^{1 / 2}\right) z^{2}}{2 i} \\
& =-3(1-x)^{1 / 4} z^{2}+3(1-x)^{3 / 4} z^{2}=-3 \Delta\left(-\frac{1}{6}, \frac{7}{12},-\frac{1}{4} ; q\right)+3 \Delta\left(\frac{1}{6}, \frac{1}{12},-\frac{1}{12} ; q\right) \\
& =\sum_{n=1}^{+\infty}\left(-3 \delta\left(-\frac{1}{6}, \frac{7}{12},-\frac{1}{4} ; n\right)+3 \delta\left(\frac{1}{6}, \frac{1}{12},-\frac{1}{12} ; n\right)\right) q^{n} .
\end{aligned}
$$

Equating coefficients and dividing both sides by -24 , we obtain the asserted identity. 


\subsection{Identities for the sums of 8 squares}

In this section we state and prove four identities relating the functions $\delta(a, b, c ; n)$ obtained in Theorems 7.1.1,7.1.2 and 7.1.3.

Theorem 8.3.1. For $n \in \mathbb{N}$, we have

$$
\delta\left(0, \frac{1}{6}, \frac{1}{6} ; n\right)=0, \text { if } n \equiv 0(\bmod 2)
$$

Proof. Let $q \in D_{\delta}$. By Theorem 2.10.2(ii) and Theorem 8.1.1(i) we have

$$
\begin{aligned}
\sum_{n=1}^{+\infty} \delta\left(0, \frac{1}{6}, \frac{1}{6} ; 2 n\right) q^{2 n}= & \frac{1}{2}\left(\sum_{n=1}^{+\infty} \delta\left(0, \frac{1}{6}, \frac{1}{6} ; n\right) q^{n}\right. \\
& \left.+\sum_{n=1}^{+\infty} \delta\left(0, \frac{1}{6}, \frac{1}{6} ; n\right)(-q)^{n}\right) \\
= & \frac{1}{2}\left(\Delta\left(0, \frac{1}{6}, \frac{1}{6} ; q\right)+\Delta\left(0, \frac{1}{6}, \frac{1}{6} ;-q\right)\right) \\
= & \frac{1}{2}\left(\frac{x \sqrt{1-x}}{16} z^{4}-\frac{x \sqrt{1-x}}{16} z^{4}\right)=0 .
\end{aligned}
$$

Equating coefficients we obtain $\delta\left(0, \frac{1}{6}, \frac{1}{6} ; n\right)=0$ if $n \equiv 0(\bmod 2)$.

Theorem 8.3.2. For $n \in \mathbb{N}$, we have

$$
\delta\left(0, \frac{1}{6}, \frac{1}{6} ; n / 2\right)=\frac{1}{8}\left(\delta\left(-\frac{1}{6}, \frac{5}{12}, \frac{1}{12} ; n\right)-\delta\left(\frac{1}{6},-\frac{1}{12}, \frac{1}{4} ; n\right)\right)
$$

Proof. Let $q \in D_{\delta}$. By Theorem 2.10.2(ii) we have $\Delta\left(0, \frac{1}{6}, \frac{1}{6} ; q\right)=\frac{x(1-x)^{1 / 2}}{16} z^{4}$.

By Theorem 8.1.1(ii)

$$
\Delta\left(0, \frac{1}{6}, \frac{1}{6} ; q^{2}\right)=\frac{(1-x)^{1 / 4}(1-\sqrt{1-x})^{2}(1+\sqrt{1-x})}{2^{7}} z^{4}
$$




$$
\begin{aligned}
& =\frac{x(1-x)^{1 / 4}(1-\sqrt{1-x})}{2^{7}} z^{4}=\frac{x(1-x)^{1 / 4}}{2^{7}} z^{4}-\frac{x(1-x)^{3 / 4}}{2^{7}} z^{4} \\
& =\frac{1}{8}\left(\Delta\left(-\frac{1}{6}, \frac{5}{12}, \frac{1}{12} ; q\right)-\Delta\left(\frac{1}{6},-\frac{1}{12}, \frac{1}{4} ; q\right)\right) .
\end{aligned}
$$

This implies that

$$
\sum_{n=1}^{+\infty} \delta\left(0, \frac{1}{6}, \frac{1}{6} ; n\right) q^{2 n}=\frac{1}{8} \sum_{n=1}^{+\infty}\left(\delta\left(-\frac{1}{6}, \frac{5}{12}, \frac{1}{12} ; n\right)-\delta\left(\frac{1}{6},-\frac{1}{12}, \frac{1}{4} ; n\right)\right) q^{n},
$$

for all $q \in D_{\delta}$. Equating the coefficients of $q^{n}$, we obtain

$$
\delta\left(0, \frac{1}{6}, \frac{1}{6} ; n / 2\right)=\frac{1}{8}\left(\delta\left(-\frac{1}{6}, \frac{5}{12}, \frac{1}{12} ; n\right)-\delta\left(\frac{1}{6},-\frac{1}{12}, \frac{1}{4} ; n\right)\right) .
$$

Theorem 8.3.3. For $n \in \mathbb{N}$, we have

$$
\left(\frac{-4}{n}\right) \delta\left(0, \frac{1}{6}, \frac{1}{6} ; n\right)=\frac{1}{2}\left(\delta\left(\frac{1}{6},-\frac{1}{12}, \frac{1}{4} ; n\right)+\delta\left(-\frac{1}{6}, \frac{5}{12}, \frac{1}{12} ; n\right)\right) .
$$

Proof. Let $q \in D_{\delta}$. By Theorem 2.10.2(ii) we have $\Delta\left(0, \frac{1}{6}, \frac{1}{6} ; q\right)=\frac{x(1-x)^{1 / 2}}{16} z^{4}$. By Theorem 8.1.2(i) we have

$$
\begin{aligned}
& \Delta\left(0, \frac{1}{6}, \frac{1}{6} ; i q\right) \\
& =\frac{(-1)^{4} i(1-x)^{1 / 4}(1-\sqrt{1-x})\left(1+i(1-x)^{1 / 4}\right)^{2}\left(1-i(1-x)^{1 / 4}\right)^{2}}{2^{5}} z^{4} \\
& =\frac{i(1-x)^{1 / 4}(1-\sqrt{1-x})(1+\sqrt{1-x})^{2}}{32} z^{4}=\frac{i x(1-x)^{1 / 4}(1+\sqrt{1-x})}{32} z^{4}
\end{aligned}
$$

and by Theorem 8.1.2(ii)

$$
\Delta\left(0, \frac{1}{6}, \frac{1}{6} ;-i q\right)
$$




$$
\begin{aligned}
& =\frac{(-1)^{5} i(1-x)^{1 / 4}(1-\sqrt{1-x})\left(1+i(1-x)^{1 / 4}\right)^{2}\left(1-i(1-x)^{1 / 4}\right)^{2}}{2^{5}} z^{4} \\
& =\frac{i(1-x)^{1 / 4}(1-\sqrt{1-x})(1+\sqrt{1-x})^{2}}{32} z^{4}=\frac{-i x(1-x)^{1 / 4}(1+\sqrt{1-x})}{32} z^{4} .
\end{aligned}
$$

Recall that

$$
\frac{i^{n}-(-i)^{n}}{2 i}=\left(\frac{-4}{n}\right)
$$

We have

$$
\begin{array}{rl}
\sum_{n=1}^{+\infty}\left(\frac{-4}{n}\right) & \delta\left(0, \frac{1}{6}, \frac{1}{6} ; n\right) q^{n}=\sum_{n=1}^{+\infty} \delta\left(0, \frac{1}{6}, \frac{1}{6} ; n\right)\left(\frac{i^{n}-(-i)^{n}}{2 i}\right) q^{n} \\
& =\frac{1}{2 i}\left(\sum_{n=1}^{+\infty} \delta\left(0, \frac{1}{6}, \frac{1}{6} ; n\right)(i q)^{n}-\sum_{n=1}^{+\infty} \delta\left(0, \frac{1}{6}, \frac{1}{6} ; n\right)(-i q)^{n}\right) \\
& =\frac{1}{2 i}\left(\Delta\left(0, \frac{1}{6}, \frac{1}{6} ; i q\right)-\Delta\left(0, \frac{1}{6}, \frac{1}{6} ;-i q\right)\right) \\
& =\frac{1}{2 i}\left(\frac{i x(1-x)^{1 / 4}(1+\sqrt{1-x})}{32} z^{4}+\frac{i x(1-x)^{1 / 4}(1+\sqrt{1-x})}{32} z^{4}\right) \\
& =\frac{1}{2 i}\left(\frac{i x(1-x)^{1 / 4}(1+\sqrt{1-x})}{16}\right) z^{4}=\frac{x(1-x)^{1 / 4}(1+\sqrt{1-x})}{32} z^{4} \\
& =\frac{1}{2}\left(\Delta\left(\frac{1}{6},-\frac{1}{12}, \frac{1}{4} ; q\right)+\Delta\left(-\frac{1}{6}, \frac{5}{12}, \frac{1}{12} ; q\right)\right) \\
32 & x(1-x)^{1 / 4} \\
z^{4}+\frac{x(1-x)^{3 / 4}}{32} z^{4}
\end{array}
$$




$$
=\frac{1}{2} \sum_{n=1}^{+\infty}\left(\delta\left(\frac{1}{6},-\frac{1}{12}, \frac{1}{4} ; n\right)+\delta\left(-\frac{1}{6}, \frac{5}{12}, \frac{1}{12} ; n\right)\right) q^{n} .
$$

Equating the coefficients of $q^{n}(n \in \mathbb{N})$, we obtain the asserted identity.

Theorem 8.3.4. For $n \in \mathbb{N}$, we have

$$
\begin{aligned}
\left(\frac{-4}{n}\right) \sigma_{3}(n)= & -\frac{1}{4} \delta\left(-\frac{1}{2}, \frac{17}{12},-\frac{7}{12} ; n\right)+\frac{1}{4} \delta\left(-\frac{1}{6}, \frac{11}{12},-\frac{5}{12} ; n\right) \\
& +\frac{7}{2} \delta\left(-\frac{1}{6}, \frac{5}{12}, \frac{1}{12} ; n\right)-\frac{1}{2} \delta\left(\frac{1}{6},-\frac{1}{12}, \frac{1}{4} ; n\right) .
\end{aligned}
$$

Proof. Let $q \in D_{\delta}$. As

$$
\frac{i^{n}-(-i)^{n}}{2 i}=\left(\frac{-4}{n}\right)
$$

we have

$$
\begin{aligned}
\frac{E_{2}(i q)-E_{2}(-i q)}{2 i} & =\frac{1}{2 i}\left(\left(1+240 \sum_{n=1}^{+\infty} \sigma_{3}(n)(i q)^{n}\right)-\left(1+240 \sum_{n=1}^{+\infty} \sigma_{3}(n)(-i q)^{n}\right)\right) \\
& =240 \sum_{n=1}^{+\infty} \sigma_{3}(n)\left(\frac{i^{n}-(-i)^{n}}{2 i}\right) q^{n} \\
& =240 \sum_{n=1}^{+\infty}\left(\frac{-4}{n}\right) \sigma_{3}(n) q^{n} .
\end{aligned}
$$

Moreover, by Theorem 8.1.4(i) and (ii), we have

$$
\begin{aligned}
& \frac{E_{2}(i q)-E_{2}(-i q)}{2 i}=-\frac{15}{4} g z^{4}+\frac{105}{2} g^{3} z^{4}-\frac{105}{2} g^{5} z^{4}+\frac{15}{2} g^{7} z^{4} \\
& =-\frac{15}{4}(1-x)^{1 / 4} z^{4}+\frac{105}{2}(1-x)^{3 / 4} z^{4}-\frac{105}{2}(1-x)^{5 / 4} z^{4}+\frac{15}{2}(1-x)^{7 / 4} z^{4}
\end{aligned}
$$




$$
\begin{aligned}
= & -\frac{15}{2}(1-x)^{1 / 4} z^{4}+\frac{105}{2}(1-x)^{3 / 4} z^{4}-\frac{105}{2}(1-x)^{1 / 4} z^{4}+\frac{105}{2} x(1-x)^{1 / 4} z^{4} \\
& +\frac{15}{2}(1-x)^{3 / 4} z^{4}-\frac{15}{2} x(1-x)^{3 / 4} z^{4} \\
= & -60(1-x)^{1 / 4} z^{4}+60(1-x)^{3 / 4} z^{4}+\frac{105}{2} x(1-x)^{1 / 4} z^{4}-\frac{15}{2} x(1-x)^{3 / 4} z^{4} \\
= & -60 \Delta\left(-\frac{1}{2}, \frac{17}{12},-\frac{7}{12} ; q\right)+60 \Delta\left(-\frac{1}{6}, \frac{11}{12},-\frac{5}{12} ; q\right) \\
+ & 840 \Delta\left(-\frac{1}{6}, \frac{5}{12}, \frac{1}{12} ; q\right)-120 \Delta\left(\frac{1}{6},-\frac{1}{12}, \frac{1}{4} ; q\right) \\
= & \sum_{n=1}^{+\infty}\left(-60 \delta\left(-\frac{1}{2}, \frac{17}{12},-\frac{7}{12} ; n\right)+60 \delta\left(-\frac{1}{6}, \frac{11}{12},-\frac{5}{12} ; n\right)\right. \\
& \left.+840 \delta\left(-\frac{1}{6}, \frac{5}{12}, \frac{1}{12} ; n\right)-120 \delta\left(\frac{1}{6},-\frac{1}{12}, \frac{1}{4} ; n\right)\right) q^{n} .
\end{aligned}
$$

Equating coefficients and dividing both sides by 240 , we obtain the asserted identity.

\subsection{Identities for the sums of 12 squares}

In this section we state and prove eight identities relating the functions $\delta(a, b, c ; n)$ obtained in Theorems 7.2.1,7.2.2 and 7.2.3.

Theorem 8.4.1. For $n \in \mathbb{N}$, we have

$$
\delta\left(0, \frac{1}{2}, 0 ; n\right)=0, \text { if } n \equiv 0(\bmod 2) .
$$


Proof. Let $q \in D_{\delta}$. By Theorem 2.10.2(ii) and Theorem 8.1.1(i), we have

$$
\begin{aligned}
2 \sum_{n=1}^{+\infty} \delta\left(0, \frac{1}{2}, 0 ; 2 n\right) q^{2 n} & =\sum_{n=1}^{\infty} \delta\left(0, \frac{1}{2}, 0 ; n\right) q^{n}+\sum_{n=1}^{\infty} \delta\left(0, \frac{1}{2}, 0 ; n\right)(-q)^{n} \\
& =\Delta\left(0, \frac{1}{2}, 0 ; q\right)+\Delta\left(0, \frac{1}{2}, 0 ;-q\right) \\
& =\frac{x(1-x)}{16} z^{6}-\frac{x(1-x)}{16} z^{6}=0 .
\end{aligned}
$$

Equating coefficients we obtain $\delta\left(0, \frac{1}{2}, 0 ; n\right)=0$ if $n \equiv 0(\bmod 2)$.

Theorem 8.4.2. For $n \in \mathbb{N}$, we have

$$
\begin{aligned}
\delta\left(-\frac{1}{3}, 1,-\frac{1}{6} ; n\right) & =8 \delta\left(0,0, \frac{1}{2} ; n\right)=8 \delta\left(0, \frac{1}{2}, 0 ; \frac{n}{2}\right)=8 \tau_{2}\left(\frac{n}{2}\right) \\
& =4 \sum_{\substack{\left(x_{1}, x_{2}, x_{3}, x_{4}\right) \in \mathbb{Z}^{4} \\
x_{1}^{2}+x_{2}^{2}+x_{3}^{2}+x_{4}^{2}=\frac{n}{2}}}\left(x_{1}^{4}-3 x_{1}^{2} x_{2}^{2}\right), \text { if } n \equiv 0(\bmod 2) .
\end{aligned}
$$

Proof. Let $q \in D_{\delta}$. By Theorem 2.10.2(ii) and Theorem 8.1.1(i) we have

$$
\begin{aligned}
\sum_{n=1}^{+\infty} \delta\left(-\frac{1}{3}, 1,-\frac{1}{6} ; 2 n\right) q^{2 n} & =\frac{1}{2}\left(\delta\left(-\frac{1}{3}, 1,-\frac{1}{6} ; n\right) q^{n}+\sum_{n=1}^{+\infty} \delta\left(-\frac{1}{3}, 1,-\frac{1}{6} ; n\right)(-q)^{n}\right) \\
& =\frac{1}{2}\left(\Delta\left(-\frac{1}{3}, 1,-\frac{1}{6} ; q\right)+\Delta\left(-\frac{1}{3}, 1,-\frac{1}{6} ;-q\right)\right) \\
& =\frac{1}{2}\left(\frac{x(1-x)^{1 / 2}}{16} z^{6}-\frac{x(1-x)^{3 / 2}}{16} z^{6}\right)=\frac{x^{2}(1-x)^{1 / 2}}{32} z^{6}
\end{aligned}
$$

and

$$
8 \sum_{n=1}^{+\infty} \delta\left(0,0, \frac{1}{2} ; 2 n\right) q^{2 n}=8\left(\frac{1}{2}\left(\sum_{n=1}^{+\infty} \delta\left(0,0, \frac{1}{2} ; n\right) q^{n}+\sum_{n=1}^{+\infty} \delta\left(0,0, \frac{1}{2} ; n\right)(-q)^{n}\right)\right)
$$




$$
\begin{aligned}
& =4\left(\Delta\left(0,0, \frac{1}{2} ; q\right)+\Delta\left(0,0,-\frac{1}{2} ;-q\right)\right) \\
& =4\left(\frac{x^{2}(1-x)^{1 / 2}}{256} z^{6}+\frac{x^{2}(1-x)^{1 / 2}}{256} z^{6}\right)=\frac{x^{2}(1-x)^{1 / 2}}{32} z^{6} .
\end{aligned}
$$

This implies

$$
\sum_{n=1}^{+\infty} \delta\left(-\frac{1}{3}, 1,-\frac{1}{6} ; 2 n\right) q^{2 n}=\sum_{n=1}^{+\infty} \delta\left(0,0, \frac{1}{2} ; 2 n\right) q^{2 n} .
$$

Equating coefficients, we obtain the asserted identity.

Theorem 8.4.3. For $n \in \mathbb{N}$, we have

$$
\delta\left(-\frac{1}{3}, 1,-\frac{1}{6} ; n\right)=0, \text { if } n \equiv 0(\bmod 4) .
$$

Proof. Let $n \in \mathbb{N}$. If $n \equiv 0(\bmod 4)$, by Theorem 8.4 .2 , we have $\delta\left(-\frac{1}{3}, 1,-\frac{1}{6} ; n\right)=8 \tau_{2}(n / 2) . \quad$ As $\frac{n}{2} \equiv 0(\bmod 2)$, by Theorem 2.11.3, we have $\tau_{2}\left(\frac{n}{2}\right)=0$. Thus $\delta\left(-\frac{1}{3}, 1,-\frac{1}{6} ; n\right)=0$ for $n \equiv 0(\bmod 4)$.

Theorem 8.4.4. For $n \in \mathbb{N}$, we have

$$
\delta\left(\frac{1}{6},-\frac{1}{4}, \frac{7}{12} ; n\right)=\delta\left(-\frac{1}{3}, 1,-\frac{1}{6} ; n / 2\right), \text { if } n \equiv 0(\bmod 2) .
$$

Proof. Let $q \in D_{\delta}$. By Theorems 2.10.2(ii) and 8.1.1(i), we have

$$
\begin{aligned}
\sum_{n=1}^{+\infty} \delta\left(\frac{1}{6},-\frac{1}{4}, \frac{7}{12} ; 2 n\right) q^{2 n}=\frac{1}{2} & \left(\sum_{n=1}^{+\infty} \delta\left(\frac{1}{6},-\frac{1}{4}, \frac{7}{12} ; n\right) q^{n}\right. \\
& \left.+\sum_{n=1}^{+\infty} \delta\left(\frac{1}{6},-\frac{1}{4}, \frac{7}{12} ; n\right)(-q)^{n}\right)
\end{aligned}
$$




$$
\begin{aligned}
& =\frac{1}{2}\left(\Delta\left(\frac{1}{6},-\frac{1}{4}, \frac{7}{12} ; q\right)+\Delta\left(\frac{1}{6},-\frac{1}{4}, \frac{7}{12} ;-q\right)\right) \\
& =\frac{1}{2}\left(\frac{x^{2}(1-x)^{3 / 4}}{256} z^{6}+\frac{x^{2}(1-x)^{1 / 4}}{256} z^{6}\right) \\
& =\frac{x^{2}(1-x)^{1 / 4}}{512} z^{6}+\frac{x^{2}(1-x)^{3 / 4}}{512} z^{6}
\end{aligned}
$$

and by Theorem 8.1.1(ii)

$$
\begin{aligned}
\sum_{n=1}^{+\infty} \delta\left(-\frac{1}{3}, 1,-\frac{1}{6} ; n\right) q^{2 n} & =\Delta\left(-\frac{1}{3}, 1,-\frac{1}{6} ; q^{2}\right) \\
& =\frac{(1-x)^{1 / 4}(1-\sqrt{1-x})^{2}(1+\sqrt{1-x})^{3}}{512} z^{6} \\
& =\frac{x^{2}(1-x)^{1 / 4}(1+\sqrt{1-x})}{512} z^{6} \\
& =\frac{x^{2}(1-x)^{1 / 4}}{512} z^{6}+\frac{x^{2}(1-x)^{3 / 4}}{512} z^{6} .
\end{aligned}
$$

This implies

$$
\sum_{n=1}^{+\infty} \delta\left(\frac{1}{6},-\frac{1}{4}, \frac{7}{12} ; 2 n\right) q^{2 n}=\sum_{n=1}^{+\infty} \delta\left(-\frac{1}{3}, 1,-\frac{1}{6} ; n\right) q^{2 n} .
$$

Equating coefficients we obtain $\delta\left(\frac{1}{6},-\frac{1}{4}, \frac{7}{12} ; 2 n\right)=\delta\left(-\frac{1}{3}, 1,-\frac{1}{6} ; n\right)$, which is the desired identity.

Theorem 8.4.5. For $n \in \mathbb{N}$, we have

$$
\delta\left(-\frac{1}{6}, \frac{1}{4}, \frac{5}{12} ; n\right)=\delta\left(-\frac{1}{3}, 1,-\frac{1}{6} ; n / 2\right) \text {, if } n \equiv 0(\bmod 2) .
$$


Proof. Let $q \in D_{\delta}$. By Theorem 2.10.2(ii) and Theorem 8.1.1(i), we have

$$
\begin{aligned}
\sum_{n=1}^{+\infty} \delta\left(-\frac{1}{6}, \frac{1}{4}, \frac{5}{12} ; 2 n\right) q^{2 n}= & \frac{1}{2}\left(\sum_{n=1}^{+\infty} \delta\left(-\frac{1}{6}, \frac{1}{4}, \frac{5}{12} ; n\right) q^{n}\right. \\
& \left.+\sum_{n=1}^{+\infty} \delta\left(-\frac{1}{6}, \frac{1}{4}, \frac{5}{12} ; n\right)(-q)^{n}\right) \\
= & \frac{1}{2}\left(\Delta\left(-\frac{1}{6}, \frac{1}{4}, \frac{5}{12} ; q\right)+\Delta\left(-\frac{1}{6}, \frac{1}{4}, \frac{5}{12} ;-q\right)\right) \\
= & \frac{1}{2}\left(\frac{x^{2}(1-x)^{1 / 4}}{256} z^{6}+\frac{x^{2}(1-x)^{3 / 4}}{256} z^{6}\right) \\
= & \frac{x^{2}(1-x)^{1 / 4}}{512} z^{6}+\frac{x^{2}(1-x)^{3 / 4}}{512} z^{6}
\end{aligned}
$$

and by Theorem 8.1.1(ii)

$$
\begin{aligned}
\sum_{n=1}^{+\infty} \delta\left(-\frac{1}{3}, 1,-\frac{1}{6} ; n\right) q^{2 n} & =\Delta\left(-\frac{1}{3}, 1,-\frac{1}{6} ; q^{2}\right) \\
& =\frac{(1-x)^{1 / 4}(1-\sqrt{1-x})^{2}(1+\sqrt{1-x})^{3}}{512} z^{6} \\
& =\frac{x^{2}(1-x)^{1 / 4}(1+\sqrt{1-x})}{512} z^{6} \\
& =\frac{x^{2}(1-x)^{1 / 4}}{512} z^{6}+\frac{x^{2}(1-x)^{3 / 4}}{512} z^{6} .
\end{aligned}
$$

This implies

$$
\sum_{n=1}^{+\infty} \delta\left(-\frac{1}{6}, \frac{1}{4}, \frac{5}{12} ; 2 n\right) q^{2 n}=\sum_{n=1}^{+\infty} \delta\left(-\frac{1}{3}, 1,-\frac{1}{6} ; n\right) q^{2 n}
$$


Equating coefficients we obtain $\delta\left(-\frac{1}{6}, \frac{1}{4}, \frac{5}{12} ; 2 n\right)=\delta\left(-\frac{1}{3}, 1,-\frac{1}{6} ; n\right)$, which is the desired identity.

Theorem 8.4.6. For $n \in \mathbb{N}$, we have

$$
\delta\left(-\frac{1}{2}, \frac{5}{4},-\frac{1}{4} ; n\right)= \begin{cases}20 \delta\left(-\frac{1}{3}, 1,-\frac{1}{6} ; n / 2\right), & \text { if } n \equiv 0(\bmod 4), \\ 12 \delta\left(-\frac{1}{3}, 1,-\frac{1}{6} ; n / 2\right), & \text { if } n \equiv 2(\bmod 4) .\end{cases}
$$

Proof. Let $q \in D_{\delta}$. By Theorem 2.10.2(ii) and Theorem 8.1.1(i), we have

$$
\begin{aligned}
\sum_{n=1}^{+\infty} \delta\left(-\frac{1}{2}, \frac{5}{4},-\frac{1}{4} ; 2 n\right) q^{2 n}= & \frac{1}{2}\left(\sum_{n=1}^{+\infty} \delta\left(-\frac{1}{2}, \frac{5}{4},-\frac{1}{4} ; n\right) q^{n}\right. \\
& \left.+\sum_{n=1}^{+\infty} \delta\left(-\frac{1}{2}, \frac{5}{4},-\frac{1}{4} ; n\right)(-q)^{n}\right) \\
= & \frac{1}{2}\left(\Delta\left(-\frac{1}{2}, \frac{5}{4},-\frac{1}{4} ; q\right)+\Delta\left(-\frac{1}{2}, \frac{5}{4},-\frac{1}{4} ;-q\right)\right) \\
= & \frac{1}{2}\left(\frac{x(1-x)^{1 / 4}}{16} z^{6}-\frac{x(1-x)^{7 / 4}}{16} z^{6}\right) \\
= & \frac{x(1-x)^{1 / 4}}{32} z^{6}-\frac{x(1-x)^{3 / 4}}{32} z^{6}+\frac{x^{2}(1-x)^{3 / 4}}{32} z^{6}
\end{aligned}
$$

and by Theorem 2.10.2(ii) and Theorem 8.1.1(ii)(iii)

$$
\begin{aligned}
& \sum_{n=1}^{+\infty} \delta\left(-\frac{1}{3}, 1,-\frac{1}{6} ; n\right)\left(16+4(-1)^{n}\right) q^{2 n} \\
& =16 \Delta\left(-\frac{1}{3}, 1,-\frac{1}{6} ; q^{2}\right)+4 \Delta\left(-\frac{1}{3}, 1,-\frac{1}{6} ;-q^{2}\right)
\end{aligned}
$$




$$
\begin{aligned}
= & \left(\frac{x^{2}(1-x)^{1 / 4}(1+\sqrt{1-x})}{32} z^{6}-\frac{x(1-x)^{3 / 4}(1-\sqrt{1-x})}{32} z^{6}\right) \\
= & \frac{x^{2}(1-x)^{1 / 4}}{32} z^{6}+\frac{x^{2}(1-x)^{3 / 4}}{32} z^{6}-\frac{x(1-x)^{3 / 4}}{32} z^{6}+\frac{x(1-x)^{5 / 4}}{32} z^{6} \\
= & \frac{x^{2}(1-x)^{1 / 4}}{32} z^{6}+\frac{x^{2}(1-x)^{3 / 4}}{32} z^{6}-\frac{x(1-x)^{3 / 4}}{32} z^{6}+\frac{x(1-x)^{1 / 4}}{32} z^{6} \\
& -\frac{x^{2}(1-x)^{1 / 4}}{32} z^{6} \\
= & \frac{x^{2}(1-x)^{3 / 4}}{32} z^{6}-\frac{x(1-x)^{3 / 4}}{32} z^{6}+\frac{x(1-x)^{1 / 4}}{32} z^{6} .
\end{aligned}
$$

This implies

$$
\sum_{n=1}^{+\infty} \delta\left(-\frac{1}{2}, \frac{5}{4},-\frac{1}{4} ; 2 n\right) q^{2 n}=\sum_{n=1}^{+\infty} \delta\left(-\frac{1}{3}, 1,-\frac{1}{6} ; n\right)\left(16+4(-1)^{n}\right) q^{2 n} .
$$

Equating coefficients we obtain

$$
\delta\left(-\frac{1}{2}, \frac{5}{4},-\frac{1}{4} ; 2 n\right)=\left(16+4(-1)^{n}\right) \delta\left(-\frac{1}{3}, 1,-\frac{1}{6} ; n\right),
$$

which is the desired identity.

Theorem 8.4.7. For $n \in \mathbb{N}$, we have

$$
\delta\left(-\frac{1}{6}, \frac{3}{4},-\frac{1}{12} ; n\right)= \begin{cases}-4 \delta\left(-\frac{1}{3}, 1,-\frac{1}{6} ; n / 2\right), & \text { if } n \equiv 0(\bmod 4), \\ 4 \delta\left(-\frac{1}{3}, 1,-\frac{1}{6} ; n / 2\right), & \text { if } n \equiv 2(\bmod 4) .\end{cases}
$$

Proof. Let $q \in D_{\delta}$. By Theorem 2.10.2(ii) and Theorem 8.1.1(i), we have

$$
\sum_{n=1}^{+\infty} \delta\left(-\frac{1}{6}, \frac{3}{4},-\frac{1}{12} ; 2 n\right) q^{2 n}=\frac{1}{2}\left(\sum_{n=1}^{+\infty} \delta\left(-\frac{1}{6}, \frac{3}{4},-\frac{1}{12} ; n\right) q^{n}\right.
$$




$$
\begin{aligned}
& \left.+\sum_{n=1}^{+\infty} \delta\left(-\frac{1}{6}, \frac{3}{4},-\frac{1}{12} ; n\right)(-q)^{n}\right) \\
= & \frac{1}{2}\left(\Delta\left(-\frac{1}{6}, \frac{3}{4},-\frac{1}{12} ; q\right)+\Delta\left(-\frac{1}{6}, \frac{3}{4},-\frac{1}{12} ;-q\right)\right) \\
= & \frac{1}{2}\left(\frac{x(1-x)^{3 / 4}}{16} z^{6}-\frac{x(1-x)^{5 / 4}}{16} z^{6}\right) \\
= & \left(\frac{x(1-x)^{3 / 4}}{32} z^{6}-\frac{x(1-x)^{5 / 4}}{32} z^{6}\right)
\end{aligned}
$$

and by Theorem 8.1.1(iii)

$$
\begin{aligned}
4 \sum_{n=1}^{+\infty} \delta\left(-\frac{1}{3}, 1,-\frac{1}{6} ; n\right)(-1)^{n} q^{2 n} & =-4 \Delta\left(-\frac{1}{3}, 1,-\frac{1}{6} ;-q^{2}\right) \\
& =\frac{(1-x)^{3 / 4}(1-\sqrt{1-x})^{2}(1+\sqrt{1-x})}{32} z^{6} \\
& =\frac{x(1-x)^{3 / 4}(1-\sqrt{1-x})}{32} z^{6} \\
& =\frac{x(1-x)^{3 / 4}}{32} z^{6}-\frac{x(1-x)^{5 / 4}}{32} z^{6} .
\end{aligned}
$$

This implies

$$
\sum_{n=1}^{+\infty} \delta\left(-\frac{1}{6}, \frac{3}{4},-\frac{1}{12} ; 2 n\right) q^{2 n}=-4 \sum_{n=1}^{+\infty} \delta\left(-\frac{1}{3}, 1,-\frac{1}{6} ; n\right)(-1)^{n} q^{2 n} .
$$

Equating coefficients we obtain $\delta\left(-\frac{1}{6}, \frac{1}{4}, \frac{5}{12} ; 2 n\right)=-4(-1)^{n} \delta\left(-\frac{1}{3}, 1,-\frac{1}{6} ; n\right)$, which is the desired identity. 
Theorem 8.4.8. For $n \in \mathbb{N}$, we have

$$
\begin{aligned}
\left(\frac{-4}{n}\right) \sigma_{5}(n)= & 2 \delta\left(-\frac{5}{6}, \frac{9}{4},-\frac{11}{12} ; n\right)-\frac{79}{2} \delta\left(-\frac{1}{2}, \frac{5}{4},-\frac{1}{4} ; n\right)+122 \delta\left(-\frac{1}{6}, \frac{1}{4}, \frac{5}{12} ; n\right) \\
& -2 \delta\left(-\frac{1}{2}, \frac{7}{4},-\frac{3}{4} ; n\right)+\frac{49}{2} \delta\left(-\frac{1}{6}, \frac{3}{4},-\frac{1}{12} ; n\right)-2 \delta\left(\frac{1}{6},-\frac{1}{4}, \frac{7}{12} ; n\right) .
\end{aligned}
$$

Proof. Let $q \in D_{\delta}$. As

$$
\frac{i^{n}-(-i)^{n}}{2 i}=\left(\frac{-4}{n}\right)
$$

we have

$$
\begin{aligned}
\frac{E_{3}(i q)-E_{3}(-i q)}{2 i} & =\frac{1}{2 i}\left(\left(1-504 \sum_{n=1}^{+\infty} \sigma_{5}(n)(i q)^{n}\right)-\left(1-504 \sum_{n=1}^{+\infty} \sigma_{5}(n)(-i q)^{n}\right)\right) \\
& =-504 \sum_{n=1}^{+\infty} \sigma_{5}(n)\left(\frac{i^{n}-(-i)^{n}}{2 i}\right) q^{n} \\
& =-504 \sum_{n=1}^{+\infty}\left(\frac{-4}{n}\right) \sigma_{5}(n) q^{n} .
\end{aligned}
$$

Moreover, by Theorem 8.1.4(iii)(iv), we have

$$
\begin{aligned}
\frac{E_{3}(i q)-E_{3}(-i q)}{2 i}= & -\frac{63}{16} g z^{6}+\frac{3843}{16} g^{3} z^{6}-\frac{6111}{8} g^{5} z^{6}+\frac{6111}{8} g^{7} z^{6}-\frac{3843}{16} g^{9} z^{6}+\frac{63}{16} g^{11} z^{6} \\
& =-\frac{63}{16}(1-x)^{1 / 4} z^{6}+\frac{3843}{16}(1-x)^{3 / 4} z^{6}-\frac{6111}{8}(1-x)^{5 / 4} z^{6} \\
& +\frac{6111}{8}(1-x)^{7 / 4} z^{6}-\frac{3843}{16}(1-x)^{9 / 4} z^{6}+\frac{63}{16}(1-x)^{11 / 4} z^{6} .
\end{aligned}
$$

Simplifying the right hand side of equation (8.4.1) using MAPLE, we obtain

$$
-1008(1-x)^{1 / 4} z^{6}+\frac{4977}{4} x(1-x)^{1 / 4} z^{6}-\frac{3843}{16} x^{2}(1-x)^{1 / 4} z^{6}
$$




$$
\begin{aligned}
& +1008(1-x)^{3 / 4} z^{6}-\frac{3087}{4} x(1-x)^{3 / 4} z^{6}+\frac{63}{16} x^{2}(1-x)^{3 / 4} z^{6} \\
= & -1008 \Delta\left(-\frac{5}{6}, \frac{9}{4},-\frac{11}{12} ; q\right)+19908 \Delta\left(-\frac{1}{2}, \frac{5}{4},-\frac{1}{4} ; q\right)-61488 \Delta\left(-\frac{1}{6}, \frac{1}{4}, \frac{5}{12} ; q\right) \\
& +1008 \Delta\left(-\frac{1}{2}, \frac{7}{4},-\frac{3}{4} ; q\right)-12348 \Delta\left(-\frac{1}{6}, \frac{3}{4},-\frac{1}{12} ; q\right)+1008 \Delta\left(\frac{1}{6},-\frac{1}{4}, \frac{7}{12} ; q\right) \\
= & \sum_{n=1}^{+\infty}\left(-1008 \delta\left(-\frac{5}{6}, \frac{9}{4},-\frac{11}{12} ; n\right)+19908 \delta\left(-\frac{1}{2}, \frac{5}{4},-\frac{1}{4} ; n\right)-61488 \delta\left(-\frac{1}{6}, \frac{1}{4}, \frac{5}{12} ; n\right)\right. \\
& \left.+1008 \delta\left(-\frac{1}{2}, \frac{7}{4},-\frac{3}{4} ; n\right)-12348 \delta\left(-\frac{1}{6}, \frac{3}{4},-\frac{1}{12} ; n\right)+1008 \delta\left(\frac{1}{6},-\frac{1}{4}, \frac{7}{12} ; n\right)\right) q^{n} .
\end{aligned}
$$

Equating coefficients and dividing both sides by -504 , we obtain the desired identity.

\subsection{Identities for the sums of 16 squares}

In this section we state and prove eight identities relating the functions $\delta(a, b, c ; n)$ obtained in Theorems 7.3.1,7.3.2 and 7.3.3.

Theorem 8.5.1. For $n \in \mathbb{N}$, we have

$$
\delta\left(0, \frac{1}{3}, \frac{1}{3} ; n\right)=\frac{1}{8} \delta\left(-\frac{1}{3}, \frac{4}{3},-\frac{1}{3} ; n\right), \text { if } n \equiv 0(\bmod 2) .
$$

Proof. Let $q \in D_{\delta}$. By Theorem 2.10.2(ii) and Theorem 8.1.1(i), we have

$$
\sum_{n=1}^{+\infty} \delta\left(0, \frac{1}{3}, \frac{1}{3} ; 2 n\right) q^{2 n}=\frac{1}{2}\left(\sum_{n=1}^{+\infty} \delta\left(0, \frac{1}{3}, \frac{1}{3} ; n\right) q^{n}\right.
$$




$$
\begin{aligned}
& \left.+\sum_{n=1}^{+\infty} \delta\left(0, \frac{1}{3}, \frac{1}{3} ; n\right)(-q)^{n}\right) \\
= & \frac{1}{2}\left(\Delta\left(0, \frac{1}{3}, \frac{1}{3} ; q\right)+\Delta\left(0, \frac{1}{3}, \frac{1}{3} ;-q\right)\right) \\
= & \frac{1}{2}\left(\frac{x^{2}(1-x)}{256} z^{8}+\frac{x^{2}(1-x)}{256} z^{8}\right) \\
= & \frac{x^{2}(1-x)}{256} z^{8}
\end{aligned}
$$

and

$$
\begin{aligned}
& \frac{1}{8} \sum_{n=1}^{+\infty} \delta\left(-\frac{1}{3}, \frac{4}{3},-\frac{1}{3} ; 2 n\right) q^{2 n}=\frac{1}{16}\left(\Delta\left(-\frac{1}{3}, \frac{4}{3},-\frac{1}{3} ; q\right)+\Delta\left(-\frac{1}{3}, \frac{4}{3},-\frac{1}{3} ;-q\right)\right) \\
& =\frac{1}{16}\left(\frac{x(1-x)}{16} z^{8}-\frac{x(1-x)^{2}}{16} z^{8}\right) \\
& =\frac{1}{16}\left(\frac{x(1-x)}{16} z^{8}-\frac{x(1-x)}{16} z^{8}+\frac{x^{2}(1-x)}{16} z^{8}\right)=\frac{x^{2}(1-x)}{256} z^{8} .
\end{aligned}
$$

This implies

$$
\sum_{n=1}^{+\infty} \delta\left(0, \frac{1}{3}, \frac{1}{3} ; 2 n\right) q^{2 n}=\frac{1}{8} \sum_{n=1}^{+\infty} \delta\left(-\frac{1}{3}, \frac{4}{3},-\frac{1}{3} ; 2 n\right) q^{2 n}
$$

Equating coefficients we obtain the desired identity.

Theorem 8.5.2. For $n \in \mathbb{N}$, we have

$$
\delta\left(0,-\frac{1}{6}, \frac{5}{6} ; n\right)=0, \text { if } n \equiv 0(\bmod 2) .
$$


Proof. Let $q \in D_{\delta}$. By Theorem 2.10.2(ii) and Theorem 8.1.1(i), we have

$$
\begin{aligned}
2 \sum_{n=1}^{+\infty} \delta\left(0,-\frac{1}{6}, \frac{5}{6} ; 2 n\right) q^{2 n} & =\sum_{n=1}^{\infty} \delta\left(0,-\frac{1}{6}, \frac{5}{6} ; n\right) q^{n}+\sum_{n=1}^{\infty} \delta\left(0,-\frac{1}{6}, \frac{5}{6} ; n\right)(-q)^{n} \\
& =\Delta\left(0,-\frac{1}{6}, \frac{5}{6} ; q\right)+\Delta\left(0,-\frac{1}{6}, \frac{5}{6} ;-q\right) \\
& =\frac{x^{3} \sqrt{1-x}}{2^{12}} z^{8}-\frac{x^{3} \sqrt{1-x}}{2^{12}} z^{8}=0
\end{aligned}
$$

Equating coefficients we obtain $\delta\left(0,-\frac{1}{6}, \frac{5}{6} ; n\right)=0$ if $n \equiv 0(\bmod 2)$.

Theorem 8.5.3. For $n \in \mathbb{N}$ such that $n \equiv 0(\bmod 4)$, we have

(i) $\delta\left(-\frac{2}{3}, \frac{11}{6},-\frac{1}{2} ; n\right)=-6 \delta\left(-\frac{1}{3}, \frac{4}{3},-\frac{1}{3} ; n\right)$,

(ii) $\delta\left(-\frac{1}{3}, \frac{5}{6}, \frac{1}{6} ; n\right)=-\frac{3}{8} \delta\left(-\frac{1}{3}, \frac{4}{3},-\frac{1}{3} ; n\right)$.

Proof. (i) Let $q \in D_{\delta}$. As

$$
\frac{(1)^{n}+(-1)^{n}+i^{n}+(-i)^{n}}{4}= \begin{cases}1, & \text { if } n \equiv 0(\bmod 4) \\ 0, & \text { otherwise. }\end{cases}
$$

we have by Theorems 2.10.2(ii), 8.1.1(i) and 8.1.2

$$
\begin{aligned}
& \sum_{\substack{n=0 \\
n \equiv 0}}^{+\infty} \delta\left(-\frac{2}{3}, \frac{11}{6},-\frac{1}{2} ; n\right) q^{n} \\
& =\frac{1}{4} \sum_{n=0}^{+\infty} \delta\left(-\frac{2}{3}, \frac{11}{6},-\frac{1}{2} ; n\right)\left((1)^{n}+(-1)^{n}+(i)^{n}+(-i)^{n}\right) q^{n} \\
& =\frac{1}{4}\left(\Delta\left(-\frac{2}{3}, \frac{11}{6},-\frac{1}{2} ; q\right)+\Delta\left(-\frac{2}{3}, \frac{11}{6},-\frac{1}{2} ;-q\right)+\Delta\left(-\frac{2}{3}, \frac{11}{6},-\frac{1}{2} ; i q\right)\right.
\end{aligned}
$$




$$
\begin{aligned}
& \left.+\Delta\left(-\frac{2}{3}, \frac{11}{6},-\frac{1}{2} ;-i q\right)\right) \\
& =\frac{1}{4}\left(\frac{x(1-x)^{1 / 2}}{16} z^{8}-\frac{x(1-x)^{5 / 2}}{16} z^{8}\right. \\
& \quad+\frac{i}{512}(1-x)^{1 / 4}(1-\sqrt{1-x})\left(1+i(1-x)^{1 / 4}\right)^{2}\left(1-i(1-x)^{1 / 4}\right)^{10} z^{8} \\
& \left.\quad-\frac{i}{512}(1-x)^{1 / 4}(1-\sqrt{1-x})\left(1+i(1-x)^{1 / 4}\right)^{10}\left(1-i(1-x)^{1 / 4}\right)^{2} z^{8}\right) .
\end{aligned}
$$

Simplifying the last quantity using MAPLE we obtain

$$
-\frac{3}{64} x^{2} z^{8}+\frac{3}{64} x^{3} z^{8}+\frac{3}{64} x^{2} \sqrt{1-x} z^{8}-\frac{3}{128} x^{3} \sqrt{1-x} z^{8} .
$$

Moreover, using the same theorems we have

$$
\begin{aligned}
\sum_{\substack{n=0 \\
n \equiv 0(\bmod 4)}}^{+\infty}-6 \delta\left(-\frac{1}{3}, \frac{4}{3},-\frac{1}{3} ; n\right) q^{n} \\
=-\frac{6}{4} \sum_{n=0}^{+\infty} \delta\left(-\frac{1}{3}, \frac{4}{3},-\frac{1}{3} ; n\right)\left((1)^{n}+(-1)^{n}+(i)^{n}+(-i)^{n}\right) q^{n} \\
=-\frac{6}{4}\left(\Delta\left(-\frac{1}{3}, \frac{4}{3},-\frac{1}{3} ; q\right)+\Delta\left(-\frac{1}{3}, \frac{4}{3},-\frac{1}{3} ;-q\right)+\Delta\left(-\frac{1}{3}, \frac{4}{3},-\frac{1}{3} ; i q\right)\right. \\
\left.\quad+\Delta\left(-\frac{1}{3}, \frac{4}{3},-\frac{1}{3} ;-i q\right)\right) \\
=-\frac{6}{4}\left(\frac{x(1-x)}{16} z^{8}-\frac{x(1-x)^{2}}{16} z^{8}\right. \\
\quad-\frac{i}{512}(1-x)^{1 / 4}(1-\sqrt{1-x})\left(1+i(1-x)^{1 / 4}\right)^{4}\left(1-i(1-x)^{1 / 4}\right)^{8} z^{8} \\
\left.\quad+\frac{i}{512}(1-x)^{1 / 4}(1-\sqrt{1-x})\left(1+i(1-x)^{1 / 4}\right)^{8}\left(1-i(1-x)^{1 / 4}\right)^{4} z^{8}\right)
\end{aligned}
$$

Simplifying the last expression using MAPLE we obtain

$$
-\frac{3}{64} x^{2} z^{8}+\frac{3}{64} x^{3} z^{8}+\frac{3}{64} x^{2} \sqrt{1-x} z^{8}-\frac{3}{128} x^{3} \sqrt{1-x} z^{8} .
$$

Thus, we have shown that

$$
\sum_{\substack{n=0 \\ n \equiv 0(\bmod 4)}}^{+\infty} \delta\left(-\frac{2}{3}, \frac{11}{6},-\frac{1}{2} ; n\right) q^{n}=\sum_{\substack{n=0 \\ n \equiv 0(\bmod 4)}}^{+\infty}-6 \delta\left(-\frac{1}{3}, \frac{4}{3},-\frac{1}{3} ; n\right) q^{n} .
$$


Equating coefficients, we obtain the desired identity. The proof for (ii) follows in exactly the same way.

Theorem 8.5.4. For $n \in \mathbb{N}$ such that $n \equiv 0(\bmod 4)$, we have

(i) $\delta\left(-\frac{1}{2}, \frac{13}{12}, \frac{1}{12} ; n\right)=\delta\left(-\frac{1}{2}, \frac{19}{12},-\frac{5}{12} ; n\right)=\frac{1}{15} \delta\left(-\frac{5}{6}, \frac{25}{12},-\frac{7}{12} ; n\right)$,

(ii)

$$
\delta\left(-\frac{1}{6}, \frac{1}{12}, \frac{3}{4} ; n\right)=-\delta\left(\frac{1}{6},-\frac{5}{12}, \frac{11}{12} ; n\right)=\frac{1}{240} \delta\left(-\frac{5}{6}, \frac{25}{12},-\frac{7}{12} ; n\right)
$$

(iii) $\delta\left(-\frac{1}{6}, \frac{7}{12}, \frac{1}{4} ; n\right)=0$.

Proof. We only prove (i) as the proofs of (ii) and (iii) are similar. Let $q \in D_{\delta}$. As

$$
\frac{1+(-1)^{n}+i^{n}+(-i)^{n}}{4}= \begin{cases}1, & \text { if } n \equiv 0(\bmod 4) \\ 0, & \text { otherwise. }\end{cases}
$$

we have by Theorems 2.10.2(ii), 8.1.1(i) and 8.1.2

$$
\begin{aligned}
& \sum_{\substack{n=0 \\
n \equiv 0(\bmod 4)}}^{+\infty} \delta\left(-\frac{1}{2}, \frac{13}{12}, \frac{1}{12} ; n\right) q^{n} \\
= & \frac{1}{4} \sum_{n=0}^{+\infty} \delta\left(-\frac{1}{2}, \frac{13}{12}, \frac{1}{12} ; n\right)\left(1+(-1)^{n}+(i)^{n}+(-i)^{n}\right) q^{n} \\
= & \frac{1}{4}\left(\Delta\left(-\frac{1}{2}, \frac{13}{12}, \frac{1}{12} ; q\right)+\Delta\left(-\frac{1}{2}, \frac{13}{12}, \frac{1}{12} ;-q\right)+\Delta\left(-\frac{1}{2}, \frac{13}{12}, \frac{1}{12} ; i q\right)\right. \\
= & \frac{1}{4}\left(\frac{x^{2}(1-x)^{1 / 4}}{256} z^{8}+\frac{x^{2}(1-x)^{7 / 4}}{256} z^{8}\right. \\
& +\frac{i}{1024} \sqrt{1-x}(1-\sqrt{1-x})^{2}\left(1+i(1-x)^{1 / 4}\right)\left(1-i(1-x)^{1 / 4}\right)^{7} z^{8} \\
& \left.\quad-\frac{i}{1024} \sqrt{1-x}(1-\sqrt{1-x})^{2}\left(1+i(1-x)^{1 / 4}\right)^{7}\left(1-i(1-x)^{1 / 4}\right) z^{8}\right) .
\end{aligned}
$$


Simplifying the last expression using MAPLE we obtain

$$
\begin{aligned}
& -\frac{1}{64} x(1-x)^{1 / 4} z^{8}+\frac{5}{256} x^{2}(1-x)^{1 / 4} z^{8}-\frac{3}{1024} x^{3}(1-x)^{1 / 4} z^{8} \\
& +\frac{1}{64} x(1-x)^{3 / 4} z^{8}-\frac{3}{256} x^{2}(1-x)^{3 / 4} z^{8}-\frac{1}{1024} x^{3}(1-x)^{3 / 4} z^{8} .
\end{aligned}
$$

Moreover, using the same theorems, we have

$$
\begin{aligned}
& \sum_{\substack{n=0 \\
n \equiv 0(\bmod 4)}}^{+\infty} \frac{1}{15} \delta\left(-\frac{5}{6}, \frac{25}{12},-\frac{7}{12} ; n\right) q^{n} \\
& =\frac{1}{60} \sum_{n=0}^{+\infty} \delta\left(-\frac{5}{6}, \frac{25}{12},-\frac{7}{12} ; n\right)\left((1)^{n}+(-1)^{n}+(i)^{n}+(-i)^{n}\right) q^{n} \\
& =\frac{1}{60}\left(\Delta\left(-\frac{5}{6}, \frac{25}{12},-\frac{7}{12} ; q\right)+\Delta\left(-\frac{5}{6}, \frac{25}{12},-\frac{7}{12} ;-q\right)+\Delta\left(-\frac{5}{6}, \frac{25}{12},-\frac{7}{12} ; i q\right)\right. \\
& \left.\quad+\Delta\left(-\frac{5}{6}, \frac{25}{12},-\frac{7}{12} ;-i q\right)\right) \\
& =\frac{1}{60}\left(\frac{x(1-x)^{1 / 4}}{16} z^{8}-\frac{x(1-x)^{11 / 4}}{256} z^{8}\right. \\
& \quad-\frac{1}{512}(1-x)^{1 / 4}(1-\sqrt{1-x})\left(1+i(1-x)^{1 / 4}\right)\left(1-i(1-x)^{1 / 4}\right)^{11} z^{8} \\
& \left.\quad-\frac{1}{512}(1-x)^{1 / 4}(1-\sqrt{1-x})\left(1+i(1-x)^{1 / 4}\right)^{11}\left(1-i(1-x)^{1 / 4}\right) z^{8}\right) .
\end{aligned}
$$

Simplifying the last expression using MAPLE we obtain

$$
\begin{aligned}
& -\frac{1}{64} x(1-x)^{1 / 4} z^{8}+\frac{5}{256} x^{2}(1-x)^{1 / 4} z^{8}-\frac{3}{1024} x^{3}(1-x)^{1 / 4} z^{8} \\
& +\frac{1}{64} x(1-x)^{3 / 4} z^{8}-\frac{3}{256} x^{2}(1-x)^{3 / 4} z^{8}-\frac{1}{1024} x^{3}(1-x)^{3 / 4} z^{8} .
\end{aligned}
$$

Thus, we have shown that

$$
\sum_{\substack{n=0 \\ n \equiv 0(\bmod 4)}}^{+\infty} \delta\left(-\frac{1}{2}, \frac{13}{12}, \frac{1}{12} ; n\right) q^{n}=\sum_{\substack{n=0 \\ n \equiv 0(\bmod 4)}}^{+\infty} \frac{1}{15} \delta\left(-\frac{5}{6}, \frac{25}{12},-\frac{7}{12} ; n\right) q^{n} .
$$

Equating coefficients, we obtain the identity

$$
\delta\left(-\frac{1}{2}, \frac{13}{12}, \frac{1}{12} ; n\right)=\frac{1}{15} \delta\left(-\frac{5}{6}, \frac{25}{12},-\frac{7}{12} ; n\right),
$$


when $n \equiv 0(\bmod 4)$. In the same way, we can obtain the identity

$$
\delta\left(-\frac{1}{2}, \frac{19}{12},-\frac{5}{12} ; n\right)=\frac{1}{15} \delta\left(-\frac{5}{6}, \frac{25}{12},-\frac{7}{12} ; n\right),
$$

when $n \equiv 0(\bmod 4)$, which proves $(\mathrm{i})$.

Theorem 8.5.5. For $n \in \mathbb{N}$, we have

$$
\begin{aligned}
\left(\frac{-4}{n}\right) \sigma_{7}(n)= & -34 \delta\left(-\frac{7}{6}, \frac{37}{12},-\frac{5}{4} ; n\right)+\frac{1905}{2} \delta\left(-\frac{5}{6}, \frac{25}{12},-\frac{7}{12} ; n\right) \\
& -6810 \delta\left(-\frac{1}{2}, \frac{13}{12}, \frac{1}{12} ; n\right)+4376 \delta\left(-\frac{1}{6}, \frac{1}{12}, \frac{3}{4} ; n\right) \\
& +34 \delta\left(-\frac{5}{6}, \frac{31}{12},-\frac{13}{12} ; n\right)-\frac{1359}{2} \delta\left(-\frac{1}{2}, \frac{19}{12},-\frac{5}{12} ; n\right) \\
& +2442 \delta\left(-\frac{1}{6}, \frac{7}{12}, \frac{1}{4} ; n\right)-8 \delta\left(\frac{1}{6},-\frac{5}{12}, \frac{11}{12} ; n\right) .
\end{aligned}
$$

Proof. Let $q \in D_{\delta}$. As

$$
\frac{i^{n}-(-i)^{n}}{2 i}=\left(\frac{-4}{n}\right)
$$

we have

$$
\begin{aligned}
\frac{E_{4}(i q)-E_{4}(-i q)}{2 i} & =\frac{1}{2 i}\left(\left(1+480 \sum_{n=1}^{+\infty} \sigma_{7}(n)(i q)^{n}\right)-\left(1+480 \sum_{n=1}^{+\infty} \sigma_{7}(n)(-i q)^{n}\right)\right) \\
& =480 \sum_{n=1}^{+\infty} \sigma_{7}(n)\left(\frac{i^{n}-(-i)^{n}}{2 i}\right) q^{n}=480 \sum_{n=1}^{+\infty}\left(\frac{-4}{n}\right) \sigma_{7}(n) q^{n} .
\end{aligned}
$$

Moreover, by Theorem 8.1.4 (v)(vi) and the use of MAPLE, we have $\frac{E_{4}(i q)-E_{4}(-i q)}{2 i}=-\frac{15}{16} g z^{8}+\frac{8205}{16} g^{3} z^{8}-\frac{73215}{16} g^{5} z^{8}+\frac{179685}{16} g^{7} z^{8}-\frac{179685}{16} g^{9} z^{8}$ 


$$
\begin{aligned}
& +\frac{73215}{16} g^{11} z^{8}-\frac{8205}{16} g^{13} z^{8}+\frac{15}{16} g^{15} z^{8} \\
= & -\frac{15}{16}(1-x)^{1 / 4} z^{8}+\frac{8205}{16}(1-x)^{3 / 4} z^{8}-\frac{73215}{16}(1-x)^{5 / 4} z^{8}+\frac{179685}{16}(1-x)^{7 / 4} z^{8} \\
& -\frac{179685}{16}(1-x)^{9 / 4} z^{8}+\frac{73215}{16}(1-x)^{11 / 4} z^{8}-\frac{8205}{16}(1-x)^{13 / 4} z^{8}+\frac{15}{16}(1-x)^{15 / 4} z^{8} .
\end{aligned}
$$

Simplifying the last expression using MAPLE, we obtain

$$
\begin{aligned}
& -16320(1-x)^{1 / 4} z^{8}+28575 x(1-x)^{1 / 4} z^{8}-\frac{51075}{4} x^{2}(1-x)^{1 / 4} z^{8}+\frac{8205}{16} x^{3}(1-x)^{1 / 4} z^{8} \\
& +16320(1-x)^{3 / 4} z^{8}-20385 x(1-x)^{3 / 4} z^{8}+\frac{18315}{4} x^{2}(1-x)^{3 / 4} z^{8}-\frac{15}{16} x^{3}(1-x)^{3 / 4} z^{8} \\
& =-16320 \Delta\left(-\frac{7}{6}, \frac{37}{12},-\frac{5}{4} ; q\right)+457200 \Delta\left(-\frac{5}{6}, \frac{25}{12},-\frac{7}{12} ; q\right) \\
& -3268800 \Delta\left(-\frac{1}{2}, \frac{13}{12}, \frac{1}{12} ; q\right)+2100480 \Delta\left(-\frac{1}{6}, \frac{1}{12}, \frac{3}{4} ; q\right) \\
& +16320 \Delta\left(-\frac{5}{6}, \frac{31}{12},-\frac{13}{12} ; q\right)-326160 \Delta\left(-\frac{1}{2}, \frac{19}{12},-\frac{5}{12} ; q\right) \\
& +1172160 \Delta\left(-\frac{1}{6}, \frac{7}{12}, \frac{1}{4} ; q\right)-3840 \Delta\left(\frac{1}{6},-\frac{5}{12}, \frac{11}{12} ; q\right) \\
& +\sum_{n=1}^{+\infty}\left(-16320 \delta\left(-\frac{7}{6}, \frac{37}{12},-\frac{5}{4} ; n\right)+457200 \delta\left(-\frac{5}{6}, \frac{25}{12},-\frac{7}{12} ; n\right)\right.
\end{aligned}
$$




$$
\begin{aligned}
& -3268800 \delta\left(-\frac{1}{2}, \frac{13}{12}, \frac{1}{12} ; n\right)+2100480 \delta\left(-\frac{1}{6}, \frac{1}{12}, \frac{3}{4} ; n\right) \\
& +16320 \delta\left(-\frac{5}{6}, \frac{31}{12},-\frac{13}{12} ; n\right)-326160 \delta\left(-\frac{1}{2}, \frac{19}{12},-\frac{5}{12} ; n\right) \\
& \left.+1172160 \delta\left(-\frac{1}{6}, \frac{7}{12}, \frac{1}{4} ; n\right)-3840 \delta\left(\frac{1}{6},-\frac{5}{12}, \frac{11}{12} ; n\right)\right) q^{n} .
\end{aligned}
$$

Equating coefficients and dividing both sides by 480 , we obtain the desired identity.

\subsection{An important identity}

The goal of this section is to prove an important delta identity that plays a key role in Chapter 9 in simplifying the results involving 16 squares.

Theorem 8.6.1.

$$
\delta(-5 / 6,25 / 12,-7 / 12 ; n):= \begin{cases}45 \delta\left(-\frac{1}{3}, \frac{4}{3},-\frac{1}{3} ; n\right), & \text { if } n \equiv 0(\bmod 8), \\ -15 \delta\left(-\frac{1}{3}, \frac{4}{3},-\frac{1}{3} ; n\right), & \text { if } n \equiv 4(\bmod 8) .\end{cases}
$$

Several lemmas are needed to prove this identity. We set

$$
\begin{aligned}
& A:=\varphi(q), \\
& B:=\varphi(-q) .
\end{aligned}
$$

With this notation, the identities of Lemmas 2.3.1 and 2.3.2, give

$$
\varphi\left(q^{2}\right)=\left(\frac{A^{2}+B^{2}}{2}\right)^{1 / 2}
$$




$$
\begin{gathered}
\varphi\left(-q^{2}\right)=(A B)^{1 / 2}, \\
\varphi\left(q^{4}\right)=\frac{A+B}{2},
\end{gathered}
$$

$$
\varphi\left(-q^{4}\right)=\left(\frac{A B\left(A^{2}+B^{2}\right)}{2}\right)^{1 / 4}
$$

$$
\varphi(i q)=\frac{(A+B)}{2}+i \frac{(A-B)}{2}
$$

$$
\varphi(-i q)=\frac{(A+B)}{2}-i \frac{(A-B)}{2}
$$

The following lemma express $\varphi(\omega q), \varphi(i \omega q), \varphi(-\omega q)$ and $\varphi(-i \omega q)$ (where $\omega=e^{\frac{2 \pi z}{8}}$ ) in terms of $A$ and $B$.

Lemma 8.6.1. For $q \in \mathbb{C}$ such that $|q|<1$ and $\omega=e^{\frac{2 \pi 2}{8}}=\frac{1+i}{\sqrt{2}}$, we have

(i) $\varphi(\omega q)=\left(\frac{A B\left(A^{2}+B^{2}\right)}{2}\right)^{1 / 4}+\frac{\omega}{2}(A-B)$,

(ii) $\varphi(i \omega q)=\left(\frac{A B\left(A^{2}+B^{2}\right)}{2}\right)^{1 / 4}+\frac{i \omega}{2}(A-B)$,

(iii) $\varphi(-\omega q)=\left(\frac{A B\left(A^{2}+B^{2}\right)}{2}\right)^{1 / 4}-\frac{\omega}{2}(A-B)$,

(iv) $\varphi(-i \omega q)=\left(\frac{A B\left(A^{2}+B^{2}\right)}{2}\right)^{1 / 4}-\frac{i \omega}{2}(A-B)$.

Proof. We only prove (i) as the proofs of (ii), (iii) and (iv) are similar. By (8.6.5) 
and (8.6.6) we have

$$
\begin{aligned}
\varphi(\omega q) & =\sum_{n=-\infty}^{+\infty}(\omega q)^{n^{2}}=\sum_{n=-\infty}^{+\infty} \omega^{n^{2}} q^{n^{2}}=\sum_{\substack{n=-\infty \\
n \equiv 0(\bmod 2)}}^{+\infty} \omega^{n^{2}} q^{n^{2}}+\sum_{\substack{n=-\infty \\
n \equiv 1(\bmod 2)}}^{+\infty} \omega^{n^{2}} q^{n^{2}} \\
& =\sum_{n=-\infty}^{+\infty}\left(-q^{4}\right)^{n^{2}}+\omega \sum_{\substack{n=-\infty \\
n \equiv 1(\bmod 2)}}^{+\infty} q^{n^{2}}=\varphi\left(-q^{4}\right)+\omega\left(\sum_{n=-\infty}^{+\infty} q^{n^{2}}-\sum_{\substack{n=-\infty \\
n \equiv 0(\bmod 2)}}^{+\infty} q^{n^{2}}\right) \\
& =\varphi\left(-q^{4}\right)+\omega\left(\varphi(q)-\varphi\left(q^{4}\right)\right)=\varphi\left(-q^{4}\right)+\omega\left(\frac{\varphi(q)-\varphi(-q)}{2}\right) \\
& =\left(\frac{A B\left(A^{2}+B^{2}\right)}{2}\right)^{1 / 4}+\frac{\omega}{2}(A-B),
\end{aligned}
$$

as asserted.

The next lemma expresses $\Delta\left(-\frac{5}{6}, \frac{25}{12},-\frac{7}{12} ; q\right)$ and $\Delta\left(-\frac{5}{6}, \frac{25}{12},-\frac{7}{12} ;-q\right)$ in terms of $A$ and $B$.

Lemma 8.6.2. For $q \in D_{\delta}$ we have

(i) $\Delta\left(-\frac{5}{6}, \frac{25}{12},-\frac{7}{12} ; q\right)=\frac{1}{16} A^{15} B-\frac{1}{16} A^{11} B^{5}$,

(ii) $\Delta\left(-\frac{5}{6}, \frac{25}{12},-\frac{7}{12} ;-q\right)=-\frac{1}{16} A^{5} B^{11}+\frac{1}{16} A B^{15}$.

Proof. We only prove (i) as the proof of (ii) is similar. By Definition 2.3.2, Theorem 2.10.2, Theorem 2.4.6(i), (8.6.1) and (8.6.2), we have

$$
\begin{aligned}
& \Delta\left(-\frac{5}{6}, \frac{25}{12},-\frac{7}{12} ; q\right)=\frac{x(1-x)^{1 / 4}}{16} z^{8}=\frac{1}{16}\left(1-\frac{\varphi^{4}(-q)}{\varphi^{4}(q)}\right) \frac{\varphi(-q)}{\varphi(q)} \varphi^{16}(q) \\
& =\frac{1}{16}\left(\varphi^{4}(q)-\varphi^{4}(-q)\right) \varphi(-q) \varphi^{11}(q)=\frac{1}{16}\left(\varphi^{15}(q) \varphi(-q)-\varphi^{11}(q) \varphi^{5}(-q)\right)
\end{aligned}
$$




$$
=\frac{1}{16} A^{15} B-\frac{1}{16} A^{11} B^{5}
$$

as required.

The next lemma expresses $\Delta\left(-\frac{5}{6}, \frac{25}{12},-\frac{7}{12} ; i q\right)+\Delta\left(-\frac{5}{6}, \frac{25}{12},-\frac{7}{12} ;-i q\right)$ in terms of $A$ and $B$.

Lemma 8.6.3. For $q \in D_{\delta}$ we have

$$
\begin{aligned}
\Delta\left(-\frac{5}{6}, \frac{25}{12},-\frac{7}{12} ; i q\right)+\Delta\left(-\frac{5}{6}, \frac{25}{12},-\frac{7}{12} ;-i q\right) & \\
=\frac{1}{256}\left(-A^{15} B+45 A^{13} B^{3}-209 A^{11} B^{5}\right. & +165 A^{9} B^{7}+165 A^{7} B^{9} \\
& \left.-209 A^{5} B^{11}+45 A^{3} B^{13}-A B^{15}\right) .
\end{aligned}
$$

Proof. Replacing $q$ by $i q$ and $-q$ by $-i q$ in Lemma 8.6.2 and using (8.6.7) and (8.6.8), we have

$$
\begin{aligned}
\Delta & \left(-\frac{5}{6}, \frac{25}{12},-\frac{7}{12} ; i q\right)+\Delta\left(-\frac{5}{6}, \frac{25}{12},-\frac{7}{12} ;-i q\right) \\
= & \frac{1}{16} \varphi^{15}(i q) \varphi(-i q)-\frac{1}{16} \varphi^{11}(i q) \varphi^{5}(-i q)+\frac{1}{16} \varphi^{15}(-i q) \varphi(i q)-\frac{1}{16} \varphi^{11}(-i q) \varphi^{5}(i q) \\
= & \frac{1}{16}\left(\frac{(A+B)}{2}+i \frac{(A-B)}{2}\right)^{15}\left(\frac{(A+B)}{2}-i \frac{(A-B)}{2}\right) \\
& -\frac{1}{16}\left(\frac{(A+B)}{2}+i \frac{(A-B)}{2}\right)^{11}\left(\frac{(A+B)}{2}-i \frac{(A-B)}{2}\right)^{5} \\
& +\frac{1}{16}\left(\frac{(A+B)}{2}-i \frac{(A-B)}{2}\right)^{15}\left(\frac{(A+B)}{2}+i \frac{(A-B)}{2}\right)^{5} \\
& -\frac{1}{16}\left(\frac{(A+B)}{2}-i \frac{(A-B)}{2}\right)^{11}\left(\frac{(A+B)}{2}+i \frac{(A-B)}{2}\right)^{5} .
\end{aligned}
$$


Simplifying the last expression with MAPLE, we obtain

$$
\begin{aligned}
\Delta\left(-\frac{5}{6}, \frac{25}{12},-\frac{7}{12} ; i q\right)+\Delta\left(-\frac{5}{6}, \frac{25}{12},-\frac{7}{12} ;-i q\right) & \\
=\frac{1}{256}\left(-A^{15} B+45 A^{13} B^{3}-209 A^{11} B^{5}\right. & +165 A^{9} B^{7}+165 A^{7} B^{9} \\
& \left.-209 A^{5} B^{11}+45 A^{3} B^{13}-A B^{15}\right)
\end{aligned}
$$

which is the desired result.

Lemmas 8.6.2 and 8.6.3 allow us to prove the following result.

Lemma 8.6.4. For $q \in D_{\delta}$ we have

$$
\begin{aligned}
& \sum_{\substack{n=0 \\
n \equiv 0(\bmod 4)}}^{+\infty} \delta\left(-\frac{5}{6}, \frac{25}{12},-\frac{7}{12} ; n\right) q^{n} \\
& =\frac{1}{1024}\left(15 A^{15} B+45 A^{13} B^{3}-225 A^{11} B^{5}+165 A^{9} B^{7}+165 A^{7} B^{9}\right. \\
& \left.-225 A^{5} B^{11}+45 A^{3} B^{13}+15 A B^{15}\right) .
\end{aligned}
$$

Proof. From Lemmas 8.6.2 and 8.6.3, we have

$$
\begin{aligned}
& \sum_{\substack{n=0 \\
n \equiv 0}}^{+\infty} \delta\left(-\frac{5}{6}, \frac{25}{12},-\frac{7}{12} ; n\right) q^{n} \\
& =\frac{1}{4} \sum_{n=0}^{+\infty} \delta\left(-\frac{5}{6}, \frac{25}{12},-\frac{7}{12} ; n\right)\left(1+(-1)^{n}+i^{n}+(-i)^{n}\right) q^{n} \\
& =\frac{1}{4}\left(\Delta\left(-\frac{5}{6}, \frac{25}{12},-\frac{7}{12} ; q\right)+\Delta\left(-\frac{5}{6}, \frac{25}{12},-\frac{7}{12} ;-q\right)+\Delta\left(-\frac{5}{6}, \frac{25}{12},-\frac{7}{12} ; i q\right)\right.
\end{aligned}
$$




$$
\begin{gathered}
\left.\quad+\Delta\left(-\frac{5}{6}, \frac{25}{12},-\frac{7}{12} ;-i q\right)\right) \\
=\frac{1}{64} A^{15} B-\frac{1}{64} A^{11} B^{5}-\frac{1}{64} A^{5} B^{11}+\frac{1}{64} A B^{15} \\
+\frac{1}{1024}\left(-A^{15} B+45 A^{13} B^{3}-209 A^{11} B^{5}+165 A^{9} B^{7}+165 A^{7} B^{9}\right. \\
\left.-209 A^{5} B^{11}+45 A^{3} B^{13}-A B^{15}\right) \\
=\frac{1}{1024}\left(15 A^{15} B+45 A^{13} B^{3}-225 A^{11} B^{5}+165 A^{9} B^{7}+165 A^{7} B^{9}\right. \\
\left.-225 A^{5} B^{11}+45 A^{3} B^{13}+15 A B^{15}\right)
\end{gathered}
$$

which is the desired result.

The next lemma expresses $\Delta\left(-\frac{1}{3}, \frac{4}{3},-\frac{1}{3} ; q\right)$ and $\Delta\left(-\frac{1}{3}, \frac{4}{3},-\frac{1}{3} ;-q\right)$ in terms of $A$ and $B$.

Lemma 8.6.5. For $q \in \mathbb{C}$ such that $|q|<1$ we have

(i) $\Delta\left(-\frac{1}{3}, \frac{4}{3},-\frac{1}{3} ; q\right)=\frac{1}{16} A^{12} B^{4}-\frac{1}{16} A^{8} B^{8}$,

(ii) $\Delta\left(-\frac{1}{3}, \frac{4}{3},-\frac{1}{3} ;-q\right)=-\frac{1}{16} A^{8} B^{8}+\frac{1}{16} A^{4} B^{12}$.

Proof. We only prove (i) as the proof of (ii) is similar. By Definition 2.3.2, Theorem 2.10.2, Theorem 2.4.6(i), (8.6.1) and (8.6.2), we have

$$
\begin{aligned}
& \Delta\left(-\frac{1}{3}, \frac{4}{3},-\frac{1}{3} ; q\right)=\frac{x(1-x)}{16} z^{8}=\frac{1}{16}\left(1-\frac{\varphi^{4}(-q)}{\varphi^{4}(q)}\right) \frac{\varphi^{4}(-q)}{\varphi^{4}(q)} \varphi^{16}(q) \\
& \quad=\frac{1}{16}\left(\varphi^{12}(q) \varphi^{4}(-q)-\varphi^{8}(q) \varphi^{8}(-q)\right)
\end{aligned}
$$




$$
=\frac{1}{16} A^{12} B^{4}-\frac{1}{16} A^{8} B^{8}
$$

as claimed.

The next lemma expresses $\Delta\left(-\frac{1}{3}, \frac{4}{3},-\frac{1}{3} ; i q\right)+\Delta\left(-\frac{1}{3}, \frac{4}{3},-\frac{1}{3} ;-i q\right)$ in terms of $A$ and $B$.

Lemma 8.6.6. For $q \in \mathbb{C}$ such that $|q|<1$ we have

$$
\begin{aligned}
& \Delta\left(-\frac{1}{3}, \frac{4}{3},-\frac{1}{3} ; i q\right)+\Delta\left(-\frac{1}{3}, \frac{4}{3},-\frac{1}{3} ;-i q\right) \\
& \quad=\frac{1}{64}\left(-A^{14} B^{2}-2 A^{12} B^{4}+A^{10} B^{6}+4 A^{8} B^{8}+A^{6} B^{10}-2 A^{4} B^{12}-A^{2} B^{14}\right) .
\end{aligned}
$$

Proof. Replacing $q$ by $i q$ and $-q$ by $-i q$ in Lemma 8.6 .5 and using (8.6.7) and (8.6.8), we have

$$
\begin{aligned}
\Delta & \left(-\frac{1}{3}, \frac{4}{3},-\frac{1}{3} ; i q\right)+\Delta\left(-\frac{1}{3}, \frac{4}{3},-\frac{1}{3} ;-i q\right) \\
= & \frac{1}{16}\left(\varphi^{12}(i q) \varphi^{4}(-i q)-2 \varphi^{8}(i q) \varphi^{8}(-i q)+\varphi^{12}(-i q) \varphi^{4}(i q)\right) \\
= & \frac{1}{16}\left(\left(\frac{(A+B)}{2}+i \frac{(A-B)}{2}\right)^{12}\left(\frac{(A+B)}{2}-i \frac{(A-B)}{2}\right)^{4}\right. \\
& -2\left(\frac{(A+B)}{2}+i \frac{(A-B)}{2}\right)^{8}\left(\frac{(A+B)}{2}-i \frac{(A-B)}{2}\right)^{8} \\
& \left.+\left(\frac{(A+B)}{2}+i \frac{(A-B)}{2}\right)^{4}\left(\frac{(A+B)}{2}-i \frac{(A-B)}{2}\right)^{12}\right) .
\end{aligned}
$$

Simplifying the last expression with MAPLE, we obtain 
$\Delta\left(-\frac{1}{3}, \frac{4}{3},-\frac{1}{3} ; i q\right)+\Delta\left(-\frac{1}{3}, \frac{4}{3},-\frac{1}{3} ;-i q\right)$
$\quad=\frac{1}{64}\left(-A^{14} B^{2}-2 A^{12} B^{4}+A^{10} B^{6}+4 A^{8} B^{8}+A^{6} B^{10}-2 A^{4} B^{12}-A^{2} B^{14}\right)$,

which is the desired result.

Lemmas 8.6.5 and 8.6.6 allow us to prove the following result.

Lemma 8.6.7. For $q \in \mathbb{C}$ such that $|q|<1$ we have

$$
\begin{aligned}
& \sum_{\substack{n=0 \\
=0(\bmod 4)}}^{+\infty} \delta\left(-\frac{1}{3}, \frac{4}{3},-\frac{1}{3} ; n\right) q^{n} \\
& =\frac{1}{256}\left(-A^{14} B^{2}+2 A^{12} B^{4}+A^{10} B^{6}-4 A^{8} B^{8}+A^{6} B^{10}+2 A^{4} B^{12}-A^{2} B^{14}\right) .
\end{aligned}
$$

Proof. From Lemmas 8.6.5 and 8.6.6, we have

$$
\begin{aligned}
& \sum_{\substack{n=0 \\
n \equiv 0(\bmod 4)}}^{+\infty} \delta\left(-\frac{1}{3}, \frac{4}{3},-\frac{1}{3} ; n\right) q^{n} \\
& =\frac{1}{4} \sum_{n=0}^{+\infty} \delta\left(-\frac{1}{3}, \frac{4}{3},-\frac{1}{3} ; n\right)\left(1+(-1)^{n}+i^{n}+(-i)^{n}\right) q^{n} \\
& =\frac{1}{4}\left(\Delta\left(-\frac{1}{3}, \frac{4}{3},-\frac{1}{3} ; q\right)+\Delta\left(-\frac{1}{3}, \frac{4}{3},-\frac{1}{3} ;-q\right)+\Delta\left(-\frac{1}{3}, \frac{4}{3},-\frac{1}{3} ; i q\right)\right. \\
& \left.=\frac{1}{64} A^{12} B^{4}-\frac{1}{64} A^{8} B^{8}-\frac{1}{64} A^{8} B^{8}+\frac{1}{64} A^{4} B^{12}+\Delta\left(-\frac{1}{3}, \frac{4}{3},-\frac{1}{3} ;-i q\right)\right) \\
& +\frac{1}{256}\left(-A^{14} B^{2}-2 A^{12} B^{4}+A^{10} B^{6}+4 A^{8} B^{8}+A^{6} B^{10}-2 A^{4} B^{12}-A^{2} B^{14}\right) \\
& =\frac{1}{256}\left(-A^{14} B^{2}+2 A^{12} B^{4}+A^{10} B^{6}-4 A^{8} B^{8}+A^{6} B^{10}+2 A^{4} B^{12}-A^{2} B^{14}\right)
\end{aligned}
$$


which is the desired result.

Lemma 8.6.8. For $q \in \mathbb{C}$ such that $|q|<1$ we have

$$
\begin{aligned}
& \sum_{\substack{n=0 \\
n \equiv 0(\bmod 4)}}^{+\infty}(-1)^{n / 4} \delta\left(-\frac{1}{3}, \frac{4}{3},-\frac{1}{3} ; n\right) q^{n} \\
& =\frac{1}{2048}\left(A^{15} B+4 A^{14} B^{2}+3 A^{13} B^{3}-8 A^{12} B^{4}-15 A^{11} B^{5}-4 A^{10} B^{6}+11 A^{9} B^{7}\right. \\
& \quad+16 A^{8} B^{8}+11 A^{7} B^{9}-4 A^{6} B^{10}-15 A^{5} B^{11}-8 A^{4} B^{12}+3 A^{3} B^{13} \\
& \left.+4 A^{2} B^{14}+A B^{15}\right) .
\end{aligned}
$$

Proof. From Lemma 8.6.7, (8.6.1) and (8.6.2), we have

$$
\begin{gathered}
\begin{array}{r}
\Delta\left(-\frac{1}{3}, \frac{4}{3},-\frac{1}{3} ; q\right)+\Delta\left(-\frac{1}{3}, \frac{4}{3},-\frac{1}{3} ;-q\right)+\Delta\left(-\frac{1}{3}, \frac{4}{3},-\frac{1}{3} ; i q\right) \\
\left.+\Delta\left(-\frac{1}{3}, \frac{4}{3},-\frac{1}{3} ;-i q\right)\right) \\
=\frac{1}{64}\left(-A^{14} B^{2}+2 A^{12} B^{4}+A^{10} B^{6}-4 A^{8} B^{8}+A^{6} B^{10}+2 A^{4} B^{12}-A^{2} B^{14}\right) \\
=\frac{1}{64}\left(-\varphi^{14}(q) \varphi^{2}(-q)+2 \varphi^{12}(q) \varphi(-q)^{4}+\varphi(q)^{10} \varphi(-q)^{6}-4 \varphi(q)^{8} \varphi^{8}(-q)\right. \\
\left.+\varphi^{6}(q) \varphi^{10}(-q)+2 \varphi^{4}(q) \varphi^{12}(-q)-\varphi^{2}(q) \varphi^{14}(-q)\right) .
\end{array}
\end{gathered}
$$


Replacing $q$ by $\omega q$ in the last equations and using Lemma 8.6.1, we obtain

$$
\begin{aligned}
& \sum_{\substack{n=0 \\
n=0(\bmod 4)}}^{+\infty}(-1)^{n / 4} \delta\left(-\frac{1}{3}, \frac{4}{3},-\frac{1}{3} ; n\right) q^{n} \\
& =\frac{1}{4}\left(\Delta\left(-\frac{1}{3}, \frac{4}{3},-\frac{1}{3} ; \omega q\right)+\Delta\left(-\frac{1}{3}, \frac{4}{3},-\frac{1}{3} ;-\omega q\right)\right. \\
& \left.+\Delta\left(-\frac{1}{3}, \frac{4}{3},-\frac{1}{3} ; i \omega q\right)+\Delta\left(-\frac{1}{3}, \frac{4}{3},-\frac{1}{3} ;-i \omega q\right)\right) \\
& =\frac{1}{256}\left(-\left(\left(\frac{A B\left(A^{2}+B^{2}\right)}{2}\right)^{1 / 4}+\frac{\omega}{2}(A-B)\right)^{14}\left(\left(\frac{A B\left(A^{2}+B^{2}\right)}{2}\right)^{1 / 4}-\frac{\omega}{2}(A-B)\right)^{2}\right. \\
& +2\left(\left(\frac{A B\left(A^{2}+B^{2}\right)}{2}\right)^{1 / 4}+\frac{\omega}{2}(A-B)\right)^{12}\left(\left(\frac{A B\left(A^{2}+B^{2}\right)}{2}\right)^{1 / 4}-\frac{\omega}{2}(A-B)\right)^{4} \\
& +\left(\left(\frac{A B\left(A^{2}+B^{2}\right)}{2}\right)^{1 / 4}+\frac{\omega}{2}(A-B)\right)^{10}\left(\left(\frac{A B\left(A^{2}+B^{2}\right)}{2}\right)^{1 / 4}-\frac{\omega}{2}(A-B)\right)^{6} \\
& -4\left(\left(\frac{A B\left(A^{2}+B^{2}\right)}{2}\right)^{1 / 4}+\frac{\omega}{2}(A-B)\right)^{8}\left(\left(\frac{A B\left(A^{2}+B^{2}\right)}{2}\right)^{1 / 4}-\frac{\omega}{2}(A-B)\right)^{8} \\
& +\left(\left(\frac{A B\left(A^{2}+B^{2}\right)}{2}\right)^{1 / 4}+\frac{\omega}{2}(A-B)\right)^{6}\left(\left(\frac{A B\left(A^{2}+B^{2}\right)}{2}\right)^{1 / 4}-\frac{\omega}{2}(A-B)\right)^{10} \\
& +2\left(\left(\frac{A B\left(A^{2}+B^{2}\right)}{2}\right)^{1 / 4}+\frac{\omega}{2}(A-B)\right)^{4}\left(\left(\frac{A B\left(A^{2}+B^{2}\right)}{2}\right)^{1 / 4}-\frac{\omega}{2}(A-B)\right)^{12} \\
& \left.-\left(\left(\frac{A B\left(A^{2}+B^{2}\right)}{2}\right)^{1 / 4}+\frac{\omega}{2}(A-B)\right)^{2}\left(\left(\frac{A B\left(A^{2}+B^{2}\right)}{2}\right)^{1 / 4}-\frac{\omega}{2}(A-B)\right)^{14}\right) \\
& =\frac{1}{2048}\left(A^{15} B+4 A^{14} B^{2}+3 A^{13} B^{3}-8 A^{12} B^{4}-15 A^{11} B^{5}-4 A^{10} B^{6}+11 A^{9} B^{7}\right. \\
& +16 A^{8} B^{8}+11 A^{7} B^{9}-4 A^{6} B^{10}-15 A^{5} B^{11}-8 A^{4} B^{12}+3 A^{3} B^{13}+4 A^{2} B^{14} \\
& \left.+A B^{15}\right)
\end{aligned}
$$

where the last equation was found using MAPLE. 
Lemma 8.6.9. For $q \in \mathbb{C}$ such that $|q|<1$ we have

$$
\begin{aligned}
& \sum_{\substack{n=0 \\
n \equiv 0(\bmod 4)}}^{+\infty}\left(15+30(-1)^{n / 4}\right) \delta\left(-\frac{1}{3}, \frac{4}{3},-\frac{1}{3} ; n\right) q^{n} \\
& =\frac{1}{1024}\left(15 A^{15} B+45 A^{13} B^{3}-225 A^{11} B^{5}+165 A^{9} B^{7}+165 A^{7} B^{9}-225 A^{5} B^{11}\right. \\
& \left.+45 A^{3} B^{13}+15 A B^{15}\right) .
\end{aligned}
$$

Proof. By Lemmas 8.6.7 and 8.6.8 we have

$$
\begin{aligned}
& \sum_{\substack{n=0 \\
n \equiv 0(\bmod 4)}}^{+\infty}\left(15+30(-1)^{n / 4}\right) \delta\left(-\frac{1}{3}, \frac{4}{3},-\frac{1}{3} ; n\right) q^{n} \\
= & 15 \sum_{\substack{n=0 \\
n \equiv 0(\bmod 4)}}^{+\infty} \delta\left(-\frac{1}{3}, \frac{4}{3},-\frac{1}{3} ; n\right) q^{n}+30 \sum_{\substack{n=0 \\
n \equiv 0(\bmod 4)}}^{+\infty}(-1)^{n / 4} \delta\left(-\frac{1}{3}, \frac{4}{3},-\frac{1}{3} ; n\right) q^{n} \\
= & \frac{15}{256}\left(-A^{14} B^{2}+2 A^{12} B^{4}+A^{10} B^{6}-4 A^{8} B^{8}+A^{6} B^{10}+2 A^{4} B^{12}-A^{2} B^{14}\right) \\
+ & \frac{30}{2048}\left(A^{15} B+4 A^{14} B^{2}+3 A^{13} B^{3}-8 A^{12} B^{4}-15 A^{11} B^{5}-4 A^{10} B^{6}+11 A^{9} B^{7}\right. \\
& \quad+16 A^{8} B^{8}+11 A^{7} B^{9}-4 A^{6} B^{10}-15 A^{5} B^{11}-8 A^{4} B^{12}+3 A^{3} B^{13}+4 A^{2} B^{14} \\
+ & \left.+A B^{15}\right) .
\end{aligned}
$$

Simplifying the last expression with MAPLE, we obtain

$$
\frac{1}{1024}\left(15 A^{15} B+45 A^{13} B^{3}-225 A^{11} B^{5}+165 A^{9} B^{7}+165 A^{7} B^{9}-225 A^{5} B^{11}\right.
$$

$$
\left.+45 A^{3} B^{13}+15 A B^{15}\right)
$$


which gives the desired result.

We are now ready to prove Theorem 8.6.1.

Proof of Theorem 8.6.1 By Lemmas 8.6.4 and 8.6.9, we have

$$
\begin{aligned}
& \sum_{\substack{n=0 \\
n=0(\bmod 4)}}^{+\infty} \delta\left(-\frac{5}{6}, \frac{25}{12},-\frac{7}{12} ; n\right) q^{n} \\
& =\frac{1}{1024}\left(15 A^{15} B+45 A^{13} B^{3}-225 A^{11} B^{5}+165 A^{9} B^{7}+165 A^{7} B^{9}\right. \\
& \left.\quad-225 A^{5} B^{11}+45 A^{3} B^{13}+15 A B^{15}\right) \\
& =\sum_{\substack{n=0 \\
n \equiv 0(\bmod 4)}}^{+\infty}\left(15+30(-1)^{n / 4}\right) \delta\left(-\frac{1}{3}, \frac{4}{3},-\frac{1}{3} ; n\right) q^{n} .
\end{aligned}
$$

Equating coefficients of $q^{n}$ we obtain

$\delta\left(-\frac{5}{6}, \frac{25}{12},-\frac{7}{12} ; n\right)=\left(15+30(-1)^{n / 4}\right) \delta\left(-\frac{1}{3}, \frac{4}{3},-\frac{1}{3} ; n\right)$, when $n \equiv 0(\bmod 4)$,

which is the desired result. 


\section{Chapter 9}

\section{Sums with 8, 12 and 16 squares - II}

In Chapter 7, we saw the application of our main results (Theorems 6.1.3, 6.2.3 and 6.3.3) to forms involving 8, 12 or 16 squares. Inevitably these results depend on a certain number of arithmetic functions $\delta(a, b, c ; n)$. The purpose of this chapter is to use the identities found in Chapter 8 to reduce the number of functions $\delta(a, b, c ; n)$ occurring in the formulae in the theorems of Chapter 7.

In Section 1 we show that when $n$ is even certain of the formulae of Theorems 7.1 .1 - 7.1.3 depend only on $\sigma_{3}(n / 2), \ldots, \sigma_{3}(n / 16)$, whereas other require in addition the quantity $\delta\left(0, \frac{1}{6}, \frac{1}{6} ; n\right)$. These results reprove those of Alaca, Alaca and Williams [5], [11] in a different way.

In Section 2 we show that when $n$ is even certain of the formulae of Theorems 7.2 .1 - 7.2 .3 depend only on $\sigma_{5}(n / 2), \ldots, \sigma_{5}(n / 16)$, whereas others require additionally the quantity $\tau_{2}(n)$. These results are new. For example we establish in Theorem 9.2.1 the elegant formula

$$
\begin{gathered}
\operatorname{card}\left\{\left(x_{1}, \cdots, x_{12}\right) \in \mathbb{Z}^{12} \mid n=x_{1}^{2}+\cdots+x_{6}^{2}+2 x_{7}^{2}+\cdots+2 x_{12}^{2}\right\} \\
=32 \sigma_{5}(n / 2)-24 \sigma_{5}(n / 4)-512 \sigma_{5}(n / 8)+40 \tau_{2}(n / 2)
\end{gathered}
$$


for all $n \in \mathbb{N}$ with $n \equiv 0(\bmod 2)$, which is an analogue of Ramanujan's beautiful formula for $r_{24}(n)$ (see (1.0.8)).

In Section 3 we do the same for Theorems 7.3.1 - 7.3.3. The results obtained are new. The following result from Theorem 9.3.2 illustrates the type of results obtained. For $n \in \mathbb{N}$ with $n \equiv 0(\bmod 8)$ we show in Theorem 9.3.2 that

$$
\begin{aligned}
& \operatorname{card}\left\{\left(x_{1}, \cdots, x_{16}\right) \in \mathbb{Z}^{16} \mid n=x_{1}^{2}+x_{2}^{2}+2 x_{3}^{2}+2 x_{4}^{2}+4 x_{5}^{2}+\cdots+4 x_{16}^{2}\right\} \\
& =480 \sigma_{7}(n / 8)+\frac{3}{8} \sum_{\substack{\left(x_{1}, \ldots, x_{8}\right) \in \mathbb{Z}^{8} \\
x_{1}^{2}++x_{8}^{2}=n}}\left(x_{1}^{4}-3 x_{1}^{2} x_{2}^{2}\right) .
\end{aligned}
$$

The following short table gives the values occurring in this formula for $n=8,16,24,32,40$.

\begin{tabular}{|c|c|c|c|}
\hline$n$ & $N([2,2,12] ; n)$ & $\sigma_{7}(n / 8)$ & $\sum_{\substack{\left(x_{1}, \ldots, x_{8}\right) \in \mathbb{Z}^{8} \\
x_{1}^{2}++x_{8}^{2}=n}}\left(x_{1}^{4}-3 x_{1}^{2} x_{2}^{2}\right)$ \\
\hline 8 & 864 & 1 & 1024 \\
16 & 58848 & 129 & -8192 \\
24 & 1054848 & 2188 & 12288 \\
32 & 7950816 & 16513 & 65536 \\
40 & 37419840 & 78126 & -215040 \\
\hline
\end{tabular}

\subsection{Sums with 8 squares}

In this section we restrict $n$ to particular congruence classes modulo 2 and modulo 4 to reduce the number of delta functions $\delta(a, b, c ; n)$ occurring in Theorems 7.1.1, 7.1.2 and 7.1.3.

We make use of the following simple properties of $\sigma_{3}$. Let $n \in \mathbb{N}$. 
If $n \equiv 0(\bmod 2)$ then

$$
\sigma_{3}(n)=9 \sigma_{3}(n / 2)-8 \sigma_{3}(n / 4)
$$

if $n \equiv 2(\bmod 4)$ then

$$
\sigma_{3}(n)=9 \sigma_{3}(n / 2)
$$

and if $n \equiv 0(\bmod 4)$ then

$$
\left\{\begin{array}{c}
\sigma_{3}(n)=73 \sigma_{3}(n / 4)-72 \sigma_{3}(n / 8), \\
\sigma_{3}(n / 2)=9 \sigma_{3}(n / 4)-8 \sigma_{3}(n / 8) .
\end{array}\right.
$$

We begin with the simplification of Theorem 7.1.1 to the cases $n \equiv 0(\bmod 2)$ and $n \equiv 2(\bmod 4)$.

Theorem 9.1.1. Let $n \in \mathbb{N}$. For $n \equiv 0(\bmod 2)$, we have

$N([6,2,0] ; n)=64 \sigma_{3}(n / 2)-80 \sigma_{3}(n / 4)+256 \sigma_{3}(n / 8)$,

$N([4,4,0] ; n)=32 \sigma_{3}(n / 2)-48 \sigma_{3}(n / 4)+256 \sigma_{3}(n / 8)$,

$N([2,6,0] ; n)=16 \sigma_{3}(n / 2)-32 \sigma_{3}(n / 4)+256 \sigma_{3}(n / 8)$.

Proof. The results follow by applying Theorem 8.3.1 to Theorem 7.1.1 and simplifying using (9.1.1).

This theorem follows from Alaca, Alaca and Williams [11, Theorem 1.1 (ii)(iv)(ix), pp. 38-39] as $c_{8}(n)=0$ for $n \equiv 0(\bmod 2)$. The second formula also follows from Williams [83, Theorem 2, p. 388].

Corollary 9.1.1. Let $n \in \mathbb{N}$. If $n \equiv 0(\bmod 2)$ then

$$
\begin{gathered}
\operatorname{card}\left\{\left(x_{1}, \cdots, x_{8}\right) \in \mathbb{Z}^{8} \mid n=x_{1}^{2}+x_{2}^{2}+2 x_{3}^{2}+\cdots+2 x_{8}^{2}, x_{1} \equiv x_{2} \equiv 1(\bmod 2)\right\} \\
=16 \sigma_{3}(n / 2)-96 \sigma_{3}(n / 4)+336 \sigma_{3}(n / 8)-256 \sigma_{3}(n / 16) .
\end{gathered}
$$


Proof. As $n \equiv 0(\bmod 2)$ the solutions of

$$
n=x_{1}^{2}+x_{2}^{2}+2 x_{3}^{2}+\cdots+2 x_{8}^{2}
$$

satisfy $x_{1} \equiv x_{2} \equiv 0(\bmod 2)$ or $x_{1} \equiv x_{2} \equiv 1(\bmod 2)$. The former satisfy

$$
\frac{n}{2}=x_{3}^{2}+\cdots+x_{8}^{2}+2\left(\frac{x_{1}}{2}\right)^{2}+2\left(\frac{x_{2}}{2}\right)^{2}
$$

so there are $N([6,2,0] ; n / 2)$ such solutions.

Hence the required number is

$$
\begin{aligned}
N([2,0,6] ; n)- & N([6,2,0] ; n / 2) \\
& =16 \sigma_{3}(n / 2)-96 \sigma_{3}(n / 4)+336 \sigma_{3}(n / 8)-256 \sigma_{3}(n / 16)
\end{aligned}
$$

by Theorem 9.1.1.

Theorem 9.1.2. Let $n \in \mathbb{N}$. For $n \equiv 2(\bmod 4)$, we have

$N([6,2,0] ; n)=64 \sigma_{3}(n / 2)$,

$N([4,4,0] ; n)=32 \sigma_{3}(n / 2)$,

$N([2,6,0] ; n)=16 \sigma_{3}(n / 2)$.

Proof. This follows from Theorem 9.1.1, the facts that $\sigma_{3}(n / 4)$ and $\sigma_{3}(n / 8)$ are both equal to 0 when $n \equiv 2(\bmod 4)$, and (9.1.2).

The next result shows that Theorem 7.1.2 can be written in terms of the function $\delta\left(0, \frac{1}{6}, \frac{1}{6} ; n\right)$. This theorem is due to Alaca, Alaca and Williams [11, Theorem $1.1(\mathrm{iii})(\mathrm{v})(\mathrm{vii})(\mathrm{viii})(\mathrm{x})(\mathrm{xi})(\mathrm{xii})(\mathrm{xiii})(\mathrm{xiv})$, pp. 38-39].

Theorem 9.1.3. For $n \in \mathbb{N}$ we have 


$$
\begin{aligned}
N([1,2,5] ; n)= & \frac{1}{4} \sigma_{3}(n)-\frac{1}{4} \sigma_{3}(n / 2)-16 \sigma_{3}(n / 8)+256 \sigma_{3}(n / 16) \\
& +\left(\frac{3}{4}+\left(\frac{-4}{n}\right)\right) \delta\left(0, \frac{1}{6}, \frac{1}{6} ; n\right)+2 \delta\left(0, \frac{1}{6}, \frac{1}{6} ; n / 2\right), \\
N([2,2,4] ; n)= & \frac{1}{2} \sigma_{3}(n)-\frac{1}{2} \sigma_{3}(n / 2)-16 \sigma_{3}(n / 8)+256 \sigma_{3}(n / 16) \\
& +\left(\frac{3}{2}+2\left(\frac{-4}{n}\right)\right) \delta\left(0, \frac{1}{6}, \frac{1}{6} ; n\right)+4 \delta\left(0, \frac{1}{6}, \frac{1}{6} ; n / 2\right),
\end{aligned}
$$

$$
\begin{aligned}
N([3,2,3] ; n)= & \sigma_{3}(n)-\sigma_{3}(n / 2)-16 \sigma_{3}(n / 8)+256 \sigma_{3}(n / 16) \\
& +\left(2+3\left(\frac{-4}{n}\right)\right) \delta\left(0, \frac{1}{6}, \frac{1}{6} ; n\right)+8 \delta\left(0, \frac{1}{6}, \frac{1}{6} ; n / 2\right),
\end{aligned}
$$

$$
\begin{aligned}
N([4,2,2] ; n)= & 2 \sigma_{3}(n)-2 \sigma_{3}(n / 2)-16 \sigma_{3}(n / 8)+256 \sigma_{3}(n / 16) \\
& +\left(2+4\left(\frac{-4}{n}\right)\right) \delta\left(0, \frac{1}{6}, \frac{1}{6} ; n\right)+12 \delta\left(0, \frac{1}{6}, \frac{1}{6} ; n / 2\right),
\end{aligned}
$$

$N([5,2,1] ; n)=4 \sigma_{3}(n)-4 \sigma_{3}(n / 2)-16 \sigma_{3}(n / 8)+256 \sigma_{3}(n / 16)$

$$
+\left(2+4\left(\frac{-4}{n}\right)\right) \delta\left(0, \frac{1}{6}, \frac{1}{6} ; n\right)+12 \delta\left(0, \frac{1}{6}, \frac{1}{6} ; n / 2\right),
$$

$$
\begin{aligned}
N([1,4,3] ; n)= & \frac{1}{2} \sigma_{3}(n)-\frac{1}{2} \sigma_{3}(n / 2)-16 \sigma_{3}(n / 8)+256 \sigma_{3}(n / 16) \\
& +\left(\frac{1}{2}+\left(\frac{-4}{n}\right)\right) \delta\left(0, \frac{1}{6}, \frac{1}{6} ; n\right)+4 \delta\left(0, \frac{1}{6}, \frac{1}{6} ; n / 2\right)
\end{aligned}
$$

$$
\begin{aligned}
N([2,4,2] ; n)= & \sigma_{3}(n)-\sigma_{3}(n / 2)-16 \sigma_{3}(n / 8)+256 \sigma_{3}(n / 16) \\
& +\left(1+2\left(\frac{-4}{n}\right)\right) \delta\left(0, \frac{1}{6}, \frac{1}{6} ; n\right)+4 \delta\left(0, \frac{1}{6}, \frac{1}{6} ; n / 2\right),
\end{aligned}
$$

$$
\begin{aligned}
N([3,4,1] ; n)= & 2 \sigma_{3}(n)-2 \sigma_{3}(n / 2)-16 \sigma_{3}(n / 8)+256 \sigma_{3}(n / 16) \\
& +\left(2+2\left(\frac{-4}{n}\right)\right) \delta\left(0, \frac{1}{6}, \frac{1}{6} ; n\right)+4 \delta\left(0, \frac{1}{6}, \frac{1}{6} ; n / 2\right),
\end{aligned}
$$

$$
\begin{aligned}
N([1,6,1] ; n)= & \sigma_{3}(n)-\sigma_{3}(n / 2)-16 \sigma_{3}(n / 8)+256 \sigma_{3}(n / 16) \\
& +\delta\left(0, \frac{1}{6}, \frac{1}{6} ; n\right)+4 \delta\left(0, \frac{1}{6}, \frac{1}{6} ; n / 2\right)
\end{aligned}
$$


Proof. We only prove the formula for $N([3,2,3] ; n)$ as the proofs for the other cases are similar. By Theorems 7.1.2, 8.3.2 and 8.3.3 we have

$$
\begin{aligned}
N([3,2,3] ; n)= & \sigma_{3}(n)-\sigma_{3}(n / 2)-16 \sigma_{3}(n / 8)+256 \sigma_{3}(n / 16) \\
& +2 \delta\left(0, \frac{1}{6}, \frac{1}{6} ; n\right)+\frac{5}{2} \delta\left(-\frac{1}{6}, \frac{5}{12}, \frac{1}{12} ; n\right) \\
& +\frac{1}{2} \delta\left(\frac{1}{6},-\frac{1}{12}, \frac{1}{4} ; n\right) \\
= & \sigma_{3}(n)-\sigma_{3}(n / 2)-16 \sigma_{3}(n / 8)+256 \sigma_{3}(n / 16)+2 \delta\left(0, \frac{1}{6}, \frac{1}{6} ; n\right) \\
& +\left(\delta\left(-\frac{1}{6}, \frac{5}{12}, \frac{1}{12} ; n\right)-\delta\left(\frac{1}{6},-\frac{1}{12}, \frac{1}{4} ; n\right)\right) \\
& +\left(\frac{3}{2} \delta\left(-\frac{1}{6}, \frac{5}{12}, \frac{1}{12} ; n\right)+\frac{3}{2} \delta\left(\frac{1}{6},-\frac{1}{12}, \frac{1}{4} ; n\right)\right) \\
= & \sigma_{3}(n)-\sigma_{3}(n / 2)-16 \sigma_{3}(n / 8)+256 \sigma_{3}(n / 16)+2 \delta\left(0, \frac{1}{6}, \frac{1}{6} ; n\right) \\
& +8 \delta(0,1 / 6,1 / 6 ; n / 2)+3\left(\frac{-4}{n}\right) \delta(0,1 / 6,1 / 6 ; n) \\
= & \sigma_{3}(n)-\sigma_{3}(n / 2)-16 \sigma_{3}(n / 8)+256 \sigma_{3}(n / 16) \\
& +\left(2+3\left(\frac{-4}{n}\right)\right) \delta(0,1 / 6,1 / 6 ; n)+8 \delta(0,1 / 6,1 / 6 ; n / 2),
\end{aligned}
$$

as asserted.

Restricting Theorem 9.1 .3 to the special cases $n \equiv 0(\bmod 4)$ and $n \equiv 2$ $(\bmod 4)$, we obtain the following two results.

Theorem 9.1.4. Let $n \in \mathbb{N}$. For $n \equiv 0(\bmod 4)$, we have $N([1,2,5] ; n)=16 \sigma_{3}(n / 4)-32 \sigma_{3}(n / 8)+256 \sigma_{3}(n / 16)$,

$N([2,2,4] ; n)=32 \sigma_{3}(n / 4)-48 \sigma_{3}(n / 8)+256 \sigma_{3}(n / 16)$, 


$$
\begin{aligned}
& N([3,2,3] ; n)=64 \sigma_{3}(n / 4)-80 \sigma_{3}(n / 8)+256 \sigma_{3}(n / 16), \\
& N([4,2,2] ; n)=128 \sigma_{3}(n / 4)-144 \sigma_{3}(n / 8)+256 \sigma_{3}(n / 16), \\
& N([5,2,1] ; n)=256 \sigma_{3}(n / 4)-272 \sigma_{3}(n / 8)+256 \sigma_{3}(n / 16), \\
& N([1,4,3] ; n)=32 \sigma_{3}(n / 4)-48 \sigma_{3}(n / 8)+256 \sigma_{3}(n / 16), \\
& N([2,4,2] ; n)=64 \sigma_{3}(n / 4)-80 \sigma_{3}(n / 8)+256 \sigma_{3}(n / 16), \\
& N([3,4,1] ; n)=128 \sigma_{3}(n / 4)-144 \sigma_{3}(n / 8)+256 \sigma_{3}(n / 16), \\
& N([1,6,1] ; n)=64 \sigma_{3}(n / 4)-80 \sigma_{3}(n / 8)+256 \sigma_{3}(n / 16) .
\end{aligned}
$$

Proof. This follows from Theorem 8.3.1, Theorem 9.1.3 and (9.1.3).

Theorem 9.1.5. Let $n \in \mathbb{N}$. For $n \equiv 2(\bmod 4)$ we have

$$
\begin{aligned}
& N([1,2,5] ; n)=2 \sigma_{3}(n / 2)+2 \delta\left(0, \frac{1}{6}, \frac{1}{6} ; n / 2\right), \\
& N([2,2,4] ; n)=4 \sigma_{3}(n / 2)+4 \delta\left(0, \frac{1}{6}, \frac{1}{6} ; n / 2\right), \\
& N([3,2,3] ; n)=8 \sigma_{3}(n / 2)+8 \delta\left(0, \frac{1}{6}, \frac{1}{6} ; n / 2\right), \\
& N([4,2,2] ; n)=16 \sigma_{3}(n / 2)+12 \delta\left(0, \frac{1}{6}, \frac{1}{6} ; n / 2\right),
\end{aligned}
$$




$$
\begin{aligned}
& N([5,2,1] ; n)=32 \sigma_{3}(n / 2)+12 \delta\left(0, \frac{1}{6}, \frac{1}{6} ; n / 2\right), \\
& N([1,4,3] ; n)=4 \sigma_{3}(n / 2)+4 \delta\left(0, \frac{1}{6}, \frac{1}{6} ; n / 2\right) \\
& N([2,4,2] ; n)=8 \sigma_{3}(n / 2)+4 \delta\left(0, \frac{1}{6}, \frac{1}{6} ; n / 2\right) \\
& N([3,4,1] ; n)=16 \sigma_{3}(n / 2)+4 \delta\left(0, \frac{1}{6}, \frac{1}{6} ; n / 2\right) \\
& N([1,6,1] ; n)=8 \sigma_{3}(n / 2)+4 \delta\left(0, \frac{1}{6}, \frac{1}{6} ; n / 2\right)
\end{aligned}
$$

Proof. The results follow from Theorem 8.3.1, Theorem 9.1.3, the fact that $\sigma_{3}(n / 8)$ and $\sigma_{3}(n / 16)$ are both equal to 0 when $n \equiv 2(\bmod 4)$, and the use of (9.1.2) for simplification.

The next result shows that Theorem 7.1 .3 can be written in terms of the function $\delta\left(0, \frac{1}{6}, \frac{1}{6} ; n\right)$ for all $n \in \mathbb{N}$. This theorem is due to Alaca, Alaca and Williams [5, Theorem 1.1, pp. 343-344].

Theorem 9.1.6. For $n \in \mathbb{N}$, we have

$$
\begin{aligned}
N([1,0,7] ; n)= & \left(\frac{1}{8}+\frac{1}{8}\left(\frac{-4}{n}\right)\right) \sigma_{3}(n)-\frac{9}{8} \sigma_{3}(n / 2)+17 \sigma_{3}(n / 4)-32 \sigma_{3}(n / 8) \\
& +256 \sigma_{3}(n / 16)+\left(\frac{7}{8}+\frac{7}{8}\left(\frac{-4}{n}\right)\right) \delta\left(0, \frac{1}{6}, \frac{1}{6} ; n\right) \\
N([2,0,6] ; n)= & \left(\frac{1}{4}+\frac{1}{4}\left(\frac{-4}{n}\right)\right) \sigma_{3}(n)-\frac{1}{4} \sigma_{3}(n / 2)-16 \sigma_{3}(n / 8) \\
& +256 \sigma_{3}(n / 16)+\left(\frac{7}{4}+\frac{7}{4}\left(\frac{-4}{n}\right)\right) \delta\left(0, \frac{1}{6}, \frac{1}{6} ; n\right) \\
& +2 \delta\left(0, \frac{1}{6}, \frac{1}{6} ; n / 2\right)
\end{aligned}
$$




$$
\begin{aligned}
N([3,0,5] ; n)= & \left(\frac{1}{2}+\frac{1}{4}\left(\frac{-4}{n}\right)\right) \sigma_{3}(n)+\frac{3}{2} \sigma_{3}(n / 2)-34 \sigma_{3}(n / 4)+16 \sigma_{3}(n / 8) \\
& +256 \sigma_{3}(n / 16)+\left(\frac{5}{2}+\frac{11}{4}\left(\frac{-4}{n}\right)\right) \delta\left(0, \frac{1}{6}, \frac{1}{6} ; n\right) \\
& +6 \delta\left(0, \frac{1}{6}, \frac{1}{6} ; n / 2\right)
\end{aligned}
$$

$$
\begin{aligned}
N([4,0,4] ; n)= & \sigma_{3}(n)+3 \sigma_{3}(n / 2)-68 \sigma_{3}(n / 4)+48 \sigma_{3}(n / 8) \\
& +256 \sigma_{3}(n / 16)+\left(3+4\left(\frac{-4}{n}\right)\right) \delta\left(0, \frac{1}{6}, \frac{1}{6} ; n\right) \\
& +12 \delta\left(0, \frac{1}{6}, \frac{1}{6} ; n / 2\right) \\
N([5,0,3] ; n)= & \left(2-\frac{1}{2}\left(\frac{-4}{n}\right)\right) \sigma_{3}(n)+2 \sigma_{3}(n / 2)-68 \sigma_{3}(n / 4)+48 \sigma_{3}(n / 8) \\
& +256 \sigma_{3}(n / 16)+\left(3+\frac{11}{2}\left(\frac{-4}{n}\right)\right) \delta\left(0, \frac{1}{6}, \frac{1}{6} ; n\right) \\
& +20 \delta\left(0, \frac{1}{6}, \frac{1}{6} ; n / 2\right) \\
& +256 \sigma_{3}(n / 16)+\left(2+7\left(\frac{-4}{n}\right)\right) \delta\left(0, \frac{1}{6}, \frac{1}{6} ; n\right) \\
N([6,0,2] ; n)= & \left.4-\left(\frac{-4}{n}\right)\right) \sigma_{3}(n)-4 \sigma_{3}(n / 2)-16 \sigma_{3}(n / 8) \\
& +28 \delta\left(0, \frac{1}{6}, \frac{1}{6} ; n / 2\right) \\
& +28 \delta\left(0, \frac{1}{6}, \frac{1}{6} ; n / 2\right) \\
& +256 \sigma_{3}(n / 16)+7\left(\frac{-4}{n}\right) \delta\left(0, \frac{1}{6}, \frac{1}{6} ; n\right) \\
N([7,0,1] ; n)= & \left(8-\left(\frac{-4}{n}\right)\right) \sigma_{3}(n)-16 \sigma_{3}(n / 2)+136 \sigma_{3}(n / 4)-144 \sigma_{3}(n / 8) \\
& +28) \\
& \\
&
\end{aligned}
$$

Proof. We only prove the formula for $N([2,0,6] ; n)$ as the proofs for the other cases 
are similar. By Theorems 7.1.1, 8.3.2, 8.3.3 and 8.3.4, we have

$$
\begin{aligned}
N & ([2,0,6] ; n) \\
= & \frac{1}{4} \sigma_{3}(n)-\frac{1}{4} \sigma_{3}(n / 2)-16 \sigma_{3}(n / 8)+256 \sigma_{3}(n / 16) \\
& +\frac{7}{4} \delta\left(0, \frac{1}{6}, \frac{1}{6} ; n\right)-\frac{1}{16} \delta\left(-\frac{1}{2}, \frac{17}{12},-\frac{7}{12} ; n\right)+\frac{1}{16} \delta\left(-\frac{1}{6}, \frac{11}{12},-\frac{5}{12} ; n\right) \\
& +2 \delta\left(-\frac{1}{6}, \frac{5}{12}, \frac{1}{12} ; n\right)+\frac{1}{2} \delta\left(\frac{1}{6},-\frac{1}{12}, \frac{1}{4} ; n\right) \\
= & \frac{1}{4} \sigma_{3}(n)-\frac{1}{4} \sigma_{3}(n / 2)-16 \sigma_{3}(n / 8)+256 \sigma_{3}(n / 16) \\
& +\frac{7}{4} \delta\left(0, \frac{1}{6}, \frac{1}{6} ; n\right)+\left(-\frac{1}{16} \delta\left(-\frac{1}{2}, \frac{17}{12},-\frac{7}{12} ; n\right)+\frac{1}{16} \delta\left(-\frac{1}{6}, \frac{11}{12},-\frac{5}{12} ; n\right)\right. \\
& \left.+\frac{7}{8} \delta\left(-\frac{1}{6}, \frac{5}{12}, \frac{1}{12} ; n\right)-\frac{1}{8} \delta\left(\frac{1}{6},-\frac{1}{12}, \frac{1}{4} ; n\right)\right) \\
& +\left(\frac{7}{8} \delta\left(-\frac{1}{6}, \frac{5}{12}, \frac{1}{12} ; n\right)+\frac{7}{8} \delta\left(\frac{1}{6},-\frac{1}{12}, \frac{1}{4} ; n\right)\right) \\
& +\left(\frac{1}{4} \delta\left(-\frac{1}{6}, \frac{5}{12}, \frac{1}{12} ; n\right)-\frac{1}{4} \delta\left(\frac{1}{6},-\frac{1}{12}, \frac{1}{4} ; n\right)\right) \\
& +2 \delta\left(0, \frac{1}{6}, \frac{1}{6} ; n / 2\right), \\
= & \frac{1}{4} \sigma_{3}(n)-\frac{1}{4} \sigma_{3}(n / 2)-16 \sigma_{3}(n / 8)+256 \sigma_{3}(n / 16) \\
+ & \left.\frac{7}{4} \delta\left(0, \frac{1}{6}, \frac{1}{6} ; n\right)+\frac{1}{4}\left(\frac{-4}{n}\right) \sigma_{3}(n)+\frac{7}{4}\left(\frac{-4}{n}\right) \delta\left(0, \frac{1}{6}, \frac{1}{6} ; n\right)\right) \\
& +2)
\end{aligned}
$$

which is the desired result.

We note that when $n \equiv 3(\bmod 4)$ the first assertion of Theorem 9.1.6 gives $N([1,0,7] ; n)=0$. This is clear as the congruence

$$
x_{1}^{2}+4 x_{2}^{2}+\cdots+4 x_{8}^{2} \equiv n \quad(\bmod 4)
$$


is insolvable for $n \equiv 3(\bmod 4)$.

In the next two theorems we restrict Theorem 9.1.6 to the cases $n \equiv 0(\bmod 4)$ and $n \equiv 2(\bmod 4)$. It is interesting that in the case $n \equiv 0(\bmod 4)$ (Theorem 9.1.7) all the formulae can be expressed in terms of $\sigma_{3}(n), \sigma_{3}(n / 2), \ldots, \sigma_{3}(n / 16)$ only.

Theorem 9.1.7. Let $n \in \mathbb{N}$. For $n \equiv 0(\bmod 4)$, we have $N([1,0,7] ; n)=16 \sigma_{3}(n / 4)-32 \sigma_{3}(n / 8)+256 \sigma_{3}(n / 16)$, $N([2,0,6] ; n)=16 \sigma_{3}(n / 4)-32 \sigma_{3}(n / 8)+256 \sigma_{3}(n / 16)$, $N([3,0,5] ; n)=16 \sigma_{3}(n / 4)-32 \sigma_{3}(n / 8)+256 \sigma_{3}(n / 16)$, $N([4,0,4] ; n)=32 \sigma_{3}(n / 4)-48 \sigma_{3}(n / 8)+256 \sigma_{3}(n / 16)$, $N([5,0,3] ; n)=96 \sigma_{3}(n / 4)-112 \sigma_{3}(n / 8)+256 \sigma_{3}(n / 16)$, $N([6,0,2] ; n)=256 \sigma_{3}(n / 4)-272 \sigma_{3}(n / 8)+256 \sigma_{3}(n / 16)$, $N([7,0,1] ; n)=576 \sigma_{3}(n / 4)-592 \sigma_{3}(n / 8)+256 \sigma_{3}(n / 16)$.

Proof. The results follow from Theorem 9.1.6, Theorem 8.3.1, the fact that $\left(\frac{-4}{n}\right)$ is equal to 0 when $n \equiv 0(\bmod 4)$, and the use of $(9.1 .3)$ for simplification.

We remark that the formula for $N([1,0,7] ; n)(n \equiv 0(\bmod 4))$ can be derived as follows from that for $r_{8}(n)$ : we have

$$
n=x_{1}^{2}+4 x_{2}^{2}+\cdots+4 x_{8}^{2} \Longleftrightarrow \frac{n}{4}=\left(\frac{x_{1}}{2}\right)^{2}+x_{2}^{2}+\cdots+x_{8}^{2}
$$


so that

$$
N([1,0,7] ; n)=r_{8}(n / 4)=16 \sigma_{3}(n / 4)-32 \sigma_{3}(n / 8)+256 \sigma_{3}(n / 16) .
$$

Similarly the formula for $N([2,0,6] ; n)(n \equiv 0(\bmod 4))$ can also be derived from that for $r_{8}(n)$. We have

$$
n=x_{1}^{2}+x_{2}^{2}+4 x_{3}^{2}+\cdots+\cdots+4 x_{8}^{2} \Longleftrightarrow \frac{n}{4}=\left(\frac{x_{1}}{2}\right)^{2}+\left(\frac{x_{2}}{2}\right)^{2} x_{2}^{2}+x_{3}^{2}+\cdots+x_{8}^{2}
$$

so that

$$
N([2,0,6] ; n)=r_{8}(n / 4)=16 \sigma_{3}(n / 4)-32 \sigma_{3}(n / 8)+256 \sigma_{3}(n / 16) .
$$

The same argument applies to $N([3,0,5] ; n)$ as $x_{1}^{2}+x_{2}^{2}+x_{3}^{2} \equiv 0(\bmod 4)$ implies $x_{1} \equiv$ $x_{2} \equiv x_{3} \equiv 0(\bmod 2)$. However we cannot apply the same argument to $N([4,4,0] ; n)$ as $x_{1}^{2}+x_{2}^{2}+x_{3}^{2}+x_{4}^{2} \equiv 0(\bmod 4)$ does not imply $x_{1} \equiv x_{2} \equiv x_{3} \equiv x_{4} \equiv 0(\bmod 2)$.

Theorem 9.1.8. Let $n \in \mathbb{N}$. For $n \equiv 2(\bmod 4)$, we have $N([1,0,7] ; n)=0$,

$$
\begin{aligned}
& N([2,0,6] ; n)=2 \sigma_{3}(n / 2)+2 \delta\left(0, \frac{1}{6}, \frac{1}{6} ; n / 2\right), \\
& N([3,0,5] ; n)=6 \sigma_{3}(n / 2)+6 \delta\left(0, \frac{1}{6}, \frac{1}{6} ; n / 2\right), \\
& N([4,0,4] ; n)=12 \sigma_{3}(n / 2)+12 \delta\left(0, \frac{1}{6}, \frac{1}{6} ; n / 2\right), \\
& N([5,0,3] ; n)=20 \sigma_{3}(n / 2)+20 \delta\left(0, \frac{1}{6}, \frac{1}{6} ; n / 2\right), \\
& N([6,0,2] ; n)=32 \sigma_{3}(n / 2)+28 \delta\left(0, \frac{1}{6}, \frac{1}{6} ; n / 2\right), \\
& N([7,0,1] ; n)=56 \sigma_{3}(n / 2)+28 \delta\left(0, \frac{1}{6}, \frac{1}{6} ; n / 2\right) .
\end{aligned}
$$


Proof. The results follow from Theorem 9.1.6, Theorem 8.3.1, the fact that $\left(\frac{-4}{n}\right), \sigma_{3}(n / 4), \sigma_{3}(n / 8)$ and $\sigma_{3}(n / 16)$ are all equal to 0 when $n \equiv 2(\bmod 4)$, and the use of (9.1.2) for simplification.

We remark that the first assertion of Theorem 9.1.8 is clear as the congruence

$$
n \equiv x_{1}^{2}+4 x_{2}^{2}+\cdots+4 x_{8}^{2} \quad(\bmod 4)
$$

is insolvable when $n \equiv 2(\bmod 4)$.

\subsection{Sums with 12 squares}

In this section we restrict $n$ to particular congruence classes modulo 4 and modulo 8 to reduce the number of delta functions $\delta(a, b, c ; n)$ occurring in Theorems 7.2.1, 7.2.2 and 7.2.3. The results obtained are new.

We make use of the following simple properties of $\sigma_{5}$. Let $n \in \mathbb{N}$.

If $n \equiv 0(\bmod 2)$ then

$$
\sigma_{5}(n)=33 \sigma_{5}(n / 2)-32 \sigma_{5}(n / 4)
$$

if $n \equiv 2(\bmod 4)$ then

$$
\sigma_{5}(n)=33 \sigma_{5}(n / 2)
$$

if $n \equiv 0(\bmod 4)$ then

$$
\left\{\begin{array}{l}
\sigma_{5}(n)=1057 \sigma_{5}(n / 4)-1056 \sigma_{5}(n / 8) \\
\sigma_{5}(n / 2)=33 \sigma_{5}(n / 4)-32 \sigma_{5}(n / 8)
\end{array}\right.
$$


and if $n \equiv 0(\bmod 8)$ then

$$
\left\{\begin{array}{l}
\sigma_{5}(n)=33825 \sigma_{5}(n / 8)-33824 \sigma_{5}(n / 16) \\
\sigma_{5}(n / 2)=1057 \sigma_{5}(n / 8)-1056 \sigma_{5}(n / 16) \\
\sigma_{5}(n / 4)=33 \sigma_{5}(n / 8)-32 \sigma_{5}(n / 16)
\end{array}\right.
$$

We begin with the simplification of Theorem 7.2 .1 when $n \equiv 0(\bmod 2)$. It is interesting that in this case the formulae depend only on $\tau_{2}(n / 2)$ in addition to $\sigma_{3}(n), \ldots, \sigma_{3}(n / 8)$.

Theorem 9.2.1. Let $n \in \mathbb{N}$. For $n \equiv 0(\bmod 2)$, we have

$N([10,2,0] ; n)=128 \sigma_{5}(n / 2)-120 \sigma_{5}(n / 4)-512 \sigma_{5}(n / 8)+56 \tau_{2}(n / 2)$,

$N([8,4,0] ; n)=64 \sigma_{5}(n / 2)-56 \sigma_{5}(n / 4)-512 \sigma_{5}(n / 8)+56 \tau_{2}(n / 2)$,

$N([6,6,0] ; n)=32 \sigma_{5}(n / 2)-24 \sigma_{5}(n / 4)-512 \sigma_{5}(n / 8)+40 \tau_{2}(n / 2)$,

$N([4,8,0] ; n)=16 \sigma_{5}(n / 2)-8 \sigma_{5}(n / 4)-512 \sigma_{5}(n / 8)+24 \tau_{2}(n / 2)$,

$N([2,10,0] ; n)=8 \sigma_{5}(n / 2)-512 \sigma_{5}(n / 8)+16 \tau_{2}(n / 2)$.

Proof. If $n \equiv 0(\bmod 2)$, we have by Theorems 7.2.1, 8.4.1 and 8.4.2

$$
\begin{aligned}
N([4,8,0] ; n)= & \frac{1}{2} \sigma_{5}(n)-\frac{1}{2} \sigma_{5}(n / 2)+8 \sigma_{5}(n / 4)-512 \sigma_{5}(n / 8) \\
& +\frac{7}{2} \delta\left(0, \frac{1}{2}, 0 ; n\right)+4 \delta\left(-\frac{1}{3}, 1,-\frac{1}{6} ; n\right)-8 \delta\left(0,0, \frac{1}{2} ; n\right) \\
= & \frac{1}{2} \sigma_{5}(n)-\frac{1}{2} \sigma_{5}(n / 2)+8 \sigma_{5}(n / 4)-512 \sigma_{5}(n / 8)
\end{aligned}
$$




$$
\begin{aligned}
& +3 \delta\left(-\frac{1}{3}, 1,-\frac{1}{6} ; n\right) \\
& =\frac{1}{2} \sigma_{5}(n)-\frac{1}{2} \sigma_{5}(n / 2)+8 \sigma_{5}(n / 4)-512 \sigma_{5}(n / 8) \\
& +24 \tau_{2}(n / 2) .
\end{aligned}
$$

Simplifying using (9.2.1), we obtain the desired result.

In the next two theorems we restrict Theorem 9.2 .1 to the cases $n \equiv 0$ $(\bmod 4)$ and $n \equiv 2(\bmod 4)$.

Theorem 9.2.2. Let $n \in \mathbb{N}$. For $n \equiv 0(\bmod 4)$, we have

$N([10,2,0] ; n)=4104 \sigma_{5}(n / 4)-4608 \sigma_{5}(n / 8)$,

$N([8,4,0] ; n)=2056 \sigma_{5}(n / 4)-2560 \sigma_{5}(n / 8)$,

$N([6,6,0] ; n)=1032 \sigma_{5}(n / 4)-1536 \sigma_{5}(n / 8)$,

$N([4,8,0] ; n)=520 \sigma_{5}(n / 4)-1024 \sigma_{5}(n / 8)$,

$N([2,10,0] ; n)=264 \sigma_{5}(n / 4)-768 \sigma_{5}(n / 8)$.

Proof. For $n \equiv 0(\bmod 4)$ we have $\frac{n}{2} \equiv 0(\bmod 2)$ so $\tau_{2}\left(\frac{n}{2}\right)=0$. The theorem now follows from Theorem 9.2.1 and (9.2.3).

Theorem 9.2.3. Let $n \in \mathbb{N}$. For $n \equiv 2(\bmod 4)$, we have $N([10,2,0] ; n)=128 \sigma_{5}(n / 2)+56 \tau_{2}(n / 2)$,

$N([8,4,0] ; n)=64 \sigma_{5}(n / 2)+56 \tau_{2}(n / 2)$, 
$N([6,6,0] ; n)=33 \sigma_{5}(n / 2)+40 \tau_{2}(n / 2)$,

$N([4,8,0] ; n)=16 \sigma_{5}(n / 2)+24 \tau_{2}(n / 2)$,

$N([2,10,0] ; n)=8 \sigma_{5}(n / 2)+16 \tau_{2}(n / 2)$.

Proof. This follows immediately from Theorem 9.2.1 as $\sigma_{5}(n / 4)=\sigma_{5}(n / 8)=0$ when $n \equiv 2(\bmod 4)$.

In the next three theorems we simplify Theorem 7.2 .2 in the cases $n \equiv 0$ $(\bmod 4), n \equiv 0(\bmod 8)$ and $n \equiv 2(\bmod 4)$.

Theorem 9.2.4. Let $n \in \mathbb{N}$. For $n \equiv 0(\bmod 4)$, we have

$N([1,2,9] ; n)=8 \sigma_{5}(n / 4)-512 \sigma_{5}(n / 16)+16 \tau_{2}(n / 4)$,

$N([2,2,8] ; n)=16 \sigma_{5}(n / 4)-8 \sigma_{5}(n / 8)-512 \sigma_{5}(n / 16)+24 \tau_{2}(n / 4)$,

$N([3,2,7] ; n)=32 \sigma_{5}(n / 4)-24 \sigma_{5}(n / 8)-512 \sigma_{5}(n / 16)+40 \tau_{2}(n / 4)$,

$N([4,2,6] ; n)=64 \sigma_{5}(n / 4)-56 \sigma_{5}(n / 8)-512 \sigma_{5}(n / 16)+72 \tau_{2}(n / 4)$,

$N([5,2,5] ; n)=128 \sigma_{5}(n / 4)-120 \sigma_{5}(n / 8)-512 \sigma_{5}(n / 16)+136 \tau_{2}(n / 4)$,

$N([6,2,4] ; n)=256 \sigma_{5}(n / 4)-248 \sigma_{5}(n / 8)-512 \sigma_{5}(n / 16)+248 \tau_{2}(n / 4)$,

$N([7,2,3] ; n)=512 \sigma_{5}(n / 4)-504 \sigma_{5}(n / 8)-512 \sigma_{5}(n / 16)+408 \tau_{2}(n / 4)$, 
$N([8,2,2] ; n)=1024 \sigma_{5}(n / 4)-1016 \sigma_{5}(n / 8)-512 \sigma_{5}(n / 16)+568 \tau_{2}(n / 4)$,

$N([9,2,1] ; n)=2048 \sigma_{5}(n / 4)-2040 \sigma_{5}(n / 8)-512 \sigma_{5}(n / 16)+568 \tau_{2}(n / 4)$,

$N([1,4,7] ; n)=16 \sigma_{5}(n / 4)-8 \sigma_{5}(n / 8)-512 \sigma_{5}(n / 16)+24 \tau_{2}(n / 4)$,

$N([2,4,6] ; n)=32 \sigma_{5}(n / 4)-24 \sigma_{5}(n / 8)-512 \sigma_{5}(n / 16)+40 \tau_{2}(n / 4)$,

$N([3,4,5] ; n)=64 \sigma_{5}(n / 4)-56 \sigma_{5}(n / 8)-512 \sigma_{5}(n / 16)+72 \tau_{2}(n / 4)$,

$N([4,4,4] ; n)=128 \sigma_{5}(n / 4)-120 \sigma_{5}(n / 8)-512 \sigma_{5}(n / 16)+120 \tau_{2}(n / 4)$,

$N([5,4,3] ; n)=256 \sigma_{5}(n / 4)-248 \sigma_{5}(n / 8)-512 \sigma_{5}(n / 16)+184 \tau_{2}(n / 4)$,

$N([6,4,2] ; n)=512 \sigma_{5}(n / 4)-504 \sigma_{5}(n / 8)-512 \sigma_{5}(n / 16)+248 \tau_{2}(n / 4)$,

$N([7,4,1] ; n)=1024 \sigma_{5}(n / 4)-1016 \sigma_{5}(n / 8)-512 \sigma_{5}(n / 16)+248 \tau_{2}(n / 4)$,

$N([1,6,5] ; n)=32 \sigma_{5}(n / 4)-24 \sigma_{5}(n / 8)-512 \sigma_{5}(n / 16)+40 \tau_{2}(n / 4)$,

$N([2,6,4] ; n)=64 \sigma_{5}(n / 4)-56 \sigma_{5}(n / 8)-512 \sigma_{5}(n / 16)+56 \tau_{2}(n / 4)$,

$N([3,6,3] ; n)=128 \sigma_{5}(n / 4)-120 \sigma_{5}(n / 8)-512 \sigma_{5}(n / 16)+88 \tau_{2}(n / 4)$,

$N([4,6,2] ; n)=256 \sigma_{5}(n / 4)-248 \sigma_{5}(n / 8)-512 \sigma_{5}(n / 16)+120 \tau_{2}(n / 4)$,

$N([5,6,1] ; n)=512 \sigma_{5}(n / 4)-504 \sigma_{5}(n / 8)-512 \sigma_{5}(n / 16)+120 \tau_{2}(n / 4)$, 
$N([1,8,3] ; n)=64 \sigma_{5}(n / 4)-56 \sigma_{5}(n / 8)-512 \sigma_{5}(n / 16)+56 \tau_{2}(n / 4)$,

$N([2,8,2] ; n)=128 \sigma_{5}(n / 4)-120 \sigma_{5}(n / 8)-512 \sigma_{5}(n / 16)+56 \tau_{2}(n / 4)$,

$N([3,8,1] ; n)=256 \sigma_{5}(n / 4)-248 \sigma_{5}(n / 8)-512 \sigma_{5}(n / 16)+56 \tau_{2}(n / 4)$,

$N([1,10,1] ; n)=128 \sigma_{5}(n / 4)-120 \sigma_{5}(n / 8)-512 \sigma_{5}(n / 16)+56 \tau_{2}(n / 4)$.

Proof. We only prove the formula for $N([9,2,1] ; n)$ as the proofs for the other cases are similar. By Theorems 7.2.2 and 8.4.1 - 8.4.7 with $n \equiv 0(\bmod 4)$, we have

$$
\begin{aligned}
N([9,2,1] ; n)= & 2 \sigma_{5}(n)-2 \sigma_{5}(n / 2)+8 \sigma_{5}(n / 8)-512 \sigma_{5}(n / 16) \\
& +4 \delta\left(0, \frac{1}{2}, 0 ; n\right)+4 \delta\left(-\frac{1}{3}, 1,-\frac{1}{6} ; n\right)-4 \delta\left(0,0, \frac{1}{2} ; n\right) \\
& +\frac{79}{16} \delta\left(-\frac{1}{2}, \frac{5}{4},-\frac{1}{4} ; n\right)-\frac{61}{4} \delta\left(-\frac{1}{6}, \frac{1}{4}, \frac{5}{12} ; n\right) \\
& +\frac{49}{16} \delta\left(-\frac{1}{6}, \frac{3}{4},-\frac{1}{12} ; n\right)-\frac{1}{4} \delta\left(\frac{1}{6},-\frac{1}{4}, \frac{7}{12} ; n\right) \\
= & 2 \sigma_{5}(n)-2 \sigma_{5}(n / 2)+8 \sigma_{5}(n / 8)-512 \sigma_{5}(n / 16) \\
& +\frac{79}{16}\left(20 \delta\left(-\frac{1}{3}, 1,-\frac{1}{6} ; n / 2\right)\right)-\frac{61}{4} \delta\left(-\frac{1}{3}, 1,-\frac{1}{6} ; n / 2\right) \\
& +\frac{49}{16}\left(-4 \delta\left(-\frac{1}{3}, 1,-\frac{1}{6} ; n / 2\right)\right)-\frac{1}{4} \delta\left(-\frac{1}{3}, 1,-\frac{1}{6} ; n / 2\right) \\
& =2 \sigma_{5}(n)-2 \sigma_{5}(n / 2)+8 \sigma_{5}(n / 8)-512 \sigma_{5}(n / 16) \\
& +71 \delta\left(-\frac{1}{3}, 1,-\frac{1}{6} ; n / 2\right) \\
= & 2 \sigma_{5}(n)-2 \sigma_{5}(n / 2)+8 \sigma_{5}(n / 8)-512 \sigma_{5}(n / 16)+568 \tau_{2}(n / 4) .
\end{aligned}
$$

Simplifying using (9.2.3), we obtain the desired result. 
Theorem 9.2.5. Let $n \in \mathbb{N}$. For $n \equiv 0(\bmod 8)$, we have

$$
\begin{aligned}
& N([1,2,9] ; n)=264 \sigma_{5}(n / 8)-768 \sigma_{5}(n / 16), \\
& N([2,2,8] ; n)=520 \sigma_{5}(n / 8)-1024 \sigma_{5}(n / 16), \\
& N([3,2,7] ; n)=8 \sigma_{5}(n / 8)-1536 \sigma_{5}(n / 16), \\
& N([4,2,6] ; n)=2056 \sigma_{5}(n / 8)-2560 \sigma_{5}(n / 16), \\
& N([5,2,5] ; n)=4101 \sigma_{5}(n / 8)-4608 \sigma_{5}(n / 16), \\
& N([6,2,4] ; n)=8200 \sigma_{5}(n / 8)-8704 \sigma_{5}(n / 16), \\
& N([7,2,3] ; n)=16392 \sigma_{5}(n / 8)-16896 \sigma_{5}(n / 16), \\
& N([8,2,2] ; n)=32776 \sigma_{5}(n / 8)-33280 \sigma_{5}(n / 16), \\
& N([2,4,6] ; n)=1032 \sigma_{5}(n / 8)-1536 \sigma_{5}(n / 16), \\
& N([1,4,7] ; n)=520 \sigma_{5}(n / 8)-1024 \sigma_{5}(n / 16), \\
& N([3,4,2,1] ; n)=65544 \sigma_{5}(n / 8)-66048 \sigma_{5}(n / 16),
\end{aligned}
$$




$$
\begin{aligned}
& N([5,4,3] ; n)=8200 \sigma_{5}(n / 8)-8704 \sigma_{5}(n / 16), \\
& N([6,4,2] ; n)=16392 \sigma_{5}(n / 8)-16896 \sigma_{5}(n / 16), \\
& N([7,4,1] ; n)=32776 \sigma_{5}(n / 8)-33280 \sigma_{5}(n / 16), \\
& N([1,6,5] ; n)=1032 \sigma_{5}(n / 8)-1536 \sigma_{5}(n / 16), \\
& N([2,6,4] ; n)=2056 \sigma_{5}(n / 8)-2560 \sigma_{5}(n / 16), \\
& N([3,6,3] ; n)=4104 \sigma_{5}(n / 8)-4608 \sigma_{5}(n / 16), \\
& N([4,6,2] ; n)=8200 \sigma_{5}(n / 8)-8704 \sigma_{5}(n / 16), \\
& N([5,6,1] ; n)=16392 \sigma_{5}(n / 8)-16896 \sigma_{5}(n / 16), \\
& N([3,8,1] ; n)=8200 \sigma_{5}(n / 8)-8704 \sigma_{5}(n / 16), \\
& N([1,8,3] ; n)=2056 \sigma_{5}(n / 8)-2560 \sigma_{5}(n / 16), \\
& N([2,8,2] ; n)=4104 \sigma_{5}(n / 8)-4608 \sigma_{5}(n / 16), \\
& N(1,1] ; n)=4104 \sigma_{5}(n / 8)-4608 \sigma_{5}(n / 16) .
\end{aligned}
$$

Proof. As $n \equiv 0(\bmod 8)$ we have $\frac{n}{4} \equiv 0(\bmod 2)$ so $\tau_{2}(n / 4)=0$. The result now follows from Theorem 9.2.4 and (9.2.4). 
In the next theorem we have chosen to leave $\delta\left(-\frac{1}{3}, 1,-\frac{1}{6} ; n\right)$ rather than replace it by $\tau_{2}(n / 2)$ as $\delta\left(-\frac{1}{3}, 1,-\frac{1}{6} ; n / 2\right)$ also occurs. We have been unable to find an expression for $\delta\left(-\frac{1}{3}, 1,-\frac{1}{6} ; n\right)$ in terms of $\tau(n), \tau_{2}(n), \tau_{4}(n)$ when $n$ is odd.

Theorem 9.2.6. Let $n \in \mathbb{N}$. For $n \equiv 2(\bmod 4)$, we have

$$
\begin{aligned}
& N([1,2,9] ; n)=\frac{1}{4} \sigma_{5}(n / 2)+\frac{7}{32} \delta\left(-\frac{1}{3}, 1,-\frac{1}{6} ; n\right)+2 \delta\left(-\frac{1}{3}, 1,-\frac{1}{6} ; n / 2\right) \\
& N([2,2,8] ; n)=\frac{1}{2} \sigma_{5}(n / 2)+\frac{7}{16} \delta\left(-\frac{1}{3}, 1,-\frac{1}{6} ; n\right)+4 \delta\left(-\frac{1}{3}, 1,-\frac{1}{6} ; n / 2\right) \\
& N([3,2,7] ; n)=\sigma_{5}(n / 2)+\frac{7}{8} \delta\left(-\frac{1}{3}, 1,-\frac{1}{6} ; n\right)+8 \delta\left(-\frac{1}{3}, 1,-\frac{1}{6} ; n / 2\right) \\
& N([4,2,6] ; n)=2 \sigma_{5}(n / 2)+\frac{3}{2} \delta\left(-\frac{1}{3}, 1,-\frac{1}{6} ; n\right)+14 \delta\left(-\frac{1}{3}, 1,-\frac{1}{6} ; n / 2\right) \\
& N([5,2,5] ; n)=4 \sigma_{5}(n / 2)+\frac{9}{4} \delta\left(-\frac{1}{3}, 1,-\frac{1}{6} ; n\right)+22 \delta\left(-\frac{1}{3}, 1,-\frac{1}{6} ; n / 2\right) \\
& N([6,2,4] ; n)=8 \sigma_{5}(n / 2)+3 \delta\left(-\frac{1}{3}, 1,-\frac{1}{6} ; n\right)+32 \delta\left(-\frac{1}{3}, 1,-\frac{1}{6} ; n / 2\right) \\
& N([7,2,3] ; n)=16 \sigma_{5}(n / 2)+\frac{7}{2} \delta\left(-\frac{1}{3}, 1,-\frac{1}{6} ; n\right)+44 \delta\left(-\frac{1}{3}, 1,-\frac{1}{6} ; n / 2\right) \\
& N([2,4,6] ; n)=\sigma_{5}(n / 2)+\frac{5}{8} \delta\left(-\frac{1}{3}, 1,-\frac{1}{6} ; n\right)+6 \delta\left(-\frac{1}{3}, 1,-\frac{1}{6} ; n / 2\right) \\
& N([8,2,2] ; n)=32 \sigma_{5}(n / 2)+\frac{7}{2} \delta\left(-\frac{1}{3}, 1,-\frac{1}{6} ; n\right)+56 \delta\left(-\frac{1}{3}, 1,-\frac{1}{6} ; n / 2\right) \\
& N([1,4,7] ; n)=\frac{1}{2} \sigma_{5}(n / 2)+\frac{7}{16} \delta\left(-\frac{1}{3}, 1,-\frac{1}{6} ; n\right)+4 \delta\left(-\frac{1}{3}, 1,-\frac{1}{6} ; n / 2\right) \\
& N([9,2,1] ; n)=64 \sigma_{5}(n / 2)+\frac{7}{2} \delta\left(-\frac{1}{3}, 1,-\frac{1}{6} ; n\right)+56 \delta\left(-\frac{1}{3}, 1,-\frac{1}{6} ; n / 2\right) \\
& N
\end{aligned}
$$




$$
\begin{aligned}
& N([3,4,5] ; n)=2 \sigma_{5}(n / 2)+\delta\left(-\frac{1}{3}, 1,-\frac{1}{6} ; n\right)+10 \delta\left(-\frac{1}{3}, 1,-\frac{1}{6} ; n / 2\right) \\
& N([4,4,4] ; n)=4 \sigma_{5}(n / 2)+\frac{3}{2} \delta\left(-\frac{1}{3}, 1,-\frac{1}{6} ; n\right)+16 \delta\left(-\frac{1}{3}, 1,-\frac{1}{6} ; n / 2\right), \\
& N([5,4,3] ; n)=8 \sigma_{5}(n / 2)+2 \delta\left(-\frac{1}{3}, 1,-\frac{1}{6} ; n\right)+24 \delta\left(-\frac{1}{3}, 1,-\frac{1}{6} ; n / 2\right), \\
& N([6,4,2] ; n)=16 \sigma_{5}(n / 2)+\frac{5}{2} \delta\left(-\frac{1}{3}, 1,-\frac{1}{6} ; n\right)+32 \delta\left(-\frac{1}{3}, 1,-\frac{1}{6} ; n / 2\right) \\
& N([7,4,1] ; n)=32 \sigma_{5}(n / 2)+\frac{7}{2} \delta\left(-\frac{1}{3}, 1,-\frac{1}{6} ; n\right)+32 \delta\left(-\frac{1}{3}, 1,-\frac{1}{6} ; n / 2\right), \\
& N([1,6,5] ; n)=\sigma_{5}(n / 2)+\frac{5}{8} \delta\left(-\frac{1}{3}, 1,-\frac{1}{6} ; n\right)+6 \delta\left(-\frac{1}{3}, 1,-\frac{1}{6} ; n / 2\right), \\
& N([2,6,4] ; n)=2 \sigma_{5}(n / 2)+\frac{3}{4} \delta\left(-\frac{1}{3}, 1,-\frac{1}{6} ; n\right)+8 \delta\left(-\frac{1}{3}, 1,-\frac{1}{6} ; n / 2\right), \\
& N([3,6,3] ; n)=4 \sigma_{5}(n / 2)+\delta\left(-\frac{1}{3}, 1,-\frac{1}{6} ; n\right)+12 \delta\left(-\frac{1}{3}, 1,-\frac{1}{6} ; n / 2\right), \\
& N([4,6,2] ; n)=8 \sigma_{5}(n / 2)+\frac{3}{2} \delta\left(-\frac{1}{3}, 1,-\frac{1}{6} ; n\right)+16 \delta\left(-\frac{1}{3}, 1,-\frac{1}{6} ; n / 2\right) \\
& N([5,6,1] ; n)=16 \sigma_{5}(n / 2)+\frac{5}{2} \delta\left(-\frac{1}{3}, 1,-\frac{1}{6} ; n\right)+16 \delta\left(-\frac{1}{3}, 1,-\frac{1}{6} ; n / 2\right) \\
& N([1,8,3] ; n)=2 \sigma_{5}(n / 2)+\frac{3}{4} \delta\left(-\frac{1}{3}, 1,-\frac{1}{6} ; n\right)+8 \delta\left(-\frac{1}{3}, 1,-\frac{1}{6} ; n / 2\right), \\
& N([2,8,2] ; n)=4 \sigma_{5}(n / 2)+\delta\left(-\frac{1}{3}, 1,-\frac{1}{6} ; n\right)+8 \delta\left(-\frac{1}{3}, 1,-\frac{1}{6} ; n / 2\right), \\
& N([3,8,1] ; n)=8 \sigma_{5}(n / 2)+\frac{3}{2} \delta\left(-\frac{1}{3}, 1,-\frac{1}{6} ; n\right)+8 \delta\left(-\frac{1}{3}, 1,-\frac{1}{6} ; n / 2\right),
\end{aligned}
$$


$N([1,10,1] ; n)=4 \sigma_{5}(n / 2)+\delta\left(-\frac{1}{3}, 1,-\frac{1}{6} ; n\right)+8 \delta\left(-\frac{1}{3}, 1,-\frac{1}{6} ; n / 2\right)$.

Proof. We only prove the formula for $N([3,6,3] ; n)$ as the proofs for the other cases are similar. By Theorems 7.2.2 and 8.4.1 - 8.4.7 with $n \equiv 2(\bmod 4)$, we have

$$
\begin{aligned}
N([3,6,3] ; n)= & \frac{1}{8} \sigma_{5}(n)-\frac{1}{8} \sigma_{5}(n / 2)+8 \sigma_{5}(n / 8)-512 \sigma_{5}(n / 16) \\
& +\frac{11}{8} \delta\left(0, \frac{1}{2}, 0 ; n\right)+\frac{3}{2} \delta\left(-\frac{1}{3}, 1,-\frac{1}{6} ; n\right)-4 \delta\left(0,0, \frac{1}{2} ; n\right) \\
& +\frac{23}{16} \delta\left(-\frac{1}{2}, \frac{5}{4},-\frac{1}{4} ; n\right)-\frac{45}{4} \delta\left(-\frac{1}{6}, \frac{1}{4}, \frac{5}{12} ; n\right) \\
& +\frac{25}{16} \delta\left(-\frac{1}{6}, \frac{3}{4},-\frac{1}{12} ; n\right)-\frac{1}{4} \delta\left(\frac{1}{6},-\frac{1}{4}, \frac{7}{12} ; n\right) \\
= & \frac{1}{8} \sigma_{5}(n)-\frac{1}{8} \sigma_{5}(n / 2) \\
& +\frac{3}{2} \delta\left(-\frac{1}{3}, 1,-\frac{1}{6} ; n\right)-4\left(\frac{1}{8} \delta\left(-\frac{1}{3}, 1,-\frac{1}{6} ; n\right)\right) \\
& +\frac{23}{16}\left(12 \delta\left(-\frac{1}{3}, 1,-\frac{1}{6} ; n / 2\right)\right)-\frac{45}{4} \delta\left(-\frac{1}{3}, 1,-\frac{1}{6} ; n / 2\right) \\
& +\frac{25}{16}\left(4 \delta\left(-\frac{1}{6}, \frac{3}{4},-\frac{1}{12} ; n\right)\right)-\frac{1}{4} \delta\left(-\frac{1}{3}, 1,-\frac{1}{6} ; n / 2\right) \\
& =\frac{1}{8} \sigma_{5}(n)-\frac{1}{8} \sigma_{5}(n / 2) \\
& +\delta\left(-\frac{1}{3}, 1,-\frac{1}{6} ; n\right)+12 \delta\left(-\frac{1}{3}, 1,-\frac{1}{6} ; n / 2\right) .
\end{aligned}
$$

Simplifying using (9.2.2), we obtain the desired result.

In the next result, we use an identity of Chapter 8 (Theorem 8.4.8) to reduce the number of delta functions $\delta(a, b, c ; n)$ occurring in Theorem 7.2 .3 by two. Note that by Theorems 2.11 .3 and 2.11 .5 we have $\delta\left(0, \frac{1}{2}, 0 ; n\right)=\tau_{2}(n)$ and $\delta\left(0,0, \frac{1}{2} ; n\right)=\tau_{2}(n / 2)$. 
Theorem 9.2.7. For $n \in \mathbb{N}$, we have

$$
\begin{aligned}
N([1,0,11] ; n)= & \left(\frac{1}{256}+\frac{1}{256}\left(\frac{-4}{n}\right)\right) \sigma_{5}(n)-\frac{33}{256} \sigma_{5}(n / 2)+\frac{65}{8} \sigma_{5}(n / 4) \\
& -512 \sigma_{5}(n / 16) \\
& +\frac{167}{256} \delta\left(0, \frac{1}{2}, 0 ; n\right)+\frac{11}{32} \delta\left(-\frac{1}{3}, 1,-\frac{1}{6} ; n\right)-\frac{11}{4} \delta\left(0,0, \frac{1}{2} ; n\right) \\
& +\frac{319}{512} \delta\left(-\frac{1}{2}, \frac{5}{4},-\frac{1}{4} ; n\right)-\frac{1133}{128} \delta\left(-\frac{1}{6}, \frac{1}{4}, \frac{5}{12} ; n\right) \\
& +\frac{191}{512} \delta\left(-\frac{1}{6}, \frac{3}{4},-\frac{1}{12} ; n\right)-\frac{15}{128} \delta\left(\frac{1}{6},-\frac{1}{4}, \frac{7}{12} ; n\right) \\
N([2,0,10] ; n)= & \left(\frac{1}{128}+\frac{1}{128}\left(\frac{-4}{n}\right)\right) \sigma_{5}(n)-\frac{1}{128} \sigma_{5}(n / 2)+8 \sigma_{5}(n / 8) \\
& -512 \sigma_{5}(n / 16) \\
& +\frac{167}{256} \delta\left(0, \frac{1}{2}, 0 ; n\right)+\frac{11}{16} \delta\left(-\frac{1}{3}, 1,-\frac{1}{6} ; n\right)-\frac{15}{4} \delta\left(0,0, \frac{1}{2} ; n\right) \\
& +\frac{255}{256} \delta\left(-\frac{1}{2}, \frac{5}{4},-\frac{1}{4} ; n\right)-\frac{877}{64} \delta\left(-\frac{1}{6}, \frac{1}{4}, \frac{5}{12} ; n\right) \\
& +\frac{255}{256} \delta\left(-\frac{1}{6}, \frac{3}{4},-\frac{1}{12} ; n\right)-\frac{15}{64} \delta\left(\frac{1}{6},-\frac{1}{4}, \frac{7}{12} ; n\right) \\
N([4,0,8] ; n)= & \frac{1}{32} \sigma_{5}(n)+\frac{15}{32} \sigma_{5}(n / 2)-\frac{65}{2} \sigma_{5}(n / 4)+40 \sigma_{5}(n / 8)-512 \sigma_{5}(n / 16) \\
& +\frac{319}{256} \delta\left(-\frac{1}{2}, \frac{5}{4},-\frac{1}{4} ; n\right)-\frac{1005}{64} \delta\left(-\frac{1}{6}, \frac{1}{4}, \frac{5}{12} ; n\right) \\
& +\frac{119}{64} \delta\left(-\frac{1}{6}, \frac{3}{4},-\frac{1}{12} ; n\right)-\frac{15}{64} \delta\left(\frac{1}{6},-\frac{1}{4}, \frac{7}{12} ; n\right) \\
N([3,0,9] ; n)= & \left(\frac{1}{64}+\frac{1}{128}\left(\frac{-4}{n}\right)\right) \sigma_{5}(n)+\frac{15}{64} \sigma_{5}(n / 2)-\frac{65}{4} \sigma_{5}(n / 4)+24 \sigma_{5}(n / 8) \\
& -512 \sigma_{5}(n / 16) \\
&
\end{aligned}
$$




$$
\begin{aligned}
& +\frac{71}{32} \delta\left(0, \frac{1}{2}, 0 ; n\right)+\frac{7}{4} \delta\left(-\frac{1}{3}, 1,-\frac{1}{6} ; n\right)-\frac{7}{2} \delta\left(0,0, \frac{1}{2} ; n\right) \\
& +\frac{23}{16} \delta\left(-\frac{1}{2}, \frac{5}{4},-\frac{1}{4} ; n\right)-\frac{61}{4} \delta\left(-\frac{1}{6}, \frac{1}{4}, \frac{5}{12} ; n\right) \\
& +\frac{41}{16} \delta\left(-\frac{1}{6}, \frac{3}{4},-\frac{1}{12} ; n\right)-\frac{1}{4} \delta\left(\frac{1}{6},-\frac{1}{4}, \frac{7}{12} ; n\right) \text {, } \\
& N([5,0,7] ; n)=\left(\frac{1}{16}-\frac{1}{64}\left(\frac{-4}{n}\right)\right) \sigma_{5}(n)+\frac{7}{64} \sigma_{5}(n / 2)-\frac{65}{2} \sigma_{5}(n / 4)+40 \sigma_{5}(n / 8) \\
& -512 \sigma_{5}(n / 16) \\
& +\frac{37}{16} \delta\left(0, \frac{1}{2}, 0 ; n\right)+\frac{21}{8} \delta\left(-\frac{1}{3}, 1,-\frac{1}{6} ; n\right)-\frac{7}{2} \delta\left(0,0, \frac{1}{2} ; n\right) \\
& +\frac{217}{128} \delta\left(-\frac{1}{2}, \frac{5}{4},-\frac{1}{4} ; n\right)-\frac{427}{32} \delta\left(-\frac{1}{6}, \frac{1}{4}, \frac{5}{12} ; n\right) \\
& +\frac{425}{128} \delta\left(-\frac{1}{6}, \frac{3}{4},-\frac{1}{12} ; n\right)-\frac{9}{32} \delta\left(\frac{1}{6},-\frac{1}{4}, \frac{7}{12} ; n\right), \\
& N([6,0,6] ; n)=\left(\frac{1}{8}-\frac{1}{32}\left(\frac{-4}{n}\right)\right) \sigma_{5}(n)-\frac{1}{8} \sigma_{5}(n / 2)+8 \sigma_{5}(n / 8) \\
& -512 \sigma_{5}(n / 16) \\
& +\frac{17}{8} \delta\left(0, \frac{1}{2}, 0 ; n\right)+\frac{15}{4} \delta\left(-\frac{1}{3}, 1,-\frac{1}{6} ; n\right)-4 \delta\left(0,0, \frac{1}{2} ; n\right) \\
& +\frac{141}{64} \delta\left(-\frac{1}{2}, \frac{5}{4},-\frac{1}{4} ; n\right)-\frac{183}{16} \delta\left(-\frac{1}{6}, \frac{1}{4}, \frac{5}{12} ; n\right) \\
& +\frac{245}{64} \delta\left(-\frac{1}{6}, \frac{3}{4},-\frac{1}{12} ; n\right)-\frac{5}{16} \delta\left(\frac{1}{6},-\frac{1}{4}, \frac{7}{12} ; n\right) \text {, } \\
& N([7,0,5] ; n)=\left(\frac{1}{4}-\frac{1}{32}\left(\frac{-4}{n}\right)\right) \sigma_{5}(n)-\frac{5}{4} \sigma_{5}(n / 2)+65 \sigma_{5}(n / 4)-56 \sigma_{5}(n / 8) \\
& -512 \sigma_{5}(n / 16) \\
& +\frac{7}{4} \delta\left(0, \frac{1}{2}, 0 ; n\right)+5 \delta\left(-\frac{1}{3}, 1,-\frac{1}{6} ; n\right)-5 \delta\left(0,0, \frac{1}{2} ; n\right) \\
& +\frac{205}{64} \delta\left(-\frac{1}{2}, \frac{5}{4},-\frac{1}{4} ; n\right)-\frac{183}{16} \delta\left(-\frac{1}{6}, \frac{1}{4}, \frac{5}{12} ; n\right)
\end{aligned}
$$




$$
\begin{aligned}
& +\frac{245}{64} \delta\left(-\frac{1}{6}, \frac{3}{4},-\frac{1}{12} ; n\right)-\frac{5}{16} \delta\left(\frac{1}{6},-\frac{1}{4}, \frac{7}{12} ; n\right), \\
& N([8,0,4] ; n)=\frac{1}{2} \sigma_{5}(n)-\frac{5}{2} \sigma_{5}(n / 2)+130 \sigma_{5}(n / 4)-120 \sigma_{5}(n / 8)-512 \sigma_{5}(n / 16) \\
& +\frac{3}{2} \delta\left(0, \frac{1}{2}, 0 ; n\right)+6 \delta\left(-\frac{1}{3}, 1,-\frac{1}{6} ; n\right)-6 \delta\left(0,0, \frac{1}{2} ; n\right) \\
& +\frac{79}{16} \delta\left(-\frac{1}{2}, \frac{5}{4},-\frac{1}{4} ; n\right)-\frac{61}{4} \delta\left(-\frac{1}{6}, \frac{1}{4}, \frac{5}{12} ; n\right) \\
& +\frac{49}{16} \delta\left(-\frac{1}{6}, \frac{3}{4},-\frac{1}{12} ; n\right)-\frac{1}{4} \delta\left(\frac{1}{6},-\frac{1}{4}, \frac{7}{12} ; n\right), \\
& N([9,0,3] ; n)=\left(1+\frac{1}{16}\left(\frac{-4}{n}\right)\right) \sigma_{5}(n)-3 \sigma_{5}(n / 2)+130 \sigma_{5}(n / 4)-120 \sigma_{5}(n / 8) \\
& -512 \sigma_{5}(n / 16) \\
& +2 \delta\left(0, \frac{1}{2}, 0 ; n\right)+6 \delta\left(-\frac{1}{3}, 1,-\frac{1}{6} ; n\right)-6 \delta\left(0,0, \frac{1}{2} ; n\right) \\
& +\frac{237}{32} \delta\left(-\frac{1}{2}, \frac{5}{4},-\frac{1}{4} ; n\right)-\frac{183}{8} \delta\left(-\frac{1}{6}, \frac{1}{4}, \frac{5}{12} ; n\right) \\
& +\frac{49}{32} \delta\left(-\frac{1}{6}, \frac{3}{4},-\frac{1}{12} ; n\right)-\frac{1}{8} \delta\left(\frac{1}{6},-\frac{1}{4}, \frac{7}{12} ; n\right), \\
& N([10,0,2] ; n)=\left(2+\frac{1}{8}\left(\frac{-4}{n}\right)\right) \sigma_{5}(n)-2 \sigma_{5}(n / 2)+8 \sigma_{5}(n / 8)-512 \sigma_{5}(n / 16) \\
& +4 \delta\left(0, \frac{1}{2}, 0 ; n\right)+4 \delta\left(-\frac{1}{3}, 1,-\frac{1}{6} ; n\right)-4 \delta\left(0,0, \frac{1}{2} ; n\right) \\
& +\frac{79}{8} \delta\left(-\frac{1}{2}, \frac{5}{4},-\frac{1}{4} ; n\right)-\frac{61}{2} \delta\left(-\frac{1}{6}, \frac{1}{4}, \frac{5}{12} ; n\right), \\
& N([11,0,1] ; n)=\left(4+\frac{1}{8}\left(\frac{-4}{n}\right)\right) \sigma_{5}(n)-260 \sigma_{5}(n / 4)+264 \sigma_{5}(n / 8) \\
& -512 \sigma_{5}(n / 16) \\
& +8 \delta\left(0, \frac{1}{2}, 0 ; n\right)+\frac{79}{8} \delta\left(-\frac{1}{2}, \frac{5}{4},-\frac{1}{4} ; n\right)
\end{aligned}
$$




$$
-\frac{61}{2} \delta\left(-\frac{1}{6}, \frac{1}{4}, \frac{5}{12} ; n\right) .
$$

Proof. We only prove the formula for $N([5,0,7] ; n)$ as the proofs for the other cases are similar. By Theorems 7.2.3 and 8.4.8, we have

$$
\begin{aligned}
& N([5,0,7] ; n) \\
& =\frac{1}{16} \sigma_{5}(n)+\frac{7}{64} \sigma_{5}(n / 2)-\frac{65}{2} \sigma_{5}(n / 4)+40 \sigma_{5}(n / 8)-512 \sigma_{5}(n / 16) \\
& +\frac{37}{16} \delta\left(0, \frac{1}{2}, 0 ; n\right)+\frac{21}{8} \delta\left(-\frac{1}{3}, 1,-\frac{1}{6} ; n\right)-\frac{7}{2} \delta\left(0,0, \frac{1}{2} ; n\right) \\
& -\frac{1}{32} \delta\left(-\frac{5}{6}, \frac{9}{4},-\frac{11}{12} ; n\right)+\frac{37}{16} \delta\left(-\frac{1}{2}, \frac{5}{4},-\frac{1}{4} ; n\right)-\frac{61}{4} \delta\left(-\frac{1}{6}, \frac{1}{4}, \frac{5}{12} ; n\right) \\
& +\frac{1}{32} \delta\left(-\frac{1}{2}, \frac{7}{4},-\frac{3}{4} ; n\right)+\frac{47}{16} \delta\left(-\frac{1}{6}, \frac{3}{4},-\frac{1}{12} ; n\right)-\frac{1}{4} \delta\left(\frac{1}{6},-\frac{1}{4}, \frac{7}{12} ; n\right) \text {. } \\
& =\frac{1}{16} \sigma_{5}(n)+\frac{7}{64} \sigma_{5}(n / 2)-\frac{65}{2} \sigma_{5}(n / 4)+40 \sigma_{5}(n / 8)-512 \sigma_{5}(n / 16) \\
& +\frac{37}{16} \delta\left(0, \frac{1}{2}, 0 ; n\right)+\frac{21}{8} \delta\left(-\frac{1}{3}, 1,-\frac{1}{6} ; n\right)-\frac{7}{2} \delta\left(0,0, \frac{1}{2} ; n\right) \\
& -\frac{1}{64}\left(2 \delta\left(-\frac{5}{6}, \frac{9}{4},-\frac{11}{12} ; n\right)-\frac{79}{2} \delta\left(-\frac{1}{2}, \frac{5}{4},-\frac{1}{4} ; n\right)+122 \delta\left(-\frac{1}{6}, \frac{1}{4}, \frac{5}{12} ; n\right)\right. \\
& \left.-2 \delta\left(-\frac{1}{2}, \frac{7}{4},-\frac{3}{4} ; n\right)+\frac{49}{2} \delta\left(-\frac{1}{6}, \frac{3}{4},-\frac{1}{12} ; n\right)-2 \delta\left(\frac{1}{6},-\frac{1}{4}, \frac{7}{12} ; n\right)\right) \\
& +\frac{217}{128} \delta\left(-\frac{1}{2}, \frac{5}{4},-\frac{1}{4} ; n\right)-\frac{427}{32} \delta\left(-\frac{1}{6}, \frac{1}{4}, \frac{5}{12} ; n\right)+\frac{425}{128} \delta\left(-\frac{1}{2}, \frac{7}{4},-\frac{3}{4} ; n\right) \\
& -\frac{9}{32} \delta\left(\frac{1}{6},-\frac{1}{4}, \frac{7}{12} ; n\right) \\
& =\left(\frac{1}{16}-\frac{1}{64}\left(\frac{-4}{n}\right)\right) \sigma_{5}(n)+\frac{7}{64} \sigma_{5}(n / 2)-\frac{65}{2} \sigma_{5}(n / 4)+40 \sigma_{5}(n / 8)-512 \sigma_{5}(n / 16) \\
& +\frac{37}{16} \delta\left(0, \frac{1}{2}, 0 ; n\right)+\frac{21}{8} \delta\left(-\frac{1}{3}, 1,-\frac{1}{6} ; n\right)-\frac{7}{2} \delta\left(0,0, \frac{1}{2} ; n\right) \\
& +\frac{217}{128} \delta\left(-\frac{1}{2}, \frac{5}{4},-\frac{1}{4} ; n\right)-\frac{427}{32} \delta\left(-\frac{1}{6}, \frac{1}{4}, \frac{5}{12} ; n\right)+\frac{425}{128} \delta\left(-\frac{1}{2}, \frac{7}{4},-\frac{3}{4} ; n\right) \\
& -\frac{9}{32} \delta\left(\frac{1}{6},-\frac{1}{4}, \frac{7}{12} ; n\right) \text {, }
\end{aligned}
$$


which is the desired result.

In the last three theorems of this section, we simplify Theorem 9.2.7 in the cases $n \equiv 0(\bmod 4), n \equiv 0(\bmod 8)$ and $n \equiv 2(\bmod 4)$.

Theorem 9.2.8. Let $n \in \mathbb{N}$. For $n \equiv 0(\bmod 4)$, we have

$$
\begin{aligned}
N([1,0,11] ; n)= & 8 \sigma_{5}(n / 4)-512 \sigma_{5}(n / 16)+16 \tau_{2}(n / 4), \\
N([2,0,10] ; n)= & 8 \sigma_{5}(n / 4)-512 \sigma_{5}(n / 16)+16 \tau_{2}(n / 4), \\
N([3,0,9] ; n)= & 8 \sigma_{5}(n / 4)-512 \sigma_{5}(n / 16)+16 \tau_{2}(n / 4), \\
N([4,0,8] ; n)= & 16 \sigma_{5}(n / 4)-8 \sigma_{5}(n / 8)-512 \sigma_{5}(n / 16)+24 \tau_{2}(n / 4), \\
N([5,0,7] ; n)= & 48 \sigma_{5}(n / 4)-40 \sigma_{5}(n / 8)-512 \sigma_{5}(n / 16)+56 \tau_{2}(n / 4), \\
N([6,0,6] ; n)= & 128 \sigma_{5}(n / 4)-120 \sigma_{5}(n / 8)-512 \sigma_{5}(n / 16) \\
& +136 \tau_{2}(n / 4), \\
& +568 \tau_{2}(n / 4), \\
& +296 \tau_{2}(n / 4), \\
N([8,0,4] ; n)= & 576 \sigma_{5}(n / 4)-568 \sigma_{5}(n / 8)-512 \sigma_{5}(n / 16) \\
N([7,0,5] ; n)= & 288 \sigma_{5}(n / 4)-280 \sigma_{5}(n / 8)-512 \sigma_{5}(n / 16) \\
& \\
& \\
& \\
&
\end{aligned}
$$




$$
\begin{aligned}
N([9,0,3] ; n)= & 1088 \sigma_{5}(n / 4)-1080 \sigma_{5}(n / 8)-512 \sigma_{5}(n / 16) \\
& +952 \tau_{2}(n / 4) \\
N([10,0,2] ; n)= & 2048 \sigma_{5}(n / 4)-2040 \sigma_{5}(n / 8)-512 \sigma_{5}(n / 16) \\
& +1336 \tau_{2}(n / 4), \\
N([11,0,1] ; n)= & 3968 \sigma_{5}(n / 4)-3960 \sigma_{5}(n / 8)-512 \sigma_{5}(n / 16) \\
& +1336 \tau_{2}(n / 4) .
\end{aligned}
$$

Proof. We only prove the formula for $N([9,0,3] ; n)$ as the proofs for the other cases are similar. By Theorems 9.2.7 and 8.4.1 - 8.4.7, and the fact that $\left(\frac{-4}{n}\right)=0$ when $n \equiv 0(\bmod 4)$, we have when $n \equiv 0(\bmod 4)$.

$$
\begin{aligned}
N([9,0,3] ; n)= & \left(1+\frac{1}{16}\left(\frac{-4}{n}\right)\right) \sigma_{5}(n)-3 \sigma_{5}(n / 2)+130 \sigma_{5}(n / 4)-120 \sigma_{5}(n / 8) \\
& -512 \sigma_{5}(n / 16) \\
& +2 \delta\left(0, \frac{1}{2}, 0 ; n\right)+6 \delta\left(-\frac{1}{3}, 1,-\frac{1}{6} ; n\right)-6 \delta\left(0,0, \frac{1}{2} ; n\right) \\
& +\frac{237}{32} \delta\left(-\frac{1}{2}, \frac{5}{4},-\frac{1}{4} ; n\right)-\frac{183}{8} \delta\left(-\frac{1}{6}, \frac{1}{4}, \frac{5}{12} ; n\right) \\
& +\frac{49}{32} \delta\left(-\frac{1}{6}, \frac{3}{4},-\frac{1}{12} ; n\right)-\frac{1}{8} \delta\left(\frac{1}{6},-\frac{1}{4}, \frac{7}{12} ; n\right) \\
= & \sigma_{5}(n)-3 \sigma_{5}(n / 2)+130 \sigma_{5}(n / 4)-120 \sigma_{5}(n / 8)-512 \sigma_{5}(n / 16) \\
& +\frac{237}{32}\left(20 \delta\left(-\frac{1}{3}, 1,-\frac{1}{6} ; n / 2\right)\right)-\frac{183}{8} \delta\left(-\frac{1}{3}, 1,-\frac{1}{6} ; n / 2\right) \\
& +\frac{49}{32}\left(-4 \delta\left(-\frac{1}{3}, 1,-\frac{1}{6} ; n / 2\right)\right)-\frac{1}{8} \delta\left(-\frac{1}{3}, 1,-\frac{1}{6} ; n / 2\right) \\
& =\sigma_{5}(n)-3 \sigma_{5}(n / 2)+130 \sigma_{5}(n / 4)-120 \sigma_{5}(n / 8)-512 \sigma_{5}(n / 16)
\end{aligned}
$$




$$
+119 \delta\left(-\frac{1}{3}, 1,-\frac{1}{6} ; n / 2\right) \text {. }
$$

Simplifying using (9.2.3), we obtain the desired result.

We remark that when $n \equiv 0(\bmod 4)$

$$
n=x_{1}^{2}+4 x_{2}^{2}+\cdots+4 x_{12}^{2} \Longleftrightarrow \frac{n}{4}=\left(\frac{x_{1}}{2}\right)^{2}+x_{2}^{2}+\cdots+x_{12}^{2}
$$

so that

$$
\begin{aligned}
N([1,0,11] ; n) & =r_{12}(n / 4) \\
& =8 \sigma_{5}(n / 4)-512 \sigma_{5}(n / 16)+16 \delta\left(0, \frac{1}{2}, 0 ; n / 4\right) \\
& =8 \sigma_{5}(n / 4)-512 \sigma_{5}(n / 16)+16 \tau_{2}(n / 4)
\end{aligned}
$$

by Liouville's twelve squares theorem and Theorem 8.4.2. The formulae for $N([2,0,10] ; n)$ and $N([3,0,9] ; n)$ can also be proved in this way when $n \equiv 0(\bmod 4)$.

Theorem 9.2.9. Let $n \in \mathbb{N}$. For $n \equiv 0(\bmod 8)$, we have

$$
\begin{aligned}
& N([1,0,11] ; n)=264 \sigma_{5}(n / 8)-768 \sigma_{5}(n / 16), \\
& N([2,0,10] ; n)=264 \sigma_{5}(n / 8)-768 \sigma_{5}(n / 16), \\
& N([3,0,9] ; n)=264 \sigma_{5}(n / 8)-768 \sigma_{5}(n / 16), \\
& N([4,0,8] ; n)=520 \sigma_{5}(n / 8)-1024 \sigma_{5}(n / 16), \\
& N([5,0,7] ; n)=1544 \sigma_{5}(n / 8)-2048 \sigma_{5}(n / 16), \\
& N([6,0,6] ; n)=4104 \sigma_{5}(n / 8)-4608 \sigma_{5}(n / 16), \\
& N([7,0,5] ; n)=9224 \sigma_{5}(n / 8)-9728 \sigma_{5}(n / 16),
\end{aligned}
$$


$N([8,0,4] ; n)=18440 \sigma_{5}(n / 8)-18944 \sigma_{5}(n / 16)$,

$N([9,0,3] ; n)=34824 \sigma_{5}(n / 8)-35328 \sigma_{5}(n / 16)$,

$N([10,0,2] ; n)=65544 \sigma_{5}(n / 8)-66048 \sigma_{5}(n / 16)$,

$N([11,0,1] ; n)=126984 \sigma_{5}(n / 8)-127488 \sigma_{5}(n / 16)$.

Proof. As $n \equiv 0(\bmod 8)$ we have $\frac{n}{4} \equiv 0(\bmod 2)$ so $\tau_{2}(n / 4)=0$. The results now follow using (9.2.4).

Theorem 9.2.10. Let $n \in \mathbb{N}$. For $n \equiv 2(\bmod 4)$, we have $N([1,0,11] ; n)=0$,

$$
\begin{aligned}
& N([2,0,10] ; n)=\frac{1}{4} \sigma_{5}(n / 2)+\frac{7}{32} \delta\left(-\frac{1}{3}, 1,-\frac{1}{6} ; n\right)+2 \delta\left(-\frac{1}{3}, 1,-\frac{1}{6} ; n / 2\right), \\
& N([3,0,9] ; n)=\frac{3}{4} \sigma_{5}(n / 2)+\frac{21}{32} \delta\left(-\frac{1}{3}, 1,-\frac{1}{6} ; n\right)+6 \delta\left(-\frac{1}{3}, 1,-\frac{1}{6} ; n / 2\right), \\
& N([4,0,8] ; n)=\frac{3}{2} \sigma_{5}(n / 2)+\frac{21}{16} \delta\left(-\frac{1}{3}, 1,-\frac{1}{6} ; n\right)+12 \delta\left(-\frac{1}{3}, 1,-\frac{1}{6} ; n / 2\right), \\
& N([5,0,7] ; n)=\frac{5}{2} \sigma_{5}(n / 2)+\frac{35}{16} \delta\left(-\frac{1}{3}, 1,-\frac{1}{6} ; n\right)+20 \delta\left(-\frac{1}{3}, 1,-\frac{1}{6} ; n / 2\right), \\
& N([6,0,6] ; n)=4 \sigma_{5}(n / 2)+\frac{13}{4} \delta\left(-\frac{1}{3}, 1,-\frac{1}{6} ; n\right)+30 \delta\left(-\frac{1}{3}, 1,-\frac{1}{6} ; n / 2\right), \\
& N([7,0,5] ; n)=7 \sigma_{5}(n / 2)+\frac{35}{8} \delta\left(-\frac{1}{3}, 1,-\frac{1}{6} ; n\right)+42 \delta\left(-\frac{1}{3}, 1,-\frac{1}{6} ; n / 2\right),
\end{aligned}
$$




$$
\begin{aligned}
& N([8,0,4] ; n)=14 \sigma_{5}(n / 2)+\frac{21}{4} \delta\left(-\frac{1}{3}, 1,-\frac{1}{6} ; n\right)+56 \delta\left(-\frac{1}{3}, 1,-\frac{1}{6} ; n / 2\right), \\
& N([9,0,3] ; n)=30 \sigma_{5}(n / 2)+\frac{21}{4} \delta\left(-\frac{1}{3}, 1,-\frac{1}{6} ; n\right)+72 \delta\left(-\frac{1}{3}, 1,-\frac{1}{6} ; n / 2\right) \\
& N([10,0,2] ; n)=64 \sigma_{5}(n / 2)+\frac{7}{2} \delta\left(-\frac{1}{3}, 1,-\frac{1}{6} ; n\right)+88 \delta\left(-\frac{1}{3}, 1,-\frac{1}{6} ; n / 2\right), \\
& N([11,0,1] ; n)=132 \sigma_{5}(n / 2)+88 \delta\left(-\frac{1}{3}, 1,-\frac{1}{6} ; n / 2\right) .
\end{aligned}
$$

Proof. We only prove the formula for $N([2,0,10] ; n)$ as the proofs for the other cases are similar. By Theorem 9.2.7 and Theorems 8.4.1 - 8.4.7, the fact that $\left(\frac{-4}{n}\right)=0$ when $n \equiv 2(\bmod 4)$, and as $\sigma_{5}(n / 4), \sigma_{5}(n / 8), \sigma_{5}(n / 16)$ are all equal to zero when $n \equiv 2(\bmod 4)$, we have for $n \equiv 2(\bmod 4)$

$$
\begin{aligned}
N([2,0,10] ; n)= & \left(\frac{1}{128}+\frac{1}{128}\left(\frac{-4}{n}\right)\right) \sigma_{5}(n)-\frac{1}{128} \sigma_{5}(n / 2)+8 \sigma_{5}(n / 8) \\
& -512 \sigma_{5}(n / 16) \\
& +\frac{167}{256} \delta\left(0, \frac{1}{2}, 0 ; n\right)+\frac{11}{16} \delta\left(-\frac{1}{3}, 1,-\frac{1}{6} ; n\right)-\frac{15}{4} \delta\left(0,0, \frac{1}{2} ; n\right) \\
& +\frac{255}{256} \delta\left(-\frac{1}{2}, \frac{5}{4},-\frac{1}{4} ; n\right)-\frac{877}{64} \delta\left(-\frac{1}{6}, \frac{1}{4}, \frac{5}{12} ; n\right) \\
& +\frac{255}{256} \delta\left(-\frac{1}{6}, \frac{3}{4},-\frac{1}{12} ; n\right)-\frac{15}{64} \delta\left(\frac{1}{6},-\frac{1}{4}, \frac{7}{12} ; n\right) \\
= & \frac{1}{128} \sigma_{5}(n)-\frac{1}{128} \sigma_{5}(n / 2) \\
& +\frac{11}{16} \delta\left(-\frac{1}{3}, 1,-\frac{1}{6} ; n\right)-\frac{15}{32} \delta\left(-\frac{1}{3}, 1,-\frac{1}{6} ; n\right) \\
& +\frac{255}{256}\left(12 \delta\left(-\frac{1}{3}, 1,-\frac{1}{6} ; n / 2\right)\right)-\frac{877}{64} \delta\left(-\frac{1}{3}, 1,-\frac{1}{6} ; n / 2\right) \\
& +\frac{255}{256}\left(4 \delta\left(-\frac{1}{3}, 1,-\frac{1}{6} ; n / 2\right)\right)-\frac{15}{64} \delta\left(-\frac{1}{3}, 1,-\frac{1}{6} ; n / 2\right) \\
& \frac{1}{128} \sigma_{5}(n)-\frac{1}{128} \sigma_{5}(n / 2)
\end{aligned}
$$


CHAPTER 9. SUMS WITH 8, 12 AND 16 SQUARES - II

$$
+\frac{7}{32} \delta\left(-\frac{1}{3}, 1,-\frac{1}{6} ; n\right)+2 \delta\left(-\frac{1}{3}, 1,-\frac{1}{6} ; n / 2\right) .
$$

Simplifying using (9.2.2), we obtain the desired result.

\subsection{Sums with 16 squares}

In this section we restrict $n$ to particular congruence classes modulo 4 and modulo 8 in order to reduce the number of delta functions $\delta(a, b, c ; n)$ occurring in Theorems 7.3.1, 7.3.2 and 7.3.3.

We make use of the following simple properties of $\sigma_{7}$. Let $n \in \mathbb{N}$.

If $n \equiv 0(\bmod 4)$ then

$$
\left\{\begin{array}{c}
\sigma_{7}(n)=16513 \sigma_{7}(n / 4)-16512 \sigma_{5}(n / 8), \\
\sigma_{7}(n / 2)=129 \sigma_{7}(n / 4)-128 \sigma_{7}(n / 8),
\end{array}\right.
$$

if $n \equiv 4(\bmod 8)$ then

$$
\left\{\begin{array}{c}
\sigma_{7}(n)=16513 \sigma_{7}(n / 4) \\
\sigma_{7}(n / 2)=129 \sigma_{7}(n / 4)
\end{array}\right.
$$

and if $n \equiv 0(\bmod 8)$ then

$$
\begin{aligned}
& \sigma_{7}(n)=2113665 \sigma_{7}(n / 8)-2113664 \sigma_{7}(n / 16), \\
& \sigma_{7}(n / 2)=16513 \sigma_{7}(n / 8)-16512 \sigma_{7}(n / 16) \\
& \sigma_{7}(n / 4)=129 \sigma_{7}(n / 8)-128 \sigma_{7}(n / 16)
\end{aligned}
$$


The quantity $\delta\left(-\frac{1}{3}, \frac{4}{3},-\frac{1}{3} ; n\right)(n \in \mathbb{N})$ will play a major role in this section. Its value is given in Theorem 2.11.6, namely

$$
\delta\left(-\frac{1}{3}, \frac{4}{3},-\frac{1}{3} ; n\right)=\frac{1}{2} \sum_{\substack{\left(x_{1}, \ldots, x_{8}\right) \in \mathbb{Z}^{8} \\ x_{1}^{2}++x_{8}^{2}=n}}\left(x_{1}^{4}-3 x_{1}^{2} x_{2}^{2}\right) .
$$

We begin with the simplification of Theorem 7.3.1 when $n \equiv 0(\bmod 4)$.

Theorem 9.3.1. Let $n \in \mathbb{N}$. For $n \equiv 0(\bmod 4)$, we have

$$
\begin{aligned}
& N([14,2,0] ; n)=\frac{262112}{17} \sigma_{7}(n / 4)-\frac{253952}{17} \sigma_{7}(n / 8)-\frac{554}{17} \delta\left(-\frac{1}{3}, \frac{4}{3},-\frac{1}{3} ; n\right), \\
& N([12,4,0] ; n)=\frac{131040}{17} \sigma_{7}(n / 4)-\frac{122880}{17} \sigma_{7}(n / 8)-\frac{630}{17} \delta\left(-\frac{1}{3}, \frac{4}{3},-\frac{1}{3} ; n\right), \\
& N([10,6,0] ; n)=\frac{65504}{17} \sigma_{7}(n / 4)-\frac{57344}{17} \sigma_{7}(n / 8)-\frac{464}{17} \delta\left(-\frac{1}{3}, \frac{4}{3},-\frac{1}{3} ; n\right), \\
& N([8,8,0] ; n)=\frac{32736}{17} \sigma_{7}(n / 4)-\frac{24576}{17} \sigma_{7}(n / 8)-\frac{296}{17} \delta\left(-\frac{1}{3}, \frac{4}{3},-\frac{1}{3} ; n\right), \\
& N([6,10,0] ; n)=\frac{16352}{17} \sigma_{7}(n / 4)-\frac{8192}{17} \sigma_{7}(n / 8)-\frac{178}{17} \delta\left(-\frac{1}{3}, \frac{4}{3},-\frac{1}{3} ; n\right), \\
& N([4,12,0] ; n)=480 \sigma_{7}(n / 4)-6 \delta\left(-\frac{1}{3}, \frac{4}{3},-\frac{1}{3} ; n\right), \\
& N([2,14,0] ; n)=\frac{4064}{17} \sigma_{7}(n / 4)-\frac{4096}{17} \sigma_{7}(n / 8)-\frac{64}{17} \delta\left(-\frac{1}{3}, \frac{4}{3},-\frac{1}{3} ; n\right) .
\end{aligned}
$$

Proof. We only prove the formula for $N([8,8,0] ; n)$ as the proofs for the other cases are similar. By Theorems 7.3.1, 8.5.1, 8.5.2 and 8.5.3 we have

$$
N([8,8,0] ; n)
$$




$$
\begin{aligned}
= & \frac{2}{17} \sigma_{7}(n)-\frac{2}{17} \sigma_{7}(n / 2)-\frac{32}{17} \sigma_{7}(n / 8)+\frac{8192}{17} \sigma_{7}(n / 16) \\
& +\frac{134}{17} \delta\left(-\frac{1}{3}, \frac{4}{3},-\frac{1}{3} ; n\right)-\frac{224}{17} \delta\left(0, \frac{1}{3}, \frac{1}{3} ; n\right)+8 \delta\left(-\frac{2}{3}, \frac{11}{6},-\frac{1}{2} ; n\right) \\
& -\frac{1104}{17} \delta\left(-\frac{1}{3}, \frac{5}{6}, \frac{1}{6} ; n\right)+\frac{128}{17} \delta\left(0,-\frac{1}{6}, \frac{5}{6} ; n\right) . \\
= & \frac{2}{17} \sigma_{7}(n)-\frac{2}{17} \sigma_{7}(n / 2)-\frac{32}{17} \sigma_{7}(n / 8)+\frac{8192}{17} \sigma_{7}(n / 16) \\
& +\frac{134}{17} \delta\left(-\frac{1}{3}, \frac{4}{3},-\frac{1}{3} ; n\right)-\frac{224}{17}\left(\frac{1}{8} \delta\left(-\frac{1}{3}, \frac{4}{3},-\frac{1}{3} ; n\right)\right) \\
& +8\left(-6 \delta\left(-\frac{2}{3}, \frac{11}{6},-\frac{1}{2} ; n\right)\right)-\frac{1104}{17}\left(-\frac{3}{8} \delta\left(-\frac{1}{3}, \frac{5}{6}, \frac{1}{6} ; n\right)\right) \\
= & \frac{2}{17} \sigma_{7}(n)-\frac{2}{17} \sigma_{7}(n / 2)-\frac{32}{17} \sigma_{7}(n / 8)+\frac{8192}{17} \sigma_{7}(n / 16) \\
& -\frac{296}{17} \delta\left(-\frac{1}{3}, \frac{4}{3},-\frac{1}{3} ; n\right) .
\end{aligned}
$$

Simplifying using (9.3.1), we obtain the desired result.

In the next two theorems we simplify Theorem 7.3 .2 to when $n \equiv 0(\bmod 8)$ and $n \equiv 4(\bmod 8)$.

Theorem 9.3.2. Let $n \in \mathbb{N}$. For $n \equiv 0(\bmod 8)$, we have

$$
\begin{aligned}
& N([1,2,13] ; n)=\frac{4064}{17} \sigma_{7}(n / 8)+\frac{4096}{17} \sigma_{7}(n / 16)+\frac{8}{17} \delta\left(-\frac{1}{3}, \frac{4}{3},-\frac{1}{3} ; n\right), \\
& N([2,2,12] ; n)=480 \sigma_{7}(n / 8)+\frac{3}{4} \delta\left(-\frac{1}{3}, \frac{4}{3},-\frac{1}{3} ; n\right), \\
& N([3,2,11] ; n)=\frac{16352}{17} \sigma_{7}(n / 8)-\frac{8192}{17} \sigma_{7}(n / 16)+\frac{89}{68} \delta\left(-\frac{1}{3}, \frac{4}{3},-\frac{1}{3} ; n\right), \\
& N([4,2,10] ; n)=\frac{32736}{17} \sigma_{7}(n / 8)-\frac{24576}{17} \sigma_{7}(n / 16)+\frac{165}{68} \delta\left(-\frac{1}{3}, \frac{4}{3},-\frac{1}{3} ; n\right),
\end{aligned}
$$




$$
\begin{aligned}
& N([5,2,9] ; n)=\frac{65504}{17} \sigma_{7}(n / 8)-\frac{57344}{17} \sigma_{7}(n / 16)+\frac{317}{68} \delta\left(-\frac{1}{3}, \frac{4}{3},-\frac{1}{3} ; n\right) \\
& N([6,2,8] ; n)=\frac{131040}{17} \sigma_{7}(n / 8)-\frac{122880}{17} \sigma_{7}(n / 16)+\frac{151}{17} \delta\left(-\frac{1}{3}, \frac{4}{3},-\frac{1}{3} ; n\right) \\
& N([7,2,7] ; n)=\frac{262112}{17} \sigma_{7}(n / 8)-\frac{253952}{17} \sigma_{7}(n / 16)+\frac{555}{34} \delta\left(-\frac{1}{3}, \frac{4}{3},-\frac{1}{3} ; n\right) \\
& N([8,2,6] ; n)=\frac{524256}{17} \sigma_{7}(n / 8)-\frac{516096}{17} \sigma_{7}(n / 16)+\frac{488}{17} \delta\left(-\frac{1}{3}, \frac{4}{3},-\frac{1}{3} ; n\right) \\
& N([9,2,5] ; n)=\frac{1048544}{17} \sigma_{7}(n / 8)-\frac{1040384}{17} \sigma_{7}(n / 16)+\frac{824}{17} \delta\left(-\frac{1}{3}, \frac{4}{3},-\frac{1}{3} ; n\right) \\
& N([10,2,4] ; n)=123360 \sigma_{7}(n / 8)-122880 \sigma_{7}(n / 16)+\frac{315}{4} \delta\left(-\frac{1}{3}, \frac{4}{3},-\frac{1}{3} ; n\right) \\
& N([11,2,3] ; n)=\frac{4194272}{17} \sigma_{7}(n / 8)-\frac{4186112}{17} \sigma_{7}(n / 16)+\frac{8249}{68} \delta\left(-\frac{1}{3}, \frac{4}{3},-\frac{1}{3} ; n\right) \\
& N([2,4,10] ; n)=\frac{16352}{17} \sigma_{7}(n / 8)-\frac{8192}{17} \sigma_{7}(n / 16)+\frac{89}{68} \delta\left(-\frac{1}{3}, \frac{4}{3},-\frac{1}{3} ; n\right) \\
& N([12,2,2] ; n)=\frac{8388576}{17} \sigma_{7}(n / 8)-\frac{8380416}{17} \sigma_{7}(n / 16)+\frac{11385}{68} \delta\left(-\frac{1}{3}, \frac{4}{3},-\frac{1}{3} ; n\right) \\
& N([1,4,11] ; n)=480 \sigma_{7}(n / 8)+\frac{3}{4} \delta\left(-\frac{1}{3}, \frac{4}{3},-\frac{1}{3} ; n\right) \\
& N([13,2,1] ; n)=\frac{16777184}{17} \sigma_{7}(n / 8)-\frac{16769024}{17} \sigma_{7}(n / 16)+\frac{11537}{68} \delta\left(-\frac{1}{3}, \frac{4}{3},-\frac{1}{3} ; n\right) \\
& N([3,4,9] ; n)=\frac{32736}{17} \sigma_{7}(n / 8)-\frac{24576}{17} \sigma_{7}(n / 16)+\frac{165}{68} \delta\left(-\frac{1}{3}, \frac{4}{3},-\frac{1}{3} ; n\right) \\
& N(7,8] ; n)=\frac{65504}{17} \sigma_{7}(n / 8)-\frac{57344}{17} \sigma_{7}(n / 16)+\frac{75}{17} \delta\left(-\frac{1}{3}, \frac{4}{3},-\frac{1}{3} ; n\right) \\
& N(n) \\
& N(n)
\end{aligned}
$$




$$
\begin{aligned}
& N([5,4,7] ; n)=\frac{131040}{17} \sigma_{7}(n / 8)-\frac{122880}{17} \sigma_{7}(n / 16)+\frac{134}{17} \delta\left(-\frac{1}{3}, \frac{4}{3},-\frac{1}{3} ; n\right), \\
& N([6,4,6] ; n)=\frac{262112}{17} \sigma_{7}(n / 8)-\frac{253952}{17} \sigma_{7}(n / 16)+\frac{235}{17} \delta\left(-\frac{1}{3}, \frac{4}{3},-\frac{1}{3} ; n\right), \\
& N([7,4,5] ; n)=\frac{524256}{17} \sigma_{7}(n / 8)-\frac{516096}{17} \sigma_{7}(n / 16)+\frac{403}{17} \delta\left(-\frac{1}{3}, \frac{4}{3},-\frac{1}{3} ; n\right), \\
& N([8,4,4] ; n)=\frac{1048544}{17} \sigma_{7}(n / 8)-\frac{1040384}{17} \sigma_{7}(n / 16)+\frac{2667}{68} \delta\left(-\frac{1}{3}, \frac{4}{3},-\frac{1}{3} ; n\right), \\
& N([9,4,3] ; n)=123360 \sigma_{7}(n / 8)-122880 \sigma_{7}(n / 16)+\frac{243}{4} \delta\left(-\frac{1}{3}, \frac{4}{3},-\frac{1}{3} ; n\right), \\
& N([10,4,2] ; n)=\frac{4194272}{17} \sigma_{7}(n / 8)-\frac{4186112}{17} \sigma_{7}(n / 16)+\frac{5597}{68} \delta\left(-\frac{1}{3}, \frac{4}{3},-\frac{1}{3} ; n\right), \\
& N([11,4,1] ; n)=\frac{8388576}{17} \sigma_{7}(n / 8)-\frac{8380416}{17} \sigma_{7}(n / 16)+\frac{5265}{68} \delta\left(-\frac{1}{3}, \frac{4}{3},-\frac{1}{3} ; n\right), \\
& N([1,6,9] ; n)=\frac{16352}{17} \sigma_{7}(n / 8)-\frac{8192}{17} \sigma_{7}(n / 16)+\frac{89}{68} \delta\left(-\frac{1}{3}, \frac{4}{3},-\frac{1}{3} ; n\right), \\
& N([2,6,8] ; n)=\frac{32736}{17} \sigma_{7}(n / 8)-\frac{24576}{17} \sigma_{7}(n / 16)+\frac{37}{17} \delta\left(-\frac{1}{3}, \frac{4}{3},-\frac{1}{3} ; n\right), \\
& N([3,6,7] ; n)=\frac{65504}{17} \sigma_{7}(n / 8)-\frac{57344}{17} \sigma_{7}(n / 16)+\frac{133}{34} \delta\left(-\frac{1}{3}, \frac{4}{3},-\frac{1}{3} ; n\right), \\
& N([4,6,6] ; n)=\frac{131040}{17} \sigma_{7}(n / 8)-\frac{122880}{17} \sigma_{7}(n / 16)+\frac{117}{17} \delta\left(-\frac{1}{3}, \frac{4}{3},-\frac{1}{3} ; n\right), \\
& N([5,6,5] ; n)=\frac{262112}{17} \sigma_{7}(n / 8)-\frac{253952}{17} \sigma_{7}(n / 16)+\frac{201}{17} \delta\left(-\frac{1}{3}, \frac{4}{3},-\frac{1}{3} ; n\right), \\
& N([6,6,4] ; n)=\frac{524256}{17} \sigma_{7}(n / 8)-\frac{516096}{17} \sigma_{7}(n / 16)+\frac{1323}{68} \delta\left(-\frac{1}{3}, \frac{4}{3},-\frac{1}{3} ; n\right),
\end{aligned}
$$




$$
\begin{aligned}
& N([7,6,3] ; n)=\frac{1048544}{17} \sigma_{7}(n / 8)-\frac{1040384}{17} \sigma_{7}(n / 16)+\frac{2021}{68} \delta\left(-\frac{1}{3}, \frac{4}{3},-\frac{1}{3} ; n\right), \\
& N([8,6,2] ; n)=123360 \sigma_{7}(n / 8)-122880 \sigma_{7}(n / 16)+\frac{157}{4} \delta\left(-\frac{1}{3}, \frac{4}{3},-\frac{1}{3} ; n\right), \\
& N([9,6,1] ; n)=\frac{4194272}{17} \sigma_{7}(n / 8)-\frac{4186112}{17} \sigma_{7}(n / 16)+\frac{2333}{68} \delta\left(-\frac{1}{3}, \frac{4}{3},-\frac{1}{3} ; n\right), \\
& N([1,8,7] ; n)=\frac{32736}{17} \sigma_{7}(n / 8)-\frac{24576}{17} \sigma_{7}(n / 16)+\frac{37}{17} \delta\left(-\frac{1}{3}, \frac{4}{3},-\frac{1}{3} ; n\right), \\
& N([2,8,6] ; n)=\frac{65504}{17} \sigma_{7}(n / 8)-\frac{57344}{17} \sigma_{7}(n / 16)+\frac{58}{17} \delta\left(-\frac{1}{3}, \frac{4}{3},-\frac{1}{3} ; n\right), \\
& N([3,8,5] ; n)=\frac{131040}{17} \sigma_{7}(n / 8)-\frac{122880}{17} \sigma_{7}(n / 16)+\frac{100}{17} \delta\left(-\frac{1}{3}, \frac{4}{3},-\frac{1}{3} ; n\right), \\
& N([4,8,4] ; n)=\frac{262112}{17} \sigma_{7}(n / 8)-\frac{253952}{17} \sigma_{7}(n / 16)+\frac{651}{68} \delta\left(-\frac{1}{3}, \frac{4}{3},-\frac{1}{3} ; n\right), \\
& N([5,8,3] ; n)=\frac{524256}{17} \sigma_{7}(n / 8)-\frac{516096}{17} \sigma_{7}(n / 16)+\frac{983}{68} \delta\left(-\frac{1}{3}, \frac{4}{3},-\frac{1}{3} ; n\right), \\
& N([6,8,2] ; n)=\frac{1048544}{17} \sigma_{7}(n / 8)-\frac{1040384}{17} \sigma_{7}(n / 16)+\frac{1273}{68} \delta\left(-\frac{1}{3}, \frac{4}{3},-\frac{1}{3} ; n\right), \\
& N([7,8,1] ; n)=123360 \sigma_{7}(n / 8)-122880 \sigma_{7}(n / 16)+\frac{61}{4} \delta\left(-\frac{1}{3}, \frac{4}{3},-\frac{1}{3} ; n\right), \\
& N([1,10,5] ; n)=\frac{65504}{17} \sigma_{7}(n / 8)-\frac{57344}{17} \sigma_{7}(n / 16)+\frac{58}{17} \delta\left(-\frac{1}{3}, \frac{4}{3},-\frac{1}{3} ; n\right), \\
& N([2,10,4] ; n)=\frac{131040}{17} \sigma_{7}(n / 8)-\frac{122880}{17} \sigma_{7}(n / 16)+\frac{315}{68} \delta\left(-\frac{1}{3}, \frac{4}{3},-\frac{1}{3} ; n\right), \\
& N([3,10,3] ; n)=\frac{262112}{17} \sigma_{7}(n / 8)-\frac{253952}{17} \sigma_{7}(n / 16)+\frac{481}{68} \delta\left(-\frac{1}{3}, \frac{4}{3},-\frac{1}{3} ; n\right),
\end{aligned}
$$




$$
\begin{aligned}
& N([4,10,2] ; n)=\frac{524256}{17} \sigma_{7}(n / 8)-\frac{516096}{17} \sigma_{7}(n / 16)+\frac{609}{68} \delta\left(-\frac{1}{3}, \frac{4}{3},-\frac{1}{3} ; n\right), \\
& N([5,10,1] ; n)=\frac{1048544}{17} \sigma_{7}(n / 8)-\frac{1040384}{17} \sigma_{7}(n / 16)+\frac{457}{68} \delta\left(-\frac{1}{3}, \frac{4}{3},-\frac{1}{3} ; n\right), \\
& N([1,12,3] ; n)=\frac{131040}{17} \sigma_{7}(n / 8)-\frac{122880}{17} \sigma_{7}(n / 16)+\frac{315}{68} \delta\left(-\frac{1}{3}, \frac{4}{3},-\frac{1}{3} ; n\right), \\
& N([2,12,2] ; n)=\frac{262112}{17} \sigma_{7}(n / 8)-\frac{253952}{17} \sigma_{7}(n / 16)+\frac{277}{68} \delta\left(-\frac{1}{3}, \frac{4}{3},-\frac{1}{3} ; n\right), \\
& N([3,12,1] ; n)=\frac{524256}{17} \sigma_{7}(n / 8)-\frac{516096}{17} \sigma_{7}(n / 16)+\frac{201}{68} \delta\left(-\frac{1}{3}, \frac{4}{3},-\frac{1}{3} ; n\right), \\
& N([1,14,1] ; n)=\frac{262112}{17} \sigma_{7}(n / 8)-\frac{253952}{17} \sigma_{7}(n / 16)+\frac{277}{68} \delta\left(-\frac{1}{3}, \frac{4}{3},-\frac{1}{3} ; n\right) .
\end{aligned}
$$

Proof. We only prove the formula for $N([4,4,8] ; n)$ as the proofs for the other cases are similar. By Theorem 7.3.2, Theorems 8.5.1 - 8.5.4, and Theorem 8.6.1 with $n \equiv 0$ $(\bmod 8)$, we have, for $n \equiv 0(\bmod 8)$.

$$
\begin{aligned}
& N([4,4,8] ; n) \\
&=\frac{1}{544} \sigma_{7}(n)-\frac{1}{544} \sigma_{7}(n / 2)-\frac{32}{17} \sigma_{7}(n / 8)+\frac{8192}{17} \sigma_{7}(n / 16) \\
&+\frac{1087}{544} \delta\left(-\frac{1}{3}, \frac{4}{3},-\frac{1}{3} ; n\right)-\frac{29}{4} \delta\left(0, \frac{1}{3}, \frac{1}{3} ; n\right)+2 \delta\left(-\frac{2}{3}, \frac{11}{6},-\frac{1}{2} ; n\right) \\
&-\frac{849}{34} \delta\left(-\frac{1}{3}, \frac{5}{6}, \frac{1}{6} ; n\right)+\frac{64}{17} \delta\left(0,-\frac{1}{6}, \frac{5}{6} ; n\right)+\frac{545}{272} \delta\left(-\frac{5}{6}, \frac{25}{12},-\frac{7}{12} ; n\right) \\
&-\frac{2181}{68} \delta\left(-\frac{1}{2}, \frac{13}{12}, \frac{1}{12} ; n\right)+\frac{547}{17} \delta\left(-\frac{1}{6}, \frac{1}{12}, \frac{3}{4} ; n\right)+\frac{543}{272} \delta\left(-\frac{1}{2}, \frac{19}{12},-\frac{5}{12} ; n\right) \\
&-\frac{1085}{68} \delta\left(-\frac{1}{6}, \frac{7}{12}, \frac{1}{4} ; n\right)+\frac{1}{17} \delta\left(\frac{1}{6},-\frac{5}{12}, \frac{11}{12} ; n\right) . \\
&= \frac{1}{544} \sigma_{7}(n)-\frac{1}{544} \sigma_{7}(n / 2)-\frac{32}{17} \sigma_{7}(n / 8)+\frac{8192}{17} \sigma_{7}(n / 16) \\
&+\frac{1087}{544} \delta\left(-\frac{1}{3}, \frac{4}{3},-\frac{1}{3} ; n\right)-\frac{29}{4}\left(\frac{1}{8} \delta\left(-\frac{1}{3}, 1,-\frac{1}{6} ; n\right)\right)
\end{aligned}
$$




$$
\begin{aligned}
& +2\left(-6 \delta\left(-\frac{1}{3}, 1,-\frac{1}{6} ; n\right)\right)-\frac{849}{34}\left(-\frac{3}{8} \delta\left(-\frac{1}{3}, 1,-\frac{1}{6} ; n\right)\right) \\
& +\frac{545}{272}\left(45 \delta\left(-\frac{1}{3}, 1,-\frac{1}{6} ; n\right)\right)-\frac{2181}{68}\left(3 \delta\left(-\frac{1}{3}, 1,-\frac{1}{6} ; n\right)\right) \\
& +\frac{547}{17}\left(\frac{3}{16} \delta\left(-\frac{1}{3}, 1,-\frac{1}{6} ; n\right)\right)+\frac{543}{272}\left(3 \delta\left(-\frac{1}{3}, 1,-\frac{1}{6} ; n\right)\right) \\
& +\frac{1}{17}\left(-\frac{3}{16} \delta\left(-\frac{1}{3}, 1,-\frac{1}{6} ; n\right)\right) \\
& =\frac{1}{544} \sigma_{7}(n)-\frac{1}{544} \sigma_{7}(n / 2)-\frac{32}{17} \sigma_{7}(n / 8)+\frac{8192}{17} \sigma_{7}(n / 16) \\
& +\frac{75}{17} \delta\left(-\frac{1}{3}, \frac{4}{3},-\frac{1}{3} ; n\right) .
\end{aligned}
$$

Simplifying using (9.3.3), we obtain the desired result.

Theorem 9.3.3. Let $n \in \mathbb{N}$. For $n \equiv 4(\bmod 8)$, we have

$$
\begin{aligned}
& N([1,2,13] ; n)=\frac{32}{17} \sigma_{7}(n / 4)-\frac{8}{17} \delta\left(-\frac{1}{3}, \frac{4}{3},-\frac{1}{3} ; n\right), \\
& N([2,2,12] ; n)=\frac{64}{17} \sigma_{7}(n / 4)-\frac{47}{68} \delta\left(-\frac{1}{3}, \frac{4}{3},-\frac{1}{3} ; n\right), \\
& N([3,2,11] ; n)=\frac{128}{17} \sigma_{7}(n / 4)-\frac{77}{68} \delta\left(-\frac{1}{3}, \frac{4}{3},-\frac{1}{3} ; n\right), \\
& N([4,2,10] ; n)=\frac{256}{17} \sigma_{7}(n / 4)-\frac{137}{68} \delta\left(-\frac{1}{3}, \frac{4}{3},-\frac{1}{3} ; n\right), \\
& N([5,2,9] ; n)=\frac{512}{17} \sigma_{7}(n / 4)-\frac{257}{68} \delta\left(-\frac{1}{3}, \frac{4}{3},-\frac{1}{3} ; n\right), \\
& N([6,2,8] ; n)=\frac{1024}{17} \sigma_{7}(n / 4)-\frac{120}{17} \delta\left(-\frac{1}{3}, \frac{4}{3},-\frac{1}{3} ; n\right), \\
& N([7,2,7] ; n)=\frac{2048}{17} \sigma_{7}(n / 4)-\frac{429}{34} \delta\left(-\frac{1}{3}, \frac{4}{3},-\frac{1}{3} ; n\right),
\end{aligned}
$$




$$
\begin{aligned}
& N([8,2,6] ; n)=\frac{4096}{17} \sigma_{7}(n / 4)-\frac{361}{17} \delta\left(-\frac{1}{3}, \frac{4}{3},-\frac{1}{3} ; n\right), \\
& N([9,2,5] ; n)=\frac{8192}{17} \sigma_{7}(n / 4)-\frac{569}{17} \delta\left(-\frac{1}{3}, \frac{4}{3},-\frac{1}{3} ; n\right), \\
& N([10,2,4] ; n)=\frac{16384}{17} \sigma_{7}(n / 4)-\frac{3345}{68} \delta\left(-\frac{1}{3}, \frac{4}{3},-\frac{1}{3} ; n\right), \\
& N([11,2,3] ; n)=\frac{32768}{17} \sigma_{7}(n / 4)-\frac{4531}{68} \delta\left(-\frac{1}{3}, \frac{4}{3},-\frac{1}{3} ; n\right), \\
& N([12,2,2] ; n)=\frac{65536}{17} \sigma_{7}(n / 4)-\frac{5475}{68} \delta\left(-\frac{1}{3}, \frac{4}{3},-\frac{1}{3} ; n\right), \\
& N([13,2,1] ; n)=\frac{131072}{17} \sigma_{7}(n / 4)-\frac{5323}{68} \delta\left(-\frac{1}{3}, \frac{4}{3},-\frac{1}{3} ; n\right) \text {, } \\
& N([1,4,11] ; n)=\frac{64}{17} \sigma_{7}(n / 4)-\frac{47}{68} \delta\left(-\frac{1}{3}, \frac{4}{3},-\frac{1}{3} ; n\right), \\
& N([2,4,10] ; n)=\frac{128}{17} \sigma_{7}(n / 4)-\frac{77}{68} \delta\left(-\frac{1}{3}, \frac{4}{3},-\frac{1}{3} ; n\right), \\
& N([3,4,9] ; n)=\frac{256}{17} \sigma_{7}(n / 4)-\frac{137}{68} \delta\left(-\frac{1}{3}, \frac{4}{3},-\frac{1}{3} ; n\right), \\
& N([4,4,8] ; n)=\frac{512}{17} \sigma_{7}(n / 4)-\frac{60}{17} \delta\left(-\frac{1}{3}, \frac{4}{3},-\frac{1}{3} ; n\right), \\
& N([5,4,7] ; n)=\frac{1024}{17} \sigma_{7}(n / 4)-\frac{103}{17} \delta\left(-\frac{1}{3}, \frac{4}{3},-\frac{1}{3} ; n\right), \\
& N([6,4,6] ; n)=\frac{2048}{17} \sigma_{7}(n / 4)-\frac{172}{17} \delta\left(-\frac{1}{3}, \frac{4}{3},-\frac{1}{3} ; n\right) \text {, } \\
& N([7,4,5] ; n)=\frac{4096}{17} \sigma_{7}(n / 4)-\frac{276}{17} \delta\left(-\frac{1}{3}, \frac{4}{3},-\frac{1}{3} ; n\right),
\end{aligned}
$$




$$
\begin{aligned}
& N([8,4,4] ; n)=\frac{8192}{17} \sigma_{7}(n / 4)-\frac{1681}{68} \delta\left(-\frac{1}{3}, \frac{4}{3},-\frac{1}{3} ; n\right), \\
& N([9,4,3] ; n)=\frac{16384}{17} \sigma_{7}(n / 4)-\frac{2393}{68} \delta\left(-\frac{1}{3}, \frac{4}{3},-\frac{1}{3} ; n\right), \\
& N([10,4,2] ; n)=\frac{32768}{17} \sigma_{7}(n / 4)-\frac{3103}{68} \delta\left(-\frac{1}{3}, \frac{4}{3},-\frac{1}{3} ; n\right), \\
& N([11,4,1] ; n)=\frac{65536}{17} \sigma_{7}(n / 4)-\frac{3435}{68} \delta\left(-\frac{1}{3}, \frac{4}{3},-\frac{1}{3} ; n\right), \\
& N([1,6,9] ; n)=\frac{128}{17} \sigma_{7}(n / 4)-\frac{77}{68} \delta\left(-\frac{1}{3}, \frac{4}{3},-\frac{1}{3} ; n\right), \\
& N([2,6,8] ; n)=\frac{256}{17} \sigma_{7}(n / 4)-\frac{30}{17} \delta\left(-\frac{1}{3}, \frac{4}{3},-\frac{1}{3} ; n\right), \\
& N([3,6,7] ; n)=\frac{512}{17} \sigma_{7}(n / 4)-\frac{103}{34} \delta\left(-\frac{1}{3}, \frac{4}{3},-\frac{1}{3} ; n\right), \\
& N([4,6,6] ; n)=\frac{1024}{17} \sigma_{7}(n / 4)-\frac{86}{17} \delta\left(-\frac{1}{3}, \frac{4}{3},-\frac{1}{3} ; n\right), \\
& N([5,6,5] ; n)=\frac{2048}{17} \sigma_{7}(n / 4)-\frac{138}{17} \delta\left(-\frac{1}{3}, \frac{4}{3},-\frac{1}{3} ; n\right), \\
& N([6,6,4] ; n)=\frac{4096}{17} \sigma_{7}(n / 4)-\frac{849}{68} \delta\left(-\frac{1}{3}, \frac{4}{3},-\frac{1}{3} ; n\right), \\
& N([7,6,3] ; n)=\frac{8192}{17} \sigma_{7}(n / 4)-\frac{1239}{68} \delta\left(-\frac{1}{3}, \frac{4}{3},-\frac{1}{3} ; n\right), \\
& N([8,6,2] ; n)=\frac{16384}{17} \sigma_{7}(n / 4)-\frac{1679}{68} \delta\left(-\frac{1}{3}, \frac{4}{3},-\frac{1}{3} ; n\right), \\
& N([9,6,1] ; n)=\frac{32768}{17} \sigma_{7}(n / 4)-\frac{2015}{68} \delta\left(-\frac{1}{3}, \frac{4}{3},-\frac{1}{3} ; n\right),
\end{aligned}
$$




$$
\begin{aligned}
& N([1,8,7] ; n)=\frac{256}{17} \sigma_{7}(n / 4)-\frac{30}{17} \delta\left(-\frac{1}{3}, \frac{4}{3},-\frac{1}{3} ; n\right), \\
& N([2,8,6] ; n)=\frac{512}{17} \sigma_{7}(n / 4)-\frac{43}{17} \delta\left(-\frac{1}{3}, \frac{4}{3},-\frac{1}{3} ; n\right), \\
& N([3,8,5] ; n)=\frac{1024}{272} \sigma_{7}(n / 4)-\frac{69}{17} \delta\left(-\frac{1}{3}, \frac{4}{3},-\frac{1}{3} ; n\right), \\
& N([4,8,4] ; n)=\frac{2048}{17} \sigma_{7}(n / 4)-\frac{433}{68} \delta\left(-\frac{1}{3}, \frac{4}{3},-\frac{1}{3} ; n\right), \\
& N([5,8,3] ; n)=\frac{4096}{17} \sigma_{7}(n / 4)-\frac{645}{68} \delta\left(-\frac{1}{3}, \frac{4}{3},-\frac{1}{3} ; n\right), \\
& N([6,8,2] ; n)=\frac{8192}{17} \sigma_{7}(n / 4)-\frac{899}{68} \delta\left(-\frac{1}{3}, \frac{4}{3},-\frac{1}{3} ; n\right), \\
& N([7,8,1] ; n)=\frac{16384}{17} \sigma_{7}(n / 4)-\frac{1135}{68} \delta\left(-\frac{1}{3}, \frac{4}{3},-\frac{1}{3} ; n\right) \\
& N([1,10,5] ; n)=\frac{512}{17} \sigma_{7}(n / 4)-\frac{43}{17} \delta\left(-\frac{1}{3}, \frac{4}{3},-\frac{1}{3} ; n\right), \\
& N([2,10,4] ; n)=\frac{1024}{17} \sigma_{7}(n / 4)-\frac{225}{68} \delta\left(-\frac{1}{3}, \frac{4}{3},-\frac{1}{3} ; n\right) \\
& N([3,10,3] ; n)=\frac{2048}{17} \sigma_{7}(n / 4)-\frac{331}{68} \delta\left(-\frac{1}{3}, \frac{4}{3},-\frac{1}{3} ; n\right) \\
& N([4,10,2] ; n)=\frac{4096}{17} \sigma_{7}(n / 4)-\frac{475}{68} \delta\left(-\frac{1}{3}, \frac{4}{3},-\frac{1}{3} ; n\right) \\
& N([5,10,1] ; n)=\frac{8192}{17} \sigma_{7}(n / 4)-\frac{627}{68} \delta\left(-\frac{1}{3}, \frac{4}{3},-\frac{1}{3} ; n\right) \\
& N([1,12,3] ; n)=\frac{1024}{17} \sigma_{7}(n / 4)-\frac{225}{68} \delta\left(-\frac{1}{3}, \frac{4}{3},-\frac{1}{3} ; n\right)
\end{aligned}
$$


$N([2,12,2] ; n)=\frac{2048}{17} \sigma_{7}(n / 4)-\frac{263}{68} \delta\left(-\frac{1}{3}, \frac{4}{3},-\frac{1}{3} ; n\right)$,

$N([3,12,1] ; n)=\frac{4096}{17} \sigma_{7}(n / 4)-\frac{339}{68} \delta\left(-\frac{1}{3}, \frac{4}{3},-\frac{1}{3} ; n\right)$,

$N([1,14,1] ; n)=\frac{2048}{17} \sigma_{7}(n / 4)-\frac{263}{68} \delta\left(-\frac{1}{3}, \frac{4}{3},-\frac{1}{3} ; n\right)$.

Proof. We only prove the formula for $N([5,8,3] ; n)$ as the proofs for the other cases are similar. By Theorem 7.3.2, Theorems 8.5.1 - 8.5.4, and Theorem 8.6.1 with $n \equiv 4$ $(\bmod 8)$ we have, for $n \equiv 4(\bmod 8)$.

$$
\begin{aligned}
N & ([5,8,3] ; n) \\
= & \frac{1}{68} \sigma_{7}(n)-\frac{1}{68} \sigma_{7}(n / 2)-\frac{32}{17} \sigma_{7}(n / 8)+\frac{8192}{17} \sigma_{7}(n / 16) \\
& +\frac{169}{68} \delta\left(-\frac{1}{3}, \frac{4}{3},-\frac{1}{3} ; n\right)-\frac{124}{17} \delta\left(0, \frac{1}{3}, \frac{1}{3} ; n\right)+\frac{5}{2} \delta\left(-\frac{2}{3}, \frac{11}{6},-\frac{1}{2} ; n\right) \\
& -\frac{450}{17} \delta\left(-\frac{1}{3}, \frac{5}{6}, \frac{1}{6} ; n\right)+\frac{64}{17} \delta\left(0,-\frac{1}{6}, \frac{5}{6} ; n\right)+\frac{681}{272} \delta\left(-\frac{5}{6}, \frac{25}{12},-\frac{7}{12} ; n\right) \\
& -\frac{2453}{68} \delta\left(-\frac{1}{2}, \frac{13}{12}, \frac{1}{12} ; n\right)+\frac{547}{17} \delta\left(-\frac{1}{6}, \frac{1}{12}, \frac{3}{4} ; n\right)+\frac{679}{272} \delta\left(-\frac{1}{2}, \frac{19}{12},-\frac{5}{12} ; n\right) \\
& -\frac{1085}{68} \delta\left(-\frac{1}{6}, \frac{7}{12}, \frac{1}{4} ; n\right)+\frac{1}{17} \delta\left(\frac{1}{6},-\frac{5}{12}, \frac{11}{12} ; n\right) \\
= & \frac{1}{68} \sigma_{7}(n)-\frac{1}{68} \sigma_{7}(n / 2) \\
& +\frac{169}{68} \delta\left(-\frac{1}{3}, \frac{4}{3},-\frac{1}{3} ; n\right)-\frac{124}{17}\left(\frac{1}{8} \delta\left(-\frac{1}{3}, \frac{4}{3},-\frac{1}{3} ; n\right)\right) \\
& +\frac{5}{2}\left(-6 \delta\left(-\frac{1}{3}, \frac{4}{3},-\frac{1}{3} ; n\right)\right)-\frac{450}{17}\left(-\frac{3}{8} \delta\left(-\frac{1}{3}, \frac{4}{3},-\frac{1}{3} ; n\right)\right) \\
& +\frac{681}{272}\left(-15 \delta\left(-\frac{1}{3}, \frac{4}{3},-\frac{1}{3} ; n\right)\right)-\frac{2453}{68}\left(-\delta\left(-\frac{1}{3}, \frac{4}{3},-\frac{1}{3} ; n\right)\right) \\
& +\frac{547}{17}\left(-\frac{1}{16} \delta\left(-\frac{1}{3}, \frac{4}{3},-\frac{1}{3} ; n\right)\right)+\frac{679}{272}\left(-\delta\left(-\frac{1}{2}, \frac{19}{12},-\frac{5}{12} ; n\right)\right) \\
& +\frac{1}{17}\left(\frac{1}{16} \delta\left(-\frac{1}{3}, \frac{4}{3},-\frac{1}{3} ; n\right)\right)
\end{aligned}
$$




$$
=\frac{1}{68} \sigma_{7}(n)-\frac{1}{68} \sigma_{7}(n / 2)-\frac{645}{68} \delta\left(-\frac{1}{3}, \frac{4}{3},-\frac{1}{3} ; n\right) .
$$

Simplifying using (9.3.2), we obtain the desired result.

In the next result, we use an identity of Chapter 8 to reduce the number of delta functions $\delta(a, b, c ; n)$ occurring in Theorem 7.3 .3 by two.

Theorem 9.3.4. For $n \in \mathbb{N}$, we have

$$
\begin{aligned}
N & {[1,0,15] ; n) } \\
= & \left(\frac{1}{17408}+\frac{1}{17408}\left(\frac{-4}{n}\right)\right) \sigma_{7}(n)-\frac{129}{17408} \sigma_{7}(n / 2)+\frac{257}{136} \sigma_{7}(n / 4)-\frac{64}{17} \sigma_{7}(n / 8) \\
& +\frac{8192}{17} \sigma_{7}(n / 16) \\
& +\frac{7751}{17408} \delta\left(-\frac{1}{3}, \frac{4}{3},-\frac{1}{3} ; n\right)-\frac{8775}{2176} \delta\left(0, \frac{1}{3}, \frac{1}{3} ; n\right)+\frac{71}{128} \delta\left(-\frac{2}{3}, \frac{11}{6},-\frac{1}{2} ; n\right) \\
& -\frac{1143}{136} \delta\left(-\frac{1}{3}, \frac{5}{6}, \frac{1}{6} ; n\right)+\frac{253}{136} \delta\left(0,-\frac{1}{6}, \frac{5}{6} ; n\right)+\frac{15359}{34816} \delta\left(-\frac{5}{6}, \frac{25}{12},-\frac{7}{12} ; n\right) \\
& -\frac{70675}{8704} \delta\left(-\frac{1}{2}, \frac{13}{12}, \frac{1}{12} ; n\right)+\frac{40909}{2176} \delta\left(-\frac{1}{6}, \frac{1}{12}, \frac{3}{4} ; n\right)+\frac{19455}{34816} \delta\left(-\frac{1}{2}, \frac{19}{12},-\frac{5}{12} ; n\right) \\
& -\frac{64485}{8704} \delta\left(-\frac{1}{6}, \frac{7}{12}, \frac{1}{4} ; n\right)-\frac{143}{2176} \delta\left(\frac{1}{6},-\frac{5}{12}, \frac{11}{12} ; n\right), \\
N & ([2,0,14] ; n) \\
= & \left(\frac{1}{8704}+\frac{1}{8704}\left(\frac{-4}{n}\right)\right) \sigma_{7}(n)-\frac{1}{8704} \sigma_{7}(n / 2)-\frac{32}{17} \sigma_{7}(n / 8)+\frac{8192}{17} \sigma_{7}(n / 16) \\
& +\frac{7751}{8704} \delta\left(-\frac{1}{3}, \frac{4}{3},-\frac{1}{3} ; n\right)-\frac{6643}{1088} \delta\left(0, \frac{1}{3}, \frac{1}{3} ; n\right)+\frac{71}{64} \delta\left(-\frac{2}{3}, \frac{11}{6},-\frac{1}{2} ; n\right) \\
& -\frac{4565}{272} \delta\left(-\frac{1}{3}, \frac{5}{6}, \frac{1}{6} ; n\right)+\frac{239}{68} \delta\left(0,-\frac{1}{6}, \frac{5}{6} ; n\right)+\frac{17199}{17408} \delta\left(-\frac{5}{6}, \frac{25}{12},-\frac{7}{12} ; n\right) \\
& -\frac{75523}{4342} \delta\left(-\frac{1}{2}, \frac{13}{12}, \frac{1}{12} ; n\right)+\frac{30653}{1088} \delta\left(-\frac{1}{6}, \frac{1}{12}, \frac{3}{4} ; n\right)+\frac{17615}{17408} \delta\left(-\frac{1}{2}, \frac{19}{12},-\frac{5}{12} ; n\right) \\
& -\frac{54613}{4352} \delta\left(-\frac{1}{6}, \frac{7}{12}, \frac{1}{4} ; n\right)+\frac{65}{1088} \delta\left(\frac{1}{6},-\frac{5}{12}, \frac{11}{12} ; n\right), \\
N & ([3,0,13] ; n) \\
= & \left(\frac{1}{4352}+\frac{1}{8704}\left(\frac{-4}{n}\right)\right) \sigma_{7}(n)+\frac{63}{4352} \sigma_{7}(n / 2)-\frac{257}{68} \sigma_{7}(n / 4)+\frac{32}{17} \sigma_{7}(n / 8) \\
&
\end{aligned}
$$




$$
\begin{aligned}
& +\frac{8192}{17} \sigma_{7}(n / 16) \\
& +\frac{5983}{4352} \delta\left(-\frac{1}{3}, \frac{4}{3},-\frac{1}{3} ; n\right)-\frac{3809}{544} \delta\left(0, \frac{1}{3}, \frac{1}{3} ; n\right)+\frac{13}{8} \delta\left(-\frac{2}{3}, \frac{11}{6},-\frac{1}{2} ; n\right) \\
& -\frac{6539}{272} \delta\left(-\frac{1}{3}, \frac{5}{6}, \frac{1}{6} ; n\right)+\frac{65}{17} \delta\left(0,-\frac{1}{6}, \frac{5}{6} ; n\right)+\frac{27535}{17408} \delta\left(-\frac{5}{6}, \frac{25}{12},-\frac{7}{12} ; n\right) \\
& -\frac{116867}{4352} \delta\left(-\frac{1}{2}, \frac{13}{12}, \frac{1}{12} ; n\right)+\frac{33917}{1088} \delta\left(-\frac{1}{6}, \frac{1}{12}, \frac{3}{4} ; n\right)+\frac{24687}{17408} \delta\left(-\frac{1}{2}, \frac{19}{12},-\frac{5}{12} ; n\right) \\
& -\frac{68757}{4352} \delta\left(-\frac{1}{6}, \frac{7}{12}, \frac{1}{4} ; n\right)+\frac{65}{1088} \delta\left(\frac{1}{6},-\frac{5}{12}, \frac{11}{12} ; n\right),
\end{aligned}
$$

$N([4,0,12] ; n)$

$$
\begin{aligned}
= & \frac{1}{2176} \sigma_{7}(n)+\frac{63}{2176} \sigma_{7}(n / 2)-\frac{257}{34} \sigma_{7}(n / 4)+\frac{96}{17} \sigma_{7}(n / 8)+\frac{8192}{17} \sigma_{7}(n / 16) \\
& +\frac{4215}{2176} \delta\left(-\frac{1}{3}, \frac{4}{3},-\frac{1}{3} ; n\right)-\frac{1965}{272} \delta\left(0, \frac{1}{3}, \frac{1}{3} ; n\right)+\frac{33}{16} \delta\left(-\frac{2}{3}, \frac{11}{6},-\frac{1}{2} ; n\right) \\
& -\frac{3993}{136} \delta\left(-\frac{1}{3}, \frac{5}{6}, \frac{1}{6} ; n\right)+\frac{66}{17} \delta\left(0,-\frac{1}{6}, \frac{5}{6} ; n\right)+\frac{149}{68} \delta\left(-\frac{5}{6}, \frac{25}{12},-\frac{7}{12} ; n\right) \\
& -\frac{1235}{34} \delta\left(-\frac{1}{2}, \frac{13}{12}, \frac{1}{12} ; n\right)+\frac{547}{17} \delta\left(-\frac{1}{6}, \frac{1}{12}, \frac{3}{4} ; n\right)+\frac{123}{68} \delta\left(-\frac{1}{2}, \frac{19}{12},-\frac{5}{12} ; n\right) \\
& -\frac{585}{34} \delta\left(-\frac{1}{6}, \frac{7}{12}, \frac{1}{4} ; n\right)+\frac{1}{17} \delta\left(\frac{1}{6},-\frac{5}{12}, \frac{11}{12} ; n\right),
\end{aligned}
$$

$N([5,0,11] ; n)$

$$
\begin{aligned}
= & \left(\frac{1}{1088}-\frac{1}{4352}\left(\frac{-4}{n}\right)\right) \sigma_{7}(n)+\frac{31}{1088} \sigma_{7}(n / 2)-\frac{257}{34} \sigma_{7}(n / 4)+\frac{96}{17} \sigma_{7}(n / 8) \\
& +\frac{8192}{17} \sigma_{7}(n / 16) \\
& +\frac{2821}{1088} \delta\left(-\frac{1}{3}, \frac{4}{3},-\frac{1}{3} ; n\right)-\frac{495}{68} \delta\left(0, \frac{1}{3}, \frac{1}{3} ; n\right)+\frac{77}{32} \delta\left(-\frac{2}{3}, \frac{11}{6},-\frac{1}{2} ; n\right) \\
& -\frac{4367}{136} \delta\left(-\frac{1}{3}, \frac{5}{6}, \frac{1}{6} ; n\right)+\frac{66}{17} \delta\left(0,-\frac{1}{6}, \frac{5}{6} ; n\right)+\frac{23969}{8704} \delta\left(-\frac{5}{6}, \frac{25}{12},-\frac{7}{12} ; n\right) \\
& -\frac{97405}{2176} \delta\left(-\frac{1}{2}, \frac{13}{12}, \frac{1}{12} ; n\right)+\frac{18051}{544} \delta\left(-\frac{1}{6}, \frac{1}{12}, \frac{3}{4} ; n\right)+\frac{19553}{8704} \delta\left(-\frac{1}{2}, \frac{19}{12},-\frac{5}{12} ; n\right) \\
& -\frac{37579}{2176} \delta\left(-\frac{1}{6}, \frac{7}{12}, \frac{1}{4} ; n\right)+\frac{31}{544} \delta\left(\frac{1}{6},-\frac{5}{12}, \frac{11}{12} ; n\right),
\end{aligned}
$$

$N([6,0,10] ; n)$

$$
=\left(\frac{1}{544}-\frac{1}{2176}\left(\frac{-4}{n}\right)\right) \sigma_{7}(n)-\frac{1}{544} \sigma_{7}(n / 2)-\frac{32}{17} \sigma_{7}(n / 8)+\frac{8192}{17} \sigma_{7}(n / 16)
$$




$$
\begin{aligned}
& +\frac{1801}{544} \delta\left(-\frac{1}{3}, \frac{4}{3},-\frac{1}{3} ; n\right)-\frac{15}{2} \delta\left(0, \frac{1}{3}, \frac{1}{3} ; n\right)+\frac{43}{16} \delta\left(-\frac{2}{3}, \frac{11}{6},-\frac{1}{2} ; n\right) \\
& -\frac{2191}{68} \delta\left(-\frac{1}{3}, \frac{5}{6}, \frac{1}{6} ; n\right)+\frac{64}{17} \delta\left(0,-\frac{1}{6}, \frac{5}{6} ; n\right)+\frac{817}{256} \delta\left(-\frac{5}{6}, \frac{25}{12},-\frac{7}{12} ; n\right) \\
& -\frac{3245}{64} \delta\left(-\frac{1}{2}, \frac{13}{12}, \frac{1}{12} ; n\right)+\frac{547}{16} \delta\left(-\frac{1}{6}, \frac{1}{12}, \frac{3}{4} ; n\right)+\frac{12225}{4352} \delta\left(-\frac{1}{2}, \frac{19}{12},-\frac{5}{12} ; n\right) \\
& -\frac{18315}{1088} \delta\left(-\frac{1}{6}, \frac{7}{12}, \frac{1}{4} ; n\right)+\frac{15}{272} \delta\left(\frac{1}{6},-\frac{5}{12}, \frac{11}{12} ; n\right) \\
& N([7,0,9] ; n) \\
& =\left(\frac{1}{272}-\frac{1}{2176}\left(\frac{-4}{n}\right)\right) \sigma_{7}(n)-\frac{1}{16} \sigma_{7}(n / 2)+\frac{257}{17} \sigma_{7}(n / 4)-\frac{288}{17} \sigma_{7}(n / 8) \\
& +\frac{8192}{17} \sigma_{7}(n / 16) \\
& +\frac{1087}{272} \delta\left(-\frac{1}{3}, \frac{4}{3},-\frac{1}{3} ; n\right)-\frac{135}{17} \delta\left(0, \frac{1}{3}, \frac{1}{3} ; n\right)+3 \delta\left(-\frac{2}{3}, \frac{11}{6},-\frac{1}{2} ; n\right) \\
& -\frac{1035}{34} \delta\left(-\frac{1}{3}, \frac{5}{6}, \frac{1}{6} ; n\right)+\frac{60}{17} \delta\left(0,-\frac{1}{6}, \frac{5}{6} ; n\right)+\frac{881}{256} \delta\left(-\frac{5}{6}, \frac{25}{12},-\frac{7}{12} ; n\right) \\
& -\frac{3373}{64} \delta\left(-\frac{1}{2}, \frac{13}{12}, \frac{1}{12} ; n\right)+\frac{547}{16} \delta\left(-\frac{1}{6}, \frac{1}{12}, \frac{3}{4} ; n\right)+\frac{15489}{4352} \delta\left(-\frac{1}{2}, \frac{19}{12},-\frac{5}{12} ; n\right) \\
& -\frac{18315}{1088} \delta\left(-\frac{1}{6}, \frac{7}{12}, \frac{1}{4} ; n\right)+\frac{15}{272} \delta\left(\frac{1}{6},-\frac{5}{12}, \frac{11}{12} ; n\right),
\end{aligned}
$$

$N([8,0,8] ; n)$

$$
\begin{aligned}
= & \frac{1}{136} \sigma_{7}(n)-\frac{1}{8} \sigma_{7}(n / 2)+\frac{514}{17} \sigma_{7}(n / 4)-32 \sigma_{7}(n / 8)+\frac{8192}{17} \sigma_{7}(n / 16) \\
& +\frac{611}{136} \delta\left(-\frac{1}{3}, \frac{4}{3},-\frac{1}{3} ; n\right)-\frac{142}{17} \delta\left(0, \frac{1}{3}, \frac{1}{3} ; n\right)+\frac{7}{2} \delta\left(-\frac{2}{3}, \frac{11}{6},-\frac{1}{2} ; n\right) \\
& -\frac{483}{17} \delta\left(-\frac{1}{3}, \frac{5}{6}, \frac{1}{6} ; n\right)+\frac{56}{17} \delta\left(0,-\frac{1}{6}, \frac{5}{6} ; n\right)+\frac{953}{272} \delta\left(-\frac{5}{6}, \frac{25}{12},-\frac{7}{12} ; n\right) \\
& -\frac{3405}{68} \delta\left(-\frac{1}{2}, \frac{13}{12}, \frac{1}{12} ; n\right)+\frac{547}{17} \delta\left(-\frac{1}{6}, \frac{1}{12}, \frac{3}{4} ; n\right)+\frac{1223}{272} \delta\left(-\frac{1}{2}, \frac{19}{12},-\frac{5}{12} ; n\right) \\
& -\frac{1221}{68} \delta\left(-\frac{1}{6}, \frac{7}{12}, \frac{1}{4} ; n\right)+\frac{1}{17} \delta\left(\frac{1}{6},-\frac{5}{12}, \frac{11}{12} ; n\right),
\end{aligned}
$$

$N([9,0,7] ; n)$

$$
\begin{aligned}
= & \left(\frac{1}{68}+\frac{1}{1088}\left(\frac{-4}{n}\right)\right) \sigma_{7}(n)-\frac{9}{68} \sigma_{7}(n / 2)+\frac{514}{17} \sigma_{7}(n / 4)-32 \sigma_{7}(n / 8) \\
& +\frac{8192}{17} \sigma_{7}(n / 16)
\end{aligned}
$$




$$
\begin{aligned}
& +\frac{627}{136} \delta\left(-\frac{1}{3}, \frac{4}{3},-\frac{1}{3} ; n\right)-\frac{140}{17} \delta\left(0, \frac{1}{3}, \frac{1}{3} ; n\right)+\frac{35}{8} \delta\left(-\frac{2}{3}, \frac{11}{6},-\frac{1}{2} ; n\right) \\
& -\frac{483}{17} \delta\left(-\frac{1}{3}, \frac{5}{6}, \frac{1}{6} ; n\right)+\frac{56}{17} \delta\left(0,-\frac{1}{6}, \frac{5}{6} ; n\right)+\frac{7623}{2176} \delta\left(-\frac{5}{6}, \frac{25}{12},-\frac{7}{12} ; n\right) \\
& -\frac{23835}{544} \delta\left(-\frac{1}{2}, \frac{13}{12}, \frac{1}{12} ; n\right)+\frac{3829}{136} \delta\left(-\frac{1}{6}, \frac{1}{12}, \frac{3}{4} ; n\right)+\frac{11959}{2176} \delta\left(-\frac{1}{2}, \frac{19}{12},-\frac{5}{12} ; n\right) \\
& -\frac{10989}{544} \delta\left(-\frac{1}{6}, \frac{7}{12}, \frac{1}{4} ; n\right)+\frac{9}{136} \delta\left(\frac{1}{6},-\frac{5}{12}, \frac{11}{12} ; n\right),
\end{aligned}
$$

$N([10,0,6] ; n)$

$$
\begin{aligned}
= & \left(\frac{1}{34}+\frac{1}{544}\left(\frac{-4}{n}\right)\right) \sigma_{7}(n)-\frac{1}{34} \sigma_{7}(n / 2)-\frac{32}{17} \sigma_{7}(n / 8)+\frac{8192}{17} \sigma_{7}(n / 16) \\
& +\frac{287}{68} \delta\left(-\frac{1}{3}, \frac{4}{3},-\frac{1}{3} ; n\right)-\frac{120}{17} \delta\left(0, \frac{1}{3}, \frac{1}{3} ; n\right)+\frac{23}{4} \delta\left(-\frac{2}{3}, \frac{11}{6},-\frac{1}{2} ; n\right) \\
& -\frac{552}{17} \delta\left(-\frac{1}{3}, \frac{5}{6}, \frac{1}{6} ; n\right)+\frac{64}{17} \delta\left(0,-\frac{1}{6}, \frac{5}{6} ; n\right)+\frac{4083}{1088} \delta\left(-\frac{5}{6}, \frac{25}{12},-\frac{7}{12} ; n\right) \\
& -\frac{10215}{272} \delta\left(-\frac{1}{2}, \frac{13}{12}, \frac{1}{12} ; n\right)+\frac{1641}{68} \delta\left(-\frac{1}{6}, \frac{1}{12}, \frac{3}{4} ; n\right)+\frac{6795}{1088} \delta\left(-\frac{1}{2}, \frac{19}{12},-\frac{5}{12} ; n\right) \\
& -\frac{6105}{272} \delta\left(-\frac{1}{6}, \frac{7}{12}, \frac{1}{4} ; n\right)+\frac{5}{68} \delta\left(\frac{1}{6},-\frac{5}{12}, \frac{11}{12} ; n\right),
\end{aligned}
$$

$N([11,0,5] ; n)$

$$
\begin{aligned}
= & \left(\frac{1}{17}+\frac{1}{544}\left(\frac{-4}{n}\right)\right) \sigma_{7}(n)+\frac{3}{17} \sigma_{7}(n / 2)-\frac{1028}{17} \sigma_{7}(n / 4)+\frac{992}{17} \sigma_{7}(n / 8) \\
& +\frac{8192}{17} \sigma_{7}(n / 16) \\
& +\frac{117}{34} \delta\left(-\frac{1}{3}, \frac{4}{3},-\frac{1}{3} ; n\right)-\frac{80}{17} \delta\left(0, \frac{1}{3}, \frac{1}{3} ; n\right)+\frac{15}{2} \delta\left(-\frac{2}{3}, \frac{11}{6},-\frac{1}{2} ; n\right) \\
& -\frac{690}{17} \delta\left(-\frac{1}{3}, \frac{5}{6}, \frac{1}{6} ; n\right)+\frac{80}{17} \delta\left(0,-\frac{1}{6}, \frac{5}{6} ; n\right)+\frac{5171}{1088} \delta\left(-\frac{5}{6}, \frac{25}{12},-\frac{7}{12} ; n\right) \\
& -\frac{10215}{272} \delta\left(-\frac{1}{2}, \frac{13}{12}, \frac{1}{12} ; n\right)+\frac{1641}{68} \delta\left(-\frac{1}{6}, \frac{1}{12}, \frac{3}{4} ; n\right)+\frac{6795}{1088} \delta\left(-\frac{1}{2}, \frac{19}{12},-\frac{5}{12} ; n\right) \\
& -\frac{6105}{272} \delta\left(-\frac{1}{6}, \frac{7}{12}, \frac{1}{4} ; n\right)+\frac{5}{68} \delta\left(\frac{1}{6},-\frac{5}{12}, \frac{11}{12} ; n\right),
\end{aligned}
$$

$N([12,0,4] ; n)$

$$
\begin{aligned}
= & \frac{2}{17} \sigma_{7}(n)+\frac{6}{17} \sigma_{7}(n / 2)-\frac{2056}{17} \sigma_{7}(n / 4)+\frac{2016}{17} \sigma_{7}(n / 8)+\frac{8192}{17} \sigma_{7}(n / 16) \\
& +\frac{49}{17} \delta\left(-\frac{1}{3}, \frac{4}{3},-\frac{1}{3} ; n\right)-\frac{32}{17} \delta\left(0, \frac{1}{3}, \frac{1}{3} ; n\right)+9 \delta\left(-\frac{2}{3}, \frac{11}{6},-\frac{1}{2} ; n\right)
\end{aligned}
$$




$$
\begin{aligned}
& -\frac{828}{17} \delta\left(-\frac{1}{3}, \frac{5}{6}, \frac{1}{6} ; n\right)+\frac{96}{17} \delta\left(0,-\frac{1}{6}, \frac{5}{6} ; n\right)+\frac{1905}{272} \delta\left(-\frac{5}{6}, \frac{25}{12},-\frac{7}{12} ; n\right) \\
& -\frac{3405}{68} \delta\left(-\frac{1}{2}, \frac{13}{12}, \frac{1}{12} ; n\right)+\frac{547}{17} \delta\left(-\frac{1}{6}, \frac{1}{12}, \frac{3}{4} ; n\right)+\frac{1359}{272} \delta\left(-\frac{1}{2}, \frac{19}{12},-\frac{5}{12} ; n\right) \\
& -\frac{1221}{68} \delta\left(-\frac{1}{6}, \frac{7}{12}, \frac{1}{4} ; n\right)+\frac{1}{17} \delta\left(\frac{1}{6},-\frac{5}{12}, \frac{11}{12} ; n\right),
\end{aligned}
$$

$N([13,0,3] ; n)$

$$
\begin{aligned}
= & \left(\frac{4}{17}-\frac{1}{272}\left(\frac{-4}{n}\right)\right) \sigma_{7}(n)+\frac{4}{17} \sigma_{7}(n / 2)-\frac{2056}{17} \sigma_{7}(n / 4)+\frac{2016}{17} \sigma_{7}(n / 8) \\
& +\frac{8192}{17} \sigma_{7}(n / 16) \\
& +\frac{64}{17} \delta\left(-\frac{1}{3}, \frac{4}{3},-\frac{1}{3} ; n\right)+9 \delta\left(-\frac{2}{3}, \frac{11}{6},-\frac{1}{2} ; n\right)-\frac{828}{17} \delta\left(-\frac{1}{3}, \frac{5}{6}, \frac{1}{6} ; n\right) \\
& +\frac{96}{17} \delta\left(0,-\frac{1}{6}, \frac{5}{6} ; n\right)+\frac{5715}{544} \delta\left(-\frac{5}{6}, \frac{25}{12},-\frac{7}{12} ; n\right)-\frac{10215}{136} \delta\left(-\frac{1}{2}, \frac{13}{12}, \frac{1}{12} ; n\right) \\
& +\frac{1641}{34} \delta\left(-\frac{1}{6}, \frac{1}{12}, \frac{3}{4} ; n\right)+\frac{1359}{544} \delta\left(-\frac{1}{2}, \frac{19}{12},-\frac{5}{12} ; n\right)-\frac{1221}{136} \delta\left(-\frac{1}{6}, \frac{7}{12}, \frac{1}{4} ; n\right) \\
& +\frac{1}{34} \delta\left(\frac{1}{6},-\frac{5}{12}, \frac{11}{12} ; n\right),
\end{aligned}
$$

$N([14,0,2] ; n)$

$$
\begin{aligned}
= & \left(\frac{8}{17}-\frac{1}{136}\left(\frac{-4}{n}\right)\right) \sigma_{7}(n)-\frac{8}{17} \sigma_{7}(n / 2)-\frac{32}{17} \sigma_{7}(n / 8)+\frac{8192}{17} \sigma_{7}(n / 16) \\
& +\frac{128}{17} \delta\left(-\frac{1}{3}, \frac{4}{3},-\frac{1}{3} ; n\right)+6 \delta\left(-\frac{2}{3}, \frac{11}{6},-\frac{1}{2} ; n\right)-\frac{552}{17} \delta\left(-\frac{1}{3}, \frac{5}{6}, \frac{1}{6} ; n\right) \\
& +\frac{64}{17} \delta\left(0,-\frac{1}{6}, \frac{5}{6} ; n\right)+\frac{1905}{136} \delta\left(-\frac{5}{6}, \frac{25}{12},-\frac{7}{12} ; n\right)-\frac{3405}{34} \delta\left(-\frac{1}{2}, \frac{13}{12}, \frac{1}{12} ; n\right) \\
& +\frac{1094}{17} \delta\left(-\frac{1}{6}, \frac{1}{12}, \frac{3}{4} ; n\right),
\end{aligned}
$$

$N([15,0,1] ; n)$

$$
\begin{aligned}
= & \left(\frac{16}{17}-\frac{1}{136}\left(\frac{-4}{n}\right)\right) \sigma_{7}(n)-\frac{32}{17} \sigma_{7}(n / 2)+\frac{4112}{17} \sigma_{7}(n / 4)-\frac{4128}{17} \sigma_{7}(n / 8) \\
& +\frac{8192}{17} \sigma_{7}(n / 16) \\
& +\frac{256}{17} \delta\left(-\frac{1}{3}, \frac{4}{3},-\frac{1}{3} ; n\right)+\frac{1905}{136} \delta\left(-\frac{5}{6}, \frac{25}{3},-\frac{7}{12} ; n\right)-\frac{3405}{34} \delta\left(-\frac{1}{2}, \frac{13}{12}, \frac{1}{12} ; n\right) \\
& +\frac{1094}{17} \delta\left(-\frac{1}{6}, \frac{1}{12}, \frac{3}{4} ; n\right) .
\end{aligned}
$$


Proof. We only prove the formula for $N([13,0,3] ; n)$ as the proofs for the other cases are similar. By Theorems 7.3.3 and 8.5.5, we have

$$
\begin{aligned}
& N([13,0,3] ; n) \\
& =\frac{4}{17} \sigma_{7}(n)+\frac{4}{17} \sigma_{7}(n / 2)-\frac{2056}{17} \sigma_{7}(n / 4)+\frac{2016}{17} \sigma_{7}(n / 8)+\frac{8192}{17} \sigma_{7}(n / 16) \\
& +\frac{64}{17} \delta\left(-\frac{1}{3}, \frac{4}{3},-\frac{1}{3} ; n\right)+9 \delta\left(-\frac{2}{3}, \frac{11}{6},-\frac{1}{2} ; n\right)-\frac{828}{17} \delta\left(-\frac{1}{3}, \frac{5}{6}, \frac{1}{6} ; n\right) \\
& +\frac{96}{17} \delta\left(0,-\frac{1}{6}, \frac{5}{6} ; n\right)+\frac{1}{8} \delta\left(-\frac{7}{6}, \frac{37}{12},-\frac{5}{4} ; n\right)+\frac{1905}{272} \delta\left(-\frac{5}{6}, \frac{25}{12},-\frac{7}{12} ; n\right) \\
& -\frac{3405}{68} \delta\left(-\frac{1}{2}, \frac{13}{12}, \frac{1}{12} ; n\right)+\frac{547}{17} \delta\left(-\frac{1}{6}, \frac{1}{12}, \frac{3}{4} ; n\right)-\frac{1}{8} \delta\left(-\frac{5}{6}, \frac{31}{12},-\frac{13}{12} ; n\right) \\
& +\frac{1359}{272} \delta\left(-\frac{1}{2}, \frac{19}{12},-\frac{5}{12} ; n\right)-\frac{1221}{68} \delta\left(-\frac{1}{6}, \frac{7}{12}, \frac{1}{4} ; n\right)+\frac{1}{17} \delta\left(\frac{1}{6},-\frac{5}{12}, \frac{11}{12} ; n\right) \text {. } \\
& =\frac{4}{17} \sigma_{7}(n)+\frac{4}{17} \sigma_{7}(n / 2)-\frac{2056}{17} \sigma_{7}(n / 4)+\frac{2016}{17} \sigma_{7}(n / 8)+\frac{8192}{17} \sigma_{7}(n / 16) \\
& +\frac{64}{17} \delta\left(-\frac{1}{3}, \frac{4}{3},-\frac{1}{3} ; n\right)+9 \delta\left(-\frac{2}{3}, \frac{11}{6},-\frac{1}{2} ; n\right)-\frac{828}{17} \delta\left(-\frac{1}{3}, \frac{5}{6}, \frac{1}{6} ; n\right) \\
& +\frac{96}{17} \delta\left(0,-\frac{1}{6}, \frac{5}{6} ; n\right) \\
& -\frac{1}{272}\left(-34 \delta\left(-\frac{7}{6}, \frac{37}{12},-\frac{5}{4} ; n\right)+\frac{1905}{2} \delta\left(-\frac{5}{6}, \frac{25}{12},-\frac{7}{12} ; n\right)\right. \\
& -6810 \delta\left(-\frac{1}{2}, \frac{13}{12}, \frac{1}{12} ; n\right)+4376 \delta\left(-\frac{1}{6}, \frac{1}{12}, \frac{3}{4} ; n\right)+34 \delta\left(-\frac{5}{6}, \frac{31}{12},-\frac{13}{12} ; n\right) \\
& \left.-\frac{1359}{2} \delta\left(-\frac{1}{2}, \frac{19}{12},-\frac{5}{12} ; n\right)+2442 \delta\left(-\frac{1}{6}, \frac{7}{12}, \frac{1}{4} ; n\right)-8 \delta\left(\frac{1}{6},-\frac{5}{12}, \frac{11}{12} ; n\right)\right) \\
& +\frac{5715}{544} \delta\left(-\frac{5}{6}, \frac{25}{12},-\frac{7}{12} ; n\right)-\frac{10215}{136} \delta\left(-\frac{1}{2}, \frac{13}{12}, \frac{1}{12} ; n\right)+\frac{1641}{34} \delta\left(-\frac{1}{6}, \frac{1}{12}, \frac{3}{4} ; n\right) \\
& +\frac{1359}{272} \delta\left(-\frac{1}{2}, \frac{19}{12},-\frac{5}{12} ; n\right)-\frac{1221}{68} \delta\left(-\frac{1}{6}, \frac{7}{12}, \frac{1}{4} ; n\right)+\frac{1}{34} \delta\left(\frac{1}{6},-\frac{5}{12}, \frac{11}{12} ; n\right) \\
& =\left(\frac{4}{17}-\frac{1}{272}\left(\frac{-4}{n}\right)\right) \sigma_{7}(n)+\frac{4}{17} \sigma_{7}(n / 2)-\frac{2056}{17} \sigma_{7}(n / 4)+\frac{2016}{17} \sigma_{7}(n / 8)
\end{aligned}
$$




$$
\begin{aligned}
& +\frac{8192}{17} \sigma_{7}(n / 16) \\
& +\frac{64}{17} \delta\left(-\frac{1}{3}, \frac{4}{3},-\frac{1}{3} ; n\right)+9 \delta\left(-\frac{2}{3}, \frac{11}{6},-\frac{1}{2} ; n\right)-\frac{828}{17} \delta\left(-\frac{1}{3}, \frac{5}{6}, \frac{1}{6} ; n\right) \\
& +\frac{96}{17} \delta\left(0,-\frac{1}{6}, \frac{5}{6} ; n\right) \\
& +\frac{5715}{544} \delta\left(-\frac{5}{6}, \frac{25}{12},-\frac{7}{12} ; n\right)-\frac{10215}{136} \delta\left(-\frac{1}{2}, \frac{13}{12}, \frac{1}{12} ; n\right)+\frac{1641}{34} \delta\left(-\frac{1}{6}, \frac{1}{12}, \frac{3}{4} ; n\right) \\
& +\frac{1359}{272} \delta\left(-\frac{1}{2}, \frac{19}{12},-\frac{5}{12} ; n\right)-\frac{1221}{68} \delta\left(-\frac{1}{6}, \frac{7}{12}, \frac{1}{4} ; n\right)+\frac{1}{34} \delta\left(\frac{1}{6},-\frac{5}{12}, \frac{11}{12} ; n\right),
\end{aligned}
$$

which is the desired result.

In the last two theorems of this section, we simplify Theorem 9.3.4 when $n \equiv 0(\bmod 8)$ and $n \equiv 4(\bmod 8)$.

Theorem 9.3.5. Let $n \in \mathbb{N}$. For $n \equiv 0(\bmod 8)$, we have

$$
\begin{aligned}
& N([1,0,15] ; n)=\frac{4064}{17} \sigma_{7}(n / 8)+\frac{4096}{17} \sigma_{7}(n / 16)+\frac{8}{17} \delta\left(-\frac{1}{3}, \frac{4}{3},-\frac{1}{3} ; n\right), \\
& N([2,0,14] ; n)=\frac{4064}{17} \sigma_{7}(n / 8)+\frac{4096}{17} \sigma_{7}(n / 16)+\frac{8}{17} \delta\left(-\frac{1}{3}, \frac{4}{3},-\frac{1}{3} ; n\right), \\
& N([3,0,13] ; n)=\frac{4064}{17} \sigma_{7}(n / 8)+\frac{4096}{17} \sigma_{7}(n / 16)+\frac{8}{17} \delta\left(-\frac{1}{3}, \frac{4}{3},-\frac{1}{3} ; n\right), \\
& N([4,0,12] ; n)=480 \sigma_{7}(n / 8)+\frac{3}{4} \delta\left(-\frac{1}{3}, \frac{4}{3},-\frac{1}{3} ; n\right) \\
& N([5,0,11] ; n)=\frac{24544}{17} \sigma_{7}(n / 8)-\frac{16384}{17} \sigma_{7}(n / 16)+\frac{127}{68} \delta\left(-\frac{1}{3}, \frac{4}{3},-\frac{1}{3} ; n\right), \\
& N([6,0,10] ; n)=\frac{65504}{17} \sigma_{7}(n / 8)-\frac{57344}{17} \sigma_{7}(n / 16)+\frac{317}{68} \delta\left(-\frac{1}{3}, \frac{4}{3},-\frac{1}{3} ; n\right), \\
& N([7,0,9] ; n)=8672 \sigma_{7}(n / 8)-8192 \sigma_{7}(n / 16)+\frac{41}{4} \delta\left(-\frac{1}{3}, \frac{4}{3},-\frac{1}{3} ; n\right), \\
& N([8,0,8] ; n)=\frac{294880}{17} \sigma_{7}(n / 8)-\frac{286720}{17} \sigma_{7}(n / 16)+\frac{341}{17} \delta\left(-\frac{1}{3}, \frac{4}{3},-\frac{1}{3} ; n\right), \\
& N([9,0,7] ; n)=\frac{557024}{17} \sigma_{7}(n / 8)-\frac{548864}{17} \sigma_{7}(n / 16)+\frac{611}{17} \delta\left(-\frac{1}{3}, \frac{4}{3},-\frac{1}{3} ; n\right), \\
& N([10,0,6] ; n)=\frac{1048544}{17} \sigma_{7}(n / 8)-\frac{1040384}{17} \sigma_{7}(n / 16)+\frac{1028}{17} \delta\left(-\frac{1}{3}, \frac{4}{3},-\frac{1}{3} ; n\right),
\end{aligned}
$$




$$
\begin{aligned}
& N([11,0,5] ; n)=\frac{2031584}{17} \sigma_{7}(n / 8)-\frac{2023424}{17} \sigma_{7}(n / 16)+\frac{1658}{17} \delta\left(-\frac{1}{3}, \frac{4}{3},-\frac{1}{3} ; n\right), \\
& N([12,0,4] ; n)=\frac{4063200}{17} \sigma_{7}(n / 8)-\frac{4055040}{17} \sigma_{7}(n / 16)+\frac{10395}{68} \delta\left(-\frac{1}{3}, \frac{4}{3},-\frac{1}{3} ; n\right), \\
& N([13,0,3] ; n)=\frac{8257504}{17} \sigma_{7}(n / 8)-\frac{8249344}{17} \sigma_{7}(n / 16)+\frac{15775}{68} \delta\left(-\frac{1}{3}, \frac{4}{3},-\frac{1}{3} ; n\right), \\
& N([14,0,2] ; n)=\frac{16777184}{17} \sigma_{7}(n / 8)-\frac{16769024}{17} \sigma_{7}(n / 16)+\frac{22145}{68} \delta\left(-\frac{1}{3}, \frac{4}{3},-\frac{1}{3} ; n\right), \\
& N([15,0,1] ; n)=\frac{33816544}{17} \sigma_{7}(n / 8)-\frac{33808384}{17} \sigma_{7}(n / 16)+\frac{24277}{68} \delta\left(-\frac{1}{3}, \frac{4}{3},-\frac{1}{3} ; n\right) .
\end{aligned}
$$

Proof. We only prove the formula for $N([11,0,5] ; n)$ as the proofs for the other cases are similar. By Theorem 9.3.4, Theorems 8.5.1 - 8.5.4, Theorem 8.6.1 and the fact that $\left(\frac{-4}{n}\right)=0$ when $n \equiv 0(\bmod 8)$, we have, for $n \equiv 0(\bmod 8)$

$$
\begin{aligned}
N & ([11,0,5] ; n) \\
= & \left(\frac{1}{17}+\frac{1}{544}\left(\frac{-4}{n}\right)\right) \sigma_{7}(n)+\frac{3}{17} \sigma_{7}(n / 2)-\frac{1028}{17} \sigma_{7}(n / 4)+\frac{992}{17} \sigma_{7}(n / 8) \\
& +\frac{8192}{17} \sigma_{7}(n / 16) \\
& +\frac{117}{34} \delta\left(-\frac{1}{3}, \frac{4}{3},-\frac{1}{3} ; n\right)-\frac{80}{17} \delta\left(0, \frac{1}{3}, \frac{1}{3} ; n\right)+\frac{15}{2} \delta\left(-\frac{2}{3}, \frac{11}{6},-\frac{1}{2} ; n\right) \\
& -\frac{690}{17} \delta\left(-\frac{1}{3}, \frac{5}{6}, \frac{1}{6} ; n\right)+\frac{80}{17} \delta\left(0,-\frac{1}{6}, \frac{5}{6} ; n\right)+\frac{5171}{1088} \delta\left(-\frac{5}{6}, \frac{25}{12},-\frac{7}{12} ; n\right) \\
& -\frac{10215}{272} \delta\left(-\frac{1}{2}, \frac{13}{12}, \frac{1}{12} ; n\right)+\frac{1641}{68} \delta\left(-\frac{1}{6}, \frac{1}{12}, \frac{3}{4} ; n\right)+\frac{6795}{1088} \delta\left(-\frac{1}{2}, \frac{19}{12},-\frac{5}{12} ; n\right) \\
& -\frac{6105}{272} \delta\left(-\frac{1}{6}, \frac{7}{12}, \frac{1}{4} ; n\right)+\frac{5}{68} \delta\left(\frac{1}{6},-\frac{5}{12}, \frac{11}{12} ; n\right) \\
= & \frac{1}{17} \sigma_{7}(n)+\frac{3}{17} \sigma_{7}(n / 2)-\frac{1028}{17} \sigma_{7}(n / 4)+\frac{992}{17} \sigma_{7}(n / 8)+\frac{8192}{17} \sigma_{7}(n / 16) \\
& +\frac{117}{34} \delta\left(-\frac{1}{3}, \frac{4}{3},-\frac{1}{3} ; n\right)-\frac{80}{17}\left(\frac{1}{8} \delta\left(-\frac{1}{3}, \frac{4}{3},-\frac{1}{3} ; n\right)\right) \\
& +\frac{15}{2}\left(-6 \delta\left(-\frac{1}{3}, \frac{4}{3},-\frac{1}{3} ; n\right)\right)-\frac{690}{17}\left(-\frac{3}{8} \delta\left(-\frac{1}{3}, \frac{4}{3},-\frac{1}{3} ; n\right)\right) \\
& +\frac{5171}{1088}\left(45 \delta\left(-\frac{1}{3}, \frac{4}{3},-\frac{1}{3} ; n\right)\right)-\frac{10215}{272}\left(3 \delta\left(-\frac{1}{3}, \frac{4}{3},-\frac{1}{3} ; n\right)\right)
\end{aligned}
$$




$$
\begin{aligned}
& +\frac{1641}{68}\left(\frac{3}{16} \delta\left(-\frac{1}{3}, \frac{4}{3},-\frac{1}{3} ; n\right)\right)+\frac{6795}{1088}\left(3 \delta\left(-\frac{1}{3}, \frac{4}{3},-\frac{1}{3} ; n\right)\right) \\
& +\frac{5}{68}\left(-\frac{3}{16} \delta\left(-\frac{1}{3}, \frac{4}{3},-\frac{1}{3} ; n\right)\right) \\
& =\frac{1}{17} \sigma_{7}(n)+\frac{3}{17} \sigma_{7}(n / 2)-\frac{1028}{17} \sigma_{7}(n / 4)+\frac{992}{17} \sigma_{7}(n / 8)+\frac{8192}{17} \sigma_{7}(n / 16) \\
& +\frac{117}{34} \delta\left(-\frac{1}{3}, \frac{4}{3},-\frac{1}{3} ; n\right) .
\end{aligned}
$$

Simplifying using (9.3.3), we obtain the desired result.

Theorem 9.3.6. Let $n \in \mathbb{N}$. For $n \equiv 4(\bmod 8)$, we have

$$
\begin{aligned}
& N([1,0,15] ; n)=\frac{32}{17} \sigma_{7}(n / 4)-\frac{8}{17} \delta\left(-\frac{1}{3}, \frac{4}{3},-\frac{1}{3} ; n\right) \\
& N([2,0,14] ; n)=\frac{32}{17} \sigma_{7}(n / 4)-\frac{8}{17} \delta\left(-\frac{1}{3}, \frac{4}{3},-\frac{1}{3} ; n\right) \\
& N([3,0,13] ; n)=\frac{32}{17} \sigma_{7}(n / 4)-\frac{8}{17} \delta\left(-\frac{1}{3}, \frac{4}{3},-\frac{1}{3} ; n\right) \\
& N([4,0,12] ; n)=\frac{64}{17} \sigma_{7}(n / 4)-\frac{47}{68} \delta\left(-\frac{1}{3}, \frac{4}{3},-\frac{1}{3} ; n\right) \\
& N([5,0,11] ; n)=\frac{192}{17} \sigma_{7}(n / 4)-\frac{107}{68} \delta\left(-\frac{1}{3}, \frac{4}{3},-\frac{1}{3} ; n\right) \\
& N([6,0,10] ; n)=\frac{512}{17} \sigma_{7}(n / 4)-\frac{257}{68} \delta\left(-\frac{1}{3}, \frac{4}{3},-\frac{1}{3} ; n\right) \\
& N([7,0,9] ; n)=\frac{1152}{17} \sigma_{7}(n / 4)-\frac{557}{68} \delta\left(-\frac{1}{3}, \frac{4}{3},-\frac{1}{3} ; n\right) \\
& N([8,0,8] ; n)=\frac{2304}{17} \sigma_{7}(n / 4)-\frac{270}{17} \delta\left(-\frac{1}{3}, \frac{4}{3},-\frac{1}{3} ; n\right) \\
& N([9,0,7] ; n)=256 \sigma_{7}(n / 4)-28 \delta\left(-\frac{1}{3}, \frac{4}{3},-\frac{1}{3} ; n\right) \\
& N([10,0,6] ; n)=\frac{8192}{17} \sigma_{7}(n / 4)-\frac{773}{17} \delta\left(-\frac{1}{3}, \frac{4}{3},-\frac{1}{3} ; n\right) \\
& N([11,0,5] ; n)=\frac{15872}{17} \sigma_{7}(n / 4)-\frac{1163}{17} \delta\left(-\frac{1}{3}, \frac{4}{3},-\frac{1}{3} ; n\right) \\
& N([12,0,4] ; n)=\frac{31744}{17} \sigma_{7}(n / 4)-\frac{6465}{68} \delta\left(-\frac{1}{3}, \frac{4}{3},-\frac{1}{3} ; n\right)
\end{aligned}
$$




$$
\begin{aligned}
& N([13,0,3] ; n)=\frac{64512}{17} \sigma_{7}(n / 4)-\frac{8157}{68} \delta\left(-\frac{1}{3}, \frac{4}{3},-\frac{1}{3} ; n\right), \\
& N([14,0,2] ; n)=\frac{131072}{17} \sigma_{7}(n / 4)-\frac{8859}{68} \delta\left(-\frac{1}{3}, \frac{4}{3},-\frac{1}{3} ; n\right), \\
& N([15,0,1] ; n)=\frac{264192}{17} \sigma_{7}(n / 4)-\frac{6727}{68} \delta\left(-\frac{1}{3}, \frac{4}{3},-\frac{1}{3} ; n\right),
\end{aligned}
$$

Proof. We only prove the formula for $N([7,0,9] ; n)$ as the proofs for the other cases are similar. By Theorem 9.3.4, Theorems 8.5.1 - 8.5.4, Theorem 8.6.1 and the fact that $\left(\frac{-4}{n}\right)=0$ when $n \equiv 4(\bmod 8)$, we have, for $n \equiv 4(\bmod 8)$

$$
\begin{aligned}
N & ([7,0,9] ; n) \\
= & \left(\frac{1}{272}-\frac{1}{2176}\left(\frac{-4}{n}\right)\right) \sigma_{7}(n)-\frac{1}{16} \sigma_{7}(n / 2)+\frac{257}{17} \sigma_{7}(n / 4)-\frac{288}{17} \sigma_{7}(n / 8) \\
& +\frac{8192}{17} \sigma_{7}(n / 16) \\
& +\frac{1087}{272} \delta\left(-\frac{1}{3}, \frac{4}{3},-\frac{1}{3} ; n\right)-\frac{135}{17} \delta\left(0, \frac{1}{3}, \frac{1}{3} ; n\right)+3 \delta\left(-\frac{2}{3}, \frac{11}{6},-\frac{1}{2} ; n\right) \\
& -\frac{1035}{34} \delta\left(-\frac{1}{3}, \frac{5}{6}, \frac{1}{6} ; n\right)+\frac{60}{17} \delta\left(0,-\frac{1}{6}, \frac{5}{6} ; n\right)+\frac{881}{256} \delta\left(-\frac{5}{6}, \frac{25}{12},-\frac{7}{12} ; n\right) \\
& -\frac{3373}{64} \delta\left(-\frac{1}{2}, \frac{13}{12}, \frac{1}{12} ; n\right)+\frac{547}{16} \delta\left(-\frac{1}{6}, \frac{1}{12}, \frac{3}{4} ; n\right)+\frac{15489}{4352} \delta\left(-\frac{1}{2}, \frac{19}{12},-\frac{5}{12} ; n\right) \\
& -\frac{18315}{1088} \delta\left(-\frac{1}{6}, \frac{7}{12}, \frac{1}{4} ; n\right)+\frac{15}{272} \delta\left(\frac{1}{6},-\frac{5}{12}, \frac{11}{12} ; n\right) \\
= & \frac{1}{272} \sigma_{7}(n)-\frac{1}{16} \sigma_{7}(n / 2)+\frac{257}{17} \sigma_{7}(n / 4) \\
& +\frac{1087}{272} \delta\left(-\frac{1}{3}, \frac{4}{3},-\frac{1}{3} ; n\right)-\frac{135}{17}\left(\frac{1}{8} \delta\left(-\frac{1}{3}, \frac{4}{3},-\frac{1}{3} ; n\right)\right) \\
& +3\left(-6 \delta\left(-\frac{1}{3}, \frac{4}{3},-\frac{1}{3} ; n\right)\right)-\frac{1035}{34}\left(-\frac{3}{8} \delta\left(-\frac{1}{3}, \frac{4}{3},-\frac{1}{3} ; n\right)\right) \\
& +\frac{881}{256}\left(-15 \delta\left(-\frac{1}{3}, \frac{4}{3},-\frac{1}{3} ; n\right)\right)-\frac{3373}{64}\left(-\delta\left(-\frac{1}{3}, \frac{4}{3},-\frac{1}{3} ; n\right)\right) \\
& +\frac{547}{16}\left(-\frac{1}{16} \delta\left(-\frac{1}{3}, \frac{4}{3},-\frac{1}{3} ; n\right)\right)+\frac{15489}{4352}\left(-\delta\left(-\frac{1}{3}, \frac{4}{3},-\frac{1}{3} ; n\right)\right) \\
& +\frac{15}{272}\left(\frac{1}{16} \delta\left(-\frac{1}{3}, \frac{4}{3},-\frac{1}{3} ; n\right)\right)
\end{aligned}
$$




$$
=\frac{1}{272} \sigma_{7}(n)-\frac{1}{16} \sigma_{7}(n / 2)+\frac{257}{17} \sigma_{7}(n / 4)-\frac{557}{68} \delta\left(-\frac{1}{3}, \frac{4}{3},-\frac{1}{3} ; n\right) .
$$

Simplifying using (9.3.2), we obtain the desired result.

We close by remarking that the first three formulae of Theorems 9.3 .5 and 9.3.6 can be derived from the Ramanujan-Mordell formula for $r_{16}(n)$, see the remark following Theorem 9.2.8. 


\section{Conclusion}

The research of this thesis suggests several possibilities for further development. First, the analogous formulae for $N([r, s, t] ; n)(r+s+t=4 k)$ should be determined when $s$ is odd. Perhaps this will involve the sums

$$
\sum_{\substack{d \in \mathbb{N} \\ d \mid n}}\left(\frac{8}{d}\right) d^{2 k-1}, \sum_{\substack{d \in \mathbb{N} \\ d \mid n}}\left(\frac{8}{d}\right)(n / d)^{2 k-1} ?
$$

Secondly the formulae for $N([r, s, t] ; n)(r+s+t=4 k+2)$ should be found. In this case it is possible that the evaluations will require sums of the form

$$
\begin{aligned}
& \sum_{\substack{d \in \mathbb{N} \\
d \mid n}}\left(\frac{-4}{d}\right) d^{2 k}, \sum_{\substack{d \in \mathbb{N} \\
d \mid n}}\left(\frac{-4}{n / d}\right) d^{2 k}, s \text { even, } \\
& \sum_{\substack{d \in \mathbb{N} \\
d \mid n}}\left(\frac{-8}{d}\right) d^{2 k}, \sum_{\substack{d \in \mathbb{N} \\
d \mid n}}\left(\frac{-8}{n / d}\right) d^{2 k}, s \text { odd. }
\end{aligned}
$$

Finally it would be interesting to determine the minimum number of $\delta$-coefficients that are required for the evaluations of $N([r, s, t] ; n)$ including the cases when $n$ is restricted to certain residue classes. 


\section{List of References}

[1] A. Alaca, S.. Alaca, M. F. Lemire and K. S. Williams, Jacobi's identity and representations of integers by certain quaternary quadratic forms, Int. J. Modern Math. 2 (2007), 143-176.

[2] A. Alaca, S. Alaca, M. F. Lemire and K. S. Williams, Nineteen quaternary quadratic forms. Acta Arith. 130 (2007), 277-310.

[3] A. Alaca, S. Alaca, M. F. Lemire and K. S. Williams, The number of representations of a positive integer by certain quaternary quadratic forms, Int. J. Number Theory 5 (2009), 13-40.

[4] A. Alaca, Ş. Alaca and K. S. Williams, The simplest proof of Jacobi's six squares theorem, Far East J. Math. Sci. 27 (2007), 187-192.

[5] A. Alaca, S. Alaca and K. S. Williams, Seven octonary quadratic forms, Acta Arith. 135 (2008), 339-350.

[6] A. Alaca, Ş. Alaca and K. S. Williams, Berndt's curious formula, Int. J. Number Theory 4 (2008), 677-689.

[7] A. Alaca, S. Alaca and K. S. Williams, The convolution sum $\sum_{m<n / 16} \sigma(m) \sigma(n-16 m)$, Canad. Math. Bull. 51 (2008), 3-14.

[8] A. Alaca, Ş. Alaca and K. S. Williams, Liouville's sextenary quadratic forms $x^{2}+y^{2}+z^{2}+t^{2}+2 u^{2}+2 v^{2}, x^{2}+y^{2}+2 z^{2}+2 t^{2}+2 u^{2}+2 v^{2}$ and $x^{2}+2 y^{2}+2 z^{2}+$ $2 t^{2}+2 u^{2}+4 v^{2}$, Far East J. Math. Soc. 30 (2008), 547-556.

[9] A. Alaca, S. Alaca and K. S. Williams, Sums of $4 k$ squares: a polynomial approach, J. Combinatorics and Number Theory 1 (2) (2009), 33-52.

[10] A. Alaca, Ş. Alaca and K. S. Williams, Some identities involving theta functions, J. Number Theory 129 (2009), 1404-1431. 
[11] A. Alaca, Ş. Alaca and K. S. Williams, Fourteen octonary quadratic forms, Int. J. Number Theory 6 (2010), 37-50.

[12] K. Ananda Rau, On the representation of a number as a sum of an even number of squares, J. Madras Univ. Part B 24 (1954), 61-89.

[13] N. C. Ankeny, Sums of 3 squares, Proc. Amer. Math. Soc. 8 (1957), 316-319.

[14] T. M. Apostol, Modular Functions and Dirichlet Series in Number Theory, Springer Verlag, New York 1976.

[15] P. T. Bateman, On the representation of a number as a sum of three squares, Trans. Amer. Math. Soc. 71 (1951), 70-101.

[16] E. T. Bell, On the number of representations of $2 n$ as a sum of $2 r$ squares, Bull. Amer. Math. Soc. 26 (1919), 19-25.

[17] E. Benz, Über die Anzahl Darstellungen einer Zahlen durch gewisse quaternäre quadratische Formen: Beweise, welche auf Identiäten aus dem Gebiete der Thetafunktionen basierren. Dissertation, Zürich 1964, in "Studien zur Theorie der quadratischen Formen", editors B. L. van der Waerden und H. Gross, Stuttgart, 1968, pp. 165-198.

[18] B. C. Berndt, Ramanujan's Notebooks, Part I (1985), Part II (1989), Part III (1991), Part IV (1994), Part V (1998), Springer-Verlag, New York.

[19] B. C. Berndt, Number Theory in the Spirit of Ramanujan, Amer Math. Soc., Providence, Rhode Island, USA, 2006.

[20] J. M. Borwein, P. B. Borwein and F. G. Garvan, Some cubic modular identities of Ramanujan, Trans. Amer. Math. Soc. 343 (1994), 35-47.

[21] V. Bulygin (V. Boulyguine), Sur une application des fonction elliptiques au problème de représentation des nombres entiers par une somme de carrés, Bull. Acad. Imp. Sci. St. Petersbourg 6 (1914), 389-404.

[22] L. Carlitz, On the representations of an integer as the sum of 24 squares, Nederl. Akad. Wetensch. Indag. Math 17 (1955), 504-506.

[23] L. Carlitz, Note on sums of 4 and 6 squares, Proc. Amer. Math. Soc. 8 (1957), 120-124.

[24] L. Carlitz, Bulygin's method for sums of squares, J. Number Theory 5 (1973), 405-412. 
[25] H. H. Chan, S. Cooper and W.-C. Liaw, On $\eta^{3}(a \tau) \eta^{3}(b \tau)$ with $a+b=8$, J. Austral. Math. Soc. 84 (2008), 301-313.

[26] H. H. Chan and K. S. Chua, Representations of integers as sums of 32 squares, Ramanujan J. 7 (2003), 79-89.

[27] N. Cheng, Convolution sums involving divisor functions, M.Sc. thesis, Carleton University, Ottawa, Canada, 2003.

[28] N. Cheng and K. S. Williams, Evaluation of some convolution sums involving the sum of divisors functions, Yokohama Math. J. 52 (2005), 39-57.

[29] J. B. Conway, Functions of One Complex Variable, Second Edition, SpringerVerlag, Providence, New York, USA, 1978.

[30] E. T. Copson, An Introduction to the Theory of Functions of a Complex Variable, Oxford University Press, 1946.

[31] S. Cooper, On sums of an even number of squares, and an even number of triangular numbers: an elementary approach based on Ramanujan's ${ }_{1} \psi_{1}$ summation formula, in $q$-series with Applications to Combinatorics, Number Theory, and Physics, editors B. C. Berndt and K. Ono, Contemporary Mathematics 291 (2001) Amer. Math. Soc., Providence, RI, 2001, pp. 115-137.

[32] S. Cooper and H. Y. Lam, Sums of two, four, six and eight squares and triangular numbers: an elementary approach, Indian J. Math. 44 (2002), 21-40.

[33] P. Deligne, Formes modulaires et représentations l-adiques, Lecture Notes in Mathematics, No. 179, Berlin: Springer-Verlag, (1971), 139-172.

[34] P. Deligne, La conjecture de Weil I, Publ. Math. IHES, 43 (1974), 273-307.

[35] P. Demuth, Die Zahl der Darstellungen einer natürlichen Zahl durch spezielle quaternäre quadratishe Formen aufgrund der Siegelschen Massformel, in "Studien zur Theorie der quadratischen Formen", editors B. L. van der Waerden und H. Gross, Stuttgart, 1968, pp. 224-254.

[36] L. E. Dickson, History of the Theory of Numbers, Vols. I-III, Chelsea, 1966.

[37] Th. Estermann, On the representations of a number as a sum of 2 squares, Acta. Arith. 2 (1936), 47-79.

[38] Th. Estermann, On the representations of a number as a sum of 3 squares, Proc. London Math. Soc. (3) 9 (1959), 575-594. 
[39] Th. Estermann, On the representations of a number as a sum of squares, Acta Arith. 2 (1936), 47-79.

[40] J. A. Ewell, A simple derivation of Jacobi's four-square formula, Proc. Amer. Math. Soc. 85 (1982), 323-326.

[41] J. A. Ewell, On sums of sixteen squares, Rocky Mountain J. Math. 17 (1987), 295-299.

[42] J. W. L. Glaisher, On the numbers of representations of a number as a sum of $2 r$ squares, where $2 r$ does not exceed eighteen, Proc. London Math. Soc. (2) 5 (1907), 479-490.

[43] J. W. L. Glaisher, On the representations of a number as a sum two, four, six, eight, ten and twelve squares, Quart. J. Pure Appl. Math. Oxford 38 (1907), $1-62$.

[44] J. W. L. Glaisher, On the representations of a number as a sum of fourteen and sixteen squares, Quart. J. Pure Appl. Math. Oxford 38 (1907), 178-236.

[45] J. W. L. Glaisher, On the representations of a number as a sum of eighteen squares, Quart. J. Pure Appl. Math. Oxford 38 (1907), 289-351.

[46] E. Grosswald, Representation of Integers as Sums of Squares, Springer-Verlag, New York, 1985.

[47] K.-B. Gundlach, On the representation of a number as a sum of squares, Glasgow Math. J. 19 (1978), 173-197.

[48] G. H. Hardy, On the representation of a number as a sum of any number of squares and in particular of five and seven, Proc. Nat. Acad. Sci., U.S.A. 4 (1918), 189-193.

[49] G. H. Hardy, On the representation of a number as a sum of any number of squares and in particular of five, Trans. Amer. Math. Soc. 21 (1920), 255-284.

[50] M. D. Hirschhorn, A simple proof of Jacobi's four-square theorem, J. Austral. Math. Soc. Ser. A 32 (1982), 61-67.

[51] J. G. Huard and K. S. Williams, Sums of twelve squares, Acta Arith. 109 (2003), 195-204.

[52] J. G. Huard and K. S. Williams, Sums of sixteen squares, Far East J. Math. Sci. 7 (2002), 147-164. 
[53] C. G. J. Jacobi, Fundamenta nova theoriae functionum ellipticarum, 1829, in Gesammelte Werke (Erster Band), Chelsea Publishing Co., New York, 1969, pp. 49-239.

[54] L. J. P. Kilford, Modular Forms, Imperial College Press, 2008.

[55] F. Klein and R. Fricke, Vorlesungen über die Theorie der elliptischen Modulfunktionen, Vol. I (1890), Vol. II (1892), Teubner, Leipzig.

[56] M. I. Knopp, Modular Functions in Analytic Number Theory, Chelsea Publishing Co., New York, 1993.

[57] J. Liouville, Extrait d'une lettre adressée à M. Besge, J. Math. Pures Appl. 9 (1864), 296-298.

[58] G. A. Lomadze, Representation of numbers by sums of the quadratic forms $x_{1}^{2}+$ $x_{1} x_{2}+x_{2}^{2}$, Acta Arith. 54 (1989), 9-36. (in Russian)

[59] Y. Martin, Multiplicative $\eta$-quotients, Trans. Amer. Math. Soc. 348 (1996), 48254856.

[60] E. McAfee and K. S. Williams, Sums of six squares, Far East J. Math. Sci. 16 (2005), 17-41.

[61] S. C. Milne, Infinite families of exact sums of squares formulas, Jacobi elliptic functions, continued fractions, and Schur functions, The Ramanujan Journal, 6 (2002), no. 1, 1-151.

[62] L. J. Mordell, On Mr. Ramanujan's empirical expansions of modular functions, Proc. Cambridge Philos. Soc. 19 (1917), 117-124.

[63] L. J. Mordell, On the representations of numbers as a sum of $2 r$ squares, Quart. J. Pure Appl. Math. 48 (1917), 93-104.

[64] L. J. Mordell, On the representations of a number as a sum of 3 squares, Rev. Math. Pures Appl. 3 (1958), 25-27.

[65] L. J. Mordell, On the representations of a number as a sum of an odd number of squares, Trans. Cambridge Philos. Soc. 22 (1919), 361-372.

[66] C. J. Moreno and S. S. Wagstaff, Sums of Squares of Integers, Chapman and Hall, Boca Raton, Florida, 2006. 
[67] K. Ono, Representations of integers as sums of squares, J. Number Theory 95 (2002), 253-258.

[68] T. Pepin, Étude sur quelques formules d'analyse utiles dans la thèorie des nombres, Atti Accad. Pont. Nuovi Lincei 38 (1884-5), 139-196.

[69] T. Pepin, Sur quelques quadratiques quaternaires, J. Math. Pures Appl. 6 (1890), 5-67.

[70] H. Petersson, Modulfunktionen und Quadraische Formen, Springer-Verlag, Berlin, 1982.

[71] S. Ramanujan, On certain arithmetical functions, Trans. Cambridge Philos. Soc. 22 (1916), 159-184.

[72] S. Ramanujan, Collected Papers, Cambridge University Press, Cambridge, 1927; reprinted by Chelsea, New York, 1962; reprinted by the American Mathematical Society, Providence, RI, 2000.

[73] S. Ramanujan, Notebooks, 2 vols, Tata Institute of Fundamental Research, Bombay, 1957.

[74] R. A. Rankin, On the representations of a number as a sum of squares and certain related identities, Proc. Cambridge Philos. Soc. 41 (1945), 1-11.

[75] R. A. Rankin, Representations of a number as the sum of a large number of squares, Proc. Roy. Soc. Edinburgh Sect. A 65 (1960/61), 318-331.

[76] R. A. Rankin, On the representation of a number as the sum of any number of squares and in particular of 20, Acta Arith. 7 (1961/62), 399-407.

[77] R. A. Rankin, Sums of squares and cusp forms, Amer. J. Math. 87 (1965), $857-860$.

[78] R. A. Rankin, Modular Forms and Functions, Cambridge University Press, Cambridge, 1977.

[79] B. K. Spearman and K. S. Williams, The simplest arithmetic proof of Jacobi's four squares theorem, Far East J. Math. Sci. 2 (2000), 433-439.

[80] B. van der Pol, The representation of numbers as sums of 8,16 and 24 squares, Indag. Math. 16 (1954), 349-361. 
[81] H. Wild, Die Anzahl der Darstellungen einer natürlichen Zahl durch die Form $x^{2}+y^{2}+z^{2}+2 t^{2}$, Abh. Math. Sem. Univ. Hamburg 40 (1974), 132-135.

[82] K. S. Williams, An arithmetic proof of Jacobi's eight squares theorem, Far East J. Math. Sci. 3 (2001), 1001-1005.

[83] K. S. Williams, The convolution sum $\sum_{m<n / 8} \sigma(m) \sigma(n-8 m)$, Pacific J. Math. 228 (2006), 387-396.

[84] K. S. Williams, On the representations of a positive integer by the forms $x^{2}+$ $y^{2}+z^{2}+2 t^{2}$ and $x^{2}+2 y^{2}+2 z^{2}+2 t^{2}$, Int. J. Modern Math. 3 (2008), 225-230.

[85] K. S. Williams, On Liouville's twelve squares theorem, Far East J. Math. Sci. 29 (2008), 239-242. 